WHOI-89-42

\title{
CTD Observations Off Northern California During the Shelf Mixed Layer Experiment, SMILE, May 1989
}

by

Richard Limeburner and Robert C. Beardsley

Woods Hole Oceanographic Institution

Woods Hole, Massachusetts 02543

September 1989

\section{Technical Report}

Funding was provided by the National Science Foundation through Grant Number OCE 87-16937

Reproduction in whole or in part is permitted for any purpose of the United States Government. This report should be cited as:

Woods Hole Oceanog. Inst. Tech. Rept., WHOI-89-42.

Approved for publication; distribution unlimited.

Approved for Distribution:

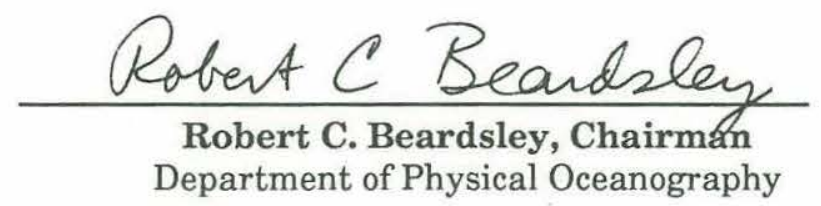




\title{
CTD OBSERVATIONS OFF NORTHERN CALIFORNIA \\ DURING THE SHELF MIXED LAYER EXPERIMENT, SMILE, MAY 1989
}

\begin{abstract}
CTD observations were made off the northern California coast during $\mathrm{R} / \mathrm{V} W e$ coma cruise W8905 May 5-14, 1989 as part of the Shelf MIxed Layer Experiment (SMILE). The surveys consisted of two sampling plans - a large-scale grid of four crossshelf transects extending to both sides of Point Arena and Point Reyes, and a small-scale grid of six cross-shelf transects located near the central SMILE mooring site. All of the cross-shelf transects extended beyond the shelf break and the maximum sampling depth at each station was near-bottom or $1500 \mathrm{~m}$. The average along-shelf separation between cross-shelf transects was about $15 \mathrm{~km}$ for the small-scale surveys and $50 \mathrm{~km}$ for the large-scale grid.

The primary objectives of the hydrographic measurement program were to observe and characterize the temperature, salinity, density, and light transmission fields and their temporal and spatial variability in the surface boundary layer along the continental shelf and slope near the SMILE moored array, and to acquire estimates of the cross- and alongshelf scales over which the mixed-layer depth varies. This report presents a summary in graphic and tabular form of the hydrographic observations made during cruise W8905 on the $\mathrm{R} / \mathrm{V}$ Wecoma.
\end{abstract}




\section{Table of Contents}

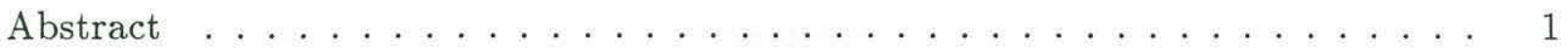

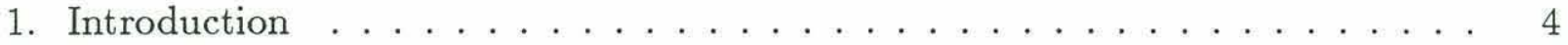

2. Instrumentation and Calibration ............... 5

3. Data Processing . . . . . . . . . . . . . . . . . 6

4. Data Presentation . . . . . . . . . . . . . . . . . 6

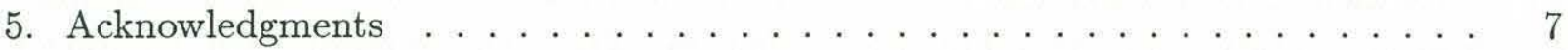

6. References........................... 7

7. Graphic Description of CTD Data . . . . . . . . . . . . . 9

A. Large-Scale Survey - May 5-8, 1989 - Stations 1 to $44 \ldots 9$

1. Station Locations ................... . . 10

2. Section Locations . . . . . . . . . . . . . . . . . 11

3. $\mathrm{T} / \mathrm{S}$ and Vertical Sections ..................... 12

4. Surface Horizontal Sections . . . . . . . . . . . . . 20

B. Small-Scale Survey - May 8-9, 1989 - Stations 47 to 86 . . . . . . . 25

1. Station Locations .................... 27

2. Section Locations . . . . . . . . . . . . . . . 27

3. $\mathrm{T} / \mathrm{S}$ and Vertical Sections ...................... 28

4. Surface Horizontal Sections . . . . . . . . . . . . . . 40

C. Section C - May 10, 1989 - Stations 94 to $100 \ldots . \ldots 45$

1. Station Locations . . . . . . . . . . . . . . . . . 47

2. Section Locations . . . . . . . . . . . . . . . . 47 
3. $\mathrm{T} / \mathrm{S}$ and Vertical Sections $\ldots \ldots \ldots \ldots \ldots \ldots \ldots$

D. Section PL - May 11, 1989 - Stations 123 to $128 \ldots \ldots \ldots \ldots$

1. Station Locations . . . . . . . . . . . . . . . 53

2. Section Location $\ldots \ldots \ldots \ldots \ldots \ldots \ldots$

3. $\mathrm{T} / \mathrm{S}$ and Vertical Sections $\ldots \ldots \ldots \ldots \ldots \ldots$

E. Small-Scale Survey - May 11-13, 1989 - Stations 129 to $170 \ldots 57$

1. Station Locations . . . . . . . . . . . . . . . . . 59

2. Section Locations . . . . . . . . . . . . . . . . 59

3. $\mathrm{T} / \mathrm{S}$ and Vertical Sections $\ldots \ldots \ldots \ldots \ldots \ldots$

4. Surface Horizontal Sections _. . . . . . . . . . . . 72

F. Section C - May 14, 1989 - Stations 256 to $262 \ldots \ldots \ldots 77$

1. Station Locations . . . . . . . . . . . . . . . . 79

2. Section Locations . . . . . . . . . . . . . . . 79

3. $\mathrm{T} / \mathrm{S}$ and Vertical Sections $\ldots \ldots \ldots \ldots \ldots \ldots$

8. Station Listings and Profiles . . . . . . . . . . . . . . . 83 


\section{Introduction}

Past hydrographic (CTD) and Acoustic Doppler Current Profiler (ADCP) mesoscale surveys of the coastal region near Point Arena, California (Huyer and Kosro, 1987; Huyer, 1984; Kosro, 1987) obtained during the Coastal Ocean Dynamics Experiment (CODE) in 1981 and 1982 have provided a description of the spatial structure of the temperature, salinity, density, and velocity fields and variability during the upwelling season (April to August). In summer, when winds were strong and persistently upwelling favorable, surface temperatures increased monotonically and surface salinities decreased with distance from the coast. Also, isopycnals, isotherms, and isohalines all slope upward toward the coast and there is on average a surface intensified, equatorward, baroclinic alongshore coastal jet found at midshelf. During wind relaxation (weak wind) events in the CODE field program, near-surface water warms appreciably, particularly nearshore where warm water is advected northward along the coast as a narrow inshore countercurrent (Send et al., 1987). Also, coherent mesoscale flow structures identified over the outer shelf and slope with shipboard acoustic Doppler current profiler surveys are closely related to the variability observed in satellite infrared imagery.

The $\underline{\text { Shelf }} \underline{\text { MIxed }} \underline{\text { Layer }}$ Experiment (SMILE) was undertaken to investigate the surface boundary layer over the continental shelf off northern California during winter. As part of the SMILE field program, a moored array was deployed on the shelf between Point Arena and Point Reyes from mid November, 1988 through May, 1989 to make longterm (6 months) observations of the atmospheric forcing and the spatial and temporal structure of the oceanic response in the surface boundary layer. Extensive shipboard CTD and ADCP measurements were also made over the shelf and slope in this region as part of SMILE during three periods: the first just following the mooring deployment operations in November, 1988; the second midway through the moored array deployment in February, 1989; and the third after the spring transition and just before the mooring recovery operations in May, 1989. The hydrographic surveys were conducted using three sampling plans - a large-scale grid of four cross-shelf transects extending to both sides of Point Arena and Point Reyes, a small-scale grid of five (or six) cross-shelf transects located near the central SMILE mooring site, and an expanded small-scale grid consisting

of nine cross-shelf transects. All of the cross-shelf transects extended beyond the shelf break and the maximum sampling depth at each station was near-bottom or $500 \mathrm{~m}$ (November), $1000 \mathrm{~m}$ (February), or $1500 \mathrm{~m}$ (May). The average along-shelf separation between cross-shelf transects was about $15 \mathrm{~km}$ for the small-scale surveys and $50 \mathrm{~km}$ for the large-scale grid. We present here in graphic and tabular form a preliminary analysis 
of the hydrographic survey observations made off northern California during the May, 1989 SMILE field program. The large-scale survey was completed during May 5-8, 1989, the small-scale survey was completed during May 8-9 and again during May 11-13, Section C was completed during May 10 and again during May 14, and Section PL was completed during May 11. Time series of CTD profiles repeated every 15 minutes were made at mooring $\mathrm{C} 1$ on the central section on May 10-11, 1989 (stations 102-121), and at mooring C3 on May 13-14 (stations 171-255). Data from these time series stations will be presented in a separate report.

\section{Instrumentation and Calibration}

A Neil Brown Instrument Systems (NBIS) model MKIII CTD fish was used as the primary profiling instrument during $\mathrm{R} / \mathrm{V}$ Wecoma cruise W8905. The instrument provided continuous sampling of temperature, conductivity, pressure, and light transmission. Salinity and density were subsequently derived from the measured variables. The instrument package consisted of the MKIII CTD underwater unit mounted one meter below a General Oceanics rosette sampler with 12 five-liter Niskin bottles. The fast response thermistor on the CTD fish was disabled and the instrument sampled at a rate of $32 \mathrm{hz}$. Lowering speeds were approximately $36 \mathrm{~m} / \mathrm{min}$ to match the response times of the temperature and conductivity sensors (Giles and McDougall, 1986).

The CTD underwater unit is part of the R/V Wecoma's scientific equipment and was calibrated at the manufacturer's calibration facility just prior to the cruise. Water samples were normally collected at each station on the up cast at a depth where the vertical stratification was minimal. The calibration samples were then processed at Woods Hole on a Guildline AutoSal salinometer to determine salinity. The individual salinities were converted to in situ conductivity and compared to the conductivity output of the instrument. A least-squares fit of the difference between the bottle and instrument conductivity gives

$$
C(B O T T L E)-C(C T D)=-0.0000106312 * X-0.0103436
$$

where $X$ is the station number which varied from 1 to 263 , and the sum of the squares

of the residuals is $0.009 \mathrm{mmho}^{2}$. This means that the CTD conductivity sensor drift and offset were small during the cruise and that the rms difference between the corrected 
CTD and bottle conductivity values was \pm 0.008 mmho. The CTD conductivity for the entire cruise has been edited using the linear regression correction given above.

\section{Data Processing}

The CTD data were recorded at sea with a PC-based data acquisition system written by Howard Saklad of the University of Alaska, Institute of Marine Science. The program acquires the raw CTD data at $32 \mathrm{hz}$, displays the data in real time, postcast data averages, and prints lists of data at each station. Normally, only the down profile is processed at sea. During post cruise data processing, the conductivity calibration was first applied to the raw data. Then an exponential recursive filter (Middleton and Foster, 1980) described by Cox was applied to pressure and conductivity to match the amplitude and phase of the platinum temperature sensor. The Cox filter has the form

$$
c^{\prime}(t)=w_{0} c^{\prime}(t-d t)+\left(1-w_{0}\right) c(t)
$$

where $c^{\prime}(t)$ is the filtered conductivity at time $t, c^{\prime}(t-d t)$ is the previous filtered conductivity, and $c(t)$ is the original unfiltered conductivity at time $t$. The filter weight is given by

$$
w_{0}=e^{-d t / t l a g}
$$

where $d t=.032 \mathrm{sec}$ is the sampling period, and tlag $=.235 \mathrm{sec}$ is the time lag of the platinum temperature sensor. A uniform pressure series of $1 \mathrm{db}$ interval was then created from the filtered data, after spurious data observations (spikes) had been replaced with interpolated data. Salinity was calculated using Lewis (1980) and a value of 42.914 was used to convert to conductivity ratio (Culkin and Smith, 1980).

\section{Data Presentation}

The hydrographic survey data are shown in the form of vertical sections, T/S correlations, horizontal sections, vertical profiles, and tabular lists at graduated depths. Plots of the mixed layer depth for each survey are defined by a vertical temperature change from the surface value of less than $0.1^{\circ} \mathrm{C}$. 


\section{Acknowledgments}

Cruise W8905 on the R/V Wecoma was supported by the NSF Grant OCE8716937. Dr. Robert Beardsley was the Chief Scientist on the hydrographic cruise. The work was a cooperative effort by R. Beardsley, A. Voorhis, R. Limeburner, S. Lentz, R. Stanley, W. Geyer, and M. Willis. Their assistance is greatly appreciated. Finally, the helpfulness of the officers and crew of the R/V Wecoma contributed significantly to the success of the cruise.

\section{References}

Culkin, F. and N. D. Smith (1980) Determination of the concentration of potassium chloride having the same electrical conductivity, at $15^{\circ} \mathrm{C}$ and infinite frequency, as standard seawater of salinity $35.000 \mathrm{ppm}$ (chlorinity $19.37394 \mathrm{ppm}$ ). IEEE Journal of Ocean Engineering, OE-5, 22-23.

Giles, A. B., and T. J. McDougall (1986) Two methods for the reduction of salinity spiking of CTDs. Deep-Sea Research, 33(9), 1253-1274.

Huyer, A. (1984) Hydrographic observations along the CODE central line off northern California, 1981. Journal of Physical Oceanography, 14, 1647-1658.

Huyer, A., and P. M. Kosro (1987) Mesoscale surveys over the shelf and slope in the upwelling region near Point Arena, California. Journal of Geophysical Research, 92, 1655-1682.

Kosro, P. M. (1987) Structure of the coastal current field off northern California during the Coastal Ocean Dynamics Experiment. Journal of Geophysical Research, 92, 1637-1654.

Lewis, L. L. (1980) The Practical Salinity Scale 1978 and its antecedents. IEEE Journal of Ocean Engineering, OE-5, 3-8.

Middleton, J. H., and T. D. Foster (1980) Fine structure measurements in a temperature compensated halocline. Journal of Geophysical Research, 85(C2), 1107-1122.

Send, U., R. C. Beardsley, and C. D. Winant (1987) Relaxation from upwelling in CODE. Journal of Geophysical Research, 92, 1683-1698. 

7. Graphic Description of CTD Data

A. Large-Scale Survey - May 5-8, 1989 Stations 1 to 44 


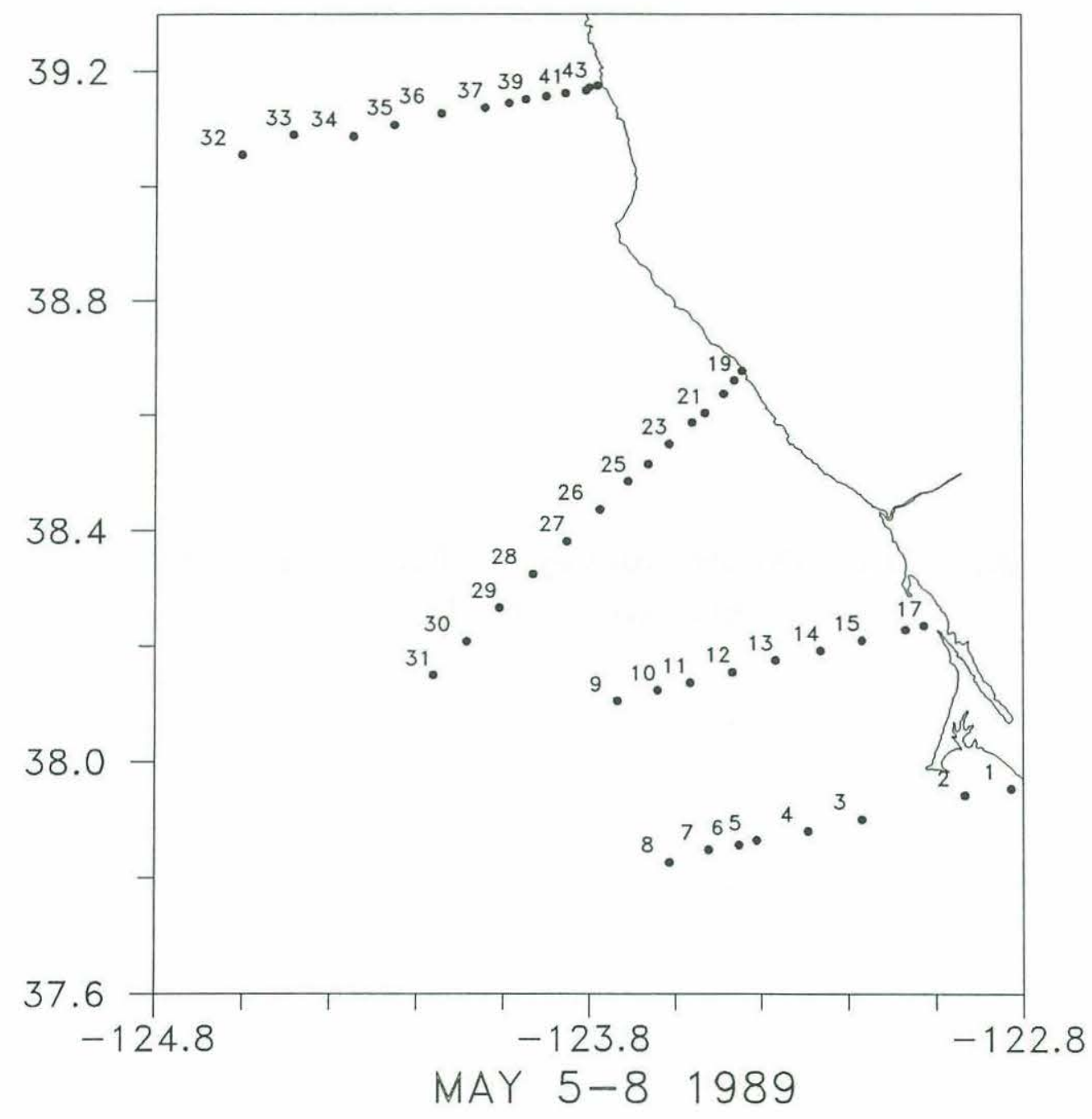




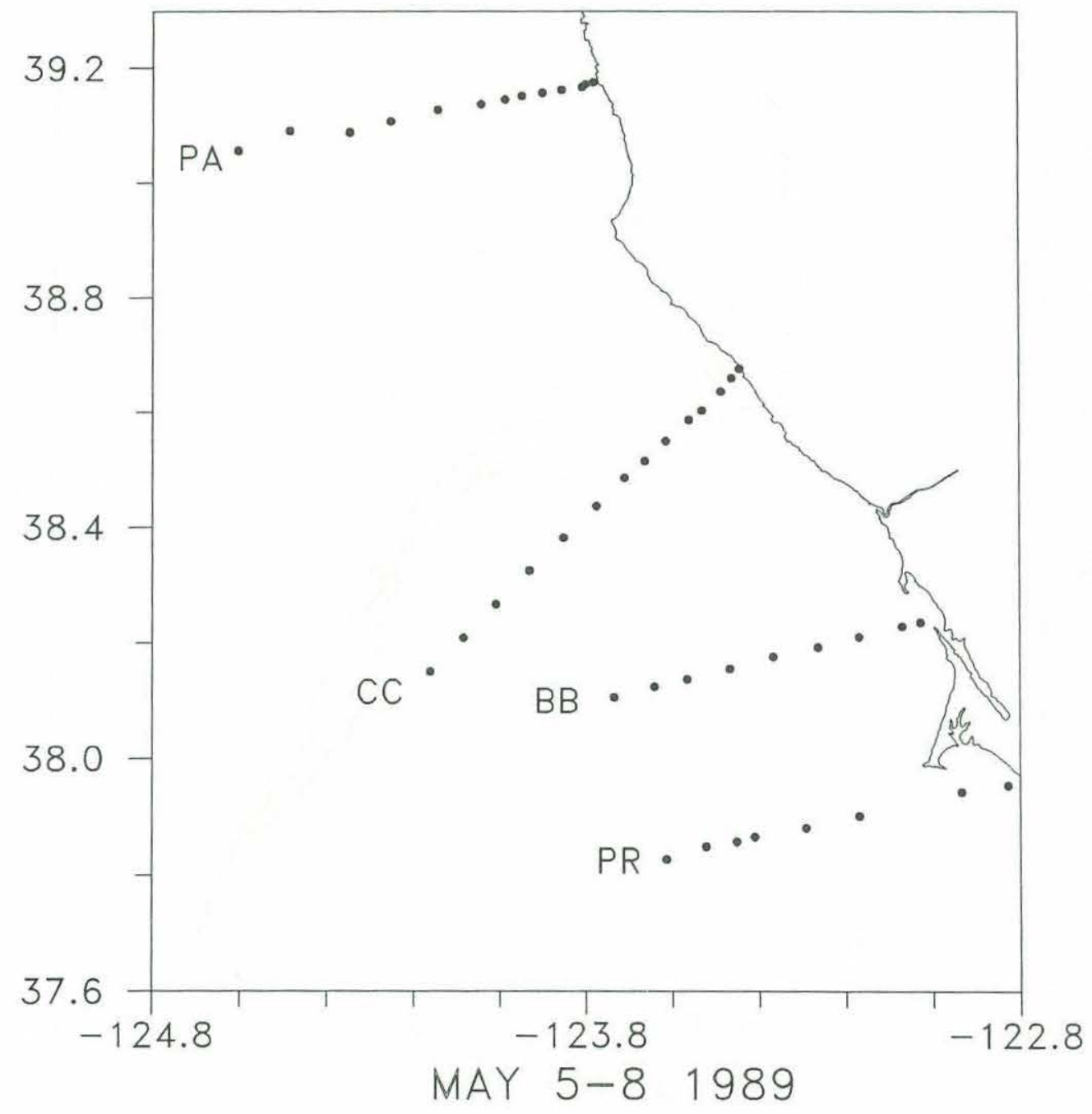




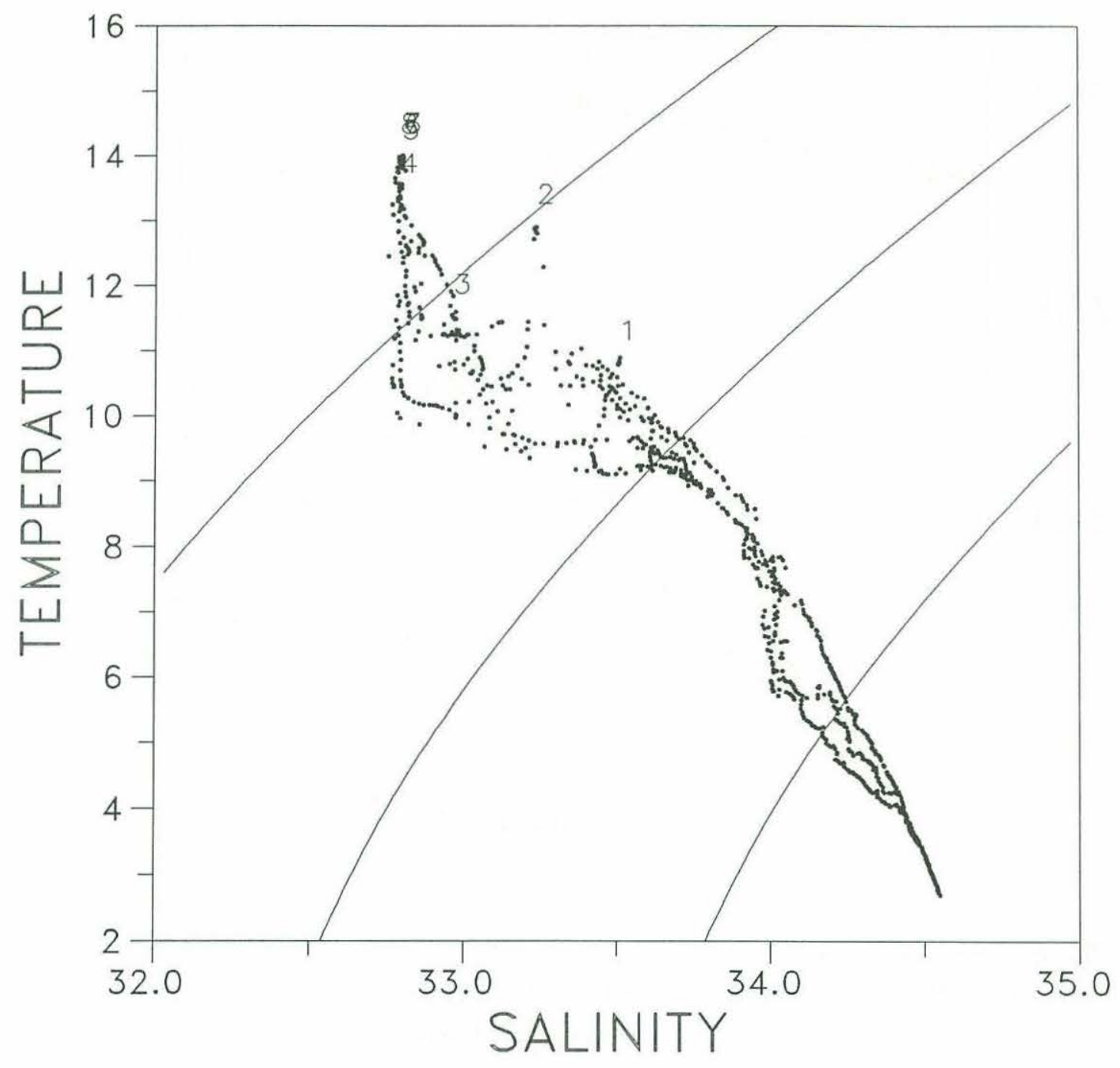


PR TEMPERATURE
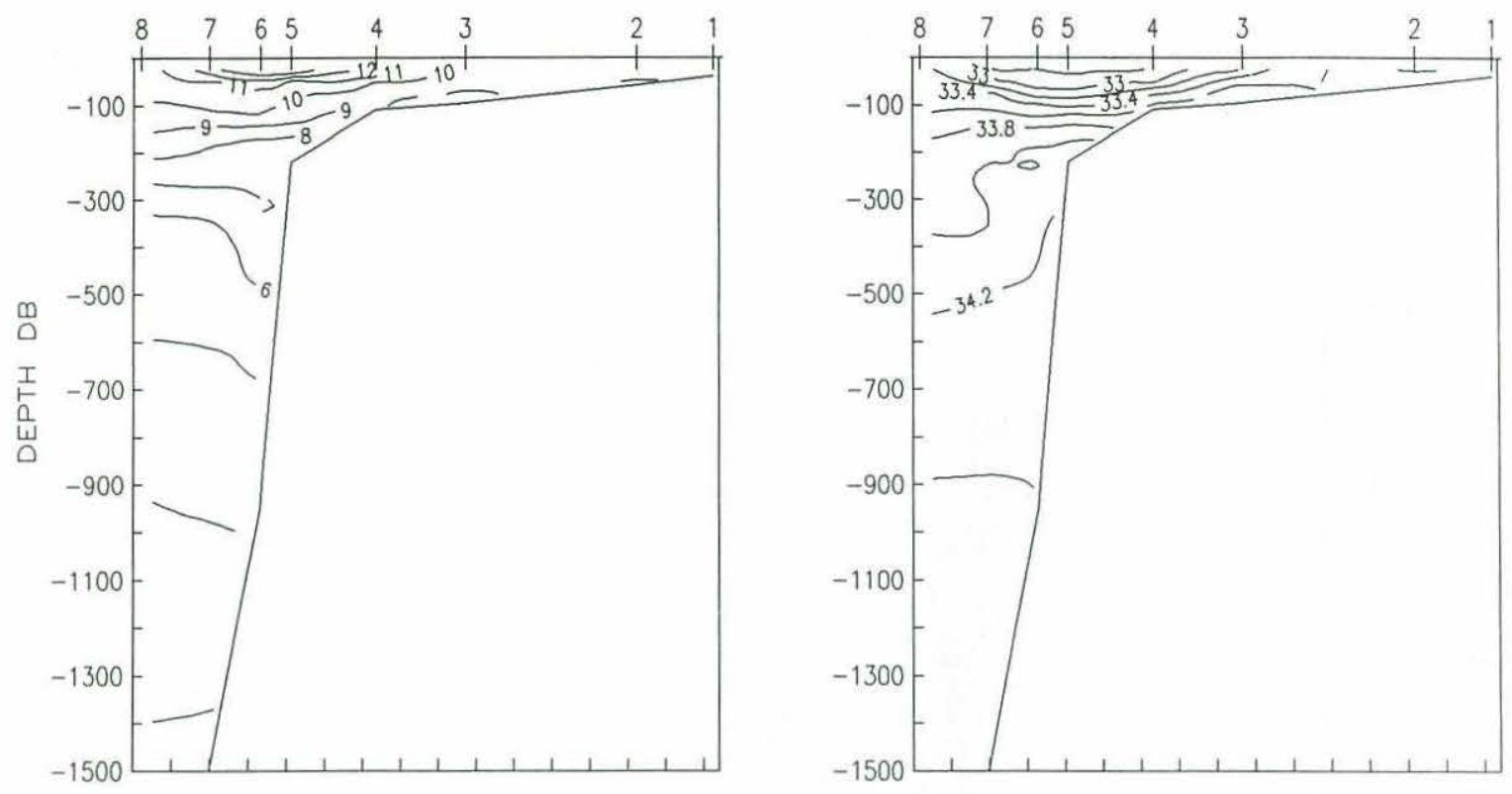

PR SIGMA-T

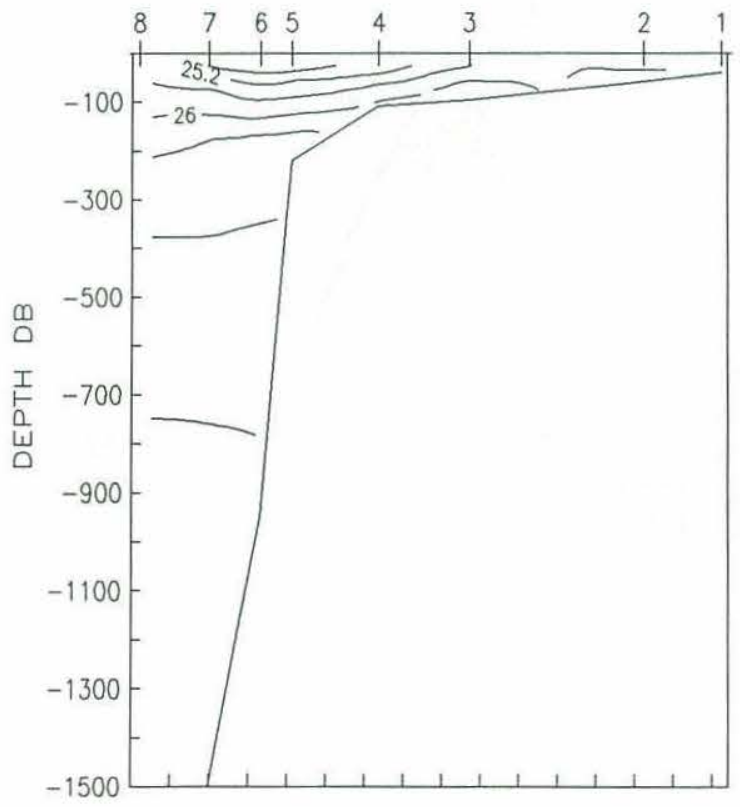




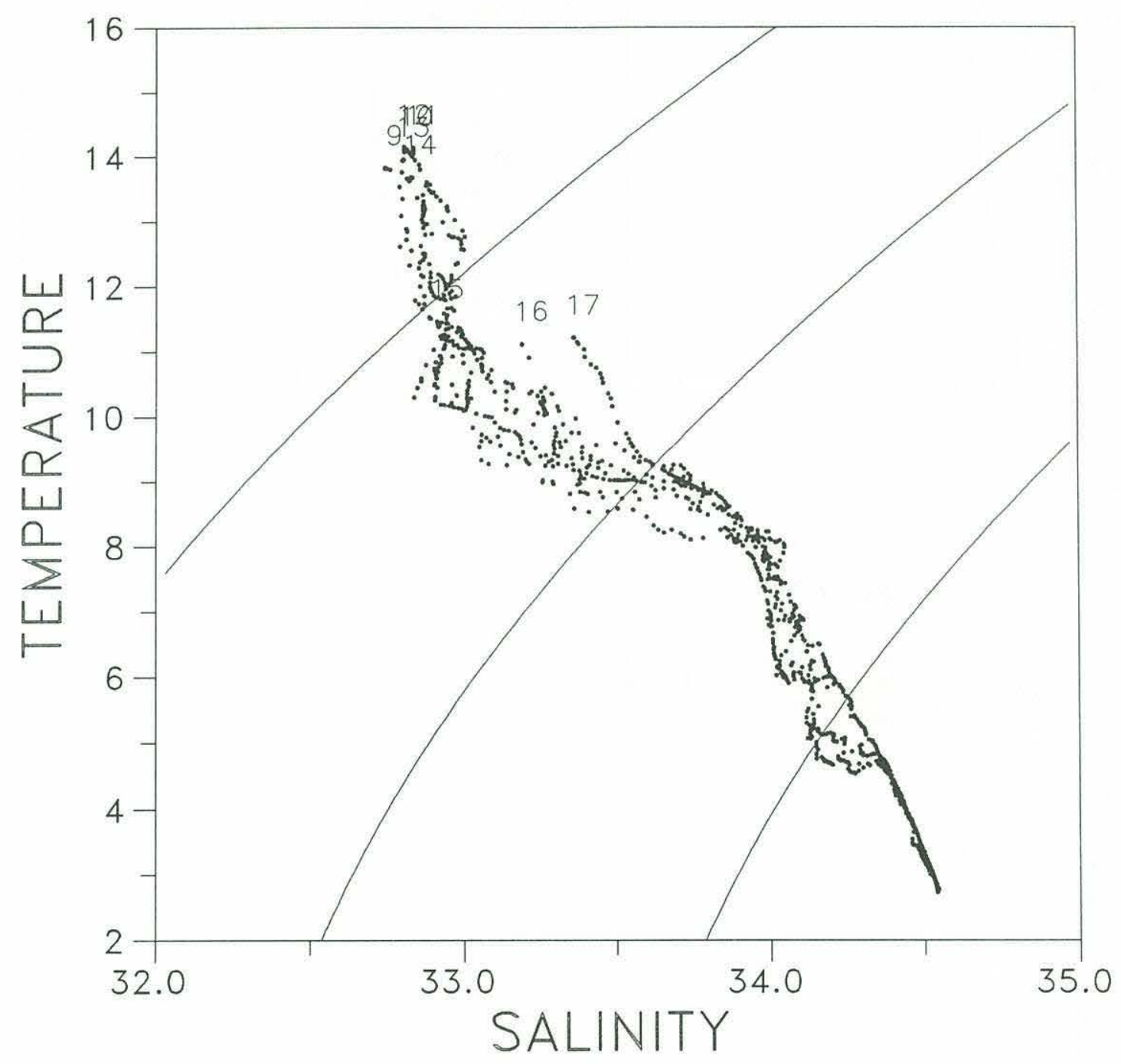


BB TEMPERATURE

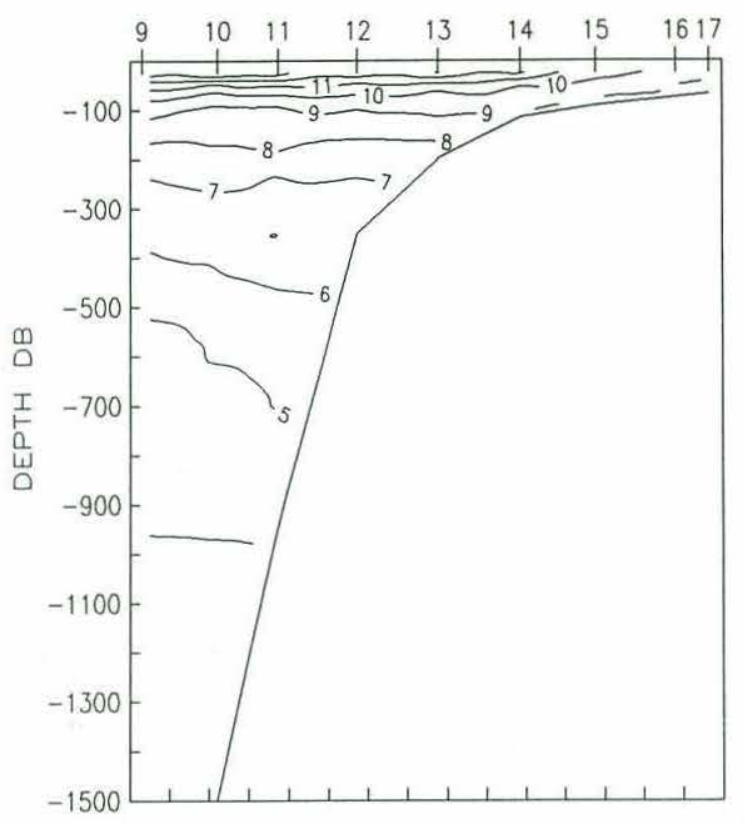

BB SIGMA-T

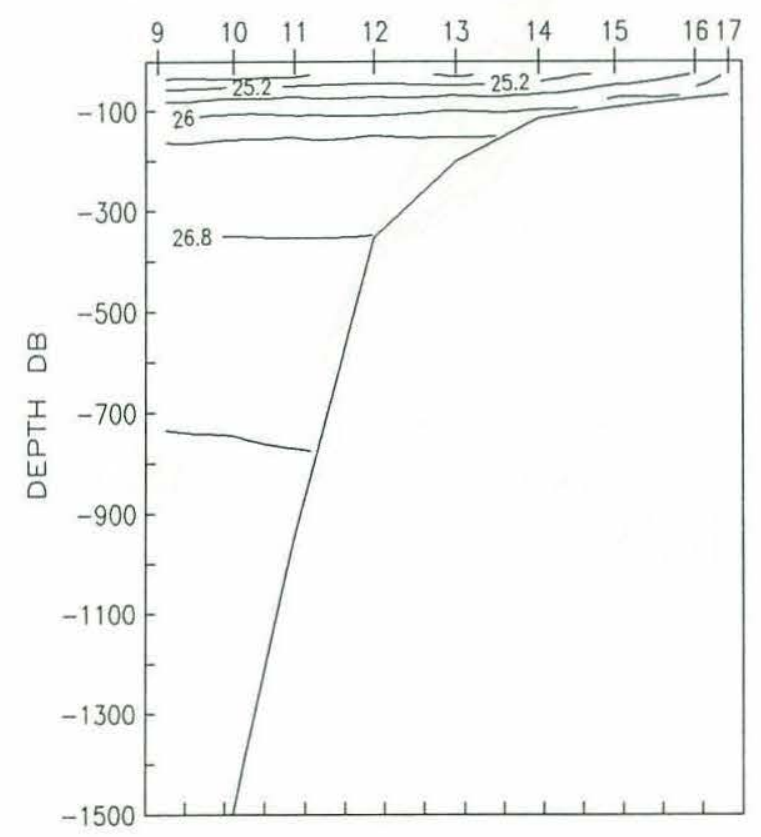

BB SALINITY

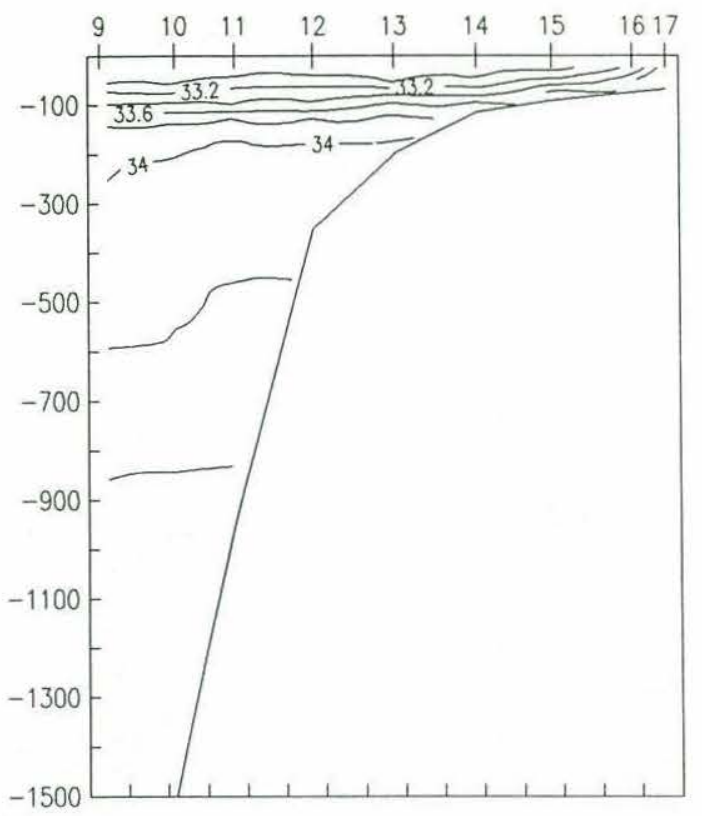




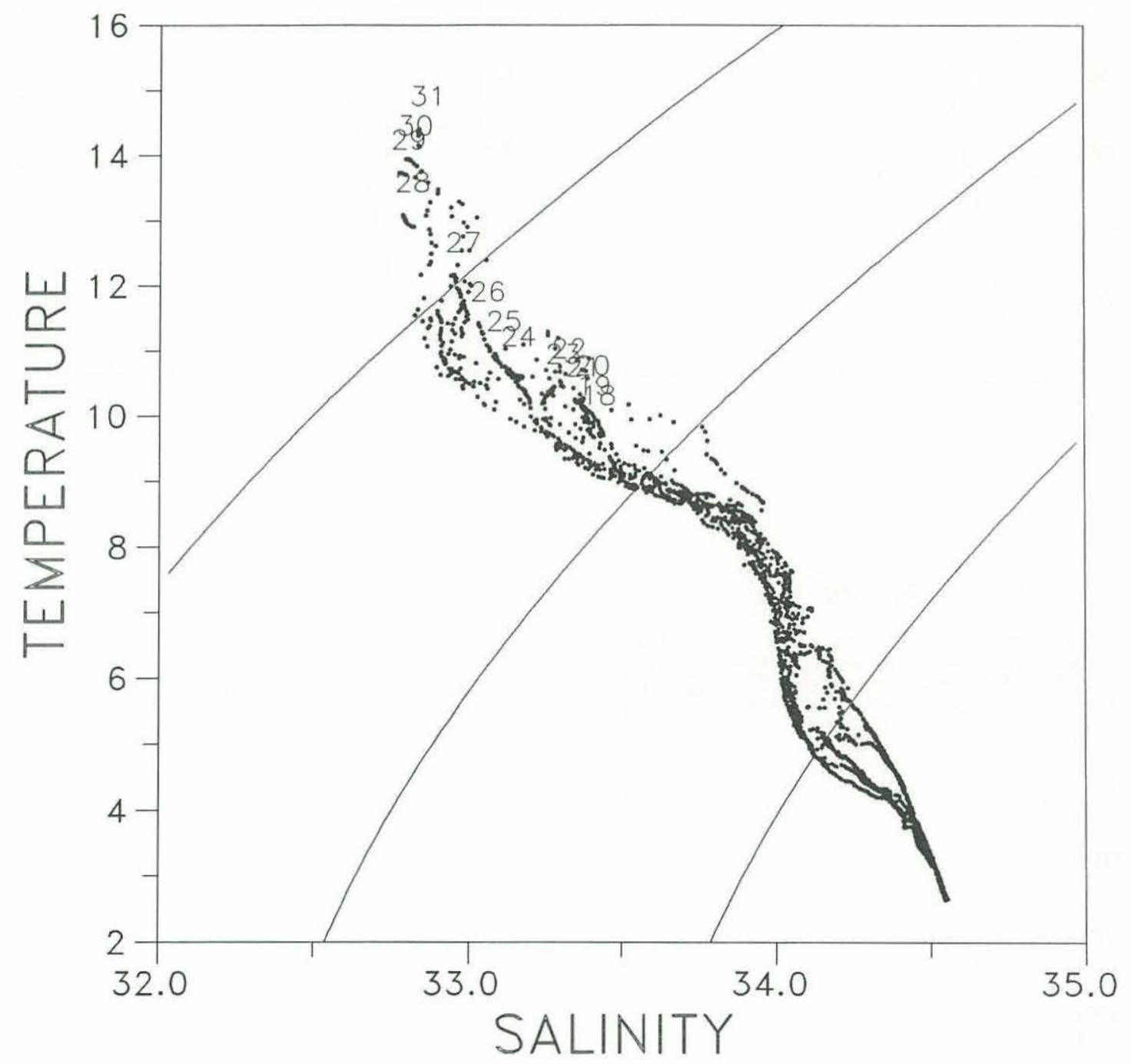



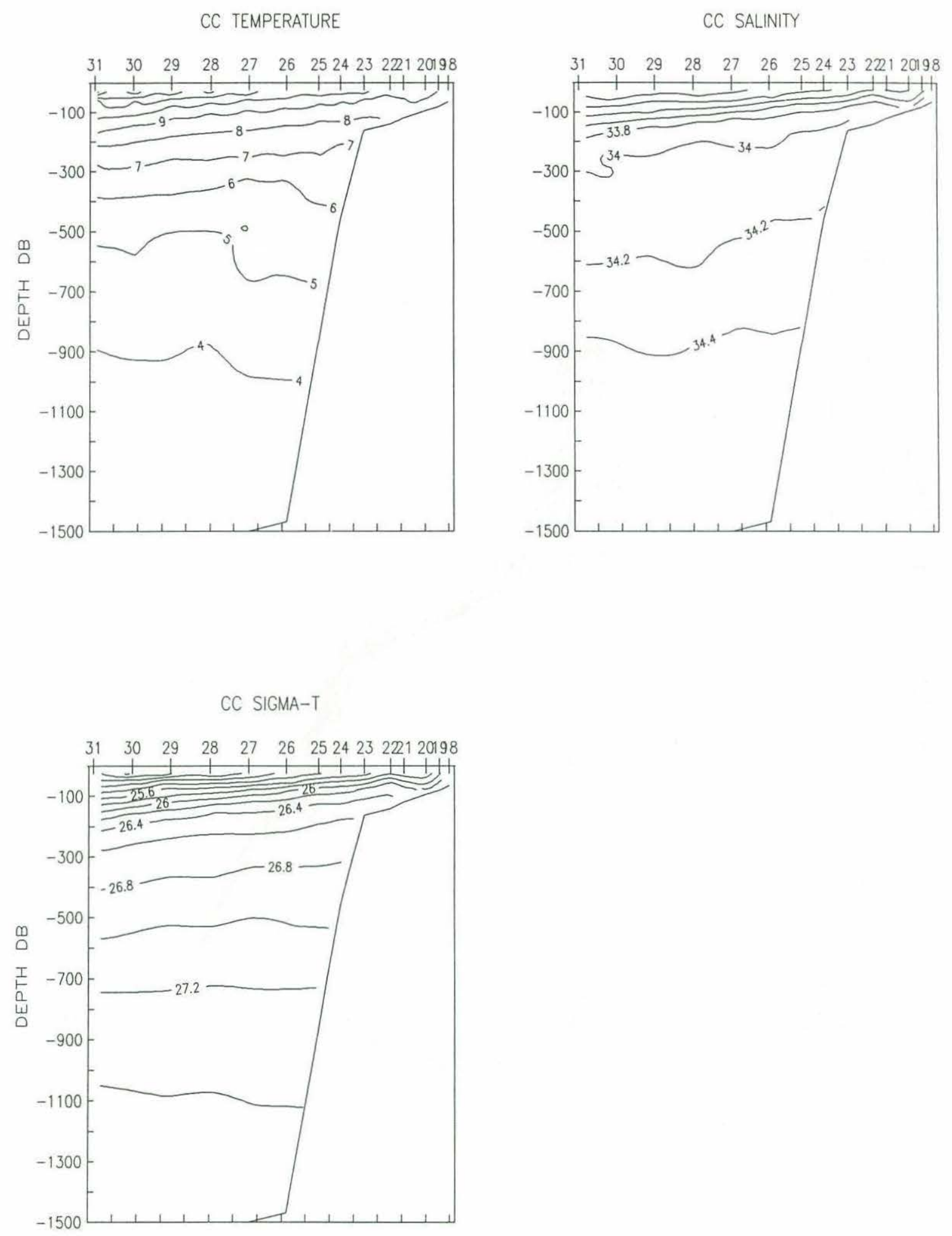


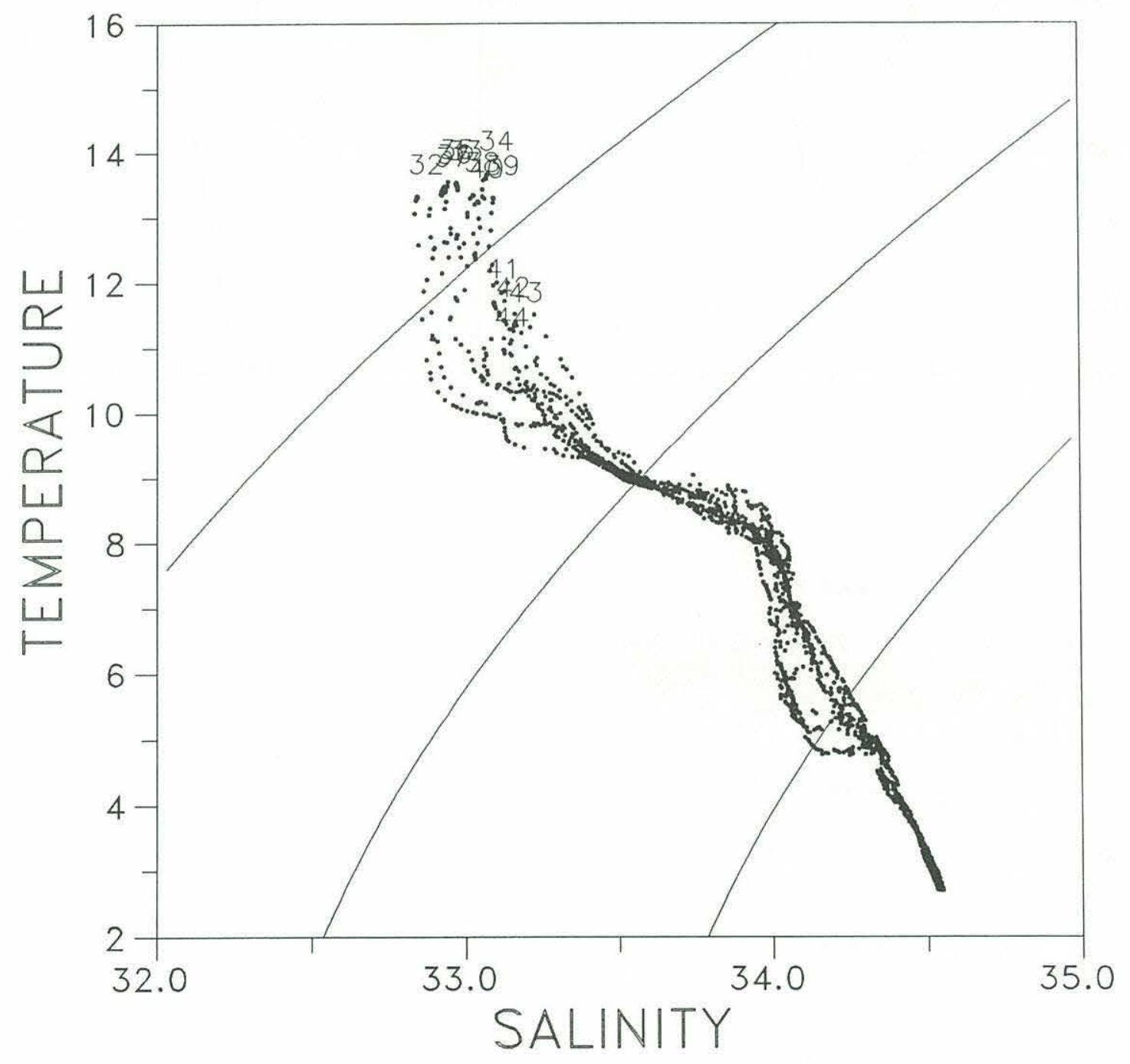



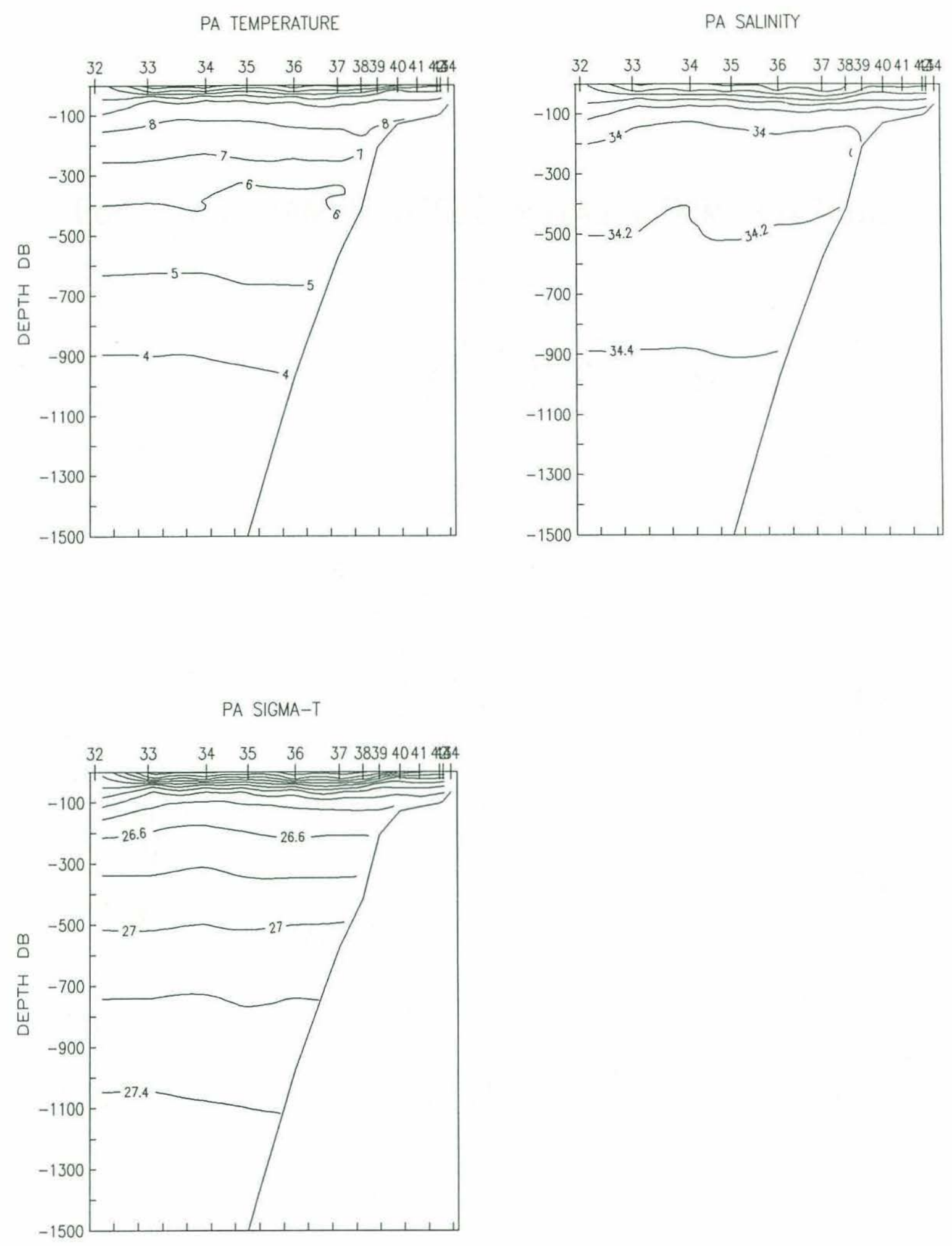
SURFACE MIXED LAYER DEPTH M MAY 5-8 1989

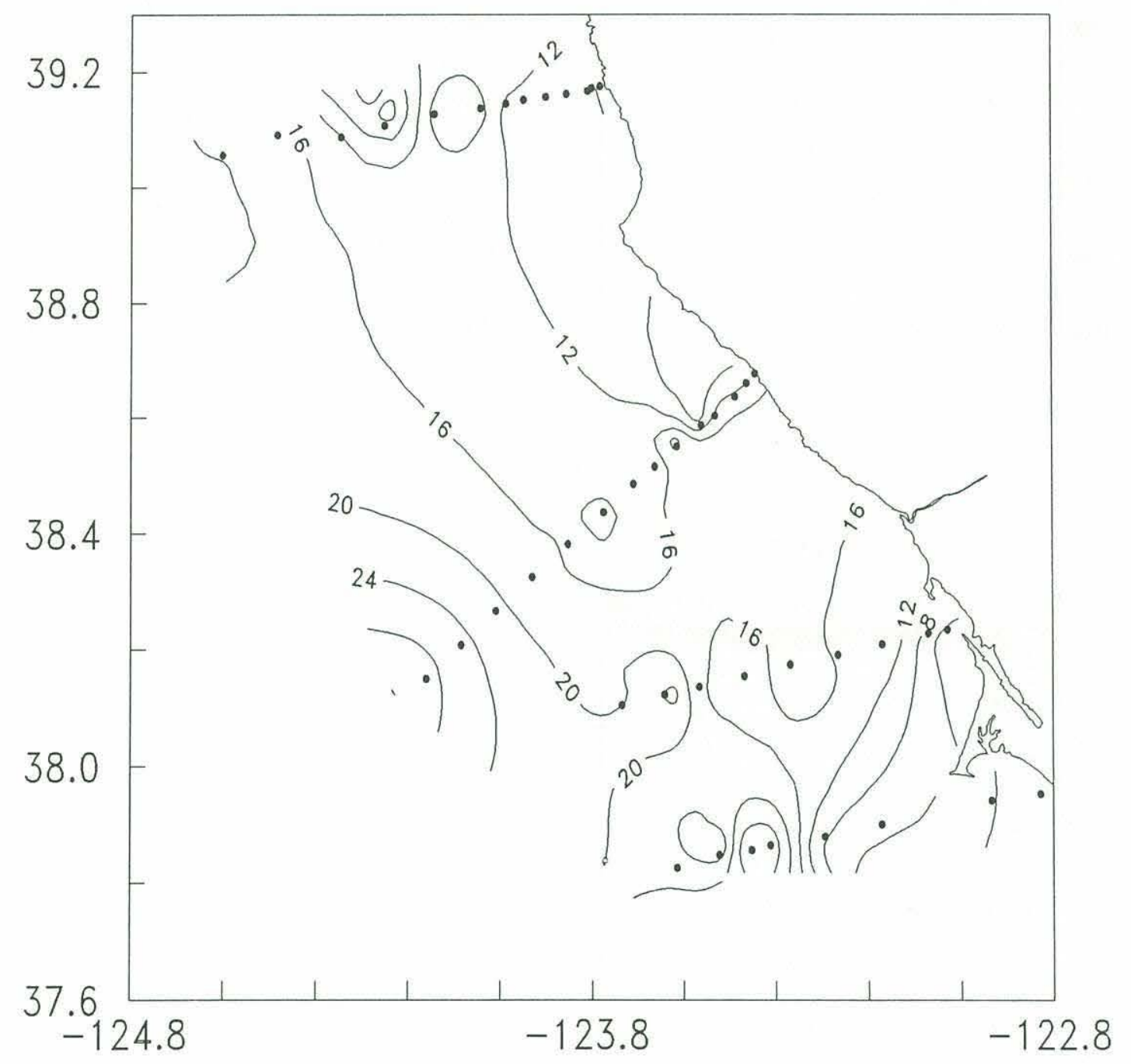


SURFACE TEMP MAY 5-8 1989

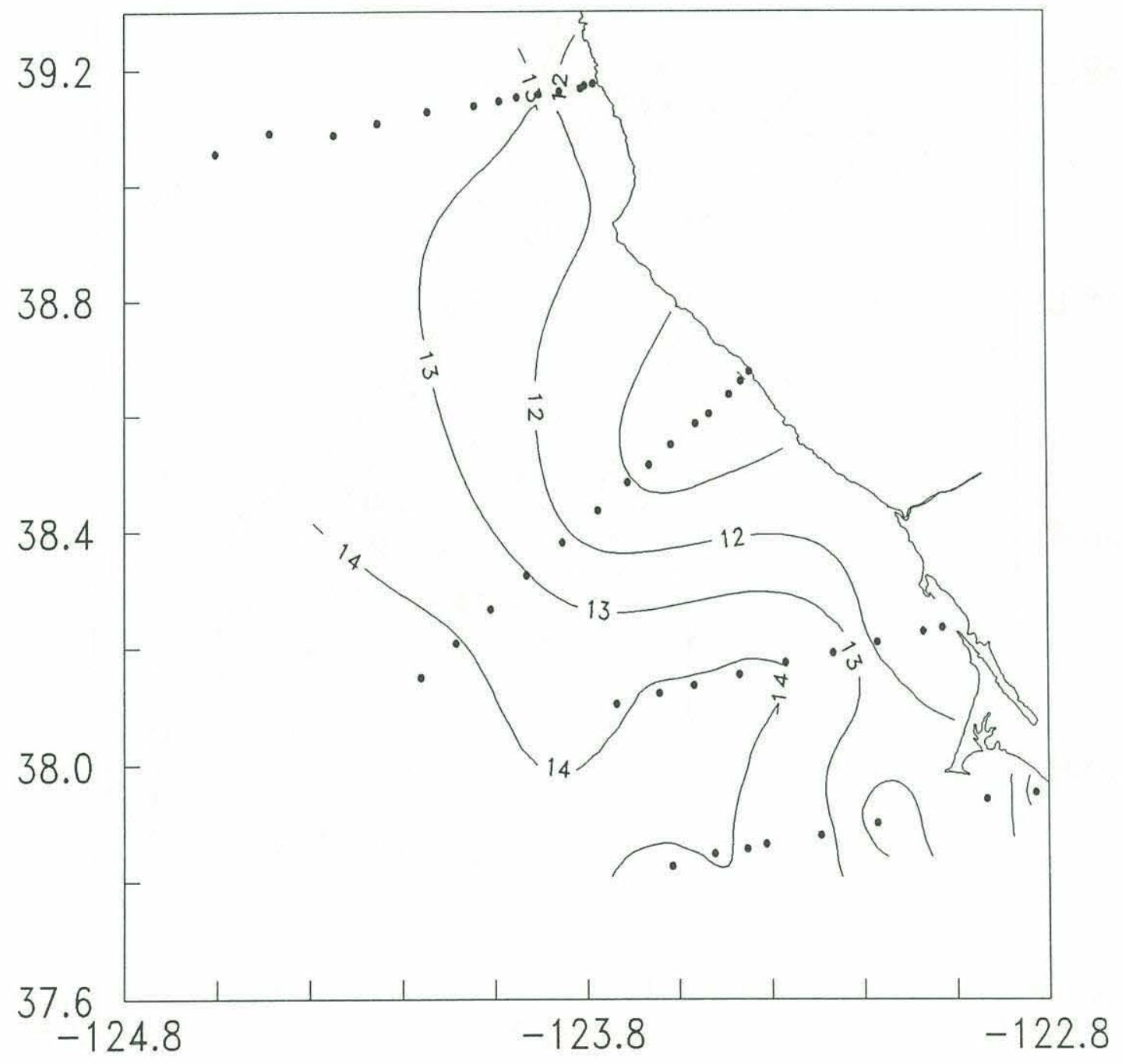


SURFACE SALINITY MAY 5-8 1989

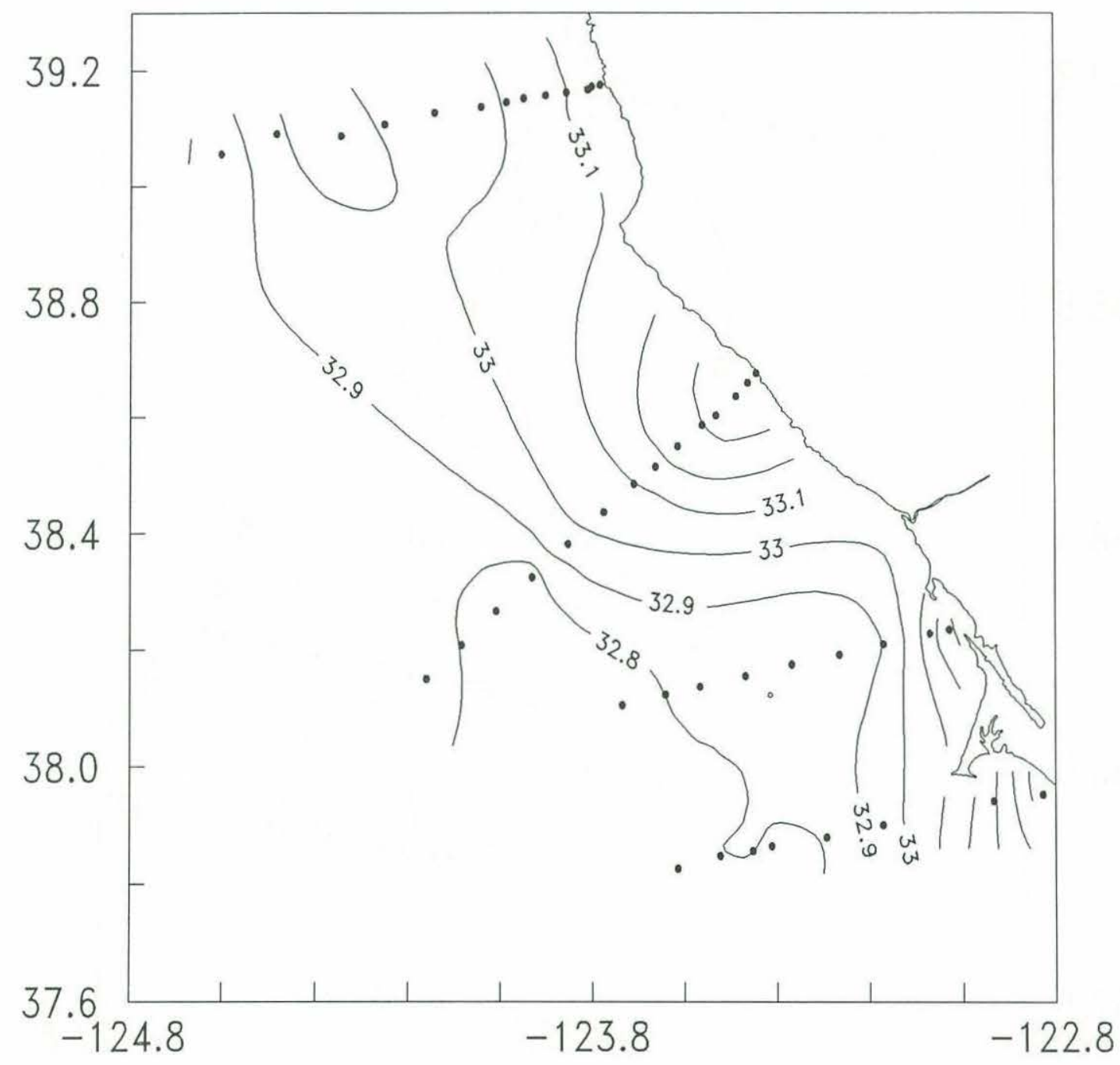


SURFACE SIGMA-T MAY 5-8 1989

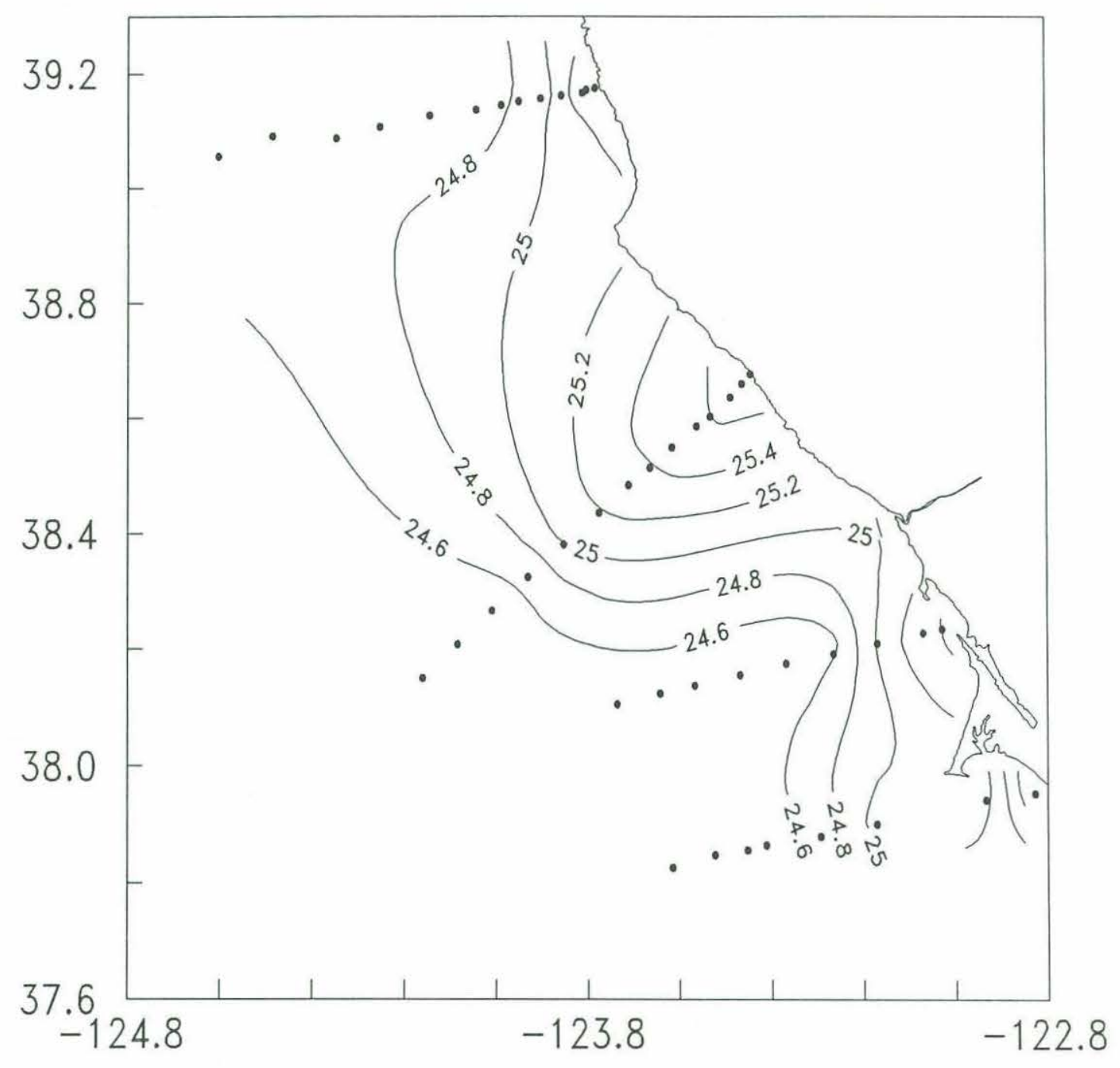



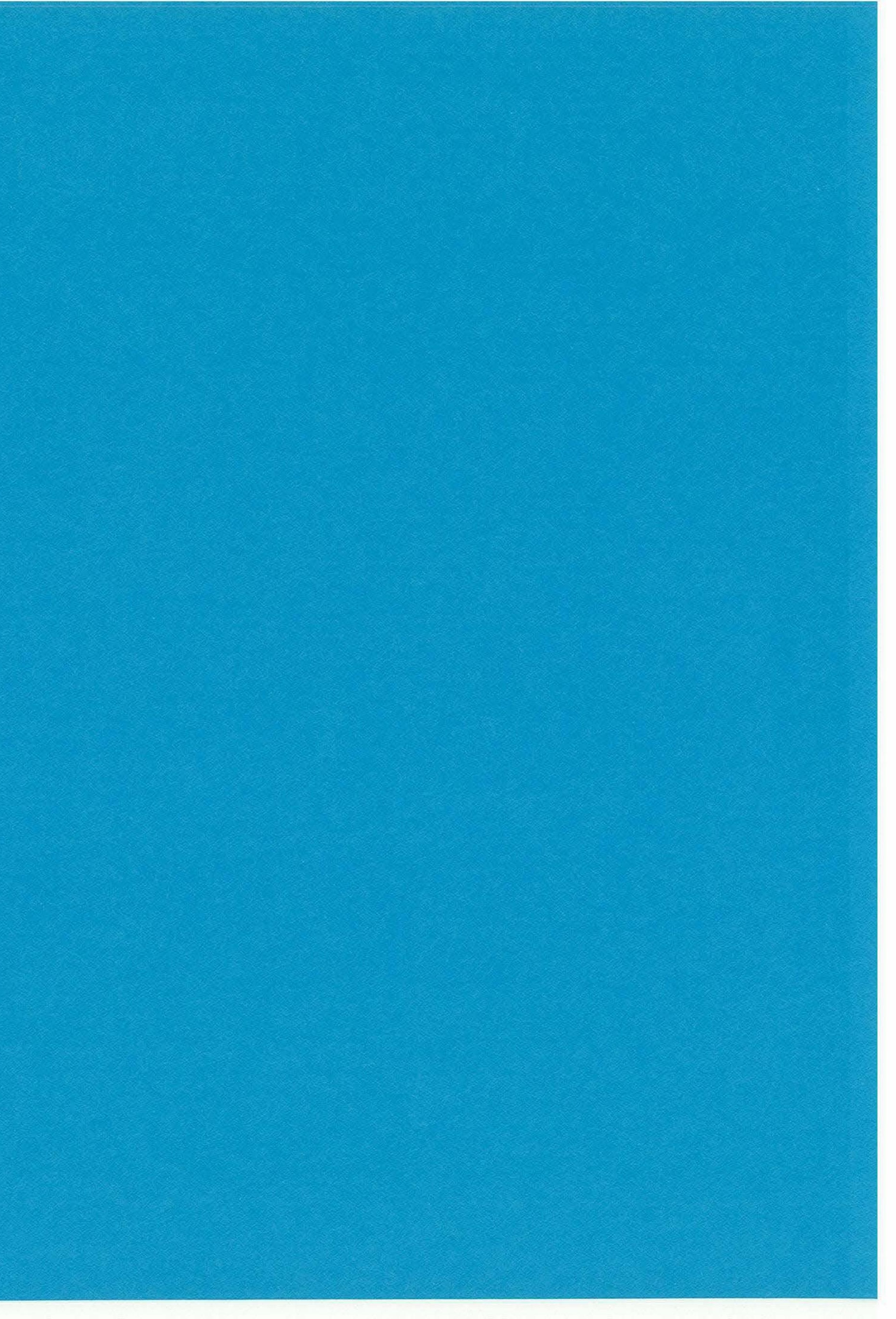
B. Small-Scale Survey - May 8-9, 1989 Stations 47 to 86 

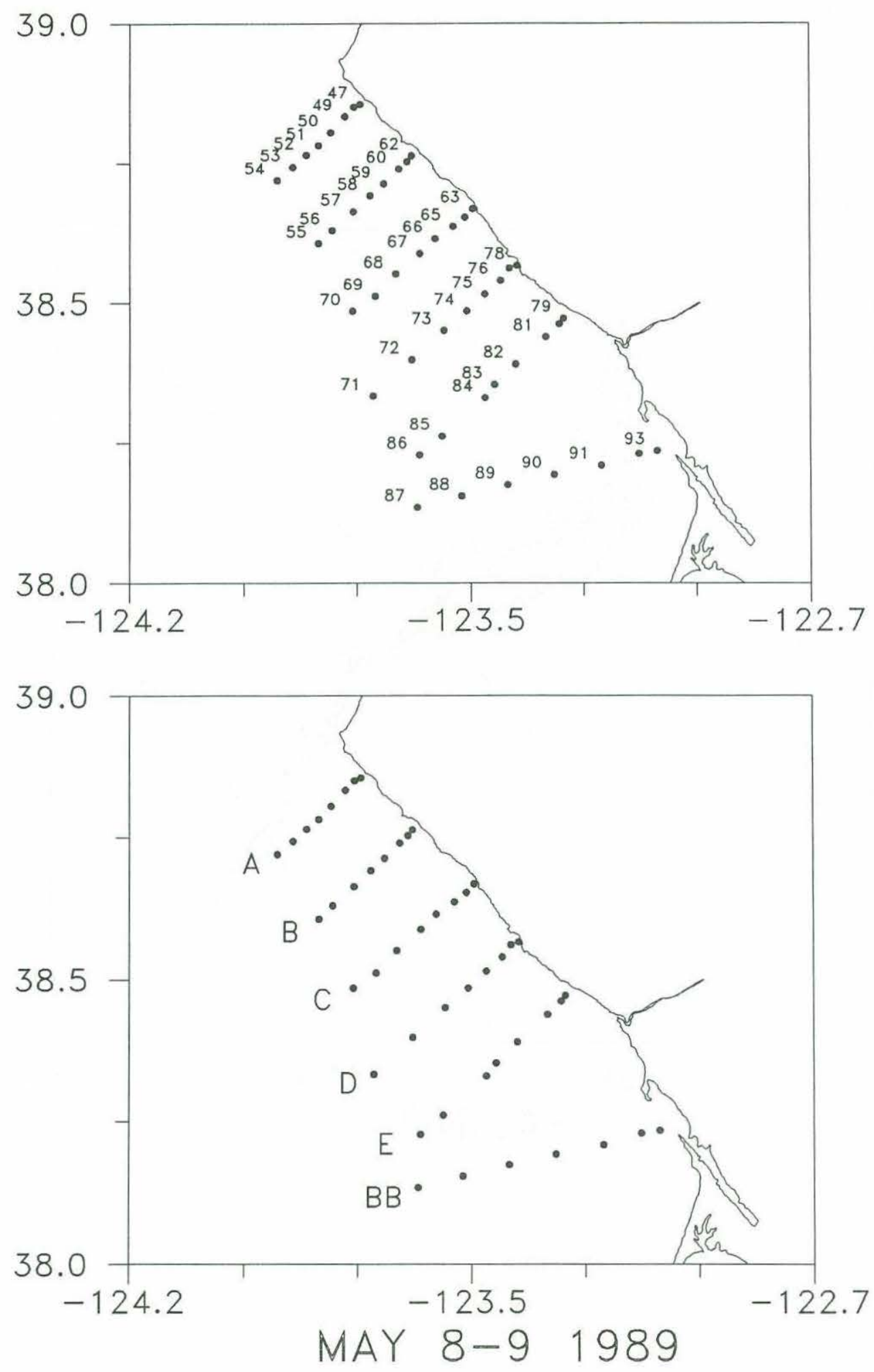


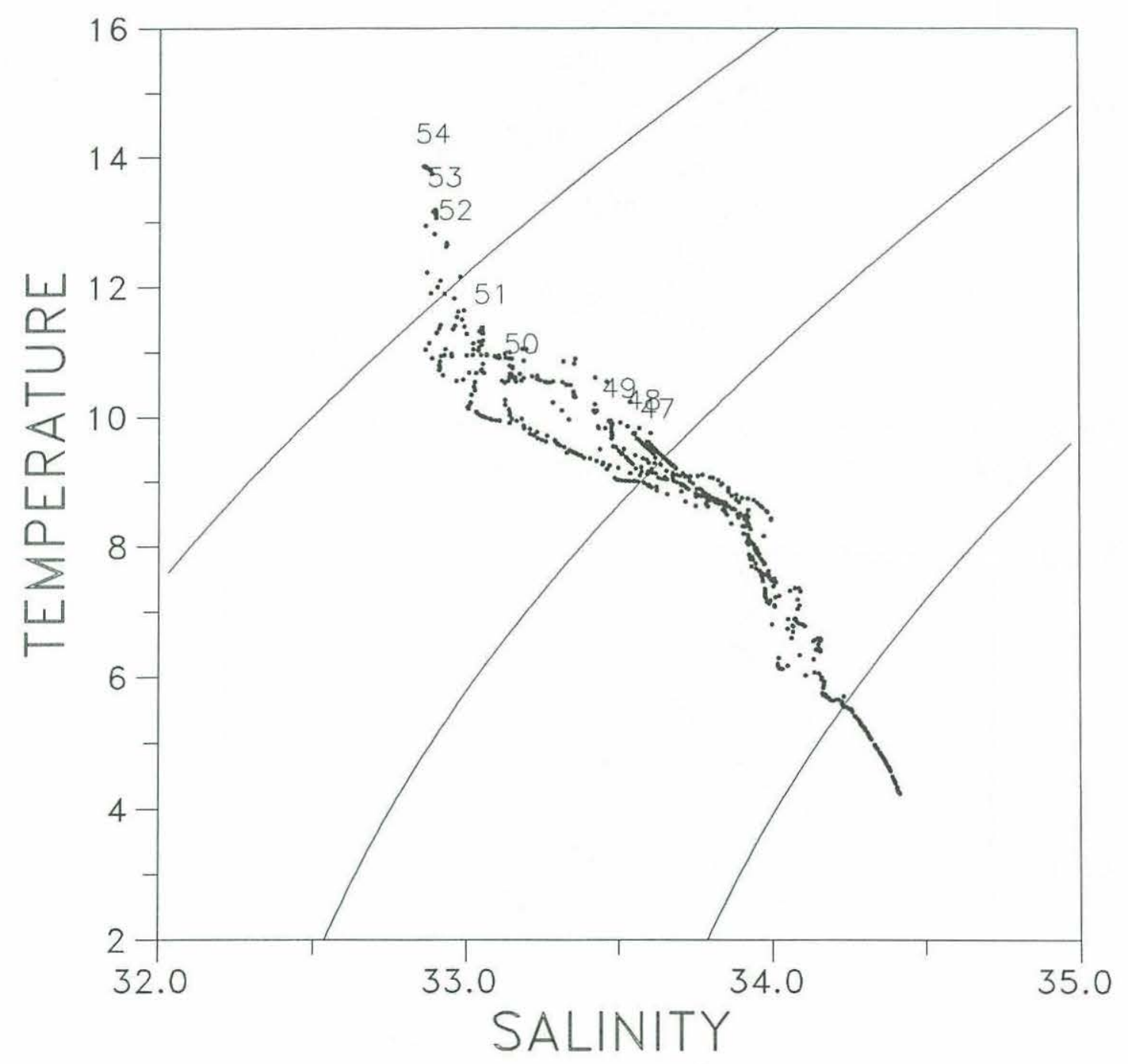


A TEMPERATURE

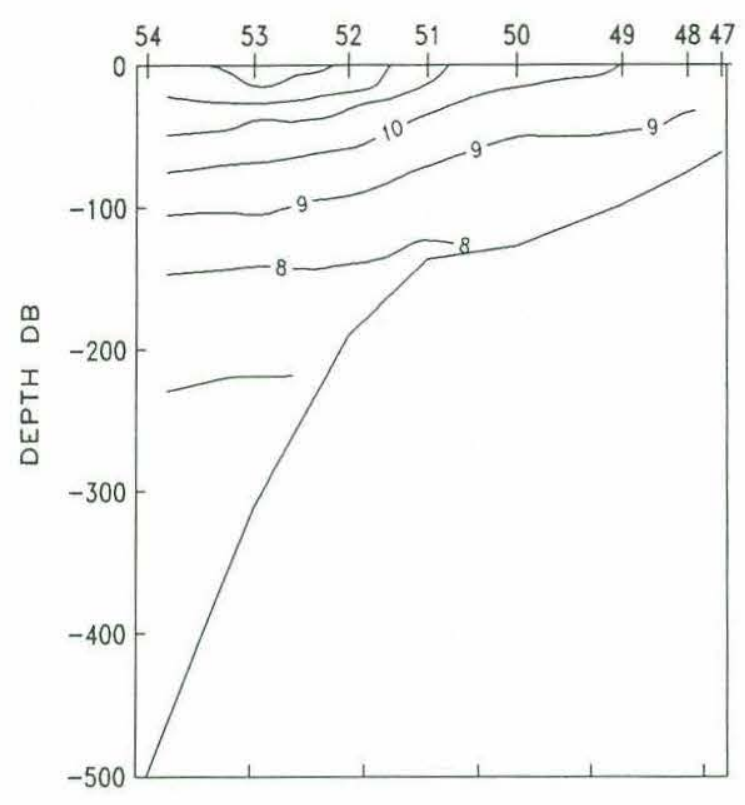

A SALINITY

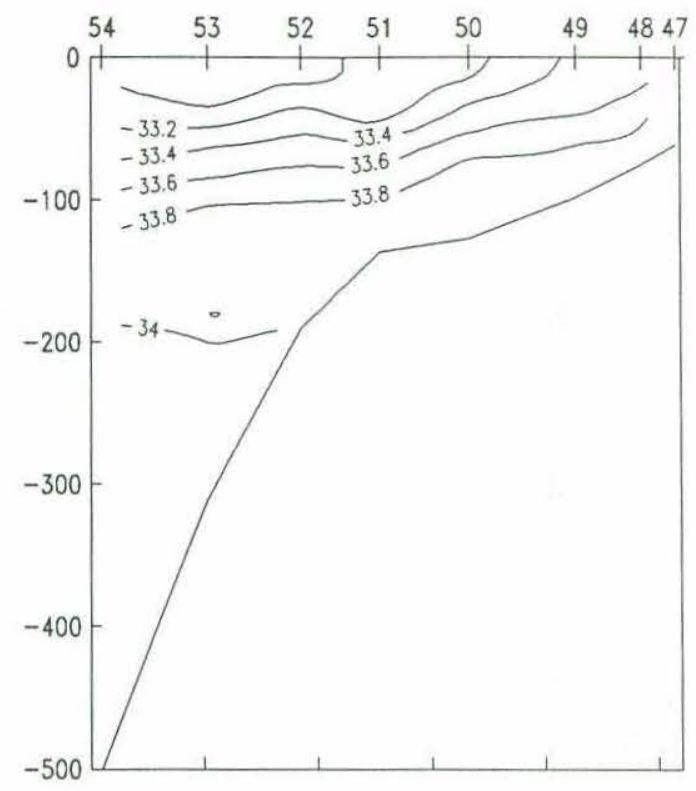

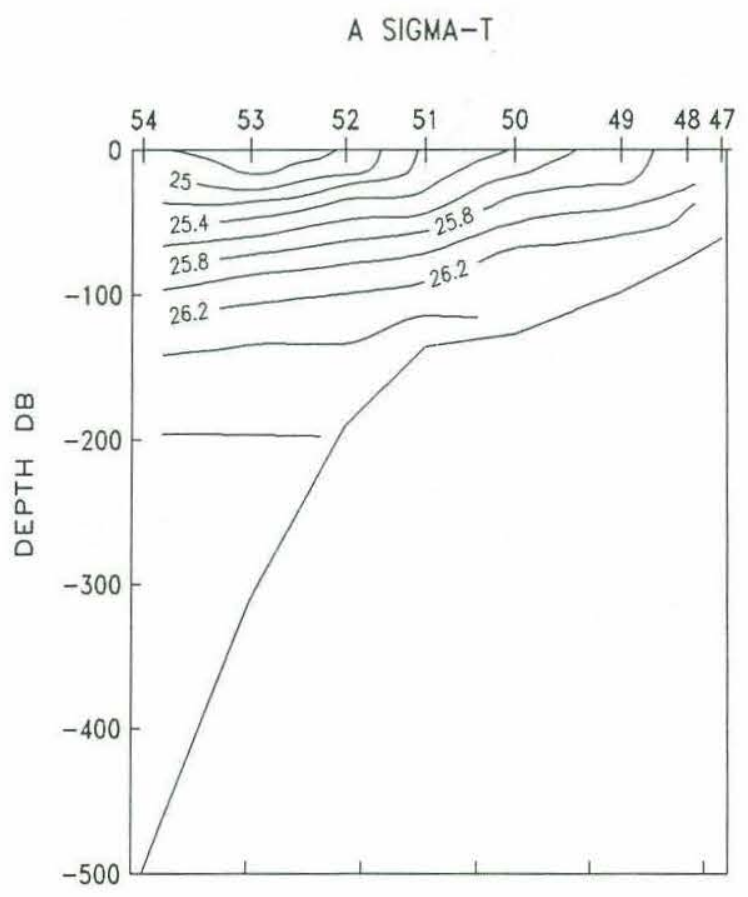




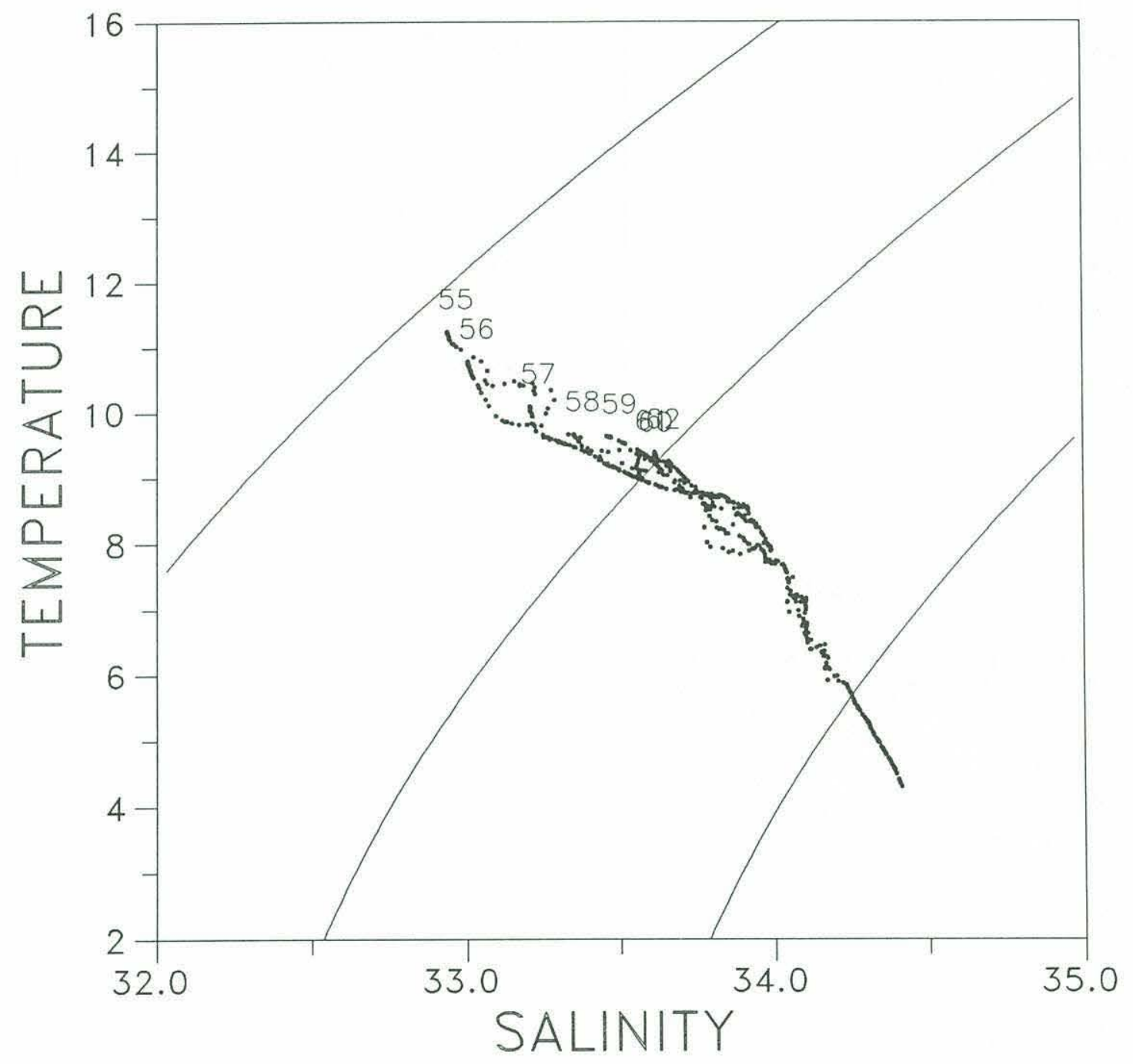



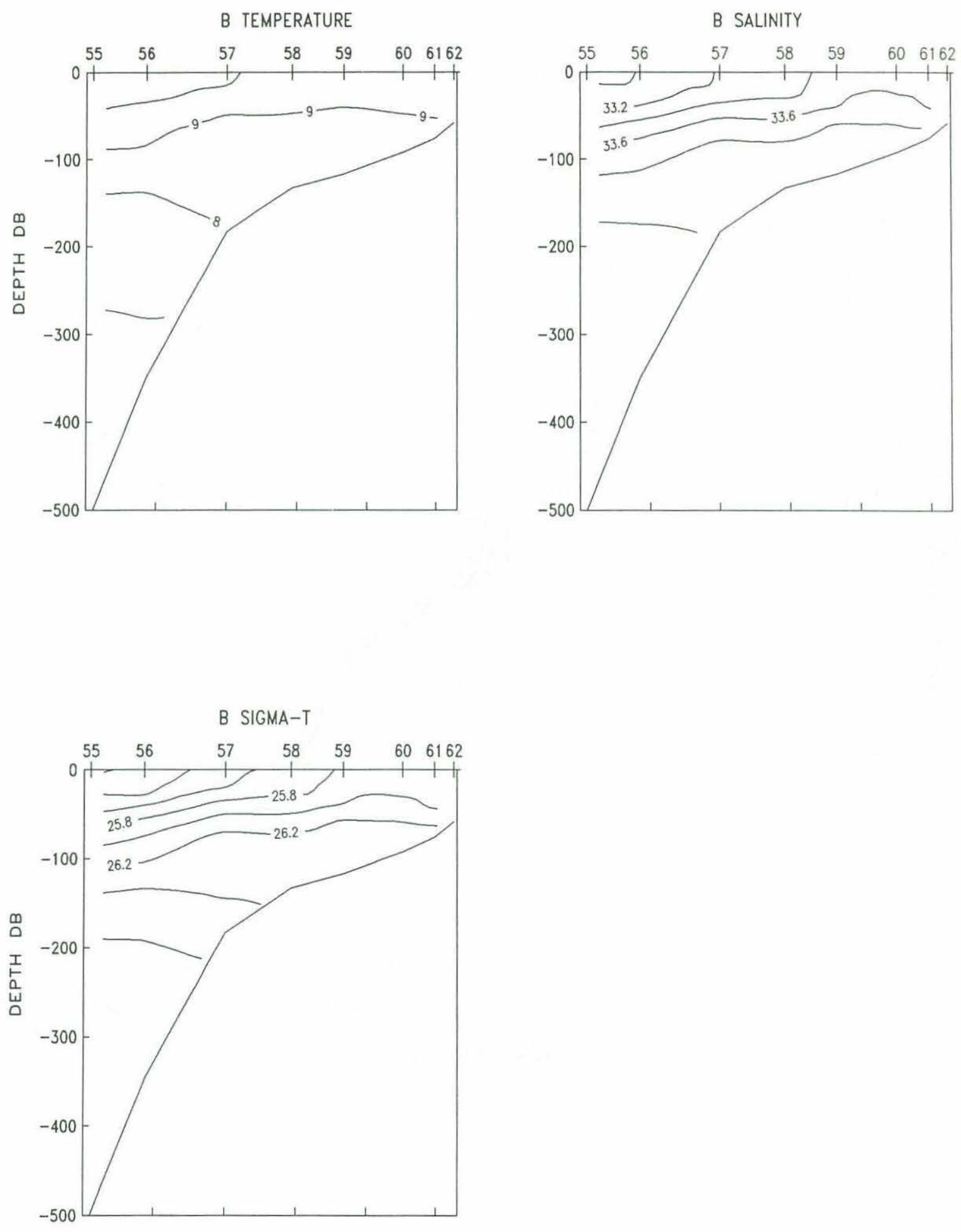


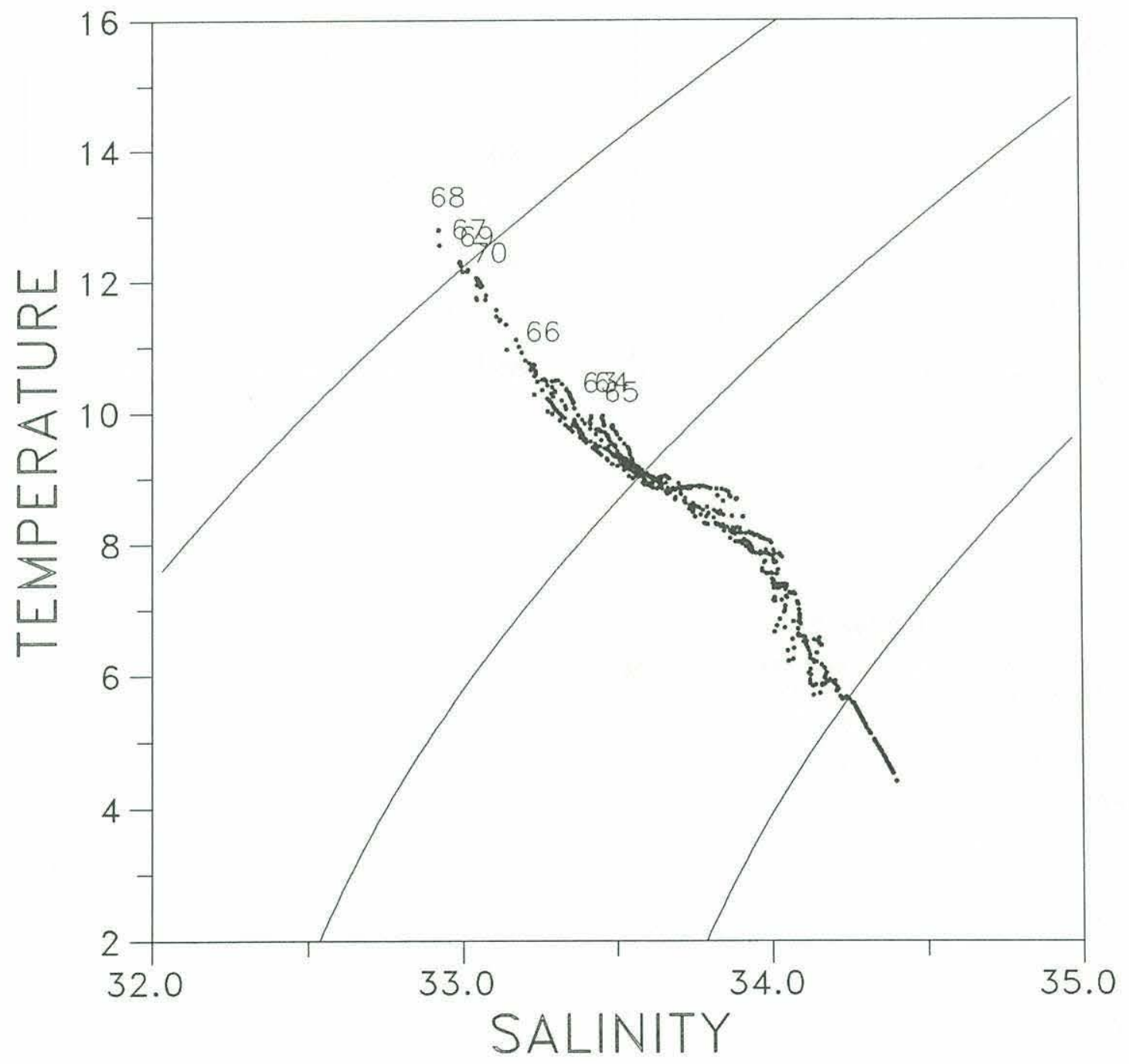


C TEMPERATURE

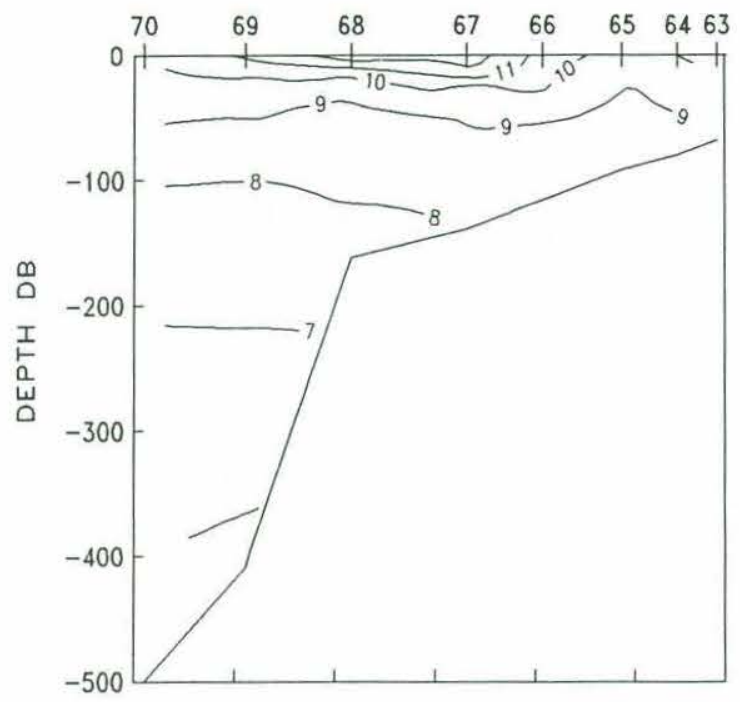

C SALINITY

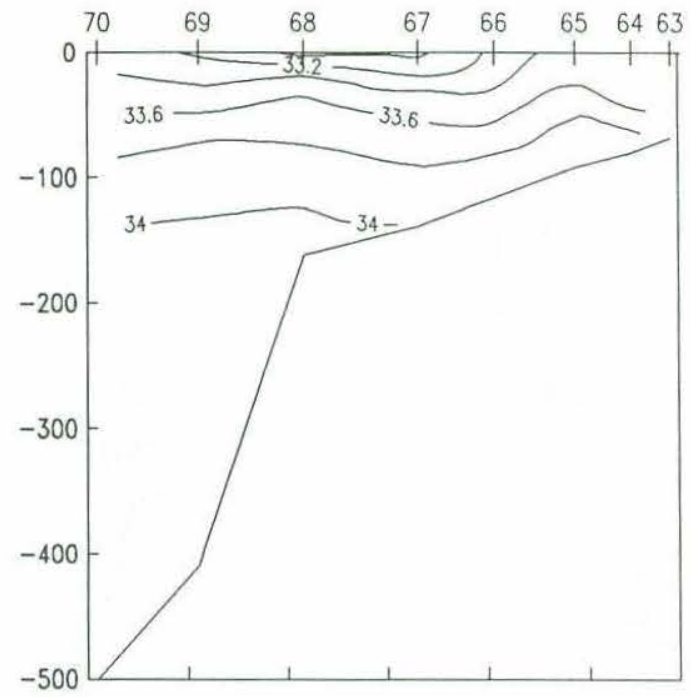

C SIGMA-T

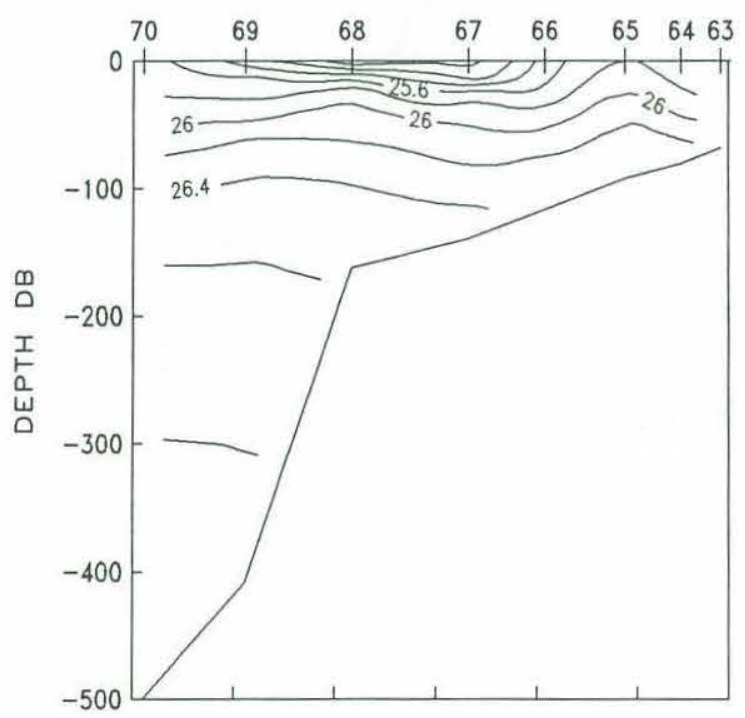




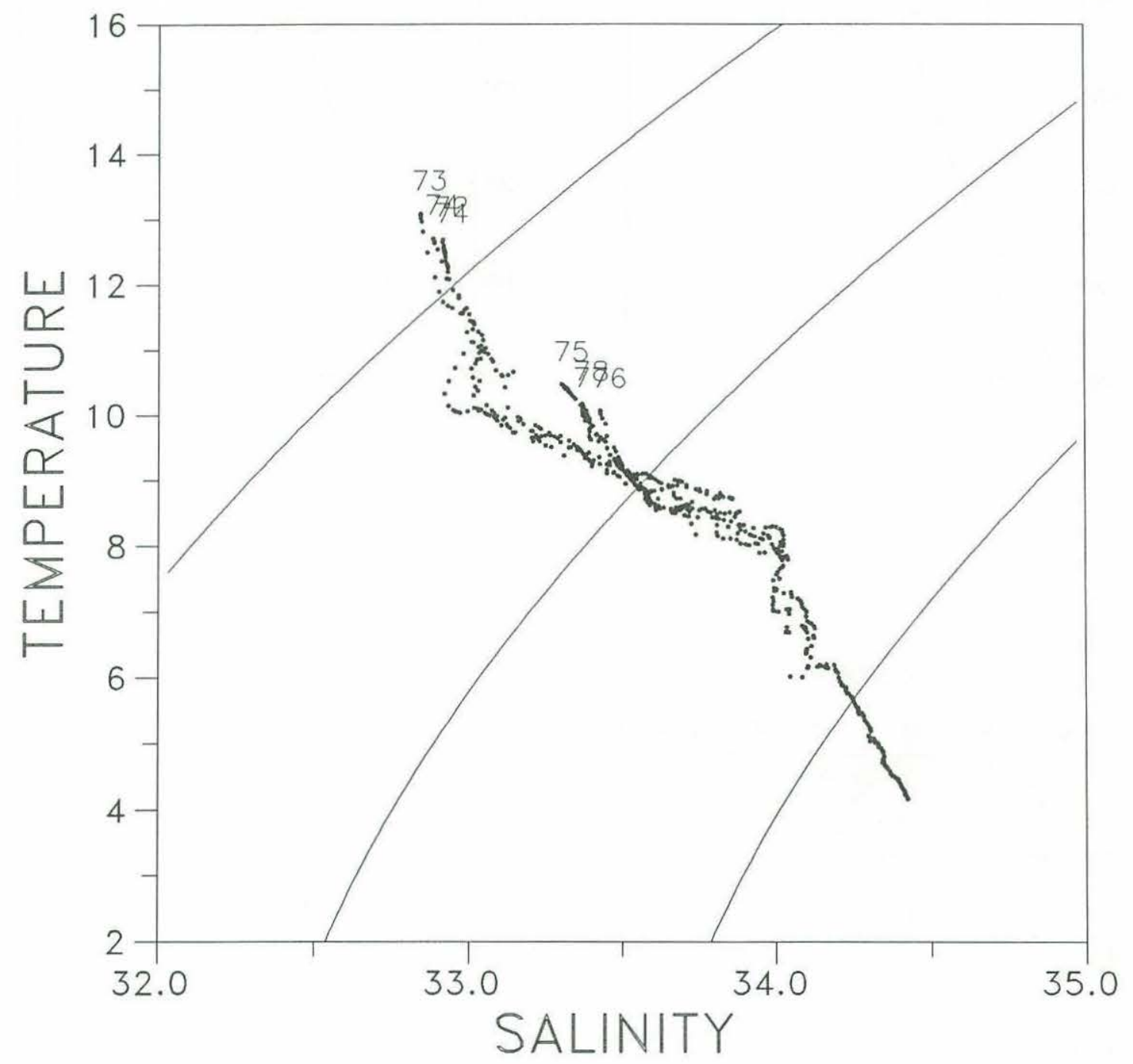


D TEMPERATURE

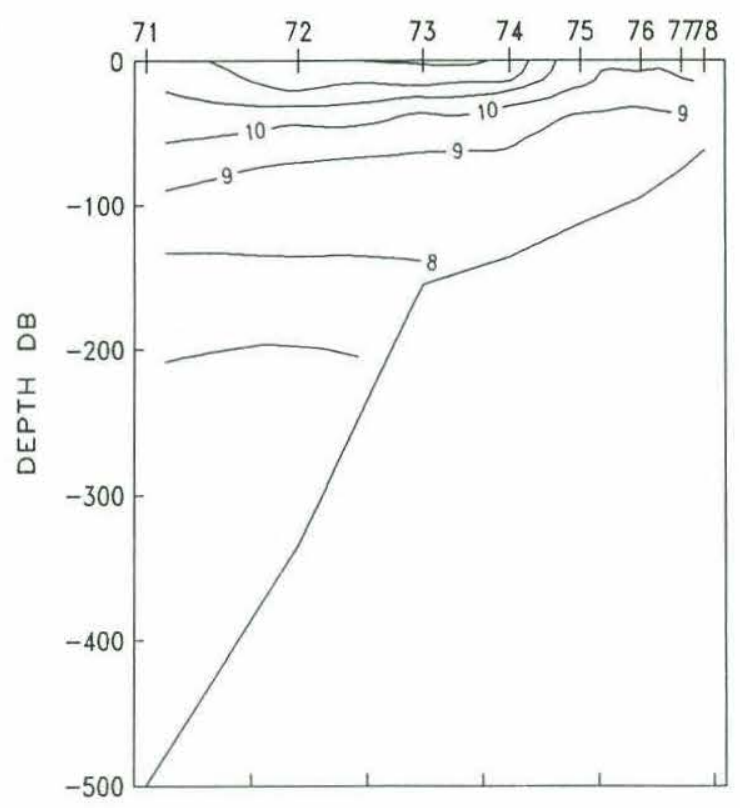

D SALINITY

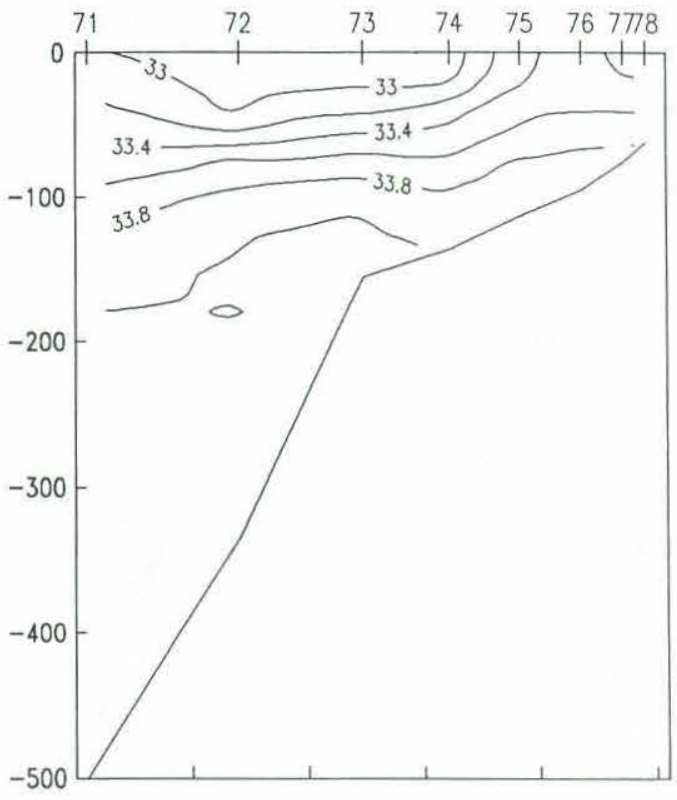

D SIGMA-T

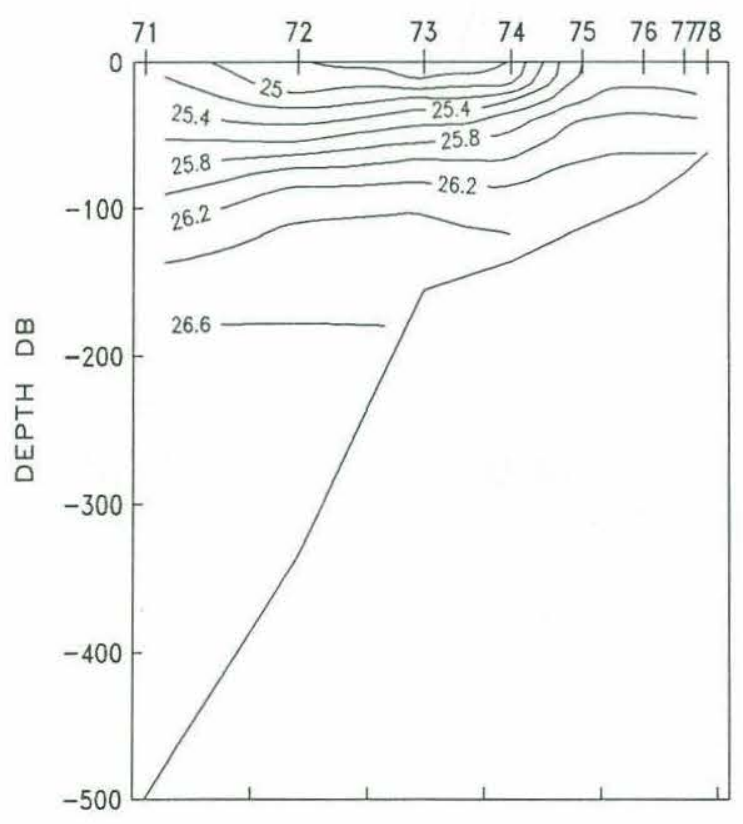




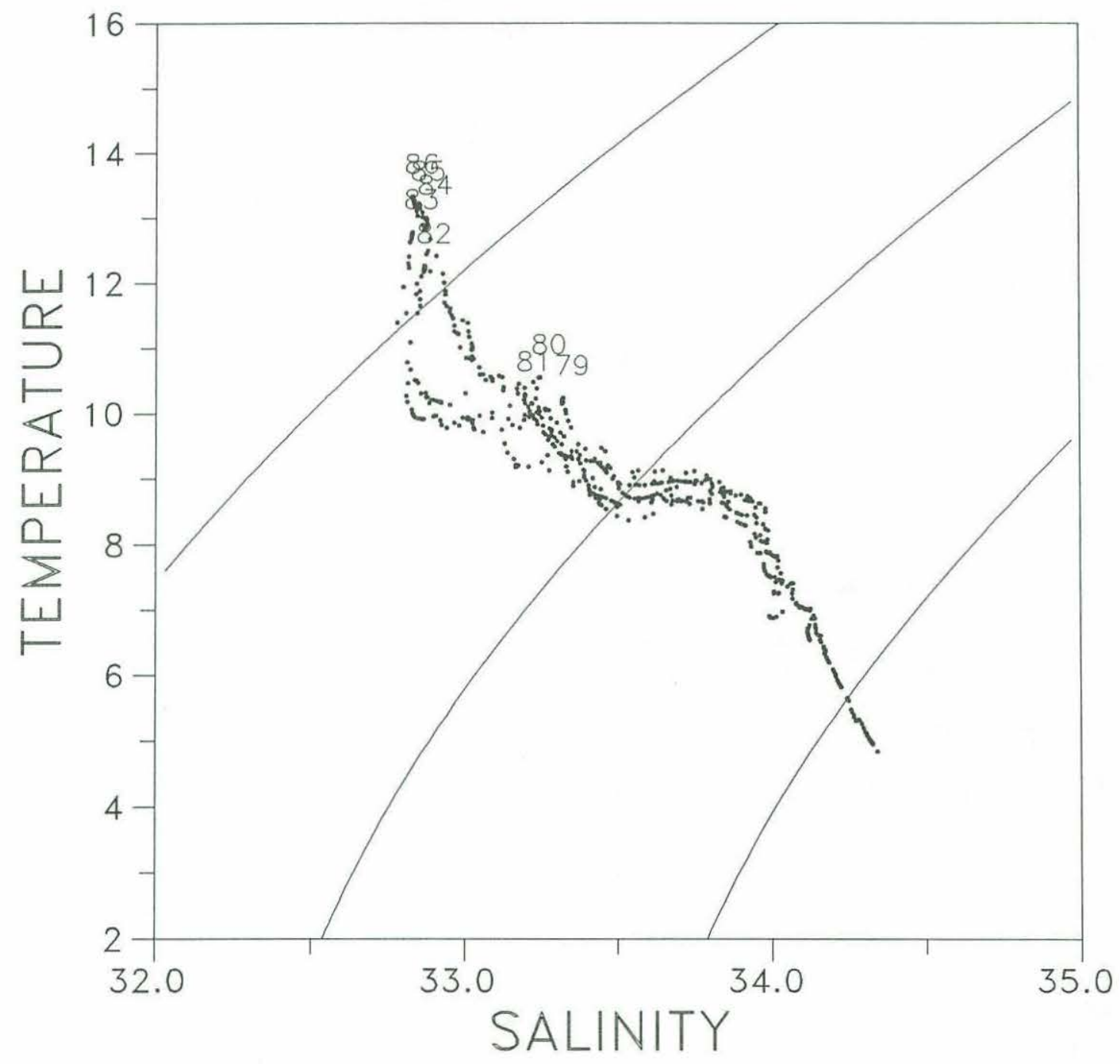



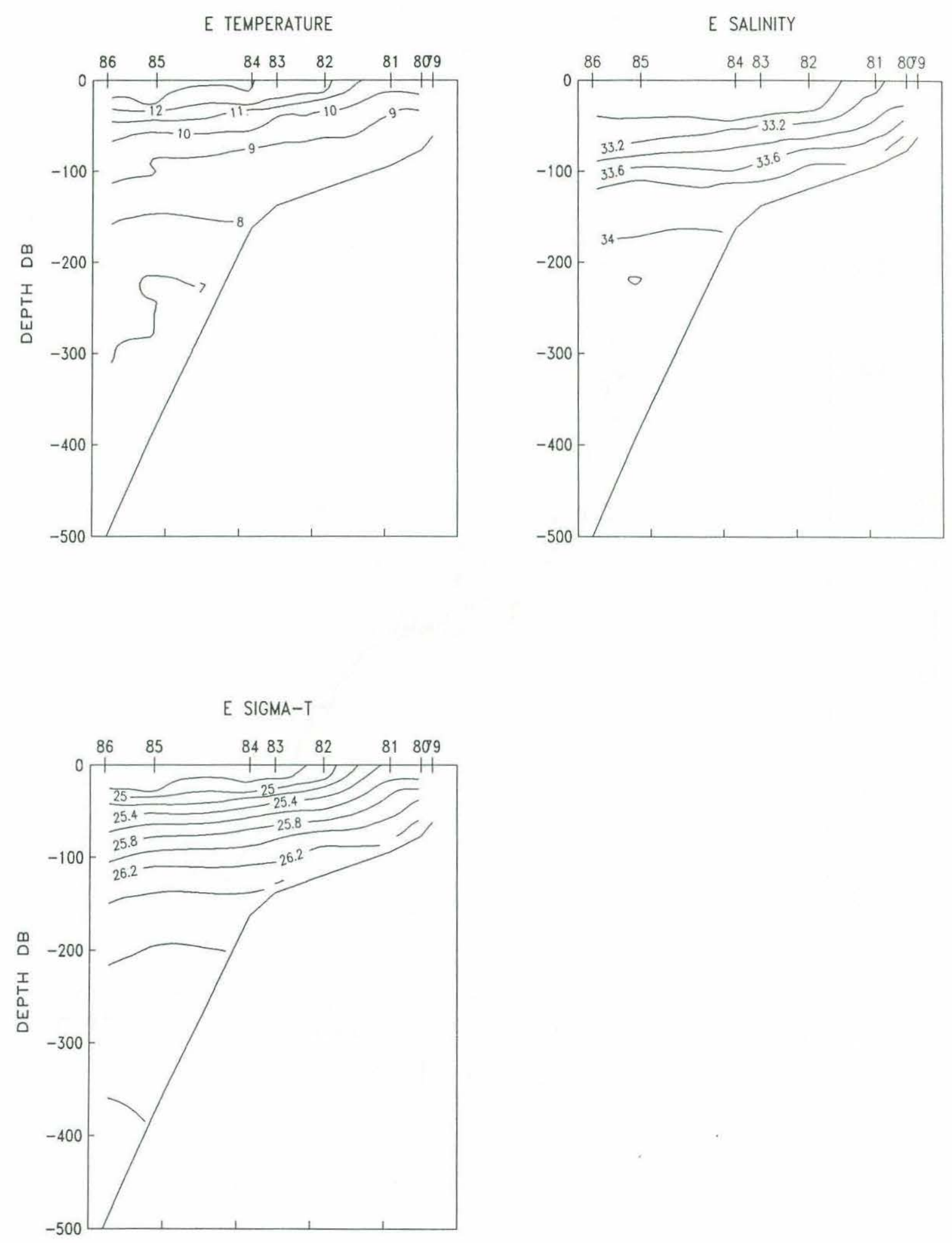


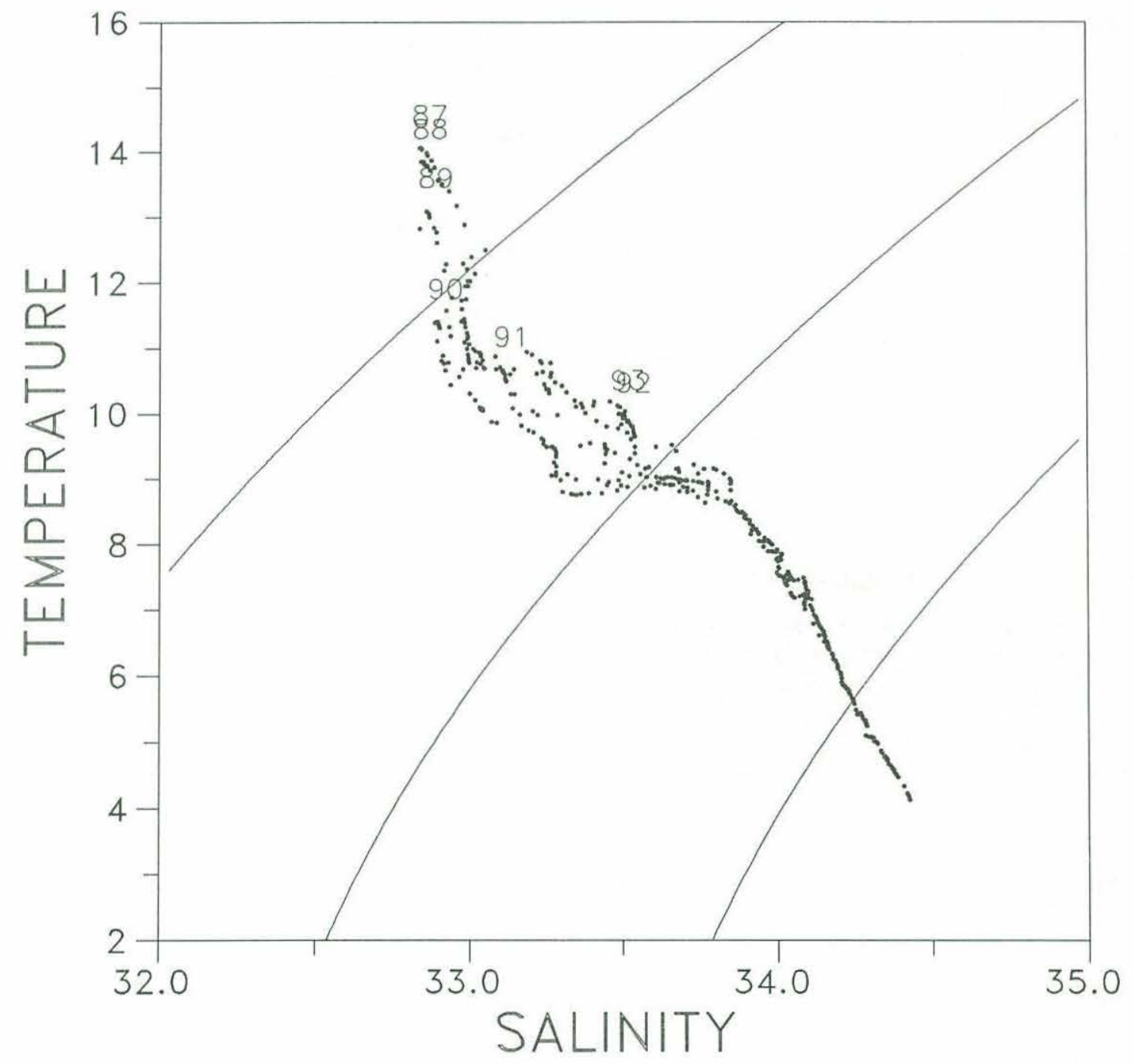


BB TEMPERATURE

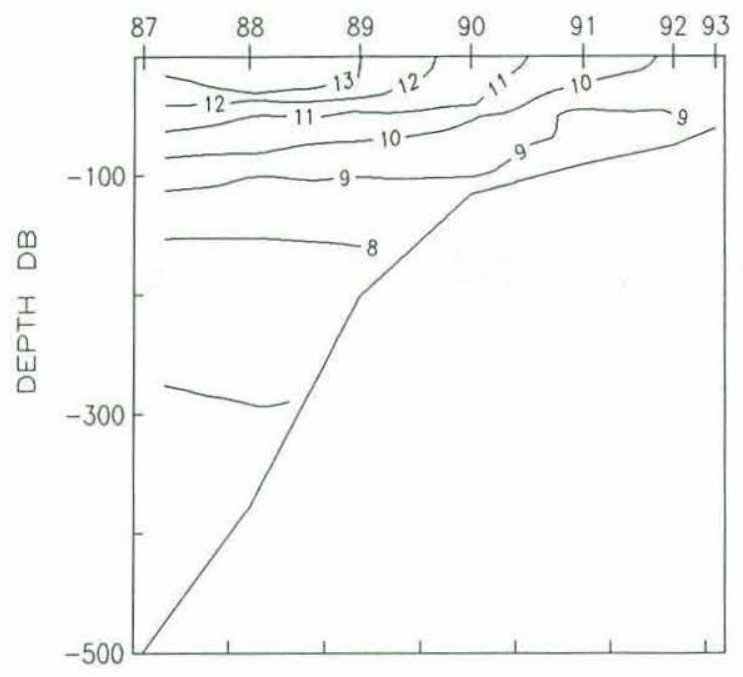

BB SALINITY

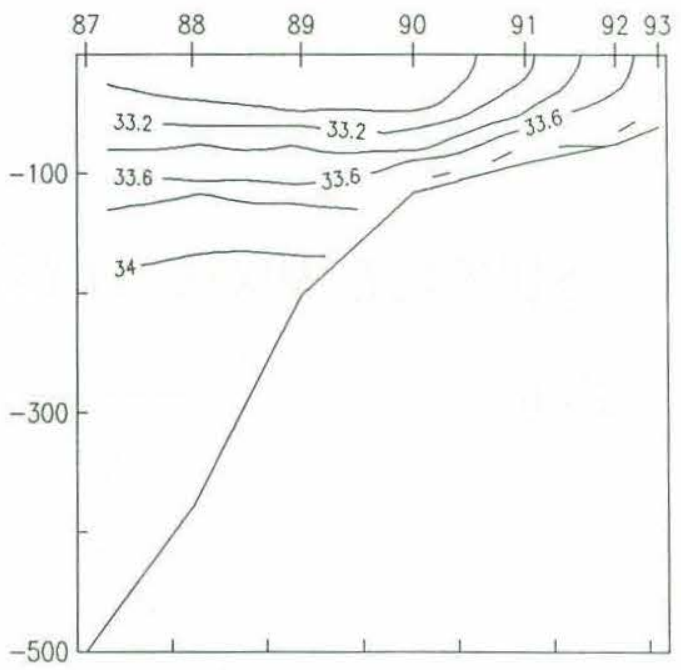

BB SIGMA-T

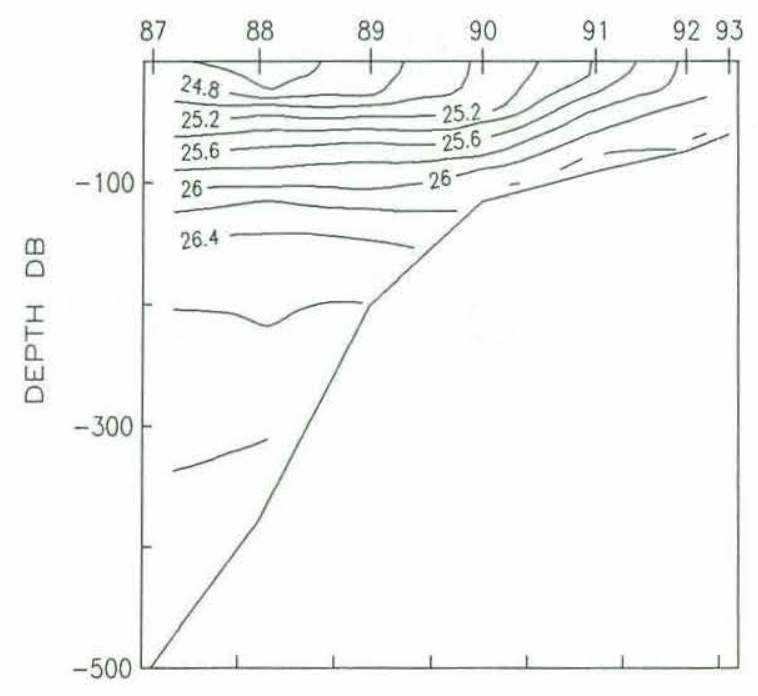




\section{SURFACE MIXED LAYER DEPTH M MAY 8-9 1989}

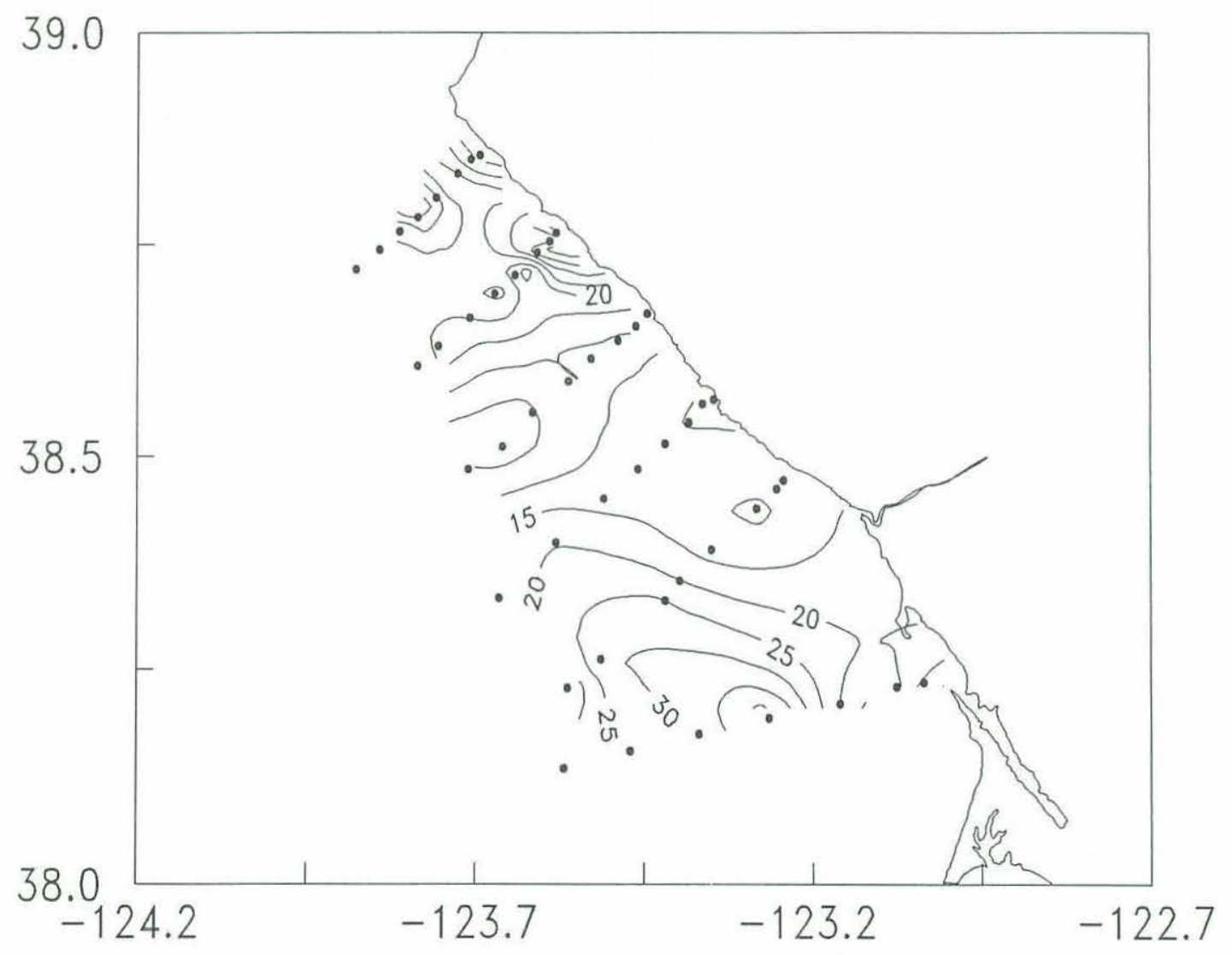


SURFACE TEMP MAY 8-9 1989

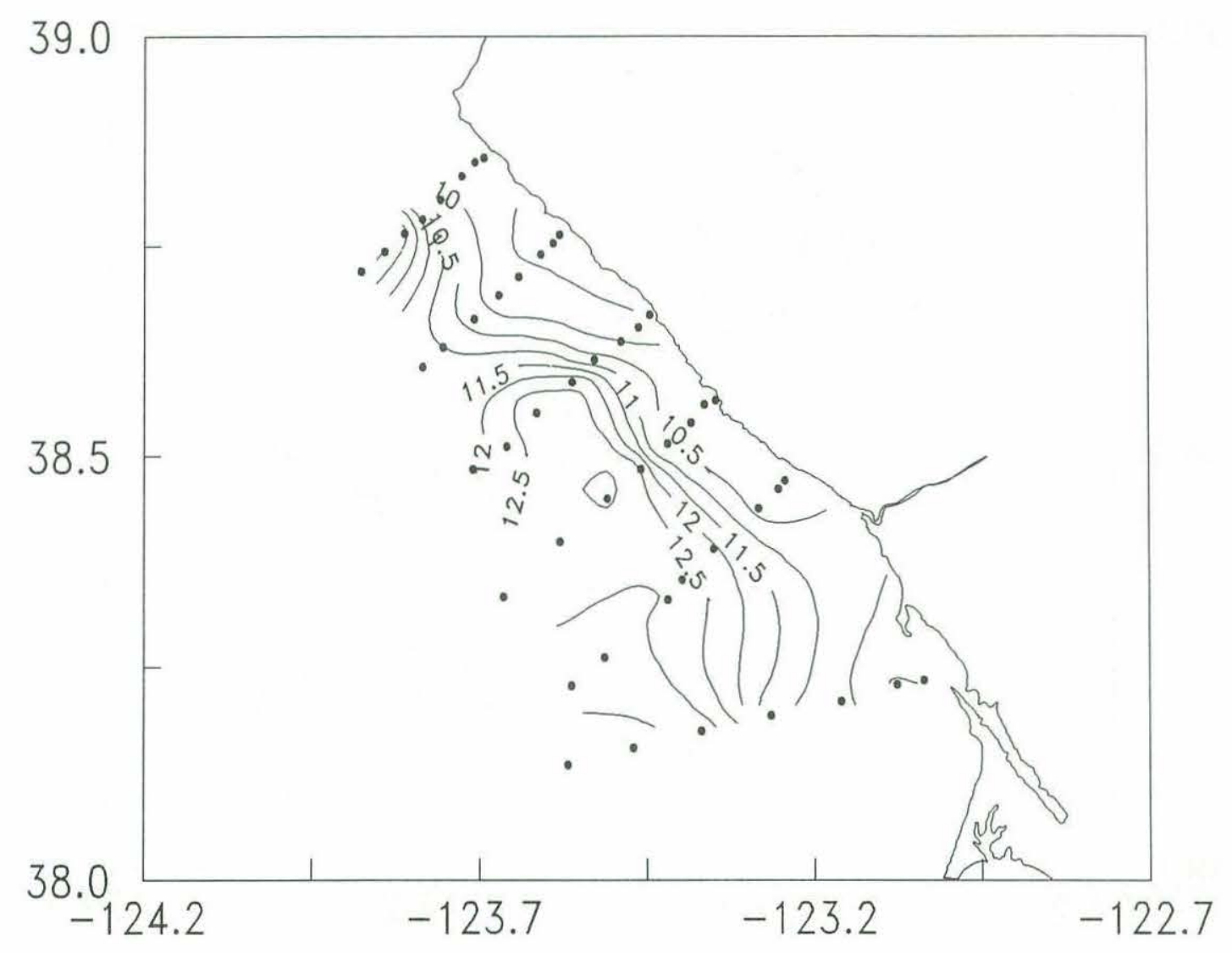


SURFACE SALINITY MAY 8-9 1989

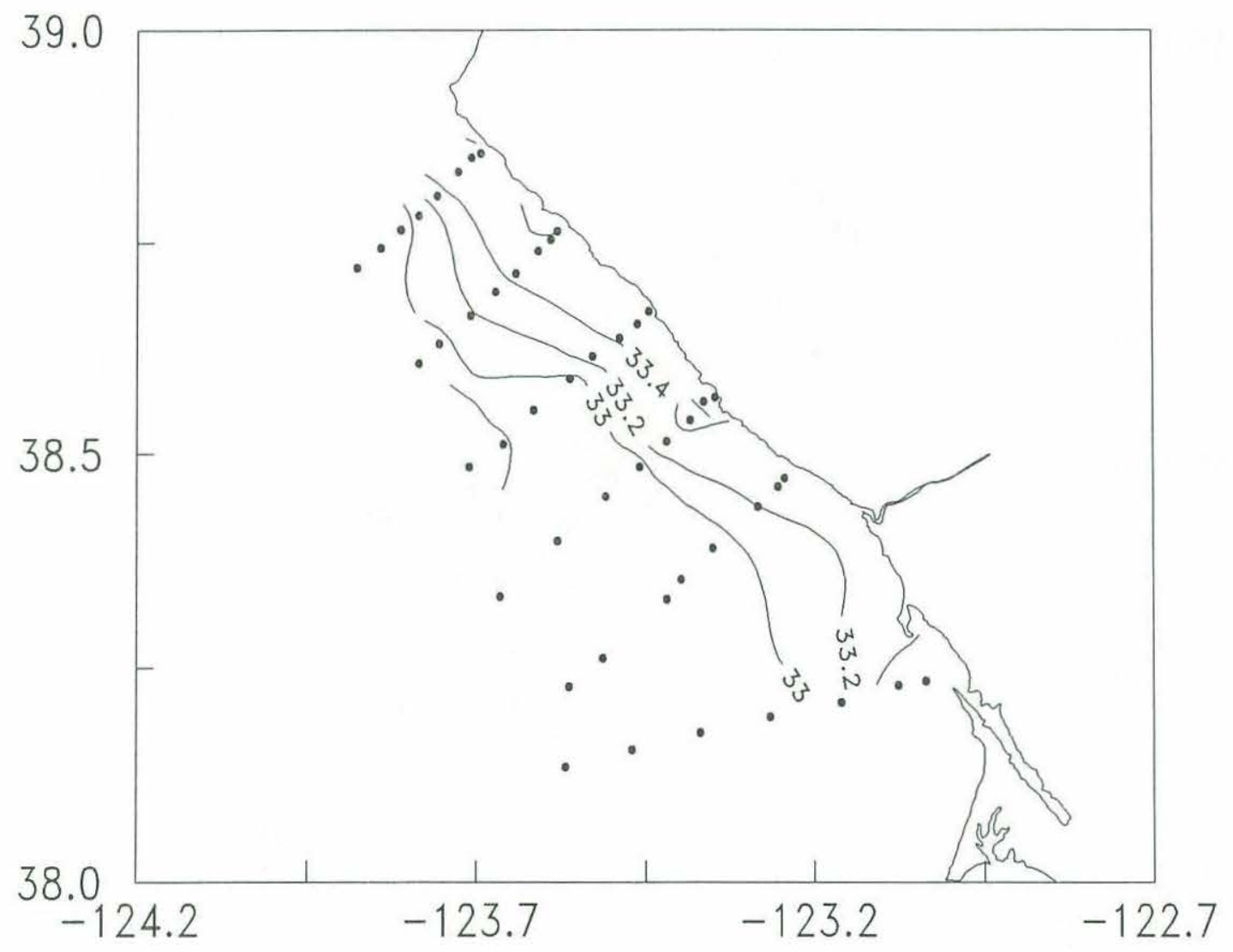


SURFACE SIGMA-T MAY 8-9 1989

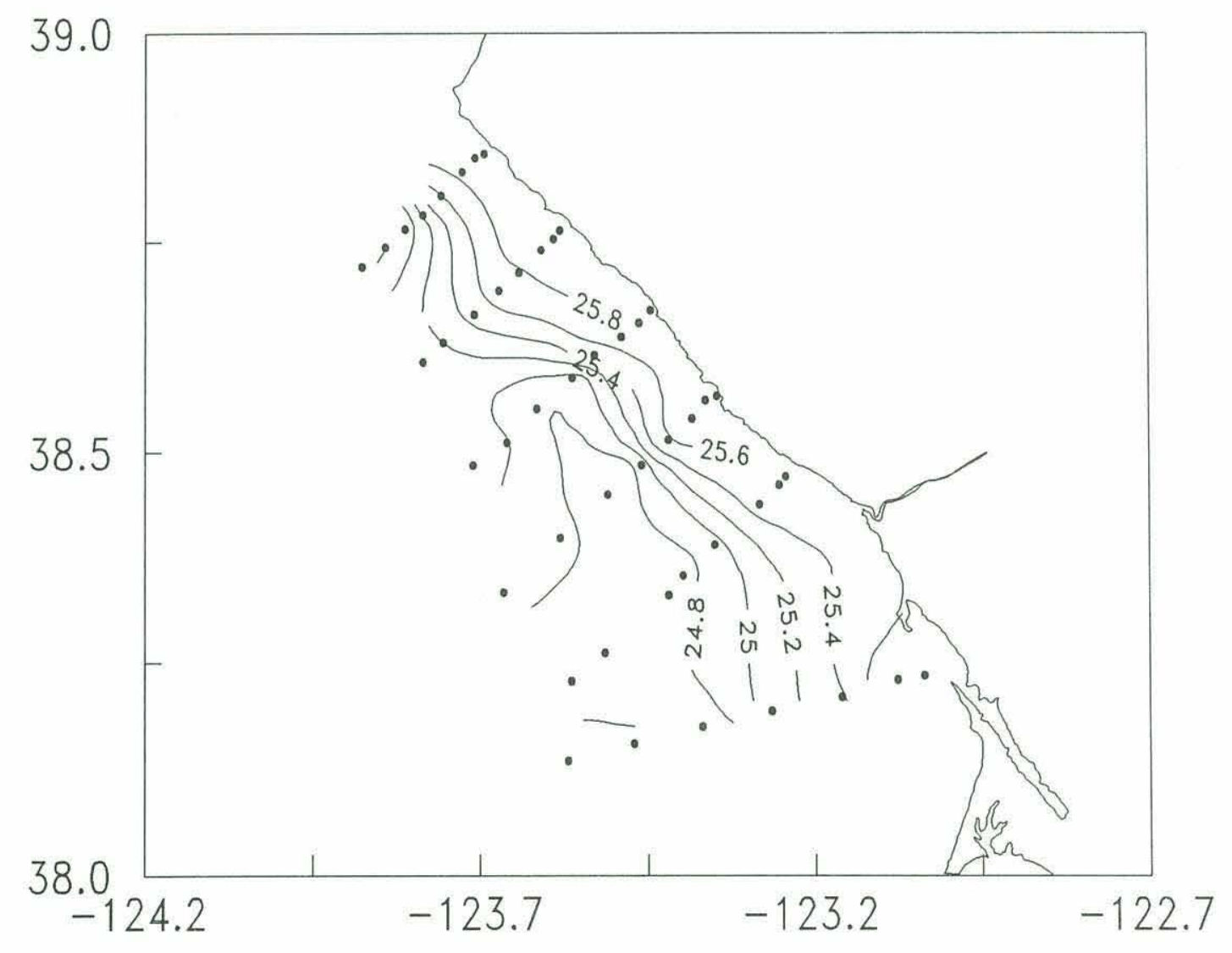




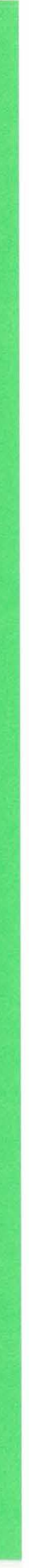




\section{Section C - May 10, 1989 Stations 94 to 100}



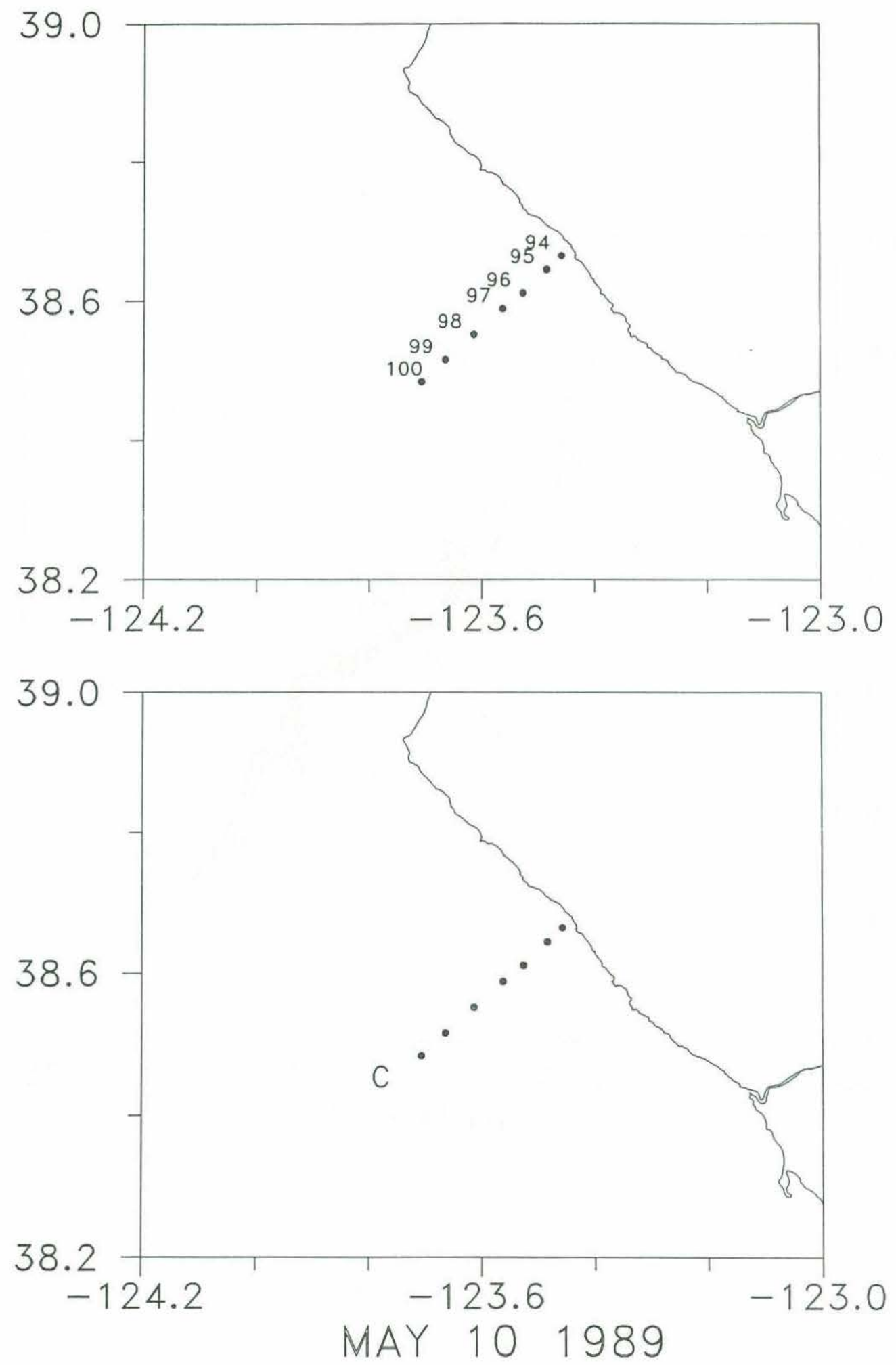


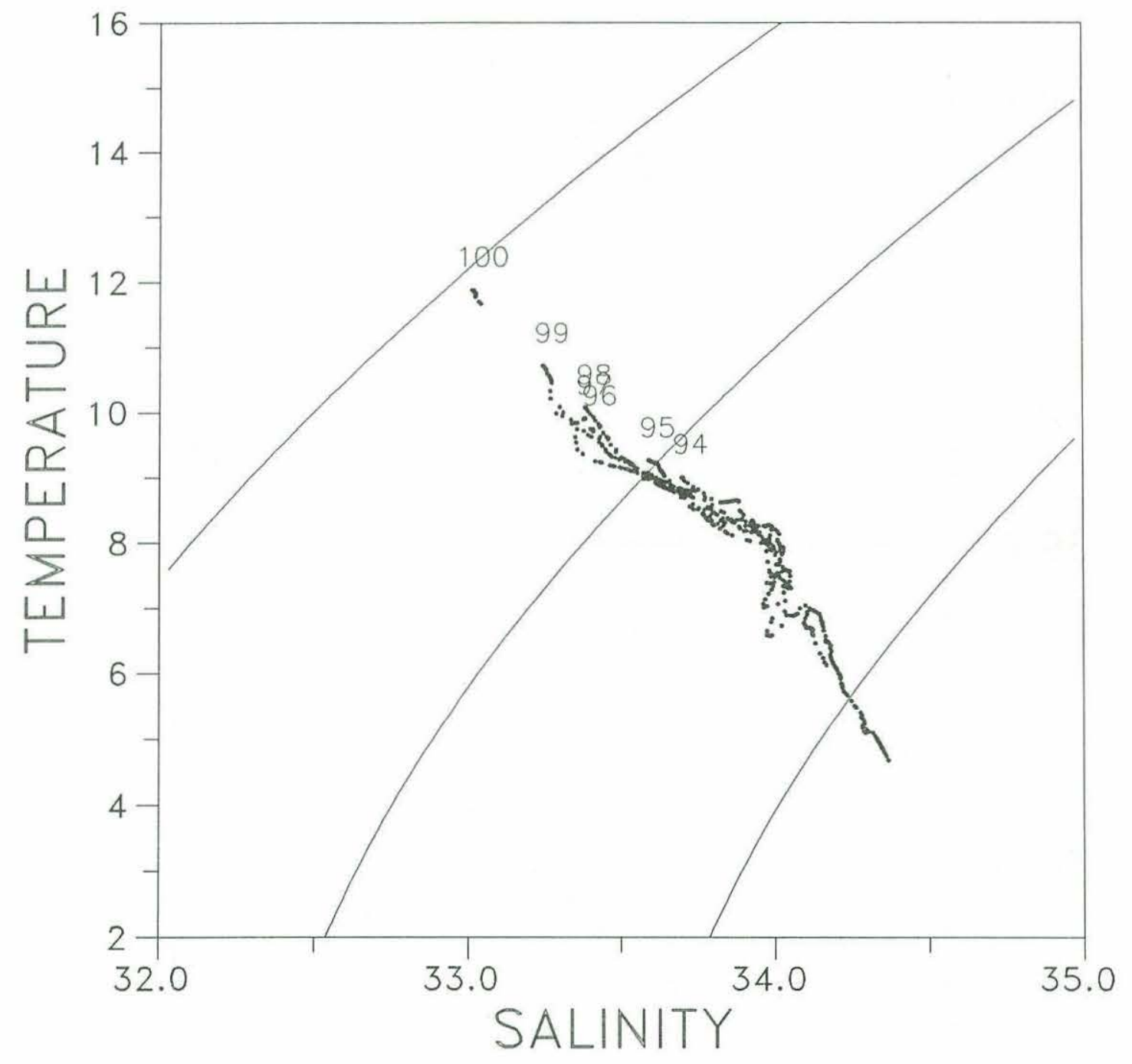



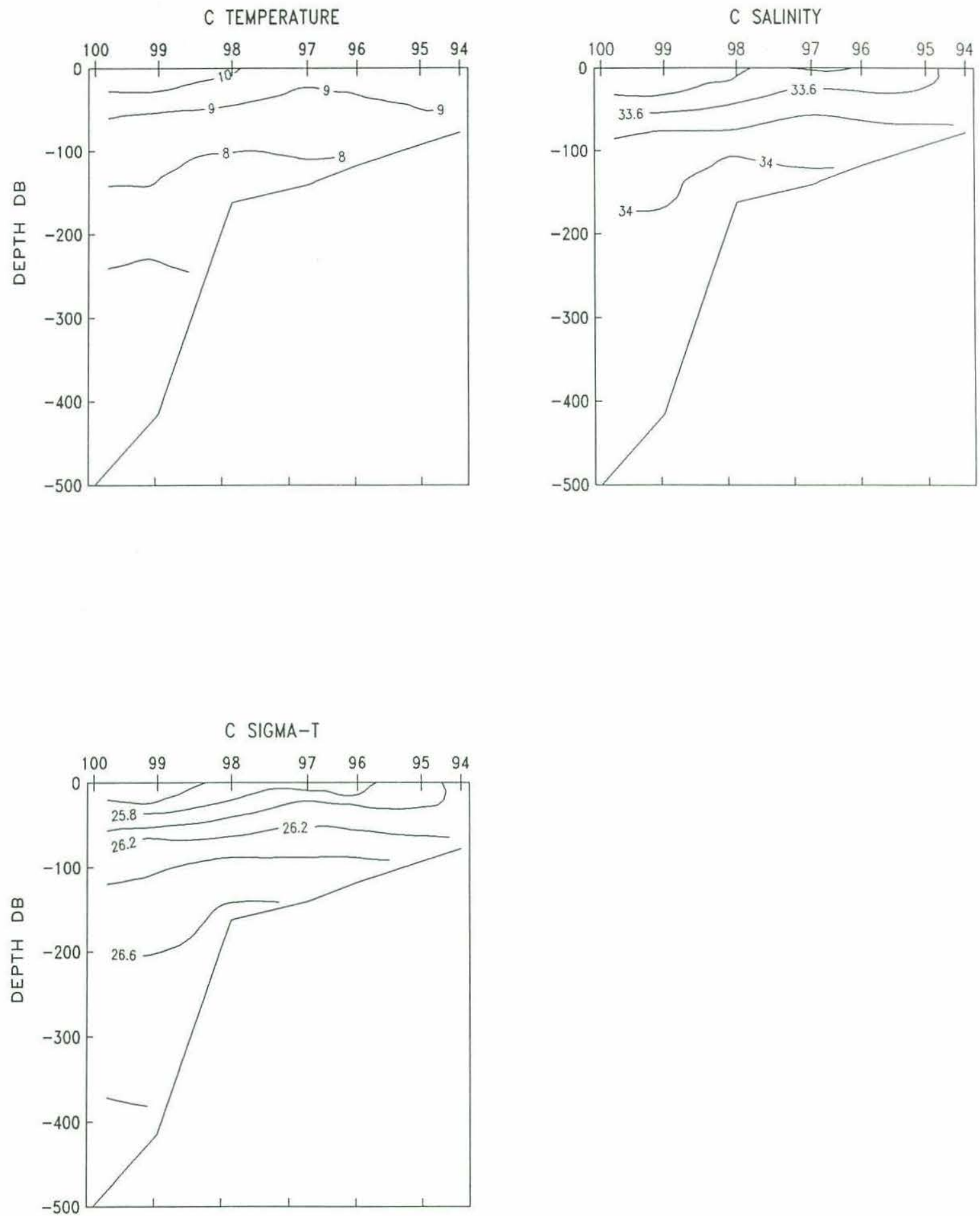

D. Section PL - May 11, 1989 - Stations 123 to 128 

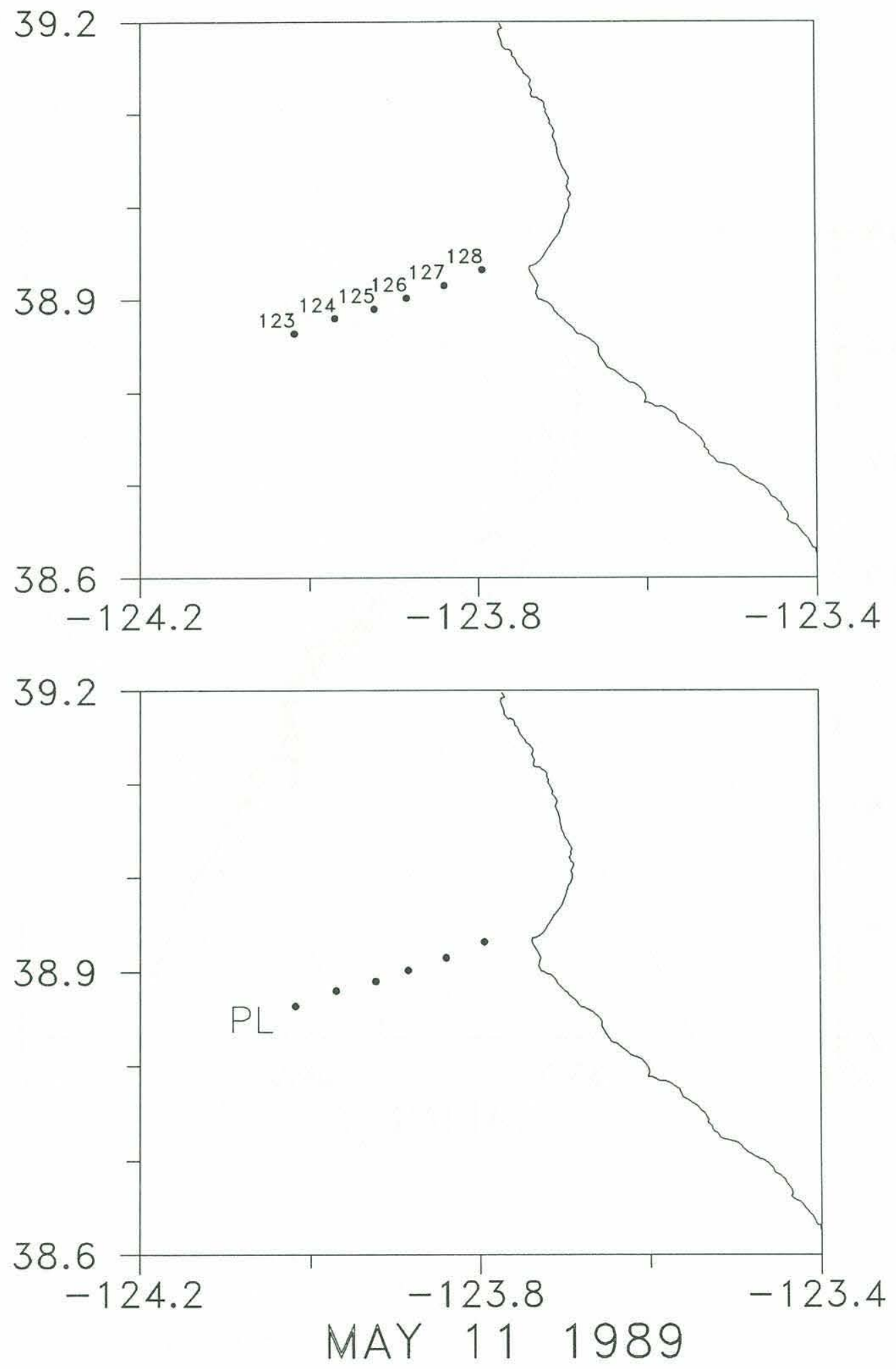


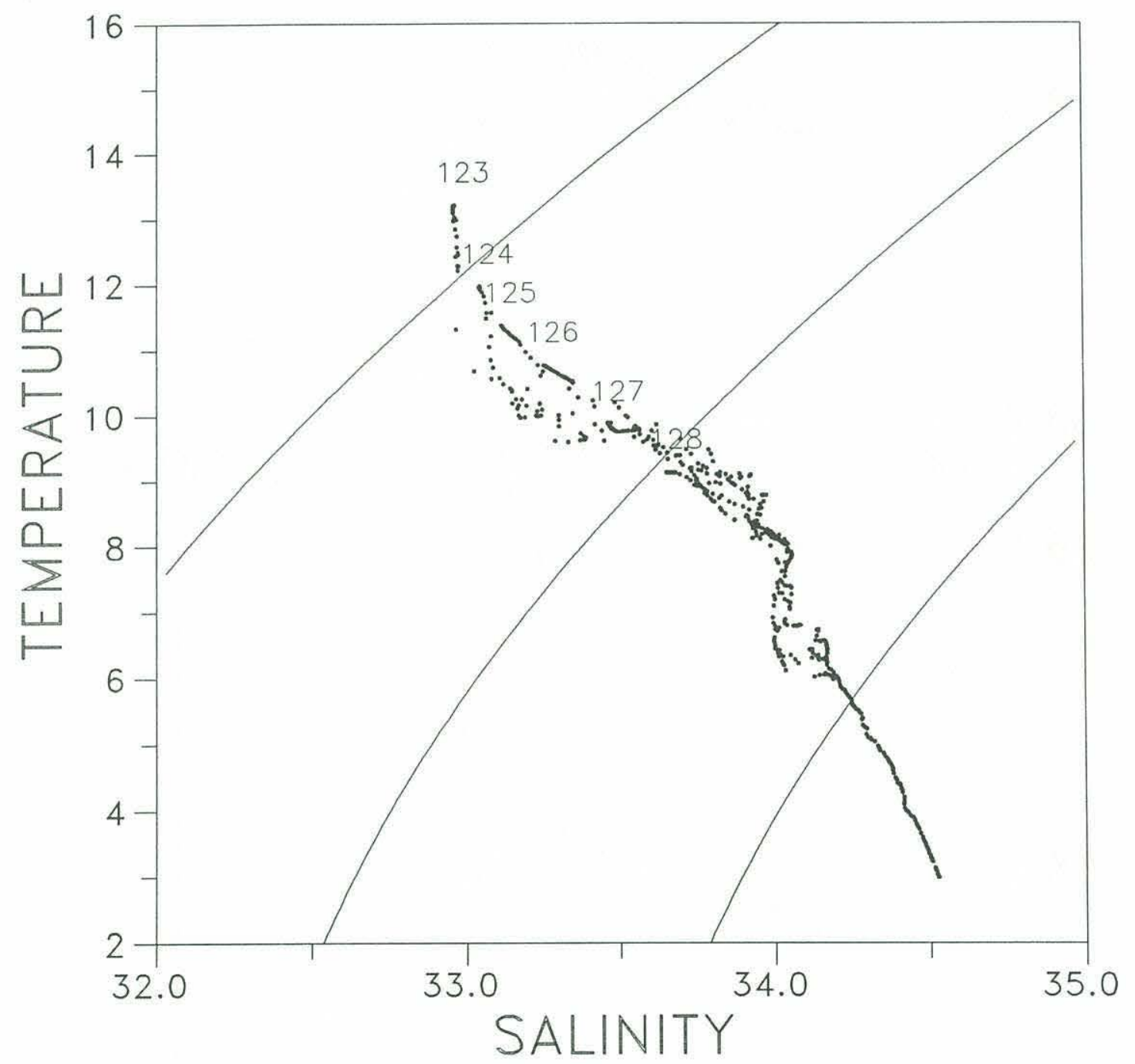


PL TEMPERATURE

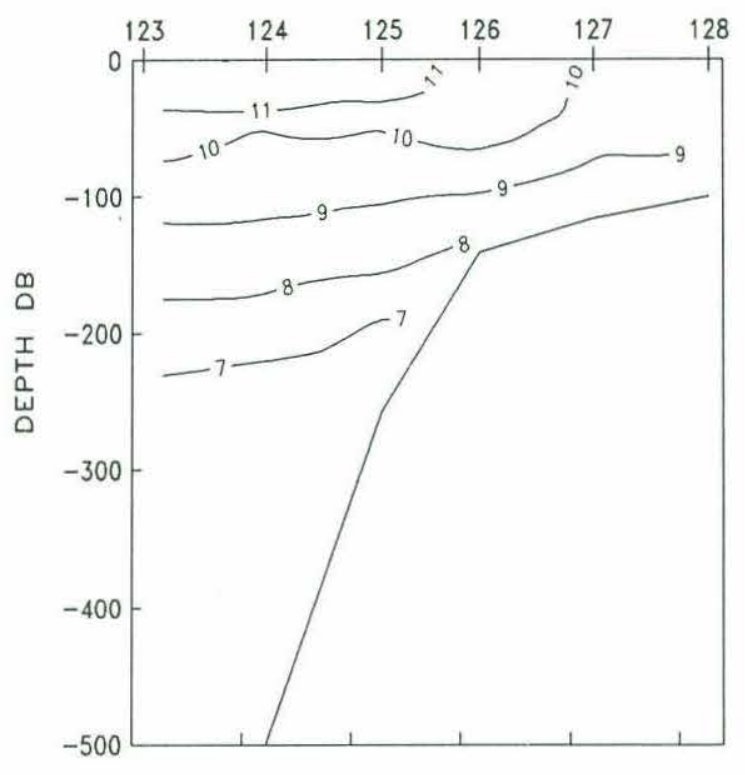

PL SIGMA-T

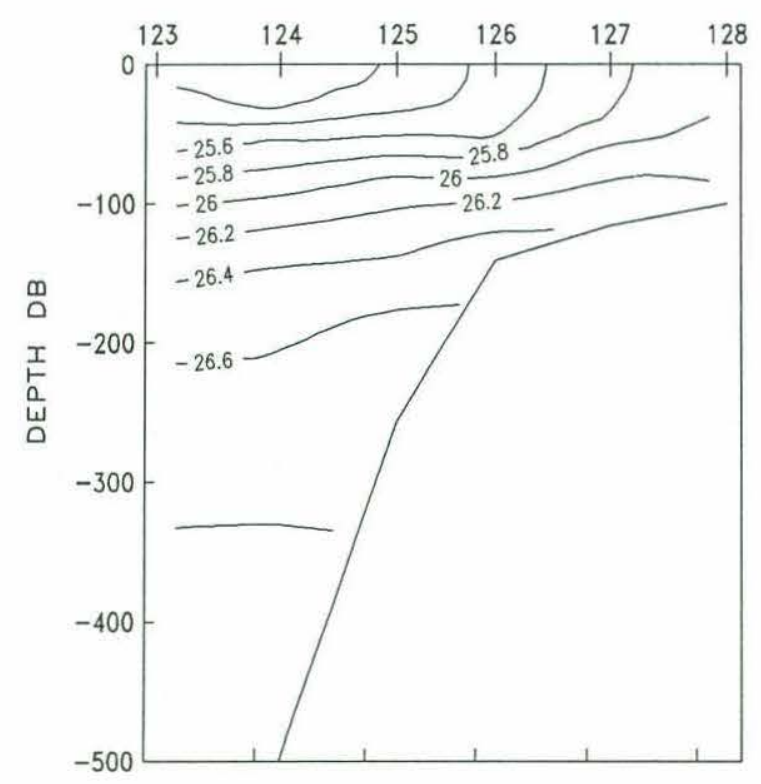

PL SALINITY

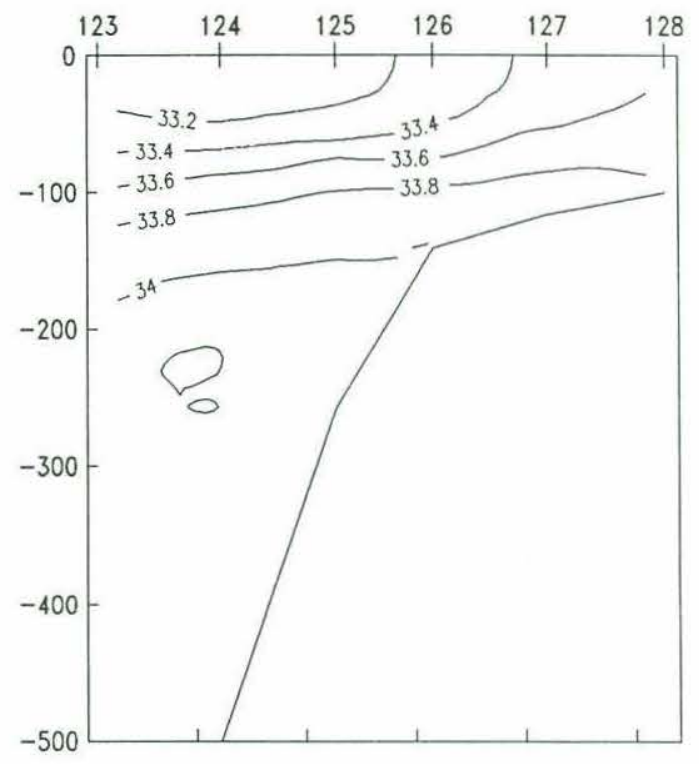





\section{E. Small-Scale Survey - May 11-13, 1989 Stations 129 to 170}



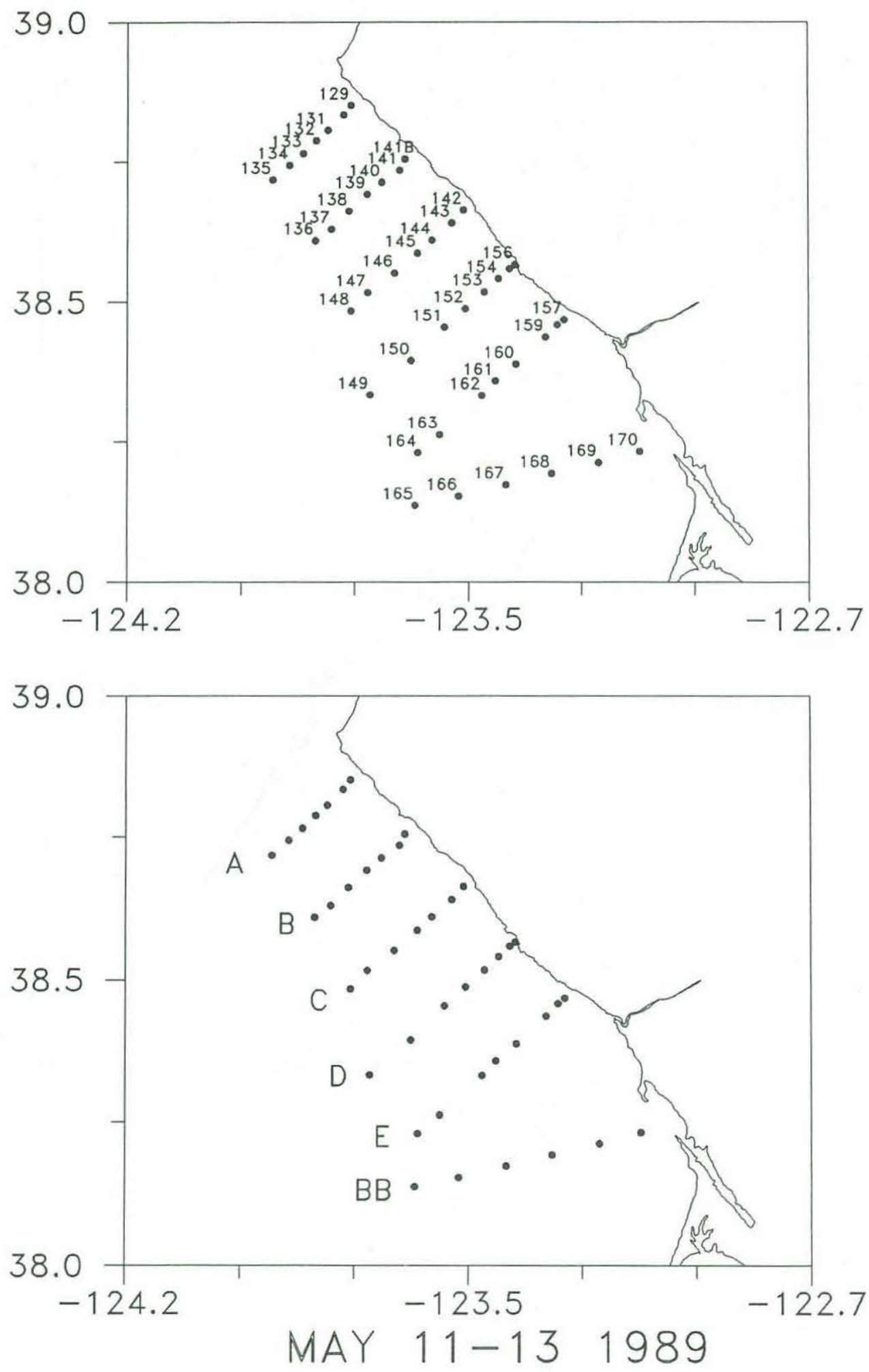


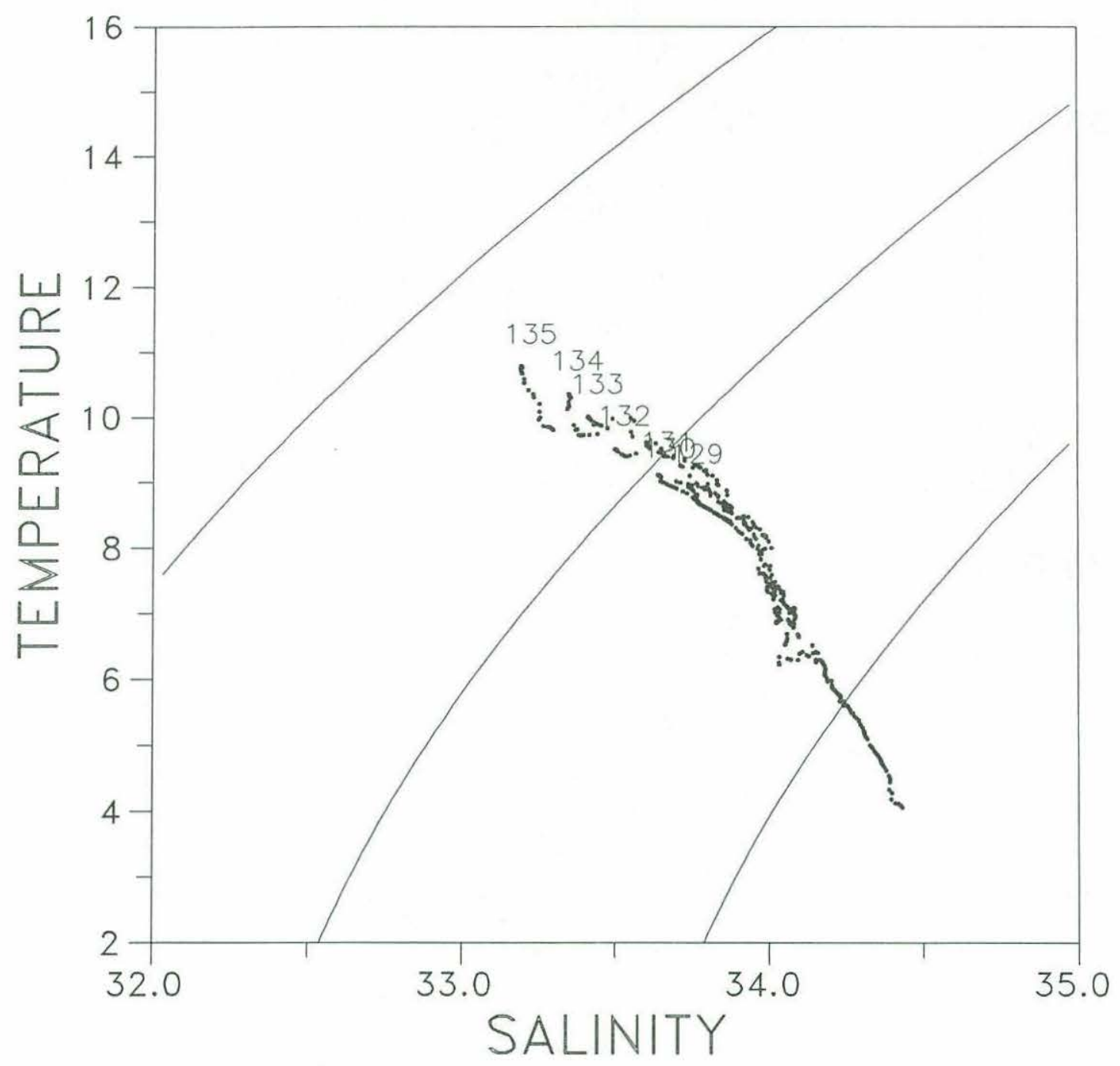



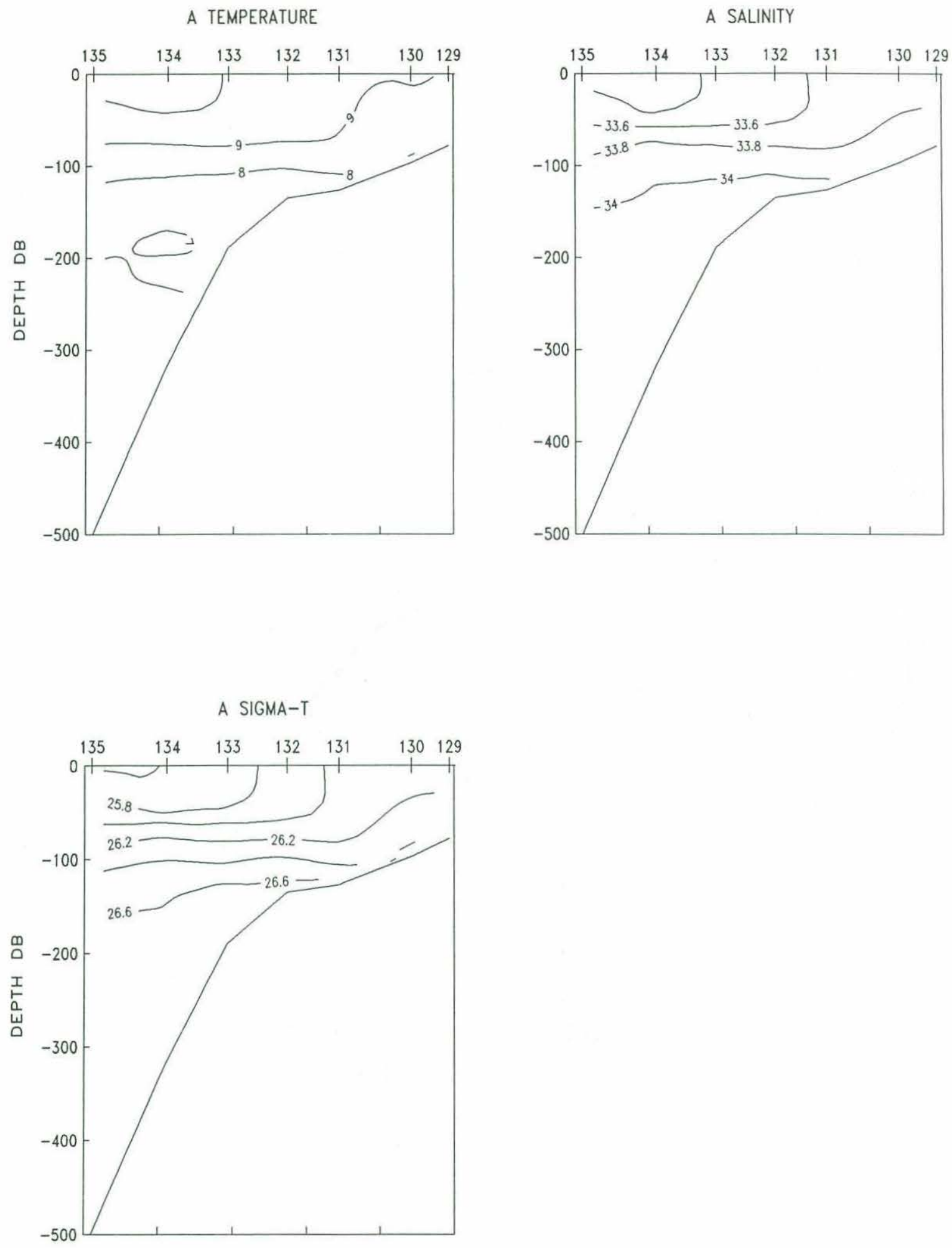


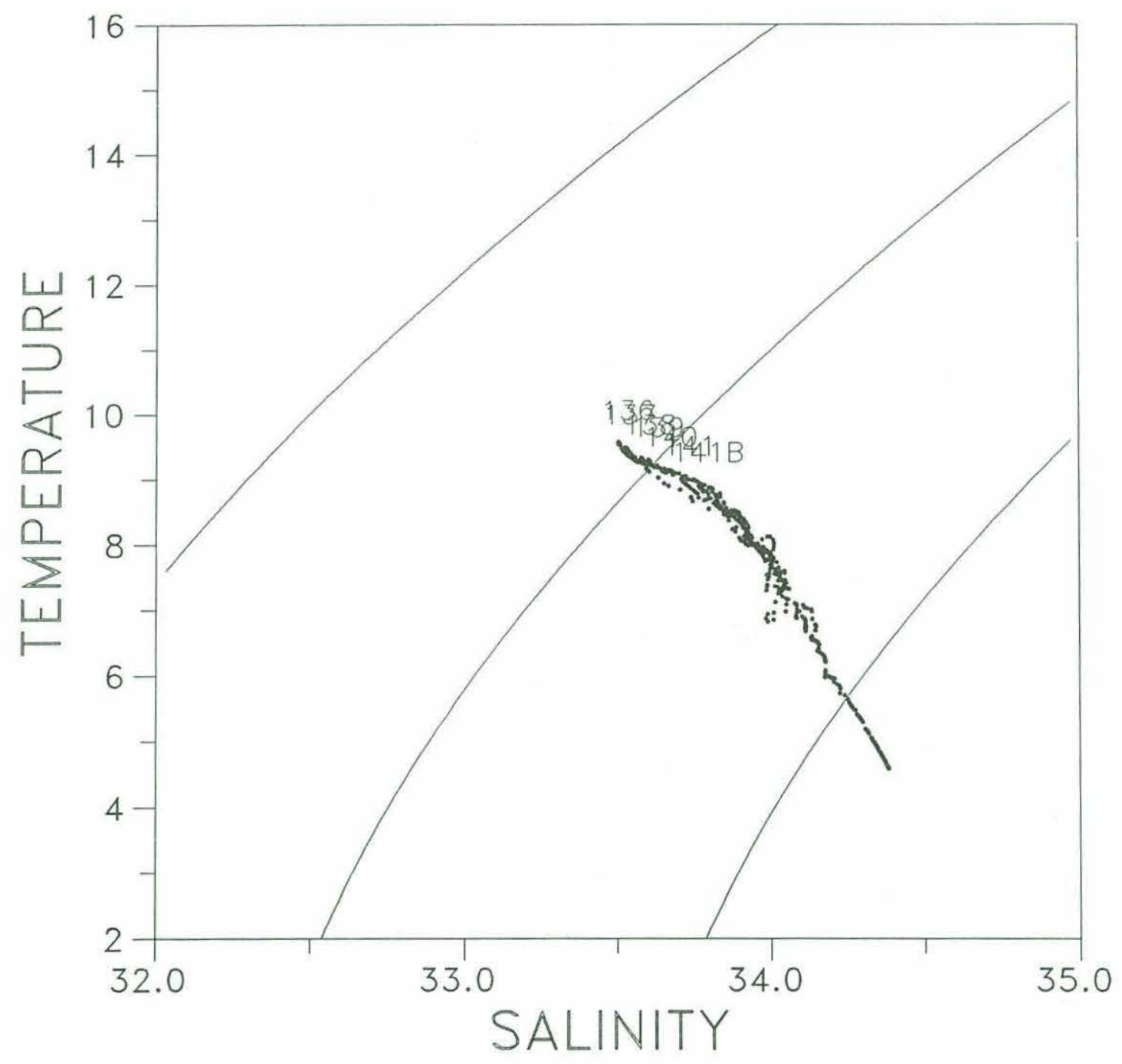



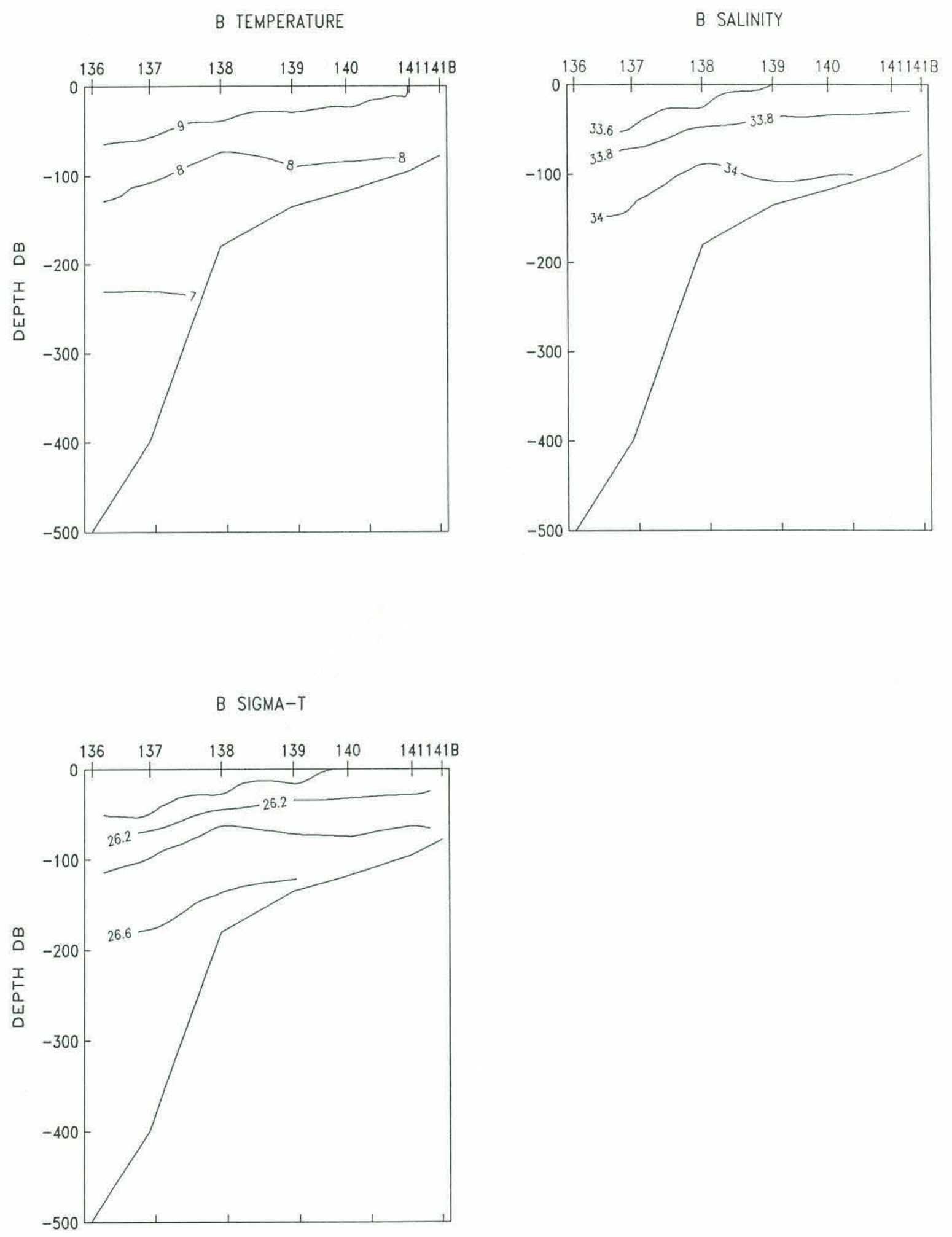


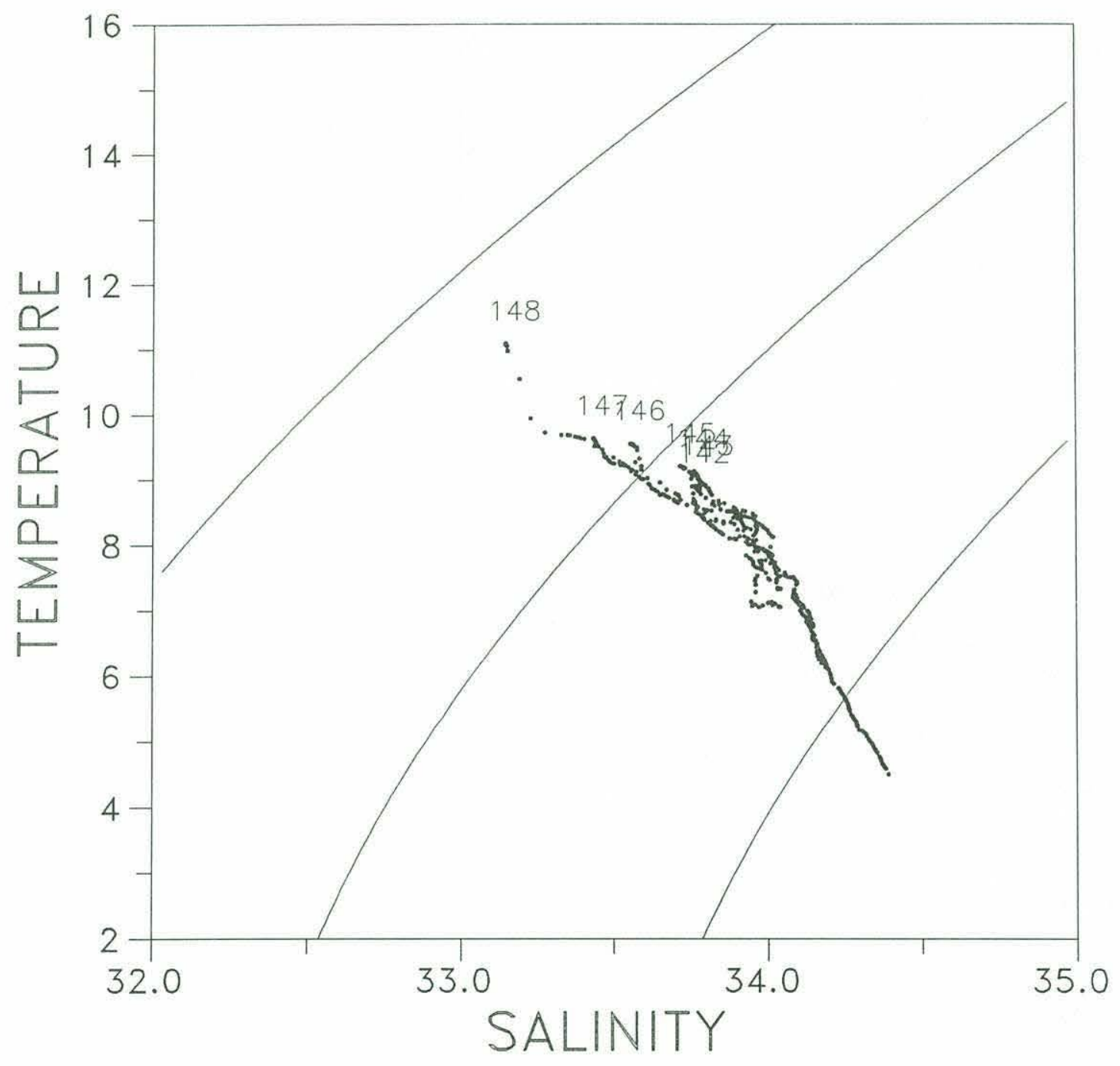



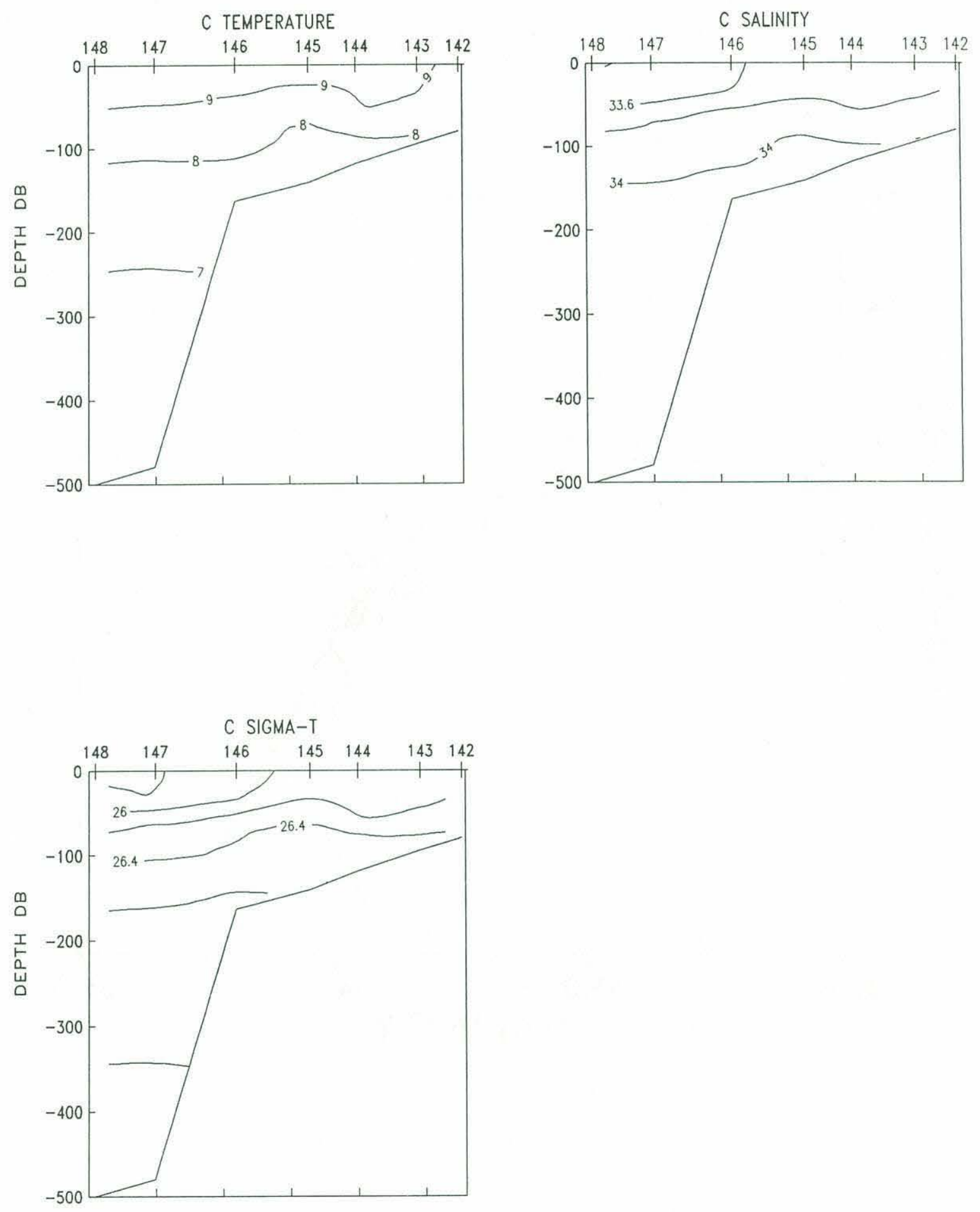


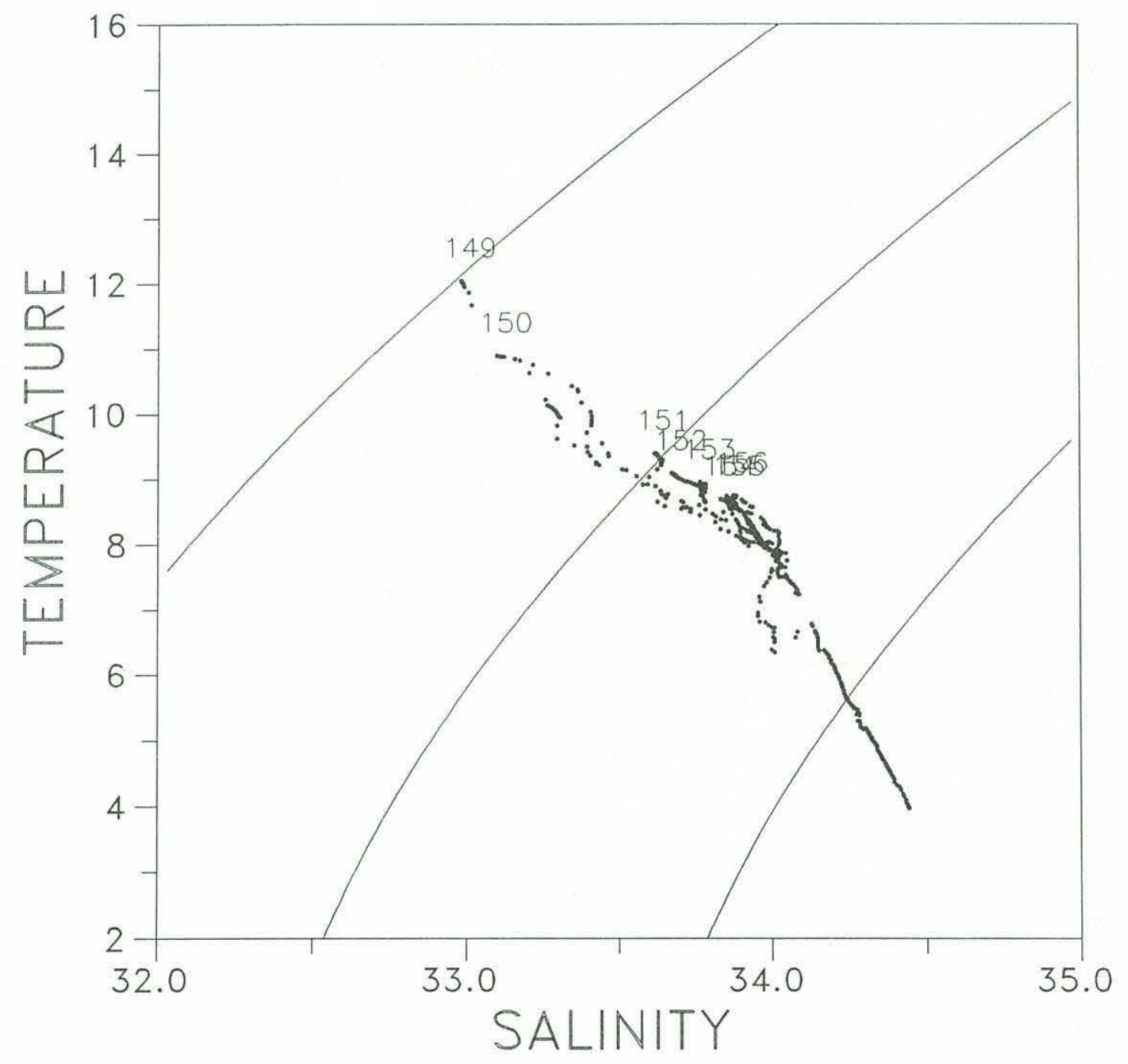



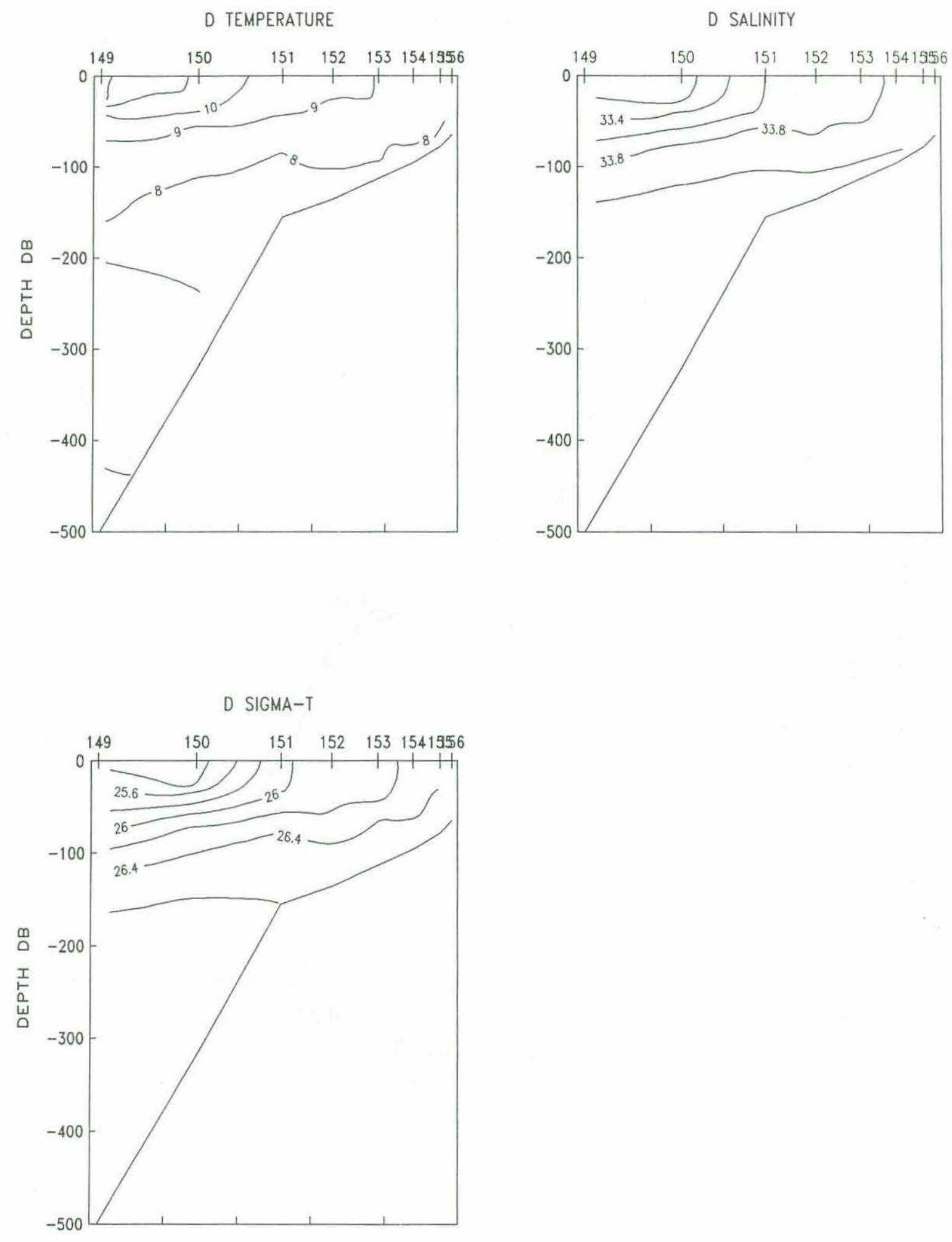


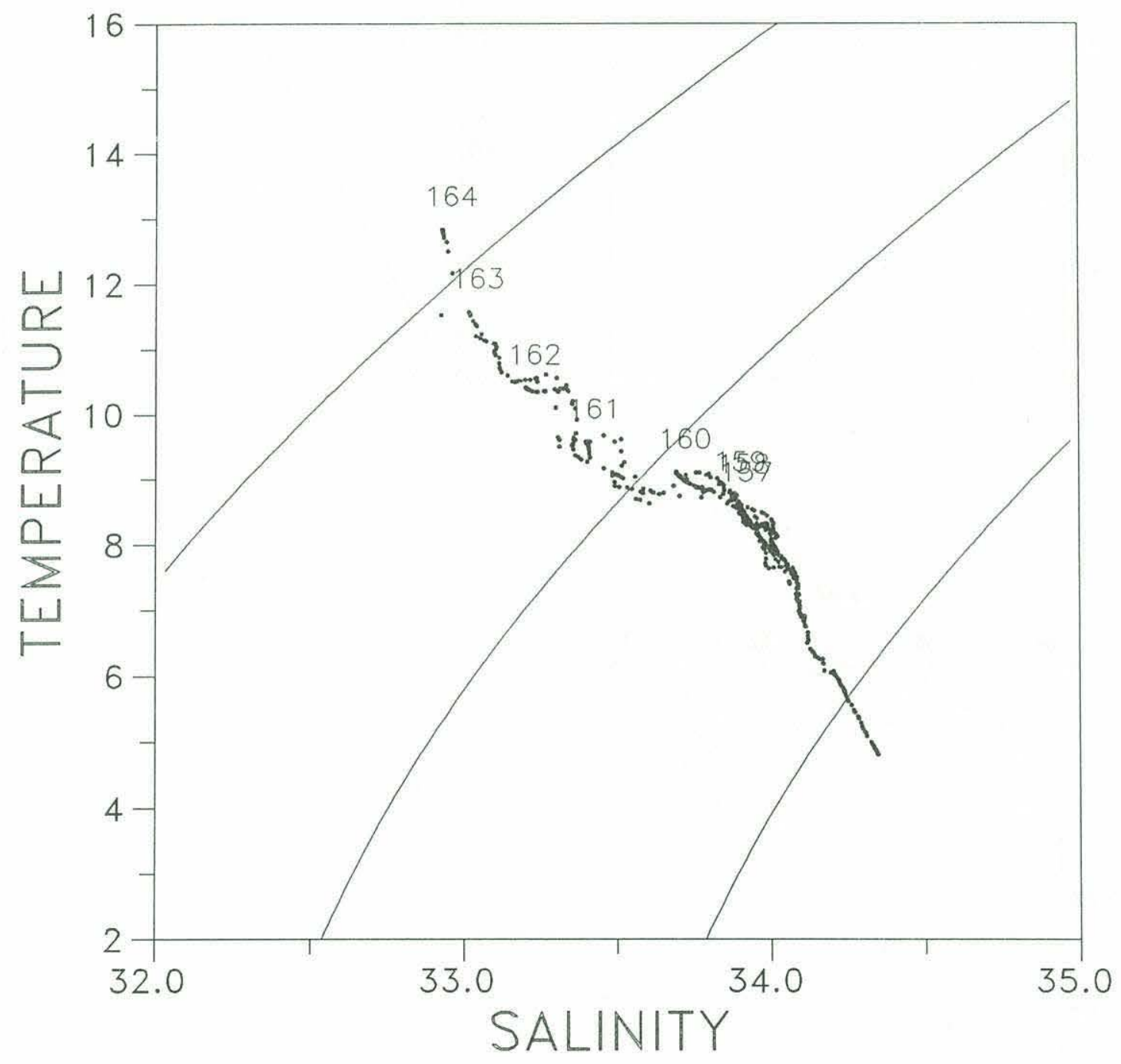




\section{E TEMPERATURE}

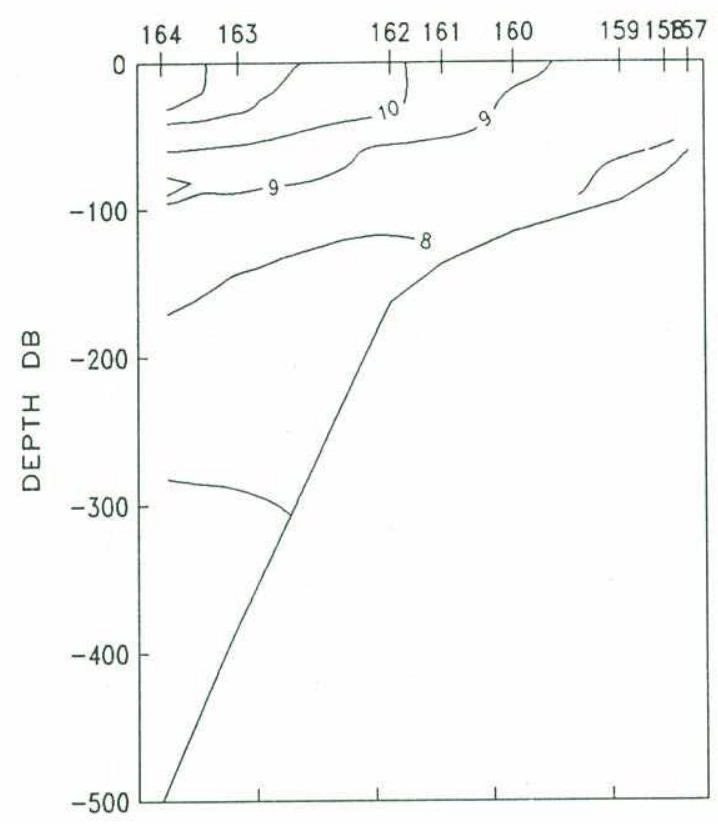

E SALINITY

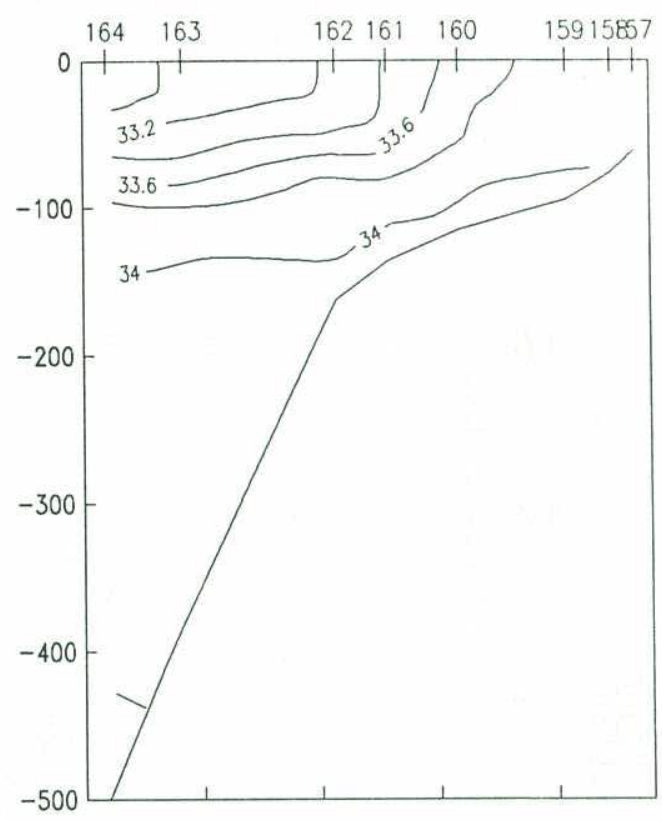

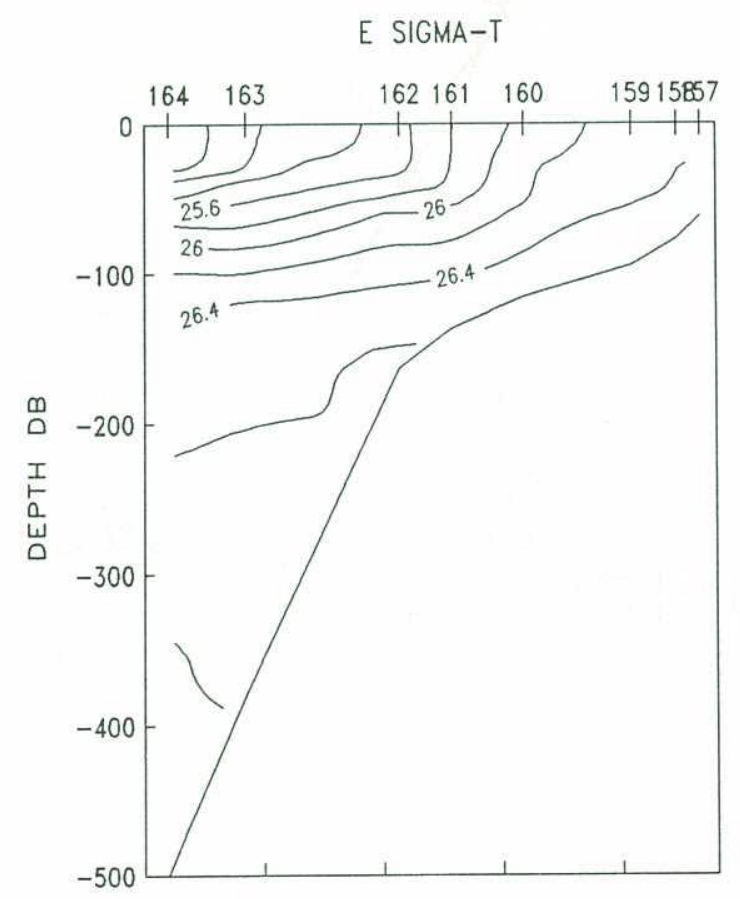




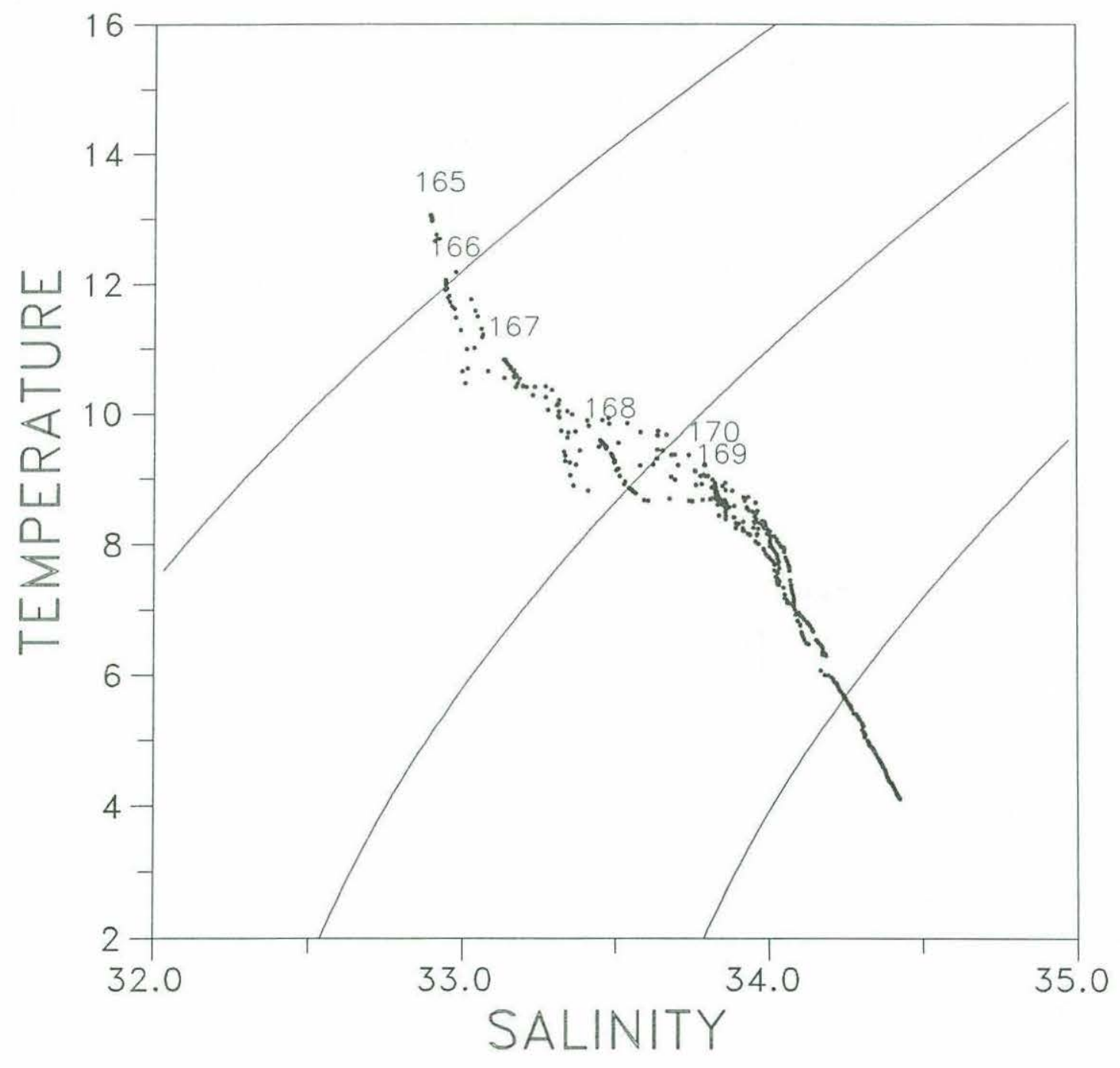


BB TEMPERATURE

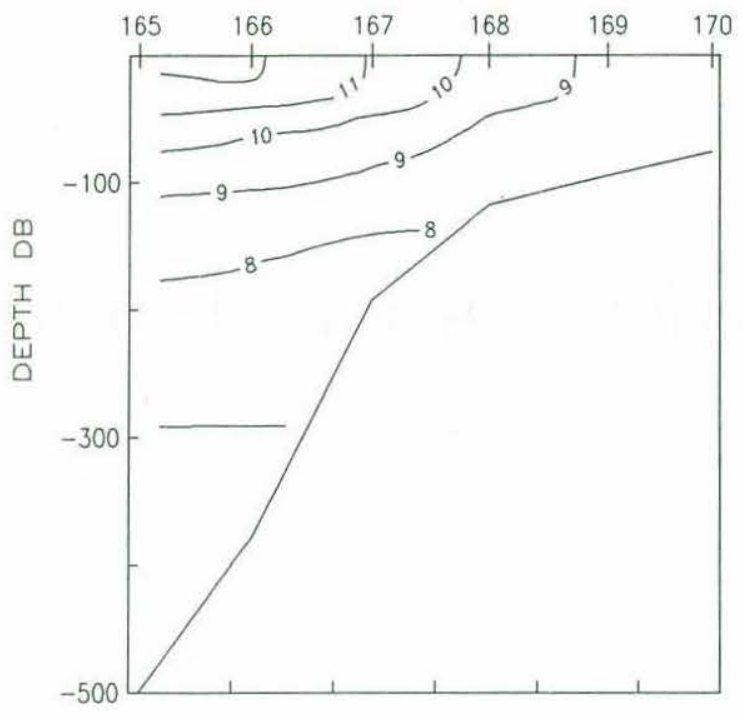

BB SALINITY

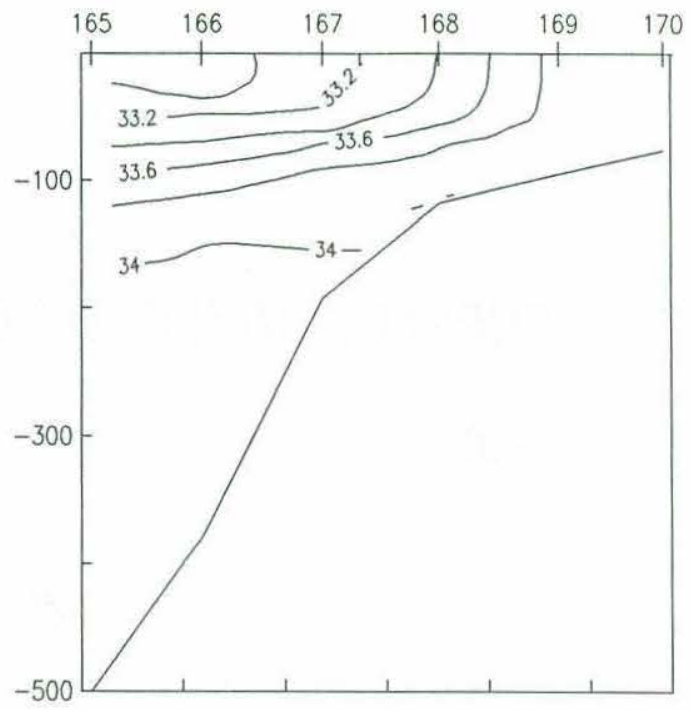

BB SIGMA-T

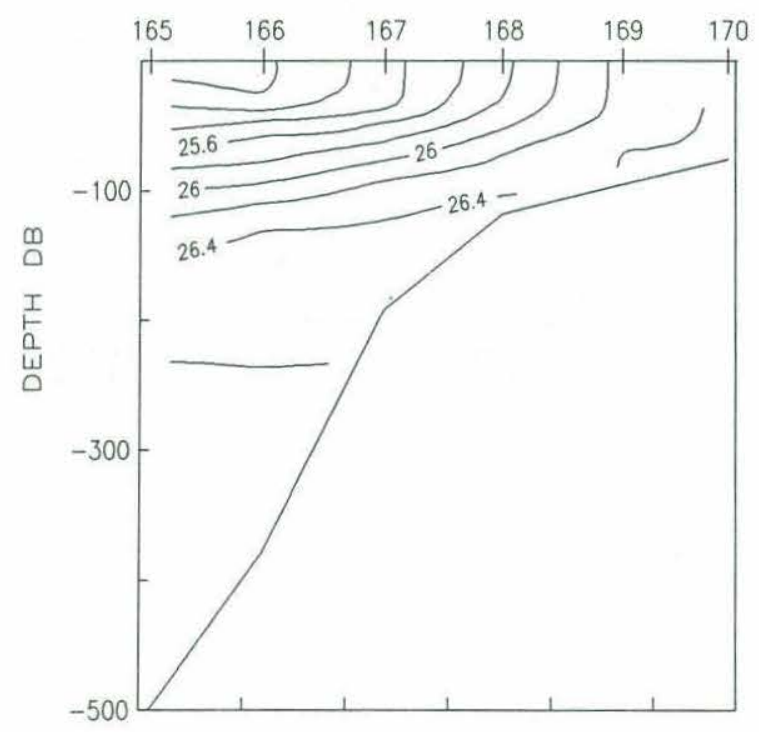


SURFACE MIXED LAYER DEPTH M MAY 11-13 1989

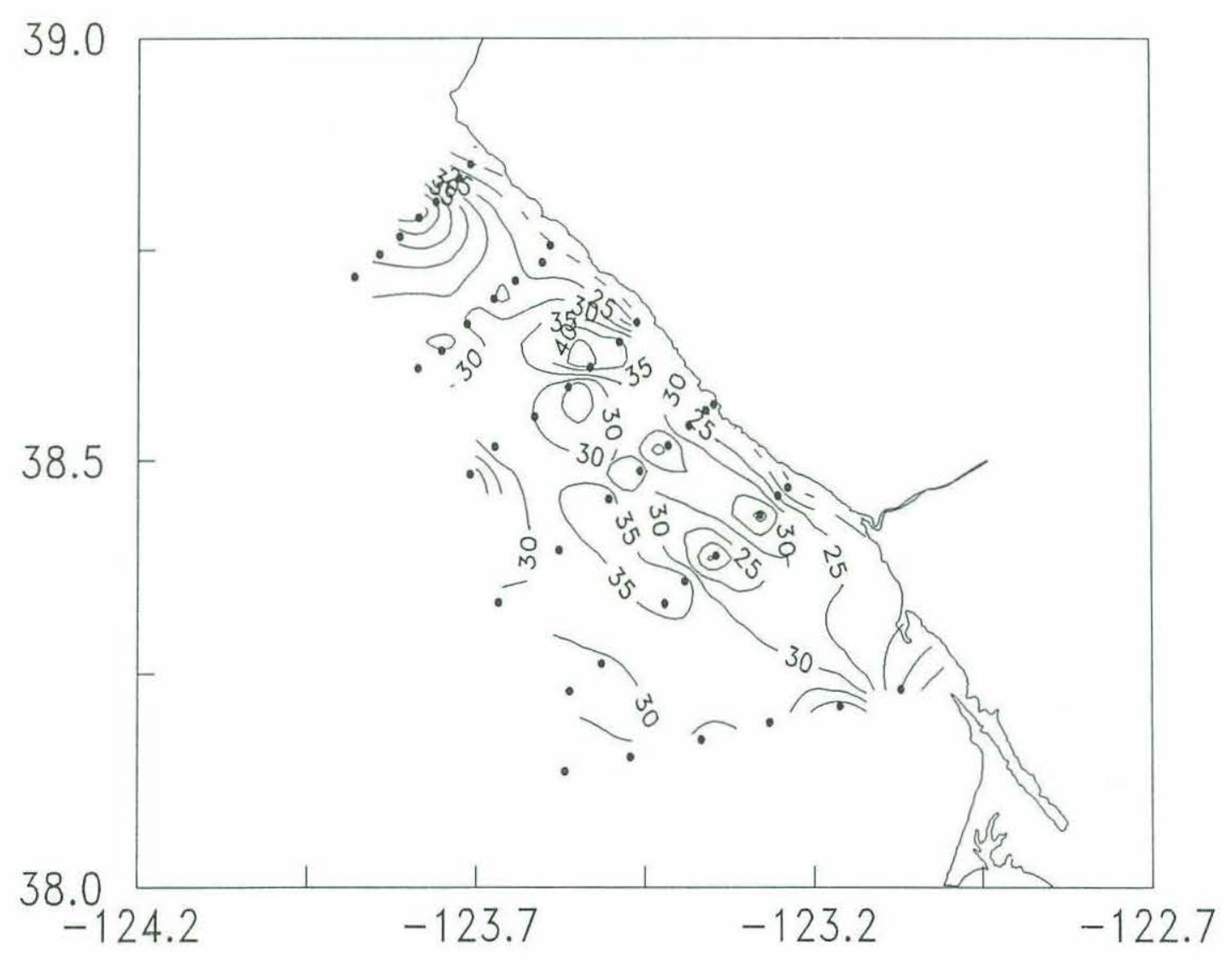


SURFACE TEMP MAY 11-13 1989

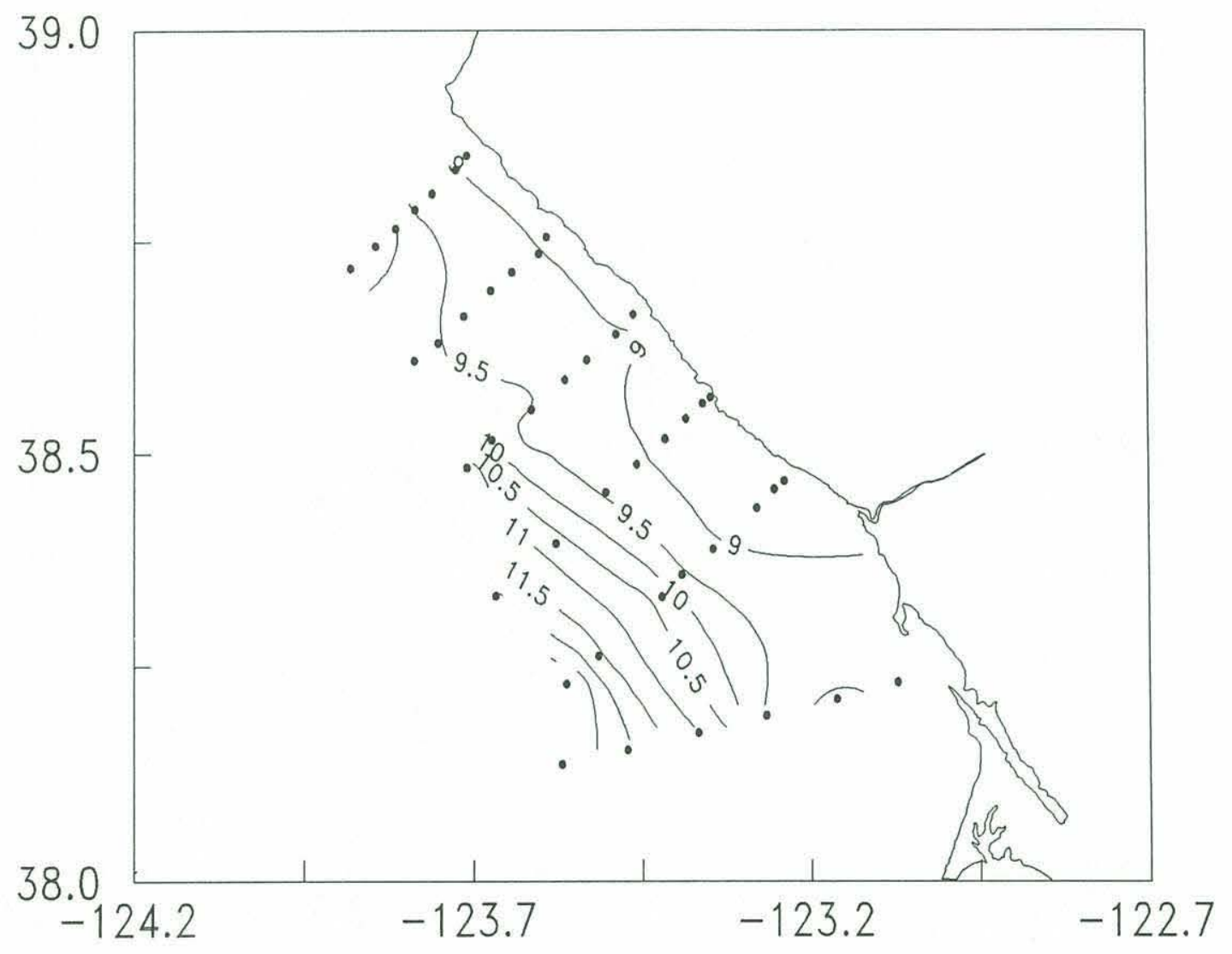


SURFACE SALINITY MAY 11-13 1989

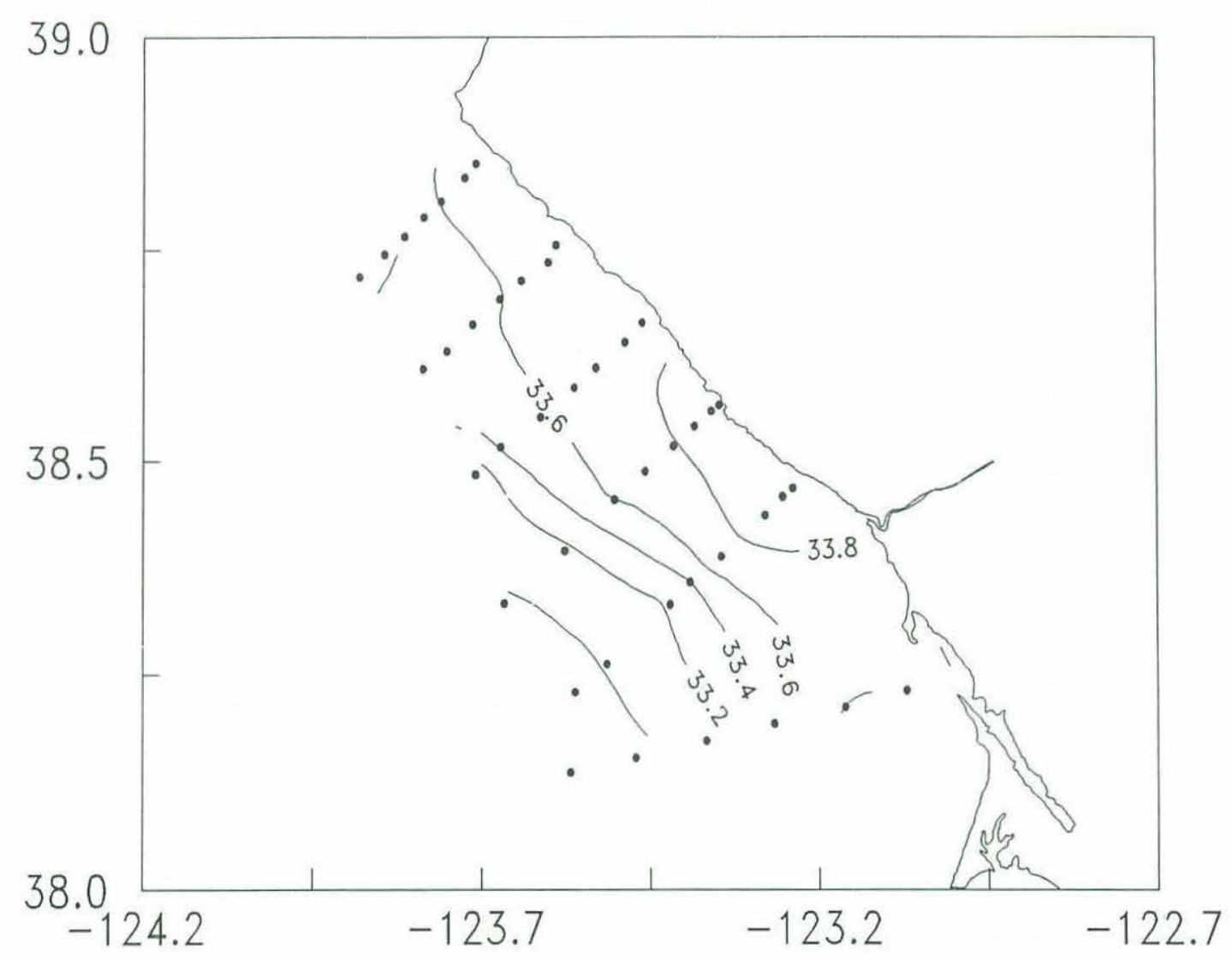


SURFACE SIGMA-T MAY 11-13 1989

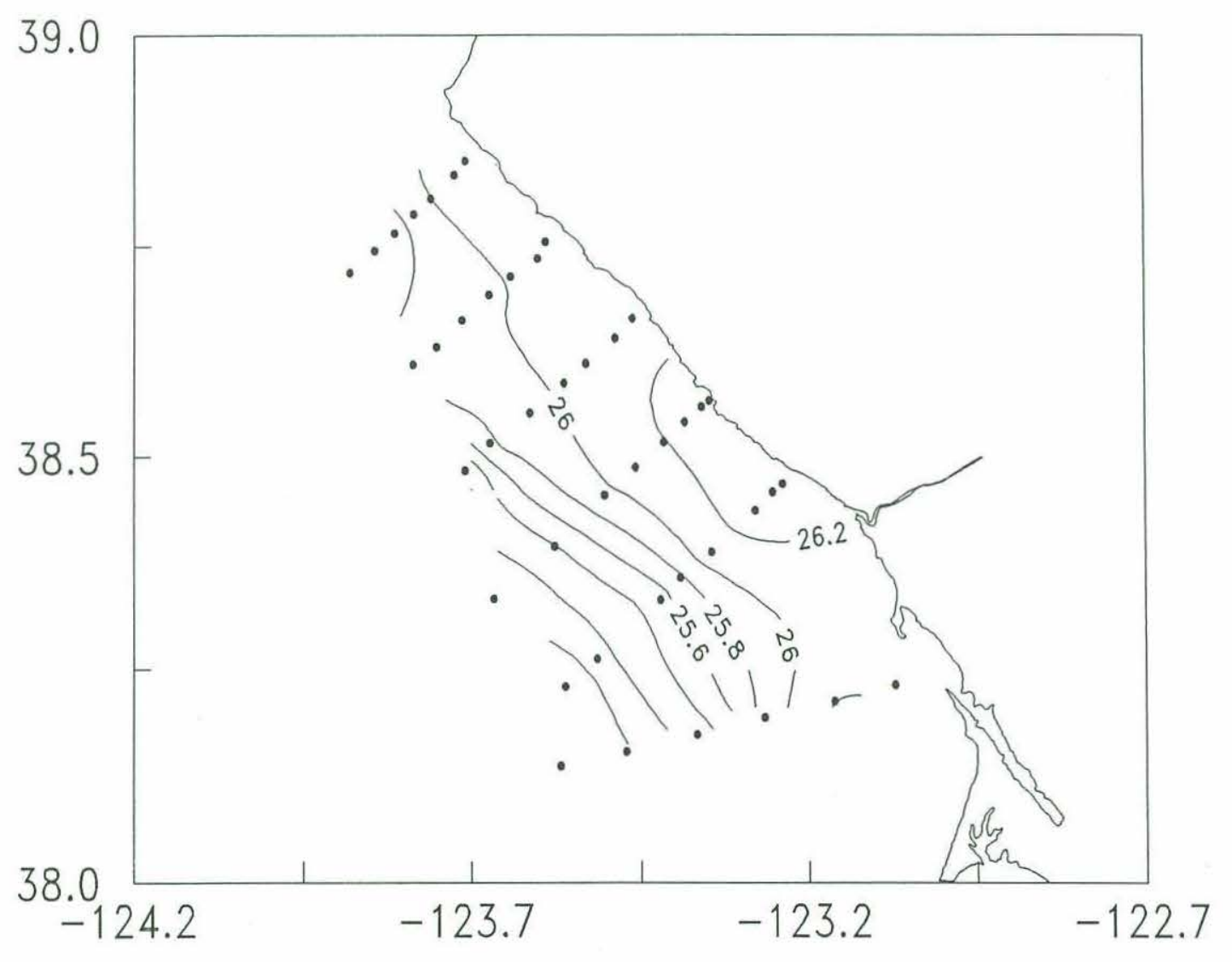



F. Section C - May 14, 1989

Stations 256 to 262 

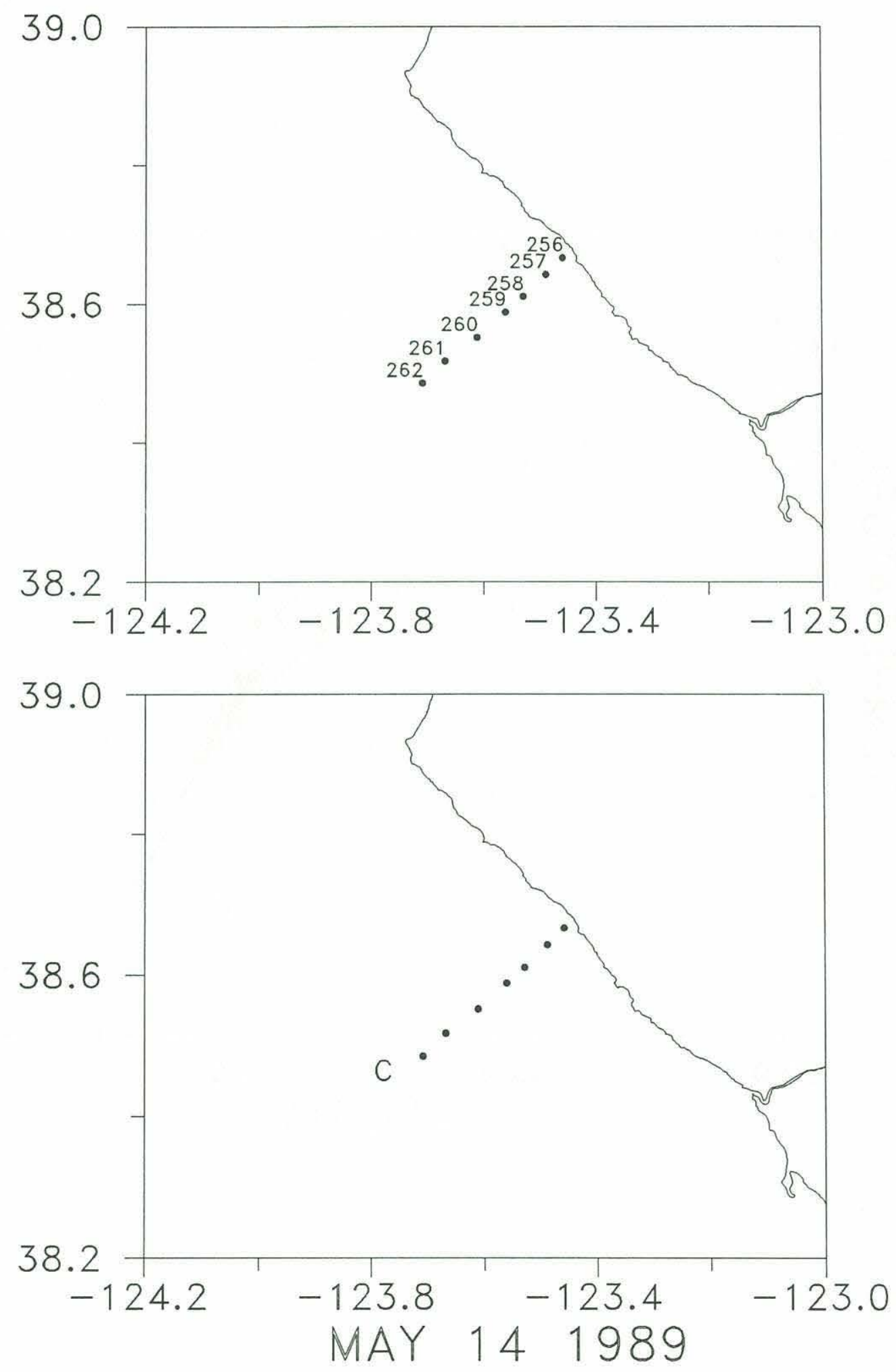


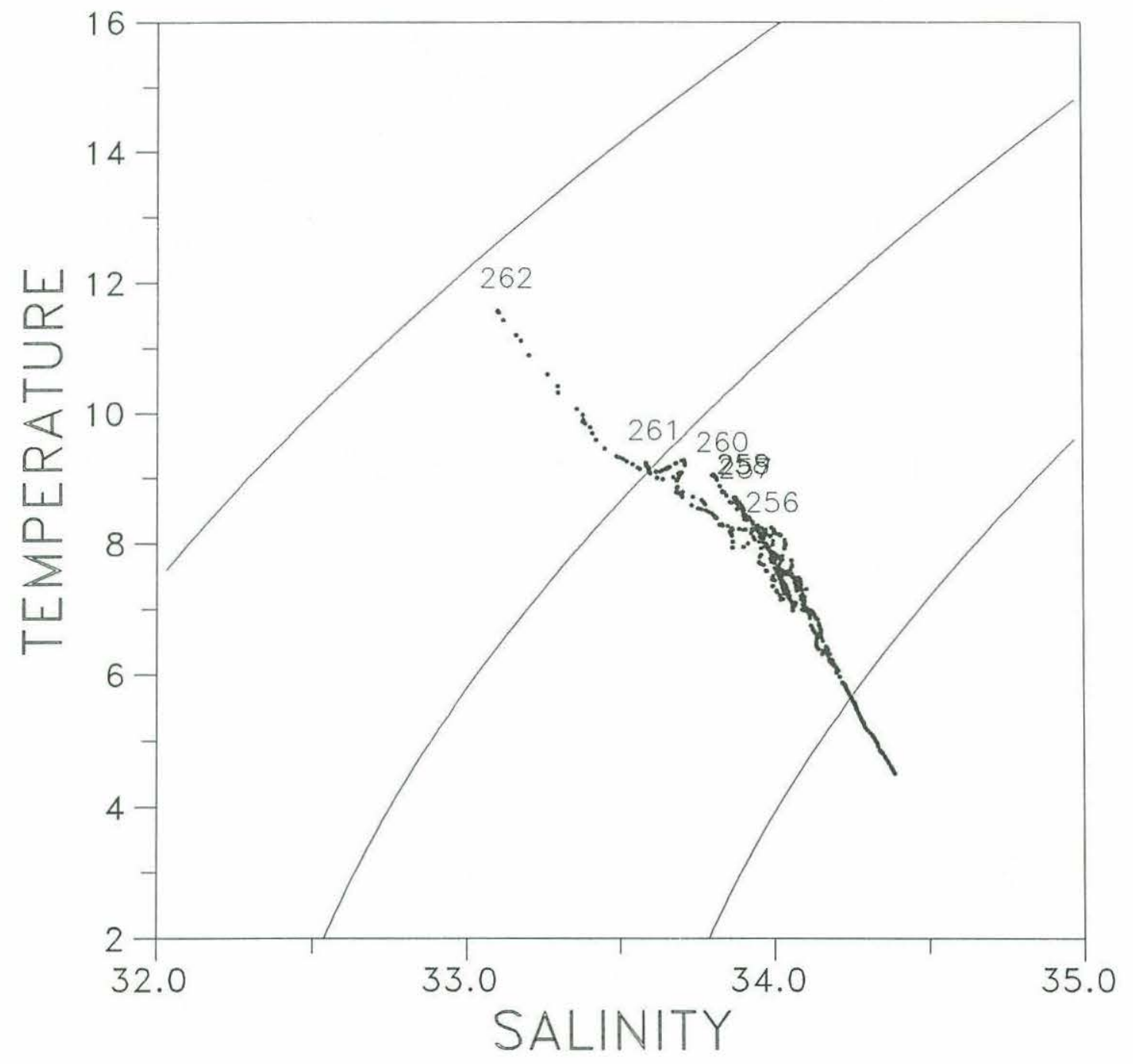



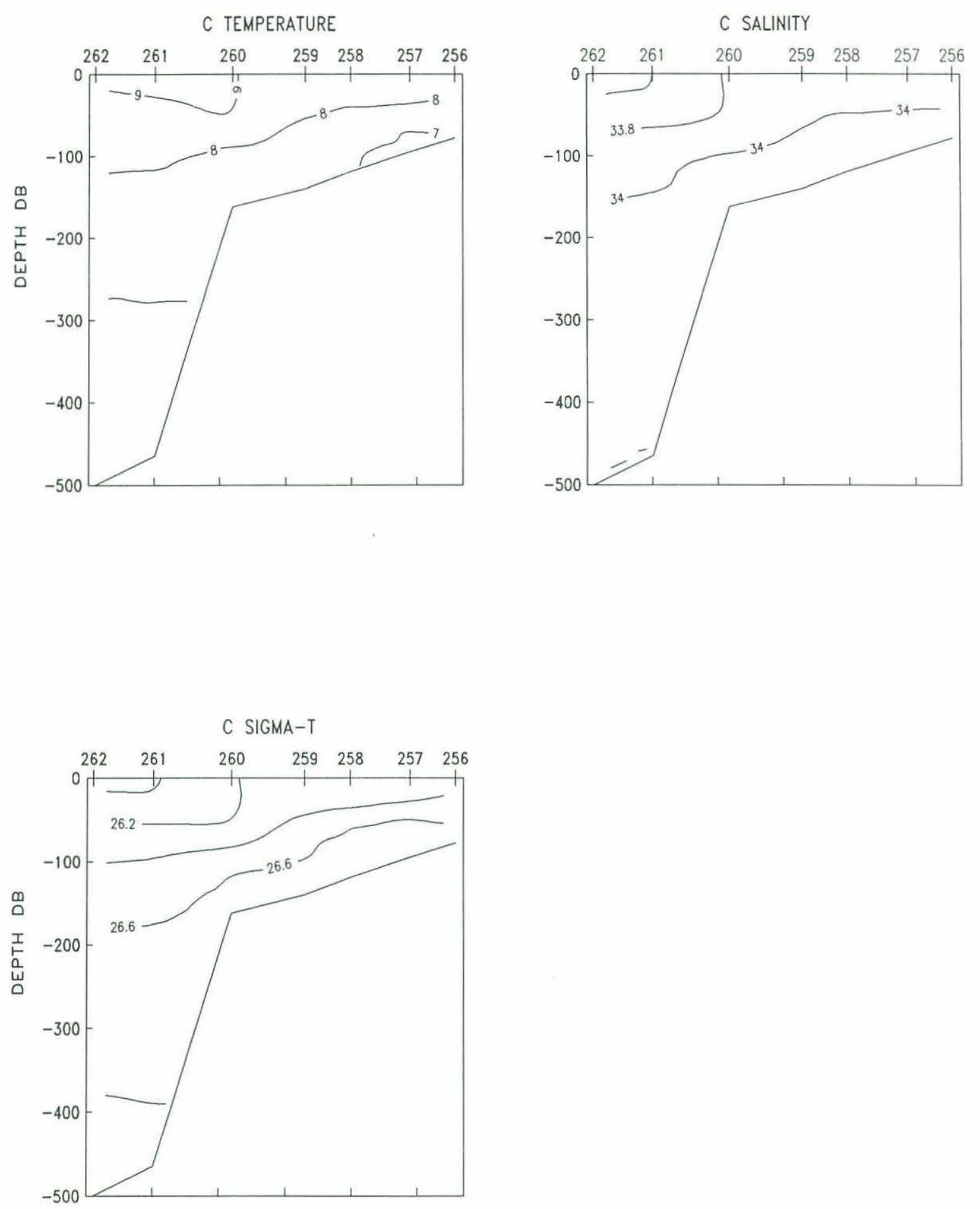
8. Station Listings and Profiles 
Cruise w8905 Station \# 1 Depth m: 39 Time: 1989-05-05 22:12

Position: $3757.20^{\prime} \mathrm{N} 12249.70^{\prime} \mathrm{W} \quad$ Depth Deck Offset: 0.00

\begin{tabular}{|c|c|c|c|c|c|c|}
\hline $\begin{array}{l}\text { PRESSURE } \\
\text { (d-bars) }\end{array}$ & Interp & $\begin{array}{c}\text { TEMP } \\
(\operatorname{deg} C)\end{array}$ & $\begin{array}{l}\text { SALINITY } \\
(0 / 00)\end{array}$ & SIGMA-T & DELTA-D & $\begin{array}{l}\text { CHANNELA } \\
\text { (\%light) }\end{array}$ \\
\hline 0.0 & E & 10.801 & 33.505 & 25.647 & 0.0000 & 0.0 \\
\hline 2.0 & & 10.801 & 33.504 & 25.647 & 0.0047 & 0.0 \\
\hline 4.0 & & 10.799 & 33.505 & 25.648 & 0.0095 & 0.0 \\
\hline 6.0 & & 10.892 & 33.510 & 25.635 & 0.0142 & 0.0 \\
\hline 8.0 & & 10.583 & 33.478 & 25.665 & 0.0189 & 0.0 \\
\hline 10.0 & & 10.303 & 33.464 & 25.702 & 0.0236 & 0.0 \\
\hline 12.0 & & 10.101 & 33.459 & 25.732 & 0.0282 & 0.0 \\
\hline 14.0 & & 9.847 & 33.442 & 25.761 & 0.0328 & 0.0 \\
\hline 16.0 & & 9.660 & 33.551 & 25.877 & 0.0372 & 0.0 \\
\hline 18.0 & & 9.650 & 33.569 & 25.893 & 0.0415 & 0.0 \\
\hline 20.0 & & 9.570 & 33.586 & 25.919 & 0.0457 & 0.0 \\
\hline 22.0 & & 9.477 & 33.613 & 25.956 & 0.0499 & 0.0 \\
\hline 24.0 & & 9.399 & 33.606 & 25.963 & 0.0540 & 0.0 \\
\hline 26.0 & & 9.309 & 33.609 & 25.980 & 0.0582 & 0.0 \\
\hline 28.0 & & 9.244 & 33.625 & 26.003 & 0.0623 & 0.0 \\
\hline 30.0 & & 9.213 & 33.661 & 26.036 & 0.0663 & 0.0 \\
\hline 32.0 & & 9.162 & 33.689 & 26.066 & 0.0703 & 0.0 \\
\hline 34.0 & & 8.996 & 33.756 & 26.145 & 0.0742 & 0.0 \\
\hline 35.0 & & 8.961 & 33.769 & 26.161 & 0.0760 & 0.0 \\
\hline
\end{tabular}

TEMPERATURE C
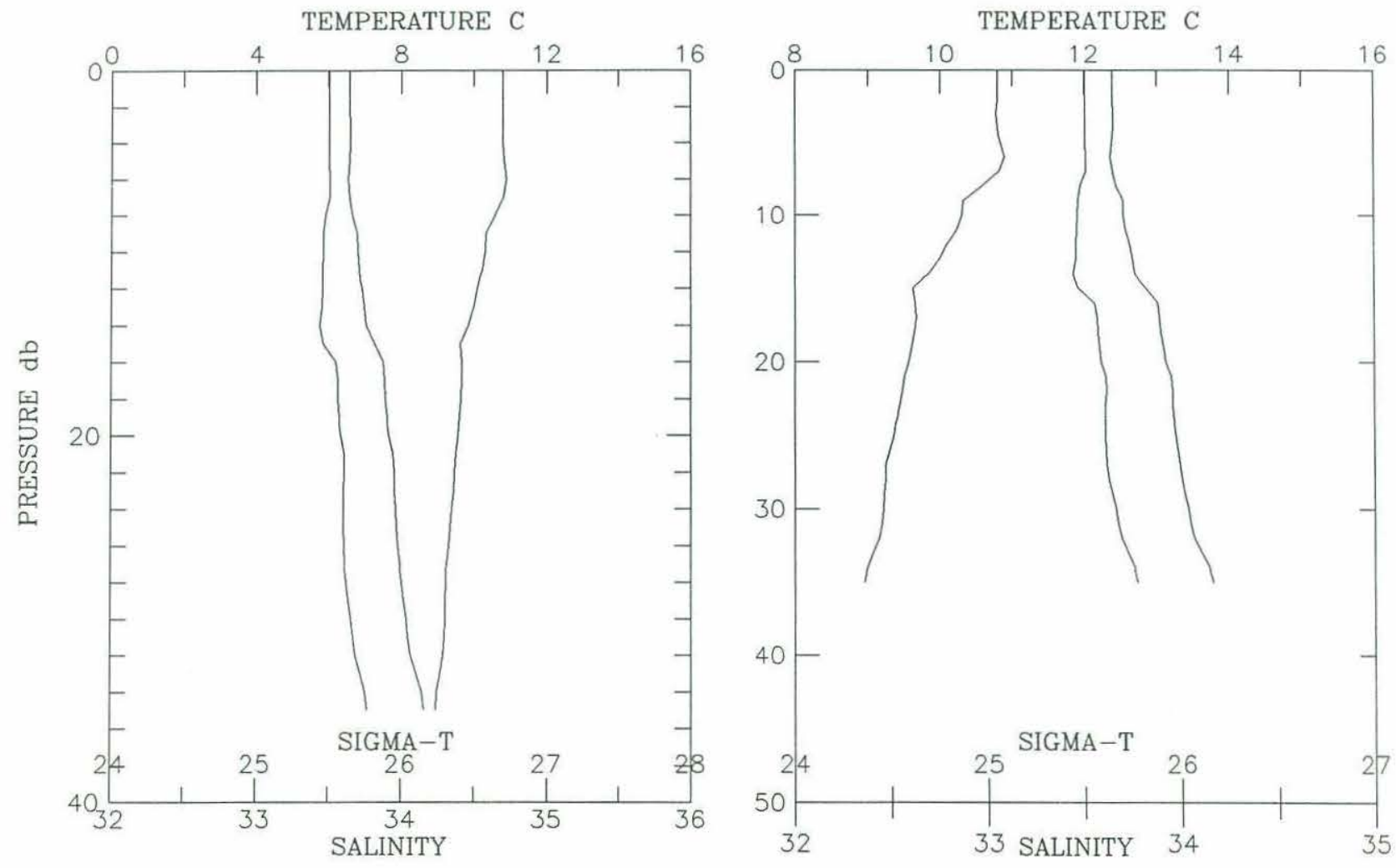


\begin{tabular}{|c|c|c|c|c|c|c|}
\hline $\begin{array}{l}\text { PRESSURE } \\
\text { (d-bars) }\end{array}$ & Interp & $\begin{array}{l}\text { TEMP } \\
(\operatorname{deg} C)\end{array}$ & $\begin{array}{l}\text { SALINITY } \\
(0 / 00)\end{array}$ & SIGMA-T & DELTA-D & $\begin{array}{l}\text { CHANNELA } \\
\text { ( }(1 \mathrm{ight})\end{array}$ \\
\hline 0.0 & E & 12.899 & 33.238 & 25.048 & 0.0000 & 0.0 \\
\hline 5.0 & & 12.824 & 33.236 & 25.062 & 0.0146 & 0.0 \\
\hline 10.0 & & 10.829 & 33.442 & 25.593 & 0.0282 & 0.0 \\
\hline 15.0 & & 10.239 & 33.484 & 25.729 & 0.0400 & 0.0 \\
\hline 20.0 & & 9.928 & 33.537 & 25.822 & 0.0512 & 0.0 \\
\hline 25.0 & & 9.461 & 33.631 & 25.972 & 0.0618 & 0.0 \\
\hline 30.0 & & 9.350 & 33.658 & 26.012 & 0.0720 & 0.0 \\
\hline 35.0 & & 9.296 & 33.719 & 26.068 & 0.0820 & 0.0 \\
\hline 40.0 & & 9.105 & 33.728 & 26.106 & 0.0918 & 0.0 \\
\hline 45.0 & & 8.841 & 33.797 & 26.202 & 0.1013 & 0.0 \\
\hline 50.0 & & 8.811 & 33.812 & 26.218 & 0.1105 & 0.0 \\
\hline 53.0 & & 8.814 & 33.813 & 26.218 & 0.1160 & 0.0 \\
\hline
\end{tabular}
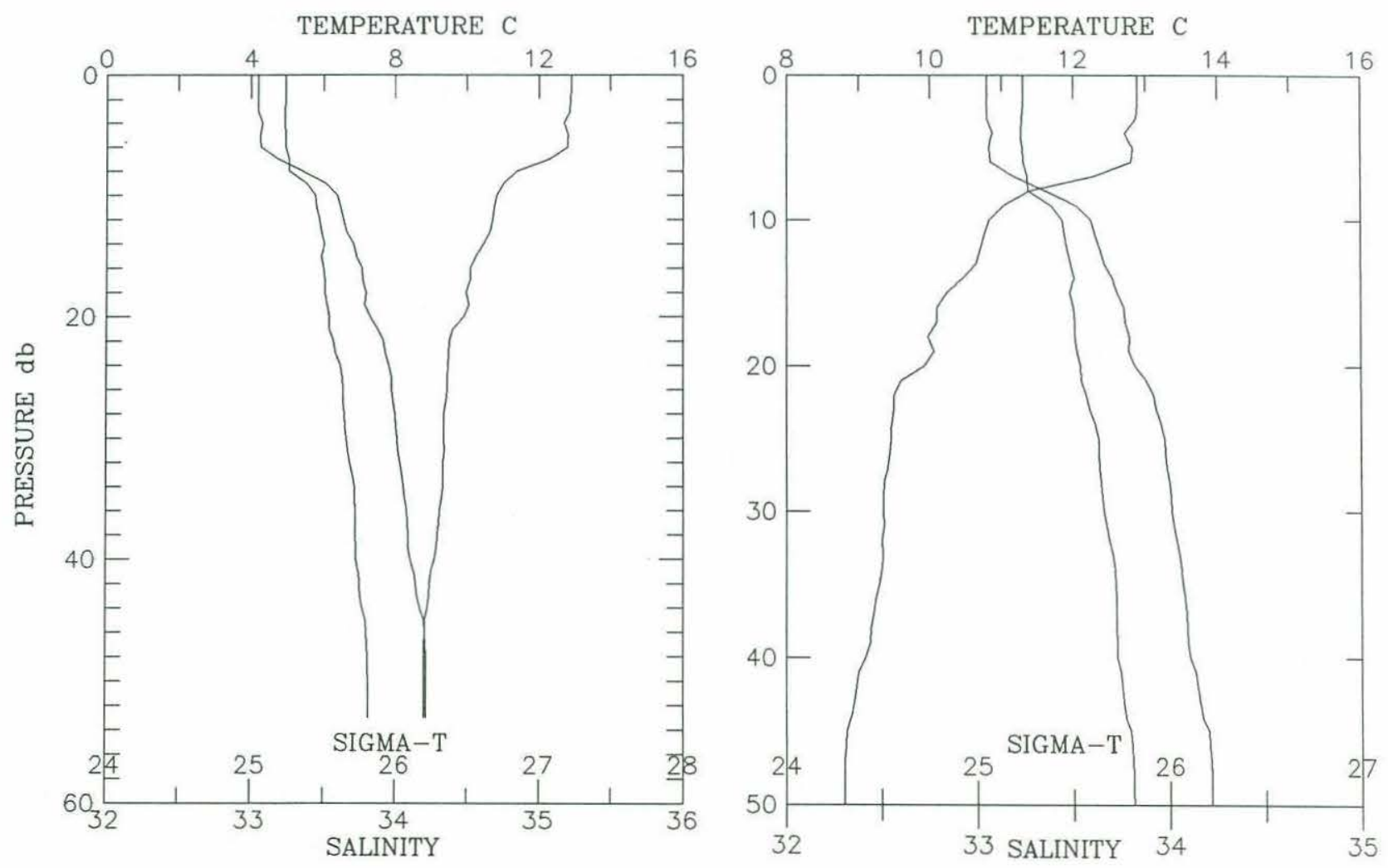
Cruise w8905 Station \# 3 Depth m: 96 Time: 1989-05-06 00:16 Position: $3754.06^{\prime} \mathrm{N} 12310.24^{\prime} \mathrm{W}$ Depth Deck offset: -0.25

\begin{tabular}{|c|c|c|c|c|c|c|}
\hline $\begin{array}{l}\text { PRESSURE } \\
\text { (d-bars) }\end{array}$ & Interp & $\begin{array}{l}\text { TEMP } \\
(\operatorname{deg} C)\end{array}$ & $\begin{array}{c}\text { SALINITY } \\
(0 / 00)\end{array}$ & SIGMA-T & DELTA-D & $\begin{array}{l}\text { CHANNELA } \\
\text { ( }(81 \text { ight) }\end{array}$ \\
\hline 0.0 & E & 11.511 & 32.965 & 25.100 & 0.0000 & 0.0 \\
\hline 5.0 & & 11.590 & 32.973 & 25.092 & 0.0144 & 0.0 \\
\hline 10.0 & & 11.071 & 33.035 & 25.234 & 0.0286 & 0.0 \\
\hline 15.0 & & 10.731 & 33.063 & 25.315 & 0.0422 & 0.0 \\
\hline 20.0 & & 10.211 & 33.095 & 25.430 & 0.0554 & 0.0 \\
\hline 25.0 & & 9.655 & 33.193 & 25.598 & 0.0679 & 0.0 \\
\hline 30.0 & & 9.580 & 33.336 & 25.722 & 0.0797 & 0.0 \\
\hline 35.0 & & 9.387 & 33.422 & 25.821 & 0.0909 & 0.0 \\
\hline 40.0 & & 9.232 & 33.430 & 25.852 & 0.1019 & 0.0 \\
\hline 45.0 & & 9.115 & 33.455 & 25.890 & 0.1127 & 0.0 \\
\hline 50.0 & & 9.140 & 33.556 & 25.965 & 0.1233 & 0.0 \\
\hline 55.0 & & 9.150 & 33.615 & 26.010 & 0.1336 & 0.0 \\
\hline 60.0 & & 9.130 & 33.671 & 26.057 & 0.1436 & 0.0 \\
\hline 65.0 & & 9.110 & 33.693 & 26.078 & 0.1535 & 0.0 \\
\hline 70.0 & & 9.052 & 33.717 & 26.105 & 0.1633 & 0.0 \\
\hline 75.0 & & 8.970 & 33.746 & 26.141 & 0.1729 & 0.0 \\
\hline 80.0 & & 8.886 & 33.791 & 26.190 & 0.1824 & 0.0 \\
\hline 85.0 & & 8.863 & 33.803 & 26.203 & 0.1917 & 0.0 \\
\hline 90.0 & & 8.858 & 33.806 & 26.206 & 0.2010 & 0.0 \\
\hline 91.0 & & 8.858 & 33.806 & 26.206 & 0.2028 & 0.0 \\
\hline
\end{tabular}

TEMPERATURE C
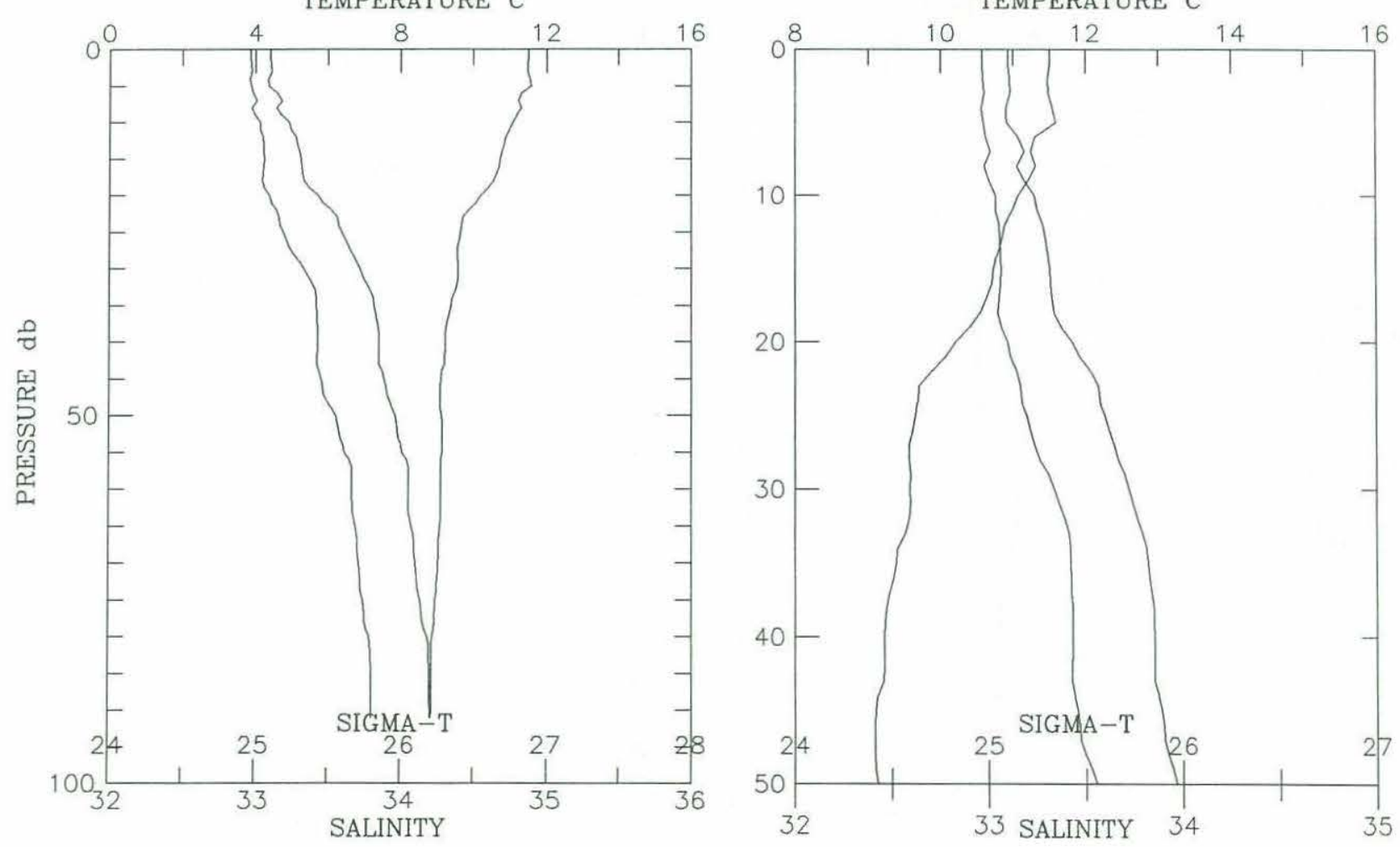
Cruise w8905 Station \# 4 Depth m: 109 Time: 1989-05-06 01:09 Position: $3752.80^{\prime} \mathrm{N} 12317.60^{\prime} \mathrm{W}$ Depth Deck Offset: -0.20

\begin{tabular}{|c|c|c|c|c|c|c|}
\hline $\begin{array}{l}\text { PRESSURE } \\
\text { (d-bars) }\end{array}$ & Interp & $\begin{array}{l}\text { TEMP } \\
(\operatorname{deg} C)\end{array}$ & $\begin{array}{l}\text { SALINITY } \\
(0 / 00)\end{array}$ & SIGMA-T & DELTA-D & $\begin{array}{l}\text { CHANNELA } \\
\text { ( }(\text { ight) }\end{array}$ \\
\hline 0.0 & E & 13.371 & 32.791 & 24.609 & 0.0000 & 0.0 \\
\hline 5.0 & & 13.357 & 32.790 & 24.611 & 0.0168 & 0.0 \\
\hline 10.0 & & 13.234 & 32.796 & 24.640 & 0.0335 & 0.0 \\
\hline 15.0 & & 13.239 & 32.797 & 24.640 & 0.0502 & 0.0 \\
\hline 20.0 & & 12.574 & 32.853 & 24.813 & 0.0666 & 0.0 \\
\hline 25.0 & & 12.026 & 32.860 & 24.923 & 0.0821 & 0.0 \\
\hline 30.0 & & 10.978 & 32.791 & 25.060 & 0.0971 & 0.0 \\
\hline 35.0 & & 10.441 & 32.796 & 25.158 & 0.1115 & 0.0 \\
\hline 40.0 & & 10.180 & 32.854 & 25.246 & 0.1255 & 0.0 \\
\hline 45.0 & & 10.144 & 32.915 & 25.300 & 0.1391 & 0.0 \\
\hline 50.0 & & 9.998 & 32.975 & 25.371 & 0.1524 & 0.0 \\
\hline 55.0 & & 9.802 & 33.086 & 25.491 & 0.1655 & 0.0 \\
\hline 60.0 & & 9.720 & 33.130 & 25.539 & 0.1779 & 0.0 \\
\hline 65.0 & & 9.526 & 33.177 & 25.607 & 0.1902 & 0.0 \\
\hline 70.0 & & 9.347 & 33.217 & 25.667 & 0.2022 & 0.0 \\
\hline 75.0 & & 9.156 & 33.392 & 25.835 & 0.2134 & 0.0 \\
\hline 80.0 & & 9.155 & 33.462 & 25.890 & 0.2243 & 0.0 \\
\hline 85.0 & & 9.226 & 33.565 & 25.959 & 0.2349 & 0.0 \\
\hline 90.0 & & 9.234 & 33.574 & 25.964 & 0.2453 & 0.0 \\
\hline 95.0 & & 9.213 & 33.602 & 25.990 & 0.2557 & 0.0 \\
\hline 100.0 & & 9.113 & 33.707 & 26.088 & 0.2658 & 0.0 \\
\hline 105.0 & & 9.040 & 33.749 & 26.132 & 0.2755 & 0.0 \\
\hline
\end{tabular}

TEMPERATURE C
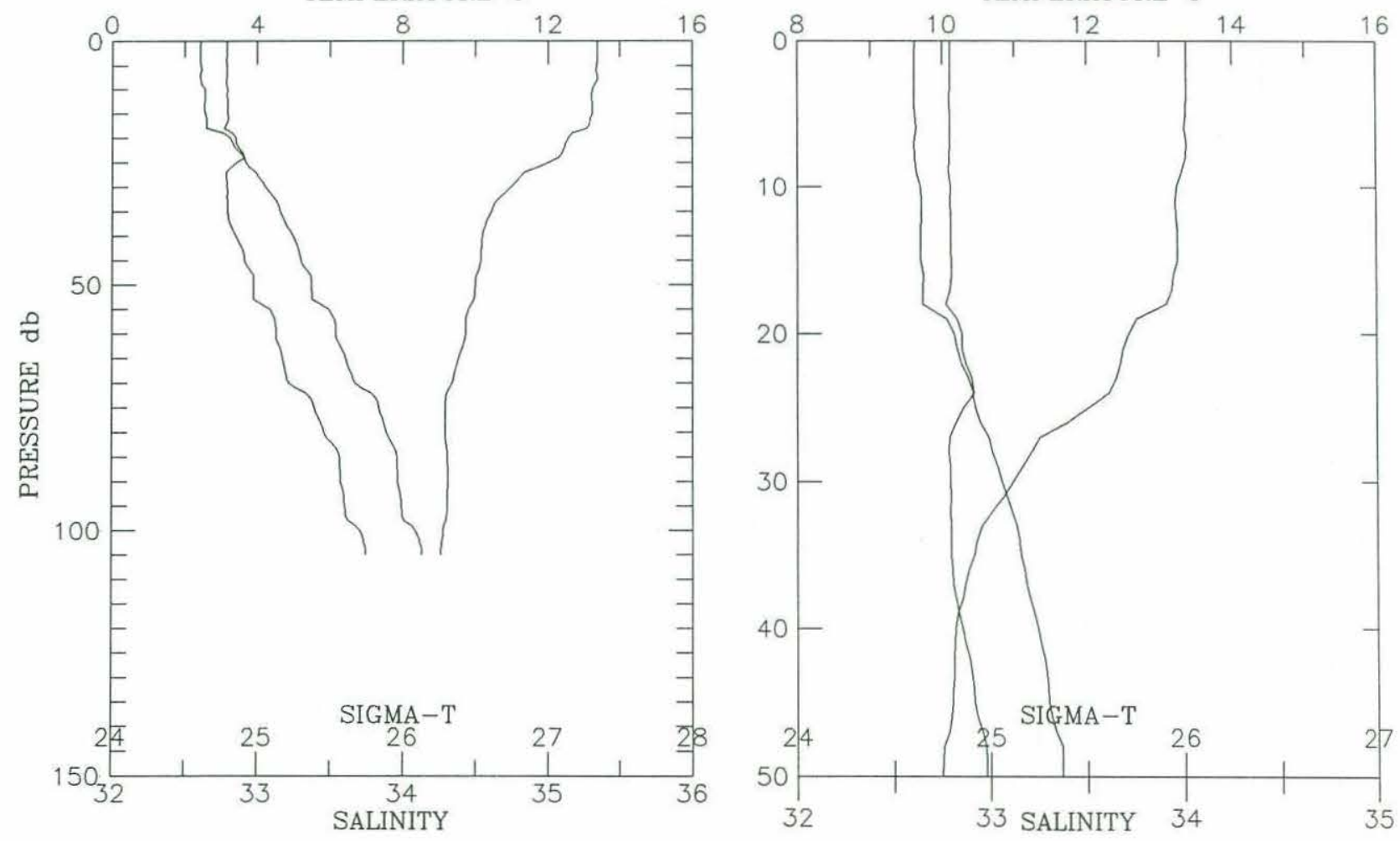
Cruise W8905 Station \# 5 Depth m: 220 Time: 1989-05-06 01:58

Position: $3751.90^{\prime} \mathrm{N} 12324.70^{\prime} \mathrm{W}$ Depth Deck Offset: -0.20

$\begin{array}{lcccc}\text { PRESSURE Interp } & \text { TEMP } \\ \text { (d-bars) } & \text { SALINITY } & \text { SIGMA-T DELTA-D } & \text { CHANNELA } \\ (\mathrm{deg} C) & (0 / 00) & & \text { ( } 1 \text { ight) }\end{array}$

\begin{tabular}{|c|c|c|c|c|c|c|}
\hline 0.0 & E & 13.894 & 32.797 & 24.507 & 0.0000 & 0.0 \\
\hline 10.0 & & 13.892 & 32.796 & 24.507 & 0.0345 & 0.0 \\
\hline 20.0 & & 13.875 & 32.796 & 24.510 & 0.0691 & 0.0 \\
\hline 30.0 & & 13.563 & 32.796 & 24.574 & 0.1035 & 0.0 \\
\hline 40.0 & & 11.688 & 32.787 & 24.929 & 0.1361 & 0.0 \\
\hline 50.0 & & 10.376 & 32.766 & 25.145 & 0.1652 & 0.0 \\
\hline 60.0 & & 10.056 & 32.968 & 25.356 & 0.1928 & 0.0 \\
\hline 80.0 & & 10.406 & 33.205 & 25.482 & 0.2447 & 0.0 \\
\hline 100.0 & & 9.622 & 33.393 & 25.760 & 0.2930 & 0.0 \\
\hline 150.0 & & 8.546 & 33.850 & 26.289 & 0.3944 & 0.0 \\
\hline 200.0 & & 7.296 & 34.052 & 26.632 & 0.4729 & 0.0 \\
\hline 206.0 & & 7.268 & 34.057 & 26.639 & 0.4816 & 0.0 \\
\hline
\end{tabular}
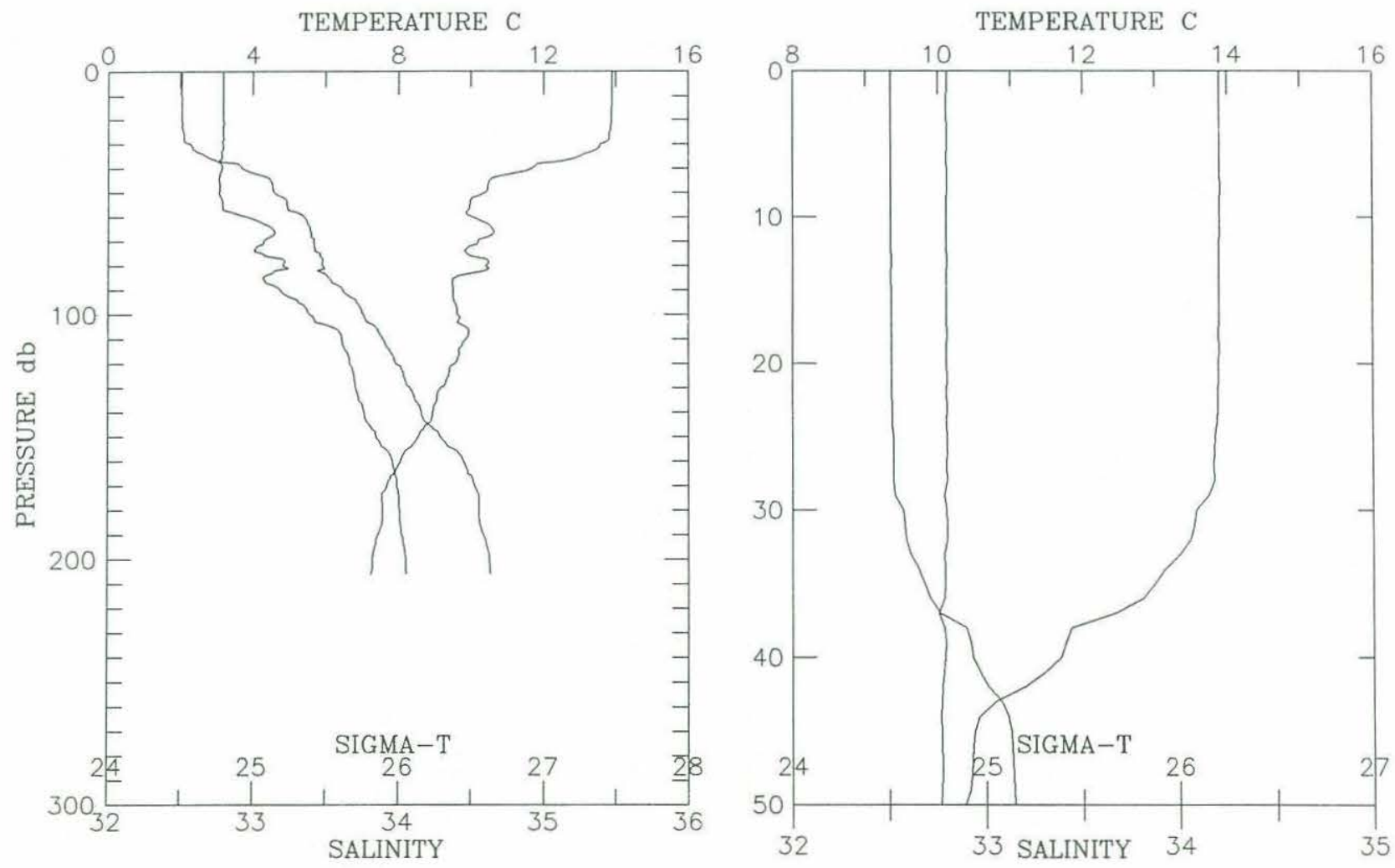
Cruise w8905 Station \# 6 Depth m: 949 Time: 1989-05-06 02:36 Position: $3751.40^{\prime} \mathrm{N} 12327.20^{\prime} \mathrm{W}$ Depth Deck Offset: -0.20

\begin{tabular}{|c|c|c|c|c|c|c|}
\hline $\begin{array}{l}\text { PRESSURE } \\
\text { (d-bars) }\end{array}$ & Interp & $\begin{array}{l}\text { TEMP } \\
(\operatorname{deg} C)\end{array}$ & $\begin{array}{c}\text { SALINITY } \\
(0 / 00)\end{array}$ & SIGMA-T & DELTA-D & $\begin{array}{l}\text { CHANNEL } \\
\text { (\%light) }\end{array}$ \\
\hline 0.0 & E & 13.982 & 32.802 & 24.493 & 0.0000 & 0.0 \\
\hline 10.0 & & 13.981 & 32.802 & 24.493 & 0.0347 & 0.0 \\
\hline 20.0 & & 13.943 & 32.801 & 24.500 & 0.0693 & 0.0 \\
\hline 30.0 & & 13.895 & 32.801 & 24.510 & 0.1039 & 0.0 \\
\hline 40.0 & & 12.519 & 32.821 & 24.799 & 0.1369 & 0.0 \\
\hline 50.0 & & 11.442 & 32.853 & 25.025 & 0.1673 & 0.0 \\
\hline 60.0 & & 10.829 & 32.982 & 25.235 & 0.1957 & 0.0 \\
\hline 80.0 & & 10.871 & 33.235 & 25.425 & 0.249 .5 & 0.0 \\
\hline 100.0 & & 10.586 & 33.500 & 25.681 & 0.2989 & 0.0 \\
\hline 150.0 & & 8.660 & 33.831 & 26.256 & 0.4057 & 0.0 \\
\hline 200.0 & & 7.860 & 34.039 & 26.540 & 0.4871 & 0.0 \\
\hline 250.0 & & 6.796 & 34.013 & 26.670 & 0.5616 & 0.0 \\
\hline 300.0 & & 6.945 & 34.115 & 26.729 & 0.6321 & 0.0 \\
\hline 400.0 & & 6.303 & 34.179 & 26.865 & 0.7648 & 0.0 \\
\hline 500.0 & & 5.921 & 34.220 & 26.946 & 0.8885 & 0.0 \\
\hline 600.0 & & 5.338 & 34.272 & 27.059 & 1.0029 & 0.0 \\
\hline 700.0 & & 4.900 & 34.332 & 27.157 & 1.1082 & 0.0 \\
\hline 800.0 & & 4.652 & 34.368 & 27.214 & 1.2077 & 0.0 \\
\hline 900.0 & & 4.367 & 34.397 & 27.268 & 1.3021 & 0.0 \\
\hline 961.0 & & 4.188 & 34.421 & 27.306 & 1.3578 & 0.0 \\
\hline
\end{tabular}
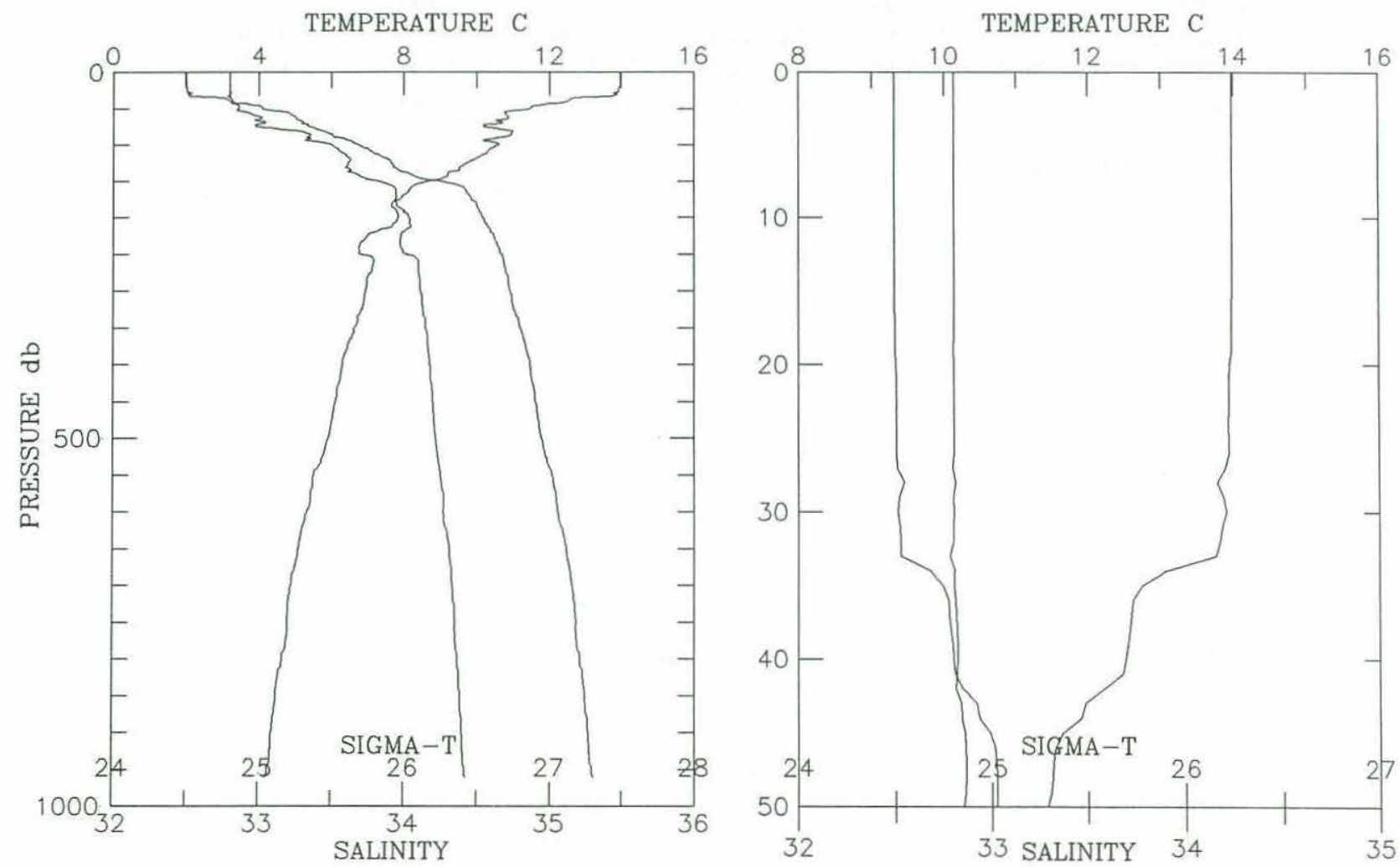
Cruise w8905 Station \# 7 Depth m: 1821 Time: 1989-05-06 03:44 Position: $3750.90^{\prime} \mathrm{N} 123 \quad 31.40^{\prime} \mathrm{W}$ Depth Deck Offset: -0.20

\begin{tabular}{|c|c|c|c|c|c|c|}
\hline $\begin{array}{l}\text { PRESSURE } \\
\text { (d-bars) }\end{array}$ & Interp & $\begin{array}{l}\text { TEMP } \\
(\operatorname{deg} C)\end{array}$ & $\begin{array}{c}\text { SALINITY } \\
(0 / 00)\end{array}$ & SIGMA-T & DELTA-D & $\begin{array}{l}\text { CHANNELA } \\
\text { (\%light) }\end{array}$ \\
\hline 0.0 & E & 14.003 & 32.800 & 24.487 & 0.0000 & 0.0 \\
\hline 10.0 & & 13.995 & 32.799 & 24.488 & 0.0347 & 0.0 \\
\hline 20.0 & & 13.450 & 32.789 & 24.591 & 0.0691 & 0.0 \\
\hline 30.0 & & 11.826 & 32.812 & 24.923 & 0.1010 & 0.0 \\
\hline 40.0 & & 11.241 & 32.966 & 25.150 & 0.1304 & 0.0 \\
\hline 50.0 & & 10.616 & 33.048 & 25.324 & 0.1585 & 0.0 \\
\hline 60.0 & & 10.527 & 33.171 & 25.435 & 0.1847 & 0.0 \\
\hline 80.0 & & 10.563 & 33.452 & 25.648 & 0.2341 & 0.0 \\
\hline 100.0 & & 10.187 & 33.528 & 25.771 & 0.2807 & 0.0 \\
\hline 150.0 & & 8.715 & 33.811 & 26.232 & 0.3839 & 0.0 \\
\hline 200.0 & & 7.852 & 33.952 & 26.473 & 0.4684 & 0.0 \\
\hline 250.0 & & 7.438 & 34.032 & 26.596 & 0.5465 & 0.0 \\
\hline 300.0 & & 6.644 & 34.014 & 26.690 & 0.6194 & 0.0 \\
\hline 400.0 & & 5.741 & 34.065 & 26.846 & 0.7545 & 0.0 \\
\hline 500.0 & & 5.642 & 34.208 & 26.971 & 0.8779 & 0.0 \\
\hline 600.0 & & 5.058 & 34.250 & 27.074 & 0.9912 & 0.0 \\
\hline 700.0 & & 4.712 & 34.321 & 27.170 & 1.0957 & 0.0 \\
\hline 800.0 & & 4.384 & 34.356 & 27.234 & 1.1938 & 0.0 \\
\hline 900.0 & & 4.222 & 34.410 & 27.294 & 1.2858 & 0.0 \\
\hline 1000.0 & & 3.889 & 34.433 & 27.347 & 1. 3734 & 0.0 \\
\hline 1100.0 & & 3.635 & 34.464 & 27.397 & 1.4561 & 0.0 \\
\hline 1200.0 & & 3.368 & 34.492 & 27.446 & 1.5342 & 0.0 \\
\hline 1300.0 & & 3.159 & 34.512 & 27.480 & 1.6087 & 0.0 \\
\hline 1400.0 & & 2.966 & 34.526 & 27.510 & 1.6803 & 0.0 \\
\hline 1500.0 & & 2.751 & 34.545 & 27.544 & 1.7492 & 0.0 \\
\hline 1501.0 & & 2.751 & 34.545 & 27.544 & 1.7499 & 0.0 \\
\hline
\end{tabular}

TEMPERATURE C
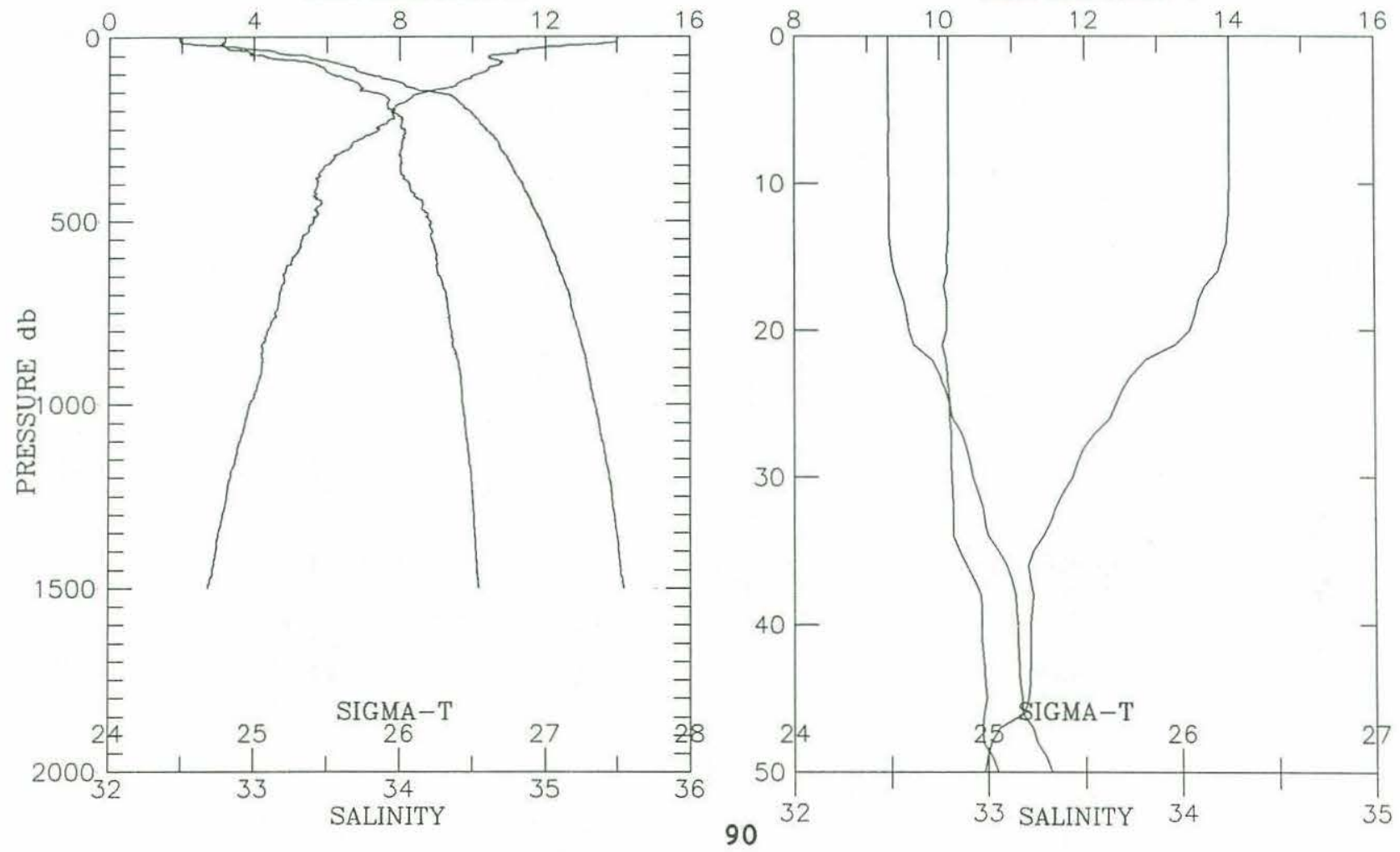
Cruise w8905 Station \# 8 Depth m: 2749 Time: 1989-05-06 05:17 Position: $3749.60^{\prime} \mathrm{N} 123 \quad 36.90^{\prime} \mathrm{W}$ Depth Deck Offset: $\quad-0.20$

\begin{tabular}{|c|c|c|c|c|c|c|}
\hline $\begin{array}{l}\text { PRESSURE } \\
\text { (d-bars) }\end{array}$ & Interp & $\begin{array}{l}\text { TEMP } \\
\text { (deg C) }\end{array}$ & $\begin{array}{l}\text { SALINITY } \\
(0 / 00)\end{array}$ & SIGMA-T & DELTA-D & $\begin{array}{l}\text { CHANNELA } \\
\text { ( } \% 1 \text { ight) }\end{array}$ \\
\hline 0.0 & E & 13.981 & 32.790 & 24.484 & 0.0000 & 0.0 \\
\hline 10.0 & & 13.974 & 32.788 & 24.484 & 0.0347 & 0.0 \\
\hline 20.0 & & 13.818 & 32.782 & 24.511 & 0.0695 & 0.0 \\
\hline 30.0 & & 12.460 & 32.897 & 24.870 & 0.1019 & 0.0 \\
\hline 40.0 & & 11.218 & 32.946 & 25.138 & 0.1317 & 0.0 \\
\hline 50.0 & & 11.440 & 33.133 & 25.244 & 0.1597 & 0.0 \\
\hline 60.0 & & 10.967 & 33.205 & 25.385 & 0.1865 & 0.0 \\
\hline 80.0 & & 10.539 & 33.385 & 25.600 & 0.2370 & 0.0 \\
\hline 100.0 & & 10.143 & 33.558 & 25.802 & 0.2841 & 0.0 \\
\hline 150.0 & & 9.168 & 33.814 & 26.163 & 0.3866 & 0.0 \\
\hline 200.0 & & 7.955 & 33.940 & 26.448 & 0.4743 & 0.0 \\
\hline 250.0 & & 7.336 & 34.010 & 26.593 & 0.5522 & 0.0 \\
\hline 300.0 & & 6.582 & 34.000 & 26.687 & 0.6255 & 0.0 \\
\hline 400.0 & & 5.896 & 34.070 & 26.831 & 0.7607 & 0.0 \\
\hline 500.0 & & 5.256 & 34.137 & 26.962 & 0.8846 & 0.0 \\
\hline 600.0 & & 4.759 & 34.207 & 27.074 & 0.9978 & 0.0 \\
\hline 700.0 & & 4.452 & 34.293 & 27.176 & 1.1012 & 0.0 \\
\hline 800.0 & & 4.173 & 34.351 & 27.252 & 1.1965 & 0.0 \\
\hline 900.0 & & 4.030 & 34.409 & 27.313 & 1.2862 & 0.0 \\
\hline 1000.0 & & 3.807 & 34.443 & 27.363 & 1.3714 & 0.0 \\
\hline 1100.0 & & 3.533 & 34.469 & 27.411 & 1.4525 & 0.0 \\
\hline 1200.0 & & 3.311 & 34.499 & 27.456 & 1.5289 & 0.0 \\
\hline 1300.0 & & 3.132 & 34.513 & 27.484 & 1.6027 & 0.0 \\
\hline 1400.0 & & 2.904 & 34.531 & 27.519 & 1.6736 & 0.0 \\
\hline 1500.0 & & 2.685 & 34.549 & 27.553 & 1.7416 & 0.0 \\
\hline 1501.0 & & 2.683 & 34.550 & 27.554 & 1.7423 & 0.0 \\
\hline
\end{tabular}

TEMPERATURE $\mathrm{C}$

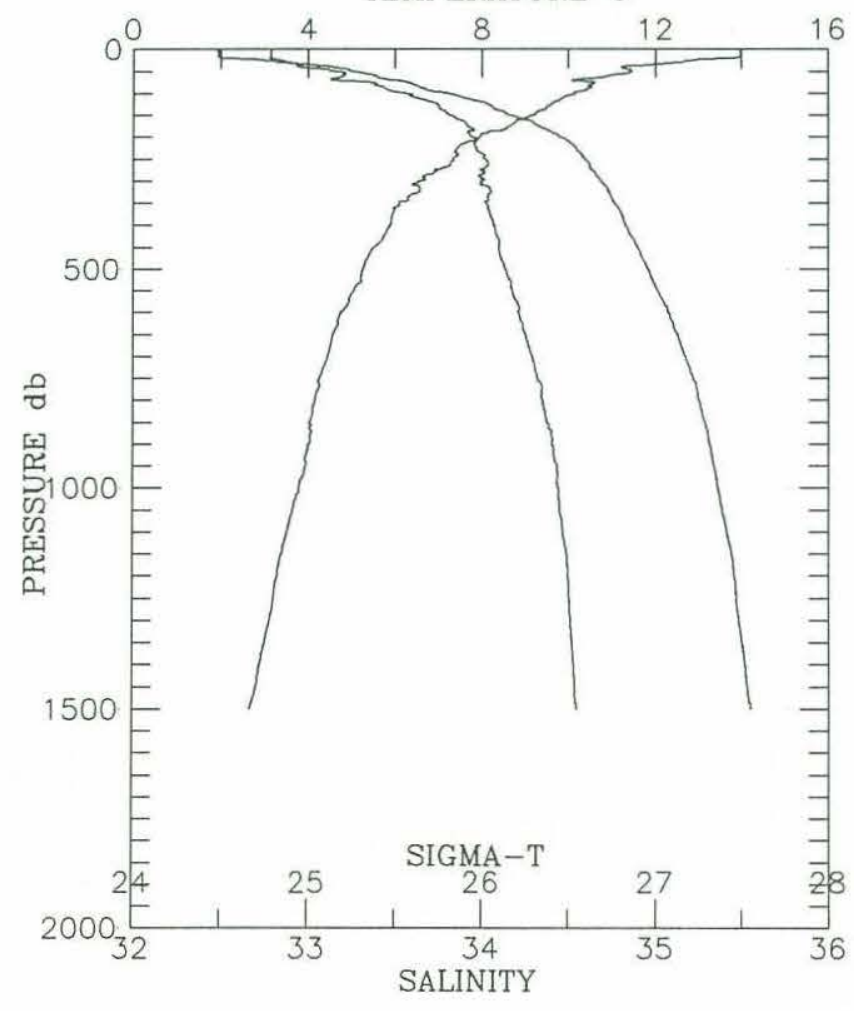

16

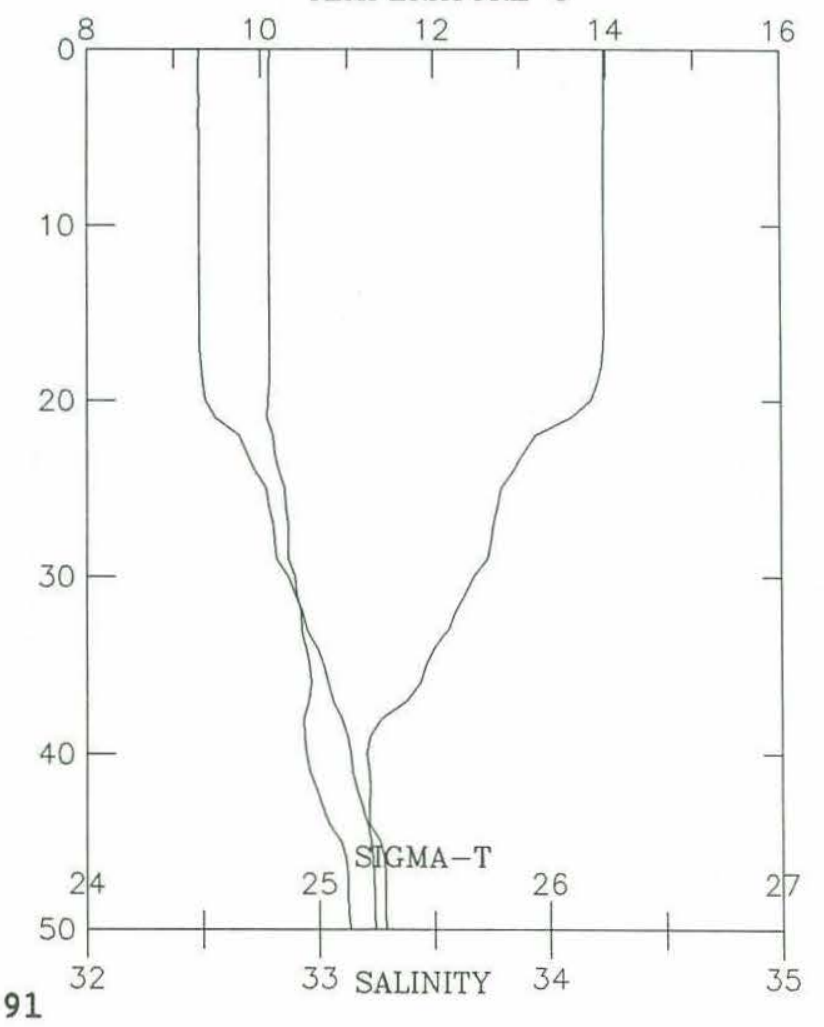


Cruise w8905 Station \# 9 Depth m: 2581 Time: 1989-05-06 08:04 Position: $38 \quad 6.30^{\prime} \mathrm{N} 123 \quad 44.10^{\prime} \mathrm{W} \quad$ Depth Deck Offset: -0.20

\begin{tabular}{|c|c|c|c|c|c|c|}
\hline $\begin{array}{l}\text { PRESSURE } \\
\text { (d-bars) }\end{array}$ & Interp & $\begin{array}{c}\text { TEMP } \\
(\operatorname{deg} \quad \mathrm{C})\end{array}$ & $\begin{array}{c}\text { SALINITY } \\
(0 / 00)\end{array}$ & SIGMA-T & DELTA-D & $\begin{array}{l}\text { CHANNELA } \\
\text { ( } \% 1 \text { ight) }\end{array}$ \\
\hline 0.0 & $\mathrm{E}$ & 13.826 & 32.745 & 24.481 & 0.0000 & 0.0 \\
\hline 10.0 & & 13.818 & 32.746 & 24.484 & 0.0348 & 0.0 \\
\hline 20.0 & & 13.800 & 32.761 & 24.499 & 0.0695 & 0.0 \\
\hline 30.0 & & 12.906 & 32.869 & 24.761 & 0.1024 & 0.0 \\
\hline 40.0 & & 11.917 & 32.896 & 24.971 & 0.1334 & 0.0 \\
\hline 50.0 & & 11.216 & 32.935 & 25.130 & 0.1628 & 0.0 \\
\hline 60.0 & & 10.651 & 33.090 & 25.350 & 0.1907 & 0.0 \\
\hline 80.0 & & 10.064 & 33.274 & 25.594 & 0.2418 & 0.0 \\
\hline 100.0 & & 9.294 & 33.440 & 25.850 & 0.2881 & 0.0 \\
\hline 150.0 & & 8.461 & 33.893 & 26.335 & 0.3855 & 0.0 \\
\hline 200.0 & & 7.478 & 33.971 & 26.542 & 0.4670 & 0.0 \\
\hline 250.0 & & 6.934 & 34.000 & 26.641 & 0.5420 & 0.0 \\
\hline 300.0 & & 6.371 & 34.009 & 26.722 & 0.6132 & 0.0 \\
\hline 400.0 & & 5.913 & 34.104 & 26.856 & 0.7459 & 0.0 \\
\hline 500.0 & & 5.170 & 34.129 & 26.965 & 0.8689 & 0.0 \\
\hline 600.0 & & 4.653 & 34.197 & 27.078 & 0.9814 & 0.0 \\
\hline 700.0 & & 4.827 & 34.346 & 27.177 & 1.0845 & 0.0 \\
\hline 800.0 & & 4.566 & 34.387 & 27.238 & 1.1815 & 0.0 \\
\hline 900.0 & & 4.108 & 34.417 & 27.312 & 1.2726 & 0.0 \\
\hline 1000.0 & & 3.917 & 34.447 & 27.355 & 1.3586 & 0.0 \\
\hline 1100.0 & & 3.475 & 34.459 & 27.409 & 1.4405 & 0.0 \\
\hline 1200.0 & & 3.288 & 34.486 & 27.448 & 1.5179 & 0.0 \\
\hline 1300.0 & & 3.094 & 34.509 & 27.484 & 1.5918 & 0.0 \\
\hline 1400.0 & & 2.905 & 34.529 & 27.517 & 1.6625 & 0.0 \\
\hline 1500.0 & & 2.702 & 34.542 & 27.546 & 1.7307 & 0.0 \\
\hline 1502.0 & & 2.700 & 34.542 & 27.546 & 1.7320 & 0.0 \\
\hline
\end{tabular}

TEMPERATURE C
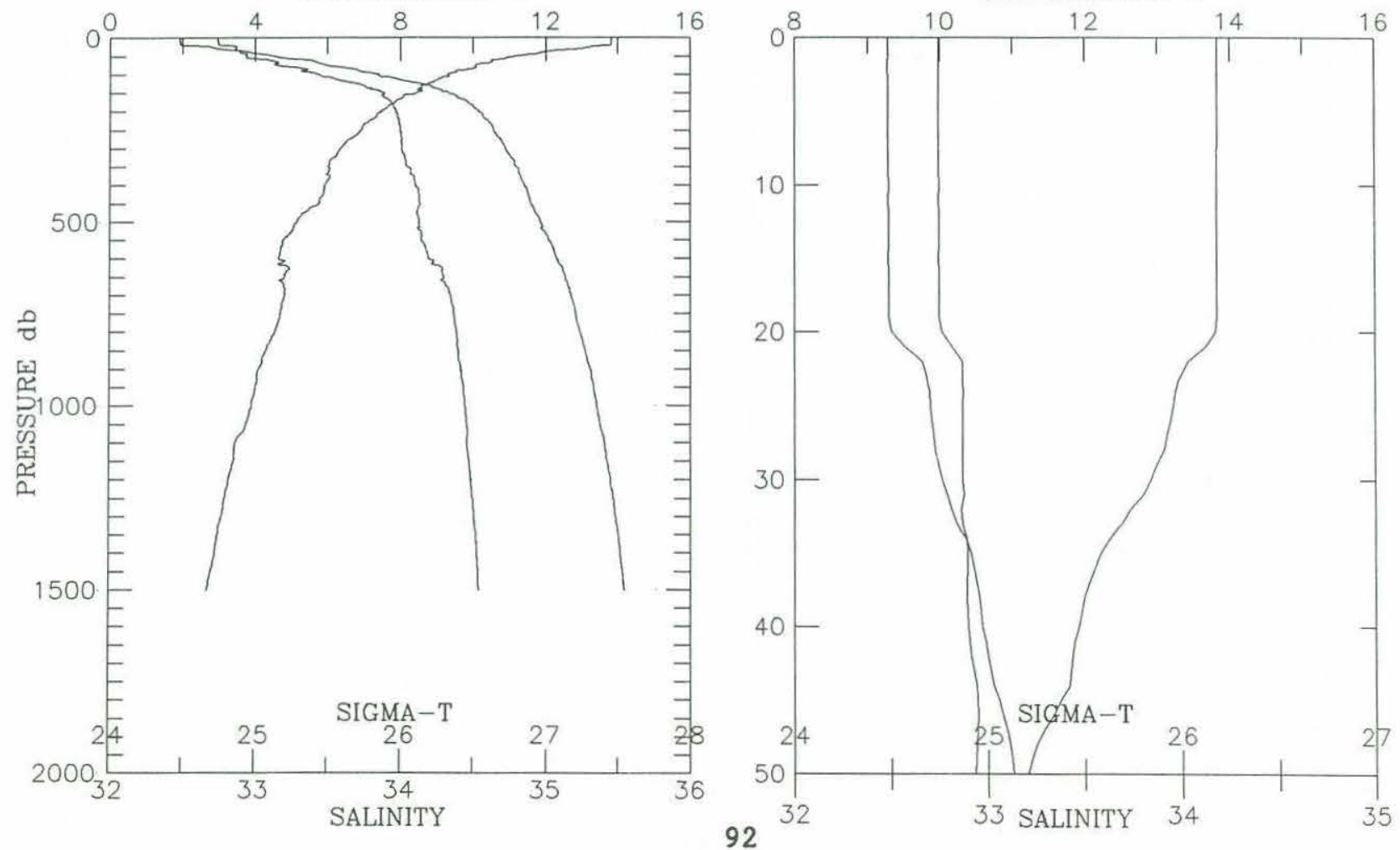
Cruise W8905 Station \# 10 Depth m: 1885 Time: 1989-05-06 09:31 Position: $38 \quad 7.40^{\prime} \mathrm{N} 123 \quad 38.50^{\prime} \mathrm{W}$ Depth Deck Offset: -0.10

\begin{tabular}{|c|c|c|c|c|c|c|}
\hline $\begin{array}{l}\text { PRESSURE } \\
\text { (d-bars) }\end{array}$ & Interp & $\begin{array}{l}\text { TEMP } \\
(\operatorname{deg} C)\end{array}$ & $\begin{array}{l}\text { SALINITY } \\
(0 / 00)\end{array}$ & SIGMA-T & DELTA-D & $\begin{array}{l}\text { CHANNELA } \\
\text { ( } ₹ 1 \text { ight) }\end{array}$ \\
\hline 0.0 & E & 14.113 & 32.816 & 24.477 & 0.0000 & 0.0 \\
\hline 10.0 & & 14.113 & 32.814 & 24.475 & 0.0348 & 0.0 \\
\hline 20.0 & & 14.106 & 32.816 & 24.478 & 0.0697 & 0.0 \\
\hline 30.0 & & 13.226 & 32.945 & 24.757 & 0.1039 & 0.0 \\
\hline 40.0 & & 11.817 & 32.959 & 25.039 & 0.1346 & 0.0 \\
\hline 50.0 & & 10.328 & 32.869 & 25.233 & 0.1631 & 0.0 \\
\hline 60.0 & & 10.093 & 32.999 & 25.374 & 0.1900 & 0.0 \\
\hline 80.0 & & 9.596 & 33.336 & 25.720 & 0.2398 & 0.0 \\
\hline 100.0 & & 8.839 & 33.500 & 25.969 & 0.2837 & 0.0 \\
\hline 150.0 & & 8.101 & 33.867 & 26.369 & 0.3761 & 0.0 \\
\hline 200.0 & & 7.687 & 33.994 & 26.530 & 0.4575 & 0.0 \\
\hline 250.0 & & 7.076 & 34.013 & 26.631 & 0.5332 & 0.0 \\
\hline 300.0 & & 6.794 & 34.087 & 26.728 & 0.6049 & 0.0 \\
\hline 400.0 & & 6.080 & 34.128 & 26.854 & 0.7374 & 0.0 \\
\hline 500.0 & & 5.273 & 34.140 & 26.962 & 0.8601 & 0.0 \\
\hline 600.0 & & 5.041 & 34.226 & 27.057 & 0.9739 & 0.0 \\
\hline 700.0 & & 4.528 & 34.275 & 27.154 & 1.0796 & 0.0 \\
\hline 800.0 & & 4.518 & 34.385 & 27.242 & 1.1771 & 0.0 \\
\hline 900.0 & & 4.212 & 34.422 & 27.305 & 1.2687 & 0.0 \\
\hline 1000.0 & & 3.919 & 34.441 & 27.350 & 1.3551 & 0.0 \\
\hline 1100.0 & & 3.675 & 34.469 & 27.397 & 1.4376 & 0.0 \\
\hline 1200.0 & & 3.463 & 34.485 & 27.431 & 1.5167 & 0.0 \\
\hline 1300.0 & & 3.214 & 34.508 & 27.472 & 1.5926 & 0.0 \\
\hline 1400.0 & & 3.014 & 34.524 & 27.504 & 1.6653 & 0.0 \\
\hline 1500.0 & & 2.764 & 34.544 & 27.542 & 1.7342 & 0.0 \\
\hline 1501.0 & & 2.764 & 34.543 & 27.541 & 1.7349 & 0.0 \\
\hline
\end{tabular}

TEMPERATURE C

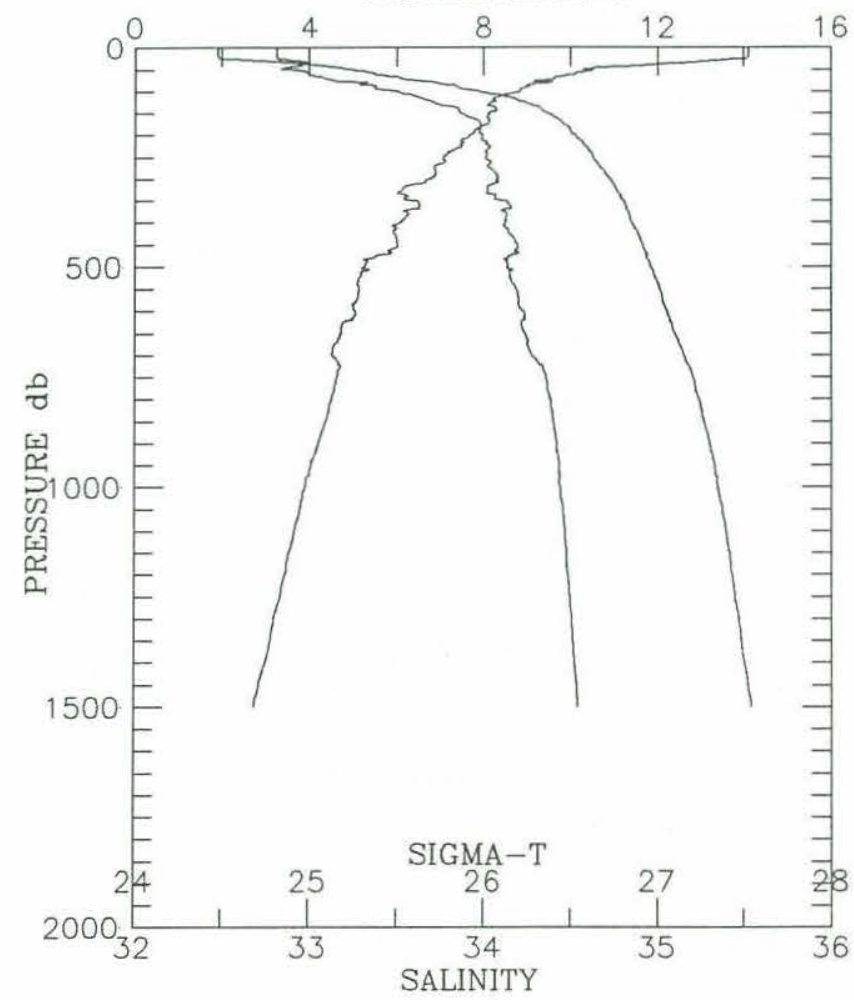

16

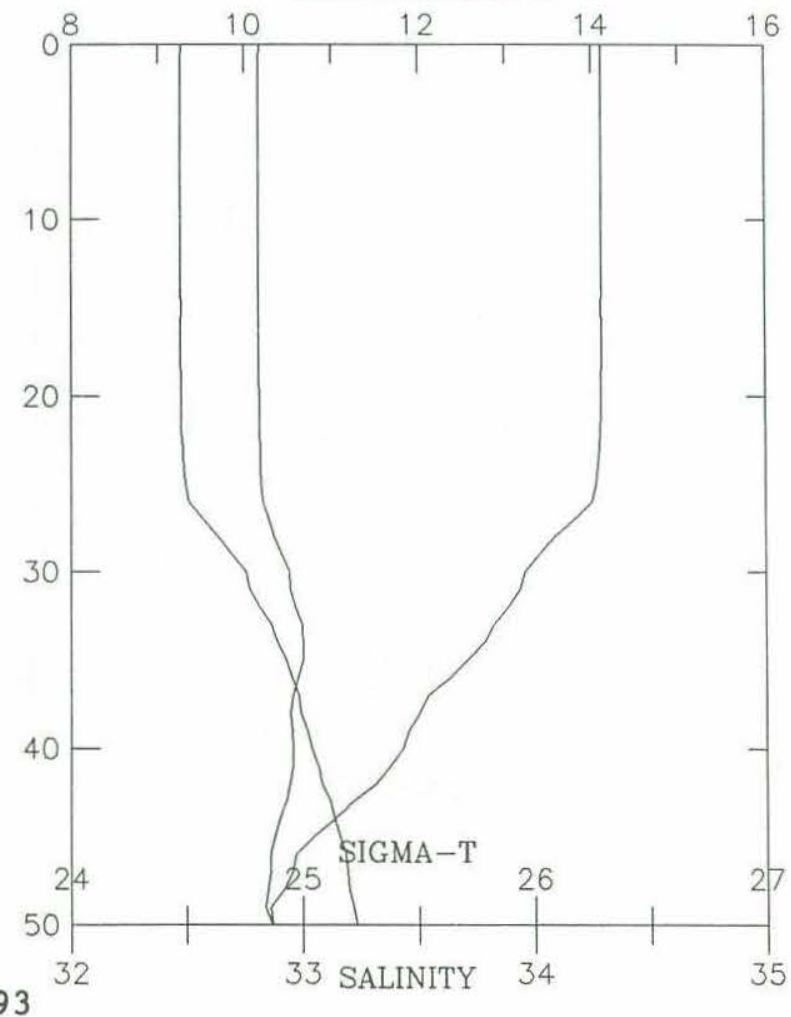




\begin{tabular}{|c|c|c|c|c|c|c|}
\hline $\begin{array}{l}\text { PRESSURE } \\
\text { (d-bars) }\end{array}$ & Interp & $\begin{array}{l}\text { TEMP } \\
(\operatorname{deg} C)\end{array}$ & $\begin{array}{c}\text { SALINITY } \\
(0 / 00)\end{array}$ & SIGMA-T & DELTA-D & $\begin{array}{l}\text { CHANNELA } \\
\text { ( } 81 \text { ight) }\end{array}$ \\
\hline 0.0 & E & 14.139 & 32.836 & 24.487 & 0.0000 & 0.0 \\
\hline 10.0 & & 14.137 & 32.836 & 24.487 & 0.0347 & 0.0 \\
\hline 20.0 & & 13.490 & 32.888 & 24.660 & 0.0692 & 0.0 \\
\hline 30.0 & & 12.759 & 32.962 & 24.862 & 0.1017 & 0.0 \\
\hline 40.0 & & 11.850 & 32.974 & 25.044 & 0.1322 & 0.0 \\
\hline 50.0 & & 10.733 & 33.001 & 25.267 & 0.1605 & 0.0 \\
\hline 60.0 & & 10.448 & 33.190 & 25.463 & 0.1867 & 0.0 \\
\hline 80.0 & & 9.117 & 33.261 & 25.738 & 0.2351 & 0.0 \\
\hline 100.0 & & 8.533 & 33.410 & 25.946 & 0.2792 & 0.0 \\
\hline 150.0 & & 8.203 & 33.930 & 26.404 & 0.3720 & 0.0 \\
\hline 200.0 & & 7.871 & 34.039 & 26.539 & 0.4528 & 0.0 \\
\hline 250.0 & & 6.791 & 34.005 & 26.663 & 0.5274 & 0.0 \\
\hline 300.0 & & 6.785 & 34.096 & 26.736 & 0.5977 & 0.0 \\
\hline 400.0 & & 6.248 & 34.175 & 26.869 & 0.7302 & 0.0 \\
\hline 500.0 & & 5.807 & 34.232 & 26.970 & 0.8533 & 0.0 \\
\hline 600.0 & & 5.288 & 34.288 & 27.077 & 0.9669 & 0.0 \\
\hline 700.0 & & 5.028 & 34.329 & 27.140 & 1.0726 & 0.0 \\
\hline 800.0 & & 4.578 & 34.380 & 27.231 & 1.1725 & 0.0 \\
\hline 860.0 & & 4.297 & 34.412 & 27.287 & 1.2279 & 0.0 \\
\hline
\end{tabular}
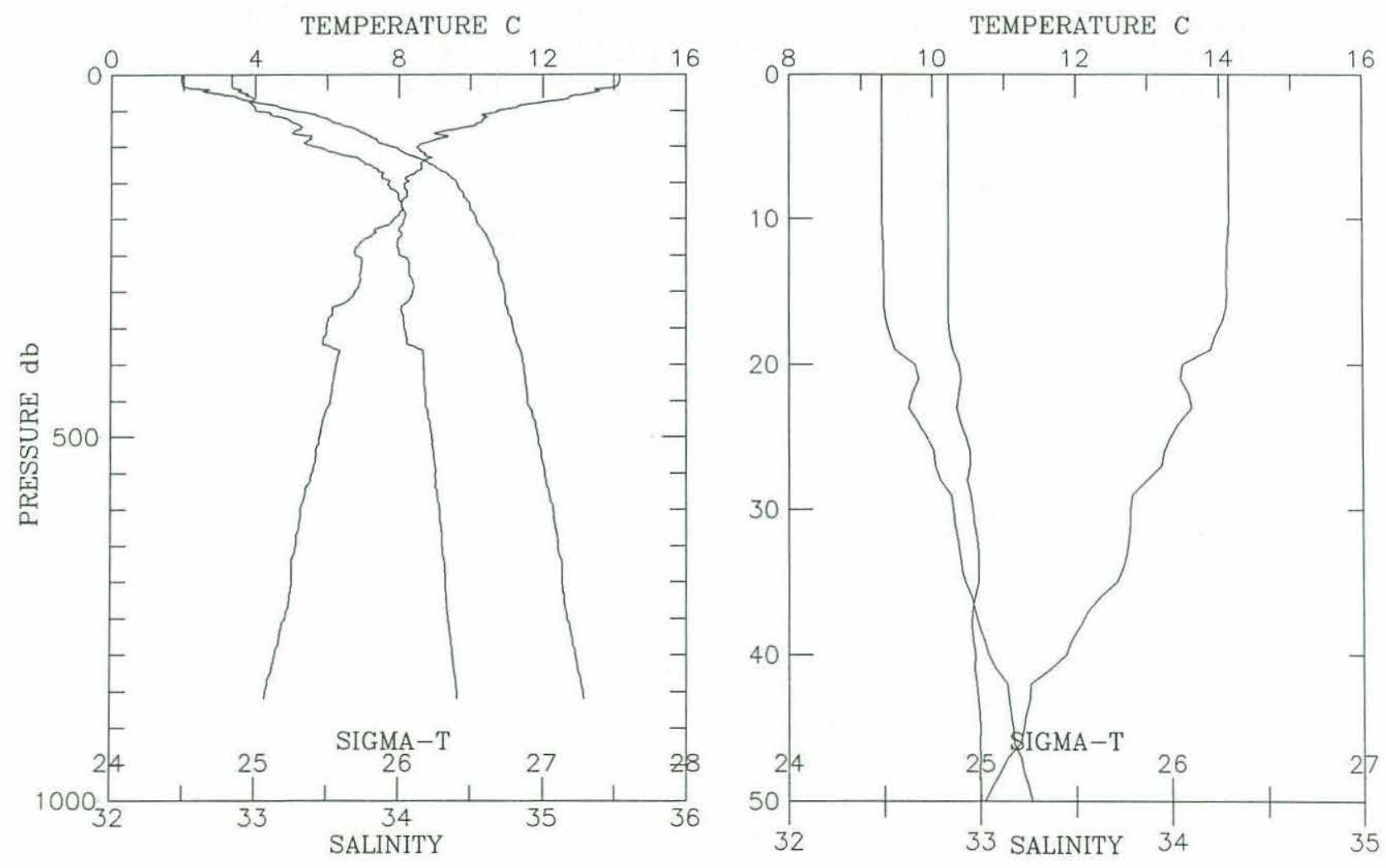


\begin{tabular}{|c|c|c|c|c|c|c|c|}
\hline Cruise W8 & $\begin{array}{r}8905 \\
\quad 38\end{array}$ & Station \# & 12 Dept & $\mathrm{m}: 353$ & $\begin{array}{l}\text { Time: } \\
\text { epth Deck }\end{array}$ & $\begin{array}{l}\text { 1989-05-06 } \\
\text { offset: }\end{array}$ & $\begin{array}{l}12: 20 \\
-0.05\end{array}$ \\
\hline $\begin{array}{l}\text { PRESSURE } \\
\text { (d-bars) }\end{array}$ & Interp & $\begin{array}{l}\text { TEMP } \\
(\operatorname{deg} C)\end{array}$ & $\begin{array}{c}\text { SALINITY } \\
(0 / 00)\end{array}$ & SIGMA-T & DELTA-D & $\begin{array}{l}\text { CHANNELA } \\
\text { ( }(1 \text { ight) }\end{array}$ & \\
\hline 0.0 & E & 14.147 & 32.807 & 24.463 & 0.0000 & 0.0 & \\
\hline 10.0 & & 14.138 & 32.807 & 24.465 & 0.0349 & 0.0 & \\
\hline 20.0 & & 13.099 & 32.866 & 24.721 & 0.0686 & 0.0 & \\
\hline 30.0 & & 11.238 & 32.923 & 25.117 & 0.0997 & 0.0 & \\
\hline 40.0 & & 11.065 & 33.025 & 25.227 & 0.1279 & 0.0 & \\
\hline 50.0 & & 10.679 & 33.072 & 25.332 & 0.1552 & 0.0 & \\
\hline 60.0 & & 10.402 & 33.205 & 25.482 & 0.1813 & 0.0 & \\
\hline 80.0 & & 9.819 & 33.384 & 25.720 & 0.2299 & 0.0 & \\
\hline 100.0 & & 8.813 & 33.406 & 25.900 & 0.2742 & 0.0 & \\
\hline 150.0 & & 8.277 & 33.958 & 26.414 & 0.3672 & 0.0 & \\
\hline 200.0 & & 7.463 & 34.037 & 26.596 & 0.4460 & 0.0 & \\
\hline 250.0 & & 6.888 & 34.067 & 26.699 & 0.5178 & 0.0 & \\
\hline 300.0 & & 6.743 & 34.092 & 26.738 & 0.5873 & 0.0 & \\
\hline 344.0 & & 6.385 & 34.116 & 26.805 & 0.6469 & 0.0 & \\
\hline
\end{tabular}
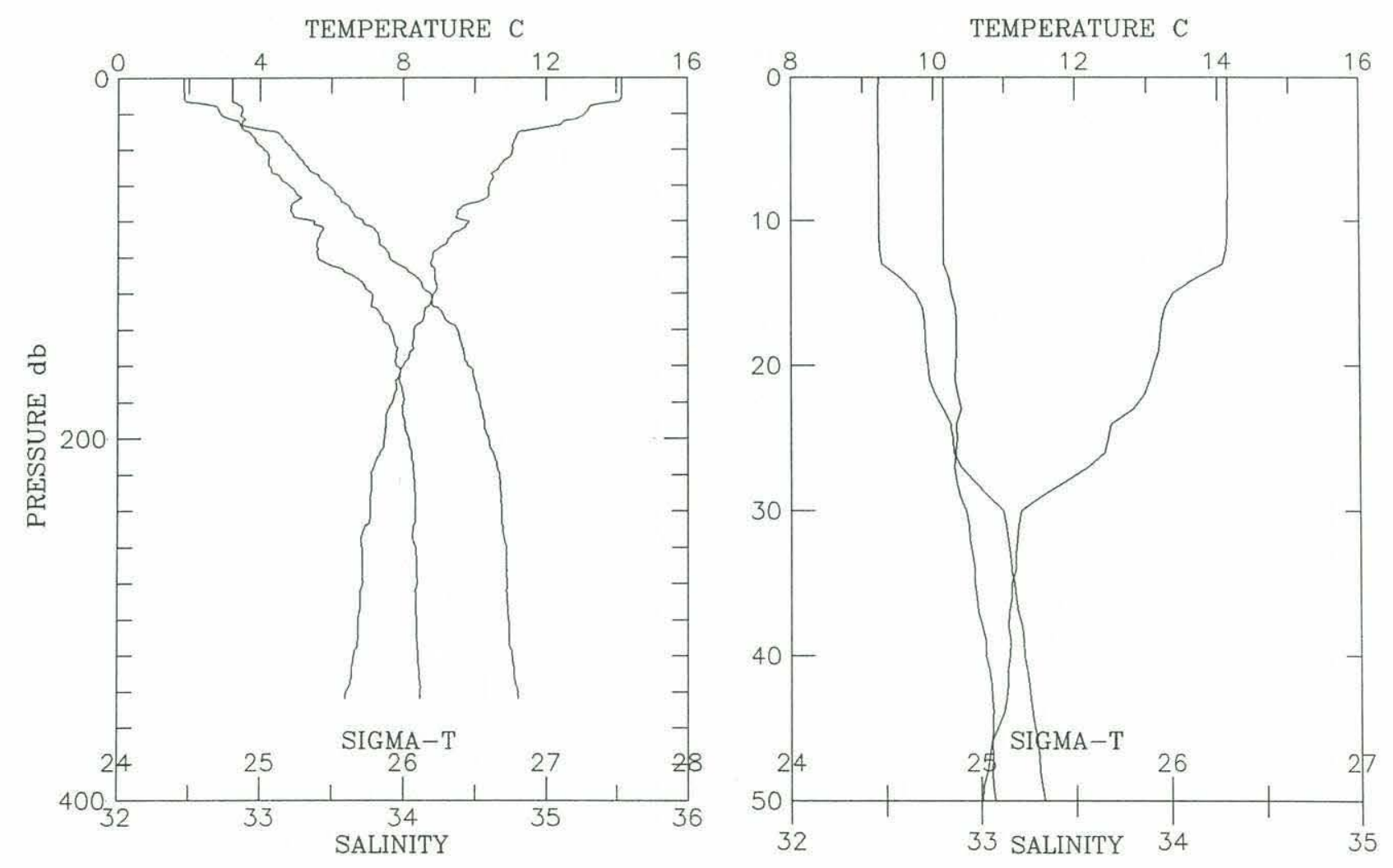
Cruise w8905 Station \# 13 Depth m: 201 Time: 1989-05-06 13:16 Position: $38 \quad 10.50^{\prime} \mathrm{N} 12322.10^{\prime} \mathrm{W}$ Depth Deck offset: -0.25

\begin{tabular}{|c|c|c|c|c|c|c|}
\hline $\begin{array}{l}\text { PRESSURE } \\
\text { (d-bars) }\end{array}$ & Interp & $\begin{array}{l}\text { TEMP } \\
(\operatorname{deg} C)\end{array}$ & $\begin{array}{c}\text { SALINITY } \\
(0 / 00)\end{array}$ & SIGMA-T & DELTA-D & $\begin{array}{l}\text { CHANNELA } \\
\text { ( }(1 \mathrm{ight})\end{array}$ \\
\hline 0.0 & E & 13.952 & 32.805 & 24.502 & 0.0000 & 0.0 \\
\hline 10.0 & & 13.953 & 32.804 & 24.501 & 0.0346 & 0.0 \\
\hline 20.0 & & 13.764 & 32.799 & 24.536 & 0.0692 & 0.0 \\
\hline 30.0 & & 11.784 & 32.841 & 24.953 & 0.1013 & 0.0 \\
\hline 40.0 & & 11.042 & 32.934 & 25.160 & 0.1306 & 0.0 \\
\hline 50.0 & & 10.196 & 32.905 & 25.284 & 0.1585 & 0.0 \\
\hline 60.0 & & 10.083 & 33.132 & 25.480 & 0.1851 & 0.0 \\
\hline 70.0 & & 9.392 & 33.251 & 25.687 & 0.2096 & 0.0 \\
\hline 80.0 & & 9.524 & 33.425 & 25.801 & 0.2325 & 0.0 \\
\hline 90.0 & & 9.332 & 33.564 & 25.941 & 0.2540 & 0.0 \\
\hline 100.0 & & 9.249 & 33.709 & 26.068 & 0.2745 & 0.0 \\
\hline 110.0 & & 9.073 & 33.744 & 26.123 & 0.2941 & 0.0 \\
\hline 120.0 & & 8.788 & 33.830 & 26.236 & 0.3127 & 0.0 \\
\hline 130.0 & & 8.693 & 33.852 & 26.268 & 0.3309 & 0.0 \\
\hline 140.0 & & 8.564 & 33.872 & 26.303 & 0.3488 & 0.0 \\
\hline 150.0 & & 8.309 & 33.916 & 26.377 & 0.3662 & 0.0 \\
\hline 160.0 & & 8.059 & 33.962 & 26.450 & 0.3827 & 0.0 \\
\hline 170.0 & & 7.836 & 33.999 & 26.512 & 0.3987 & 0.0 \\
\hline 180.0 & & 7.697 & 34.019 & 26.548 & 0.4142 & 0.0 \\
\hline 183.0 & & 7.682 & 34.024 & 26.554 & 0.4189 & 0.0 \\
\hline
\end{tabular}
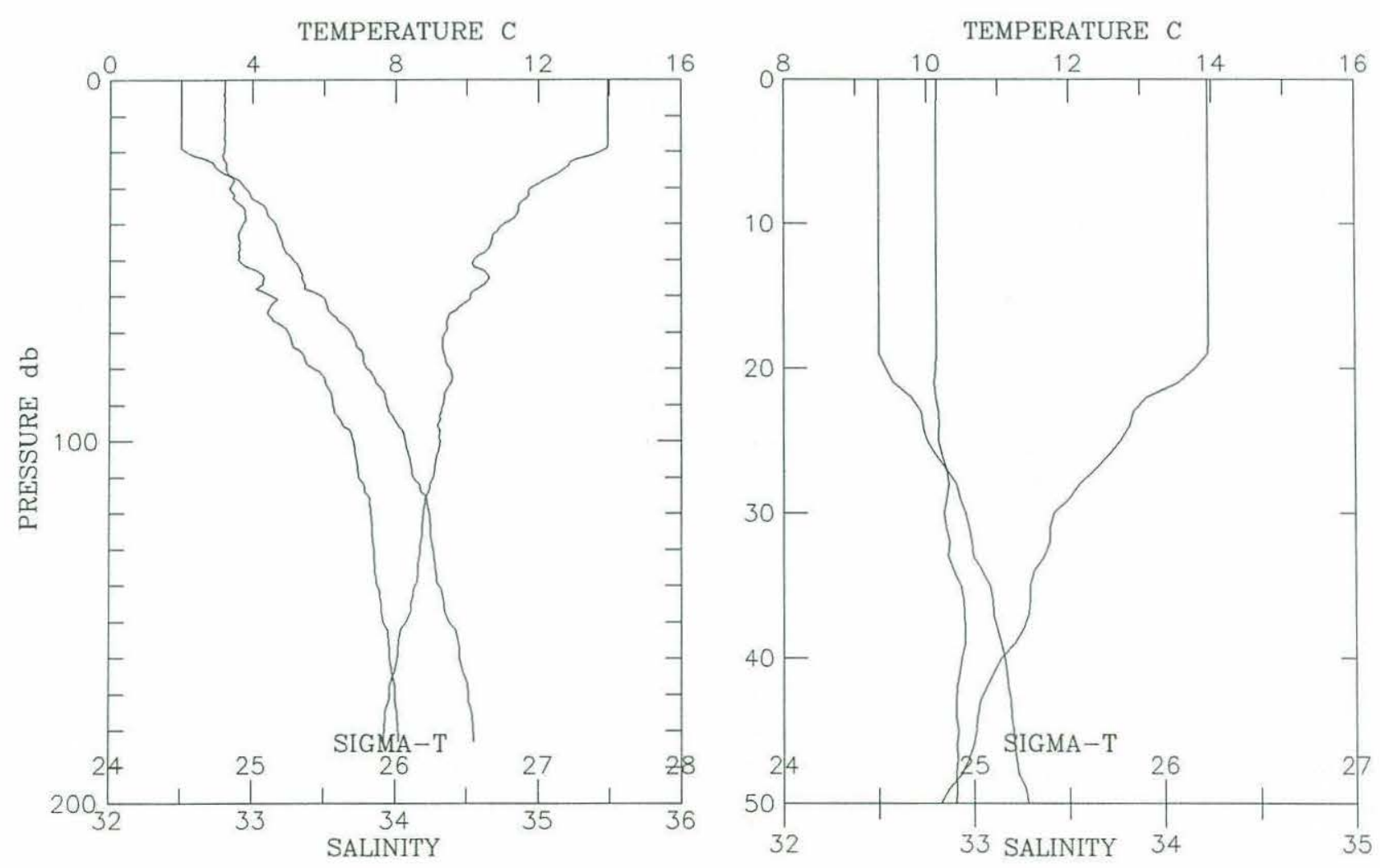
Cruise w8905 Station \# 14 Depth m: 115 Time: 1989-05-06 14:04 Position: $3811.50^{\prime} \mathrm{N} 12315.90^{\prime} \mathrm{W}$ Depth Deck Offset: -0.25

\begin{tabular}{|c|c|c|c|c|c|c|}
\hline $\begin{array}{l}\text { PRESSURE } \\
\text { (d-bars) }\end{array}$ & Interp & $\begin{array}{l}\text { TEMP } \\
(\operatorname{deg} \mathrm{C})\end{array}$ & $\begin{array}{c}\text { SALINITY } \\
(0 / 00)\end{array}$ & SIGMA-T & DELTA-D & $\begin{array}{l}\text { CHANNELA } \\
\text { ( }(1 \mathrm{ght})\end{array}$ \\
\hline 0.0 & E & 13.682 & 32.828 & 24.575 & 0.0000 & 0.0 \\
\hline 10.0 & & 13.673 & 32.828 & 24.577 & 0.0339 & 0.0 \\
\hline 20.0 & & 12.185 & 32.918 & 24.938 & 0.0668 & 0.0 \\
\hline 30.0 & & 11.238 & 32.991 & 25.170 & 0.0964 & 0.0 \\
\hline 40.0 & & 10.548 & 33.016 & 25.310 & 0.1242 & 0.0 \\
\hline 50.0 & & 9.980 & 32.990 & 25.386 & 0.1508 & 0.0 \\
\hline 60.0 & & 9.369 & 33.052 & 25.535 & 0.1761 & 0.0 \\
\hline 70.0 & & 9.409 & 33.269 & 25.698 & 0.2003 & 0.0 \\
\hline 80.0 & & 9.279 & 33.393 & 25.816 & 0.2232 & 0.0 \\
\hline 90.0 & & 9.316 & 33.598 & 25.970 & 0.2448 & 0.0 \\
\hline 100.0 & & 9.079 & 33.718 & 26.102 & 0.2650 & 0.0 \\
\hline 106.0 & & 8.894 & 33.784 & 26.183 & 0.2766 & 0.0 \\
\hline
\end{tabular}

TEMPERATURE C

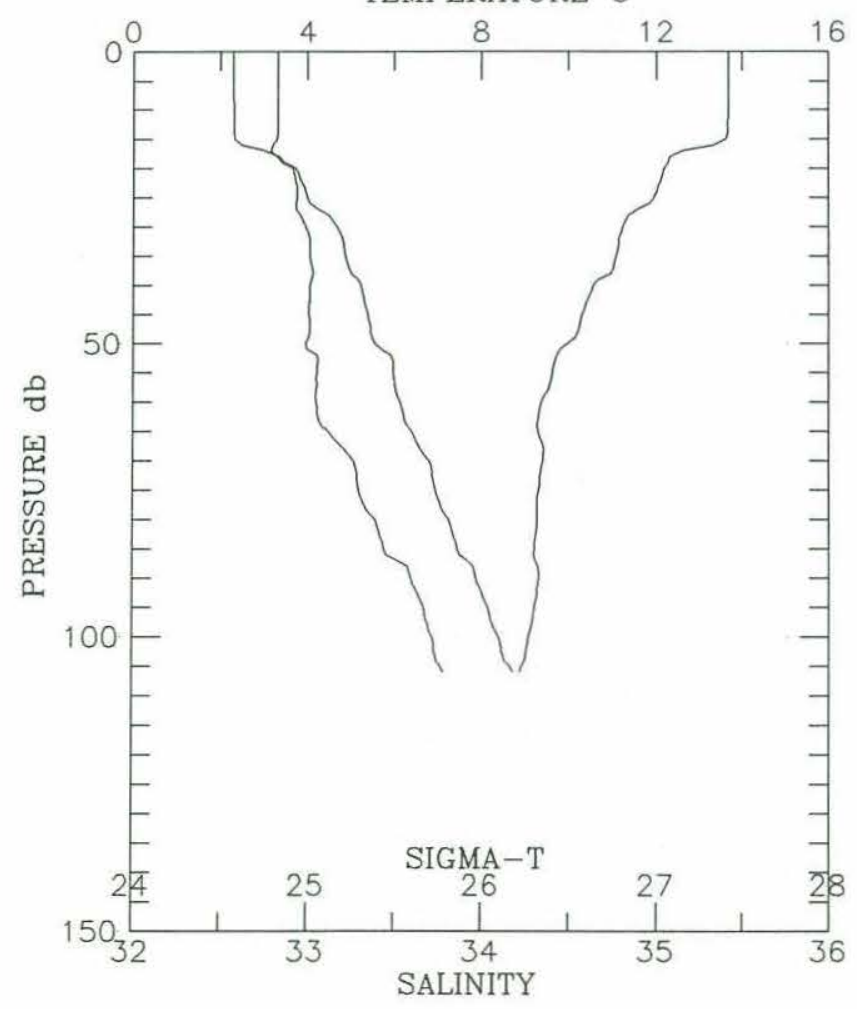

TEMPERATURE C

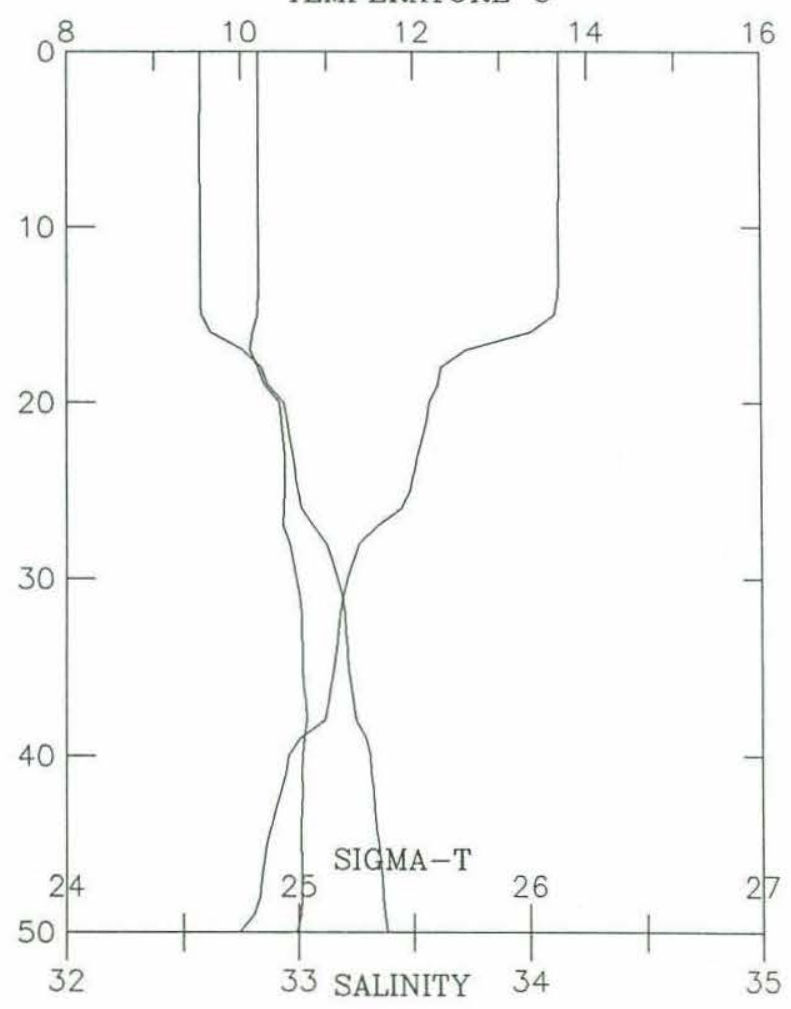


Cruise w8905 Station \# 15 Depth m: 92 Time: 1989-05-06 14:52

Position: $38 \quad 12.60^{\prime} \mathrm{N} 123 \quad 10.20^{\prime} \mathrm{W} \quad$ Depth Deck Offset: -0.25

\begin{tabular}{|c|c|c|c|c|c|c|}
\hline $\begin{array}{l}\text { PRESSURE } \\
\text { (d-bars) }\end{array}$ & Interp & $\begin{array}{l}\text { TEMP } \\
(\operatorname{deg} C)\end{array}$ & $\begin{array}{c}\text { SALINITY } \\
(0 / 00)\end{array}$ & SIGMA-T & DELTA-D & $\begin{array}{l}\text { CHANNELA } \\
\text { (\&light) }\end{array}$ \\
\hline 0.0 & E & 11.452 & 32.915 & 25.072 & 0.0000 & 0.0 \\
\hline 5.0 & & 11.454 & 32.914 & 25.071 & 0.0146 & 0.0 \\
\hline 10.0 & & 11.389 & 32.918 & 25.086 & 0.0291 & 0.0 \\
\hline 15.0 & & 11.373 & 32.918 & 25.089 & 0.0437 & 0.0 \\
\hline 20.0 & & 10.931 & 32.924 & 25.172 & 0.0579 & 0.0 \\
\hline 25.0 & & 10.449 & 32.913 & 25.247 & 0.0719 & 0.0 \\
\hline 30.0 & & 10.146 & 32.968 & 25.341 & 0.0854 & 0.0 \\
\hline 35.0 & & 9.999 & 33.081 & 25.454 & 0.0985 & 0.0 \\
\hline 40.0 & & 9.787 & 33.146 & 25.540 & 0.1111 & 0.0 \\
\hline 45.0 & & 9.578 & 33.189 & 25.608 & 0.1233 & 0.0 \\
\hline 50.0 & & 9.241 & 33.226 & 25.691 & 0.1351 & 0.0 \\
\hline 55.0 & & 8.972 & 33.293 & 25.786 & 0.1465 & 0.0 \\
\hline 60.0 & & 9.135 & 33.455 & 25.887 & 0.1575 & 0.0 \\
\hline 65.0 & & 9.190 & 33.552 & 25.954 & 0.1682 & 0.0 \\
\hline 70.0 & & 9.063 & 33.617 & 26.025 & 0.1784 & 0.0 \\
\hline 75.0 & & 8.989 & 33.682 & 26.088 & 0.1883 & 0.0 \\
\hline 80.0 & & 8.957 & 33.746 & 26.143 & 0.1980 & 0.0 \\
\hline 82.0 & & 8.894 & 33.782 & 26.181 & 0.2018 & 0.0 \\
\hline
\end{tabular}
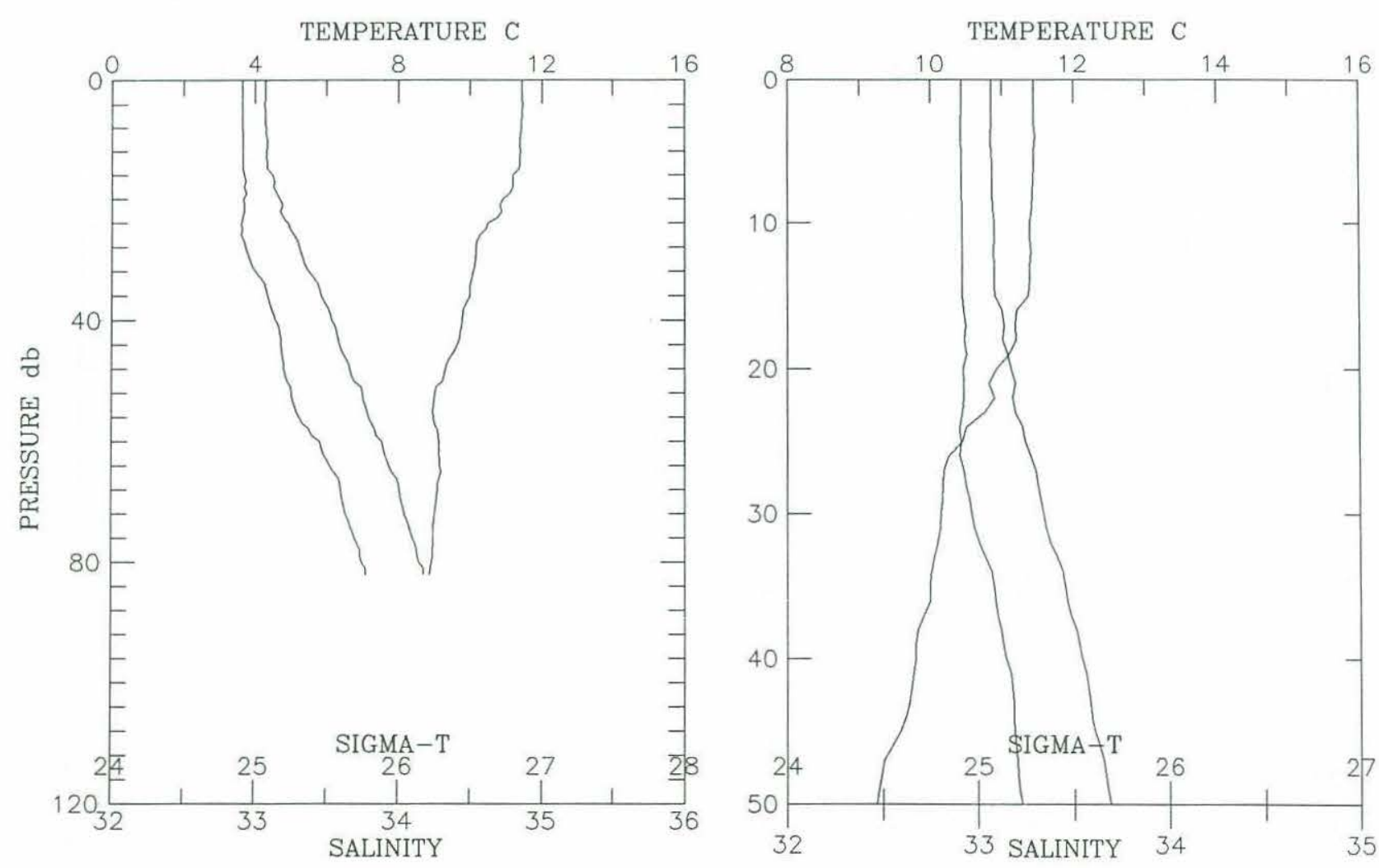


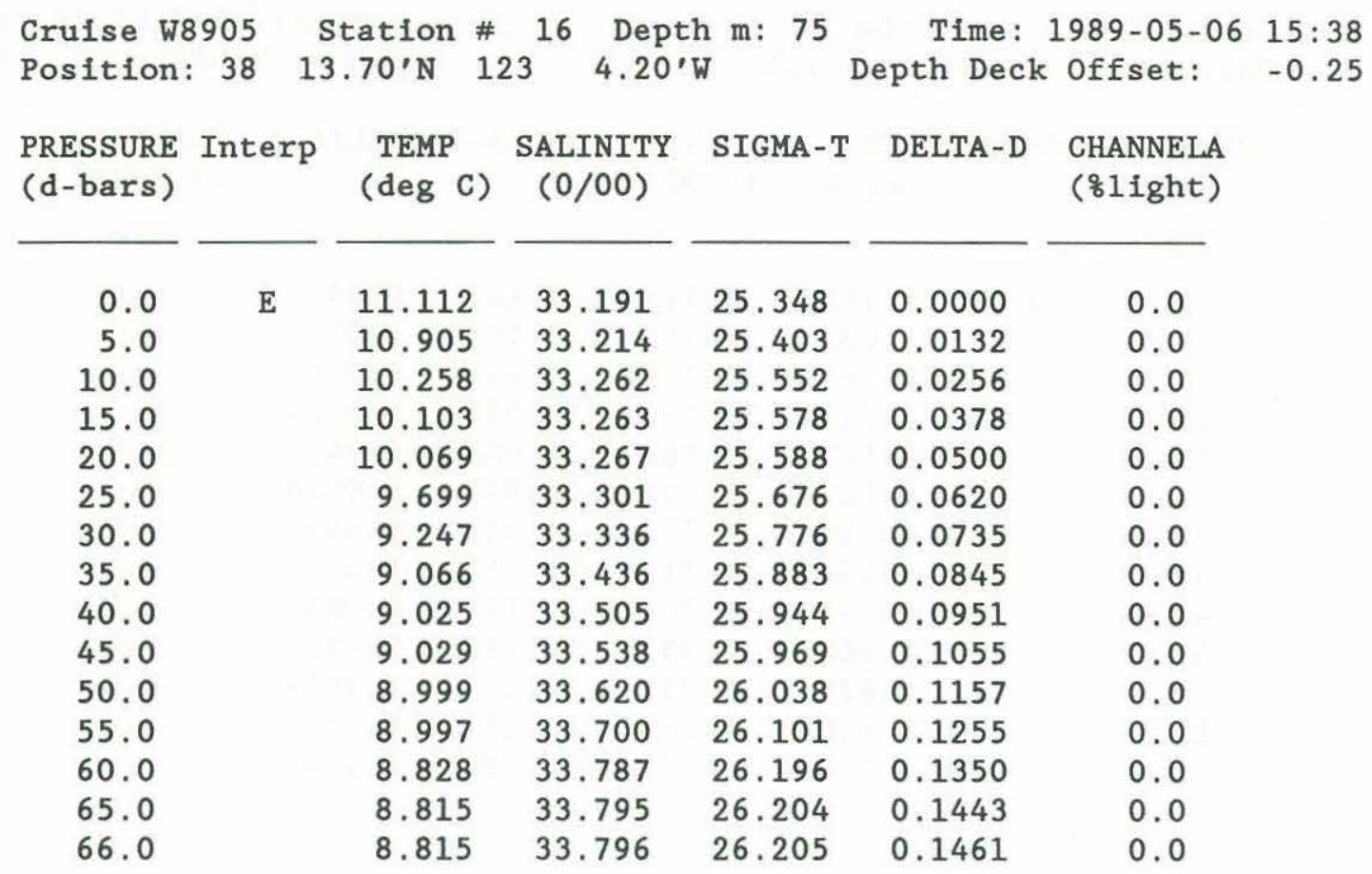
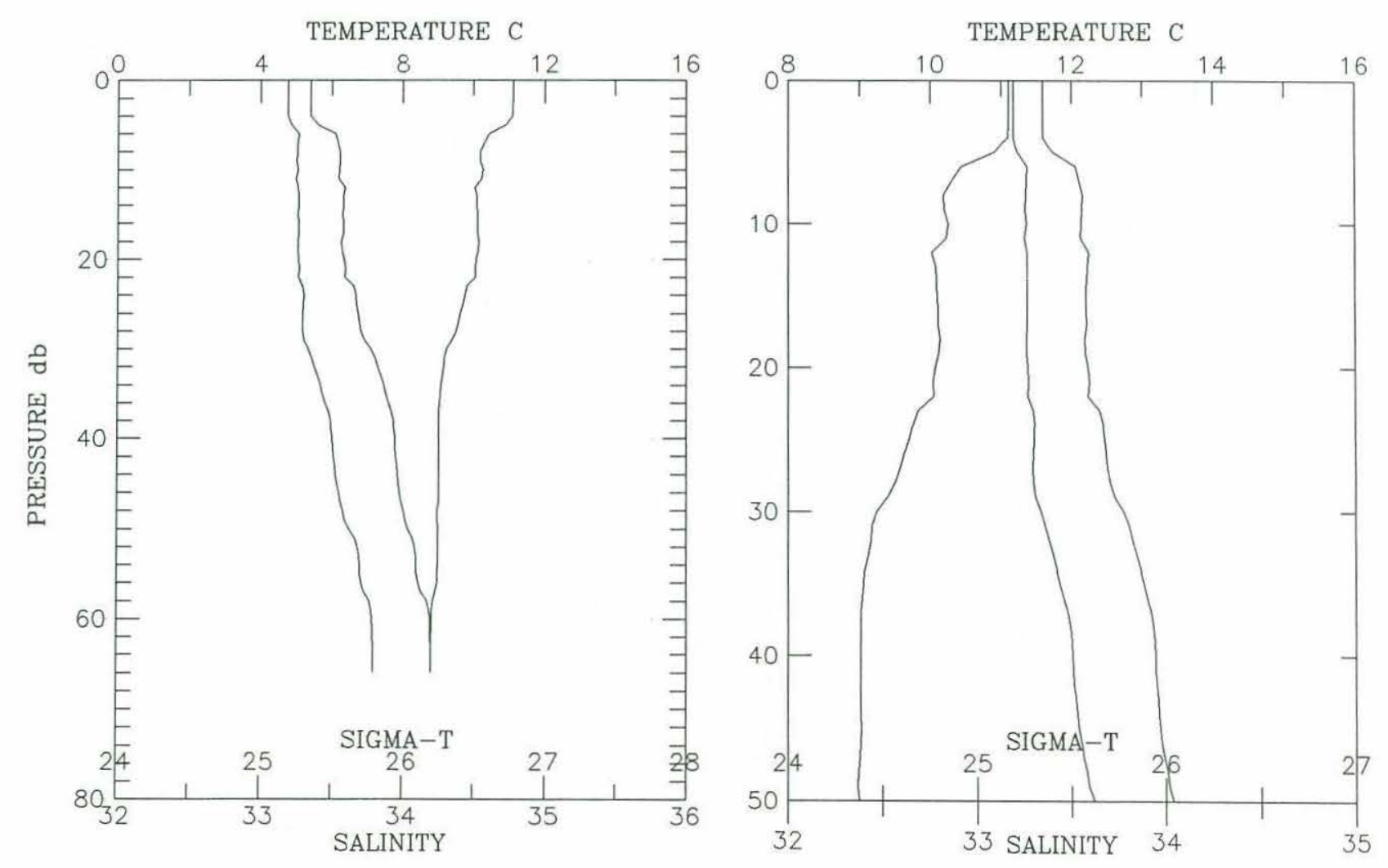
Cruise w8905 Station \# 17 Depth m: 68 Time: 1989-05-06 16:05 Position: $3814.10^{\prime} \mathrm{N} 123 \quad 1.70^{\prime} \mathrm{W}$ Depth Deck Offset: -0.25

\begin{tabular}{|c|c|c|c|c|c|c|}
\hline $\begin{array}{l}\text { PRESSURE } \\
\text { (d-bars) }\end{array}$ & Interp & $\begin{array}{l}\text { TEMP } \\
(\operatorname{deg} C)\end{array}$ & $\begin{array}{c}\text { SALINITY } \\
(0 / 00)\end{array}$ & SIGMA-T & DELTA-D & $\begin{array}{l}\text { CHANNELA } \\
\text { ( } \% 1 \mathrm{ight})\end{array}$ \\
\hline 0.0 & E & 11.212 & 33.361 & 25.462 & 0.0000 & 0.0 \\
\hline 5.0 & & 11.033 & 33.395 & 25.521 & 0.0126 & 0.0 \\
\hline 10.0 & & 10.591 & 33.454 & 25.644 & 0.0248 & 0.0 \\
\hline 15.0 & & 9.911 & 33.503 & 25.798 & 0.0363 & 0.0 \\
\hline 20.0 & & 9.463 & 33.566 & 25.921 & 0.0471 & 0.0 \\
\hline 25.0 & & 9.167 & 33.657 & 26.040 & 0.0574 & 0.0 \\
\hline 30.0 & & 9.054 & 33.698 & 26.090 & 0.0672 & 0.0 \\
\hline 35.0 & & 9.002 & 33.719 & 26.115 & 0.0769 & 0.0 \\
\hline 40.0 & & 8.941 & 33.746 & 26.146 & 0.0865 & 0.0 \\
\hline 45.0 & & 8.865 & 33.781 & 26.185 & 0.0959 & 0.0 \\
\hline 50.0 & & 8.837 & 33.795 & 26.201 & 0.1052 & 0.0 \\
\hline 55.0 & & 8.816 & 33.804 & 26.211 & 0.1144 & 0.0 \\
\hline 57.0 & & 8.820 & 33.765 & 26.180 & 0.1181 & 0.0 \\
\hline
\end{tabular}

TEMPERATURE C
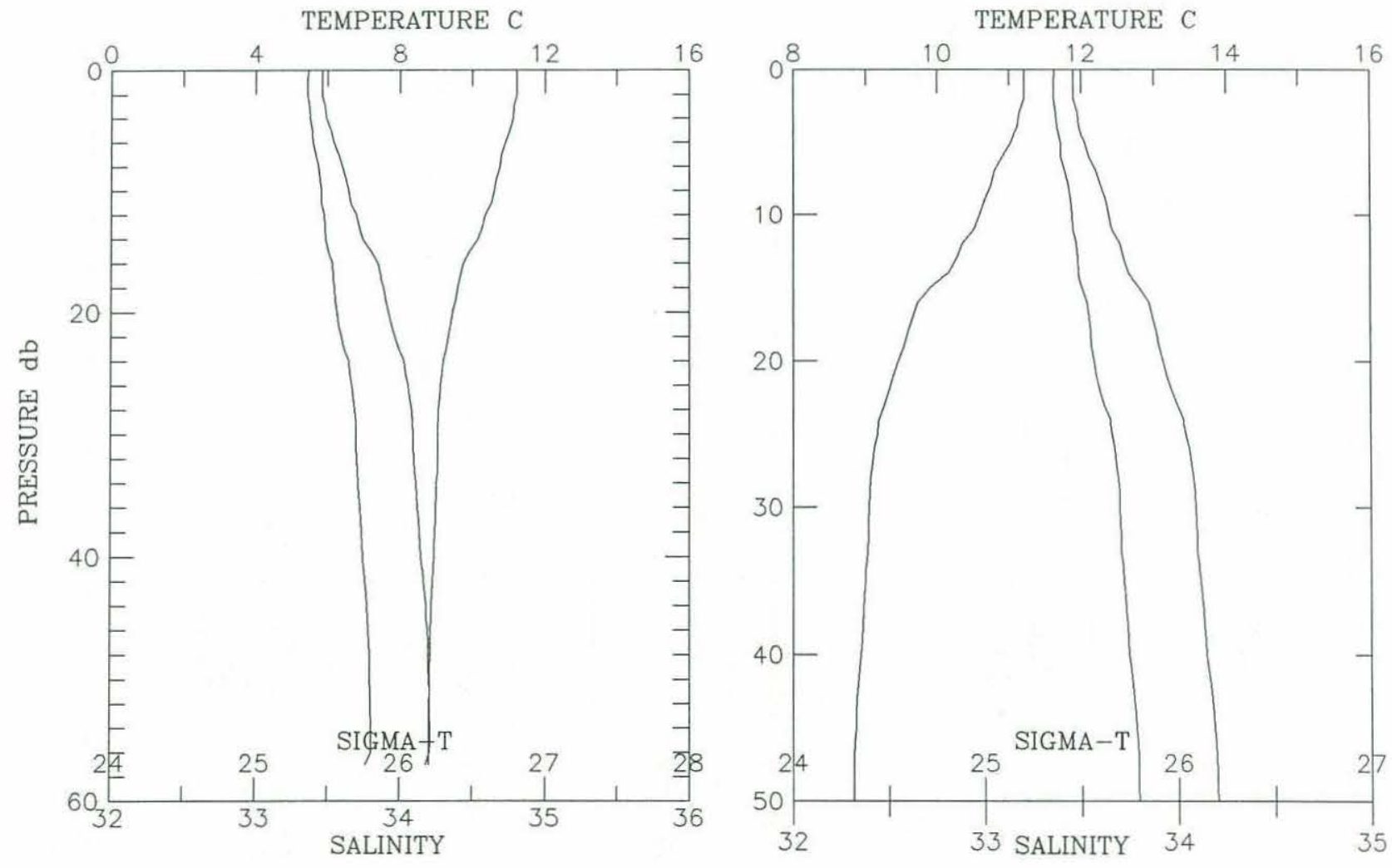
Cruise w8905 Station \# 18 Depth m: 65 Time: 1989-05-06 19:34 Position: $38 \quad 40.60^{\prime} \mathrm{N} 12326.70^{\prime} \mathrm{W}$ Depth Deck Offset: -0.20

\begin{tabular}{|c|c|c|c|c|c|c|}
\hline $\begin{array}{l}\text { PRESSURE } \\
\text { (d-bars) }\end{array}$ & Interp & $\begin{array}{l}\text { TEMP } \\
(\operatorname{deg} C)\end{array}$ & $\begin{array}{c}\text { SALINITY } \\
(0 / 00)\end{array}$ & SIGMA-T & DELTA-D & $\begin{array}{l}\text { CHANNELA } \\
\text { ( } 81 \text { ight) }\end{array}$ \\
\hline 0.0 & E & 9.806 & 33.395 & 25.731 & 0.0000 & 0.0 \\
\hline 5.0 & & 9.792 & 33.397 & 25.735 & 0.0114 & 0.0 \\
\hline 10.0 & & 9.670 & 33.409 & 25.765 & 0.0228 & 0.0 \\
\hline 15.0 & & 9.234 & 33.496 & 25.904 & 0.0338 & 0.0 \\
\hline 20.0 & & 9.101 & 33.579 & 25.990 & 0.0441 & 0.0 \\
\hline 25.0 & & 8.852 & 33.689 & 26.115 & 0.0542 & 0.0 \\
\hline 30.0 & & 8.828 & 33.709 & 26.135 & 0.0638 & 0.0 \\
\hline 35.0 & & 8.811 & 33.763 & 26.179 & 0.0732 & 0.0 \\
\hline 40.0 & & 8.800 & 33.786 & 26.199 & 0.0825 & 0.0 \\
\hline 45.0 & & 8.793 & 33.789 & 26.203 & 0.0918 & 0.0 \\
\hline 50.0 & & 8.790 & 33.791 & 26.205 & 0.1010 & 0.0 \\
\hline 55.0 & & 8.780 & 33.795 & 26.210 & 0.1102 & 0.0 \\
\hline 60.0 & & 8.772 & 33.799 & 26.214 & 0.1194 & 0.0 \\
\hline
\end{tabular}
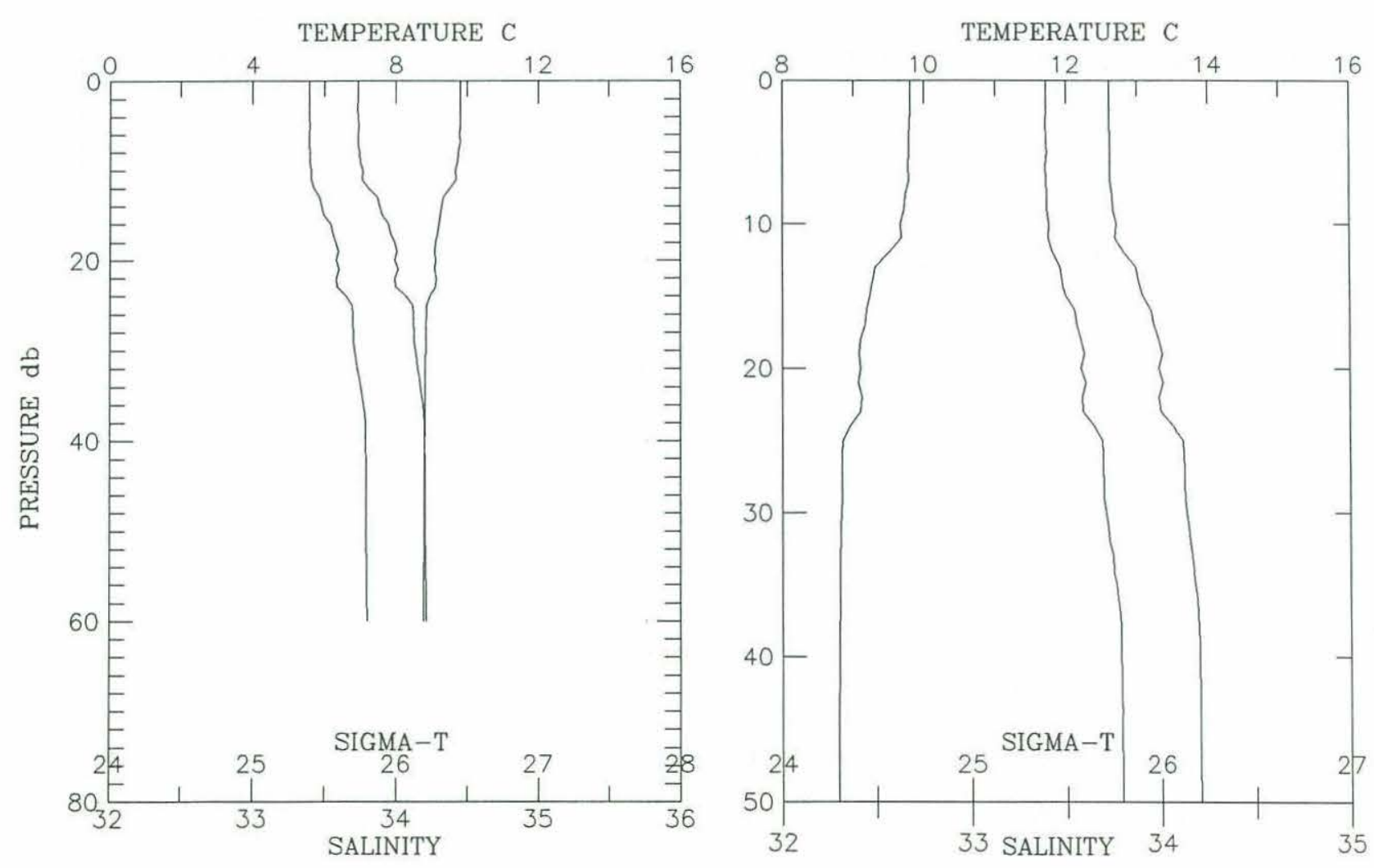


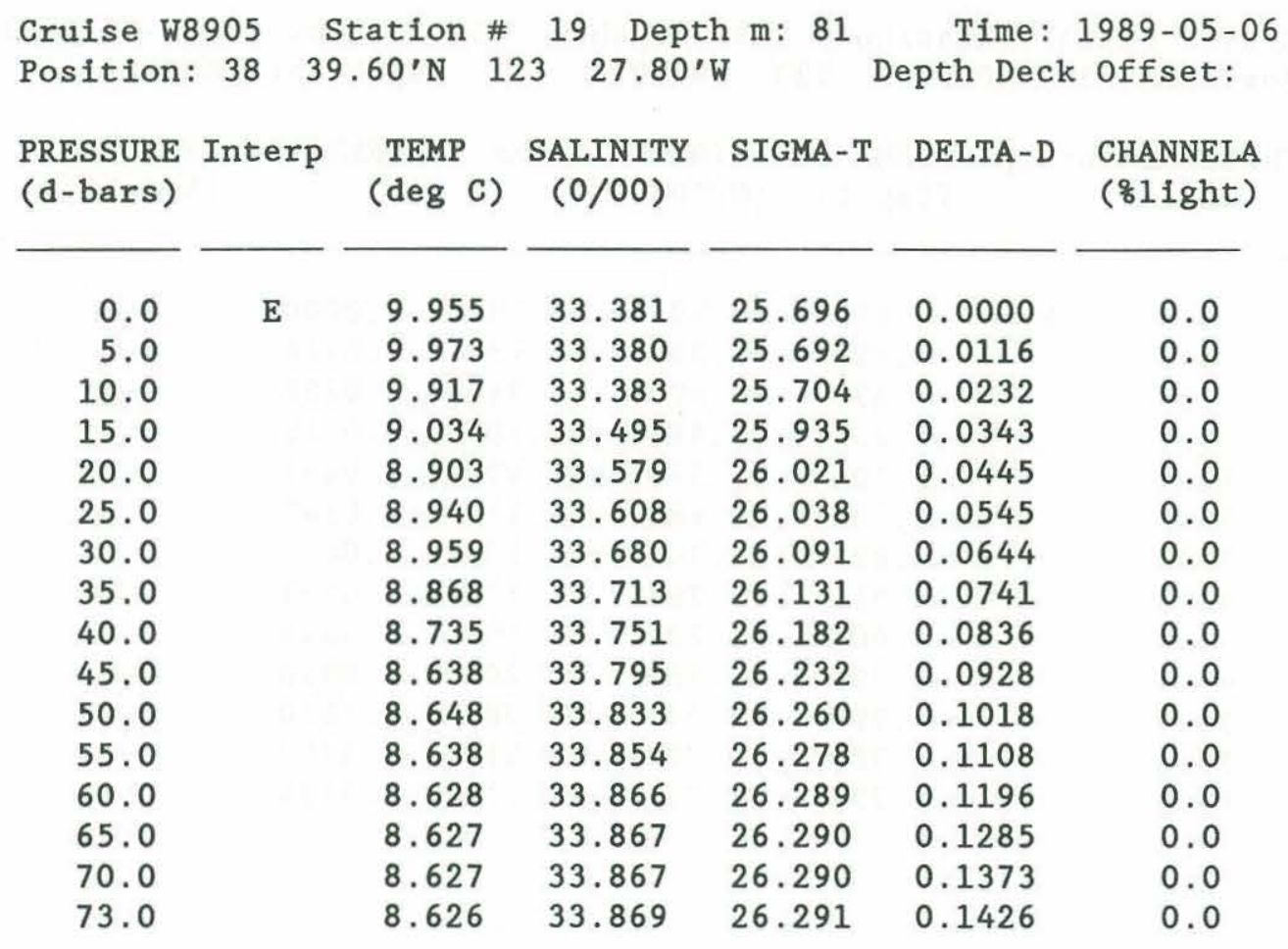
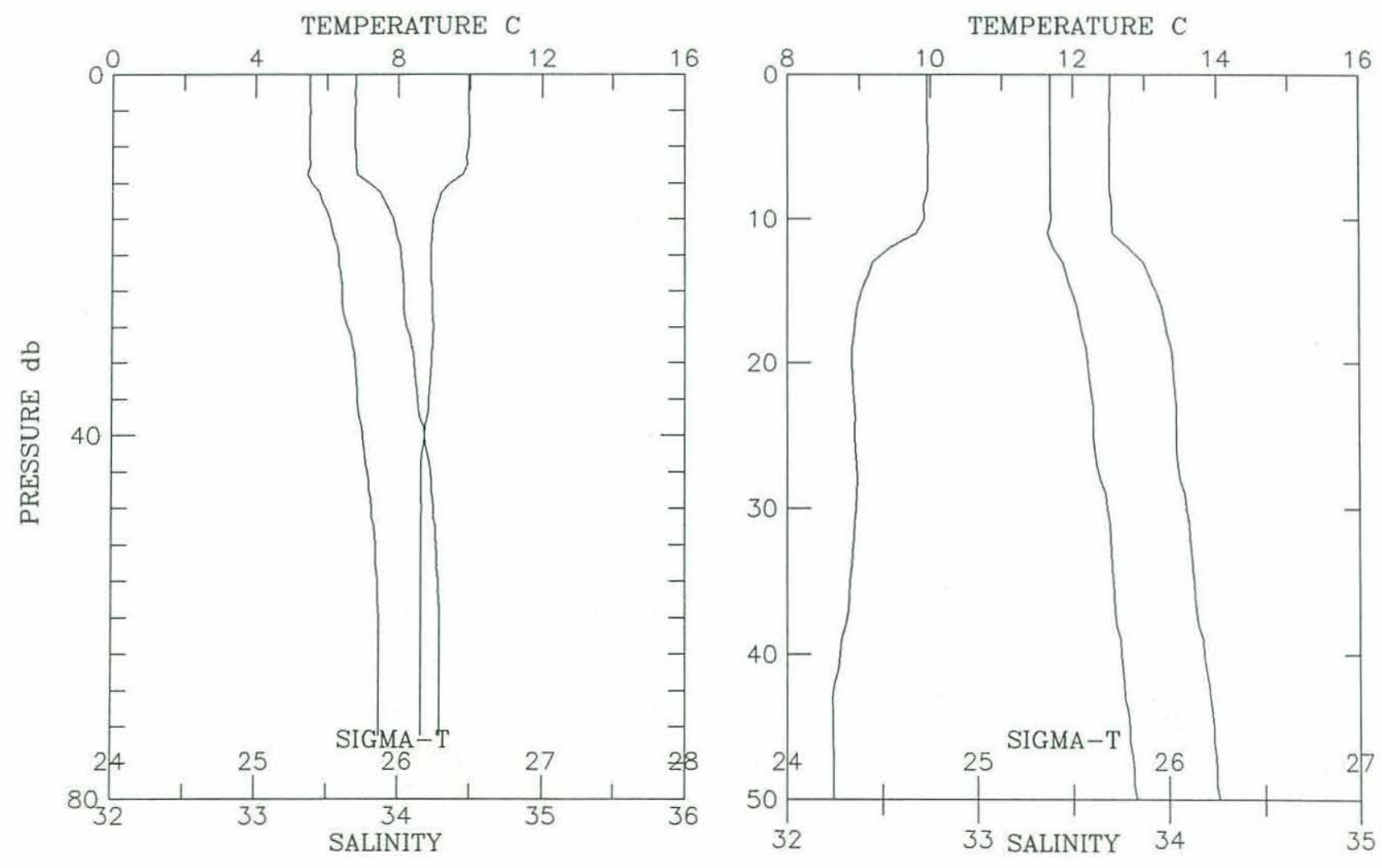
Cruise w8905 Station \# 20 Depth m: 95 Time: 1989-05-06 20:30 Position: 38 38.20'N $12329.30^{\prime} \mathrm{W} \quad$ Depth Deck Offset: $\quad-0.43$

\begin{tabular}{|c|c|c|c|c|c|c|}
\hline $\begin{array}{l}\text { PRESSURE } \\
\text { (d-bars) }\end{array}$ & Interp & $\begin{array}{l}\text { TEMP } \\
(\operatorname{deg} C)\end{array}$ & $\begin{array}{c}\text { SALINITY } \\
(0 / 00)\end{array}$ & SIGMA-T & DELTA-D & $\begin{array}{l}\text { CHANNELA } \\
\text { ( } 81 \text { ight) }\end{array}$ \\
\hline 0.0 & E & 10.265 & 33.376 & 25.639 & 0.0000 & 0.0 \\
\hline 5.0 & & 10.263 & 33.375 & 25.639 & 0.0119 & 0.0 \\
\hline 10.0 & & 10.226 & 33.371 & 25.642 & 0.0238 & 0.0 \\
\hline 15.0 & & 10.214 & 33.372 & 25.645 & 0.0356 & 0.0 \\
\hline 20.0 & & 9.901 & 33.403 & 25.722 & 0.0473 & 0.0 \\
\hline 25.0 & & 9.719 & 33.428 & 25.772 & 0.0588 & 0.0 \\
\hline 30.0 & & 9.485 & 33.445 & 25.823 & 0.0700 & 0.0 \\
\hline 35.0 & & 9.307 & 33.470 & 25.872 & 0.0808 & 0.0 \\
\hline 40.0 & & 9.272 & 33.473 & 25.880 & 0.0916 & 0.0 \\
\hline 45.0 & & 9.228 & 33.475 & 25.888 & 0.1024 & 0.0 \\
\hline 50.0 & & 9.016 & 33.519 & 25.956 & 0.1130 & 0.0 \\
\hline 55.0 & & 8.947 & 33.545 & 25.988 & 0.1233 & 0.0 \\
\hline 60.0 & & 8.922 & 33.580 & 26.019 & 0.1335 & 0.0 \\
\hline 65.0 & & 8.802 & 33.608 & 26.060 & 0.1436 & 0.0 \\
\hline 70.0 & & 8.667 & 33.695 & 26.149 & 0.1532 & 0.0 \\
\hline 75.0 & & 8.716 & 33.806 & 26.228 & 0.1626 & 0.0 \\
\hline 80.0 & & 8.663 & 33.879 & 26.294 & 0.1715 & 0.0 \\
\hline 85.0 & & 8.642 & 33.887 & 26.303 & 0.1804 & 0.0 \\
\hline 89.0 & & 8.637 & 33.890 & 26.306 & 0.1874 & 0.0 \\
\hline
\end{tabular}
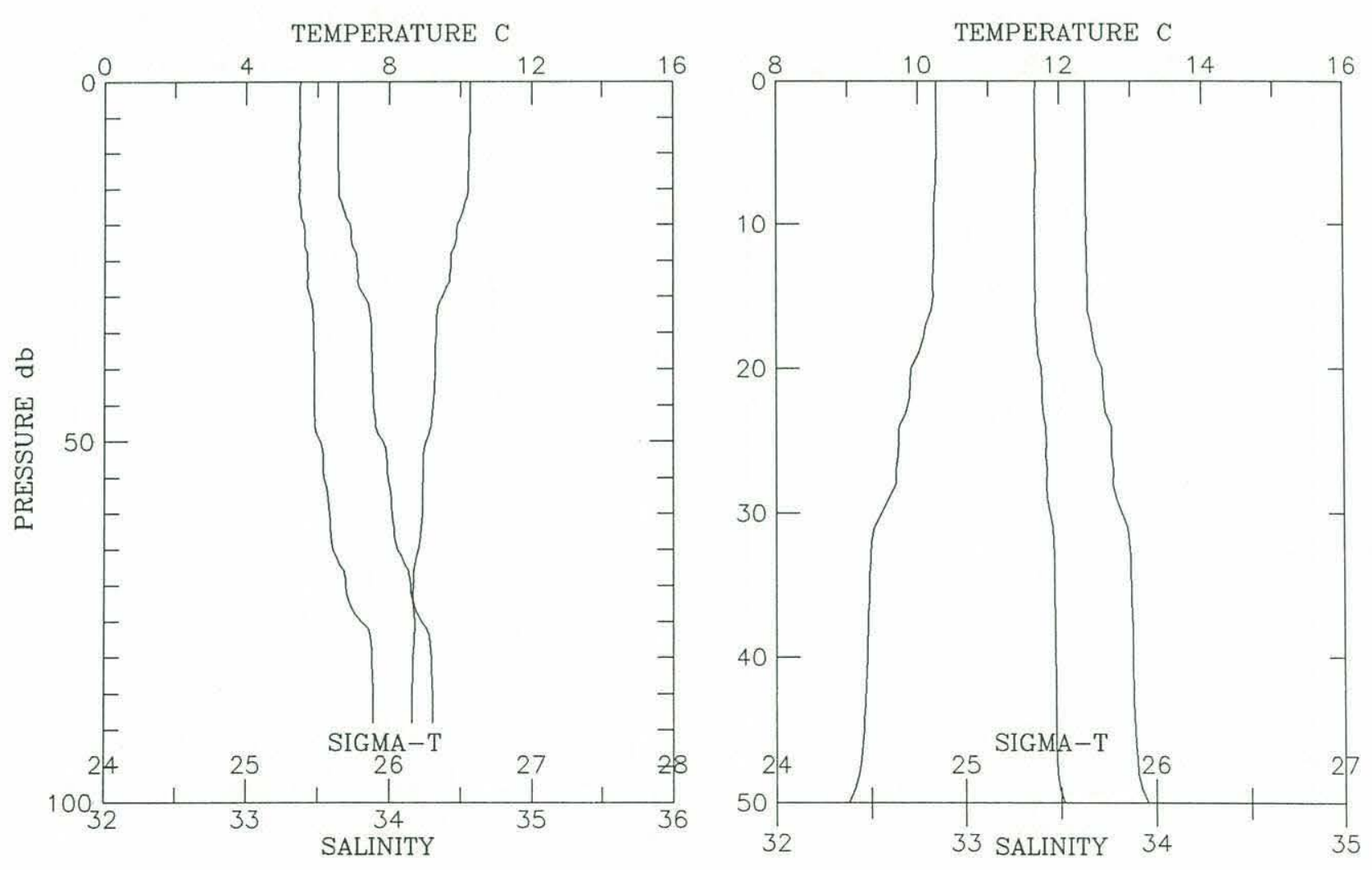
Cruise w8905 Station \# 21 Depth m: 120 Time: 1989-05-06 21:05

Position: $3836.20^{\prime} \mathrm{N} 12331.90^{\prime} \mathrm{W}$ Depth Deck Offset: -0.25

\begin{tabular}{|c|c|c|c|c|c|c|}
\hline $\begin{array}{l}\text { PRESSURE } \\
\text { (d-bars) }\end{array}$ & Interp & $\begin{array}{c}\text { TEMP } \\
(\operatorname{deg} C)\end{array}$ & $\begin{array}{c}\text { SALINITY } \\
(0 / 00)\end{array}$ & SIGMA-T & DELTA-D & $\begin{array}{l}\text { CHANNELA } \\
\text { ( } \& 1 \text { ight) }\end{array}$ \\
\hline 0.0 & E & 10.248 & 33.347 & 25.620 & 0.0000 & 0.0 \\
\hline 10.0 & & 10.202 & 33.352 & 25.631 & 0.0239 & 0.0 \\
\hline 20.0 & & 10.075 & 33.393 & 25.685 & 0.0476 & 0.0 \\
\hline 30.0 & & 9.809 & 33.430 & 25.758 & 0.0706 & 0.0 \\
\hline 40.0 & & 9.739 & 33.439 & 25.777 & 0.0932 & 0.0 \\
\hline 50.0 & & 8.755 & 33.662 & 26.109 & 0.1141 & 0.0 \\
\hline 60.0 & & 8.694 & 33.721 & 26.165 & 0.1331 & 0.0 \\
\hline 70.0 & & 8.577 & 33.779 & 26.228 & 0.1517 & 0.0 \\
\hline 80.0 & & 8.567 & 33.867 & 26.299 & 0.1695 & 0.0 \\
\hline 90.0 & & 8.530 & 33.894 & 26.326 & 0.1870 & 0.0 \\
\hline 100.0 & & 8.464 & 33.920 & 26.356 & 0.2043 & 0.0 \\
\hline 110.0 & & 8.392 & 33.928 & 26.374 & 0.2214 & 0.0 \\
\hline 113.0 & & 8.382 & 33.929 & 26.376 & 0.2265 & 0.0 \\
\hline
\end{tabular}

TEMPERATURE C

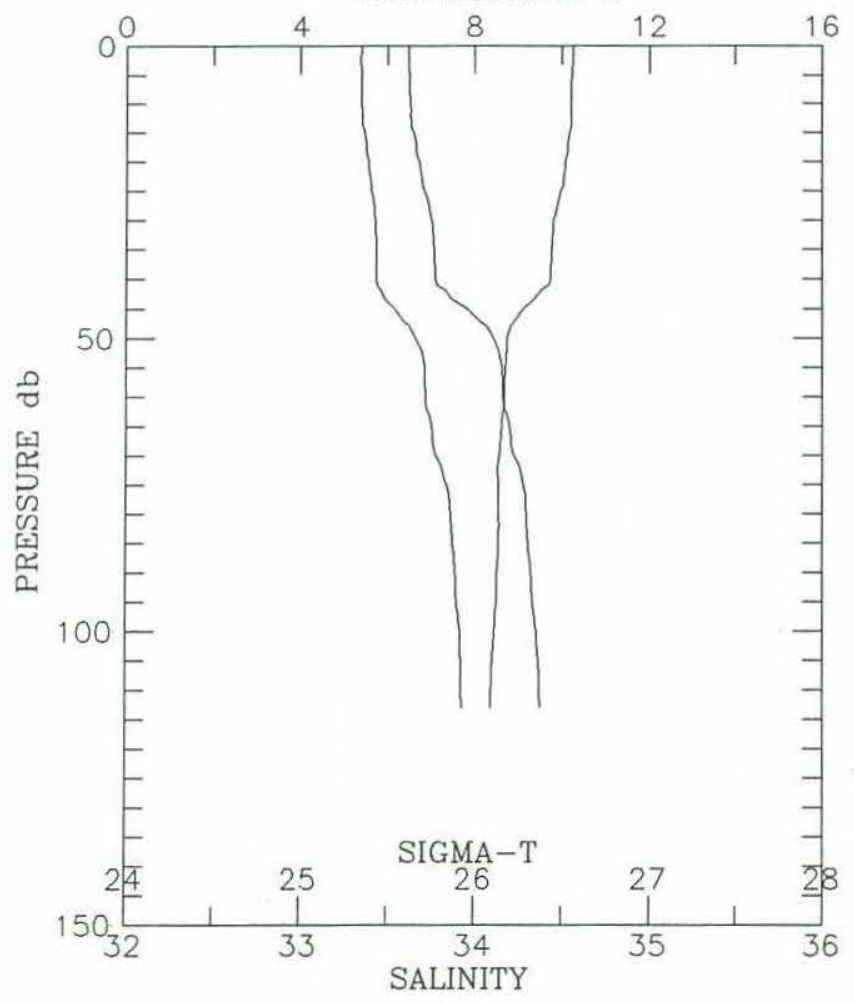

TEMPERATURE $C$

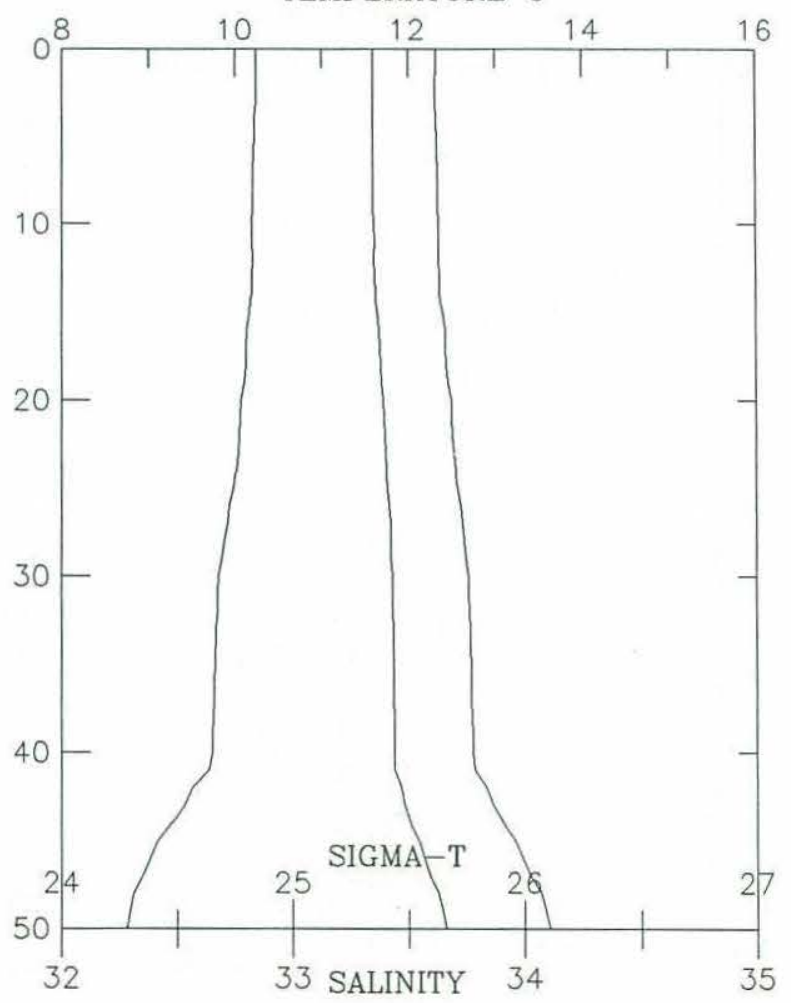


Cruise w8905 Station \# 22 Depth m: 141 Time: 1989-05-06 21:34 Position: $3835.20^{\prime} \mathrm{N} 123 \quad 33.70^{\prime} \mathrm{W}$ Depth Deck Offset: -0.28

\begin{tabular}{|c|c|c|c|c|c|c|}
\hline $\begin{array}{l}\text { PRESSURE } \\
\text { (d-bars) }\end{array}$ & Interp & $\begin{array}{c}\text { TEMP } \\
(\operatorname{deg} C)\end{array}$ & $\begin{array}{c}\text { SALINITY } \\
(0 / 00)\end{array}$ & SIGMA-T & DELTA-D & $\begin{array}{l}\text { CHANNELA } \\
\text { ( }(\mathrm{l} \text { ight) }\end{array}$ \\
\hline 0.0 & E & 10.529 & 33.299 & 25.534 & 0.0000 & 0.0 \\
\hline 10.0 & & 10.115 & 33.377 & 25.666 & 0.0243 & 0.0 \\
\hline 20.0 & & 9.792 & 33.390 & 25.730 & 0.0476 & 0.0 \\
\hline 30.0 & & 9.041 & 33.461 & 25.907 & 0.0697 & 0.0 \\
\hline 40.0 & & 8.754 & 33.638 & 26.091 & 0.0900 & 0.0 \\
\hline 50.0 & & 8.585 & 33.745 & 26.201 & 0.1090 & 0.0 \\
\hline 60.0 & & 8.479 & 33.794 & 26.255 & 0.1274 & 0.0 \\
\hline 70.0 & & 8.388 & 33.853 & 26.315 & 0.1451 & 0.0 \\
\hline 80.0 & & 8.299 & 33.904 & 26.369 & 0.1623 & 0.0 \\
\hline 90.0 & & 8.229 & 33.925 & 26.396 & 0.1792 & 0.0 \\
\hline 100.0 & & 8.211 & 33.933 & 26.405 & 0.1959 & 0.0 \\
\hline 110.0 & & 8.142 & 33.948 & 26.427 & 0.2124 & 0.0 \\
\hline 120.0 & & 8.086 & 33.953 & 26.439 & 0.2289 & 0.0 \\
\hline 130.0 & & 8.059 & 33.956 & 26.446 & 0.2452 & 0.0 \\
\hline 131.0 & & 8.059 & 33.956 & 26.446 & 0.2469 & 0.0 \\
\hline
\end{tabular}
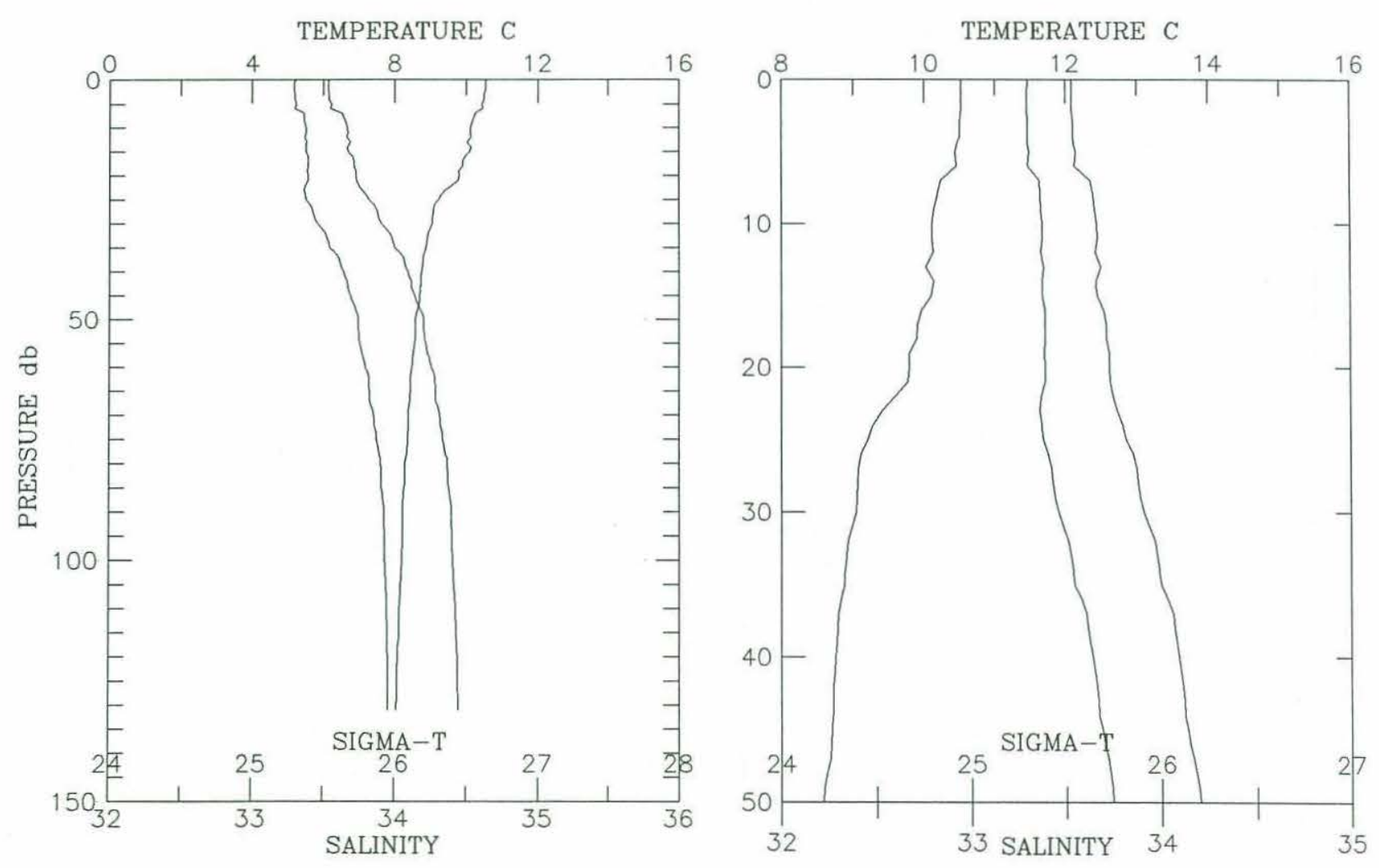


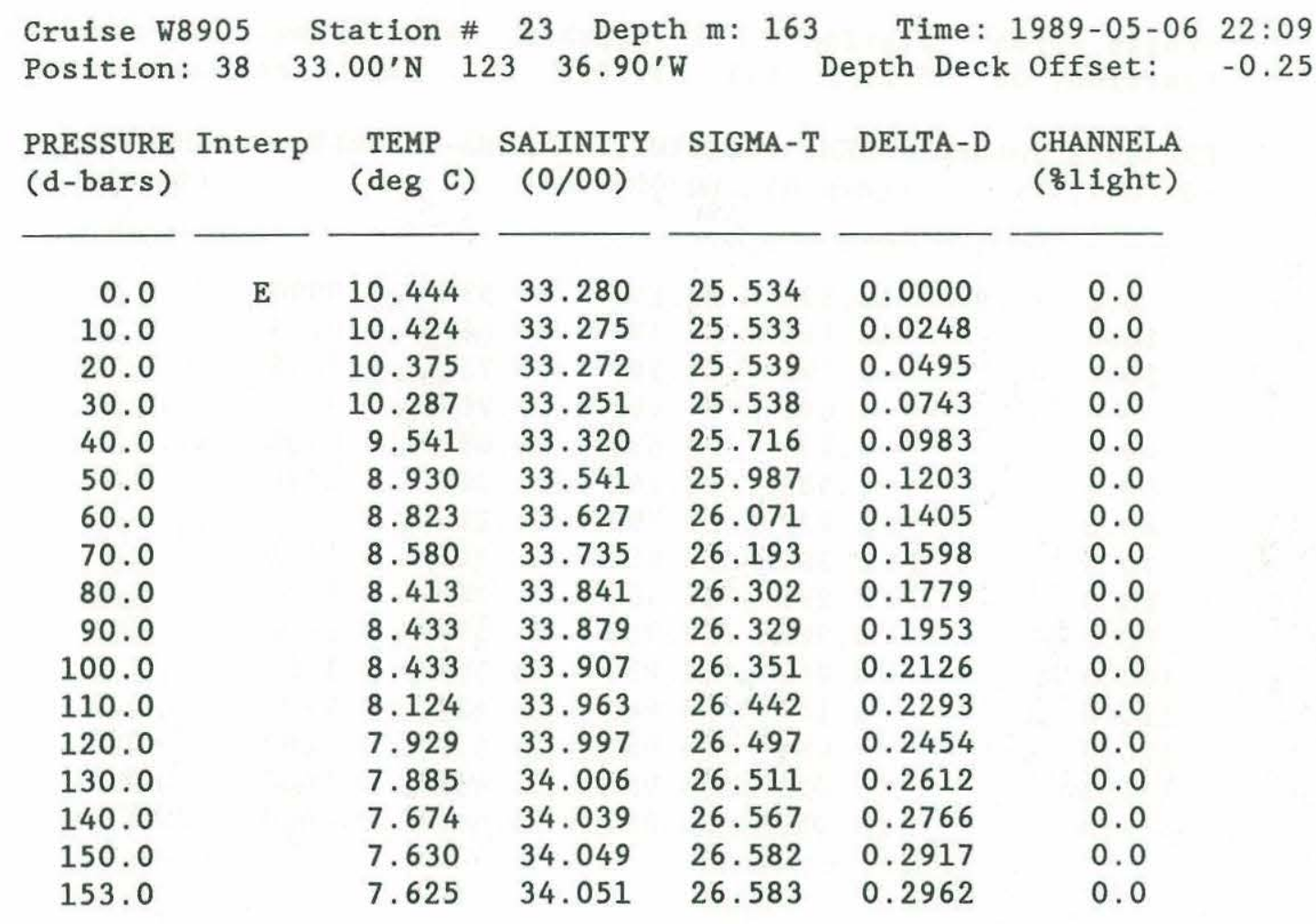
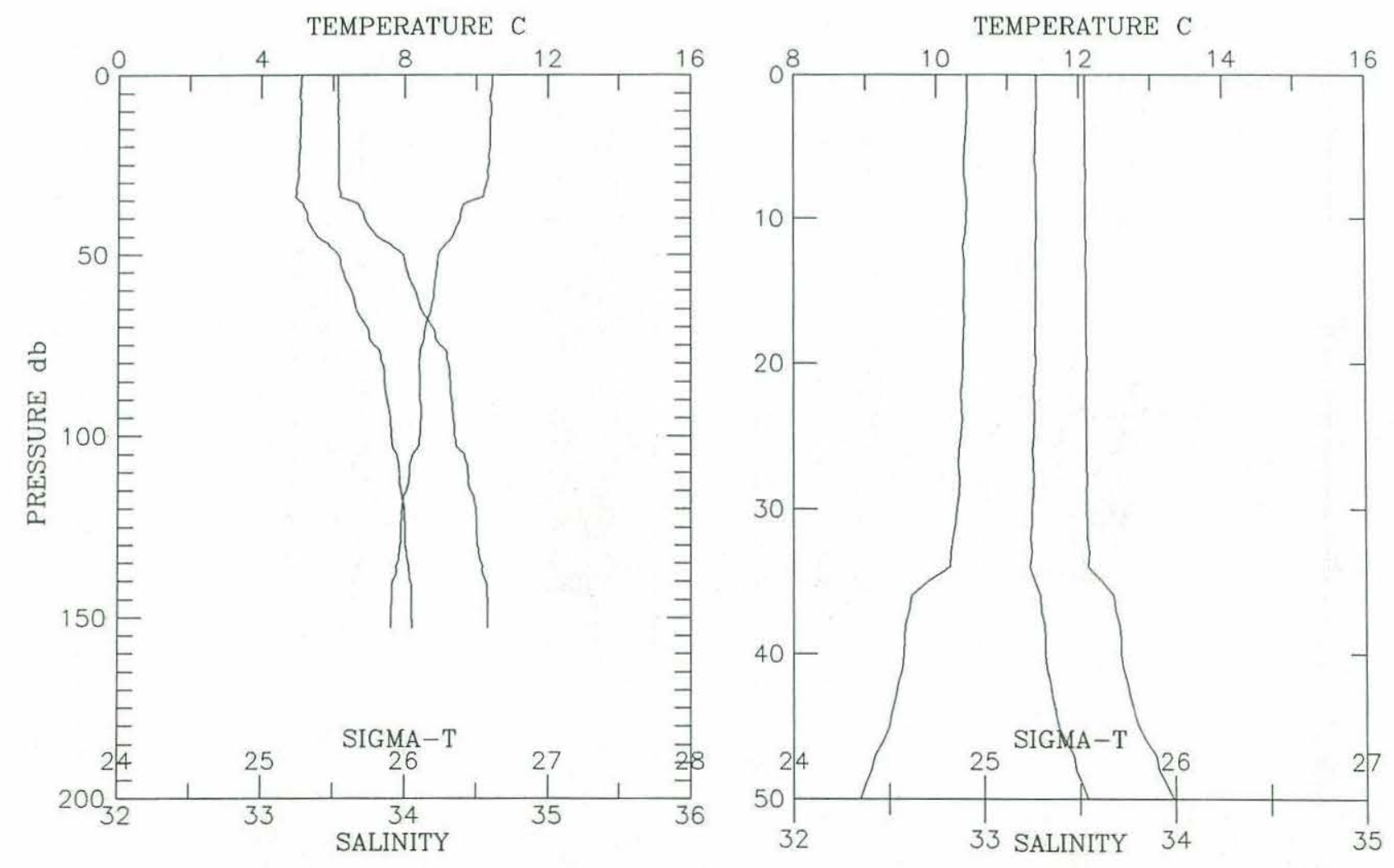
Cruise w8905 Station \# 24 Depth m: 455 Time: 1989-05-06 22:49 Position: $3830.90^{\prime} \mathrm{N} 123 \quad 39.80^{\prime} \mathrm{W}$ Depth Deck Offset: -0.28

\begin{tabular}{|c|c|c|c|c|c|c|}
\hline $\begin{array}{l}\text { PRESSURE } \\
\text { (d-bars) }\end{array}$ & Interp & $\begin{array}{l}\text { TEMP } \\
(\operatorname{deg} C)\end{array}$ & $\begin{array}{c}\text { SALINITY } \\
(0 / 00)\end{array}$ & SIGMA-T & DELTA-D & $\begin{array}{l}\text { CHANNELA } \\
\text { ( } \delta 1 \text { ight) }\end{array}$ \\
\hline 0.0 & E & 10.712 & 33.133 & 25.373 & 0.0000 & 0.0 \\
\hline 10.0 & & 10.675 & 33.133 & 25.379 & 0.0263 & 0.0 \\
\hline 20.0 & & 10.390 & 33.182 & 25.467 & 0.0520 & 0.0 \\
\hline 30.0 & & 10.293 & 33.193 & 25.492 & 0.0774 & 0.0 \\
\hline 40.0 & & 9.818 & 33.237 & 25.606 & 0.1024 & 0.0 \\
\hline 50.0 & & 9.240 & 33.409 & 25.835 & 0.1251 & 0.0 \\
\hline 60.0 & & 9.030 & 33.526 & 25.960 & 0.1464 & 0.0 \\
\hline 80.0 & & 8.824 & 33.695 & 26.124 & 0.1864 & 0.0 \\
\hline 100.0 & & 8.459 & 33.858 & 26.308 & 0.2233 & 0.0 \\
\hline 150.0 & & 7.867 & 33.999 & 26.508 & 0.3068 & 0.0 \\
\hline 200.0 & & 7.019 & 34.009 & 26.635 & 0.3820 & 0.0 \\
\hline 250.0 & & 6.673 & 34.038 & 26.705 & 0.4535 & 0.0 \\
\hline 300.0 & & 6.494 & 34.090 & 26.770 & 0.5228 & 0.0 \\
\hline 400.0 & & 6.132 & 34.190 & 26.896 & 0.6524 & 0.0 \\
\hline 446.0 & & 5.950 & 34.215 & 26.939 & 0.7085 & 0.0 \\
\hline
\end{tabular}
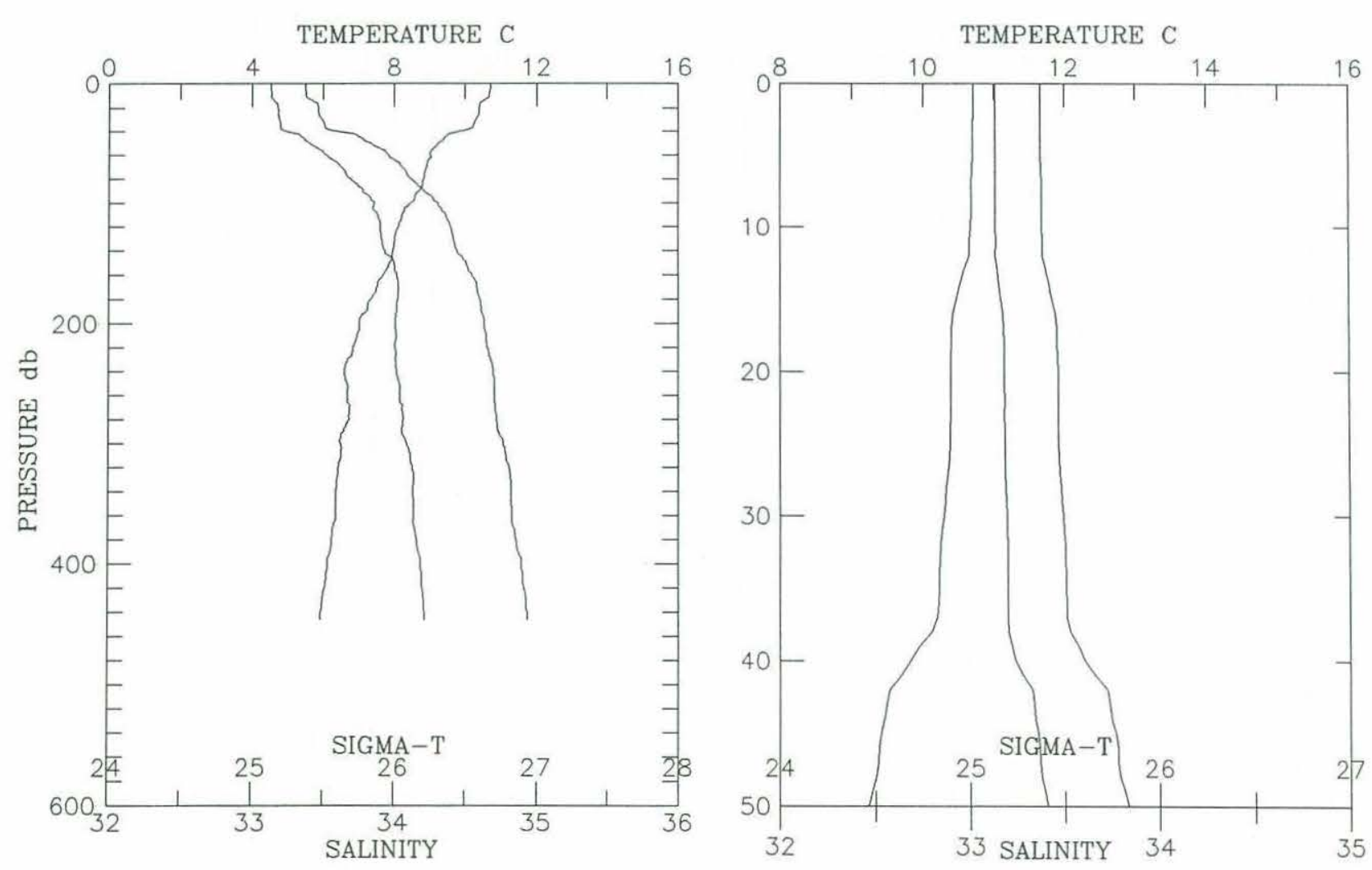
Cruise w8905 Station \# 25 Depth m: 865 Time: 1989-05-06 23:33

Position: 38 29.10'N $123 \quad 42.60^{\prime} \mathrm{W}$ Depth Deck Offset: -0.17

\begin{tabular}{|c|c|c|c|c|c|c|}
\hline $\begin{array}{l}\text { PRESSURE } \\
\text { (d-bars) }\end{array}$ & Interp & $\begin{array}{l}\text { TEMP } \\
(\operatorname{deg} C)\end{array}$ & $\begin{array}{c}\text { SALINITY } \\
(0 / 00)\end{array}$ & SIGMA-T & DELTA-D & $\begin{array}{l}\text { CHANNELA } \\
\text { (olight) }\end{array}$ \\
\hline 0.0 & E & 10.949 & 33.086 & 25.294 & 0.0000 & 0.0 \\
\hline 10.0 & & 10.949 & 33.085 & 25.294 & 0.0270 & 0.0 \\
\hline 20.0 & & 10.682 & 33.123 & 25.370 & 0.0539 & 0.0 \\
\hline 30.0 & & 10.614 & 33.169 & 25.418 & 0.0800 & 0.0 \\
\hline 40.0 & & 9.904 & 33.220 & 25.578 & 0.1053 & 0.0 \\
\hline 50.0 & & 9.481 & 33.316 & 25.723 & 0.1289 & 0.0 \\
\hline 60.0 & & 9.120 & 33.456 & 25.890 & 0.1510 & 0.0 \\
\hline 80.0 & & 8.819 & 33.677 & 26.111 & 0.1917 & 0.0 \\
\hline 100.0 & & 8.617 & 33.825 & 26.258 & 0.2292 & 0.0 \\
\hline 150.0 & & 7.793 & 33.950 & 26.480 & 0.3132 & 0.0 \\
\hline 200.0 & & 7.398 & 34.041 & 26.608 & 0.3900 & 0.0 \\
\hline 250.0 & & 7.043 & 34.074 & 26.683 & 0.4629 & 0.0 \\
\hline 300.0 & & 6.471 & 34.077 & 26.763 & 0.5323 & 0.0 \\
\hline 400.0 & & 6.134 & 34.178 & 26.886 & 0.6623 & 0.0 \\
\hline 500.0 & & 5.681 & 34.227 & 26.982 & 0.7825 & 0.0 \\
\hline 600.0 & & 5.378 & 34.282 & 27.062 & 0.8968 & 0.0 \\
\hline 700.0 & & 4.888 & 34.345 & 27.169 & 1.0024 & 0.0 \\
\hline 800.0 & & 4.518 & 34.390 & 27.246 & 1.0989 & 0.0 \\
\hline 850.0 & & 4.270 & 34.412 & 27.291 & 1.1446 & 0.0 \\
\hline
\end{tabular}
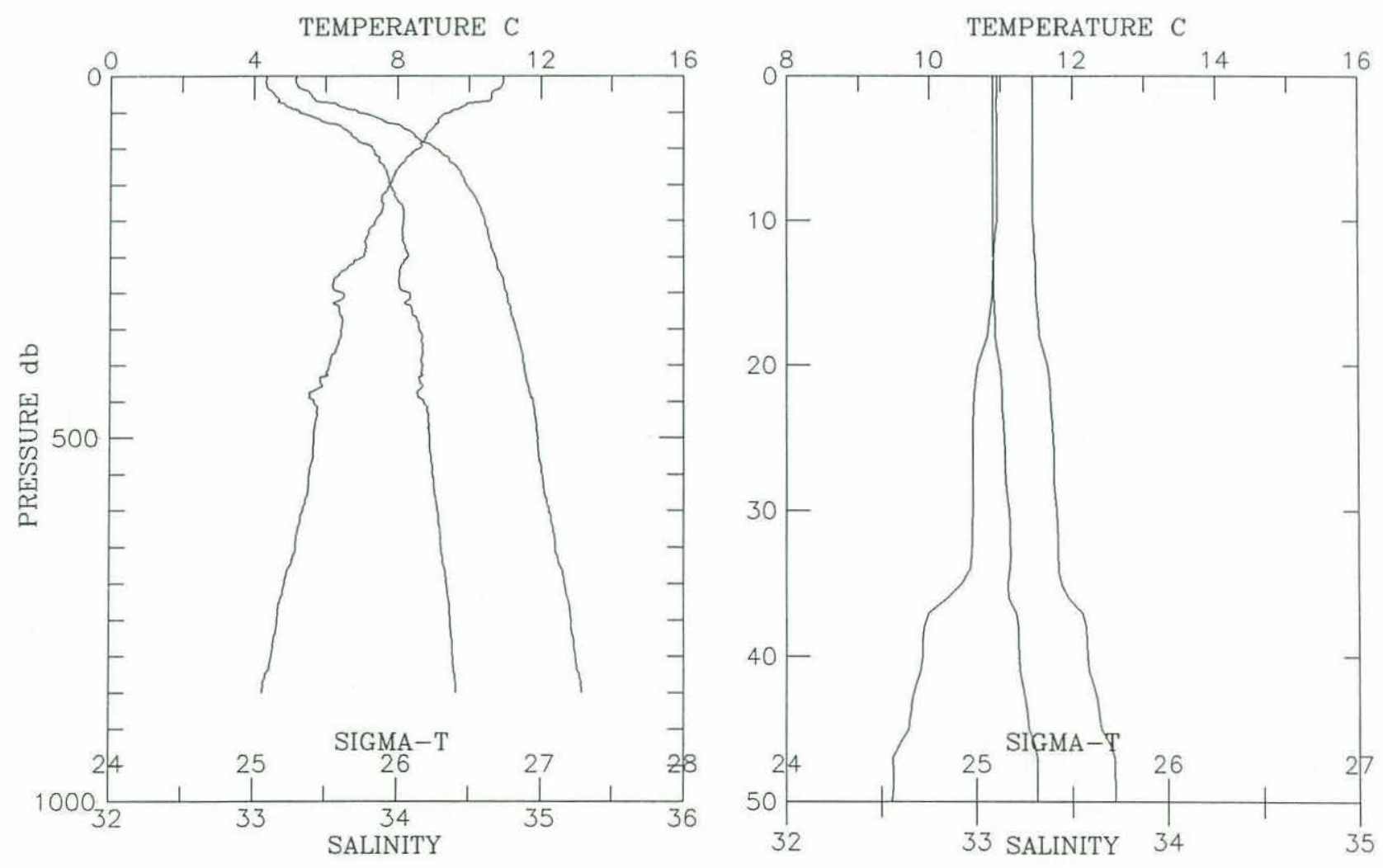
Cruise w8905 Station \# 26 Depth m: 1469 Time: 1989-05-07 00:39

Position: 38 26.20'N 123 46.50'W Depth Deck Offset: -0.20

\begin{tabular}{|c|c|c|c|c|c|c|}
\hline $\begin{array}{l}\text { PRESSURE } \\
\text { (d-bars) }\end{array}$ & Interp & $\begin{array}{c}\text { TEMP } \\
(\operatorname{deg} G)\end{array}$ & $\begin{array}{c}\text { SALINITY } \\
(0 / 00)\end{array}$ & SIGMA-T & DELTA-D & $\begin{array}{l}\text { CHANNELA } \\
\text { ( } \delta 1 \text { ight) }\end{array}$ \\
\hline 0.0 & E & 11.396 & 33.033 & 25.174 & 0.0000 & 0.0 \\
\hline 10.0 & & 11.366 & 33.036 & 25.182 & 0.0282 & 0.0 \\
\hline 20.0 & & 10.991 & 33.069 & 25.274 & 0.0558 & 0.0 \\
\hline 30.0 & & 10.756 & 33.117 & 25.353 & 0.0827 & 0.0 \\
\hline 40.0 & & 10.654 & 33.143 & 25.391 & 0.1091 & 0.0 \\
\hline 50.0 & & 10.523 & 33.164 & 25.430 & 0.1351 & 0.0 \\
\hline 60.0 & & 9.510 & 33.315 & 25.718 & 0.1593 & 0.0 \\
\hline 80.0 & & 9.093 & 33.601 & 26.008 & 0.2020 & 0.0 \\
\hline 100.0 & & 8.684 & 33.721 & 26.167 & 0.2414 & 0.0 \\
\hline 150.0 & & 7.921 & 33.912 & 26.432 & 0.3293 & 0.0 \\
\hline 200.0 & & 7.377 & 33.981 & 26.563 & 0.4085 & 0.0 \\
\hline 250.0 & & 6.947 & 34.034 & 26.665 & 0.4826 & 0.0 \\
\hline 300.0 & & 6.360 & 34.062 & 26.766 & 0.5520 & 0.0 \\
\hline 400.0 & & 5.521 & 34.057 & 26.867 & 0.6826 & 0.0 \\
\hline 500.0 & & 5.541 & 34.202 & 26.979 & 0.8038 & 0.0 \\
\hline 600.0 & & 5.006 & 34.256 & 27.085 & 0.9162 & 0.0 \\
\hline 700.0 & & 4.851 & 34.340 & 27.169 & 1.0201 & 0.0 \\
\hline 800.0 & & 4.552 & 34.390 & 27.242 & 1.1169 & 0.0 \\
\hline 900.0 & & 4.325 & 34.414 & 27.286 & 1.2095 & 0.0 \\
\hline 1000.0 & & 3.985 & 34.439 & 27.341 & 1.2975 & 0.0 \\
\hline 1100.0 & & 3.678 & 34.458 & 27.387 & 1.3811 & 0.0 \\
\hline 1200.0 & & 3.330 & 34.480 & 27.439 & 1.4599 & 0.0 \\
\hline 1300.0 & & 3.118 & 34.515 & 27.487 & 1.5342 & 0.0 \\
\hline 1400.0 & & 2.967 & 34.527 & 27.510 & 1.6054 & 0.0 \\
\hline 1452.0 & & 2.950 & 34.529 & 27.514 & 1.6420 & 0.0 \\
\hline
\end{tabular}
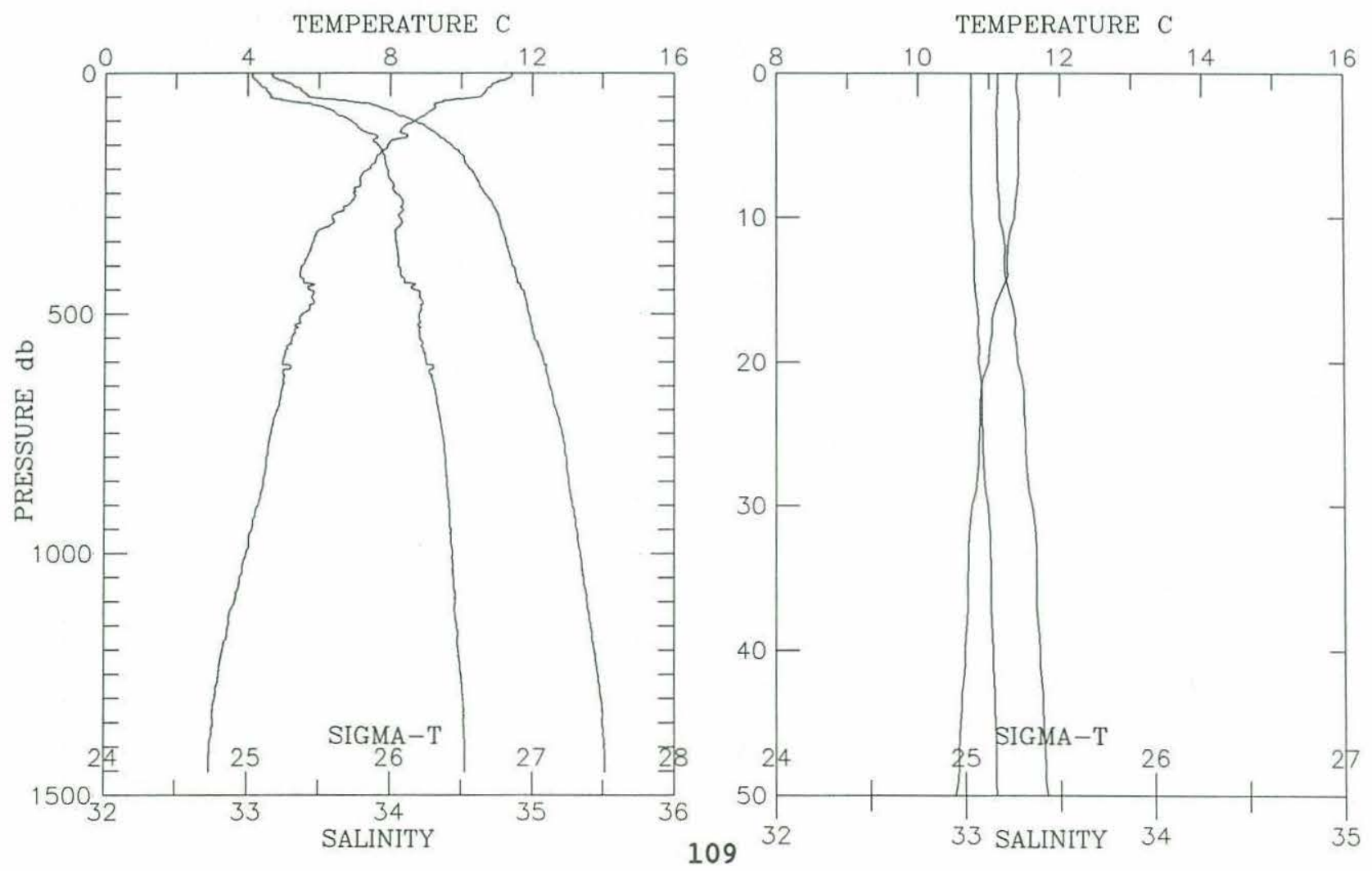
Cruise w8905 Station \# 27 Depth m: 1925 Time: 1989-05-07 02:10 Position: $3822.90^{\prime} \mathrm{N} 12351.10^{\prime} \mathrm{W}$ Depth Deck Offset: -0.28

\begin{tabular}{|c|c|c|c|c|c|c|}
\hline $\begin{array}{l}\text { PRESSURE } \\
\text { (d-bars) }\end{array}$ & Interp & $\begin{array}{l}\text { TEMP } \\
(\operatorname{deg} C)\end{array}$ & $\begin{array}{l}\text { SALINITY } \\
(0 / 00)\end{array}$ & SIGMA-T & DELTA-D & $\begin{array}{l}\text { CHANNELA } \\
\text { (\&light) }\end{array}$ \\
\hline 0.0 & E & 12.159 & 32.953 & 24.970 & 0.0000 & 0.0 \\
\hline 10.0 & & 12.160 & 32.952 & 24.969 & 0.0301 & 0.0 \\
\hline 20.0 & & 11.925 & 32.969 & 25.027 & 0.0600 & 0.0 \\
\hline 30.0 & & 11.611 & 32.992 & 25.103 & 0.0893 & 0.0 \\
\hline 40.0 & & 11.298 & 32.977 & 25.148 & 0.1181 & 0.0 \\
\hline 50.0 & & 10.046 & 33.107 & 25.467 & 0.1451 & 0.0 \\
\hline 60.0 & & 9.806 & 33.197 & 25.577 & 0.1700 & 0.0 \\
\hline 80.0 & & 9.183 & 33.396 & 25.834 & 0.2164 & 0.0 \\
\hline 100.0 & & 8.853 & 33.663 & 26.095 & 0.2583 & 0.0 \\
\hline 150.0 & & 8.144 & 33.895 & 26.385 & 0.3482 & 0.0 \\
\hline 200.0 & & 7.457 & 33.964 & 26.539 & 0.4292 & 0.0 \\
\hline 250.0 & & 7.119 & 34.041 & 26.647 & 0.5044 & 0.0 \\
\hline 300.0 & & 6.292 & 34.016 & 26.738 & 0.5753 & 0.0 \\
\hline 400.0 & & 5.450 & 34.073 & 26.888 & 0.7055 & 0.0 \\
\hline 500.0 & & 4.926 & 34.132 & 26.996 & 0.8251 & 0.0 \\
\hline 600.0 & & 5.208 & 34.305 & 27.100 & 0.9349 & 0.0 \\
\hline 700.0 & & 4.890 & 34.349 & 27.172 & 1.0377 & 0.0 \\
\hline 800.0 & & 4.524 & 34.392 & 27.247 & 1.1343 & 0.0 \\
\hline 900.0 & & 4.221 & 34.421 & 27.303 & 1.2256 & 0.0 \\
\hline 1000.0 & & 3.952 & 34.443 & 27.348 & 1.3129 & 0.0 \\
\hline 1100.0 & & 3.601 & 34.456 & 27.394 & 1.3955 & 0.0 \\
\hline 1200.0 & & 3.290 & 34.488 & 27.449 & 1.4736 & 0.0 \\
\hline 1300.0 & & 3.098 & 34.515 & 27.489 & 1.5474 & 0.0 \\
\hline 1400.0 & & 2.942 & 34.530 & 27.515 & 1.6182 & 0.0 \\
\hline 1500.0 & & 2.718 & 34.546 & 27.548 & 1.6866 & 0.0 \\
\hline 1501.0 & & 2.718 & 34.546 & 27.548 & 1.6873 & 0.0 \\
\hline
\end{tabular}

TEMPERATURE $\mathrm{C}$
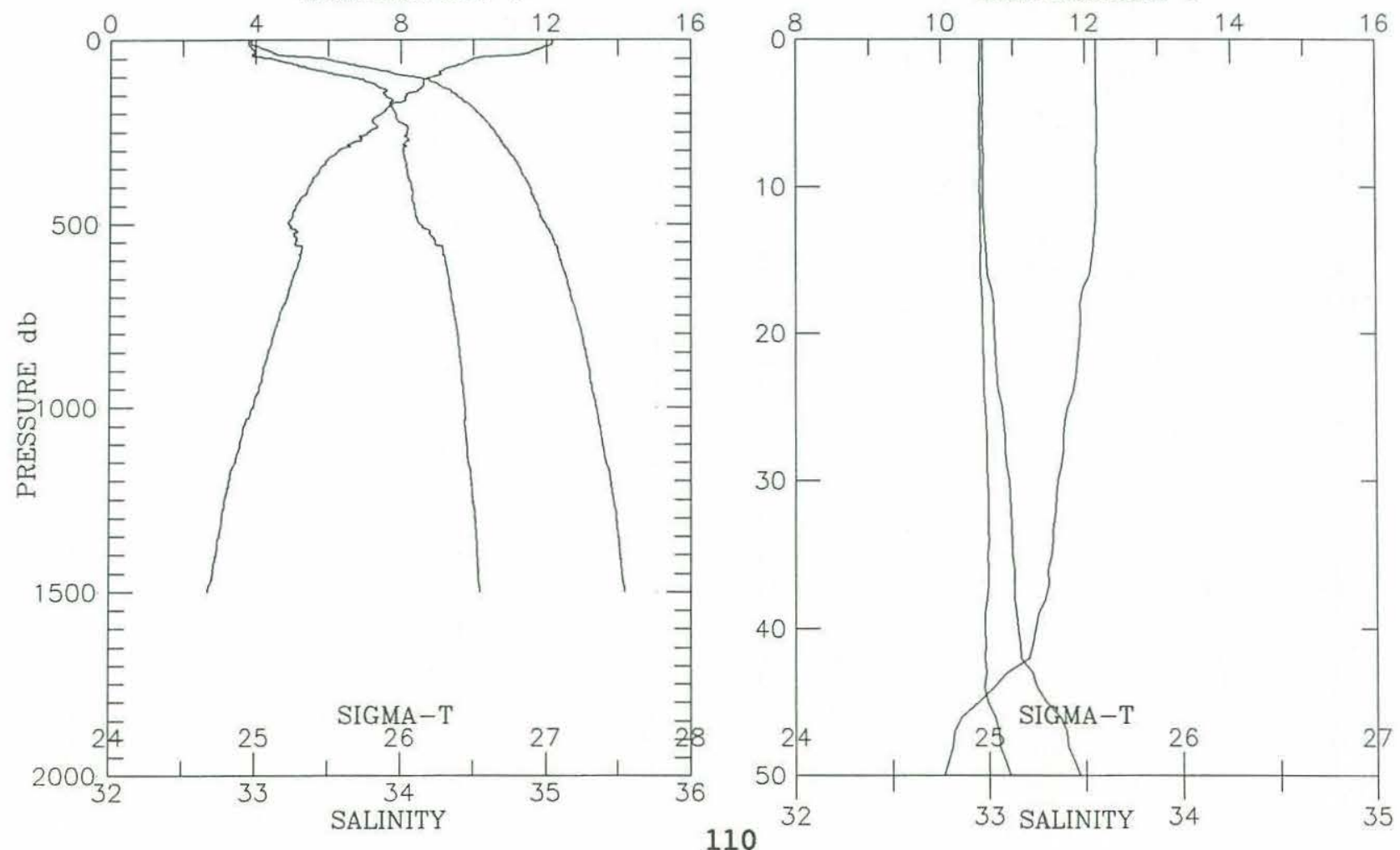
Cruise W8905 Station \# 28 Depth m: 2709 Time: 1989-05-07 03:43 Position: $3819.50^{\prime} \mathrm{N} 12355.80^{\prime} \mathrm{W} \quad$ Depth Deck Offset: 0.00

PRESSURE Interp TEMP SALINITY SIGMA-T DELTA-D CHANNELA (d-bars) ( $\quad(\operatorname{deg} C)(0 / 00) \quad$ (\%light)

\begin{tabular}{|c|c|c|c|c|c|c|}
\hline 0.0 & E & 13.080 & 32.789 & 24.665 & 0.0000 & 0.0 \\
\hline 10.0 & & 13.086 & 32.786 & 24.662 & 0.0330 & 0.0 \\
\hline 20.0 & & 12.925 & 32.812 & 24.714 & 0.0659 & 0.0 \\
\hline 30.0 & & 11.681 & 32.978 & 25.079 & 0.0966 & 0.0 \\
\hline 40.0 & & 10.949 & 32.934 & 25.176 & 0.1254 & 0.0 \\
\hline 50.0 & & 10.529 & 33.047 & 25.338 & 0.1529 & 0.0 \\
\hline 60.0 & & 10.216 & 33.188 & 25.501 & 0.1792 & 0.0 \\
\hline 80.0 & & 9.518 & 33.292 & 25.698 & 0.2277 & 0.0 \\
\hline 100.0 & & 9.071 & 33.507 & 25.938 & 0.2719 & 0.0 \\
\hline 150.0 & & 8.343 & 33.924 & 26.378 & 0.3657 & 0.0 \\
\hline 200.0 & & 7.611 & 34.002 & 26.547 & 0.4468 & 0.0 \\
\hline 250.0 & & 7.107 & 34.036 & 26.645 & 0.5220 & 0.0 \\
\hline 300.0 & & 6.458 & 34.023 & 26.722 & 0.5932 & 0.0 \\
\hline 400.0 & & 5.716 & 34.052 & 26.839 & 0.7270 & 0.0 \\
\hline 500.0 & & 5.022 & 34.103 & 26.962 & 0.8495 & 0.0 \\
\hline 600.0 & & 4.559 & 34.181 & 27.076 & 0.9619 & 0.0 \\
\hline 700.0 & & 4.310 & 34.284 & 27.184 & 1.0644 & 0.0 \\
\hline 800.0 & & 4.189 & 34.362 & 27.259 & 1.1592 & 0.0 \\
\hline 900.0 & & 3.957 & 34.409 & 27.320 & 1.2484 & 0.0 \\
\hline 1000.0 & & 3.741 & 34.440 & 27.367 & 1.3333 & 0.0 \\
\hline 1100.0 & & 3.455 & 34.458 & 27.409 & 1.4139 & 0.0 \\
\hline 1200.0 & & 3.252 & 34.491 & 27.455 & 1.4905 & 0.0 \\
\hline 1300.0 & & 3.085 & 34.518 & 27.492 & 1.5637 & 0.0 \\
\hline 1400.0 & & 2.854 & 34.537 & 27.529 & 1.6338 & 0.0 \\
\hline 1500.0 & & 2.645 & 34.553 & 27.560 & 1.7004 & 0.0 \\
\hline 1502.0 & & 2.644 & 34.553 & 27.560 & 1.7017 & 0.0 \\
\hline
\end{tabular}

TEMPERATURE C
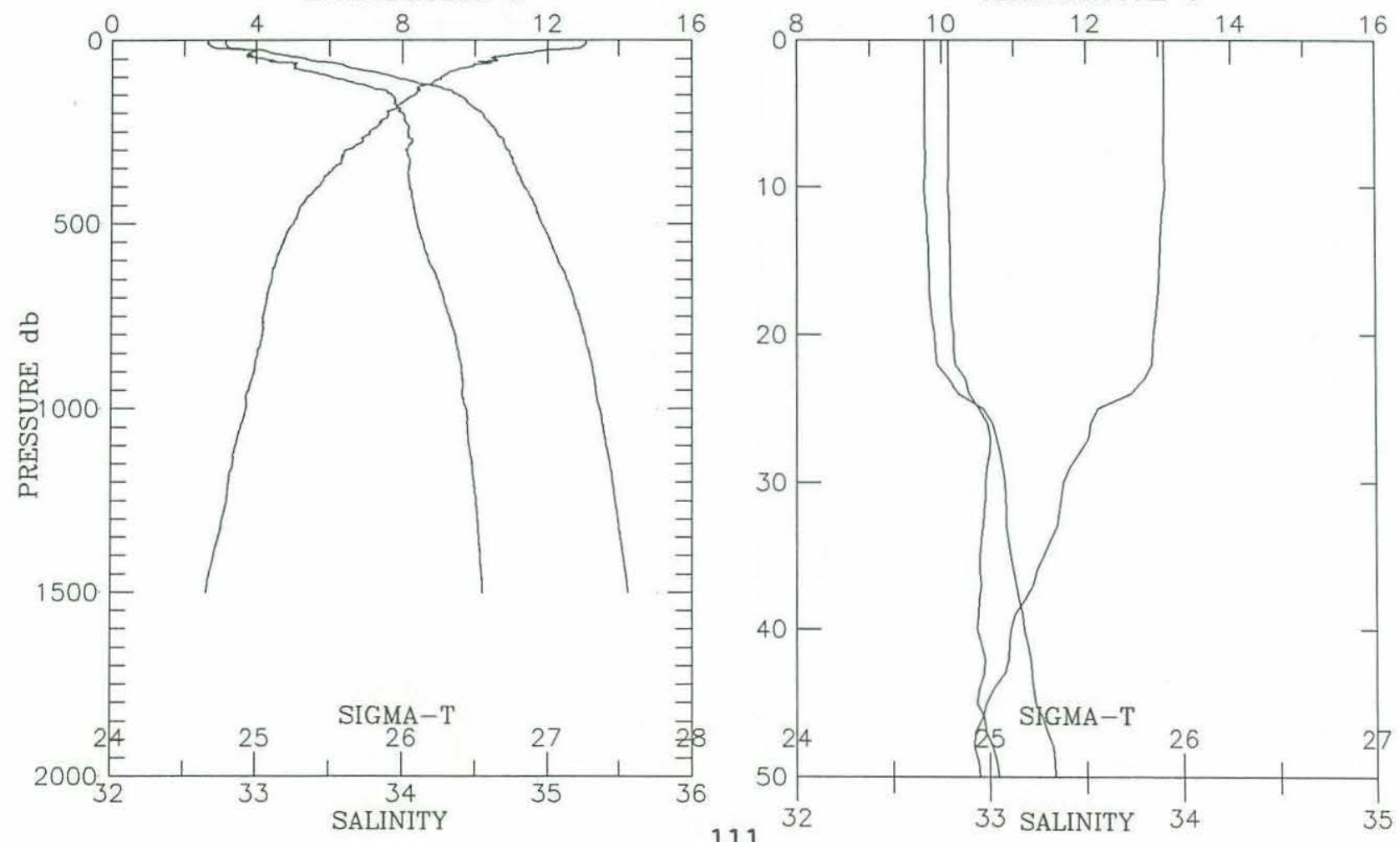
Cruise w8905 Station \# 29 Depth m: 3701 Time: 1989-05-07 05:19 Position: $38 \quad 16.00^{\prime} \mathrm{N} 124 \quad 0.50^{\prime} \mathrm{W}$ Depth Deck Offset: -0.28

\begin{tabular}{|c|c|c|c|c|c|c|}
\hline $\begin{array}{l}\text { PRESSURE } \\
\text { (d-bars) }\end{array}$ & Interp & $\begin{array}{c}\text { TEMP } \\
(\operatorname{deg} C)\end{array}$ & $\begin{array}{l}\text { SALINITY } \\
(0 / 00)\end{array}$ & SIGMA-T & DELTA-D & $\begin{array}{l}\text { CHANNELA } \\
\text { ( } \delta 1 \text { ight) }\end{array}$ \\
\hline 0.0 & E & 13.734 & 32.776 & 24.524 & 0.0000 & 0.0 \\
\hline 10.0 & & 13.733 & 32.776 & 24.524 & 0.0344 & 0.0 \\
\hline 20.0 & & 13.714 & 32.792 & 24.541 & 0.0687 & 0.0 \\
\hline 30.0 & & 11.707 & 32.975 & 25.072 & 0.1008 & 0.0 \\
\hline 40.0 & & 10.804 & 32.927 & 25.196 & 0.1293 & 0.0 \\
\hline 50.0 & & 10.555 & 33.026 & 25.317 & 0.1567 & 0.0 \\
\hline 60.0 & & 10.746 & 33.267 & 25.471 & 0.1829 & 0.0 \\
\hline 80.0 & & 10.215 & 33.377 & 25.649 & 0.2321 & 0.0 \\
\hline 100.0 & & 9.308 & 33.397 & 25.814 & 0.2781 & 0.0 \\
\hline 150.0 & & 8.668 & 33.807 & 26.236 & 0.3789 & 0.0 \\
\hline 200.0 & & 7.661 & 33.935 & 26.488 & 0.4646 & 0.0 \\
\hline 250.0 & & 7.071 & 33.987 & 26.611 & 0.5417 & 0.0 \\
\hline 300.0 & & 6.568 & 34.027 & 26.710 & 0.6137 & 0.0 \\
\hline 400.0 & & 5.716 & 34.058 & 26.844 & 0.7485 & 0.0 \\
\hline 500.0 & & 5.055 & 34.100 & 26.956 & 0.8719 & 0.0 \\
\hline 600.0 & & 4.667 & 34.220 & 27.095 & 0.9837 & 0.0 \\
\hline 700.0 & & 4.446 & 34.278 & 27.165 & 1.0865 & 0.0 \\
\hline 800.0 & & 4.241 & 34.348 & 27.242 & 1.1829 & 0.0 \\
\hline 900.0 & & 4.043 & 34.394 & 27.299 & 1.2739 & 0.0 \\
\hline 1000.0 & & 3.843 & 34.439 & 27.356 & 1.3602 & 0.0 \\
\hline 1100.0 & & 3.615 & 34.473 & 27.406 & 1.4420 & 0.0 \\
\hline 1200.0 & & 3.300 & 34.500 & 27.458 & 1.5190 & 0.0 \\
\hline 1300.0 & & 3.077 & 34.519 & 27.494 & 1.5921 & 0.0 \\
\hline 1400.0 & & 2.837 & 34.538 & 27.531 & 1.6618 & 0.0 \\
\hline 1500.0 & & 2.639 & 34.547 & 27.556 & 1.7286 & 0.0 \\
\hline 1502.0 & & 2.637 & 34.547 & 27.556 & 1.7299 & 0.0 \\
\hline
\end{tabular}
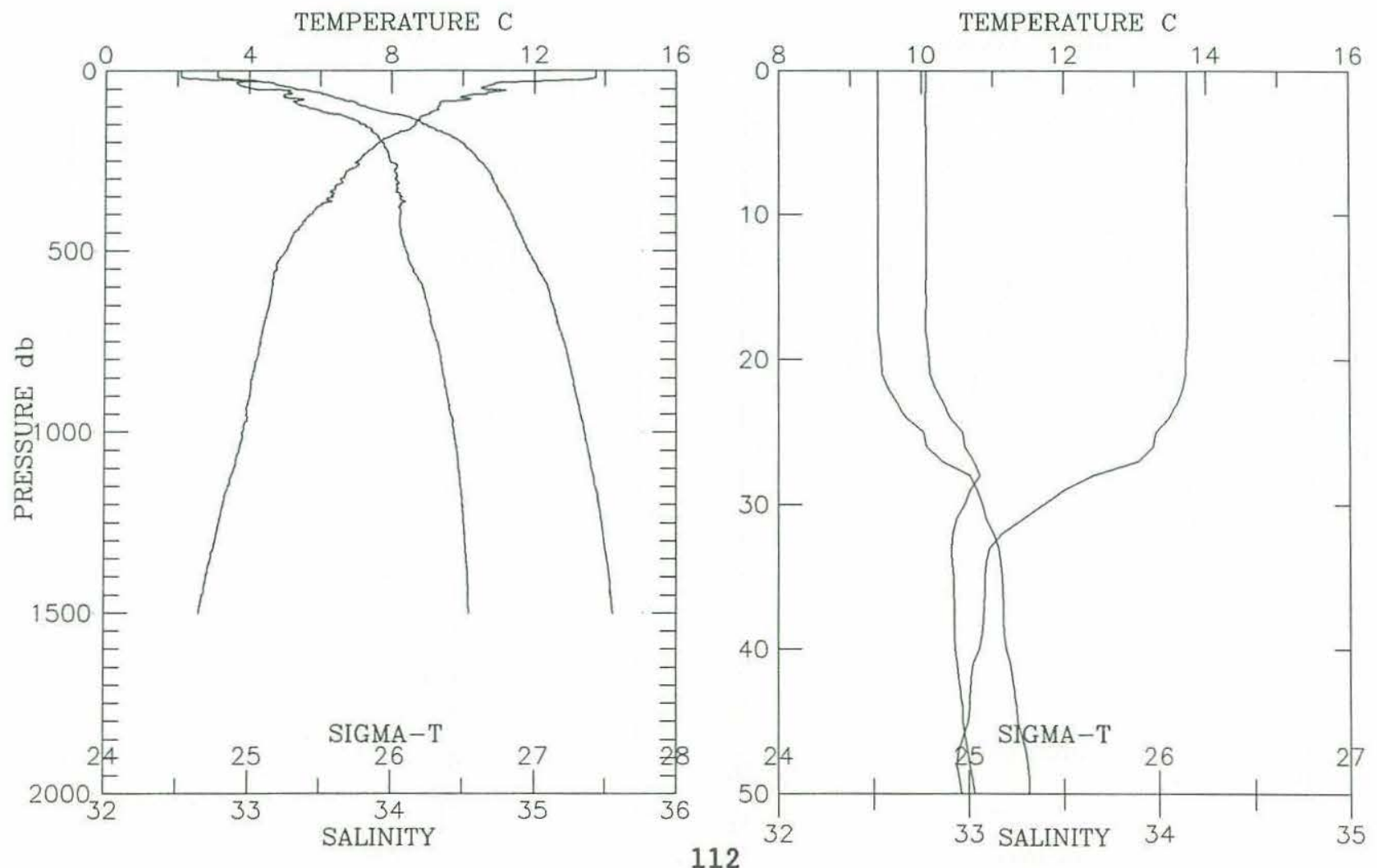
Cruise w8905 Station \# 30 Depth m: 3781 Time: 1989-05-07 06:58 Position: $38 \quad 12.50^{\prime} \mathrm{N} 124 \quad 5.00^{\prime} \mathrm{W}$ Depth Deck Offset: -0.20

\begin{tabular}{|c|c|c|c|c|c|c|}
\hline $\begin{array}{l}\text { PRESSURE } \\
\text { (d-bars) }\end{array}$ & Interp & $\begin{array}{l}\text { TEMP } \\
(\operatorname{deg} C)\end{array}$ & $\begin{array}{c}\text { SALINITY } \\
(0 / 00)\end{array}$ & SIGMA-T & DELTA-D & $\begin{array}{l}\text { CHANNELA } \\
\text { ( } \delta 1 \text { ight) }\end{array}$ \\
\hline 0.0 & E & 13.948 & 32.797 & 24.496 & 0.0000 & 0.0 \\
\hline 10.0 & & 13.949 & 32.804 & 24.501 & 0.0346 & 0.0 \\
\hline 20.0 & & 13.945 & 32.805 & 24.503 & 0.0692 & 0.0 \\
\hline 30.0 & & 13.068 & 32.945 & 24.788 & 0.1031 & 0.0 \\
\hline 40.0 & & 11.611 & 32.898 & 25.030 & 0.1337 & 0.0 \\
\hline 50.0 & & 11.132 & 32.908 & 25.124 & 0.1629 & 0.0 \\
\hline 60.0 & & 10.821 & 32.922 & 25.189 & 0.1913 & 0.0 \\
\hline 80.0 & & 11.057 & 33.237 & 25.393 & 0.2456 & 0.0 \\
\hline 100.0 & & 10.327 & 33.363 & 25.619 & 0.2958 & 0.0 \\
\hline 150.0 & & 8.669 & 33.665 & 26.125 & 0.4047 & 0.0 \\
\hline 200.0 & & 7.989 & 33.909 & 26.419 & 0.4937 & 0.0 \\
\hline 250.0 & & 7.516 & 34.029 & 26.582 & 0.5737 & 0.0 \\
\hline 300.0 & & 6.871 & 34.000 & 26.649 & 0.6482 & 0.0 \\
\hline 400.0 & & 5.765 & 34.024 & 26.811 & 0.7872 & 0.0 \\
\hline 500.0 & & 5.126 & 34.082 & 26.933 & 0.9134 & 0.0 \\
\hline 600.0 & & 4.877 & 34.191 & 27.048 & 1.0291 & 0.0 \\
\hline 700.0 & & 4.508 & 34.285 & 27.164 & 1.1341 & 0.0 \\
\hline 800.0 & & 4.288 & 34.365 & 27.250 & 1.2307 & 0.0 \\
\hline 900.0 & & 4.062 & 34.416 & 27.315 & 1.3210 & 0.0 \\
\hline 1000.0 & & 3.813 & 34.451 & 27.368 & 1.4060 & 0.0 \\
\hline 1100.0 & & 3.531 & 34.481 & 27.420 & 1.4865 & 0.0 \\
\hline 1200.0 & & 3.254 & 34.505 & 27.466 & 1.5626 & 0.0 \\
\hline 1300.0 & & 3.045 & 34.523 & 27.500 & 1.6346 & 0.0 \\
\hline 1400.0 & & 2.809 & 34.532 & 27.529 & 1.7041 & 0.0 \\
\hline 1500.0 & & 2.645 & 34.548 & 27.556 & 1.7711 & 0.0 \\
\hline 1501.0 & & 2.644 & 34.548 & 27.556 & 1.7717 & 0.0 \\
\hline
\end{tabular}
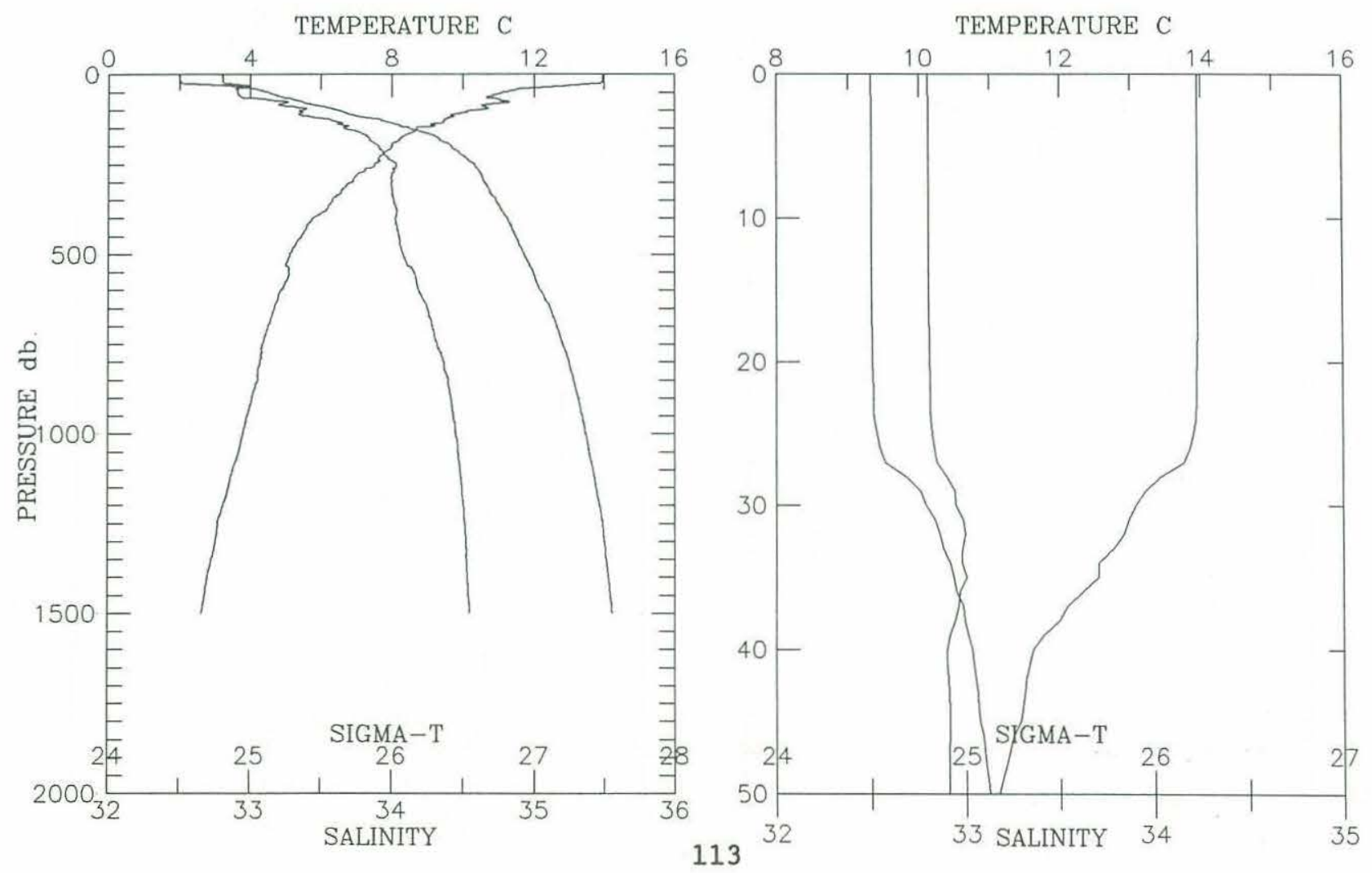
$\begin{array}{lllll}\text { Cruise w8905 } & \text { Station \# } 31 & \text { Depth m: } 3685 \text { Time: 1989-05-07 } 08: 27 \\ \text { Position: } 38 & 9.00^{\prime} \mathrm{N} 124 & 9.60^{\prime} \mathrm{W} & \text { Depth Deck Offset: } & -0.15\end{array}$

\begin{tabular}{|c|c|c|c|c|c|c|}
\hline $\begin{array}{l}\text { PRESSURE } \\
\text { (d-bars) }\end{array}$ & Interp & $\begin{array}{c}\text { TEMP } \\
(\operatorname{deg} C)\end{array}$ & $\begin{array}{c}\text { SALINITY } \\
(0 / 00)\end{array}$ & SIGMA-T & DELTA-D & $\begin{array}{l}\text { CHANNELA } \\
\text { ( } \& 1 \text { ight) }\end{array}$ \\
\hline 0.0 & E & 14.398 & 32.838 & 24.434 & 0.0000 & 0.0 \\
\hline 10.0 & & 14.394 & 32.837 & 24.434 & 0.0352 & 0.0 \\
\hline 20.0 & & 14.387 & 32.837 & 24.436 & 0.0705 & 0.0 \\
\hline 30.0 & & 14.306 & 32.836 & 24.452 & 0.1057 & 0.0 \\
\hline 40.0 & & 12.324 & 32.872 & 24.876 & 0.1383 & 0.0 \\
\hline 50.0 & & 11.304 & 32.863 & 25.058 & 0.1683 & 0.0 \\
\hline 60.0 & & 10.639 & 32.908 & 25.210 & 0.1971 & 0.0 \\
\hline 80.0 & & 10.456 & 33.059 & 25.359 & 0.2516 & 0.0 \\
\hline 100.0 & & 10.709 & 33.370 & 25.558 & 0.3026 & 0.0 \\
\hline 150.0 & & 9.315 & 33.803 & 26.131 & 0.4119 & 0.0 \\
\hline 200.0 & & 8.348 & 33.943 & 26.392 & 0.5021 & 0.0 \\
\hline 250.0 & & 7.383 & 33.978 & 26.560 & 0.5828 & 0.0 \\
\hline 300.0 & & 6.755 & 34.004 & 26.667 & 0.6574 & 0.0 \\
\hline 400.0 & & 5.856 & 34.021 & 26.797 & 0.7959 & 0.0 \\
\hline 500.0 & & 5.230 & 34.130 & 26.959 & 0.9209 & 0.0 \\
\hline 600.0 & & 4.859 & 34.201 & 27.058 & 1.0345 & 0.0 \\
\hline 700.0 & & 4.531 & 34.278 & 27.156 & 1.1394 & 0.0 \\
\hline 800.0 & & 4.275 & 34.345 & 27.236 & 1.2366 & 0.0 \\
\hline 900.0 & & 3.985 & 34.396 & 27.307 & 1.3274 & 0.0 \\
\hline 1000.0 & & 3.818 & 34.457 & 27.373 & 1.4125 & 0.0 \\
\hline 1100.0 & & 3.566 & 34.479 & 27.415 & 1.4930 & 0.0 \\
\hline 1200.0 & & 3.318 & 34.501 & 27.457 & 1.5697 & 0.0 \\
\hline 1300.0 & & 3.050 & 34.515 & 27.493 & 1.6430 & 0.0 \\
\hline 1400.0 & & 2.866 & 34.527 & 27.520 & 1.7134 & 0.0 \\
\hline 1500.0 & & 2.681 & 34.546 & 27.551 & 1.7810 & 0.0 \\
\hline 1501.0 & & 2.681 & 34.545 & 27.551 & 1.7817 & 0.0 \\
\hline
\end{tabular}

TEMPERATURE C

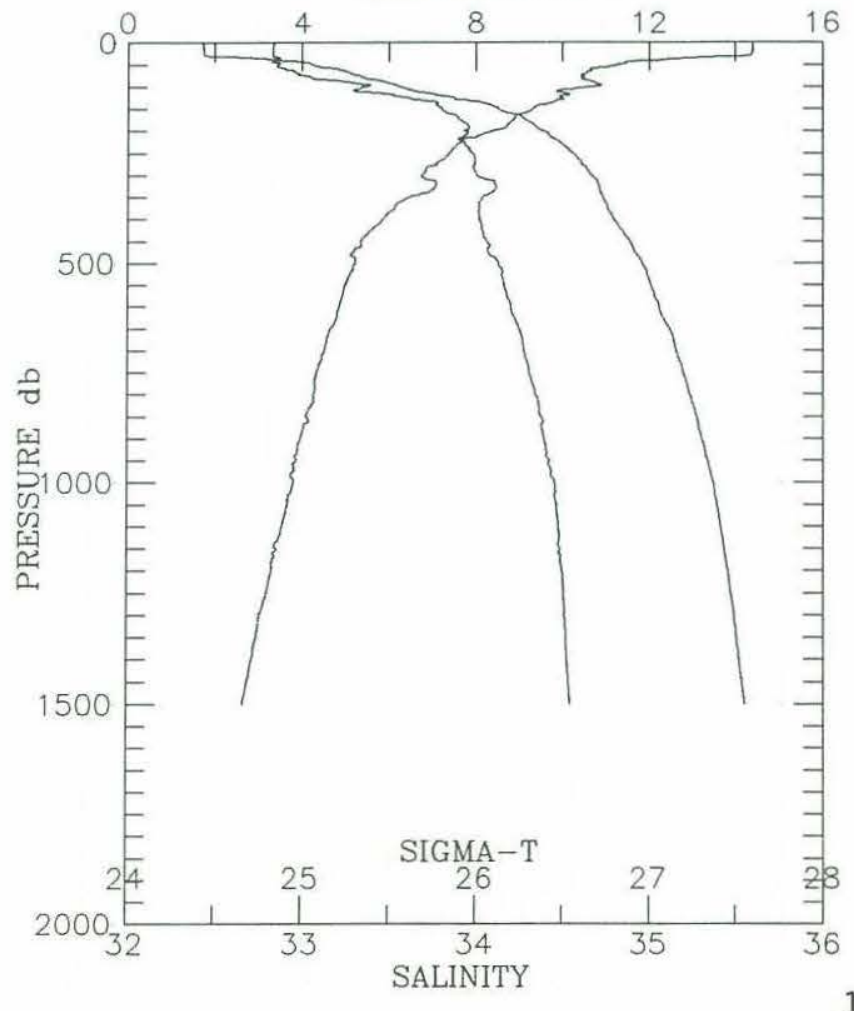

TEMPERATURE C

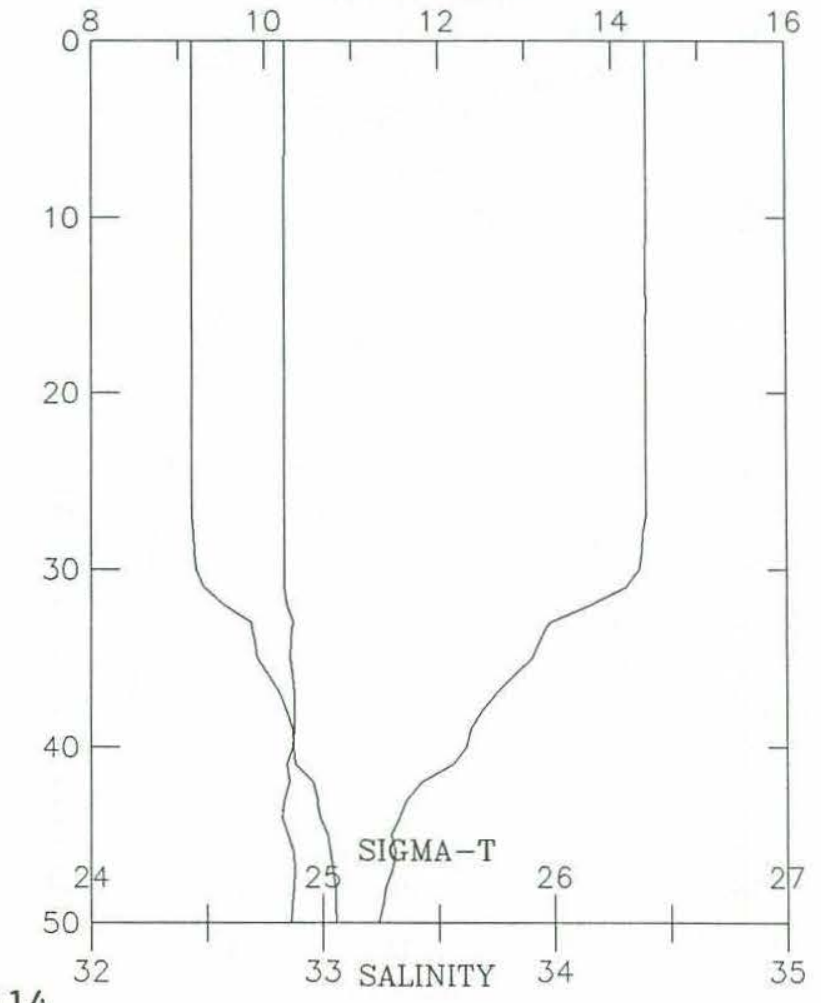


Cruise w8905 Station \# 32 Depth m: 3605 Time: 1989-05-07 15:20

Position: $39 \quad 3.30^{\prime} \mathrm{N} 12436.20^{\prime} \mathrm{W} \quad$ Depth Deck Offset: -0.15

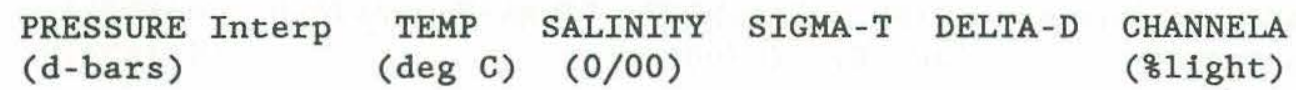
(d-bars) ( $\quad(\operatorname{deg} C) \quad(0 / 00)$

\begin{tabular}{|c|c|c|c|c|c|c|}
\hline 0.0 & E & 13.321 & 32.845 & 24.661 & 0.0000 & 0.0 \\
\hline 10.0 & & 13.307 & 32.843 & 24.662 & 0.0330 & 0.0 \\
\hline 20.0 & & 11.549 & 32.890 & 25.035 & 0.0655 & 0.0 \\
\hline 30.0 & & 10.150 & 33.037 & 25.394 & 0.0935 & 0.0 \\
\hline 40.0 & & 9.779 & 33.128 & 25.528 & 0.1193 & 0.0 \\
\hline 50.0 & & 9.367 & 33.303 & 25.731 & 0.1433 & 0.0 \\
\hline 60.0 & & 9.303 & 33.399 & 25.817 & 0.1659 & 0.0 \\
\hline 80.0 & & 8.906 & 33.609 & 26.044 & 0.2079 & 0.0 \\
\hline 100.0 & & 8.557 & 33.758 & 26.215 & 0.2463 & 0.0 \\
\hline 150.0 & & 8.191 & 33.954 & 26.425 & 0.3334 & 0.0 \\
\hline 200.0 & & 7.723 & 34.028 & 26.551 & 0.4136 & 0.0 \\
\hline 250.0 & & 7.273 & 34.055 & 26.636 & 0.4889 & 0.0 \\
\hline 300.0 & & 6.896 & 34.084 & 26.712 & 0.5606 & 0.0 \\
\hline 400.0 & & 5.639 & 34.063 & 26.857 & 0.6932 & 0.0 \\
\hline 500.0 & & 4.986 & 34.114 & 26.975 & 0.8151 & 0.0 \\
\hline 600.0 & & 4.832 & 34.243 & 27.095 & 0.9262 & 0.0 \\
\hline 700.0 & & 4.831 & 34.353 & 27.182 & 1.0293 & 0.0 \\
\hline 800.0 & & 4.236 & 34.359 & 27.251 & 1.1251 & 0.0 \\
\hline 900.0 & & 3.998 & 34.422 & 27.326 & 1.2145 & 0.0 \\
\hline 1000.0 & & 3.723 & 34.453 & 27.379 & 1.2984 & 0.0 \\
\hline 1100.0 & & 3.413 & 34.483 & 27.433 & 1.3772 & 0.0 \\
\hline 1200.0 & & 3.207 & 34.504 & 27.470 & 1.4523 & 0.0 \\
\hline 1300.0 & & 3.026 & 34.521 & 27.500 & 1.5243 & 0.0 \\
\hline 1400.0 & & 2.850 & 34.533 & 27.526 & 1.5938 & 0.0 \\
\hline 1500.0 & & 2.678 & 34.549 & 27.554 & 1.6611 & 0.0 \\
\hline 1502.0 & & 2.676 & 34.549 & 27.554 & 1.6624 & 0.0 \\
\hline
\end{tabular}
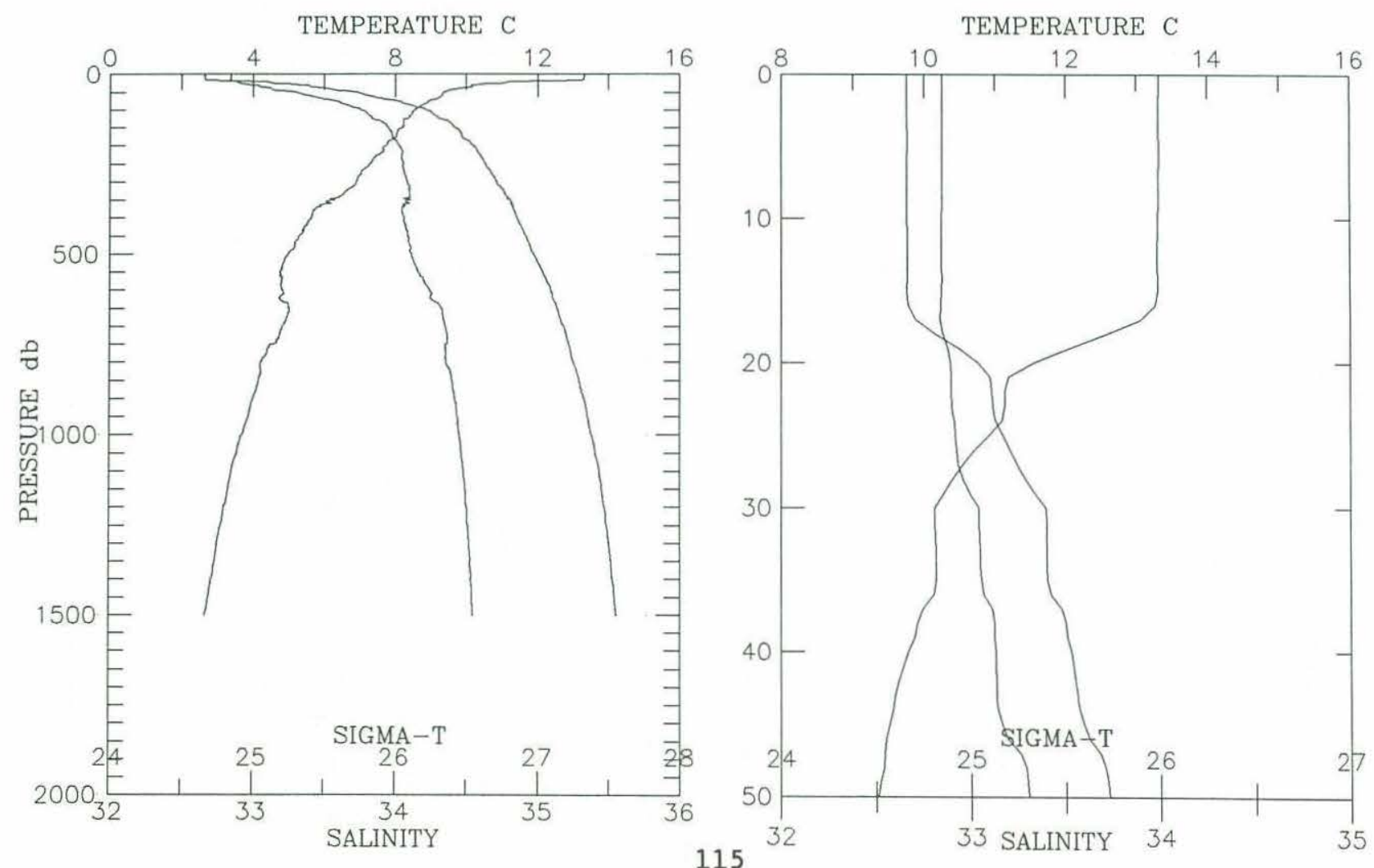


\begin{tabular}{|c|c|c|c|c|c|c|}
\hline $\begin{array}{l}\text { PRESSURE } \\
\text { (d-bars) }\end{array}$ & Interp & $\begin{array}{c}\text { TEMP } \\
(\operatorname{deg} C)\end{array}$ & $\begin{array}{c}\text { SALINITY } \\
(0 / 00)\end{array}$ & SIGMA-T & DELTA-D & $\begin{array}{l}\text { CHANNELA } \\
\text { (\&light) }\end{array}$ \\
\hline 0.0 & E & 13.530 & 32.971 & 24.716 & 0.0000 & 0.0 \\
\hline 10.0 & & 13.512 & 32.971 & 24.720 & 0.0325 & 0.0 \\
\hline 20.0 & & 13.010 & 33.008 & 24.849 & 0.0649 & 0.0 \\
\hline 30.0 & & 10.301 & 33.372 & 25.630 & 0.0919 & 0.0 \\
\hline 40.0 & & 9.364 & 33.515 & 25.898 & 0.1146 & 0.0 \\
\hline 50.0 & & 8.830 & 33.629 & 26.072 & 0.1350 & 0.0 \\
\hline 60.0 & & 8.627 & 33.728 & 26.181 & 0.1541 & 0.0 \\
\hline 80.0 & & 8.479 & 33.831 & 26.284 & 0.1905 & 0.0 \\
\hline 100.0 & & 8.346 & 33.877 & 26.341 & 0.2257 & 0.0 \\
\hline 150.0 & & 7.844 & 34.016 & 26.525 & 0.3074 & 0.0 \\
\hline 200.0 & & 7.404 & 34.040 & 26.606 & 0.3834 & 0.0 \\
\hline 250.0 & & 6.972 & 34.065 & 26.686 & 0.4562 & 0.0 \\
\hline 300.0 & & 6.716 & 34.096 & 26.745 & 0.5260 & 0.0 \\
\hline 400.0 & & 5.938 & 34.146 & 26.886 & 0.6568 & 0.0 \\
\hline 500.0 & & 5.467 & 34.198 & 26.985 & 0.7769 & 0.0 \\
\hline 600.0 & & 5.053 & 34.263 & 27.085 & 0.8891 & 0.0 \\
\hline 700.0 & & 4.839 & 34.346 & 27.175 & 0.9923 & 0.0 \\
\hline 800.0 & & 4.305 & 34.361 & 27.245 & 1.0891 & 0.0 \\
\hline 900.0 & & 4.030 & 34.410 & 27.313 & 1.1794 & 0.0 \\
\hline 1000.0 & & 3.701 & 34.451 & 27.380 & 1.2638 & 0.0 \\
\hline 1100.0 & & 3.494 & 34.477 & 27.421 & 1.3434 & 0.0 \\
\hline 1200.0 & & 3.292 & 34.494 & 27.454 & 1.4198 & 0.0 \\
\hline 1300.0 & & 3.049 & 34.516 & 27.494 & 1.4932 & 0.0 \\
\hline 1400.0 & & 2.827 & 34.526 & 27.522 & 1.5632 & 0.0 \\
\hline 1500.0 & & 2.686 & 34.546 & 27.551 & 1.6308 & 0.0 \\
\hline
\end{tabular}

TEMPERATURE C
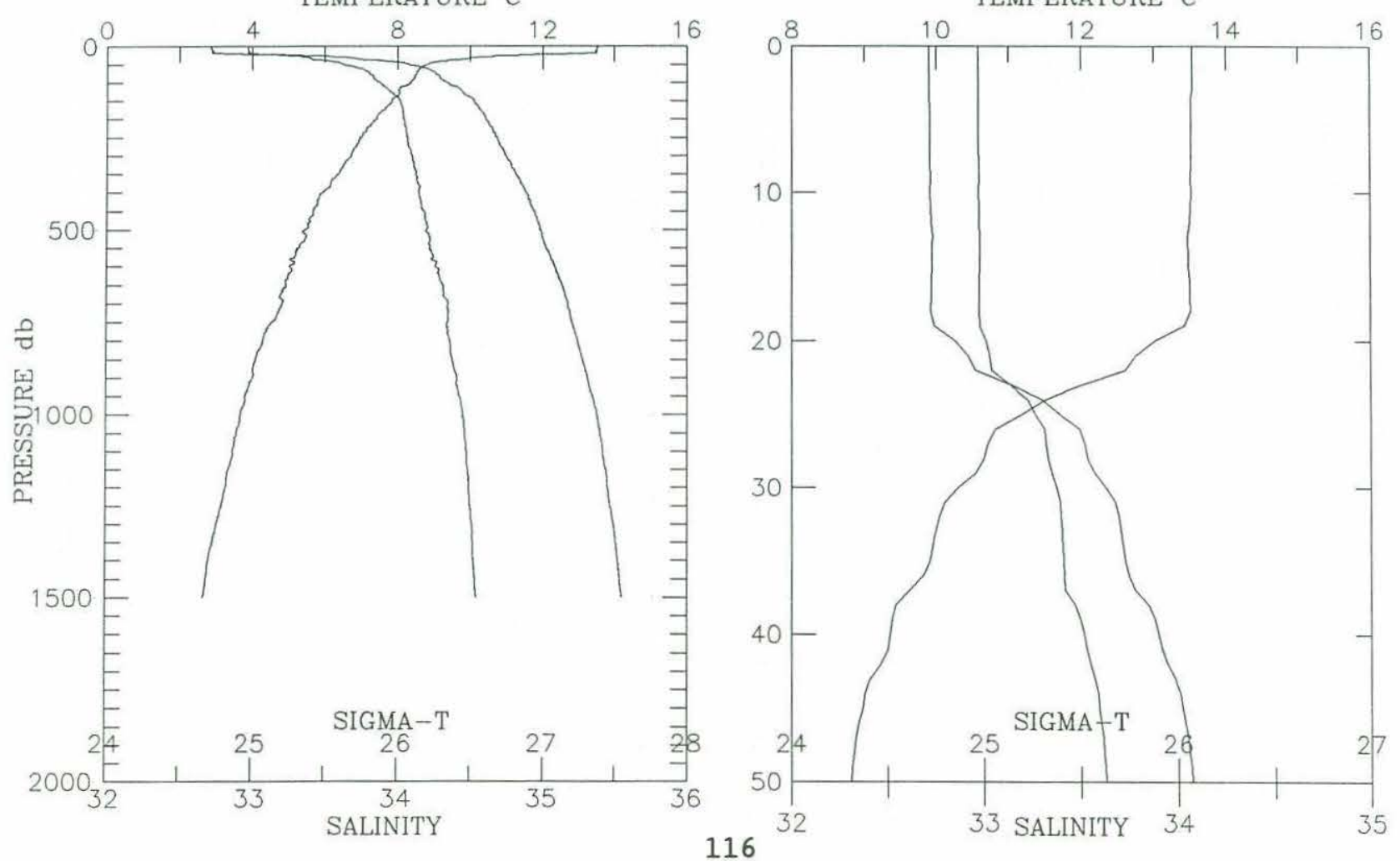
Cruise W8905

Position: 39
Station \# 34 Depth m: 3045 Time: 1989-05-07 18:54 5.20'N $12420.80^{\prime} \mathrm{W} \quad$ Depth Deck Offset: -0.15

\begin{tabular}{|c|c|c|c|c|c|c|}
\hline $\begin{array}{l}\text { PRESSURE } \\
\text { (d-bars) }\end{array}$ & Interp & $\begin{array}{c}\text { TEMP } \\
(\operatorname{deg} C)\end{array}$ & $\begin{array}{c}\text { SALINITY } \\
(0 / 00)\end{array}$ & SIGMA-T & DELTA-D & $\begin{array}{l}\text { CHANNELA } \\
\text { ( } \delta 1 \text { ight) }\end{array}$ \\
\hline 0.0 & E & 13.682 & 33.074 & 24.765 & 0.0000 & 0.0 \\
\hline 10.0 & & 13.663 & 33.070 & 24.766 & 0.0321 & 0.0 \\
\hline 20.0 & & 12.005 & 33.103 & 25.116 & 0.0634 & 0.0 \\
\hline 30.0 & & 10.345 & 33.307 & 25.572 & 0.0898 & 0.0 \\
\hline 40.0 & & 9.458 & 33.456 & 25.836 & 0.1129 & 0.0 \\
\hline 50.0 & & 8.852 & 33.621 & 26.062 & 0.1338 & 0.0 \\
\hline 60.0 & & 8.650 & 33.695 & 26.151 & 0.1532 & 0.0 \\
\hline 80.0 & & 8.342 & 33.836 & 26.309 & 0.1897 & 0.0 \\
\hline 100.0 & & 8.160 & 33.950 & 26.426 & 0.2235 & 0.0 \\
\hline 150.0 & & 7.740 & 34.021 & 26.543 & 0.3028 & 0.0 \\
\hline 200.0 & & 7.290 & 34.052 & 26.632 & 0.3776 & 0.0 \\
\hline 250.0 & & 6.822 & 34.085 & 26.722 & 0.4488 & 0.0 \\
\hline 300.0 & & 6.596 & 34.118 & 26.779 & 0.5168 & 0.0 \\
\hline 400.0 & & 6.055 & 34.199 & 26.913 & 0.6449 & 0.0 \\
\hline 500.0 & & 5.564 & 34.239 & 27.006 & 0.7638 & 0.0 \\
\hline 600.0 & & 5.181 & 34.281 & 27.085 & 0.8754 & 0.0 \\
\hline 700.0 & & 4.864 & 34.350 & 27.176 & 0.9788 & 0.0 \\
\hline 800.0 & & 4.378 & 34.369 & 27.244 & 1.0750 & 0.0 \\
\hline 900.0 & & 4.030 & 34.411 & 27.314 & 1.1653 & 0.0 \\
\hline 1000.0 & & 3.781 & 34.438 & 27.361 & 1.2506 & 0.0 \\
\hline 1100.0 & & 3.546 & 34.469 & 27.409 & 1. 3318 & 0.0 \\
\hline 1200.0 & & 3.321 & 34.491 & 27.449 & 1.4091 & 0.0 \\
\hline 1300.0 & & 3.048 & 34.507 & 27.487 & 1.4832 & 0.0 \\
\hline 1400.0 & & 2.905 & 34.524 & 27.514 & 1.5541 & 0.0 \\
\hline 1500.0 & & 2.700 & 34.535 & 27.541 & 1.6225 & 0.0 \\
\hline
\end{tabular}
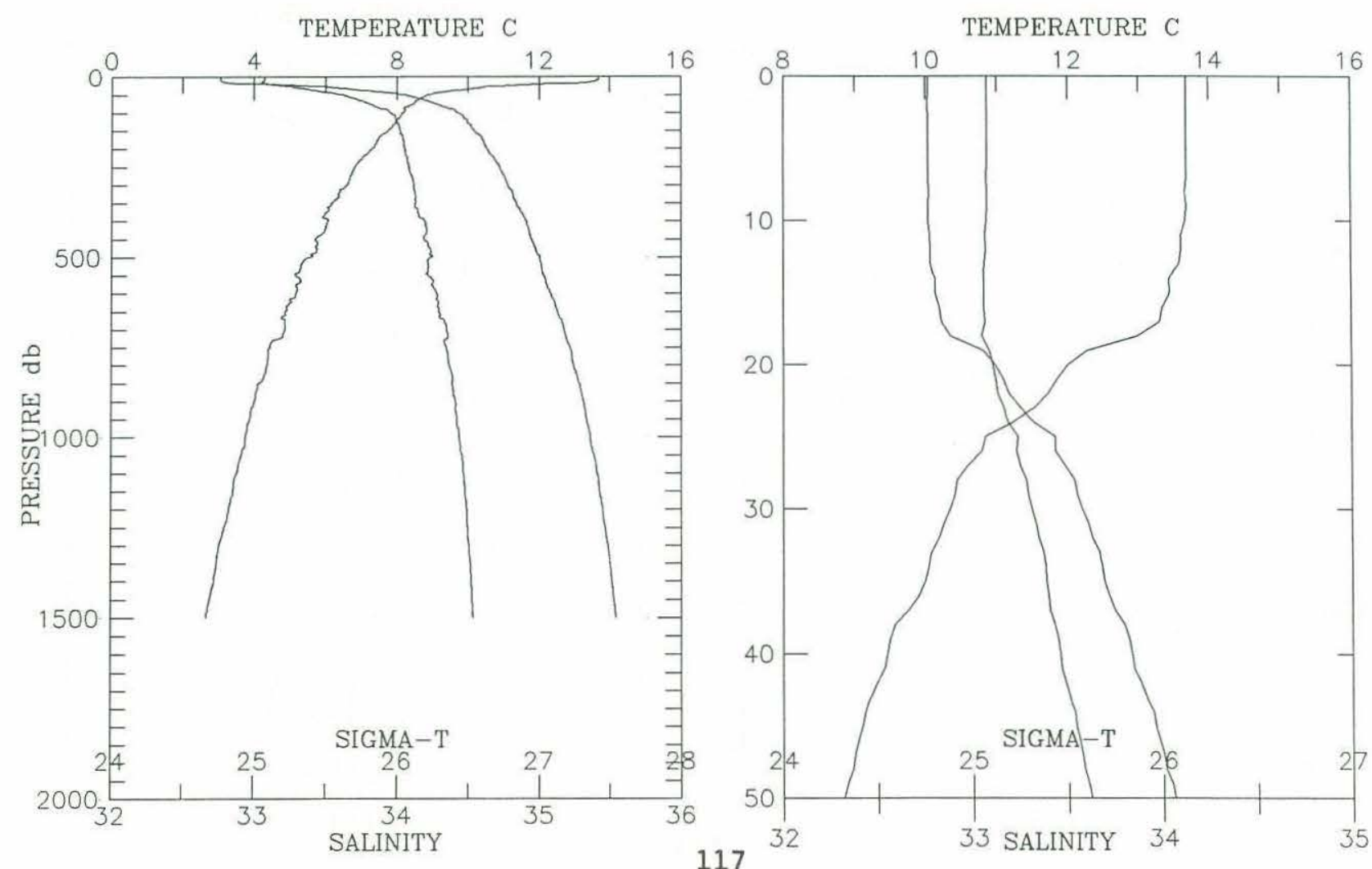
Cruise W8905 Station \# 35 Depth m: 1901 Time: 1989-05-07 20:15

Position: $396.40^{\prime} \mathrm{N} 124 \quad 15.10^{\prime} \mathrm{W}$ Depth Deck Offset: -0.23

PRESSURE Interp TEMP SALINITY SIGMA-T DELTA-D CHANNELA

$(d$-bars $) \quad(\operatorname{deg} C)(0 / 00) \quad$ (\%light)

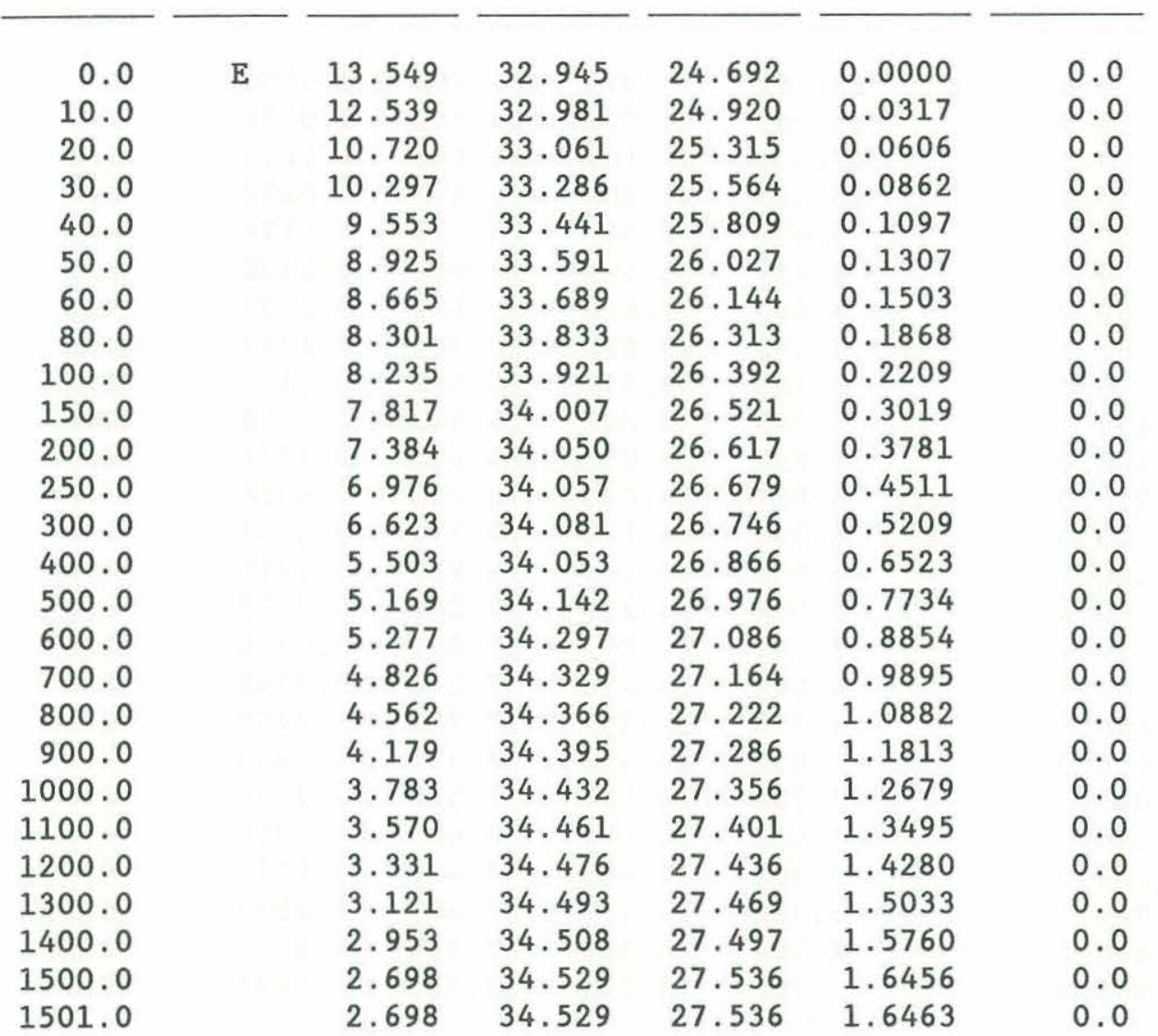

TEMPERATURE C
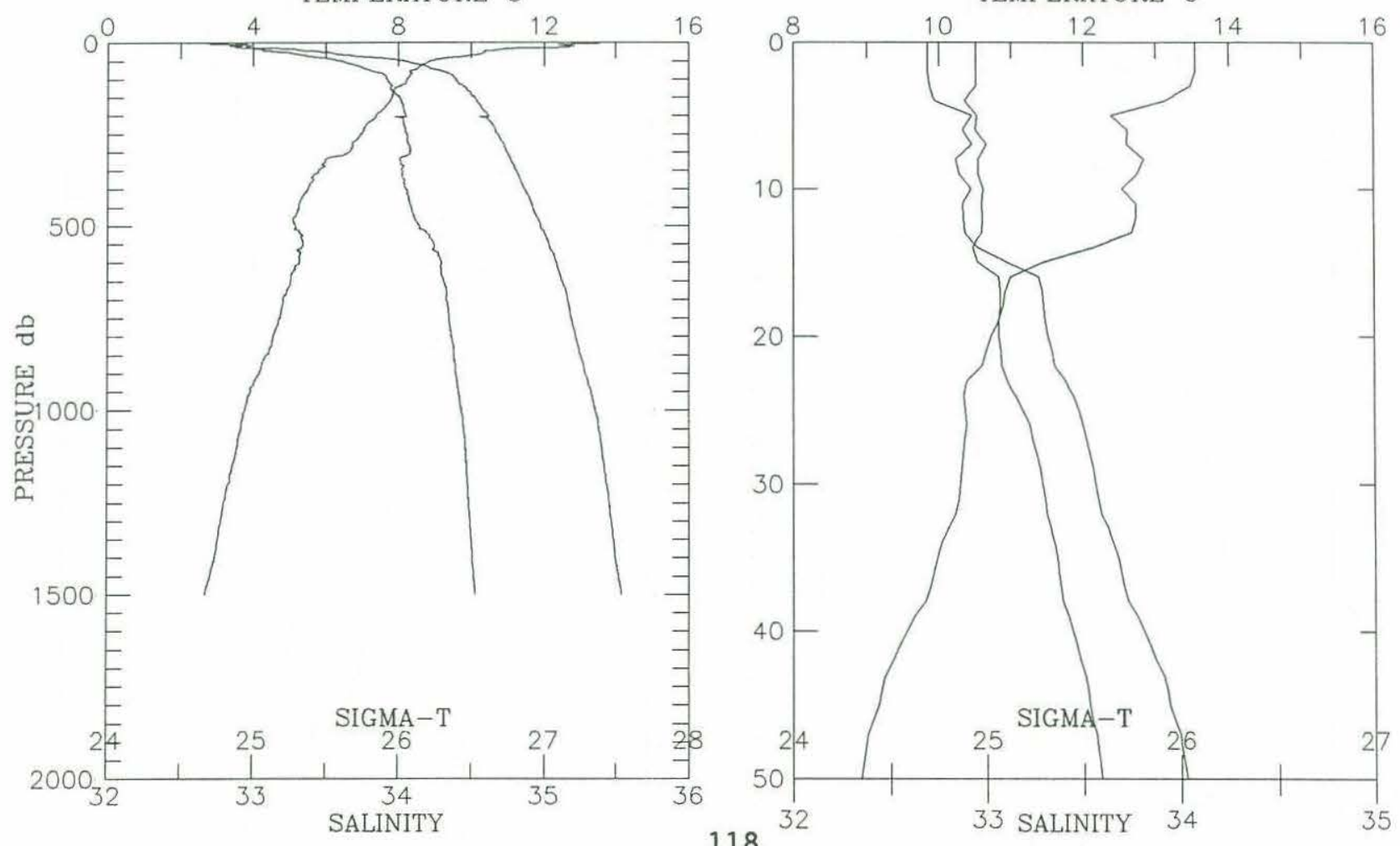
Cruise W8905 Station \# 36 Depth m: 973 Time: 1989-05-07 21:40 Position: $39 \quad 7.60^{\prime} \mathrm{N} 124 \quad 8.60^{\prime} \mathrm{W} \quad$ Depth Deck Offset: $\quad-0.20$

\begin{tabular}{|c|c|c|c|c|c|c|}
\hline $\begin{array}{l}\text { PRESSURE } \\
\text { (d-bars) }\end{array}$ & Interp & $\begin{array}{l}\text { TEMP } \\
(\operatorname{deg} C)\end{array}$ & $\begin{array}{l}\text { SALINITY } \\
(0 / 00)\end{array}$ & SIGMA-T & DELTA-D & $\begin{array}{l}\text { CHANNELA } \\
\text { (\&light) }\end{array}$ \\
\hline 0.0 & E & 13.476 & 32.937 & 24.701 & 0.0000 & 0.0 \\
\hline 10.0 & & 13.475 & 32.935 & 24.699 & 0.0327 & 0.0 \\
\hline 20.0 & & 13.246 & 32.939 & 24.749 & 0.0653 & 0.0 \\
\hline 30.0 & & 10.491 & 33.057 & 25.352 & 0.0940 & 0.0 \\
\hline 40.0 & & 9.829 & 33.266 & 25.627 & 0.1192 & 0.0 \\
\hline 50.0 & & 9.173 & 33.480 & 25.901 & 0.1419 & 0.0 \\
\hline 60.0 & & 8.947 & 33.571 & 26.008 & 0.1627 & 0.0 \\
\hline 80.0 & & 8.460 & 33.760 & 26.232 & 0.2005 & 0.0 \\
\hline 100.0 & & 8.306 & 33.873 & 26.344 & 0.2362 & 0.0 \\
\hline 150.0 & & 7.889 & 33.981 & 26.490 & 0.3189 & 0.0 \\
\hline 200.0 & & 7.498 & 34.007 & 26.567 & 0.3968 & 0.0 \\
\hline 250.0 & & 6.890 & 34.000 & 26.646 & 0.4713 & 0.0 \\
\hline 300.0 & & 6.485 & 34.025 & 26.720 & 0.5424 & 0.0 \\
\hline 400.0 & & 5.595 & 34.066 & 26.865 & 0.6746 & 0.0 \\
\hline 500.0 & & 5.676 & 34.246 & 26.998 & 0.7953 & 0.0 \\
\hline 600.0 & & 5.320 & 34.292 & 27.077 & 0.9065 & 0.0 \\
\hline 700.0 & & 4.858 & 34.339 & 27.168 & 1.0112 & 0.0 \\
\hline 800.0 & & 4.584 & 34.371 & 27.223 & 1.1090 & 0.0 \\
\hline 900.0 & & 4.289 & 34.403 & 27.281 & 1.2029 & 0.0 \\
\hline 901.0 & & 4.289 & 34.402 & 27.280 & 1.2038 & 0.0 \\
\hline
\end{tabular}

TEMPERATURE $\mathrm{C}$
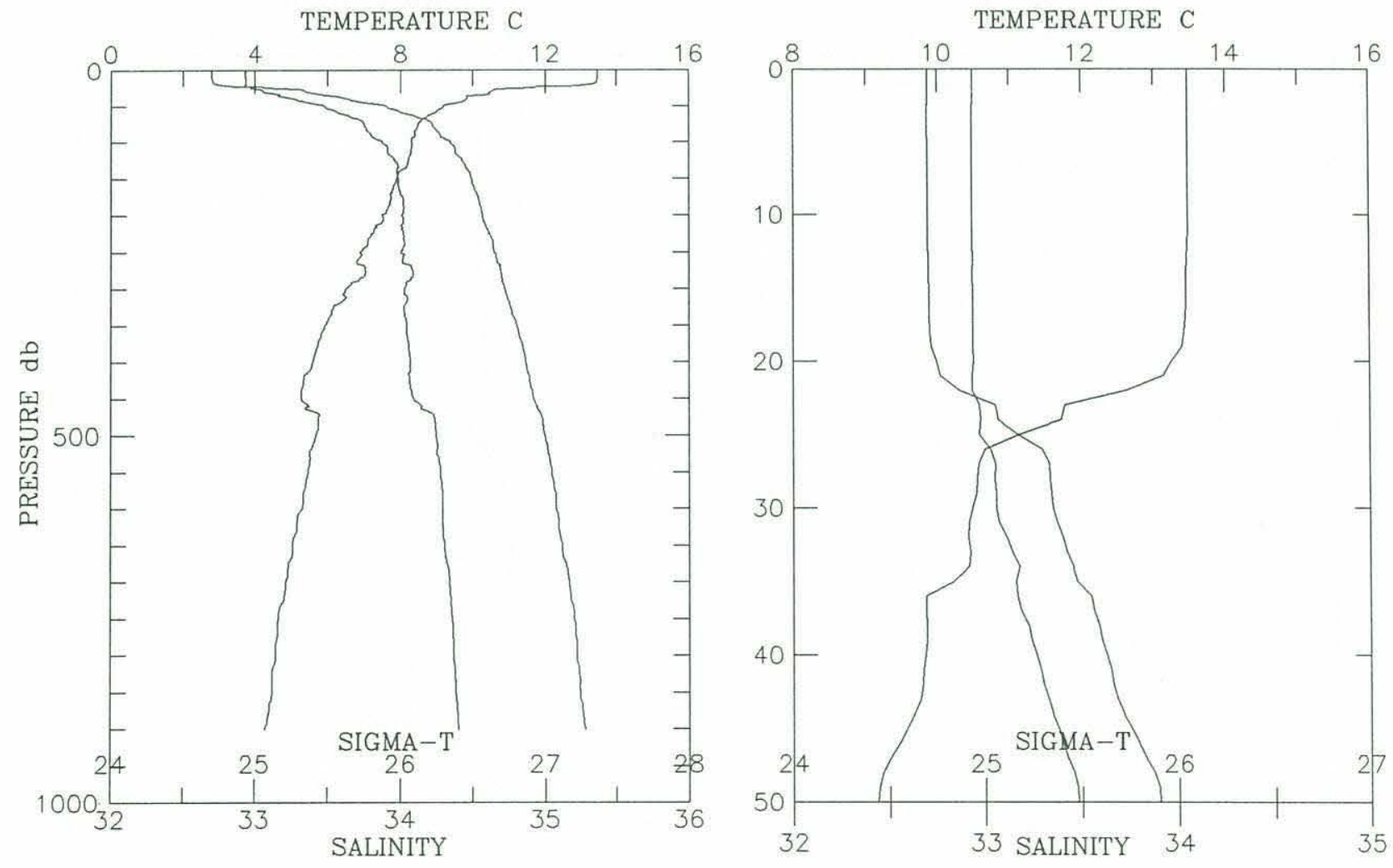


\begin{tabular}{|c|c|c|c|c|c|c|c|}
\hline $\begin{array}{l}\text { Cruise W } \\
\text { Position }\end{array}$ & $\begin{array}{r}3905 \\
\quad 39\end{array}$ & $\begin{array}{l}\text { tation \# } \\
.20^{\prime} \mathrm{N} 1\end{array}$ & $\begin{array}{rr}37 & \text { Dep } \\
24 & 2.50\end{array}$ & h m: 575 & $\begin{array}{r}\text { Time: } \\
\text { epth Dec }\end{array}$ & $\begin{array}{l}1989-05-07 \\
\text { Offset: }\end{array}$ & $\begin{array}{l}22: 47 \\
-0.20\end{array}$ \\
\hline $\begin{array}{l}\text { PRESSURE } \\
\text { (d-bars) }\end{array}$ & Interp & $\begin{array}{l}\text { TEMP } \\
(\operatorname{deg} C)\end{array}$ & $\begin{array}{c}\text { SALINITY } \\
(0 / 00)\end{array}$ & SIGMA-T & DELTA-D & $\begin{array}{l}\text { CHANNELA } \\
\text { ( }(\delta 1 \text { ight) }\end{array}$ & \\
\hline 0.0 & $\mathrm{E}$ & 13.427 & 32.929 & 24.704 & 0.0000 & 0.0 & \\
\hline 10.0 & & 13.409 & 32.924 & 24.704 & 0.0326 & 0.0 & \\
\hline 20.0 & & 12.514 & 32.899 & 24.861 & 0.0649 & 0.0 & \\
\hline 30.0 & & 10.103 & 32.972 & 25.352 & 0.0934 & 0.0 & \\
\hline 40.0 & & 9.903 & 33.116 & 25.497 & 0.1192 & 0.0 & \\
\hline 50.0 & & 9.500 & 33.344 & 25.742 & 0.1431 & 0.0 & \\
\hline 60.0 & & 9.138 & 33.494 & 25.917 & 0.1651 & 0.0 & \\
\hline 80.0 & & 8.754 & 33.767 & 26.192 & 0.2050 & 0.0 & \\
\hline 100.0 & & 8.807 & 33.882 & 26.274 & 0.2417 & 0.0 & \\
\hline 150.0 & & 8.031 & 33.999 & 26.484 & 0.3257 & 0.0 & \\
\hline 200.0 & & 7.596 & 34.051 & 26.587 & 0.4034 & 0.0 & \\
\hline 250.0 & & 7.076 & 34.076 & 26.681 & 0.4769 & 0.0 & \\
\hline 300.0 & & 6.259 & 34.013 & 26.740 & 0.5472 & 0.0 & \\
\hline 400.0 & & 6.246 & 34.179 & 26.873 & 0.6789 & 0.0 & \\
\hline 500.0 & & 5.518 & 34.233 & 27.006 & 0.7993 & 0.0 & \\
\hline 560.0 & & 5.493 & 34.265 & 27.035 & 0.8670 & 0.0 & \\
\hline
\end{tabular}
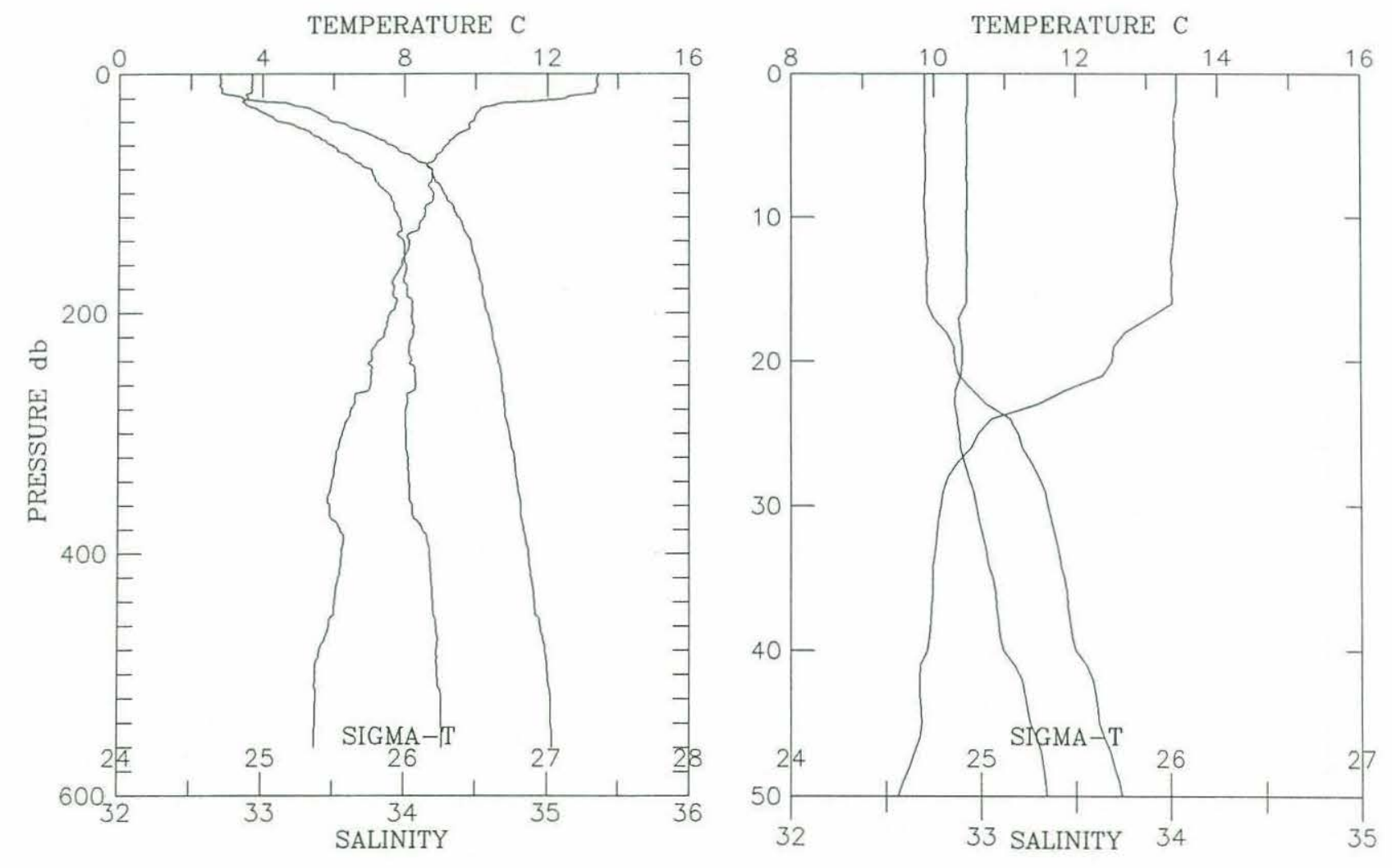
Cruise w8905 Station \# 38 Depth m: 415 Time: 1989-05-07 23:33

Position: 39

$8.70^{\prime} \mathrm{N} \quad 123 \quad 59.20^{\prime} \mathrm{W}$

Depth Deck Offset:

$-0.35$

\begin{tabular}{|c|c|c|c|c|c|c|}
\hline $\begin{array}{l}\text { PRESSURE } \\
\text { (d-bars) }\end{array}$ & Interp & $\begin{array}{l}\text { TEMP } \\
(\operatorname{deg} C)\end{array}$ & $\begin{array}{c}\text { SALINITY } \\
(0 / 00)\end{array}$ & SIGMA-T & DELTA-D & $\begin{array}{l}\text { CHANNELA } \\
\text { ( }(\delta 1 \text { ight) }\end{array}$ \\
\hline 0.0 & E & 13.343 & 33.028 & 24.798 & 0.0000 & 0.0 \\
\hline 10.0 & & 13.295 & 33.016 & 24.798 & 0.0318 & 0.0 \\
\hline 20.0 & & 11.477 & 32.953 & 25.096 & 0.0618 & 0.0 \\
\hline 30.0 & & 10.322 & 33.166 & 25.466 & 0.0885 & 0.0 \\
\hline 40.0 & & 9.476 & 33.390 & 25.782 & 0.1127 & 0.0 \\
\hline 50.0 & & 9.195 & 33.472 & 25.891 & 0.1346 & 0.0 \\
\hline 60.0 & & 8.895 & 33.563 & 26.010 & 0.1553 & 0.0 \\
\hline 80.0 & & 8.768 & 33.768 & 26.190 & 0.1945 & 0.0 \\
\hline 100.0 & & 8.763 & 33.871 & 26.272 & 0.2312 & 0.0 \\
\hline 150.0 & & 8.147 & 34.010 & 26.475 & 0.3152 & 0.0 \\
\hline 200.0 & & 7.477 & 34.023 & 26.583 & 0.3929 & 0.0 \\
\hline 250.0 & & 6.752 & 33.998 & 26.663 & 0.4665 & 0.0 \\
\hline 300.0 & & 6.786 & 34.113 & 26.749 & 0.5366 & 0.0 \\
\hline 400.0 & & 6.241 & 34.184 & 26.877 & 0.6680 & 0.0 \\
\hline 401.0 & & 6.242 & 34.183 & 26.876 & 0.6692 & 0.0 \\
\hline
\end{tabular}
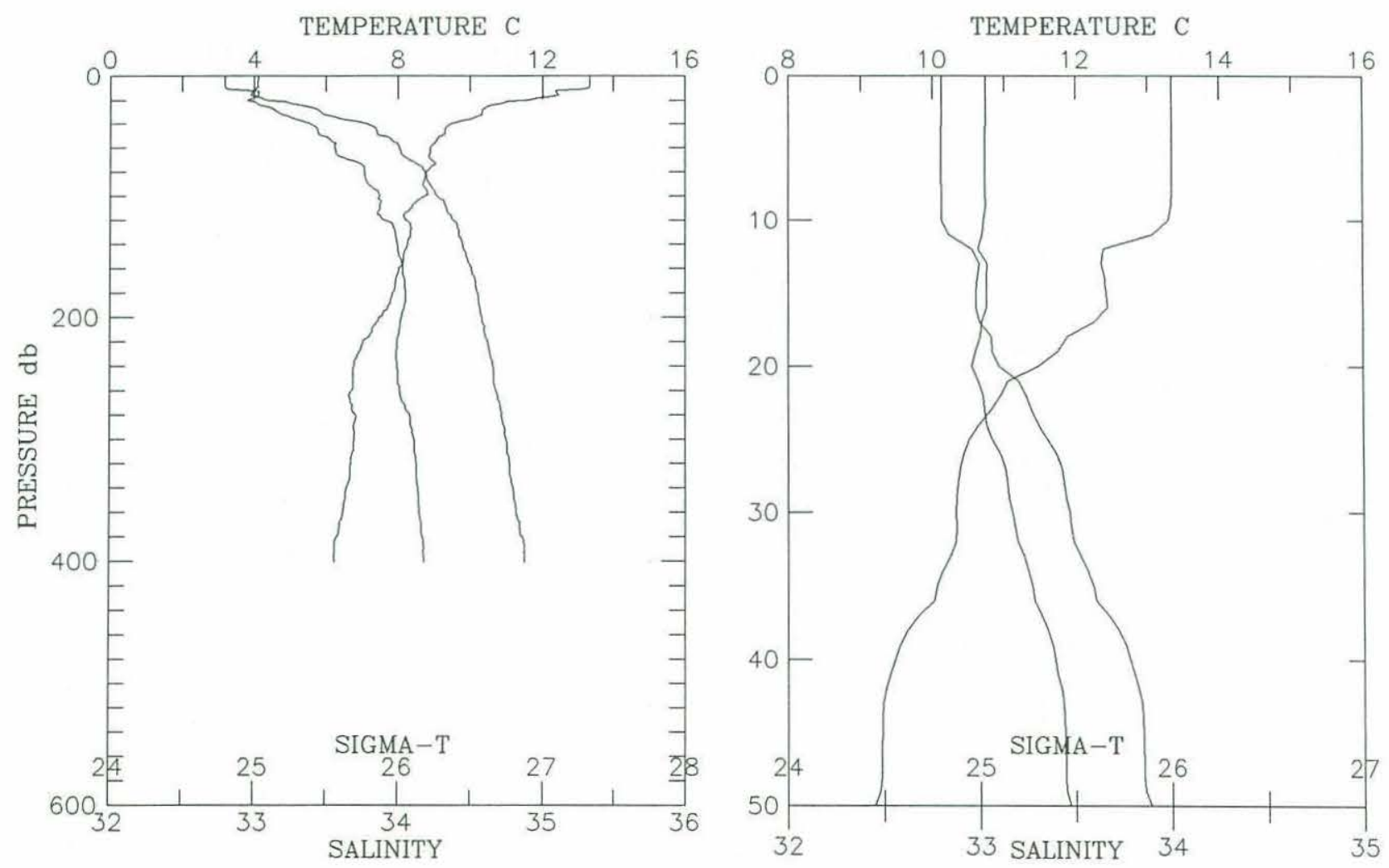


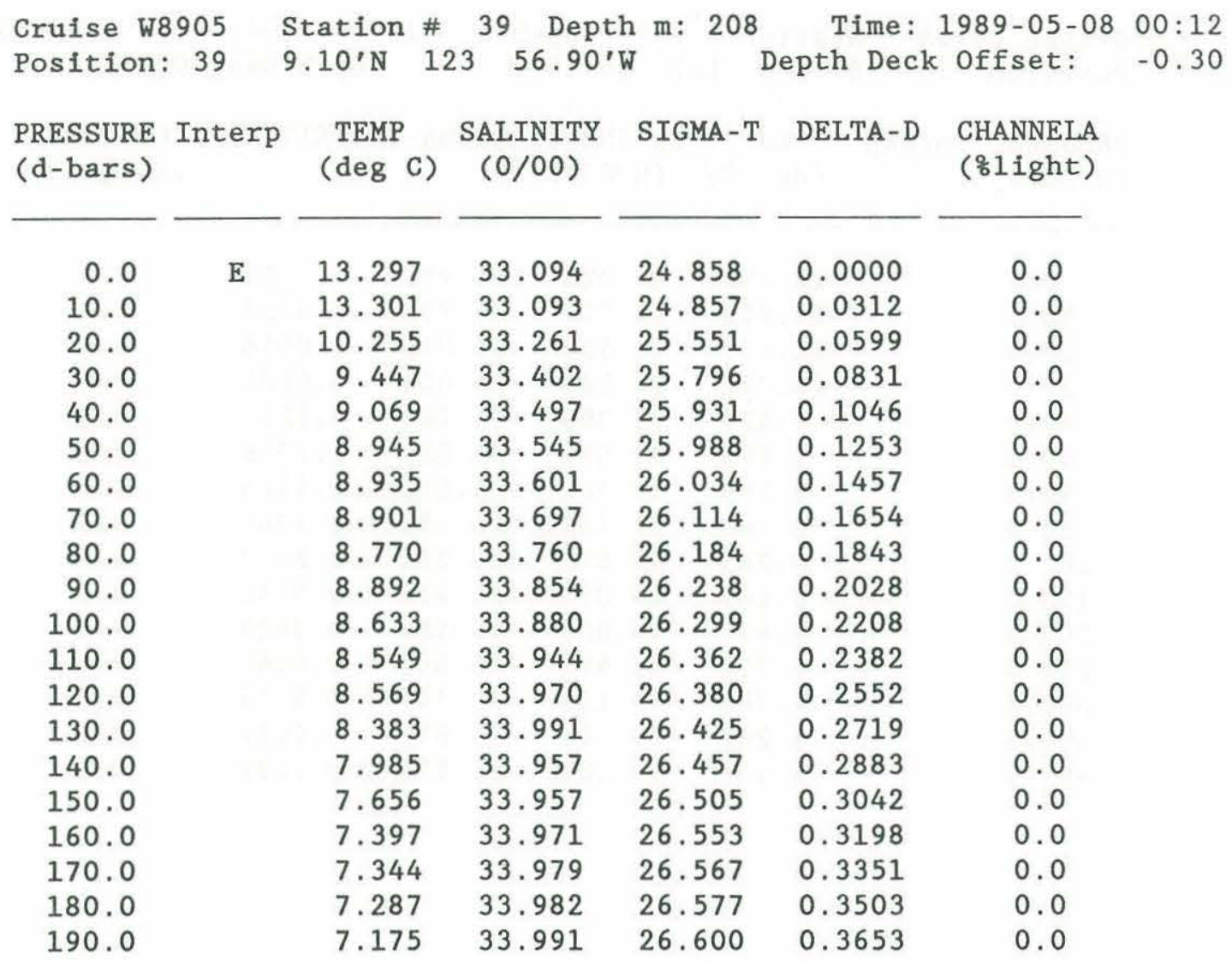
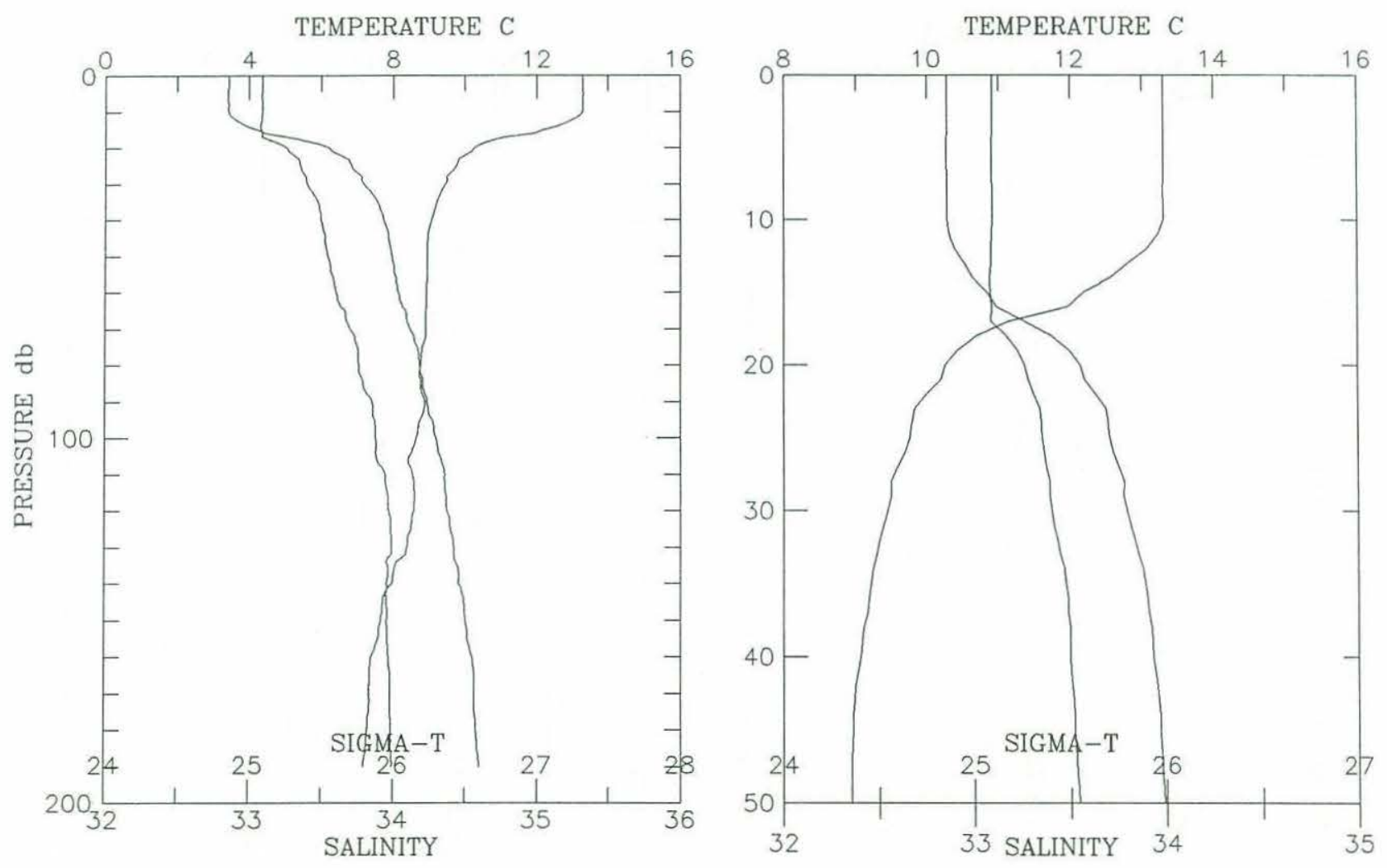


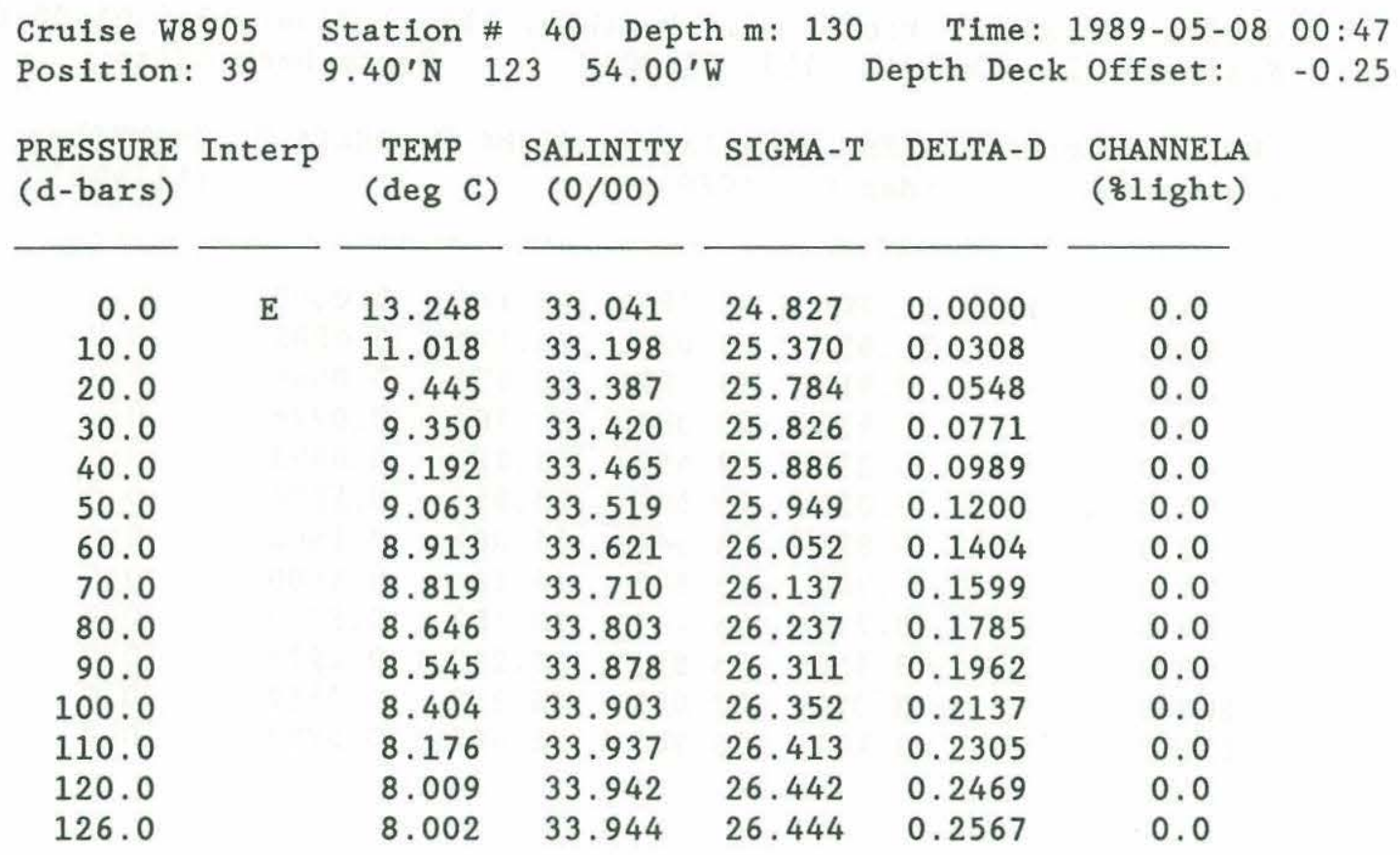
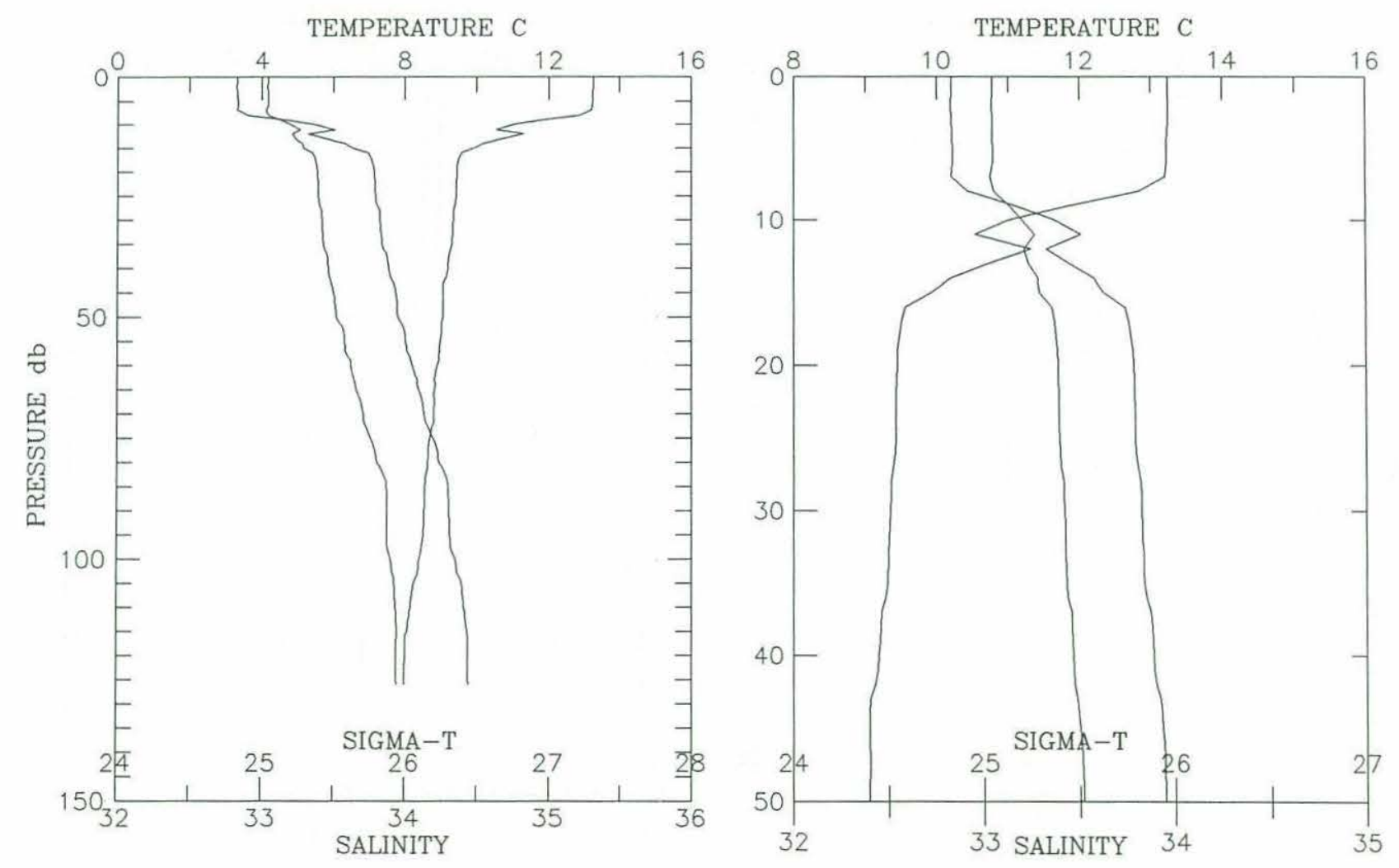
Cruise w8905 Station \# 41 Depth m: 117 Time: 1989-05-08 01:15 Position: $39 \quad 9.70^{\prime} \mathrm{N} 12351.30^{\prime} \mathrm{W}$ Depth Deck Offset: -0.30

\begin{tabular}{|c|c|c|c|c|c|c|}
\hline $\begin{array}{l}\text { PRESSURE } \\
\text { (d-bars) }\end{array}$ & Interp & $\begin{array}{l}\text { TEMP } \\
(\operatorname{deg} C)\end{array}$ & $\begin{array}{c}\text { SALINITY } \\
(0 / 00)\end{array}$ & SIGMA-T & DELTA-D & $\begin{array}{l}\text { CHANNELA } \\
\text { ( }(\mathrm{i} 1 \mathrm{ght} \text { ) }\end{array}$ \\
\hline 0.0 & E & 11.701 & 33.095 & 25.166 & 0.0000 & 0.0 \\
\hline 10.0 & & 11.655 & 33.096 & 25.175 & 0.0282 & 0.0 \\
\hline 20.0 & & 9.917 & 33.298 & 25.637 & 0.0546 & 0.0 \\
\hline 30.0 & & 9.459 & 33.387 & 25.782 & 0.0774 & 0.0 \\
\hline 40.0 & & 9.230 & 33.456 & 25.873 & 0.0995 & 0.0 \\
\hline 50.0 & & 9.030 & 33.546 & 25.975 & 0.1204 & 0.0 \\
\hline 60.0 & & 8.839 & 33.646 & 26.084 & 0.1405 & 0.0 \\
\hline 70.0 & & 8.780 & 33.685 & 26.124 & 0.1600 & 0.0 \\
\hline 80.0 & & 8.713 & 33.747 & 26.182 & 0.1790 & 0.0 \\
\hline 90.0 & & 8.558 & 33.816 & 26.260 & 0.1975 & 0.0 \\
\hline 100.0 & & 8.325 & 33.861 & 26.331 & 0.2152 & 0.0 \\
\hline 110.0 & & 8.101 & 33.909 & 26.402 & 0.2323 & 0.0 \\
\hline
\end{tabular}
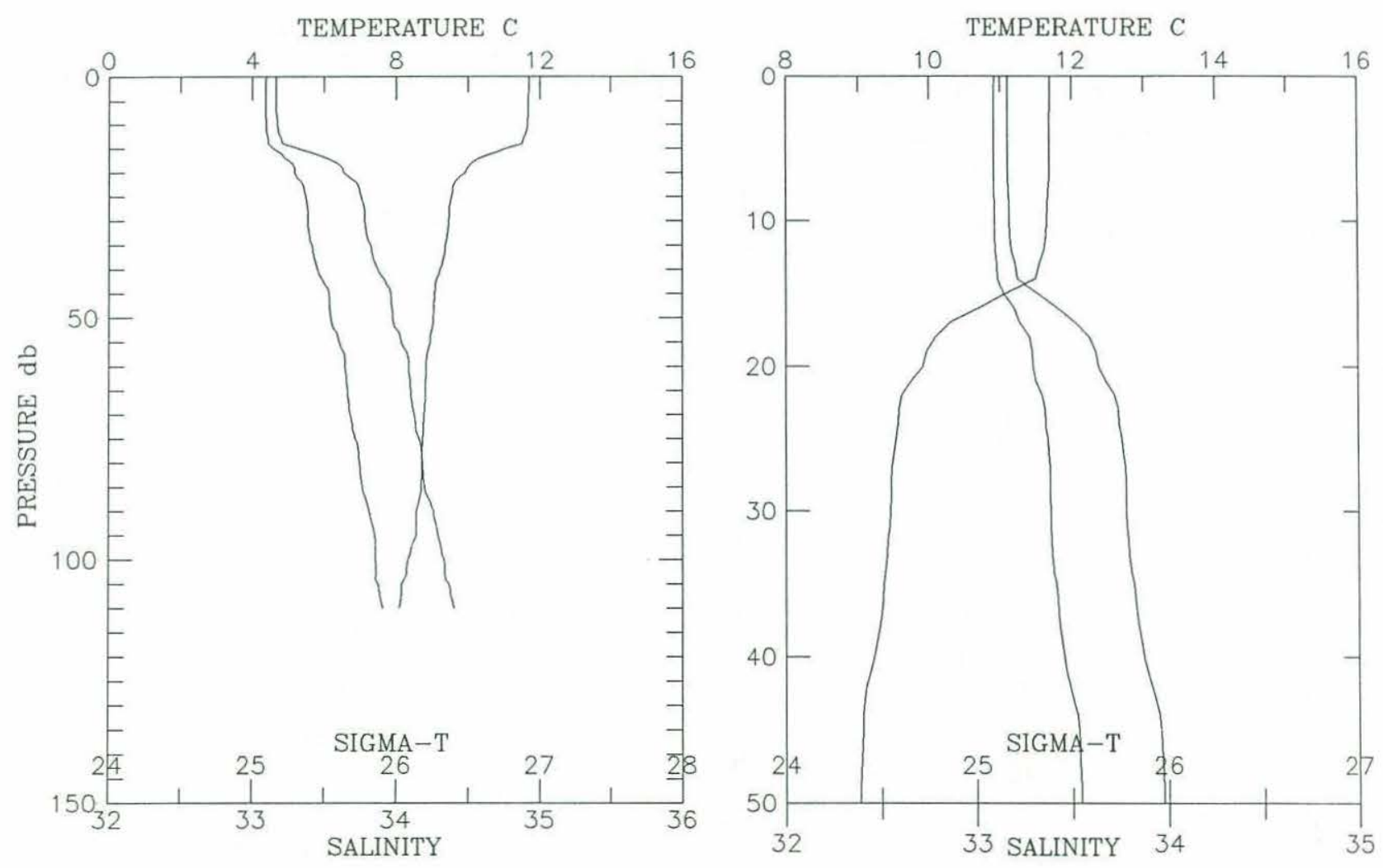
Cruise w8905 Station \# 42 Depth m: 104 Time: 1989-05-08 01:45 Position: $3910.00^{\prime} \mathrm{N} 12348.50^{\prime} \mathrm{W}$ Depth Deck Offset: -0.30

\begin{tabular}{|c|c|c|c|c|c|c|}
\hline $\begin{array}{l}\text { PRESSURE } \\
\text { (d-bars) }\end{array}$ & Interp & $\begin{array}{l}\text { TEMP } \\
(\operatorname{deg} C)\end{array}$ & $\begin{array}{c}\text { SALINITY } \\
(0 / 00)\end{array}$ & SIGMA-T & DELTA-D & $\begin{array}{l}\text { CHANNELA } \\
\text { ( } \% 1 \text { ight) }\end{array}$ \\
\hline 0.0 & E & 11.424 & 33.128 & 25.242 & 0.0000 & 0.0 \\
\hline 5.0 & & 11.423 & 33.126 & 25.240 & 0.0138 & 0.0 \\
\hline 10.0 & & 11.406 & 33.131 & 25.248 & 0.0275 & 0.0 \\
\hline 15.0 & & 10.636 & 33.175 & 25.419 & 0.0410 & 0.0 \\
\hline 20.0 & & 9.974 & 33.253 & 25.593 & 0.0535 & 0.0 \\
\hline 25.0 & & 9.419 & 33.394 & 25.794 & 0.0651 & 0.0 \\
\hline 30.0 & & 9.315 & 33.423 & 25.834 & 0.0761 & 0.0 \\
\hline 35.0 & & 9.194 & 33.475 & 25.894 & 0.0869 & 0.0 \\
\hline 40.0 & & 9.108 & 33.504 & 25.930 & 0.0976 & 0.0 \\
\hline 45.0 & & 8.978 & 33.551 & 25.988 & 0.1080 & 0.0 \\
\hline 50.0 & & 8.932 & 33.585 & 26.021 & 0.1182 & 0.0 \\
\hline 55.0 & & 8.851 & 33.629 & 26.069 & 0.1282 & 0.0 \\
\hline 60.0 & & 8.803 & 33.649 & 26.092 & 0.1380 & 0.0 \\
\hline 65.0 & & 8.714 & 33.686 & 26.135 & 0.1477 & 0.0 \\
\hline 70.0 & & 8.696 & 33.733 & 26.174 & 0.1572 & 0.0 \\
\hline 75.0 & & 8.626 & 33.807 & 26.243 & 0.1665 & 0.0 \\
\hline 80.0 & & 8.372 & 33.851 & 26.316 & 0.1754 & 0.0 \\
\hline 85.0 & & 8.279 & 33.877 & 26.351 & 0.1840 & 0.0 \\
\hline 90.0 & & 8.281 & 33.877 & 26.351 & 0.1926 & 0.0 \\
\hline 91.0 & & 8.281 & 33.877 & 26.351 & 0.1943 & 0.0 \\
\hline
\end{tabular}

TEMPERATURE C
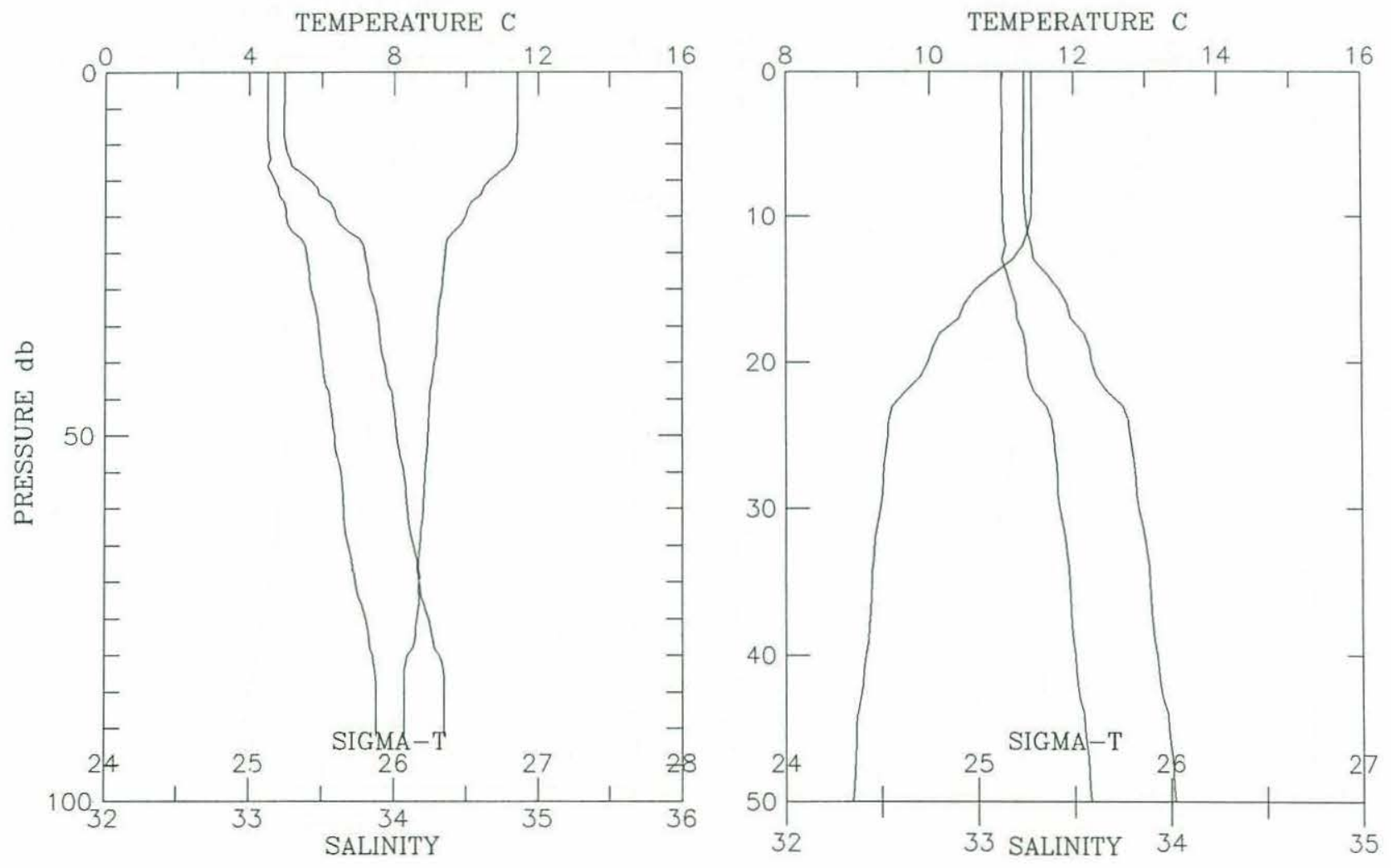
Cruise w8905 Station \# 43 Depth m: 97 Time: 1989-05-08 02:05 Position: $3910.30^{\prime} \mathrm{N} 123 \quad 48.00^{\prime} \mathrm{W}$ Depth Deck Offset: -0.43

\begin{tabular}{|c|c|c|c|c|c|c|}
\hline $\begin{array}{l}\text { PRESSURE } \\
\text { (d-bars) }\end{array}$ & Interp & $\begin{array}{l}\text { TEMP } \\
(\operatorname{deg} C)\end{array}$ & $\begin{array}{l}\text { SALINITY } \\
(0 / 00)\end{array}$ & SIGMA-T & DELTA-D & $\begin{array}{l}\text { CHANNELA } \\
\text { ( }(\mathrm{l} \text { ight) }\end{array}$ \\
\hline 0.0 & E & 11.344 & 33.168 & 25.288 & 0.0000 & 0.0 \\
\hline 5.0 & & 11.406 & 33.163 & 25.272 & 0.0136 & 0.0 \\
\hline 10.0 & & 11.119 & 33.151 & 25.315 & 0.0271 & 0.0 \\
\hline 15.0 & & 10.193 & 33.225 & 25.534 & 0.0400 & 0.0 \\
\hline 20.0 & & 9.639 & 33.297 & 25.683 & 0.0520 & 0.0 \\
\hline 25.0 & & 9.325 & 33.386 & 25.803 & 0.0633 & 0.0 \\
\hline 30.0 & & 9.263 & 33.412 & 25.833 & 0.0744 & 0.0 \\
\hline 35.0 & & 9.164 & 33.449 & 25.878 & 0.0853 & 0.0 \\
\hline 40.0 & & 9.000 & 33.506 & 25.949 & 0.0959 & 0.0 \\
\hline 45.0 & & 8.907 & 33.560 & 26.006 & 0.1062 & 0.0 \\
\hline 50.0 & & 8.829 & 33.612 & 26.059 & 0.1163 & 0.0 \\
\hline 55.0 & & 8.800 & 33.676 & 26.113 & 0.1260 & 0.0 \\
\hline 60.0 & & 8.818 & 33.711 & 26.138 & 0.1356 & 0.0 \\
\hline 65.0 & & 8.692 & 33.769 & 26.203 & 0.1450 & 0.0 \\
\hline 70.0 & & 8.635 & 33.800 & 26.236 & 0.1542 & 0.0 \\
\hline 75.0 & & 8.564 & 33.828 & 26.269 & 0.1632 & 0.0 \\
\hline 80.0 & & 8.359 & 33.867 & 26.331 & 0.1720 & 0.0 \\
\hline 85.0 & & 8.349 & 33.873 & 26.337 & 0.1807 & 0.0 \\
\hline 90.0 & & 8.349 & 33.877 & 26.340 & 0.1893 & 0.0 \\
\hline
\end{tabular}

TEMPERATURE C
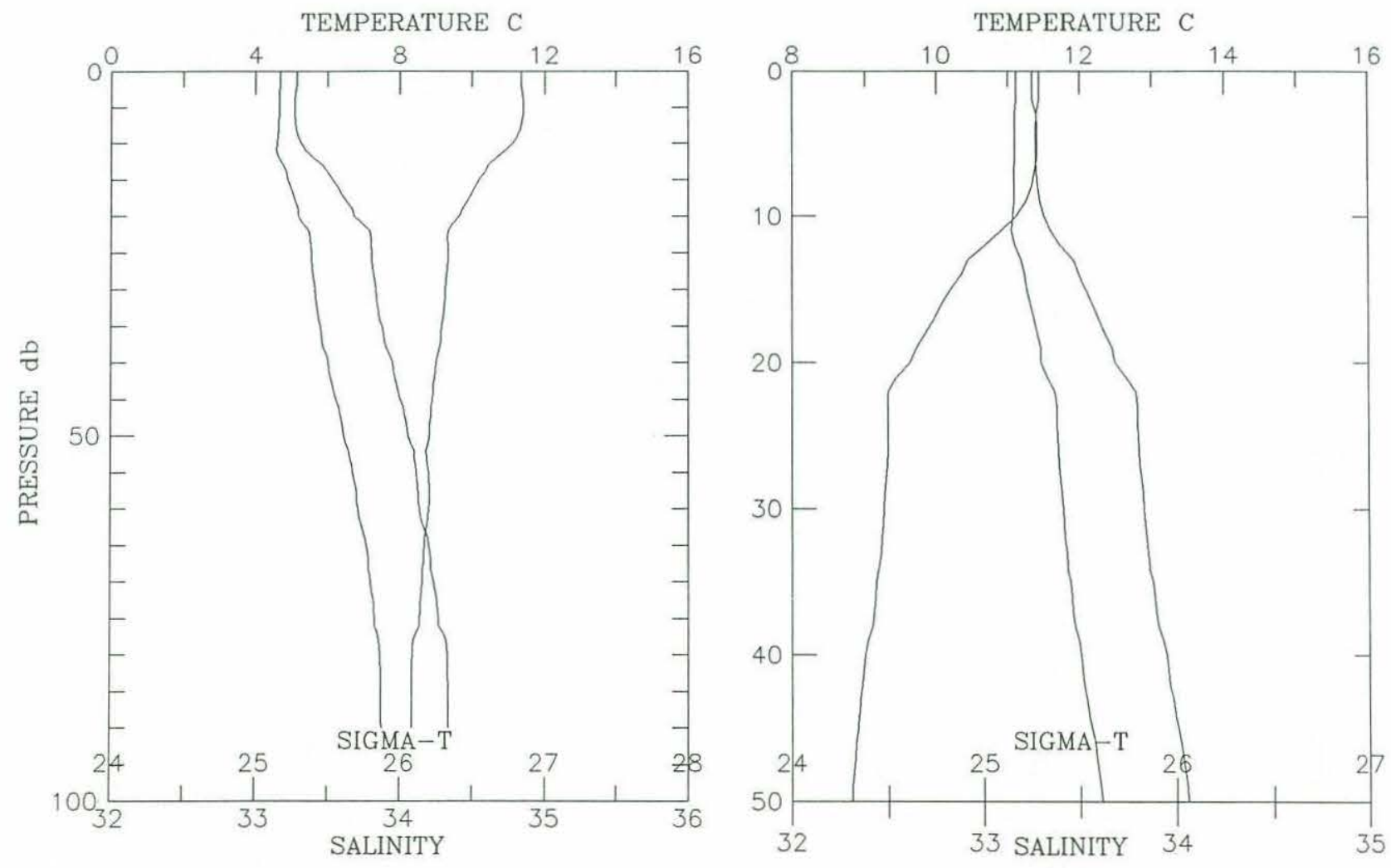
Cruise w8905 Station \# 44 Depth m: 68 Time: 1989-05-08 02:27

Position: 3910.50 'N $123 \quad 46.90^{\prime} \mathrm{W}$ Depth Deck Offset: -0.43

\begin{tabular}{|c|c|c|c|c|c|c|}
\hline $\begin{array}{l}\text { PRESSURE } \\
\text { (d-bars) }\end{array}$ & Interp & $\begin{array}{c}\text { TEMP } \\
(\operatorname{deg} C)\end{array}$ & $\begin{array}{c}\text { SALINITY } \\
(0 / 00)\end{array}$ & SIGMA-T & DELTA-D & $\begin{array}{l}\text { CHANNELA } \\
\text { ( } \delta 1 \text { ight) }\end{array}$ \\
\hline 0.0 & E & 10.969 & 33.124 & 25.321 & 0.0000 & 0.0 \\
\hline 5.0 & & 10.963 & 33.127 & 25.324 & 0.0134 & 0.0 \\
\hline 10.0 & & 10.262 & 33.207 & 25.508 & 0.0264 & 0.0 \\
\hline 15.0 & & 9.573 & 33.320 & 25.711 & 0.0385 & 0.0 \\
\hline 20.0 & & 9.390 & 33.372 & 25.782 & 0.0498 & 0.0 \\
\hline 25.0 & & 9.238 & 33.443 & 25.862 & 0.0609 & 0.0 \\
\hline 30.0 & & 9.150 & 33.465 & 25.893 & 0.0716 & 0.0 \\
\hline 35.0 & & 9.021 & 33.501 & 25.942 & 0.0822 & 0.0 \\
\hline 40.0 & & 8.981 & 33.534 & 25.974 & 0.0926 & 0.0 \\
\hline 45.0 & & 8.907 & 33.648 & 26.075 & 0.1027 & 0.0 \\
\hline 50.0 & & 8.842 & 33.695 & 26.122 & 0.1124 & 0.0 \\
\hline 55.0 & & 8.760 & 33.741 & 26.171 & 0.1219 & 0.0 \\
\hline 60.0 & & 8.729 & 33.754 & 26.185 & 0.1312 & 0.0 \\
\hline 65.0 & & 8.725 & 33.758 & 26.189 & 0.1405 & 0.0 \\
\hline
\end{tabular}

TEMPERATURE C
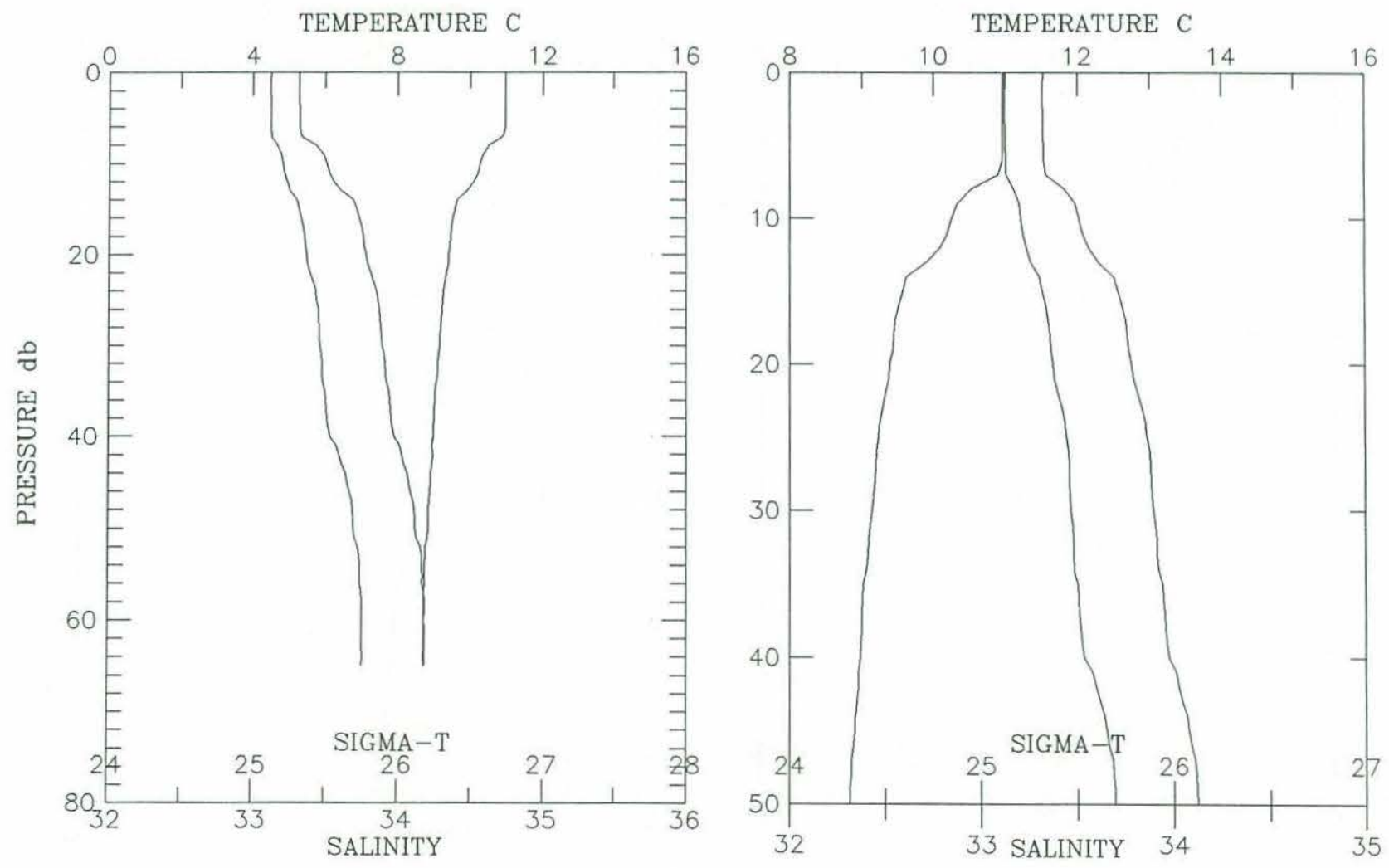
Cruise W8905

Position: 39
Station \# 45 Depth m: 95 $3.50^{\prime} \mathrm{N} \quad 123 \quad 48.50^{\prime} \mathrm{W}$
Time: 1989-05-08 03:27

Depth Deck Offset: -0.43

\begin{tabular}{|c|c|c|c|c|c|c|}
\hline $\begin{array}{l}\text { PRESSURE } \\
\text { (d-bars) }\end{array}$ & Interp & $\begin{array}{l}\text { TEMP } \\
(\operatorname{deg} C)\end{array}$ & $\begin{array}{c}\text { SALINITY } \\
(0 / 00)\end{array}$ & SIGMA-T & DELTA-D & $\begin{array}{l}\text { CHANNELA } \\
\text { ( } 81 \text { ight) }\end{array}$ \\
\hline 0.0 & E & 13.072 & 33.016 & 24.843 & 0.0000 & 0.0 \\
\hline 5.0 & & 13.082 & 33.011 & 24.837 & 0.0157 & 0.0 \\
\hline 10.0 & & 12.862 & 33.007 & 24.877 & 0.0312 & 0.0 \\
\hline 15.0 & & 11.790 & 33.133 & 25.179 & 0.0460 & 0.0 \\
\hline 20.0 & & 9.994 & 33.176 & 25.529 & 0.0591 & 0.0 \\
\hline 25.0 & & 9.638 & 33.265 & 25.658 & 0.0712 & 0.0 \\
\hline 30.0 & & 9.622 & 33.264 & 25.660 & 0.0830 & 0.0 \\
\hline 35.0 & & 9.418 & 33.338 & 25.751 & 0.0946 & 0.0 \\
\hline 40.0 & & 9.338 & 33.378 & 25.795 & 0.1059 & 0.0 \\
\hline 45.0 & & 9.253 & 33.416 & 25.838 & 0.1169 & 0.0 \\
\hline 50.0 & & 9.220 & 33.445 & 25.866 & 0.1279 & 0.0 \\
\hline 55.0 & & 9.161 & 33.499 & 25.918 & 0.1385 & 0.0 \\
\hline 60.0 & & 9.088 & 33.544 & 25.965 & 0.1491 & 0.0 \\
\hline 65.0 & & 8.860 & 33.611 & 26.053 & 0.1592 & 0.0 \\
\hline 70.0 & & 8.844 & 33.627 & 26.068 & 0.1691 & 0.0 \\
\hline 75.0 & & 8.840 & 33.636 & 26.076 & 0.1790 & 0.0 \\
\hline 80.0 & & 8.751 & 33.664 & 26.112 & 0.1888 & 0.0 \\
\hline
\end{tabular}
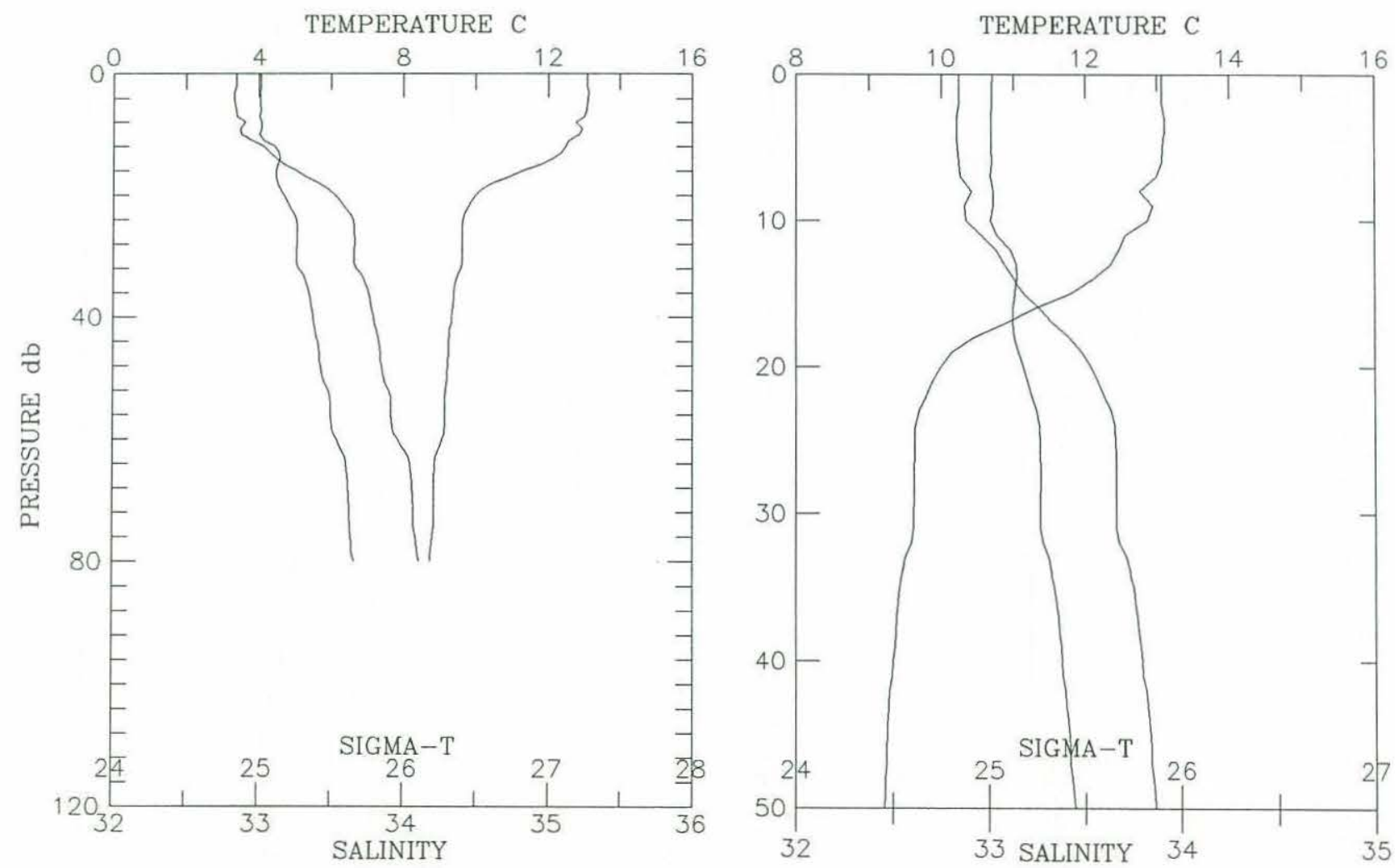
Cruise w8905 Station \# 46 Depth m: 101 Time: 1989-05-08 04:17 Position: $38 \quad 56.90^{\prime} \mathrm{N} 12348.50^{\prime} \mathrm{W}$ Depth Deck Offset: -0.43

\begin{tabular}{|c|c|c|c|c|c|c|}
\hline $\begin{array}{l}\text { PRESSURE } \\
\text { (d-bars) }\end{array}$ & Interp & $\begin{array}{l}\text { TEMP } \\
(\operatorname{deg} C)\end{array}$ & $\begin{array}{l}\text { SALINITY } \\
(0 / 00)\end{array}$ & SIGMA-T & DELTA-D & $\begin{array}{l}\text { CHANNELA } \\
\text { (\&light) }\end{array}$ \\
\hline 0.0 & $\mathrm{E}$ & 12.750 & 32.996 & 24.891 & 0.0000 & 0.0 \\
\hline 5.0 & & 12.764 & 32.995 & 24.887 & 0.0154 & 0.0 \\
\hline 10.0 & & 12.589 & 32.995 & 24.921 & 0.0309 & 0.0 \\
\hline 15.0 & & 10.194 & 33.043 & 25.392 & 0.0451 & 0.0 \\
\hline 20.0 & & 9.725 & 33.182 & 25.579 & 0.0576 & 0.0 \\
\hline 25.0 & & 9.634 & 33.244 & 25.642 & 0.0696 & 0.0 \\
\hline 30.0 & & 9.501 & 33.314 & 25.718 & 0.0814 & 0.0 \\
\hline 35.0 & & 9.378 & 33.350 & 25.766 & 0.0927 & 0.0 \\
\hline 40.0 & & 9.290 & 33.409 & 25.827 & 0.1039 & 0.0 \\
\hline 45.0 & & 9.285 & 33.428 & 25.843 & 0.1149 & 0.0 \\
\hline 50.0 & & 9.198 & 33.488 & 25.904 & 0.1257 & 0.0 \\
\hline 55.0 & & 8.921 & 33.594 & 26.030 & 0.1360 & 0.0 \\
\hline 60.0 & & 8.876 & 33.603 & 26.044 & 0.1461 & 0.0 \\
\hline 65.0 & & 8.714 & 33.650 & 26.107 & 0.1560 & 0.0 \\
\hline 70.0 & & 8.731 & 33.761 & 26.191 & 0.1655 & 0.0 \\
\hline 75.0 & & 8.662 & 33.841 & 26.264 & 0.1746 & 0.0 \\
\hline 80.0 & & 8.645 & 33.853 & 26.276 & 0.1835 & 0.0 \\
\hline 85.0 & & 8.641 & 33.855 & 26.278 & 0.1924 & 0.0 \\
\hline 90.0 & & 8.640 & 33.855 & 26.278 & 0.2014 & 0.0 \\
\hline 91.0 & & 8.641 & 33.855 & 26.278 & 0.2032 & 0.0 \\
\hline
\end{tabular}
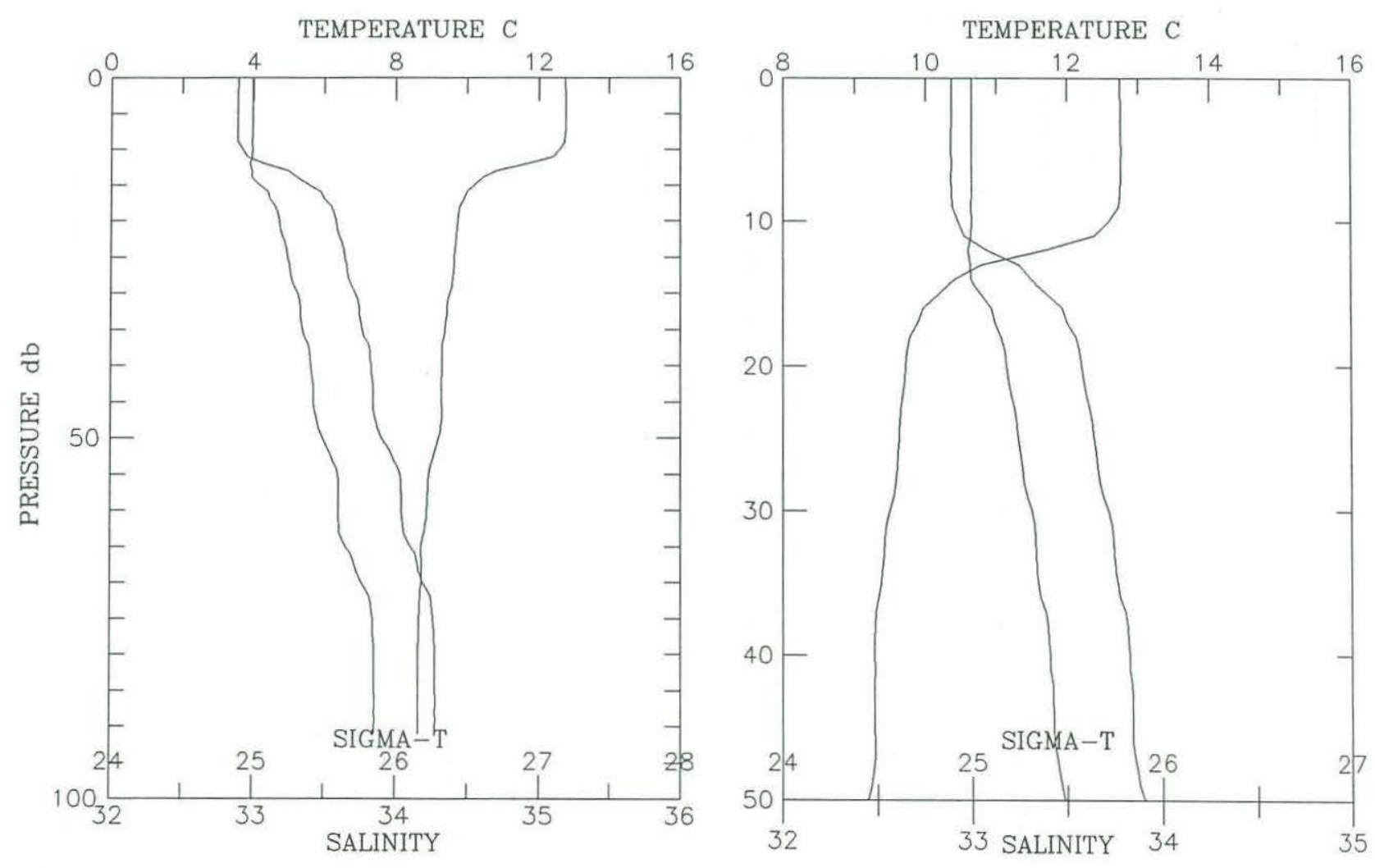


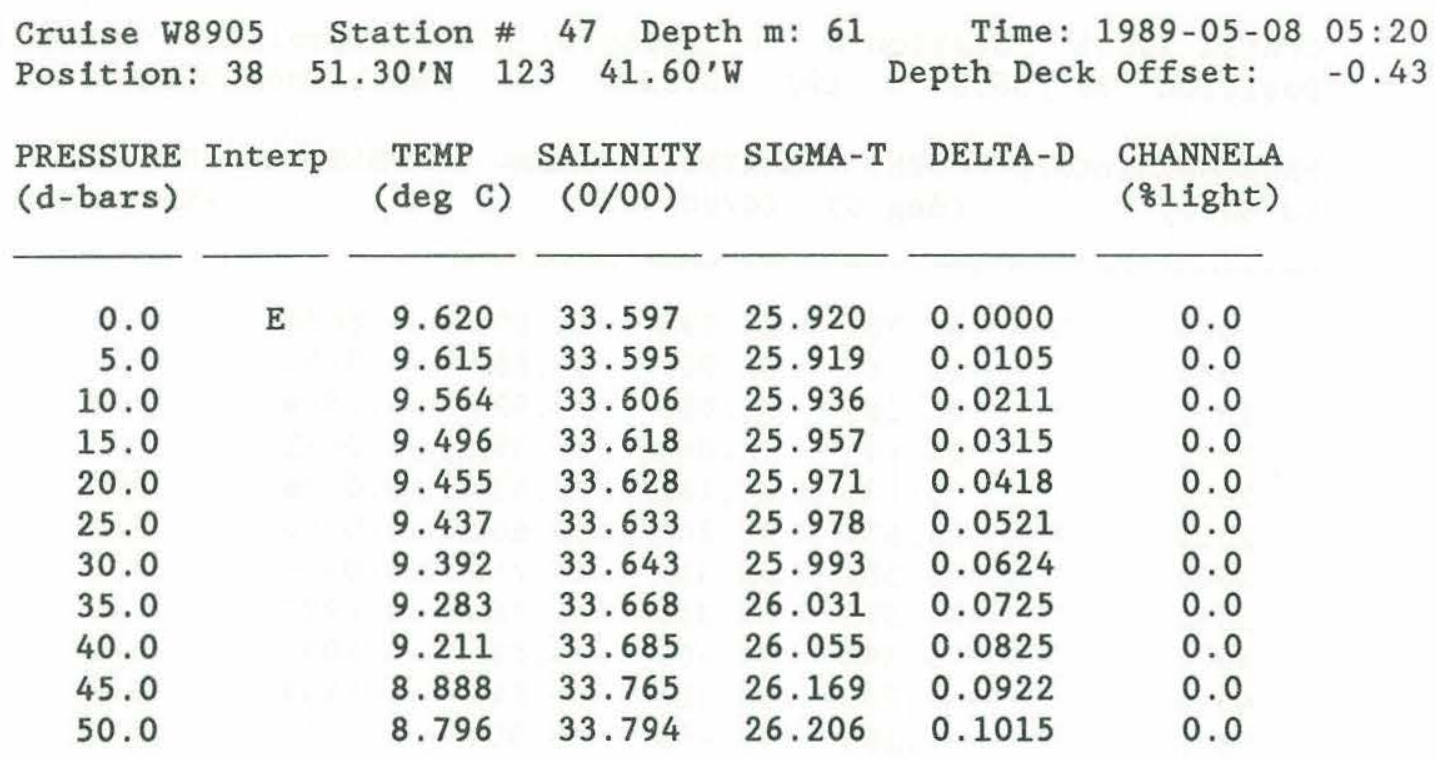
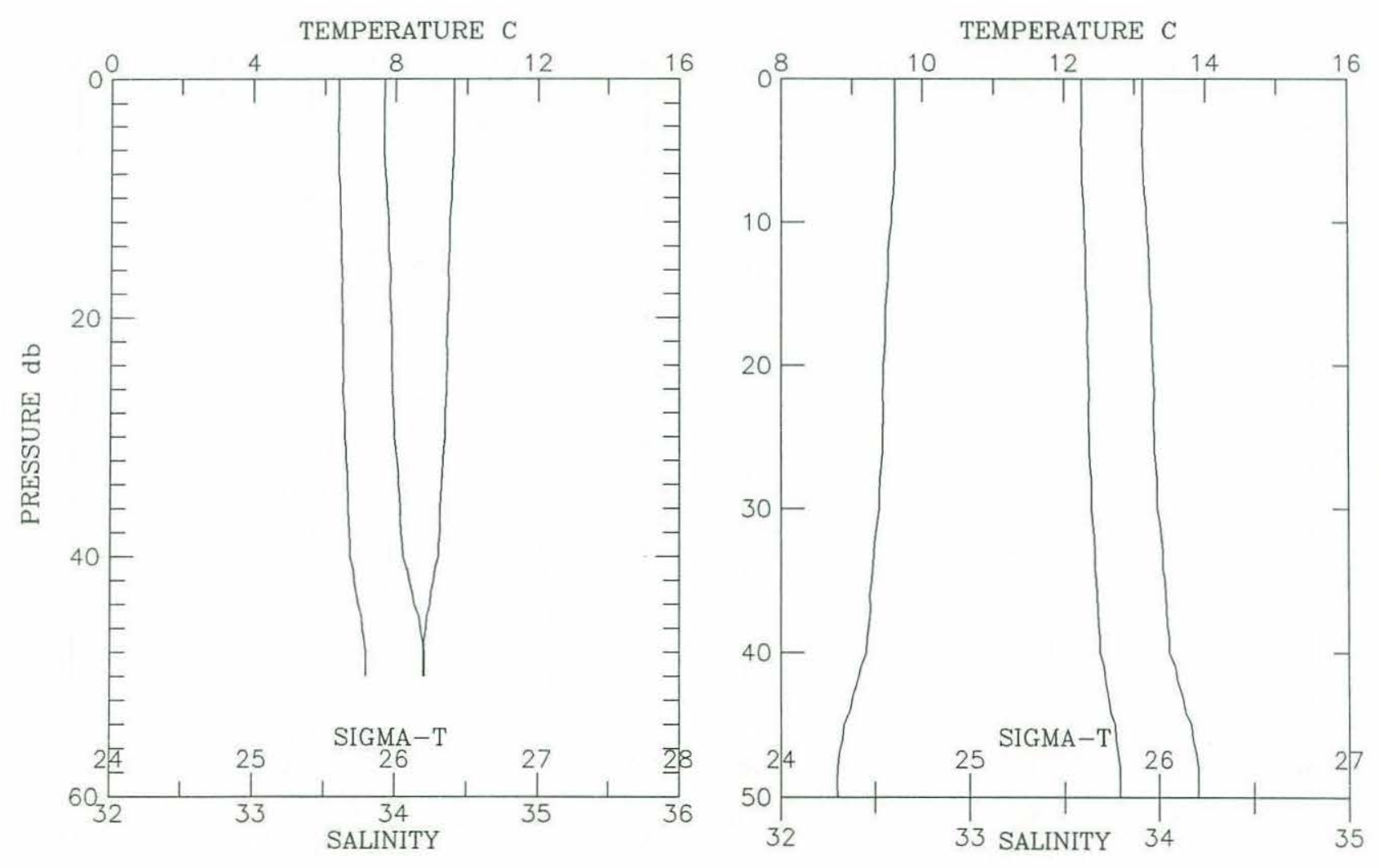
Cruise w8905 Station \# 48 Depth m: 75 Time: 1989-05-08 05:41 Position: $38 \quad 51.00^{\prime} \mathrm{N} 12342.40^{\prime} \mathrm{W}$ Depth Deck Offset: -0.35

\begin{tabular}{|c|c|c|c|c|c|c|}
\hline $\begin{array}{l}\text { PRESSURE } \\
\text { (d-bars) }\end{array}$ & Interp & $\begin{array}{l}\text { TEMP } \\
(\operatorname{deg} C)\end{array}$ & $\begin{array}{c}\text { SALINITY } \\
(0 / 00)\end{array}$ & SIGMA-T & DELTA-D & $\begin{array}{l}\text { CHANNELA } \\
\text { ( } ₹ 1 \text { ight) }\end{array}$ \\
\hline 0.0 & E & 9.757 & 33.552 & 25.862 & 0.0000 & 0.0 \\
\hline 5.0 & & 9.741 & 33.551 & 25.864 & 0.0108 & 0.0 \\
\hline 10.0 & & 9.671 & 33.558 & 25.881 & 0.0216 & 0.0 \\
\hline 15.0 & & 9.565 & 33.583 & 25.918 & 0.0323 & 0.0 \\
\hline 20.0 & & 9.516 & 33.595 & 25.936 & 0.0428 & 0.0 \\
\hline 25.0 & & 9.438 & 33.611 & 25.961 & 0.0532 & 0.0 \\
\hline 30.0 & & 8.948 & 33.704 & 26.112 & 0.0633 & 0.0 \\
\hline 35.0 & & 8.792 & 33.765 & 26.184 & 0.0727 & 0.0 \\
\hline 40.0 & & 8.746 & 33.807 & 26.224 & 0.0819 & 0.0 \\
\hline 45.0 & & 8.726 & 33.819 & 26.237 & 0.0910 & 0.0 \\
\hline 50.0 & & 8.720 & 33.821 & 26.239 & 0.1001 & 0.0 \\
\hline 55.0 & & 8.711 & 33.824 & 26.243 & 0.1092 & 0.0 \\
\hline 60.0 & & 8.704 & 33.830 & 26.249 & 0.1182 & 0.0 \\
\hline 65.0 & & 8.694 & 33.838 & 26.257 & 0.1273 & 0.0 \\
\hline 70.0 & & 8.674 & 33.849 & 26.269 & 0.1362 & 0.0 \\
\hline
\end{tabular}
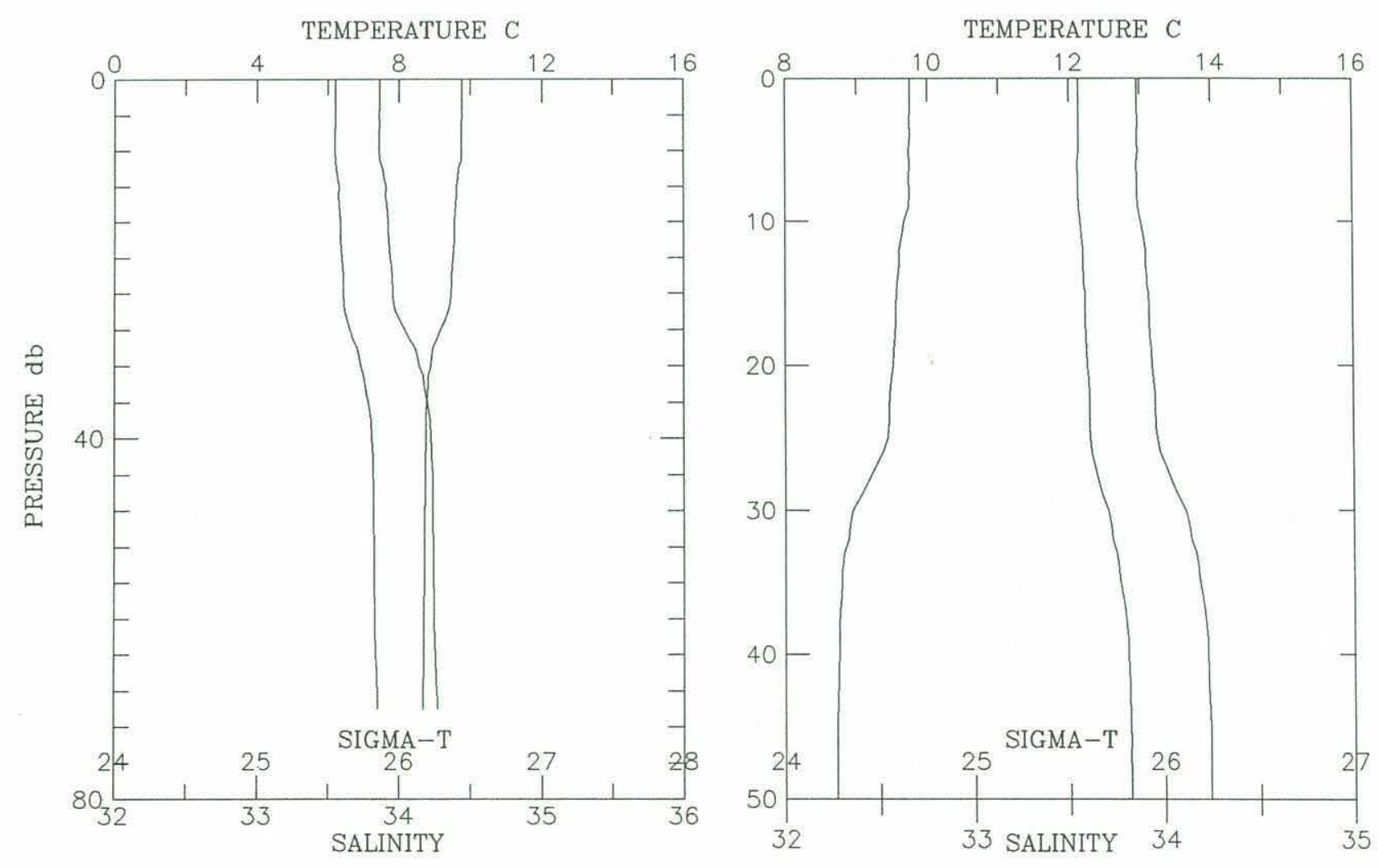


\begin{tabular}{|c|c|c|c|c|c|c|}
\hline $\begin{array}{l}\text { PRESSURE } \\
\text { (d-bars) }\end{array}$ & Interp & $\begin{array}{l}\text { TEMP } \\
(\operatorname{deg} C)\end{array}$ & $\begin{array}{c}\text { SALINITY } \\
(0 / 00)\end{array}$ & SIGMA-T & DELTA-D & $\begin{array}{l}\text { CHANNELA } \\
\text { ( }(\text { IIght) }\end{array}$ \\
\hline 0.0 & E & 9.941 & 33.473 & 25.770 & 0.0000 & 0.0 \\
\hline 5.0 & & 9.944 & 33.469 & 25.766 & 0.0113 & 0.0 \\
\hline 10.0 & & 9.948 & 33.470 & 25.766 & 0.0225 & 0.0 \\
\hline 15.0 & & 9.921 & 33.473 & 25.773 & 0.0338 & 0.0 \\
\hline 20.0 & & 9.911 & 33.476 & 25.777 & 0.0450 & 0.0 \\
\hline 25.0 & & 9.831 & 33.475 & 25.790 & 0.0563 & 0.0 \\
\hline 30.0 & & 9.537 & 33.491 & 25.851 & 0.0673 & 0.0 \\
\hline 35.0 & & 9.303 & 33.538 & 25.926 & 0.0781 & 0.0 \\
\hline 40.0 & & 9.065 & 33.629 & 26.035 & 0.0884 & 0.0 \\
\hline 45.0 & & 8.977 & 33.701 & 26.105 & 0.0983 & 0.0 \\
\hline 50.0 & & 8.913 & 33.731 & 26.139 & 0.1079 & 0.0 \\
\hline 55.0 & & 8.718 & 33.788 & 26.214 & 0.1173 & 0.0 \\
\hline 60.0 & & 8.692 & 33.816 & 26.240 & 0.1264 & 0.0 \\
\hline 65.0 & & 8.658 & 33.830 & 26.256 & 0.1354 & 0.0 \\
\hline 70.0 & & 8.620 & 33.854 & 26.281 & 0.1444 & 0.0 \\
\hline 75.0 & & 8.582 & 33.865 & 26.295 & 0.1532 & 0.0 \\
\hline 80.0 & & 8.575 & 33.876 & 26.305 & 0.1620 & 0.0 \\
\hline 85.0 & & 8.571 & 33.878 & 26.307 & 0.1708 & 0.0 \\
\hline 90.0 & & 8.573 & 33.878 & 26.307 & 0.1796 & 0.0 \\
\hline
\end{tabular}
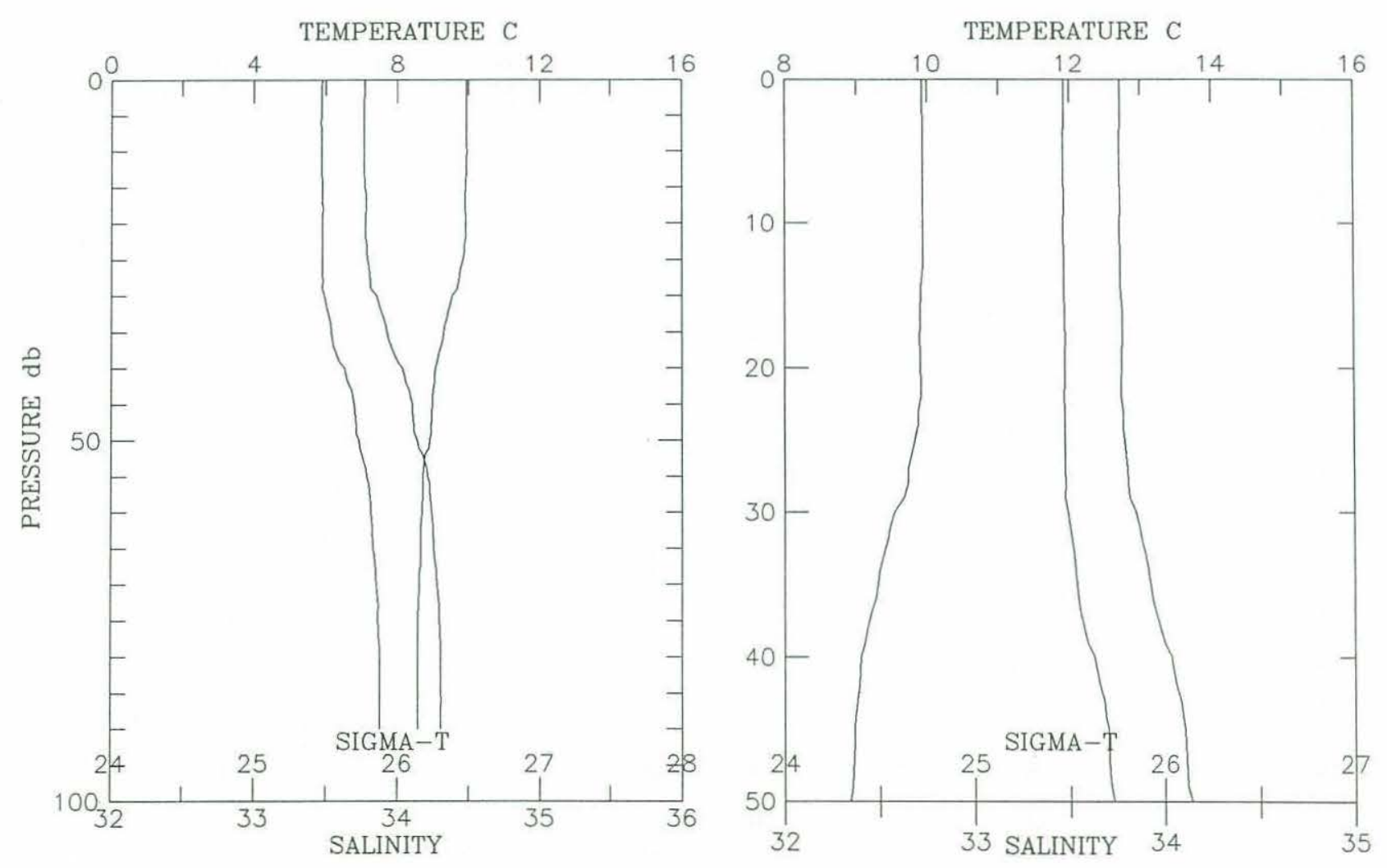
Cruise w8905 Station \# 50 Depth m: 127 Time: 1989-05-08 06:42 Position: $3848.30^{\prime} \mathrm{N} 12345.50^{\prime} \mathrm{W}$ Depth Deck Offset: -0.35

\begin{tabular}{|c|c|c|c|c|c|c|}
\hline $\begin{array}{l}\text { PRESSURE } \\
\text { (d-bars) }\end{array}$ & Interp & $\begin{array}{c}\text { TEMP } \\
(\operatorname{deg} C)\end{array}$ & $\begin{array}{c}\text { SALINITY } \\
(0 / 00)\end{array}$ & SIGMA-T & DELTA-D & $\begin{array}{l}\text { CHANNELA } \\
\text { ( } \% 1 \text { ight) }\end{array}$ \\
\hline 0.0 & $E$ & 10.622 & 33.153 & 25.404 & 0.0000 & 0.0 \\
\hline 10.0 & & 10.271 & 33.128 & 25.445 & 0.0260 & 0.0 \\
\hline 20.0 & & 9.714 & 33.265 & 25.645 & 0.0509 & 0.0 \\
\hline 30.0 & & 9.471 & 33.348 & 25.750 & 0.0740 & 0.0 \\
\hline 40.0 & & 9.198 & 33.461 & 25.882 & 0.0964 & 0.0 \\
\hline 50.0 & & 9.000 & 33.570 & 25.999 & 0.1172 & 0.0 \\
\hline 60.0 & & 8.873 & 33.676 & 26.102 & 0.1373 & 0.0 \\
\hline 70.0 & & 8.714 & 33.795 & 26.220 & 0.1560 & 0.0 \\
\hline 80.0 & & 8.633 & 33.853 & 26.278 & 0.1741 & 0.0 \\
\hline 90.0 & & 8.485 & 33.903 & 26.339 & 0.1917 & 0.0 \\
\hline 100.0 & & 8.354 & 33.921 & 26.373 & 0.2088 & 0.0 \\
\hline 110.0 & & 8.326 & 33.923 & 26.379 & 0.2257 & 0.0 \\
\hline 116.0 & & 8.267 & 33.928 & 26.392 & 0.2358 & 0.0 \\
\hline
\end{tabular}
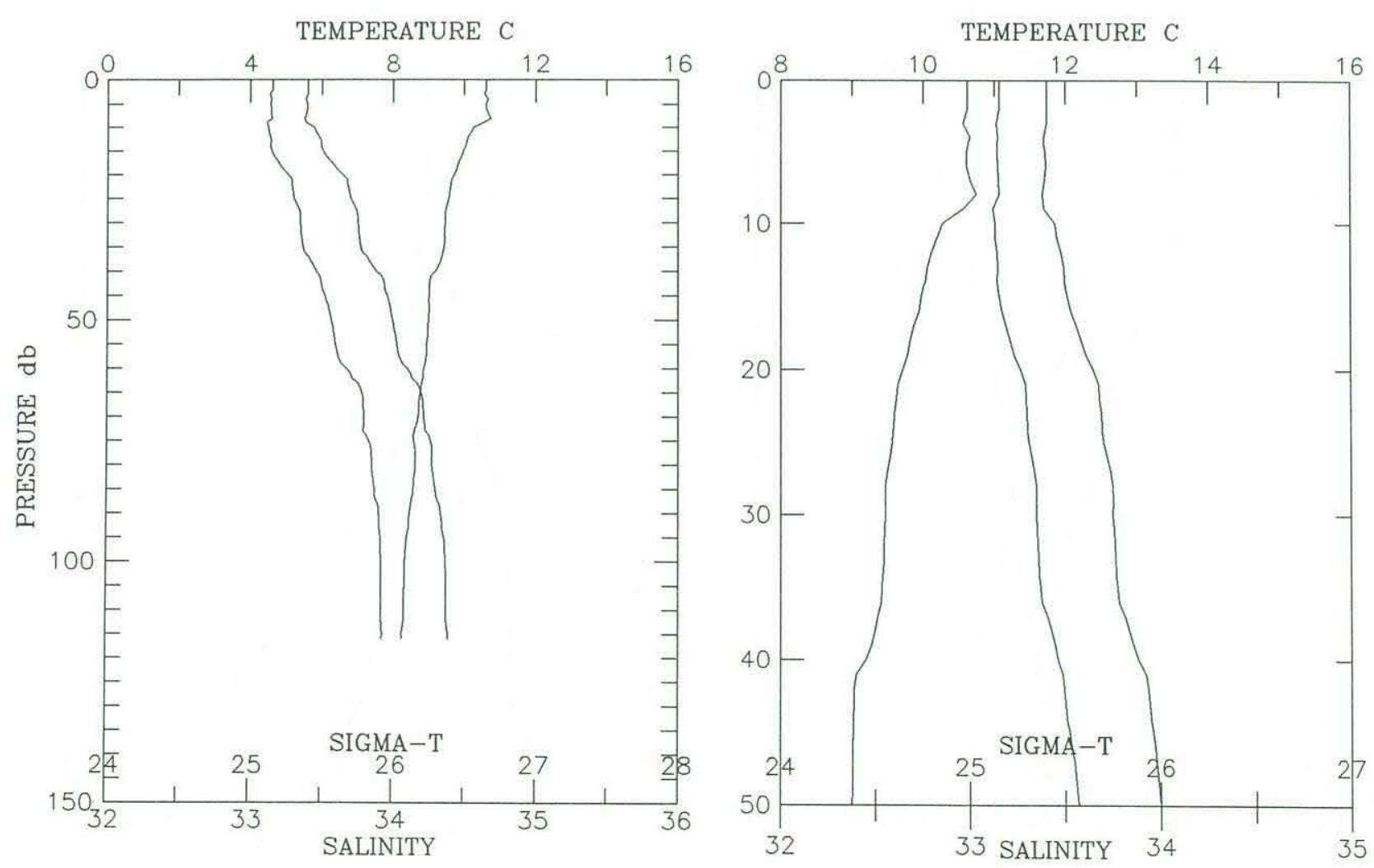
Cruise W8905 Station \# 51 Depth m: 136 Time: 1989-05-08 07:20 Position: $3846.90^{\prime} \mathrm{N} 12347.10^{\prime} \mathrm{W}$ Depth Deck Offset: -0.35

\begin{tabular}{|c|c|c|c|c|c|c|}
\hline $\begin{array}{l}\text { PRESSURE } \\
\text { (d-bars) }\end{array}$ & Interp & $\begin{array}{l}\text { TEMP } \\
(\operatorname{deg} C)\end{array}$ & $\begin{array}{c}\text { SALINITY } \\
(0 / 00)\end{array}$ & SIGMA-T & DELTA-D & $\begin{array}{l}\text { CHANNELA } \\
\text { ( } 81 \text { ight) }\end{array}$ \\
\hline 0.0 & E & 11.382 & 33.054 & 25.192 & 0.0000 & 0.0 \\
\hline 10.0 & & 11.327 & 33.053 & 25.201 & 0.0280 & 0.0 \\
\hline 20.0 & & 10.823 & 33.055 & 25.293 & 0.0557 & 0.0 \\
\hline 30.0 & & 10.138 & 33.008 & 25.374 & 0.0825 & 0.0 \\
\hline 40.0 & & 9.955 & 33.099 & 25.476 & 0.1082 & 0.0 \\
\hline 50.0 & & 9.566 & 33.294 & 25.692 & 0.1326 & 0.0 \\
\hline 60.0 & & 9.269 & 33.465 & 25.874 & 0.1550 & 0.0 \\
\hline 70.0 & & 9.100 & 33.569 & 25.982 & 0.1762 & 0.0 \\
\hline 80.0 & & 8.897 & 33.610 & 26.047 & 0.1964 & 0.0 \\
\hline 90.0 & & 8.656 & 33.737 & 26.184 & 0.2159 & 0.0 \\
\hline 100.0 & & 8.567 & 33.838 & 26.276 & 0.2342 & 0.0 \\
\hline 110.0 & & 8.308 & 33.885 & 26.352 & 0.2518 & 0.0 \\
\hline 120.0 & & 8.033 & 33.938 & 26.435 & 0.2685 & 0.0 \\
\hline 130.0 & & 7.886 & 33.958 & 26.472 & 0.2848 & 0.0 \\
\hline
\end{tabular}
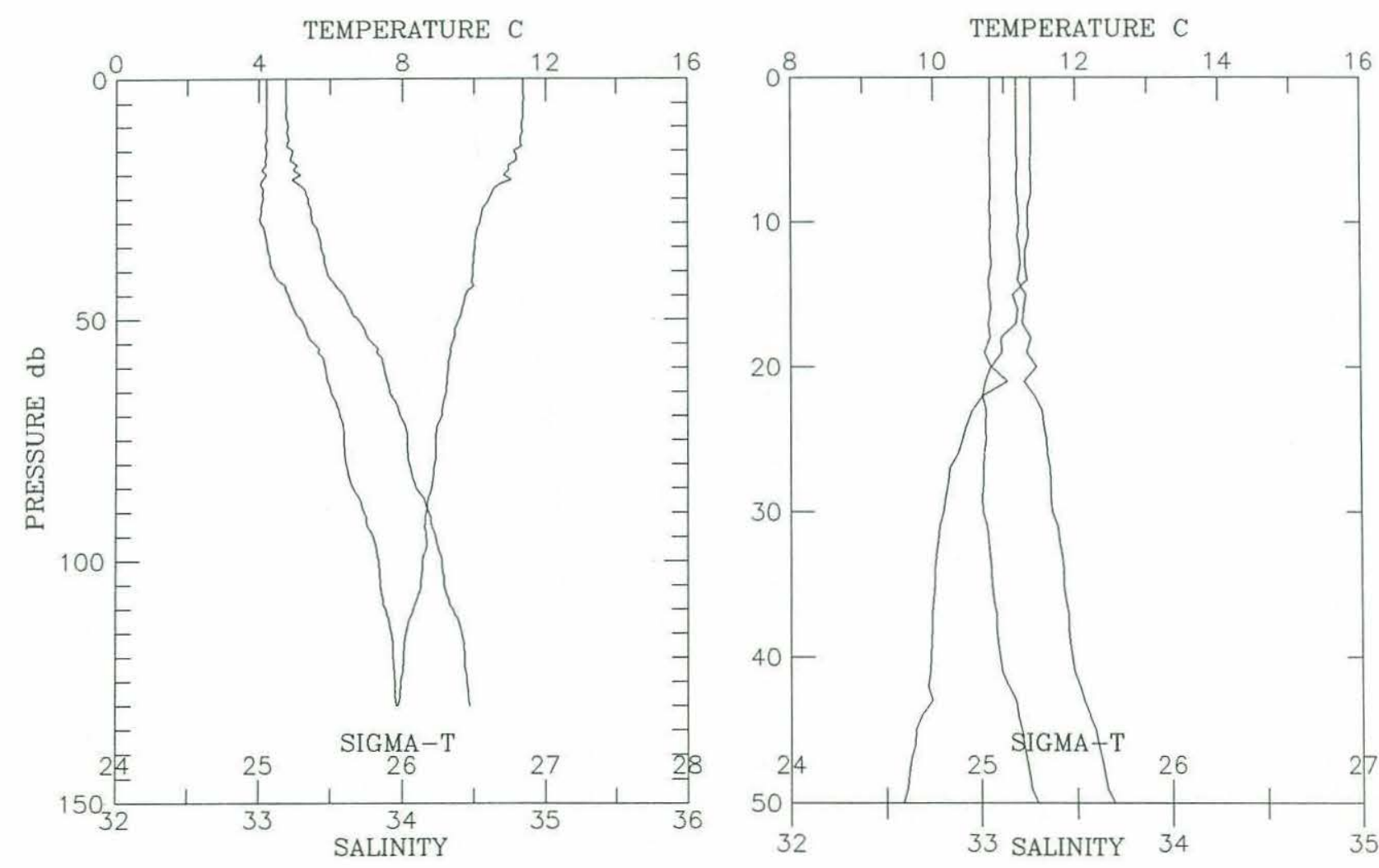
Cruise w8905 Station \# 52 Depth m: 190 Time: 1989-05-08 07:48 Position: $38 \quad 45.90^{\prime} \mathrm{N} 12348.70^{\prime} \mathrm{W} \quad$ Depth Deck Offset: -0.35

\begin{tabular}{|c|c|c|c|c|c|c|}
\hline $\begin{array}{l}\text { PRESSURE } \\
\text { (d-bars) }\end{array}$ & Interp & $\begin{array}{c}\text { TEMP } \\
(\operatorname{deg} C)\end{array}$ & $\begin{array}{c}\text { SALINITY } \\
(0 / 00)\end{array}$ & SIGMA-T & DELTA-D & $\begin{array}{l}\text { CHANNELA } \\
\text { ( }(z 1 i g h t)\end{array}$ \\
\hline 0.0 & $\mathrm{E}$ & 12.675 & 32.937 & 24.859 & 0.0000 & 0.0 \\
\hline 10.0 & & 12.678 & 32.936 & 24.858 & 0.0312 & 0.0 \\
\hline 20.0 & & 11.646 & 32.993 & 25.097 & 0.0621 & 0.0 \\
\hline 30.0 & & 11.051 & 33.184 & 25.353 & 0.0898 & 0.0 \\
\hline 40.0 & & 10.538 & 33.266 & 25.507 & 0.1155 & 0.0 \\
\hline 50.0 & & 10.319 & 33.365 & 25.622 & 0.1400 & 0.0 \\
\hline 60.0 & & 9.786 & 33.437 & 25.768 & 0.1631 & 0.0 \\
\hline 70.0 & & 9.414 & 33.554 & 25.920 & 0.1852 & 0.0 \\
\hline 80.0 & & 9.208 & 33.645 & 26.025 & 0.2060 & 0.0 \\
\hline 90.0 & & 9.105 & 33.718 & 26.098 & 0.2259 & 0.0 \\
\hline 100.0 & & 8.684 & 33.814 & 26.240 & 0.2447 & 0.0 \\
\hline 110.0 & & 8.562 & 33.878 & 26.309 & 0.2627 & 0.0 \\
\hline 120.0 & & 8.472 & 33.889 & 26.330 & 0.2801 & 0.0 \\
\hline 130.0 & & 8.177 & 33.920 & 26.399 & 0.2974 & 0.0 \\
\hline 140.0 & & 7.951 & 33.945 & 26.453 & 0.3138 & 0.0 \\
\hline 150.0 & & 7.853 & 33.956 & 26.476 & 0.3300 & 0.0 \\
\hline 160.0 & & 7.769 & 33.970 & 26.499 & 0.3459 & 0.0 \\
\hline 170.0 & & 7.541 & 33.988 & 26.546 & 0.3615 & 0.0 \\
\hline 180.0 & & 7.509 & 33.993 & 26.554 & 0.3769 & 0.0 \\
\hline 185.0 & & 7.483 & 33.996 & 26.561 & 0.3846 & 0.0 \\
\hline
\end{tabular}

TEMPERATURE C
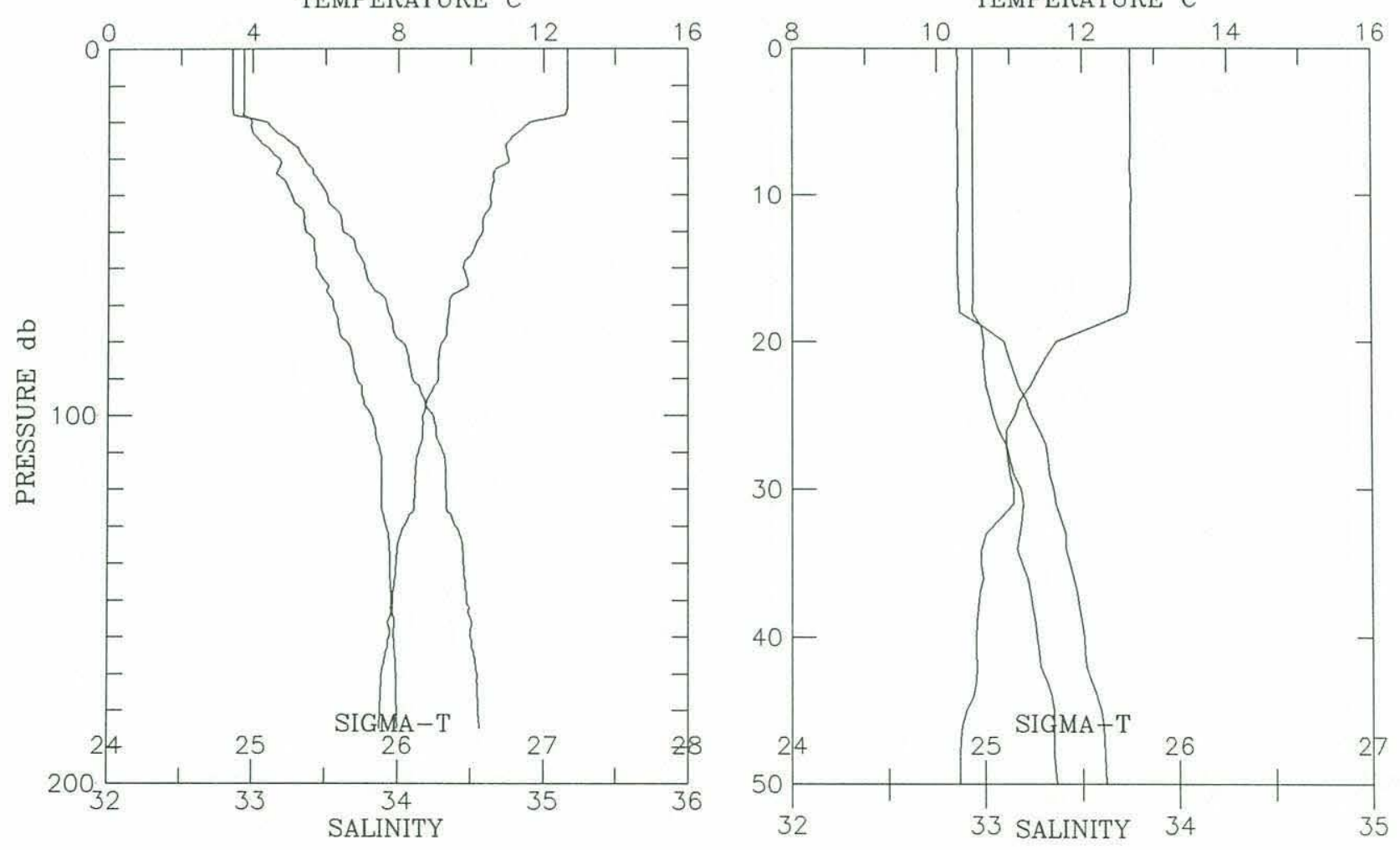


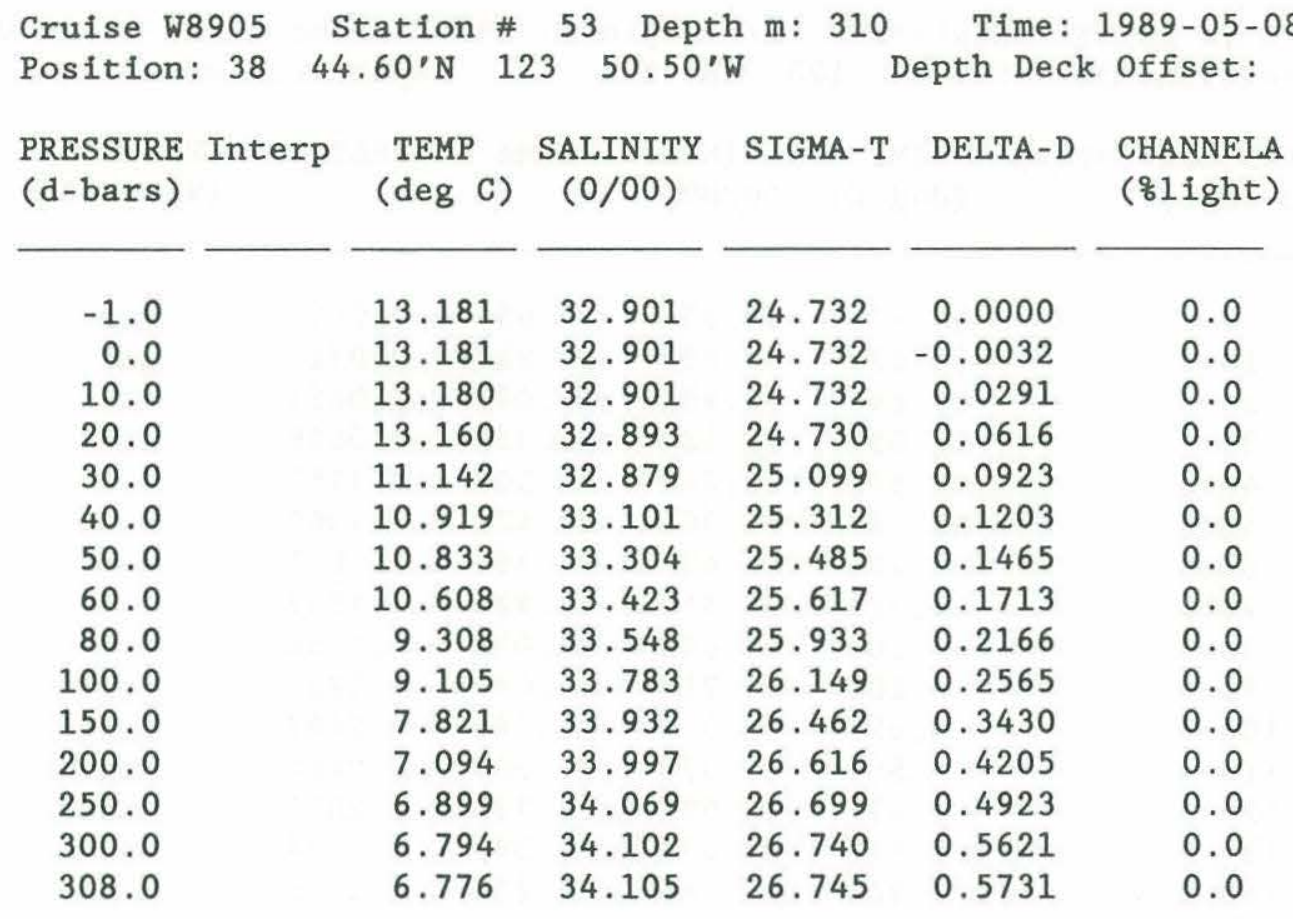

TEMPERATURE C

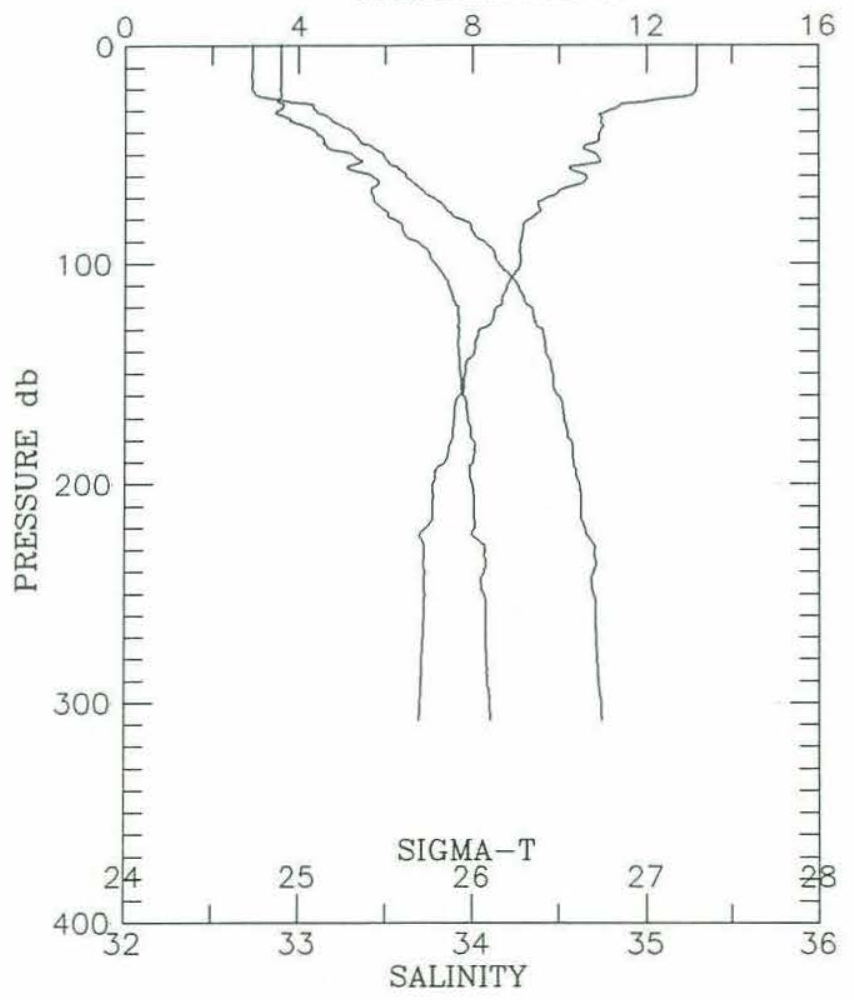

TEMPERATURE C

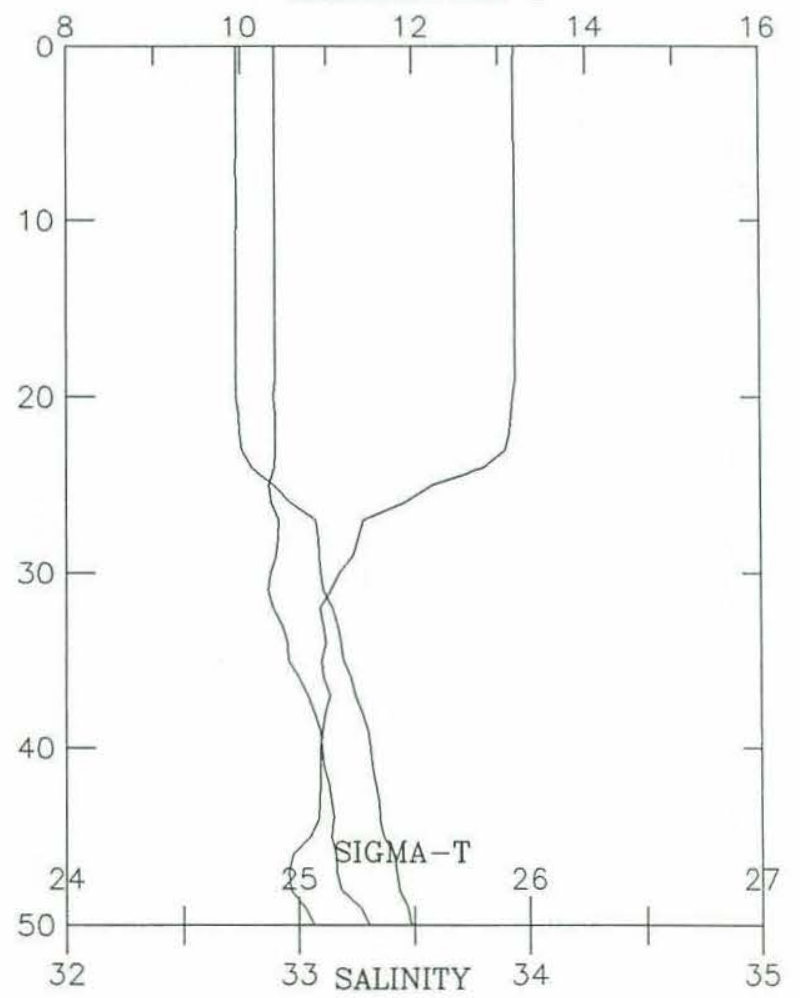


Cruise w8905 Station \# 54 Depth m: 957 Time: 1989-05-08 08:53

Position: $38 \quad 43.20^{\prime} \mathrm{N} 12352.60^{\prime} \mathrm{W} \quad$ Depth Deck Offset: -0.28

\begin{tabular}{|c|c|c|c|c|c|c|}
\hline $\begin{array}{l}\text { PRESSURE } \\
\text { (d-bars) }\end{array}$ & Interp & $\begin{array}{l}\text { TEMP } \\
(\operatorname{deg} C)\end{array}$ & $\begin{array}{c}\text { SALINITY } \\
(0 / 00)\end{array}$ & SIGMA-T & DELTA-D & $\begin{array}{l}\text { CHANNELA } \\
\text { ( } \delta 1 \text { ight) }\end{array}$ \\
\hline 0.0 & E & 13.858 & 32.864 & 24.567 & 0.0000 & 0.0 \\
\hline 10.0 & & 13.857 & 32.865 & 24.568 & 0.0340 & 0.0 \\
\hline 20.0 & & 13.843 & 32.869 & 24.574 & 0.0679 & 0.0 \\
\hline 30.0 & & 11.896 & 32.929 & 25.001 & 0.1007 & 0.0 \\
\hline 40.0 & & 10.767 & 32.913 & 25.192 & 0.1294 & 0.0 \\
\hline 50.0 & & 10.734 & 33.101 & 25.344 & 0.1567 & 0.0 \\
\hline 60.0 & & 10.890 & 33.349 & 25.510 & 0.1827 & 0.0 \\
\hline 80.0 & & 9.845 & 33.539 & 25.838 & 0.2299 & 0.0 \\
\hline 100.0 & & 9.090 & 33.717 & 26.100 & 0.2714 & 0.0 \\
\hline 150.0 & & 8.422 & 33.995 & 26.421 & 0.3609 & 0.0 \\
\hline 200.0 & & 7.259 & 33.977 & 26.577 & 0.4399 & 0.0 \\
\hline 250.0 & & 7.283 & 34.101 & 26.672 & 0.5139 & 0.0 \\
\hline 300.0 & & 6.324 & 34.023 & 26.740 & 0.5841 & 0.0 \\
\hline 400.0 & & 6.432 & 34.151 & 26.827 & 0.7174 & 0.0 \\
\hline 500.0 & & 5.725 & 34.174 & 26.935 & 0.8434 & 0.0 \\
\hline 600.0 & & 5.418 & 34.266 & 27.045 & 0.9601 & 0.0 \\
\hline 700.0 & & 4.973 & 34.333 & 27.149 & 1.0678 & 0.0 \\
\hline 800.0 & & 4.576 & 34.384 & 27.235 & 1.1664 & 0.0 \\
\hline 900.0 & & 4.305 & 34.406 & 27.281 & 1.2583 & 0.0 \\
\hline 950.0 & & 4.183 & 34.421 & 27.306 & 1.3034 & 0.0 \\
\hline
\end{tabular}

TEMPERATURE $\mathrm{C}$
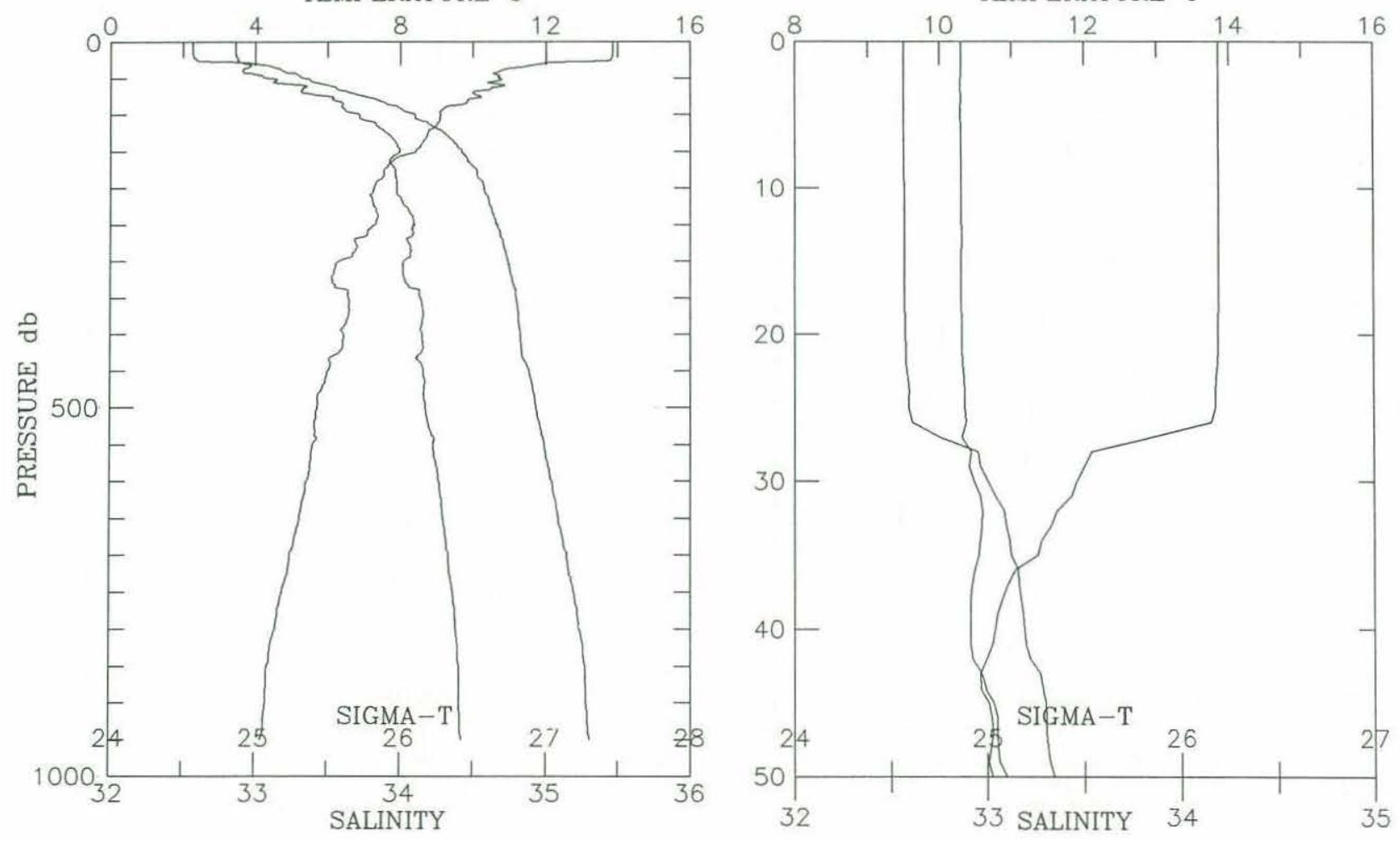
Cruise w8905 Station \# 55 Depth m: 893 Time: 1989-05-08 10:18

Position: $38 \quad 36.40^{\prime} \mathrm{N} 123 \quad 47.10^{\prime} \mathrm{W}$ Depth Deck Offset: -0.28

\begin{tabular}{|c|c|c|c|c|c|c|}
\hline $\begin{array}{l}\text { PRESSURE } \\
\text { (d-bars) }\end{array}$ & Interp & $\begin{array}{c}\text { TEMP } \\
(\operatorname{deg} C)\end{array}$ & $\begin{array}{l}\text { SALINITY } \\
(0 / 00)\end{array}$ & SIGMA-T & DELTA-D & $\begin{array}{l}\text { CHANNELA } \\
\text { ( }(81 \text { ight) }\end{array}$ \\
\hline 0.0 & E & 11.240 & 32.939 & 25.128 & 0.0000 & 0.0 \\
\hline 10.0 & & 11.243 & 32.938 & 25.127 & 0.0286 & 0.0 \\
\hline 20.0 & & 11.202 & 32.941 & 25.137 & 0.0572 & 0.0 \\
\hline 30.0 & & 10.965 & 32.983 & 25.212 & 0.0855 & 0.0 \\
\hline 40.0 & & 10.416 & 33.174 & 25.456 & 0.1120 & 0.0 \\
\hline 50.0 & & 9.976 & 33.256 & 25.595 & 0.1369 & 0.0 \\
\hline 60.0 & & 9.640 & 33.345 & 25.720 & 0.1605 & 0.0 \\
\hline 80.0 & & 9.417 & 33.535 & 25.905 & 0.2047 & 0.0 \\
\hline 100.0 & & 8.841 & 33.703 & 26.128 & 0.2454 & 0.0 \\
\hline 150.0 & & 7.999 & 33.962 & 26.459 & 0.3324 & 0.0 \\
\hline 200.0 & & 7.487 & 34.061 & 26.611 & 0.4106 & 0.0 \\
\hline 250.0 & & 7.009 & 34.062 & 26.679 & 0.4833 & 0.0 \\
\hline 300.0 & & 6.782 & 34.103 & 26.742 & 0.5534 & 0.0 \\
\hline 400.0 & & 6.335 & 34.161 & 26.847 & 0.6861 & 0.0 \\
\hline 500.0 & & 5.863 & 34.223 & 26.956 & 0.8115 & 0.0 \\
\hline 600.0 & & 5.388 & 34.283 & 27.061 & 0.9264 & 0.0 \\
\hline 700.0 & & 4.930 & 34.340 & 27.160 & 1.0321 & 0.0 \\
\hline 800.0 & & 4.630 & 34.377 & 27.223 & 1.1303 & 0.0 \\
\hline 900.0 & & 4.391 & 34.400 & 27.267 & 1.2250 & 0.0 \\
\hline 941.0 & & 4.288 & 34.409 & 27.286 & 1.2626 & 0.0 \\
\hline
\end{tabular}
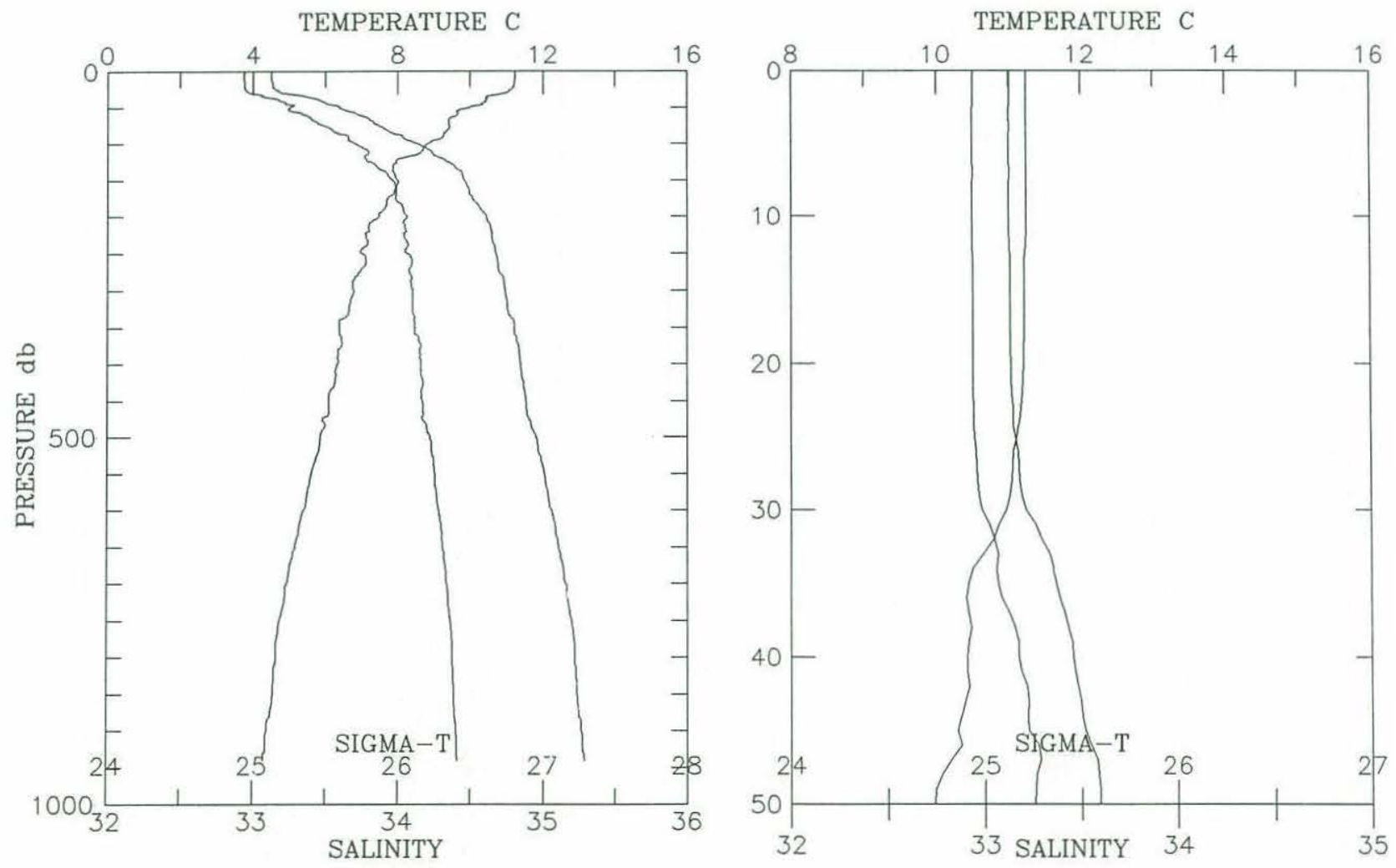
Cruise w8905 Station \# 56 Depth m: 349 Time: 1989-05-08 11:37 Position: $38 \quad 37.80^{\prime} \mathrm{N} 123 \quad 45.30^{\prime} \mathrm{W}$ Depth Deck Offset: -0.30

\begin{tabular}{|c|c|c|c|c|c|c|}
\hline $\begin{array}{l}\text { PRESSURE } \\
\text { (d-bars) }\end{array}$ & Interp & $\begin{array}{l}\text { TEMP } \\
(\operatorname{deg} G)\end{array}$ & $\begin{array}{c}\text { SALINITY } \\
(0 / 00)\end{array}$ & SIGMA-T & DELTA-D & $\begin{array}{l}\text { CHANNELA } \\
\text { ( }(\text { light) }\end{array}$ \\
\hline 0.0 & E & 10.780 & 33.006 & 25.262 & 0.0000 & 0.0 \\
\hline 10.0 & & 10.770 & 33.006 & 25.264 & 0.0273 & 0.0 \\
\hline 20.0 & & 10.641 & 33.015 & 25.293 & 0.0546 & 0.0 \\
\hline 30.0 & & 10.198 & 33.063 & 25.407 & 0.0813 & 0.0 \\
\hline 40.0 & & 9.821 & 33.219 & 25.592 & 0.1063 & 0.0 \\
\hline 50.0 & & 9.423 & 33.378 & 25.781 & 0.1296 & 0.0 \\
\hline 60.0 & & 9.245 & 33.492 & 25.899 & 0.1518 & 0.0 \\
\hline 80.0 & & 9.068 & 33.630 & 26.035 & 0.1932 & 0.0 \\
\hline 100.0 & & 8.774 & 33.751 & 26.176 & 0.2321 & 0.0 \\
\hline 150.0 & & 7.895 & 33.961 & 26.473 & 0.3183 & 0.0 \\
\hline 200.0 & & 7.365 & 34.050 & 26.620 & 0.3959 & 0.0 \\
\hline 250.0 & & 7.174 & 34.100 & 26.686 & 0.4683 & 0.0 \\
\hline 300.0 & & 6.758 & 34.090 & 26.735 & 0.5386 & 0.0 \\
\hline 341.0 & & 6.472 & 34.107 & 26.787 & 0.5940 & 0.0 \\
\hline
\end{tabular}
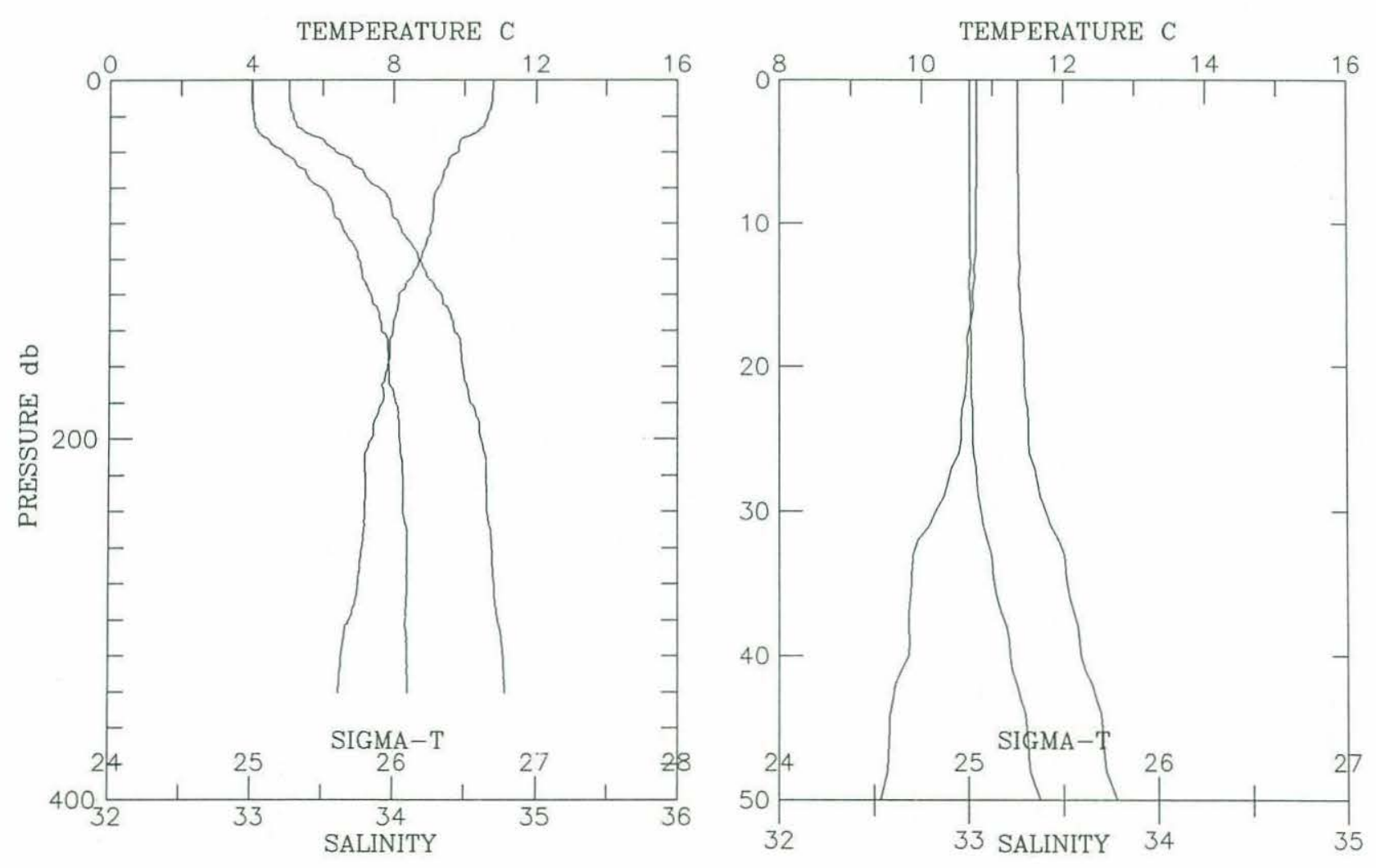


\begin{tabular}{|c|c|c|c|c|c|c|}
\hline $\begin{array}{l}\text { PRESSURE } \\
\text { (d-bars) }\end{array}$ & Interp & $\begin{array}{l}\text { TEMP } \\
(\operatorname{deg} C)\end{array}$ & $\begin{array}{c}\text { SALINITY } \\
(0 / 00)\end{array}$ & SIGMA-T & DELTA-D & $\begin{array}{l}\text { CHANNELA } \\
\text { ( }(\delta 1 \text { ight) }\end{array}$ \\
\hline 0.0 & E & 10.095 & 33.206 & 25.536 & 0.0000 & 0.0 \\
\hline 10.0 & & 10.091 & 33.207 & 25.537 & 0.0247 & 0.0 \\
\hline 20.0 & & 10.069 & 33.208 & 25.542 & 0.0495 & 0.0 \\
\hline 30.0 & & 9.553 & 33.308 & 25.705 & 0.0734 & 0.0 \\
\hline 40.0 & & 9.166 & 33.482 & 25.904 & 0.0956 & 0.0 \\
\hline 50.0 & & 8.972 & 33.573 & 26.006 & 0.1165 & 0.0 \\
\hline 60.0 & & 8.804 & 33.698 & 26.130 & 0.1360 & 0.0 \\
\hline 70.0 & & 8.702 & 33.760 & 26.194 & 0.1551 & 0.0 \\
\hline 80.0 & & 8.276 & 33.807 & 26.296 & 0.1733 & 0.0 \\
\hline 90.0 & & 8.229 & 33.844 & 26.332 & 0.1908 & 0.0 \\
\hline 100.0 & & 8.431 & 33.875 & 26.326 & 0.2080 & 0.0 \\
\hline 110.0 & & 8.422 & 33.892 & 26.340 & 0.2254 & 0.0 \\
\hline 120.0 & & 8.336 & 33.908 & 26.366 & 0.2426 & 0.0 \\
\hline 130.0 & & 8.325 & 33.928 & 26.383 & 0.2596 & 0.0 \\
\hline 140.0 & & 8.333 & 33.935 & 26.388 & 0.2765 & 0.0 \\
\hline 150.0 & & 8.274 & 33.945 & 26.404 & 0.2933 & 0.0 \\
\hline 160.0 & & 8.153 & 33.960 & 26.434 & 0.3099 & 0.0 \\
\hline 170.0 & & 8.045 & 33.970 & 26.458 & 0.3263 & 0.0 \\
\hline 174.0 & & 7.901 & 33.992 & 26.497 & 0.3327 & 0.0 \\
\hline
\end{tabular}
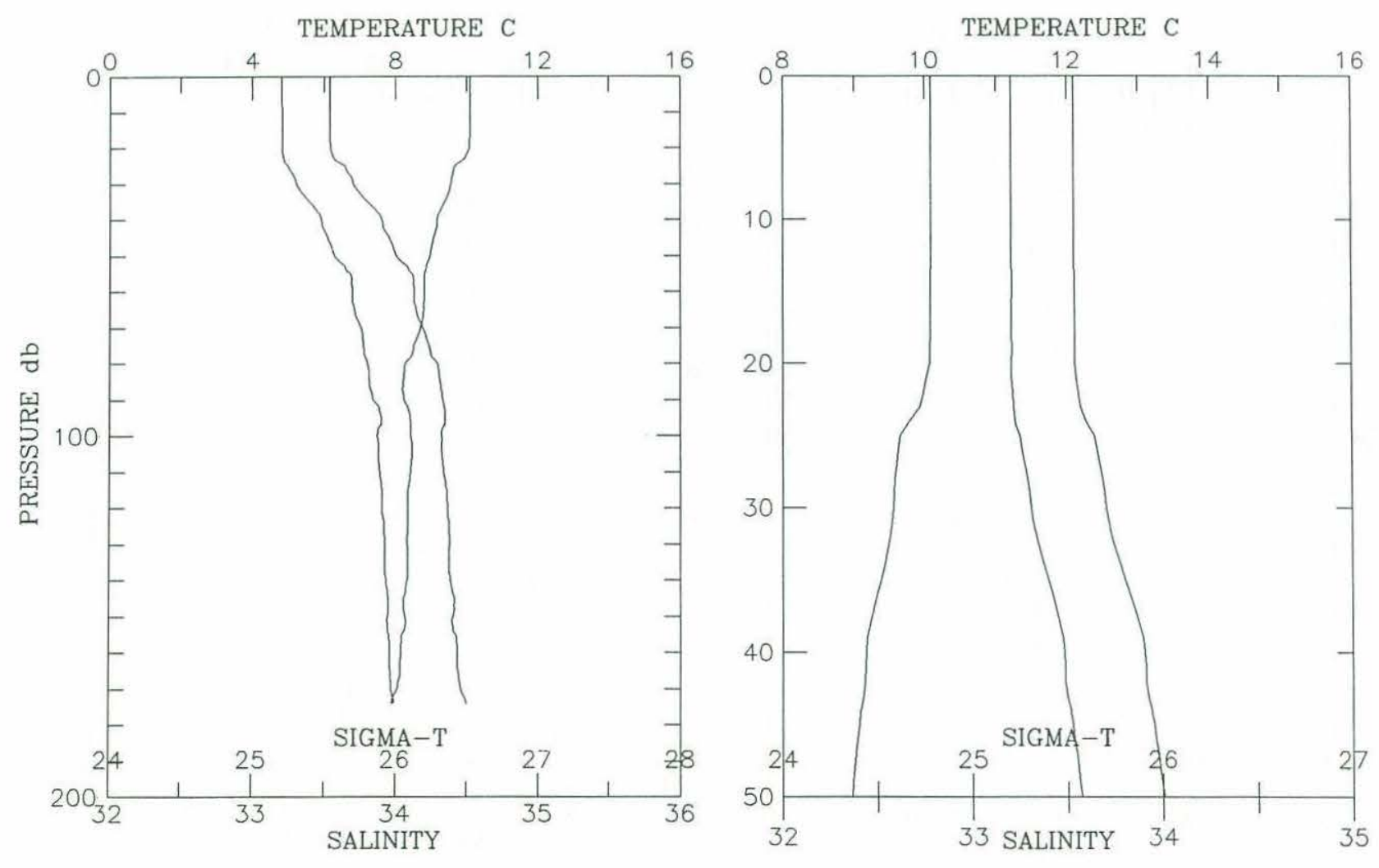


\begin{tabular}{|c|c|c|c|c|c|c|c|}
\hline \multicolumn{2}{|c|}{$\begin{array}{l}\text { Cruise w8905 } \\
\text { Position: } 38\end{array}$} & $\begin{array}{l}\text { ation \# } \\
50^{\prime} \mathrm{N} 1\end{array}$ & \multicolumn{2}{|c|}{58 Depth $\mathrm{m}: 133$} & Time: & $1989-05-08$ & \multirow[t]{2}{*}{$\begin{array}{l}13: 02 \\
-0.30\end{array}$} \\
\hline $\begin{array}{l}\text { PRESSURE } \\
\text { (d-bars) }\end{array}$ & Interp & $\begin{array}{c}\text { TEMP } \\
(\operatorname{deg} C)\end{array}$ & $\begin{array}{l}\text { SALINITY } \\
(0 / 00)\end{array}$ & SIGMA-T & DELTA-D & $\begin{array}{l}\text { CHANNELA } \\
\text { ( }(\text { light) }\end{array}$ & \\
\hline 0.0 & E & 9.665 & 33.350 & 25.720 & 0.0000 & 0.0 & \\
\hline 10.0 & & 9.662 & 33.349 & 25.719 & 0.0230 & 0.0 & \\
\hline 20.0 & & 9.641 & 33.352 & 25.725 & 0.0460 & 0.0 & \\
\hline 30.0 & & 9.559 & 33.366 & 25.750 & 0.0688 & 0.0 & \\
\hline 40.0 & & 9.038 & 33.535 & 25.966 & 0.0903 & 0.0 & \\
\hline 50.0 & & 8.914 & 33.599 & 26.035 & 0.1108 & 0.0 & \\
\hline 60.0 & & 8.839 & 33.655 & 26.091 & 0.1306 & 0.0 & \\
\hline 70.0 & & 8.581 & 33.777 & 26.226 & 0.1494 & 0.0 & \\
\hline 80.0 & & 8.561 & 33.800 & 26.247 & 0.1676 & 0.0 & \\
\hline 90.0 & & 8.542 & 33.883 & 26.315 & 0.1855 & 0.0 & \\
\hline 100.0 & & 8.556 & 33.910 & 26.334 & 0.2029 & 0.0 & \\
\hline 110.0 & & 8.527 & 33.914 & 26.341 & 0.2202 & 0.0 & \\
\hline 120.0 & & 8.296 & 33.935 & 26.393 & 0.2373 & 0.0 & \\
\hline 124.0 & & 8.247 & 33.940 & 26.405 & 0.2440 & 0.0 & \\
\hline
\end{tabular}
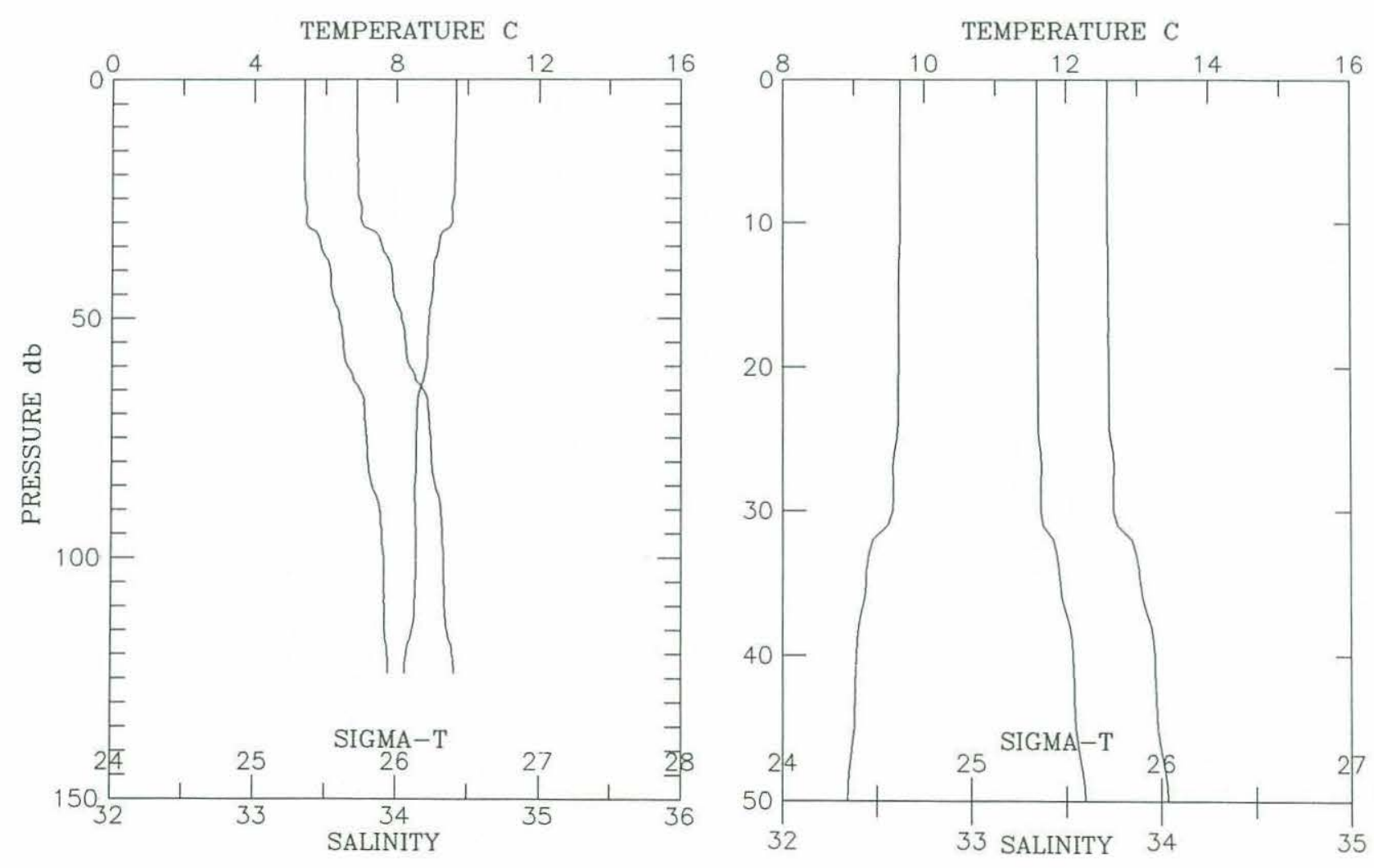


\begin{tabular}{|c|c|c|c|c|c|c|}
\hline $\begin{array}{l}\text { PRESSURE } \\
\text { (d-bars) }\end{array}$ & Interp & $\begin{array}{l}\text { TEMP } \\
(\operatorname{deg} C)\end{array}$ & $\begin{array}{c}\text { SALINITY } \\
(0 / 00)\end{array}$ & SIGMA-T & DELTA-D & $\begin{array}{l}\text { CHANNELA } \\
\text { ( }(i 1 \text { ght) }\end{array}$ \\
\hline 0.0 & E & 9.624 & 33.470 & 25.820 & 0.0000 & 0.0 \\
\hline 10.0 & & 9.618 & 33.470 & 25.821 & 0.0221 & 0.0 \\
\hline 20.0 & & 9.397 & 33.565 & 25.932 & 0.0436 & 0.0 \\
\hline 30.0 & & 9.358 & 33.566 & 25.939 & 0.0646 & 0.0 \\
\hline 40.0 & & 8.983 & 33.573 & 26.004 & 0.0853 & 0.0 \\
\hline 50.0 & & 8.762 & 33.731 & 26.162 & 0.1048 & 0.0 \\
\hline 60.0 & & 8.734 & 33.826 & 26.240 & 0.1233 & 0.0 \\
\hline 70.0 & & 8.702 & 33.841 & 26.257 & 0.1413 & 0.0 \\
\hline 80.0 & & 8.619 & 33.865 & 26.289 & 0.1592 & 0.0 \\
\hline 90.0 & & 8.579 & 33.901 & 26.323 & 0.1767 & 0.0 \\
\hline 100.0 & & 8.564 & 33.903 & 26.327 & 0.1942 & 0.0 \\
\hline 108.0 & & 8.541 & 33.909 & 26.335 & 0.2081 & 0.0 \\
\hline
\end{tabular}
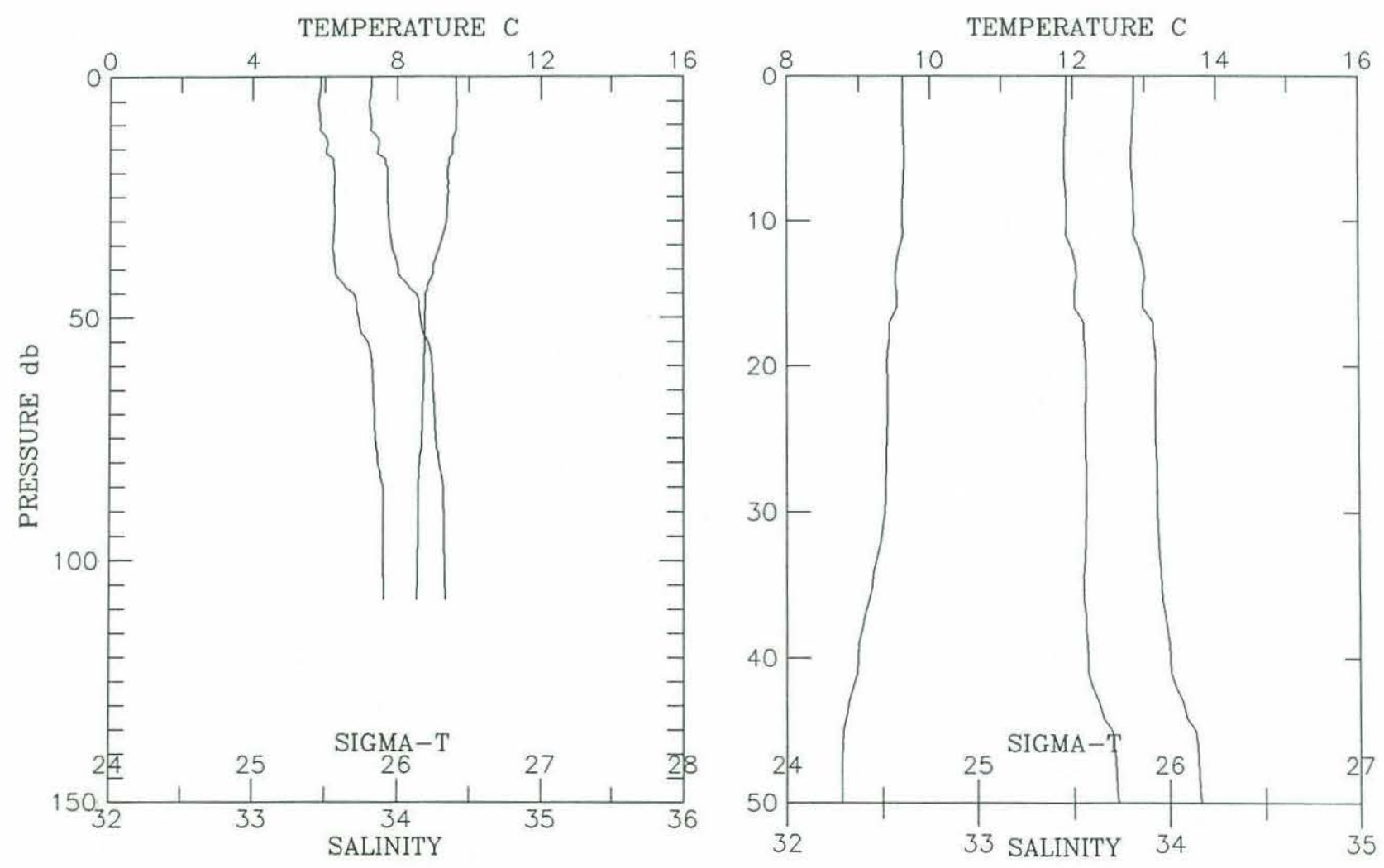
Cruise w8905 Station \# 60 Depth m: 92 Time: 1989-05-08 14:04 Position: $38 \quad 44.40^{\prime} \mathrm{N} 123 \quad 36.50^{\prime} \mathrm{W}$ Depth Deck Offset: $\quad-0.30$

\begin{tabular}{|c|c|c|c|c|c|c|}
\hline $\begin{array}{l}\text { PRESSURE } \\
\text { (d-bars) }\end{array}$ & Interp & $\begin{array}{l}\text { TEMP } \\
(\operatorname{deg} C)\end{array}$ & $\begin{array}{l}\text { SALINITY } \\
(0 / 00)\end{array}$ & SIGMA-T & DELTA-D & $\begin{array}{l}\text { CHANNELA } \\
\text { ( } 81 \text { ight) }\end{array}$ \\
\hline 0.0 & E & 9.344 & 33.586 & 25.957 & 0.0000 & 0.0 \\
\hline 5.0 & & 9.341 & 33.584 & 25.956 & 0.0104 & 0.0 \\
\hline 10.0 & & 9.342 & 33.583 & 25.955 & 0.0207 & 0.0 \\
\hline 15.0 & & 9.341 & 33.584 & 25.956 & 0.0311 & 0.0 \\
\hline 20.0 & & 9.322 & 33.590 & 25.963 & 0.0415 & 0.0 \\
\hline 25.0 & & 9.299 & 33.600 & 25.975 & 0.0519 & 0.0 \\
\hline 30.0 & & 9.264 & 33.616 & 25.993 & 0.0621 & 0.0 \\
\hline 35.0 & & 9.242 & 33.645 & 26.019 & 0.0723 & 0.0 \\
\hline 40.0 & & 9.163 & 33.677 & 26.057 & 0.0823 & 0.0 \\
\hline 45.0 & & 9.012 & 33.707 & 26.104 & 0.0921 & 0.0 \\
\hline 50.0 & & 8.915 & 33.729 & 26.137 & 0.1018 & 0.0 \\
\hline 55.0 & & 8.756 & 33.781 & 26.202 & 0.1112 & 0.0 \\
\hline 60.0 & & 8.711 & 33.802 & 26.225 & 0.1204 & 0.0 \\
\hline 65.0 & & 8.636 & 33.849 & 26.274 & 0.1294 & 0.0 \\
\hline 70.0 & & 8.627 & 33.863 & 26.286 & 0.1383 & 0.0 \\
\hline 75.0 & & 8.622 & 33.868 & 26.291 & 0.1472 & 0.0 \\
\hline 80.0 & & 8.618 & 33.868 & 26.292 & 0.1560 & 0.0 \\
\hline 83.0 & & 8.617 & 33.868 & 26.292 & 0.1613 & 0.0 \\
\hline
\end{tabular}
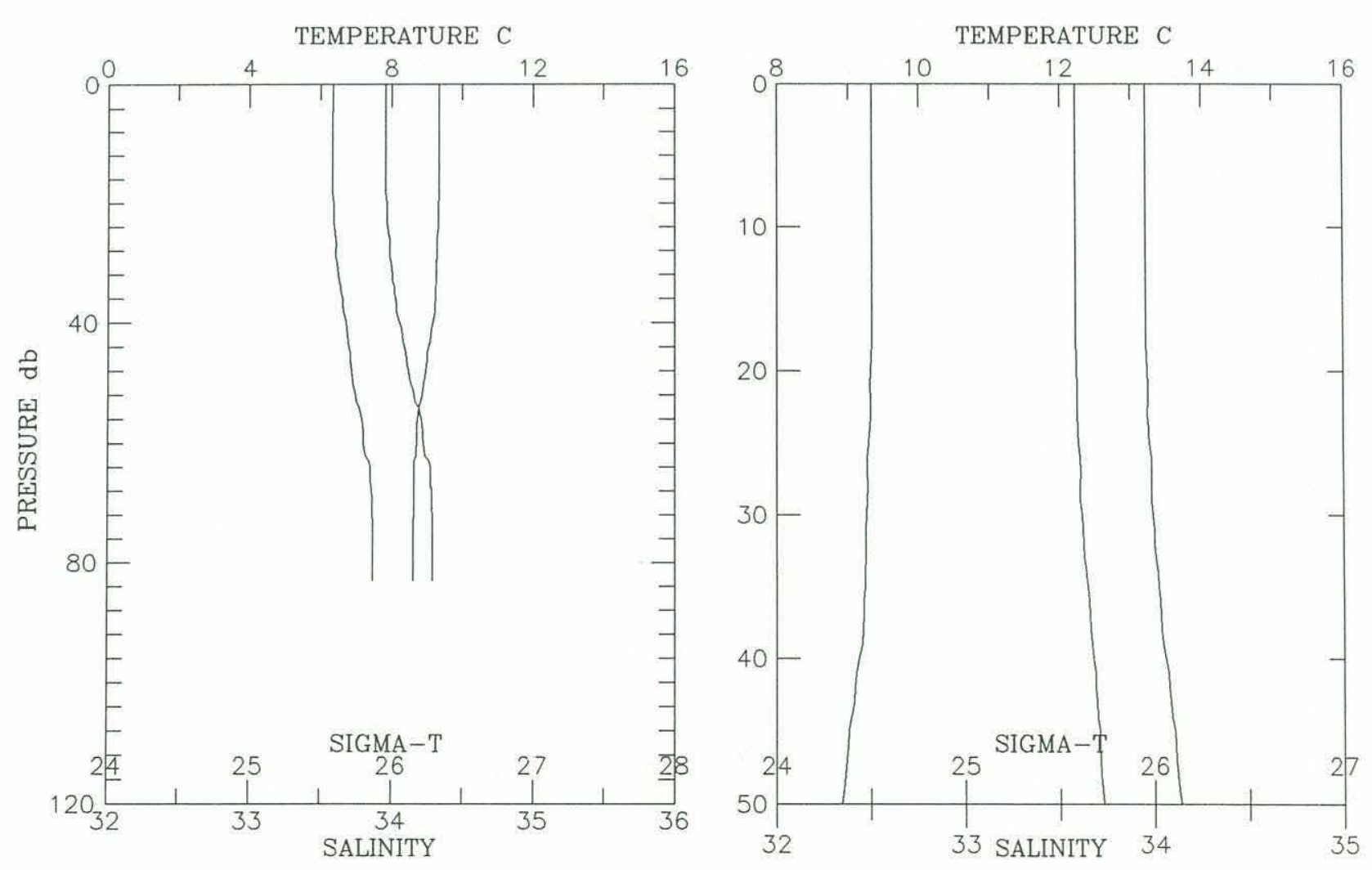


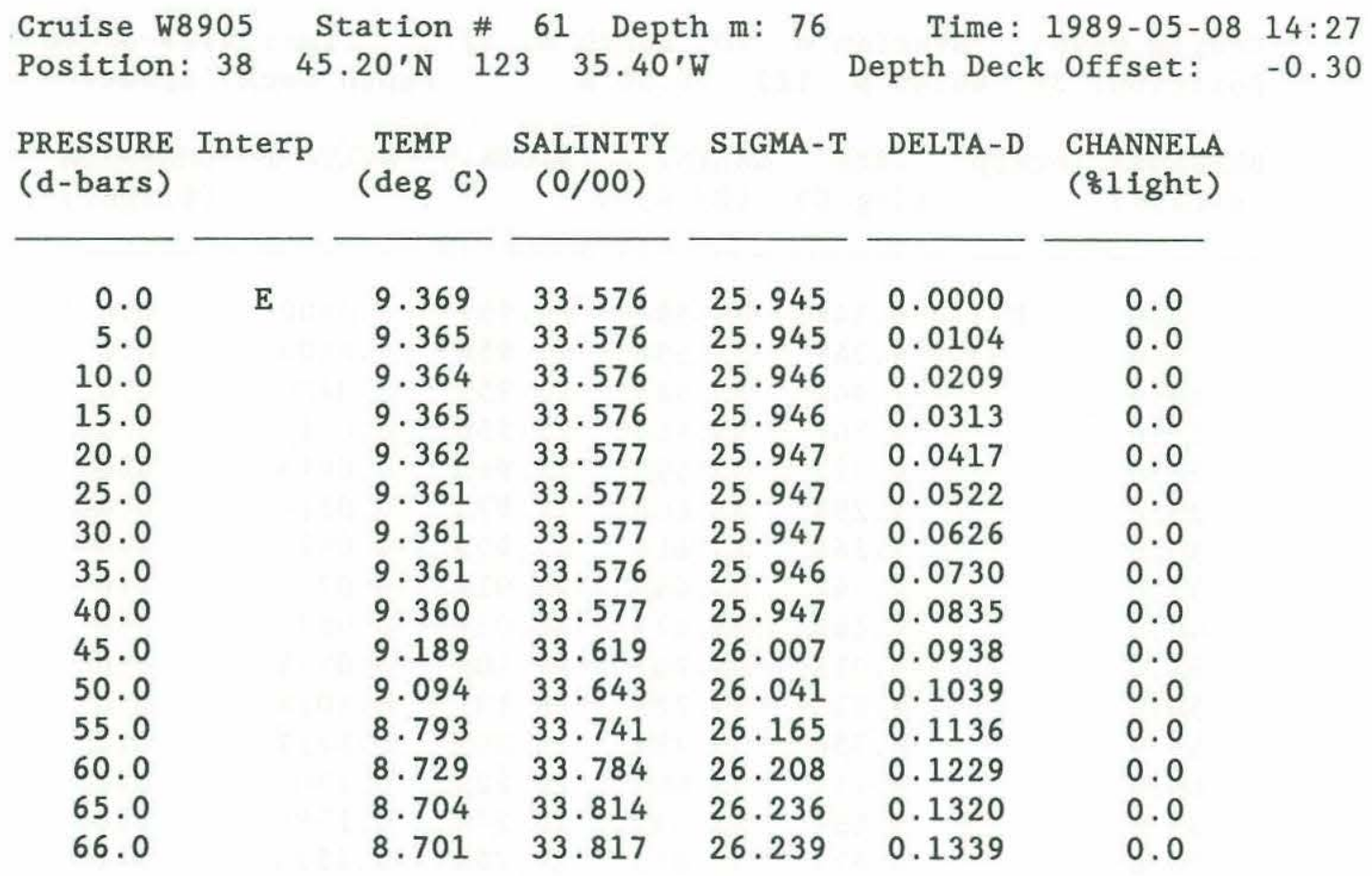
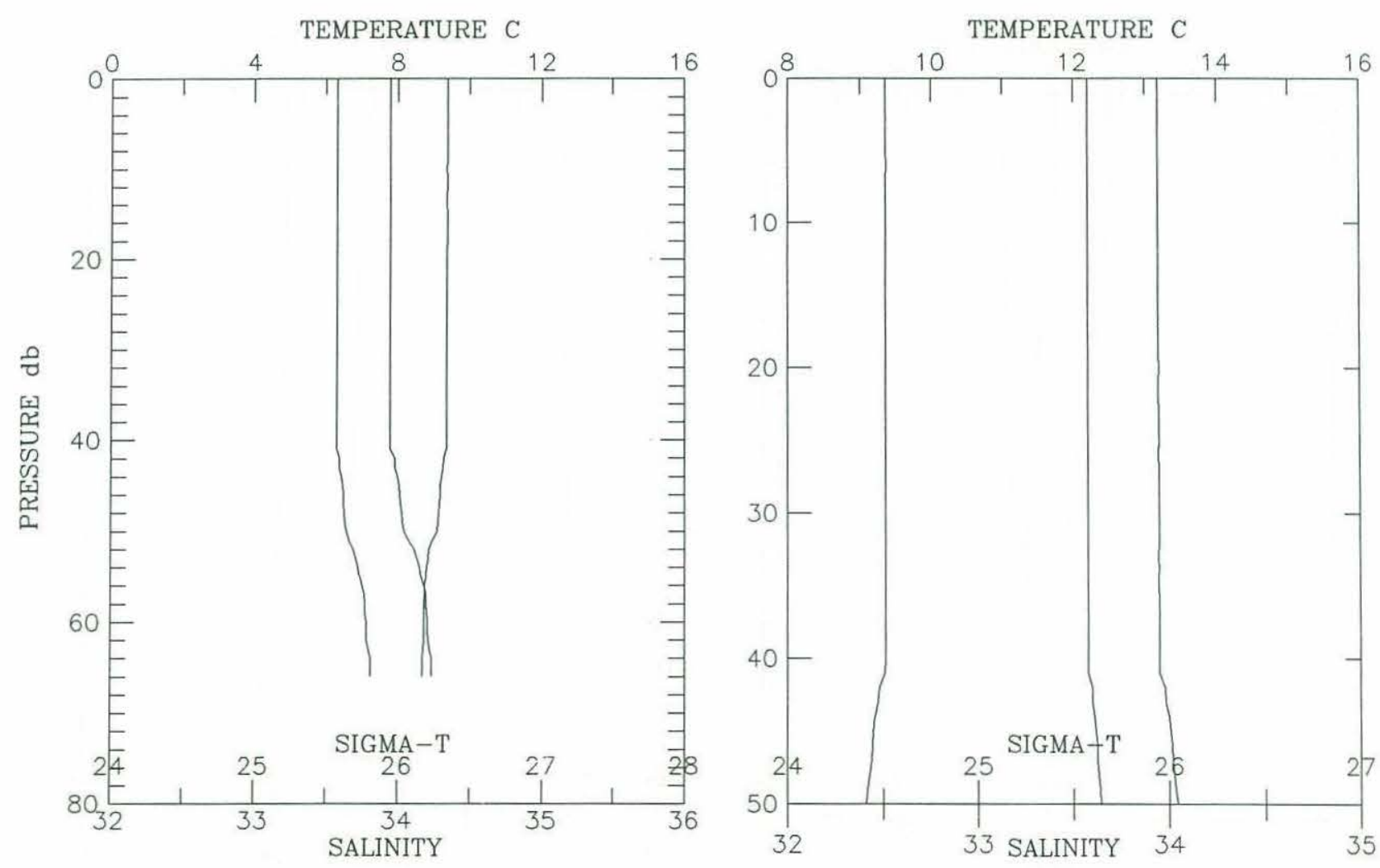


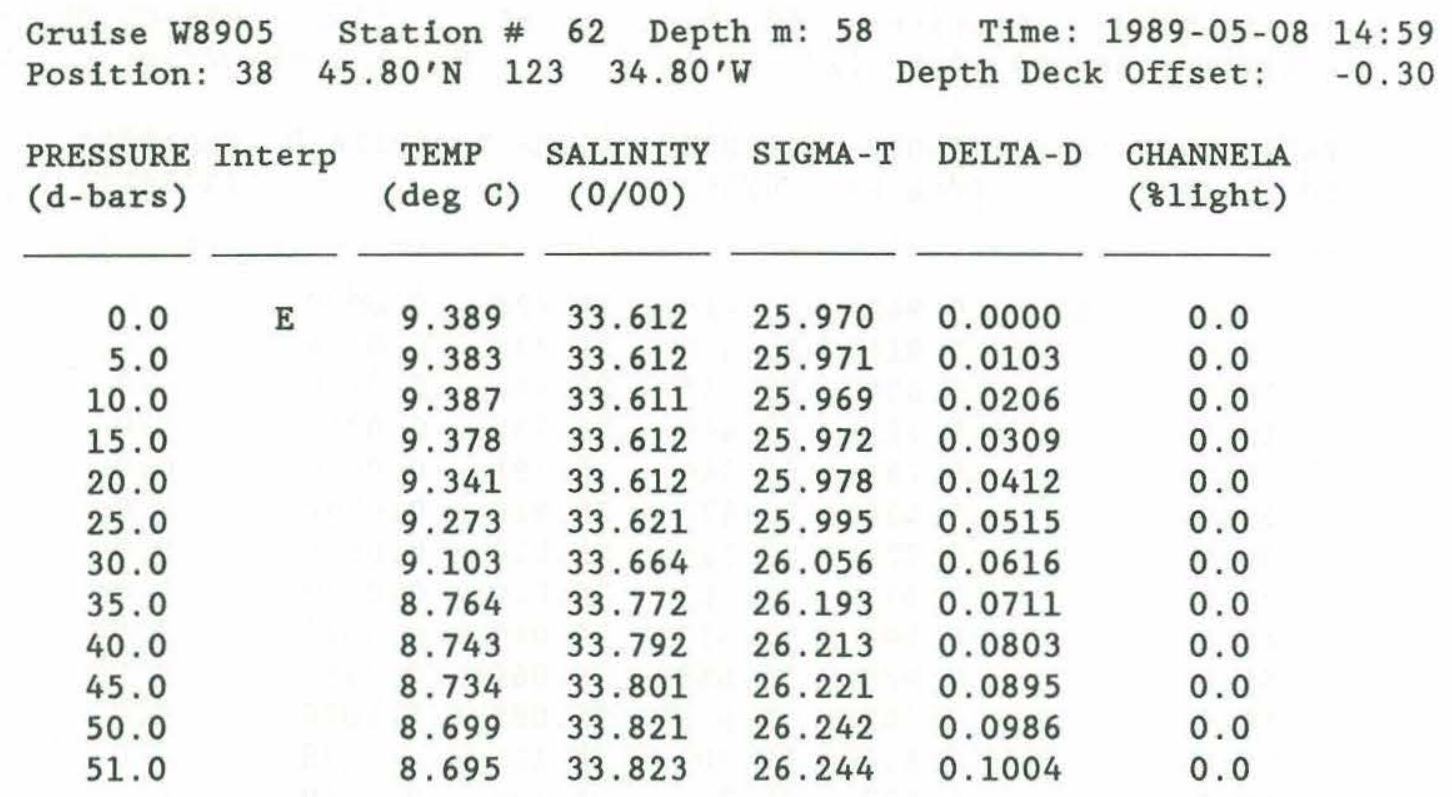
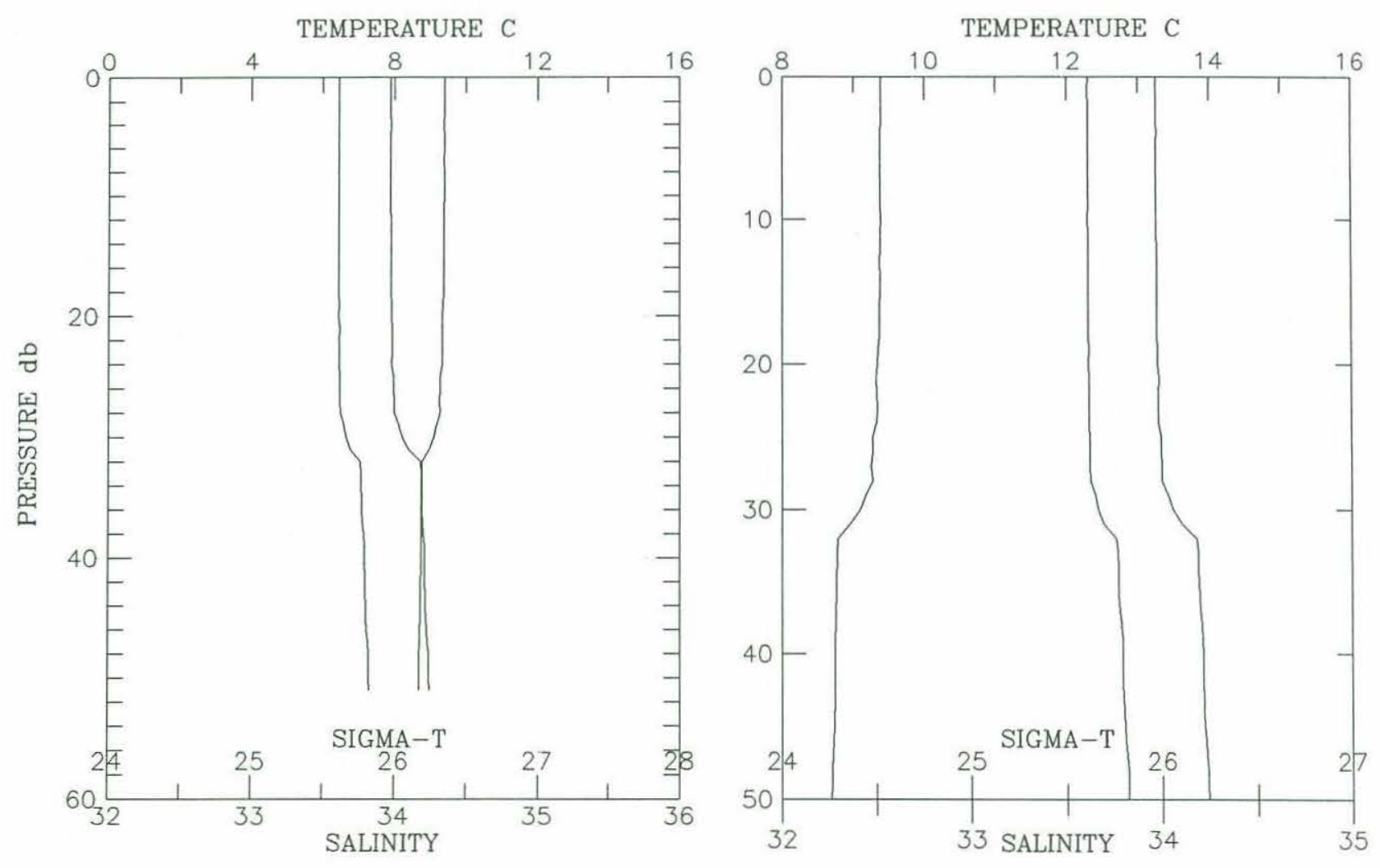


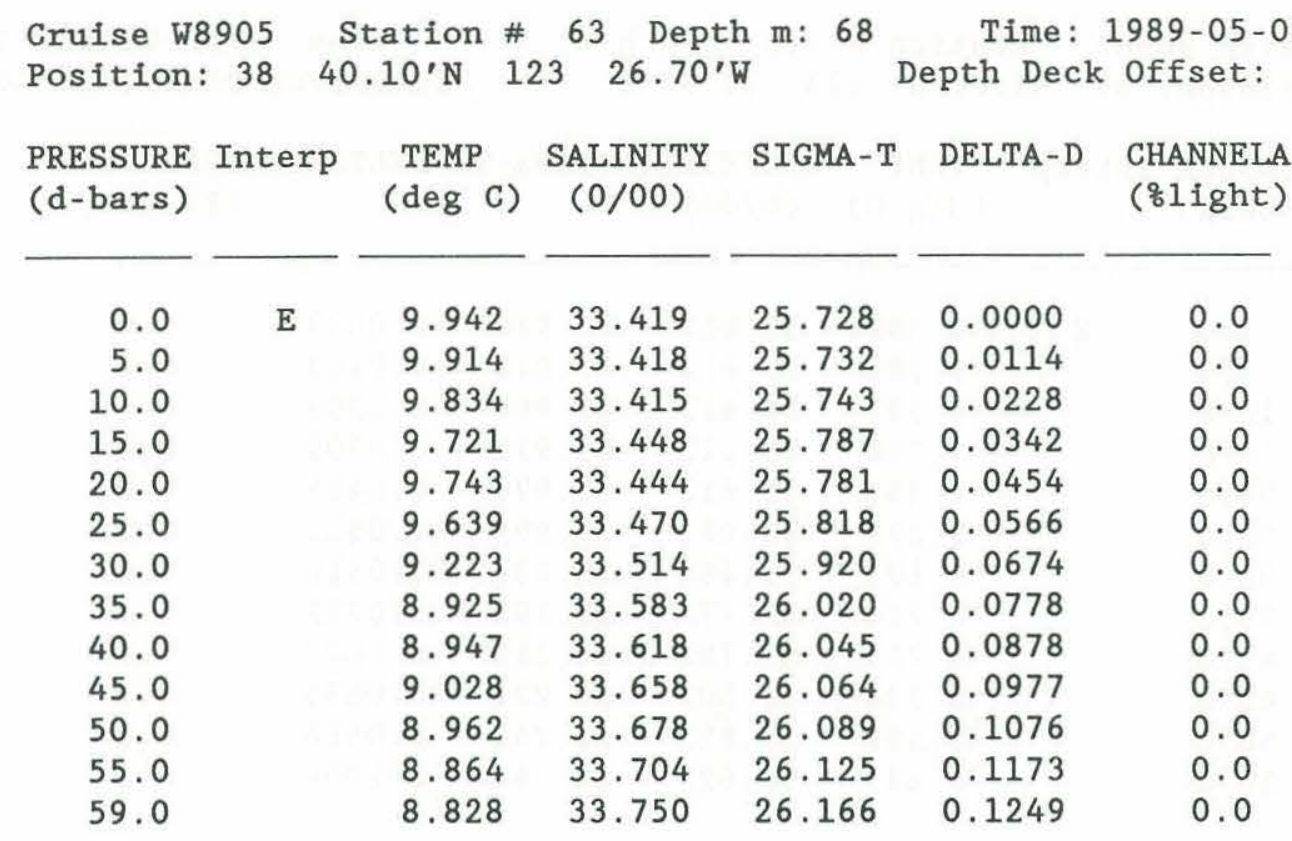
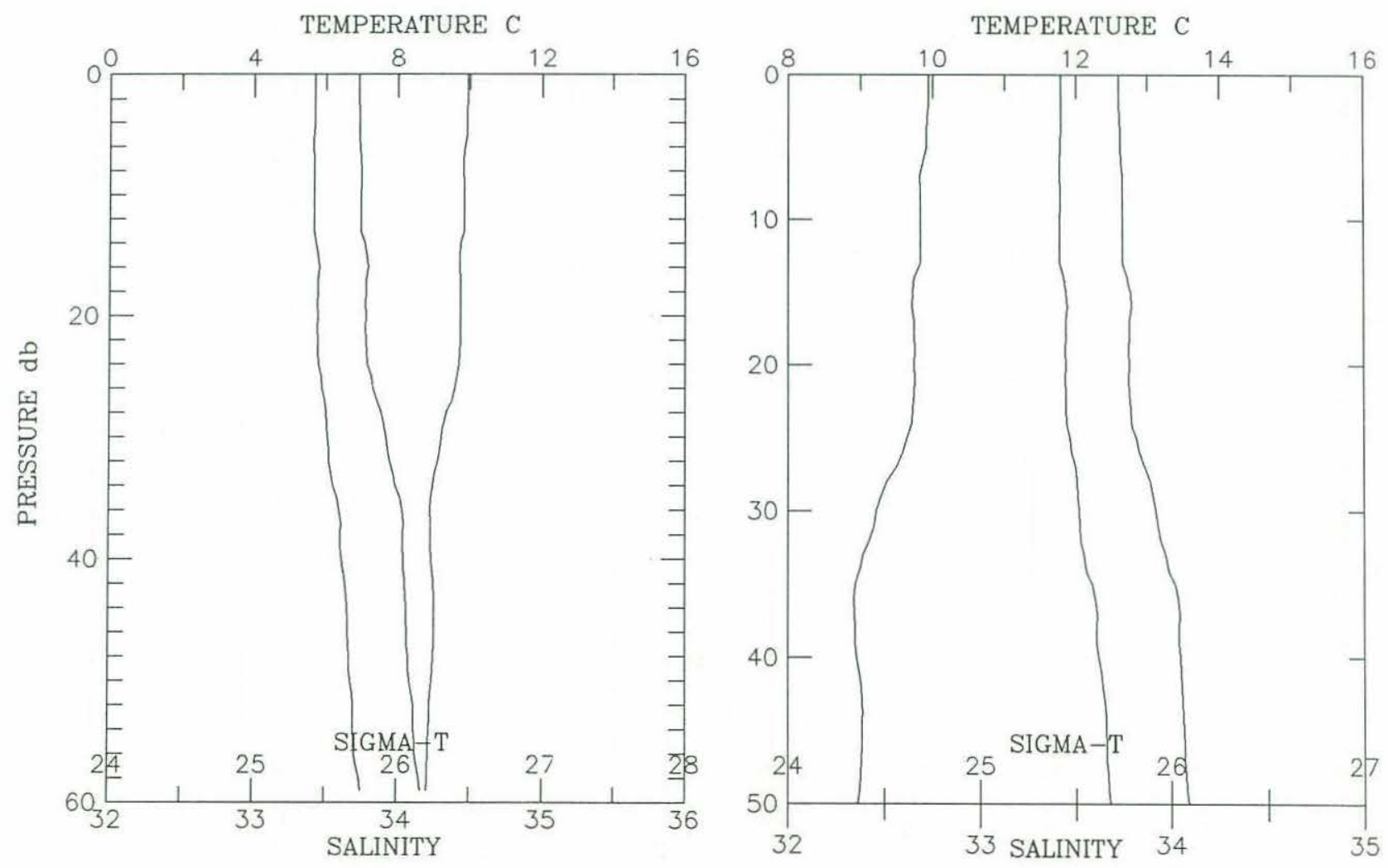
Cruise W8905 Station \# 64 Depth m: 80 Time: 1989-05-08 16:35 Position: $38 \quad 39.20^{\prime} \mathrm{N} 123 \quad 27.70^{\prime} \mathrm{W}$ Depth Deck Offset: -0.30

\begin{tabular}{|c|c|c|c|c|c|c|}
\hline $\begin{array}{l}\text { PRESSURE } \\
\text { (d-bars) }\end{array}$ & Interp & $\begin{array}{l}\text { TEMP } \\
(\operatorname{deg} C)\end{array}$ & $\begin{array}{c}\text { SALINITY } \\
(0 / 00)\end{array}$ & SIGMA-T & DELTA-D & $\begin{array}{l}\text { CHANNELA } \\
\text { ( } \% 1 \text { ight) }\end{array}$ \\
\hline 0.0 & $\mathrm{E}$ & 9.944 & 33.456 & 25.756 & 0.0000 & 0.0 \\
\hline 5.0 & & 9.937 & 33.455 & 25.757 & 0.0113 & 0.0 \\
\hline 10.0 & & 9.895 & 33.455 & 25.764 & 0.0226 & 0.0 \\
\hline 15.0 & & 9.666 & 33.468 & 25.812 & 0.0338 & 0.0 \\
\hline 20.0 & & 9.584 & 33.476 & 25.832 & 0.0448 & 0.0 \\
\hline 25.0 & & 9.516 & 33.488 & 25.852 & 0.0558 & 0.0 \\
\hline 30.0 & & 9.359 & 33.511 & 25.896 & 0.0666 & 0.0 \\
\hline 35.0 & & 9.151 & 33.543 & 25.954 & 0.0771 & 0.0 \\
\hline 40.0 & & 9.022 & 33.571 & 25.996 & 0.0875 & 0.0 \\
\hline 45.0 & & 8.843 & 33.694 & 26.120 & 0.0974 & 0.0 \\
\hline 50.0 & & 8.835 & 33.740 & 26.157 & 0.1069 & 0.0 \\
\hline 55.0 & & 8.846 & 33.803 & 26.205 & 0.1163 & 0.0 \\
\hline 60.0 & & 8.825 & 33.835 & 26.233 & 0.1255 & 0.0 \\
\hline 65.0 & & 8.805 & 33.856 & 26.253 & 0.1345 & 0.0 \\
\hline 70.0 & & 8.732 & 33.865 & 26.271 & 0.1435 & 0.0 \\
\hline 73.0 & & 8.712 & 33.869 & 26.278 & 0.1489 & 0.0 \\
\hline
\end{tabular}
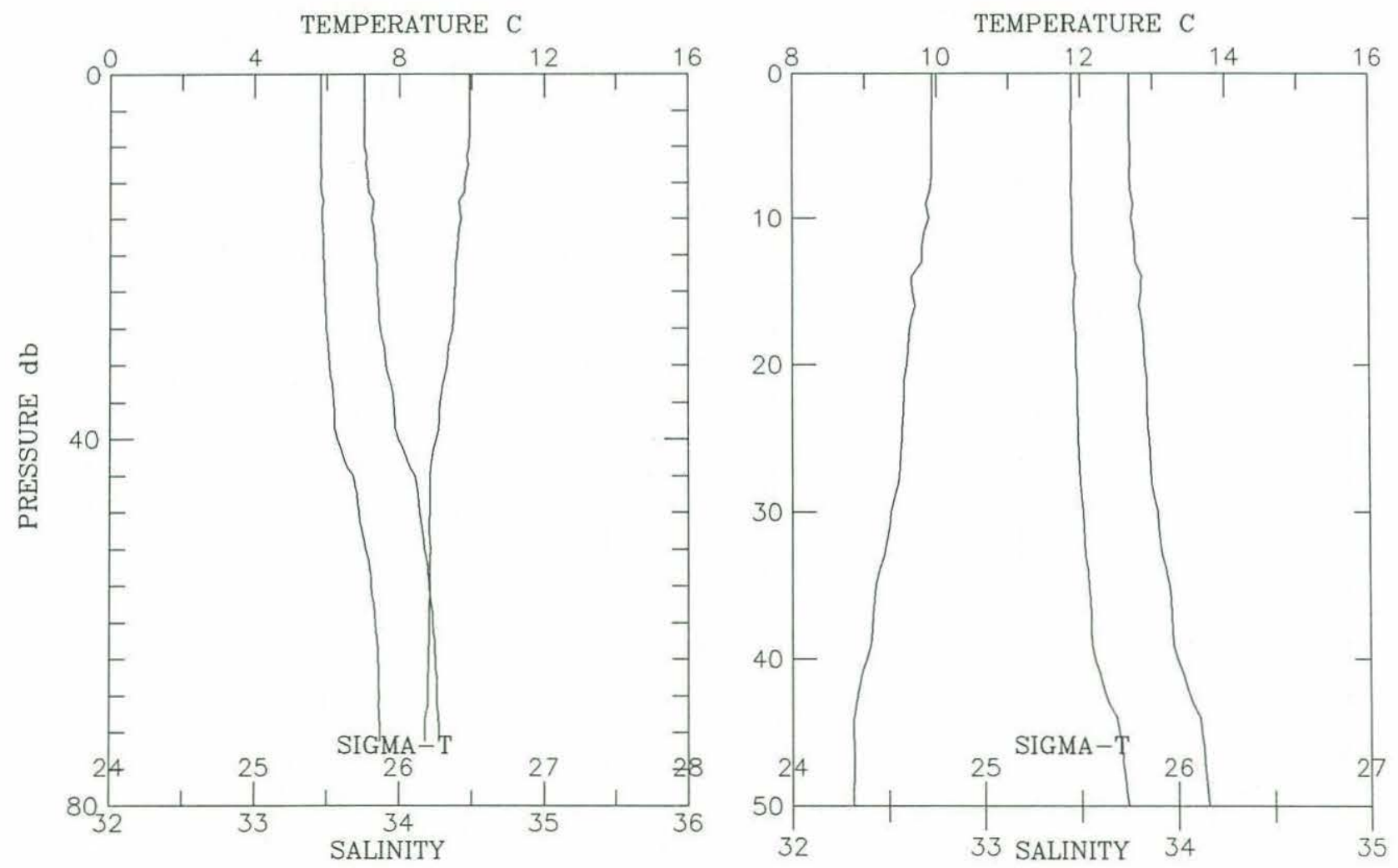
Cruise W8905 Station \# 65 Depth m: 92 Time: 1989-05-08 16:58

Position: $38 \quad 38.20^{\prime} \mathrm{N} 12329.30^{\prime} \mathrm{W} \quad$ Depth Deck Offset: -0.30

\begin{tabular}{|c|c|c|c|c|c|c|}
\hline $\begin{array}{l}\text { PRESSURE } \\
\text { (d-bars) }\end{array}$ & Interp & $\begin{array}{c}\text { TEMP } \\
(\operatorname{deg} C)\end{array}$ & $\begin{array}{c}\text { SALINITY } \\
(0 / 00)\end{array}$ & SIGMA-T & DELTA-D & $\begin{array}{l}\text { CHANNELA } \\
\text { ( } 81 \text { ight) }\end{array}$ \\
\hline 0.0 & E & 9.800 & 33.488 & 25.805 & 0.0000 & 0.0 \\
\hline 5.0 & & 9.802 & 33.487 & 25.804 & 0.0111 & 0.0 \\
\hline 10.0 & & 9.667 & 33.502 & 25.838 & 0.0221 & 0.0 \\
\hline 15.0 & & 9.388 & 33.543 & 25.916 & 0.0329 & 0.0 \\
\hline 20.0 & & 9.181 & 33.561 & 25.963 & 0.0433 & 0.0 \\
\hline 25.0 & & 9.048 & 33.580 & 25.999 & 0.0536 & 0.0 \\
\hline 30.0 & & 8.900 & 33.599 & 26.037 & 0.0637 & 0.0 \\
\hline 35.0 & & 8.834 & 33.689 & 26.118 & 0.0735 & 0.0 \\
\hline 40.0 & & 8.872 & 33.735 & 26.148 & 0.0831 & 0.0 \\
\hline 45.0 & & 8.878 & 33.778 & 26.181 & 0.0925 & 0.0 \\
\hline 50.0 & & 8.830 & 33.807 & 26.211 & 0.1018 & 0.0 \\
\hline 55.0 & & 8.651 & 33.844 & 26.267 & 0.1108 & 0.0 \\
\hline 60.0 & & 8.679 & 33.887 & 26.297 & 0.1197 & 0.0 \\
\hline 65.0 & & 8.683 & 33.888 & 26.297 & 0.1285 & 0.0 \\
\hline 70.0 & & 8.686 & 33.888 & 26.297 & 0.1373 & 0.0 \\
\hline 75.0 & & 8.686 & 33.888 & 26.297 & 0.1461 & 0.0 \\
\hline 80.0 & & 8.690 & 33.888 & 26.296 & 0.1549 & 0.0 \\
\hline 84.0 & & 8.688 & 33.888 & 26.296 & 0.1620 & 0.0 \\
\hline
\end{tabular}

TEMPERATURE $\mathrm{C}$
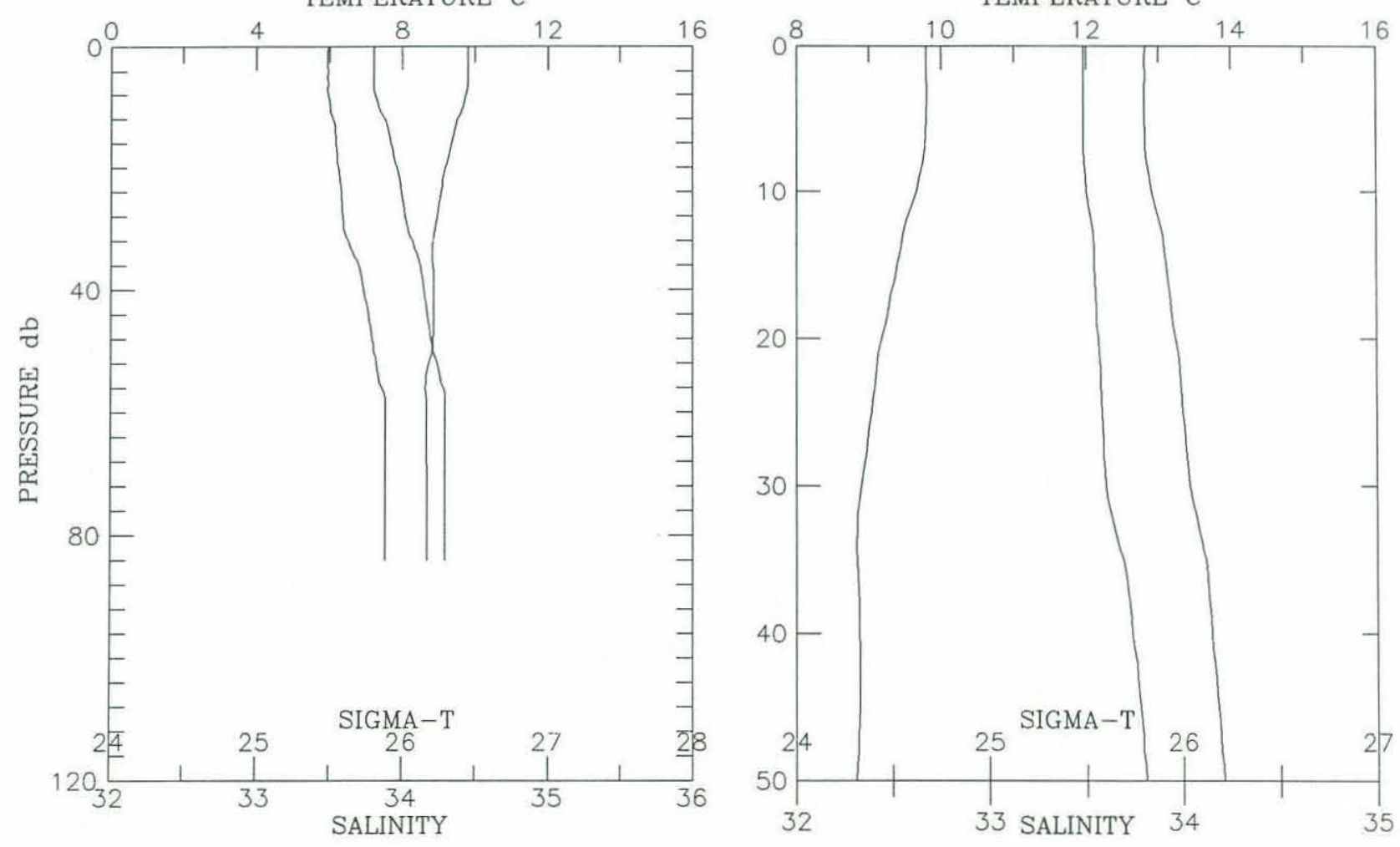
Cruise W8905 Station \# 66 Depth m: 116 Time: 1989-05-08 17:29

Position: $3836.90^{\prime} \mathrm{N} 123 \quad 31.70^{\prime} \mathrm{W}$ Depth Deck Offset: -0.30

\begin{tabular}{|c|c|c|c|c|c|c|}
\hline $\begin{array}{l}\text { PRESSURE } \\
\text { (d-bars) }\end{array}$ & Interp & $\begin{array}{l}\text { TEMP } \\
(\operatorname{deg} C)\end{array}$ & $\begin{array}{c}\text { SALINITY } \\
(0 / 00)\end{array}$ & SIGMA-T & DELTA-D & $\begin{array}{l}\text { CHANNELA } \\
\text { ( }(\text { light) }\end{array}$ \\
\hline 0.0 & E & 10.720 & 33.234 & 25.450 & 0.0000 & 0.0 \\
\hline 10.0 & & 10.579 & 33.239 & 25.479 & 0.0255 & 0.0 \\
\hline 20.0 & & 10.300 & 33.346 & 25.610 & 0.0501 & 0.0 \\
\hline 30.0 & & 10.142 & 33.360 & 25.648 & 0.0739 & 0.0 \\
\hline 40.0 & & 9.340 & 33.512 & 25.900 & 0.0966 & 0.0 \\
\hline 50.0 & & 9.063 & 33.564 & 25.985 & 0.1176 & 0.0 \\
\hline 60.0 & & 8.850 & 33.631 & 26.070 & 0.1377 & 0.0 \\
\hline 70.0 & & 8.676 & 33.689 & 26.142 & 0.1572 & 0.0 \\
\hline 80.0 & & 8.539 & 33.799 & 26.250 & 0.1758 & 0.0 \\
\hline 90.0 & & 8.446 & 33.839 & 26.295 & 0.1936 & 0.0 \\
\hline 100.0 & & 8.411 & 33.908 & 26.354 & 0.2110 & 0.0 \\
\hline 110.0 & & 8.408 & 33.910 & 26.357 & 0.2281 & 0.0 \\
\hline 111.0 & & 8.408 & 33.910 & 26.357 & 0.2298 & 0.0 \\
\hline
\end{tabular}
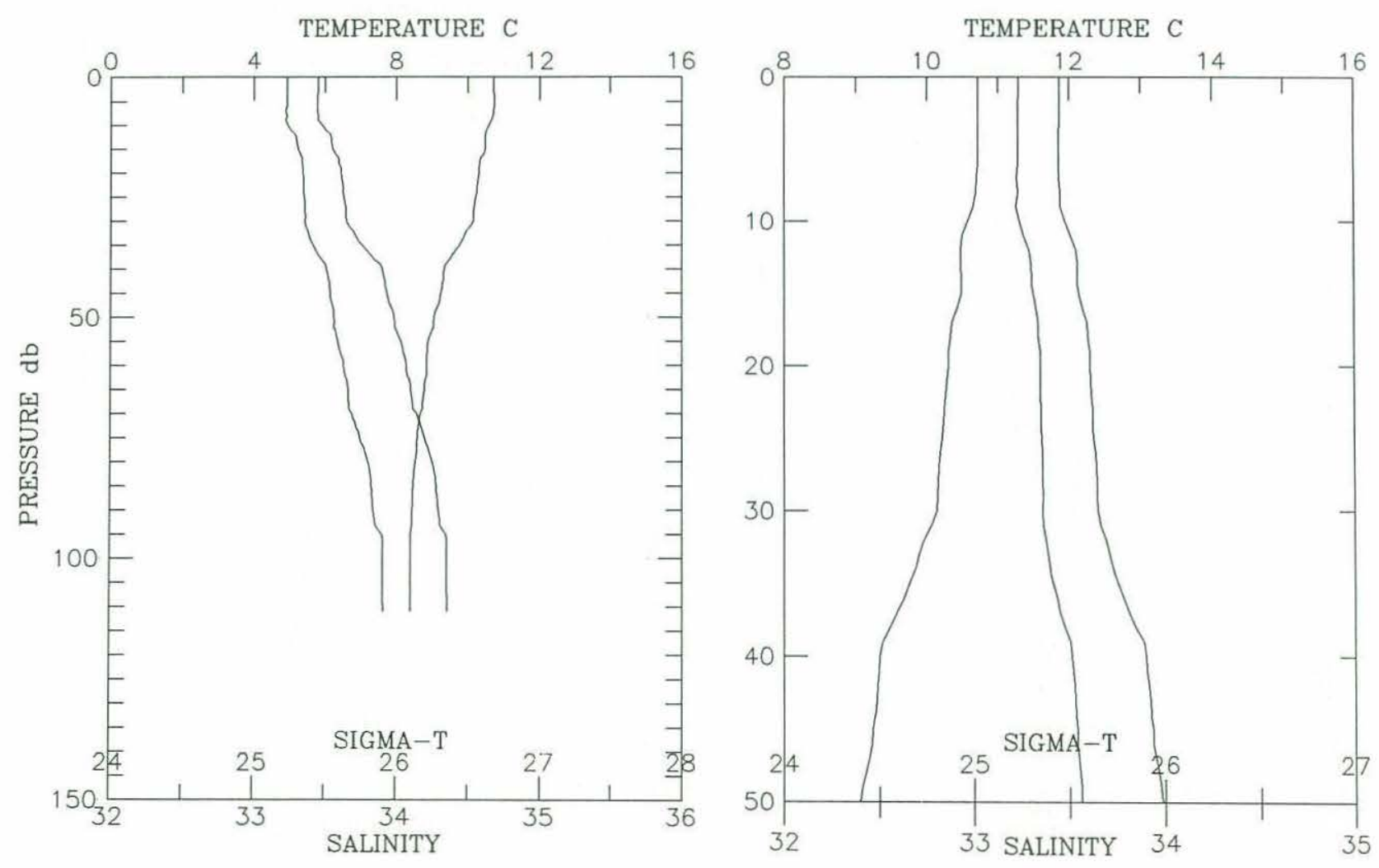
Cruise w8905 Station \# 67 Depth m: 139 Time: 1989-05-08 17:59

Position: 38 35.30'N $123 \quad 33.70^{\prime} \mathrm{W}$ Depth Deck Offset: $\quad-0.30$

\begin{tabular}{|c|c|c|c|c|c|c|}
\hline $\begin{array}{l}\text { PRESSURE } \\
\text { (d-bars) }\end{array}$ & Interp & $\begin{array}{c}\text { TEMP } \\
(\operatorname{deg} C)\end{array}$ & $\begin{array}{c}\text { SALINITY } \\
(0 / 00)\end{array}$ & SIGMA-T & DELTA-D & $\begin{array}{l}\text { CHANNELA } \\
\text { ( } \delta 1 i g h t)\end{array}$ \\
\hline 0.0 & E & 12.291 & 32.995 & 24.978 & 0.0000 & 0.0 \\
\hline 10.0 & & 12.234 & 32.999 & 24.991 & 0.0300 & 0.0 \\
\hline 20.0 & & 10.274 & 33.233 & 25.527 & 0.0583 & 0.0 \\
\hline 30.0 & & 9.543 & 33.402 & 25.781 & 0.0816 & 0.0 \\
\hline 40.0 & & 9.213 & 33.486 & 25.900 & 0.1035 & 0.0 \\
\hline 50.0 & & 9.075 & 33.576 & 25.991 & 0.1243 & 0.0 \\
\hline 60.0 & & 8.988 & 33.618 & 26.038 & 0.1445 & 0.0 \\
\hline 70.0 & & 8.848 & 33.683 & 26.111 & 0.1644 & 0.0 \\
\hline 80.0 & & 8.584 & 33.755 & 26.208 & 0.1835 & 0.0 \\
\hline 90.0 & & 8.465 & 33.791 & 26.255 & 0.2018 & 0.0 \\
\hline 100.0 & & 8.257 & 33.857 & 26.338 & 0.2195 & 0.0 \\
\hline 110.0 & & 8.192 & 33.906 & 26.386 & 0.2366 & 0.0 \\
\hline 120.0 & & 8.144 & 33.947 & 26.426 & 0.2533 & 0.0 \\
\hline 130.0 & & 8.076 & 33.975 & 26.458 & 0.2696 & 0.0 \\
\hline
\end{tabular}
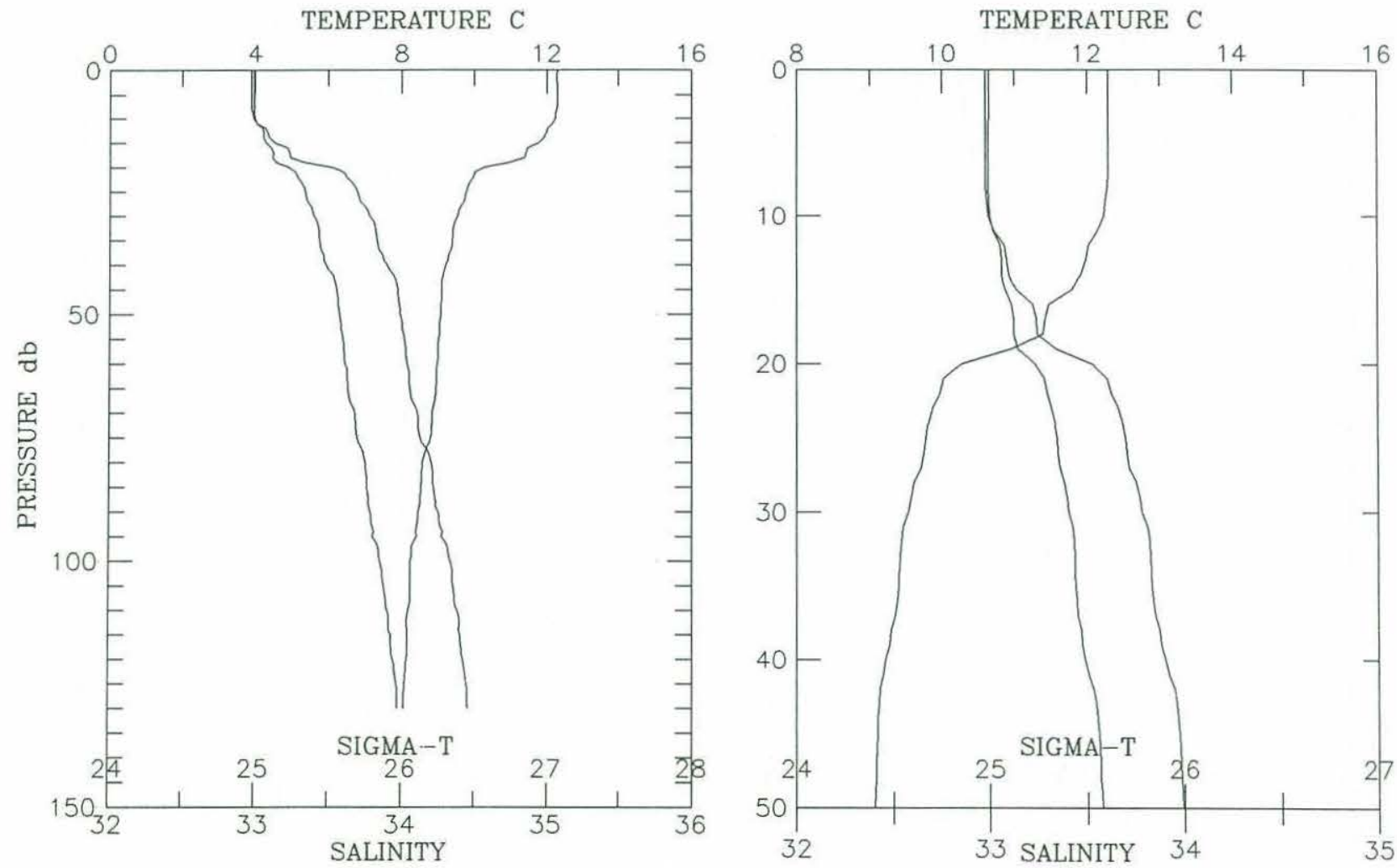


\begin{tabular}{|c|c|c|c|c|c|c|c|}
\hline \multicolumn{2}{|c|}{ Cruise W8905 } & $\begin{array}{l}\text { Station } \\
33.10^{\prime} \mathrm{N}\end{array}$ & \multicolumn{2}{|c|}{68 Depth $\mathrm{m}: 162$} & Time: & $1989-05-08$ & $\begin{array}{l}18: 43 \\
-0.30\end{array}$ \\
\hline $\begin{array}{l}\text { PRESSURE } \\
\text { (d-bars) }\end{array}$ & Interp & $\begin{array}{l}\text { TEMP } \\
(\operatorname{deg} C)\end{array}$ & $\begin{array}{c}\text { SALINITY } \\
(0 / 00)\end{array}$ & SIGMA-T & DELTA-D & $\begin{array}{l}\text { CHANNELA } \\
\text { ( } 8 \text { Iight) }\end{array}$ & \\
\hline 0.0 & $\mathrm{E}$ & 12.785 & 32.926 & 24.829 & 0.0000 & 0.0 & \\
\hline 10.0 & & 11.747 & 33.046 & 25.119 & 0.0307 & 0.0 & \\
\hline 20.0 & & 9.421 & 33.475 & 25.858 & 0.0540 & 0.0 & \\
\hline 30.0 & & 9.099 & 33.578 & 25.989 & 0.0751 & 0.0 & \\
\hline 40.0 & & 8.984 & 33.620 & 26.040 & 0.0952 & 0.0 & \\
\hline 50.0 & & 8.797 & 33.670 & 26.109 & 0.1151 & 0.0 & \\
\hline 60.0 & & 8.568 & 33.725 & 26.187 & 0.1341 & 0.0 & \\
\hline 70.0 & & 8.442 & 33.771 & 26.242 & 0.1525 & 0.0 & \\
\hline 80.0 & & 8.272 & 33.840 & 26.322 & 0.1703 & 0.0 & \\
\hline 90.0 & & 8.171 & 33.895 & 26.381 & 0.1875 & 0.0 & \\
\hline 100.0 & & 8.149 & 33.940 & 26.419 & 0.2042 & 0.0 & \\
\hline 110.0 & & 8.088 & 33.970 & 26.452 & 0.2207 & 0.0 & \\
\hline 120.0 & & 8.022 & 33.999 & 26.485 & 0.2367 & 0.0 & \\
\hline 130.0 & & 7.762 & 34.005 & 26.527 & 0.2525 & 0.0 & \\
\hline 140.0 & & 7.704 & 34.009 & 26.539 & 0.2680 & 0.0 & \\
\hline 150.0 & & 7.727 & 34.015 & 26.540 & 0.2834 & 0.0 & \\
\hline
\end{tabular}
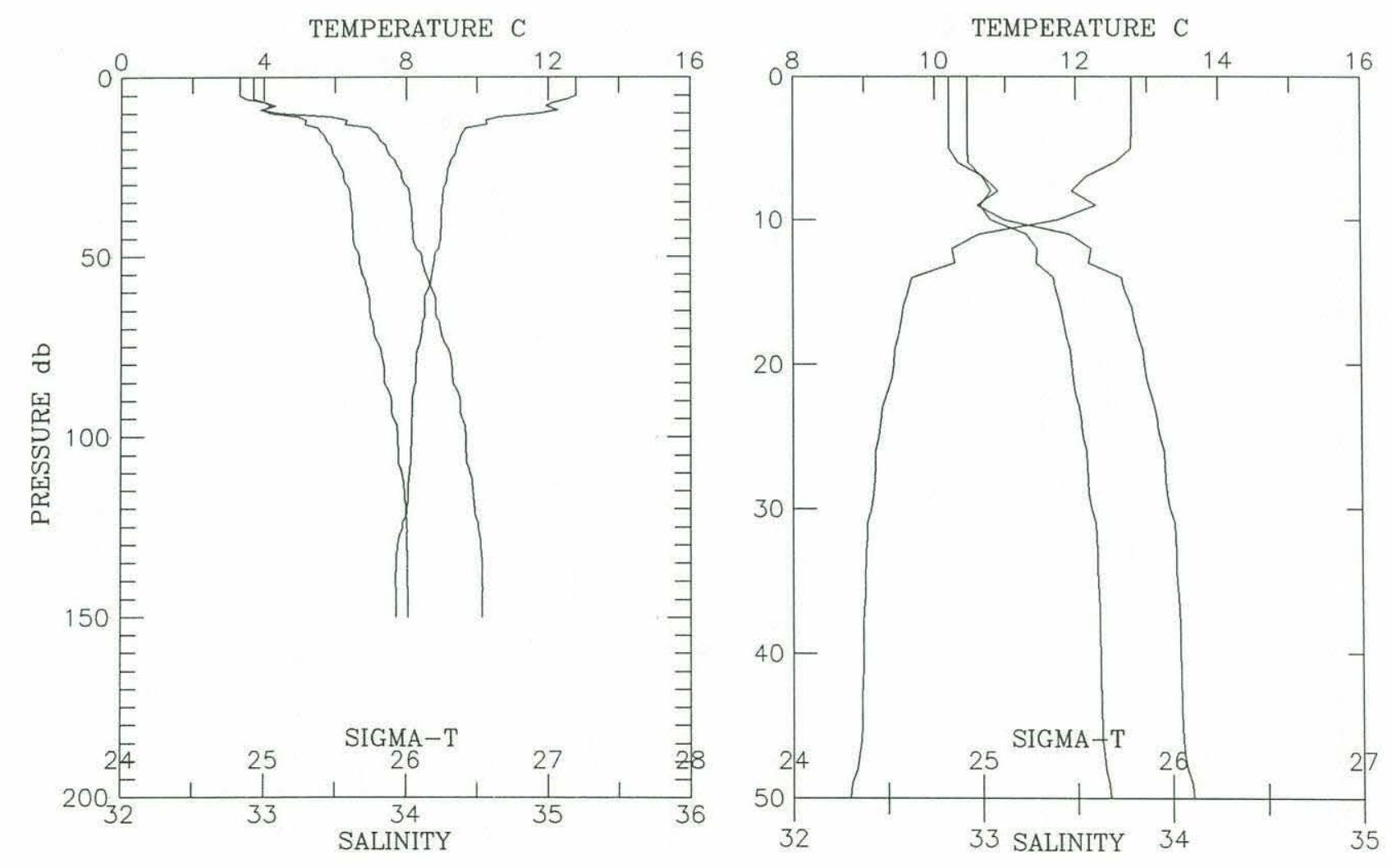


\begin{tabular}{|c|c|c|c|c|c|c|}
\hline $\begin{array}{l}\text { PRESSURE } \\
\text { (d-bars) }\end{array}$ & Interp & $\begin{array}{l}\text { TEMP } \\
(\operatorname{deg} C)\end{array}$ & $\begin{array}{c}\text { SALINITY } \\
(0 / 00)\end{array}$ & SIGMA-T & DELTA-D & $\begin{array}{l}\text { CHANNELA } \\
\text { ( } 8 \text { light) }\end{array}$ \\
\hline 0.0 & E & 12.179 & 33.020 & 25.018 & 0.0000 & 0.0 \\
\hline 10.0 & & 10.381 & 33.292 & 25.554 & 0.0266 & 0.0 \\
\hline 20.0 & & 9.837 & 33.367 & 25.705 & 0.0504 & 0.0 \\
\hline 30.0 & & 9.601 & 33.401 & 25.770 & 0.0734 & 0.0 \\
\hline 40.0 & & 9.210 & 33.552 & 25.952 & 0.0949 & 0.0 \\
\hline 50.0 & & 8.970 & 33.630 & 26.050 & 0.1154 & 0.0 \\
\hline 60.0 & & 8.441 & 33.746 & 26.223 & 0.1346 & 0.0 \\
\hline 80.0 & & 8.227 & 33.854 & 26.340 & 0.1701 & 0.0 \\
\hline 100.0 & & 7.997 & 33.931 & 26.435 & 0.2036 & 0.0 \\
\hline 150.0 & & 7.415 & 34.003 & 26.576 & 0.2824 & 0.0 \\
\hline 200.0 & & 7.203 & 34.076 & 26.663 & 0.3560 & 0.0 \\
\hline 250.0 & & 6.697 & 34.087 & 26.741 & 0.4258 & 0.0 \\
\hline 300.0 & & 6.456 & 34.110 & 26.791 & 0.4928 & 0.0 \\
\hline 400.0 & & 5.898 & 34.174 & 26.912 & 0.6188 & 0.0 \\
\hline 415.0 & & 5.929 & 34.185 & 26.917 & 0.6371 & 0.0 \\
\hline
\end{tabular}
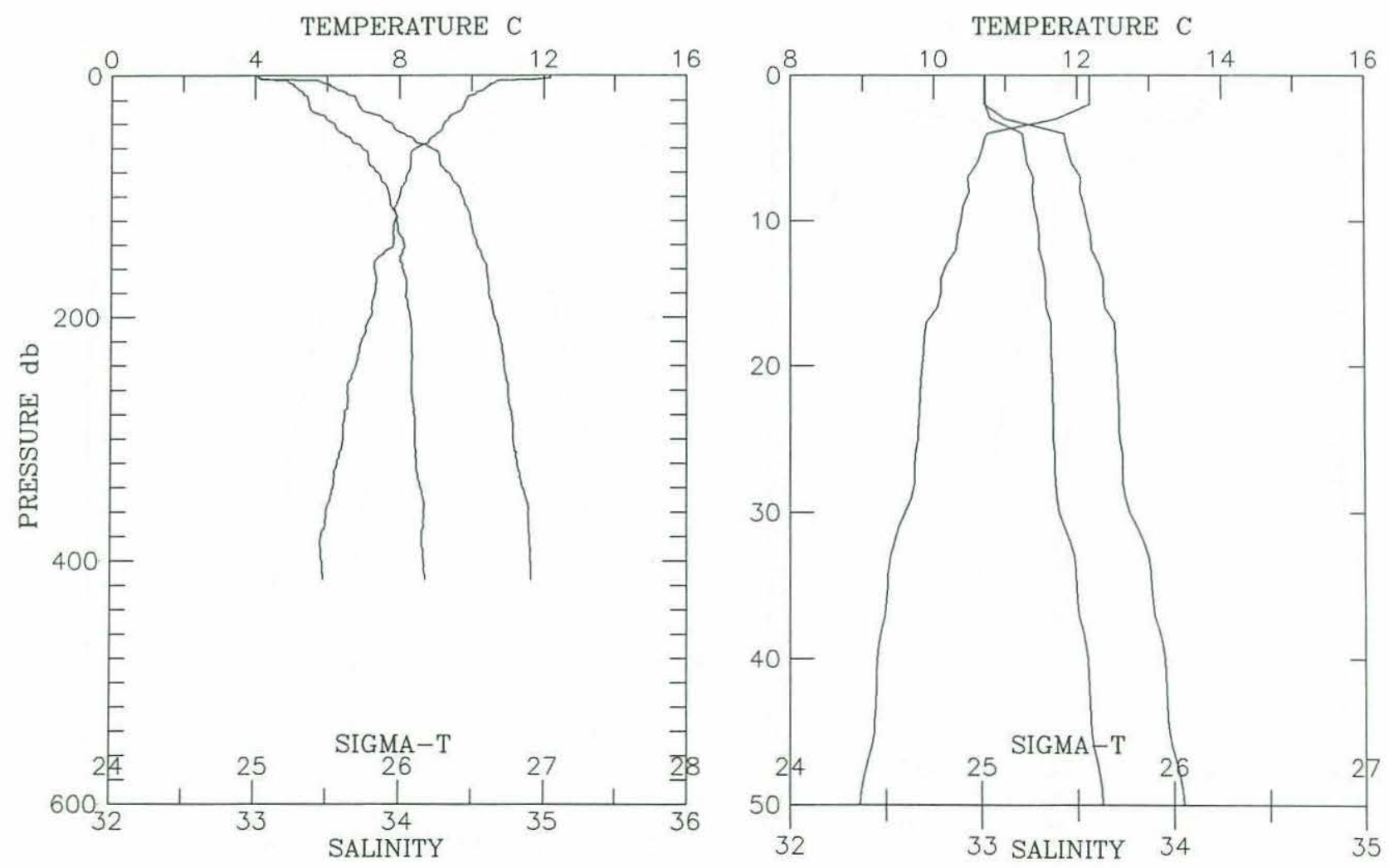
Cruise w8905 Station \# 70 Depth m: 869 Time: 1989-05-08 20:02 Position: $3829.10^{\prime} \mathrm{N} 12342.60^{\prime} \mathrm{W}$ Depth Deck Offset: -0.30

\begin{tabular}{|c|c|c|c|c|c|c|}
\hline $\begin{array}{l}\text { PRESSURE } \\
\text { (d-bars) }\end{array}$ & Interp & $\begin{array}{l}\text { TEMP } \\
(\operatorname{deg} C)\end{array}$ & $\begin{array}{l}\text { SALINITY } \\
(0 / 00)\end{array}$ & SIGMA-T & DELTA-D & $\begin{array}{l}\text { CHANNELA } \\
\text { ( } 81 \text { ight) }\end{array}$ \\
\hline 0.0 & $E$ & 11.928 & 33.062 & 25.098 & 0.0000 & 0.0 \\
\hline 10.0 & & 11.948 & 33.060 & 25.093 & 0.0291 & 0.0 \\
\hline 20.0 & & 10.341 & 33.259 & 25.536 & 0.0557 & 0.0 \\
\hline 30.0 & & 10.068 & 33.299 & 25.613 & 0.0799 & 0.0 \\
\hline 40.0 & & 9.976 & 33.318 & 25.643 & 0.1039 & 0.0 \\
\hline 50.0 & & 9.313 & 33.502 & 25.896 & 0.1267 & 0.0 \\
\hline 60.0 & & 9.131 & 33.559 & 25.969 & 0.1478 & 0.0 \\
\hline 80.0 & & 8.882 & 33.665 & 26.091 & 0.1880 & 0.0 \\
\hline 100.0 & & 8.499 & 33.835 & 26.284 & 0.2253 & 0.0 \\
\hline 150.0 & & 7.643 & 33.970 & 26.517 & 0.3080 & 0.0 \\
\hline 200.0 & & 7.139 & 34.014 & 26.623 & 0.3838 & 0.0 \\
\hline 250.0 & & 6.707 & 34.011 & 26.680 & 0.4561 & 0.0 \\
\hline 300.0 & & 6.560 & 34.103 & 26.772 & 0.5252 & 0.0 \\
\hline 400.0 & & 5.876 & 34.130 & 26.881 & 0.6550 & 0.0 \\
\hline 500.0 & & 5.657 & 34.232 & 26.988 & 0.7744 & 0.0 \\
\hline 600.0 & & 5.391 & 34.282 & 27.060 & 0.8874 & 0.0 \\
\hline 700.0 & & 4.993 & 34.330 & 27.145 & 0.9952 & 0.0 \\
\hline 800.0 & & 4.648 & 34.373 & 27.218 & 1.0945 & 0.0 \\
\hline 861.0 & & 4.336 & 34.401 & 27.274 & 1.1523 & 0.0 \\
\hline
\end{tabular}
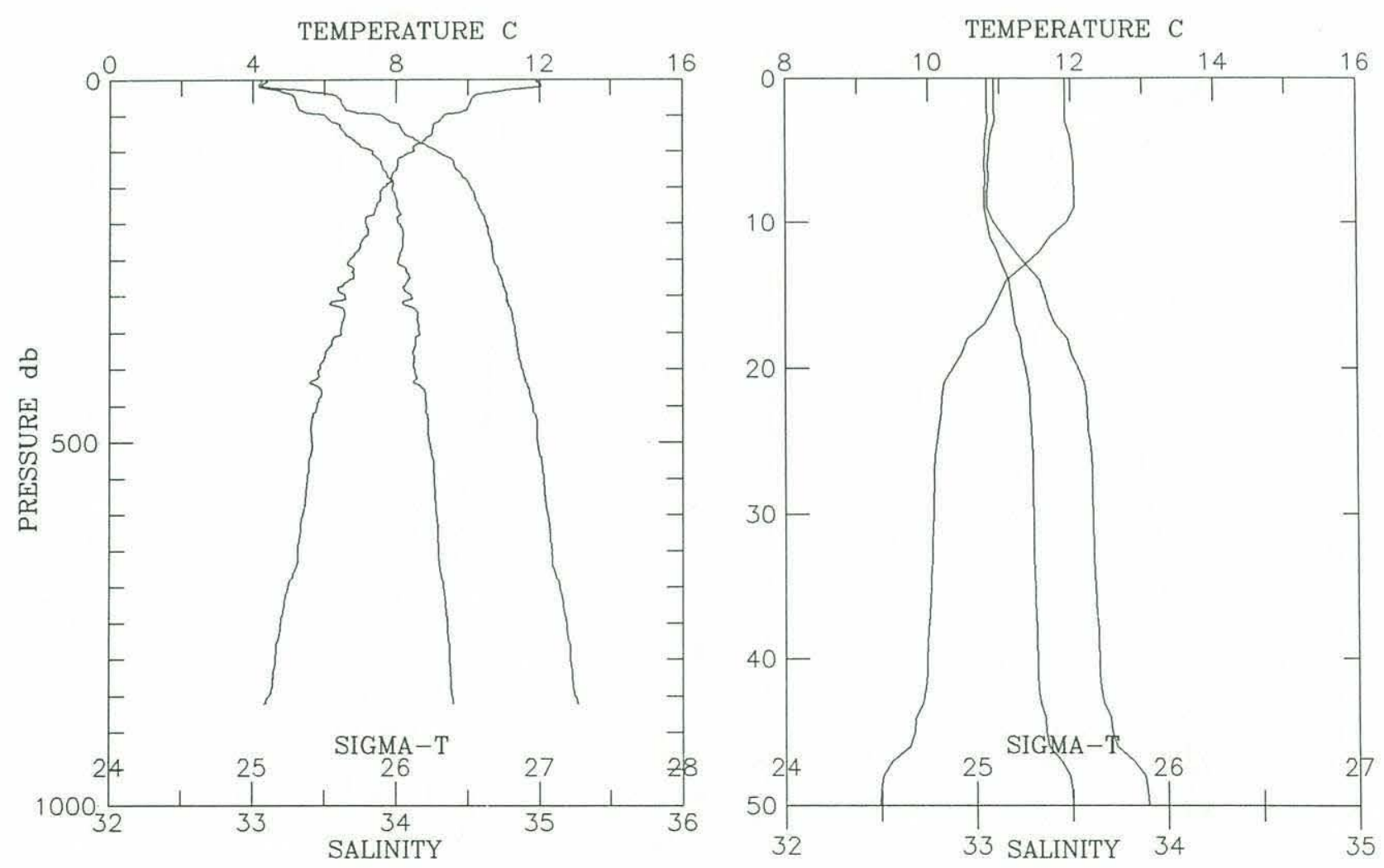
Cruise w8905 Station \# 71 Depth m: 1005 Time: 1989-05-08 21:35

Position: 38 20.00'N $123 \quad 39.90^{\prime} \mathrm{W} \quad$ Depth Deck Offset: -0.25

\begin{tabular}{|c|c|c|c|c|c|c|}
\hline $\begin{array}{l}\text { PRESSURE } \\
\text { (d-bars) }\end{array}$ & Interp & $\begin{array}{c}\text { TEMP } \\
(\operatorname{deg} C)\end{array}$ & $\begin{array}{c}\text { SALINITY } \\
(0 / 00)\end{array}$ & SIGMA-T & DELTA-D & $\begin{array}{l}\text { CHANNEL } \\
\text { ( } \delta 1 \text { ight) }\end{array}$ \\
\hline 0.0 & E & 12.585 & 32.919 & 24.862 & 0.0000 & 0.0 \\
\hline 10.0 & & 12.563 & 32.919 & 24.867 & 0.0311 & 0.0 \\
\hline 20.0 & & 12.315 & 32.929 & 24.922 & 0.0621 & 0.0 \\
\hline 30.0 & & 10.943 & 33.063 & 25.278 & 0.0916 & 0.0 \\
\hline 40.0 & & 9.964 & 33.160 & 25.522 & 0.1179 & 0.0 \\
\hline 50.0 & & 9.590 & 33.232 & 25.640 & 0.1422 & 0.0 \\
\hline 60.0 & & 9.292 & 33.388 & 25.810 & 0.1652 & 0.0 \\
\hline 80.0 & & 8.892 & 33.661 & 26.087 & 0.2069 & 0.0 \\
\hline 100.0 & & 8.514 & 33.818 & 26.268 & 0.2445 & 0.0 \\
\hline 150.0 & & 7.940 & 34.005 & 26.501 & 0.3276 & 0.0 \\
\hline 200.0 & & 7.294 & 34.029 & 26.613 & 0.4041 & 0.0 \\
\hline 250.0 & & 7.094 & 34.085 & 26.686 & 0.4765 & 0.0 \\
\hline 300.0 & & 6.764 & 34.125 & 26.762 & 0.5460 & 0.0 \\
\hline 400.0 & & 6.107 & 34.190 & 26.900 & 0.6753 & 0.0 \\
\hline 500.0 & & 5.688 & 34.246 & 26.996 & 0.7943 & 0.0 \\
\hline 600.0 & & 5.308 & 34.293 & 27.079 & 0.9067 & 0.0 \\
\hline 700.0 & & 4.941 & 34.336 & 27.156 & 1.0116 & 0.0 \\
\hline 800.0 & & 4.523 & 34.372 & 27.231 & 1.1106 & 0.0 \\
\hline 900.0 & & 4.343 & 34.404 & 27.276 & 1.2038 & 0.0 \\
\hline 992.0 & & 4.139 & 34.425 & 27.314 & 1.2865 & 0.0 \\
\hline
\end{tabular}

TEMPERATURE C
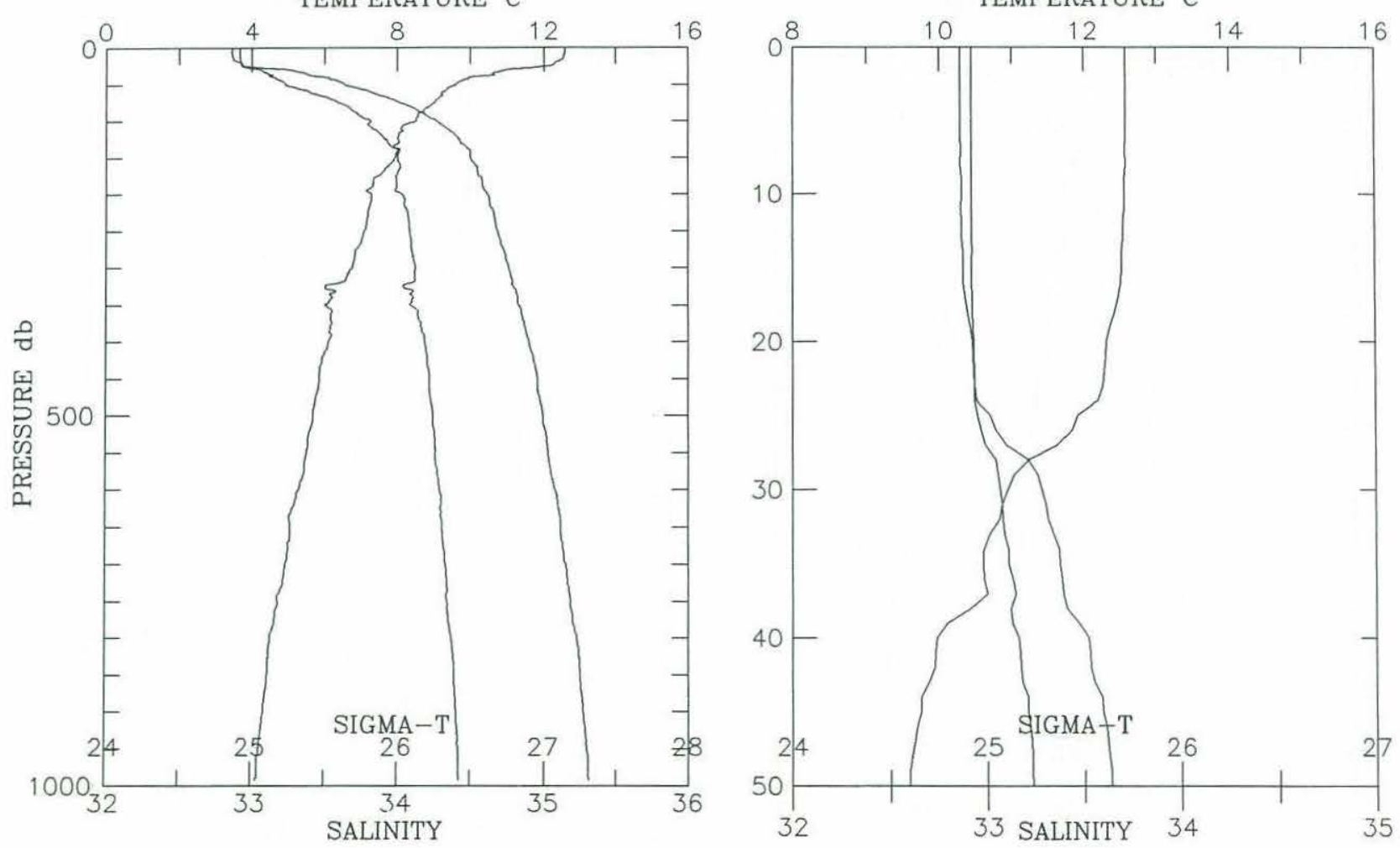
Cruise W8905 Station \# 72 Depth m: 335 Time: 1989-05-08 23:11 Position: $3823.90^{\prime} \mathrm{N} 123 \quad 34.80^{\prime} \mathrm{W}$ Depth Deck Offset: -0.25

\begin{tabular}{|c|c|c|c|c|c|c|}
\hline $\begin{array}{l}\text { PRESSURE } \\
\text { (d-bars) }\end{array}$ & Interp & $\begin{array}{l}\text { TEMP } \\
(\operatorname{deg} C)\end{array}$ & $\begin{array}{l}\text { SALINITY } \\
(0 / 00)\end{array}$ & SIGMA-T & DELTA-D & $\begin{array}{l}\text { CHANNELA } \\
\text { ( }(1 \mathrm{ight})\end{array}$ \\
\hline 0.0 & E & 12.689 & 32.915 & 24.839 & 0.0000 & 0.0 \\
\hline 10.0 & & 12.692 & 32.915 & 24.839 & 0.0314 & 0.0 \\
\hline 20.0 & & 12.550 & 32.920 & 24.870 & 0.0627 & 0.0 \\
\hline 30.0 & & 11.318 & 33.013 & 25.172 & 0.0919 & 0.0 \\
\hline 40.0 & & 10.033 & 32.971 & 25.363 & 0.1191 & 0.0 \\
\hline 50.0 & & 9.768 & 33.140 & 25.539 & 0.1448 & 0.0 \\
\hline 60.0 & & 9.523 & 33.311 & 25.713 & 0.1688 & 0.0 \\
\hline 80.0 & & 8.665 & 33.685 & 26.141 & 0.2106 & 0.0 \\
\hline 100.0 & & 8.306 & 33.881 & 26.349 & 0.2468 & 0.0 \\
\hline 150.0 & & 7.830 & 34.020 & 26.529 & 0.3281 & 0.0 \\
\hline 200.0 & & 7.038 & 34.044 & 26.661 & 0.4031 & 0.0 \\
\hline 250.0 & & 6.765 & 34.094 & 26.737 & 0.4728 & 0.0 \\
\hline 300.0 & & 6.400 & 34.096 & 26.787 & 0.5403 & 0.0 \\
\hline 315.0 & & 6.371 & 34.096 & 26.791 & 0.5602 & 0.0 \\
\hline
\end{tabular}
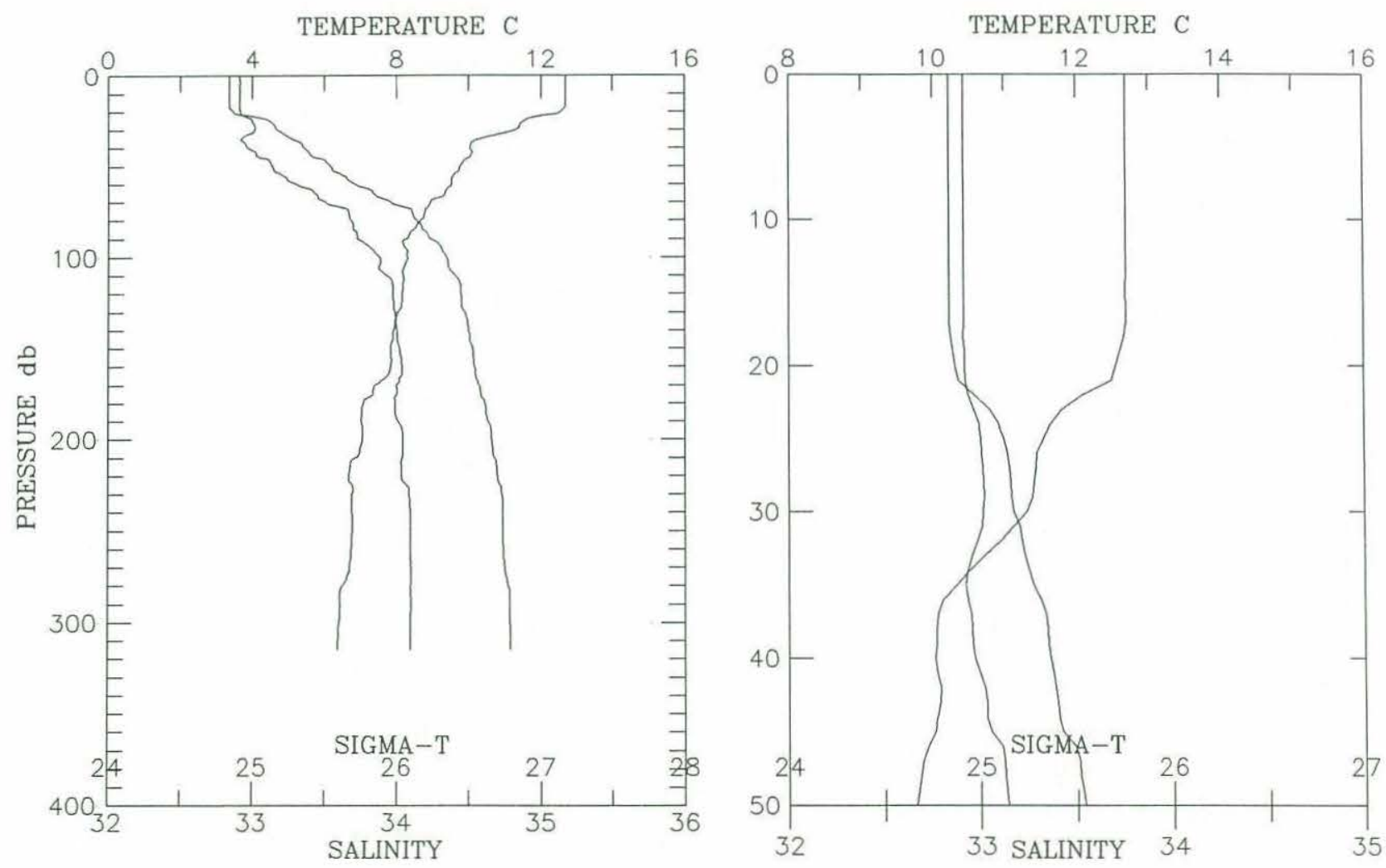


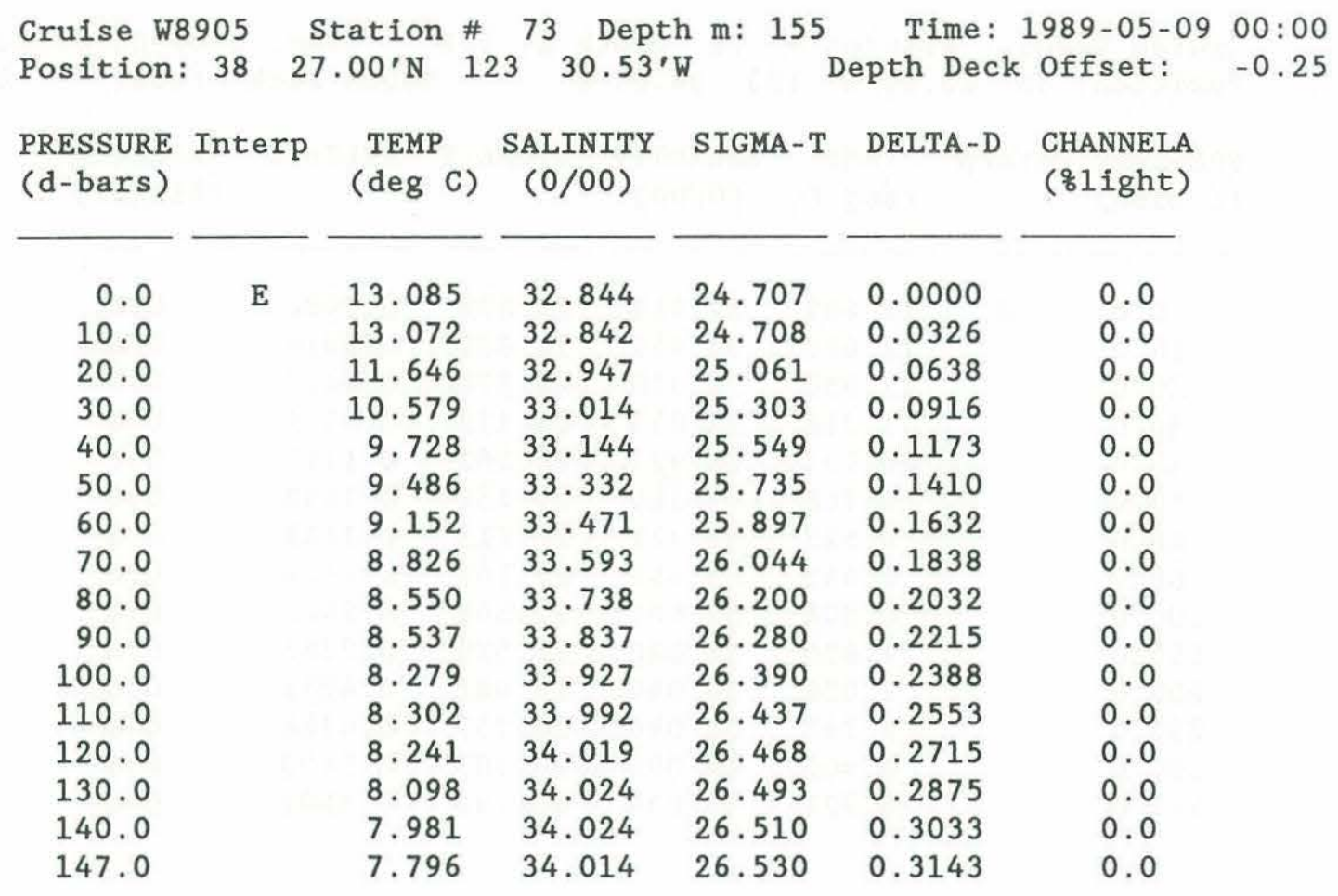
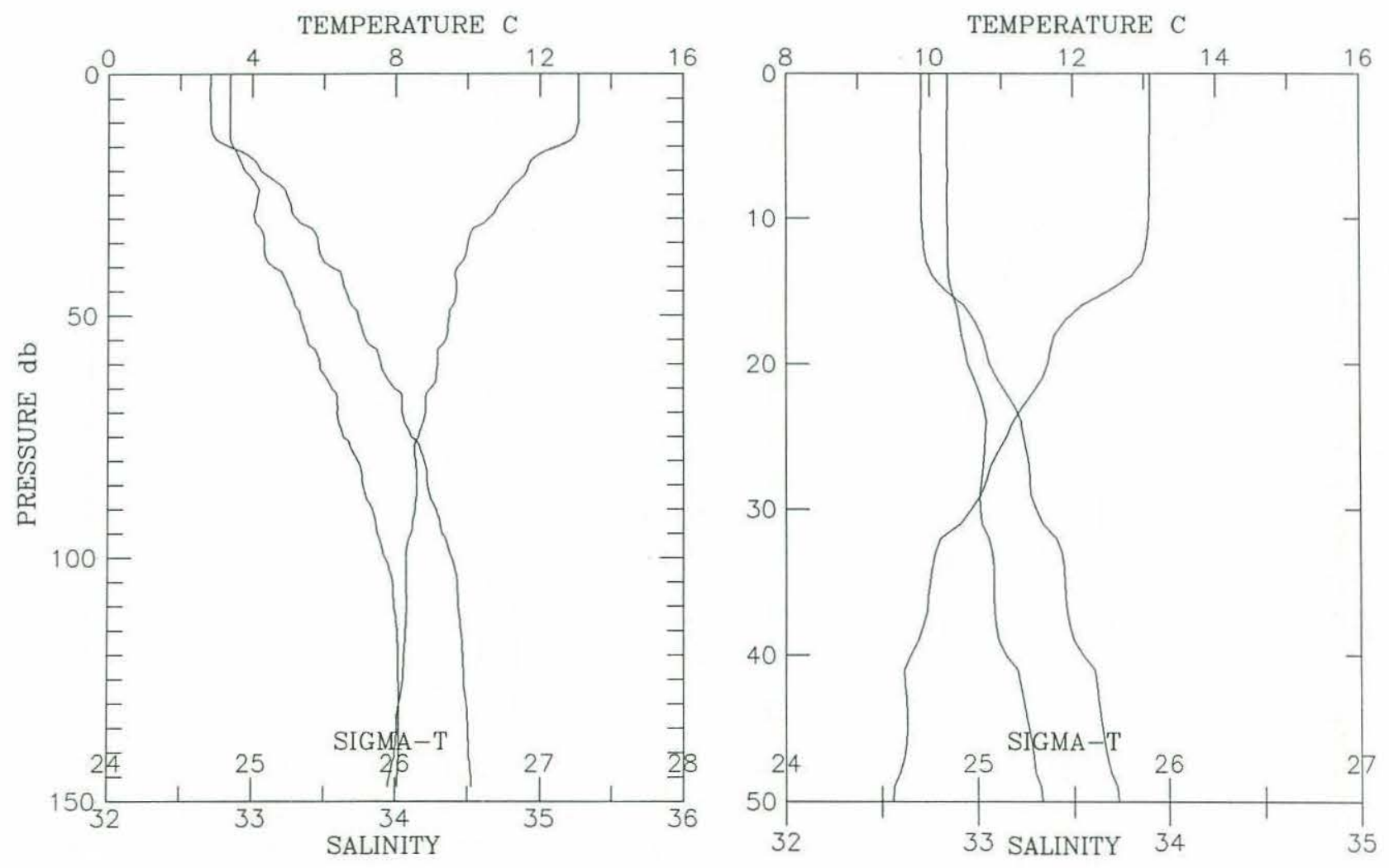
Cruise W8905 Station \# 74 Depth m: 136 Time: 1989-05-09 00:33

Position: $38 \quad 29.10^{\prime} \mathrm{N} \quad 12327.50^{\prime} \mathrm{W}$ Depth Deck Offset: -0.25

\begin{tabular}{|c|c|c|c|c|c|c|}
\hline $\begin{array}{l}\text { PRESSURE } \\
\text { (d-bars) }\end{array}$ & Interp & $\begin{array}{l}\text { TEMP } \\
(\operatorname{deg} C)\end{array}$ & $\begin{array}{c}\text { SALINITY } \\
(0 / 00)\end{array}$ & SIGMA-T & DELTA-D & $\begin{array}{l}\text { CHANNELA } \\
\text { ( } \delta 1 \text { ight) }\end{array}$ \\
\hline 0.0 & $\mathrm{E}$ & 12.713 & 32.885 & 24.811 & 0.0000 & 0.0 \\
\hline 10.0 & & 12.699 & 32.885 & 24.814 & 0.0316 & 0.0 \\
\hline 20.0 & & 11.048 & 33.031 & 25.234 & 0.0618 & 0.0 \\
\hline 30.0 & & 9.984 & 33.093 & 25.466 & 0.0884 & 0.0 \\
\hline 40.0 & & 9.517 & 33.351 & 25.745 & 0.1124 & 0.0 \\
\hline 50.0 & & 9.196 & 33.419 & 25.849 & 0.1348 & 0.0 \\
\hline 60.0 & & 9.065 & 33.489 & 25.925 & 0.1562 & 0.0 \\
\hline 70.0 & & 8.715 & 33.574 & 26.046 & 0.1768 & 0.0 \\
\hline 80.0 & & 8.504 & 33.675 & 26.158 & 0.1961 & 0.0 \\
\hline 90.0 & & 8.442 & 33.784 & 26.253 & 0.2146 & 0.0 \\
\hline 100.0 & & 8.402 & 33.830 & 26.295 & 0.2326 & 0.0 \\
\hline 110.0 & & 8.281 & 33.896 & 26.365 & 0.2499 & 0.0 \\
\hline 120.0 & & 8.144 & 33.937 & 26.418 & 0.2667 & 0.0 \\
\hline 130.0 & & 8.105 & 33.958 & 26.440 & 0.2831 & 0.0 \\
\hline
\end{tabular}
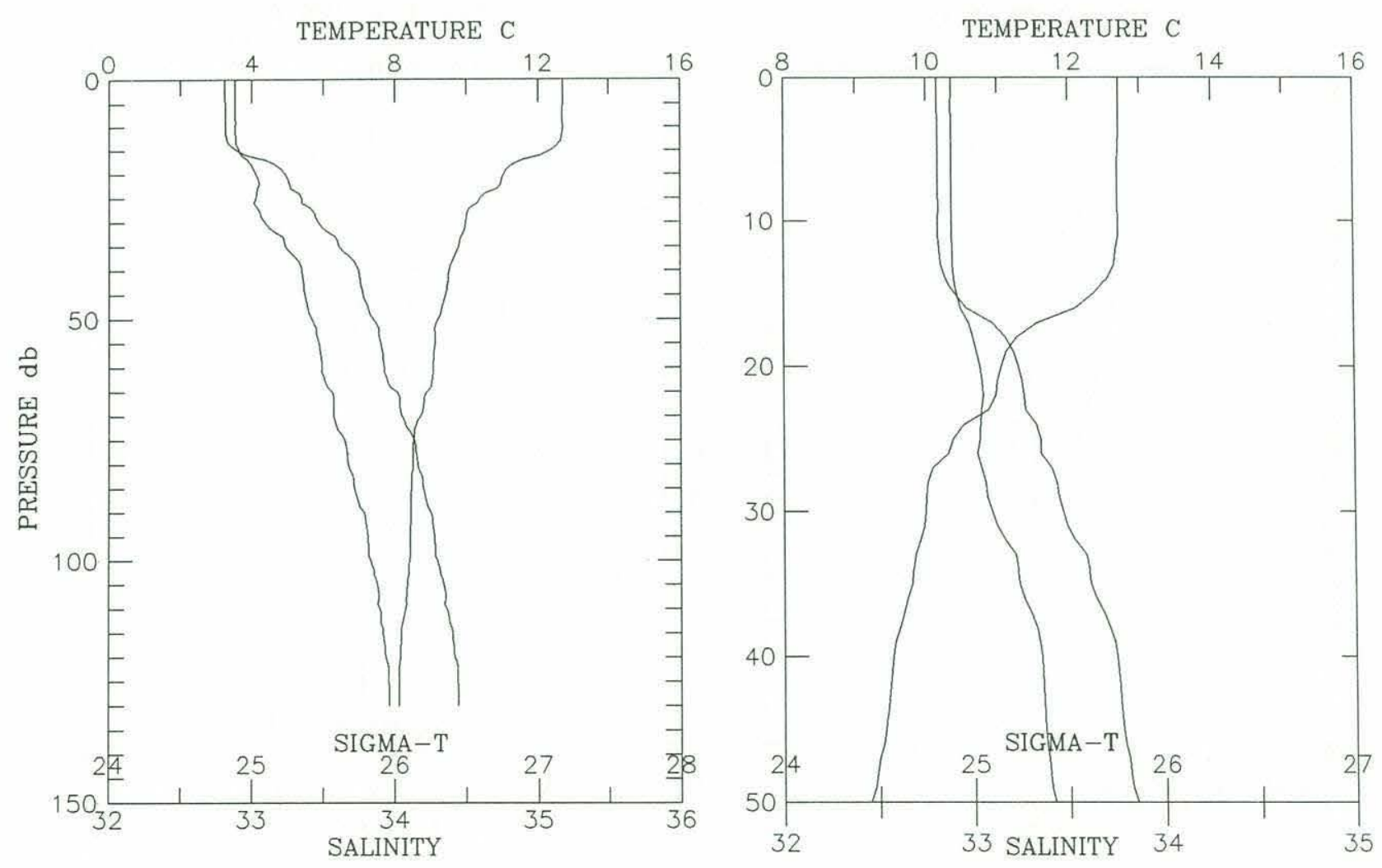
Cruise w8905 Station \# 75 Depth m: 113 Time: 1989-05-09 01:10

Position: $38 \quad 30.90^{\prime} \mathrm{N} 12325.10^{\prime} \mathrm{W}$ Depth Deck Offset: -0.25

\begin{tabular}{|c|c|c|c|c|c|c|}
\hline $\begin{array}{l}\text { PRESSURE } \\
\text { (d-bars) }\end{array}$ & Interp & $\begin{array}{c}\text { TEMP } \\
(\operatorname{deg} C)\end{array}$ & $\begin{array}{c}\text { SALINITY } \\
(0 / 00)\end{array}$ & SIGMA-T & DELTA-D & $\begin{array}{l}\text { CHANNELA } \\
\text { ( }(\delta 1 i g h t)\end{array}$ \\
\hline 0.0 & E & 10.465 & 33.305 & 25.550 & 0.0000 & 0.0 \\
\hline 10.0 & & 10.399 & 33.321 & 25.574 & 0.0245 & 0.0 \\
\hline 20.0 & & 10.136 & 33.368 & 25.655 & 0.0487 & 0.0 \\
\hline 30.0 & & 9.425 & 33.391 & 25.791 & 0.0717 & 0.0 \\
\hline 40.0 & & 8.913 & 33.560 & 26.004 & 0.0929 & 0.0 \\
\hline 50.0 & & 8.661 & 33.582 & 26.061 & 0.1129 & 0.0 \\
\hline 60.0 & & 8.562 & 33.636 & 26.118 & 0.1325 & 0.0 \\
\hline 70.0 & & 8.550 & 33.750 & 26.210 & 0.1514 & 0.0 \\
\hline 80.0 & & 8.493 & 33.838 & 26.287 & 0.1694 & 0.0 \\
\hline 90.0 & & 8.533 & 33.880 & 26.314 & 0.1869 & 0.0 \\
\hline 100.0 & & 8.529 & 33.881 & 26.315 & 0.2045 & 0.0 \\
\hline 107.0 & & 8.526 & 33.881 & 26.316 & 0.2167 & 0.0 \\
\hline
\end{tabular}

TEMPERATURE C
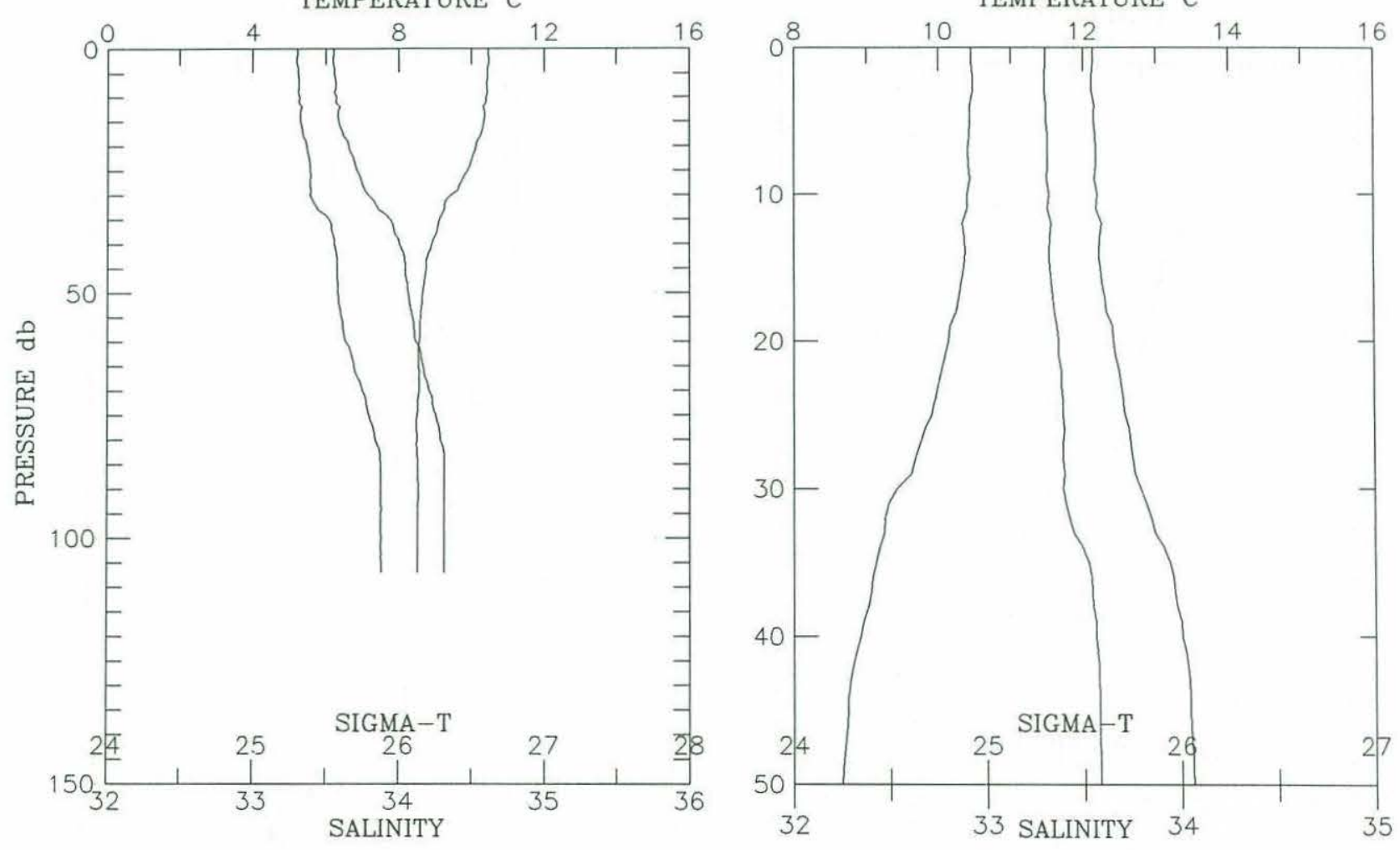
Cruise w8905 Station \# 76 Depth m: 95 Time: 1989-05-09 01:41 Position: 3832.40 'N $12323.00^{\prime} \mathrm{W}$ Depth Deck Offset: -0.25

PRESSURE InterP TEMP SALINITY SIGMA-T DELTA-D CHANNELA (d-bars) ( $\operatorname{deg} C)(0 / 00) \quad$ (olight)

\begin{tabular}{|c|c|c|c|c|c|c|}
\hline 0.0 & $\mathrm{E}$ & 10.058 & 33.428 & 25.715 & 0.0000 & 0.0 \\
\hline 5.0 & & 10.060 & 33.428 & 25.715 & 0.0115 & 0.0 \\
\hline 10.0 & & 10.064 & 33.427 & 25.714 & 0.0230 & 0.0 \\
\hline 15.0 & & 10.018 & 33.428 & 25.722 & 0.0346 & 0.0 \\
\hline 20.0 & & 9.443 & 33.478 & 25.856 & 0.0458 & 0.0 \\
\hline 25.0 & & 9.245 & 33.498 & 25.903 & 0.0565 & 0.0 \\
\hline 30.0 & & 9.133 & 33.509 & 25.930 & 0.0671 & 0.0 \\
\hline 35.0 & & 8.918 & 33.548 & 25.994 & 0.0775 & 0.0 \\
\hline 40.0 & & 8.732 & 33.587 & 26.054 & 0.0875 & 0.0 \\
\hline 45.0 & & 8.583 & 33.635 & 26.114 & 0.0974 & 0.0 \\
\hline 50.0 & & 8.598 & 33.687 & 26.153 & 0.1069 & 0.0 \\
\hline 55.0 & & 8.616 & 33.699 & 26.159 & 0.1164 & 0.0 \\
\hline 60.0 & & 8.666 & 33.722 & 26.170 & 0.1258 & 0.0 \\
\hline 65.0 & & 8.730 & 33.803 & 26.223 & 0.1351 & 0.0 \\
\hline 70.0 & & 8.741 & 33.848 & 26.257 & 0.1442 & 0.0 \\
\hline 75.0 & & 8.726 & 33.855 & 26.265 & 0.1532 & 0.0 \\
\hline 80.0 & & 8.722 & 33.857 & 26.267 & 0.1622 & 0.0 \\
\hline 85.0 & & 8.722 & 33.858 & 26.268 & 0.1711 & 0.0 \\
\hline 90.0 & & 8.722 & 33.858 & 26.268 & 0.1801 & 0.0 \\
\hline 91.0 & & 8.722 & 33.858 & 26.268 & 0.1819 & 0.0 \\
\hline
\end{tabular}
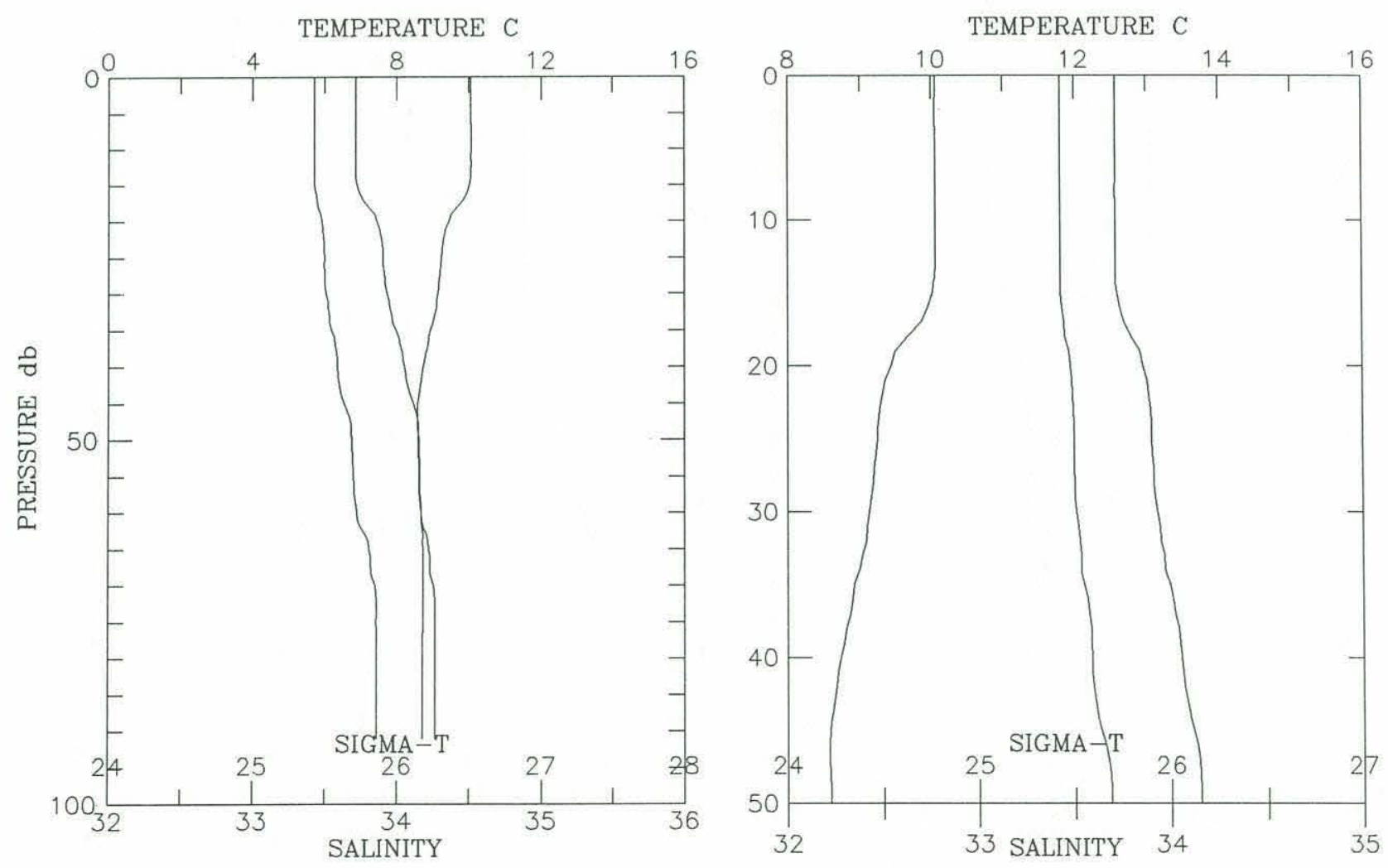
Cruise w8905 Station \# 77 Depth m: 76 Time: 1989-05-09 02:08 Position: $38 \quad 33.70^{\prime} \mathrm{N} 12321.80^{\prime} \mathrm{W}$ Depth Deck Offset: -0.30

\begin{tabular}{|c|c|c|c|c|c|c|}
\hline $\begin{array}{l}\text { PRESSURE } \\
\text { (d-bars) }\end{array}$ & Interp & $\begin{array}{l}\text { TEMP } \\
(\operatorname{deg} C)\end{array}$ & $\begin{array}{c}\text { SALINITY } \\
(0 / 00)\end{array}$ & SIGMA-T & DELTA-D & $\begin{array}{l}\text { CHANNELA } \\
\text { ( }(\text { Ilight) }\end{array}$ \\
\hline 0.0 & E & 10.073 & 33.370 & 25.668 & 0.0000 & 0.0 \\
\hline 5.0 & & 10.070 & 33.369 & 25.667 & 0.0117 & 0.0 \\
\hline 10.0 & & 10.061 & 33.370 & 25.670 & 0.0235 & 0.0 \\
\hline 15.0 & & 10.050 & 33.371 & 25.672 & 0.0352 & 0.0 \\
\hline 20.0 & & 9.780 & 33.393 & 25.735 & 0.0469 & 0.0 \\
\hline 25.0 & & 9.493 & 33.450 & 25.826 & 0.0581 & 0.0 \\
\hline 30.0 & & 9.229 & 33.499 & 25.906 & 0.0688 & 0.0 \\
\hline 35.0 & & 8.998 & 33.530 & 25.968 & 0.0793 & 0.0 \\
\hline 40.0 & & 8.857 & 33.554 & 26.008 & 0.0896 & 0.0 \\
\hline 45.0 & & 8.845 & 33.603 & 26.049 & 0.0996 & 0.0 \\
\hline 50.0 & & 8.924 & 33.670 & 26.089 & 0.1095 & 0.0 \\
\hline 55.0 & & 8.905 & 33.709 & 26.122 & 0.1192 & 0.0 \\
\hline 60.0 & & 8.879 & 33.765 & 26.170 & 0.1287 & 0.0 \\
\hline 65.0 & & 8.790 & 33.823 & 26.230 & 0.1380 & 0.0 \\
\hline 70.0 & & 8.752 & 33.829 & 26.240 & 0.1471 & 0.0 \\
\hline 71.0 & & 8.753 & 33.828 & 26.239 & 0.1489 & 0.0 \\
\hline
\end{tabular}

TEMPERATURE C
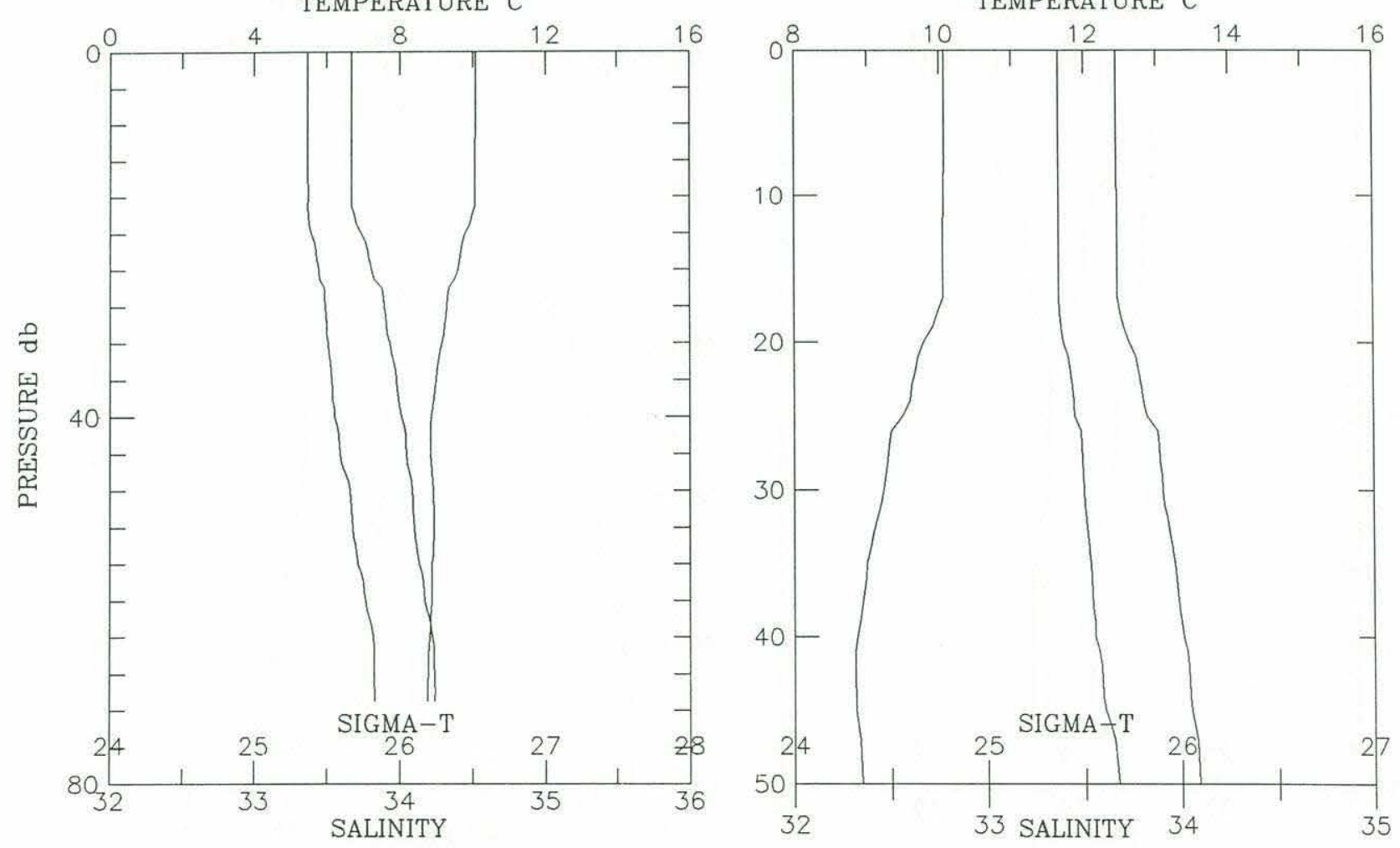
Cruise W8905 Station \# 78 Depth m: 62

Time: 1989-05-09 02:29

Position: $38 \quad 34.00^{\prime} \mathrm{N} 12320.80^{\prime} \mathrm{W}$ Depth Deck Offset: -0.30

\begin{tabular}{|c|c|c|c|c|c|c|}
\hline $\begin{array}{l}\text { PRESSURE } \\
\text { (d-bars) }\end{array}$ & Interp & $\begin{array}{l}\text { TEMP } \\
(\operatorname{deg} C)\end{array}$ & $\begin{array}{c}\text { SALINITY } \\
(0 / 00)\end{array}$ & SIGMA-T & DELTA-D & $\begin{array}{l}\text { CHANNELA } \\
\text { ( } \% 1 \text { ight) }\end{array}$ \\
\hline 0.0 & $\mathrm{E}$ & 10.168 & 33.370 & 25.652 & 0.0000 & 0.0 \\
\hline 5.0 & & 10.169 & 33.369 & 25.651 & 0.0118 & 0.0 \\
\hline 10.0 & & 10.156 & 33.369 & 25.653 & 0.0236 & 0.0 \\
\hline 15.0 & & 10.089 & 33.371 & 25.666 & 0.0354 & 0.0 \\
\hline 20.0 & & 9.697 & 33.414 & 25.765 & 0.0470 & 0.0 \\
\hline 25.0 & & 9.292 & 33.476 & 25.878 & 0.0579 & 0.0 \\
\hline 30.0 & & 9.130 & 33.506 & 25.928 & 0.0686 & 0.0 \\
\hline 35.0 & & 9.108 & 33.571 & 25.982 & 0.0789 & 0.0 \\
\hline 40.0 & & 8.963 & 33.627 & 26.049 & 0.0891 & 0.0 \\
\hline 45.0 & & 8.929 & 33.709 & 26.119 & 0.0989 & 0.0 \\
\hline 50.0 & & 8.775 & 33.786 & 26.203 & 0.1083 & 0.0 \\
\hline 55.0 & & 8.754 & 33.814 & 26.228 & 0.1175 & 0.0 \\
\hline 57.0 & & 8.747 & 33.816 & 26.231 & 0.1211 & 0.0 \\
\hline
\end{tabular}
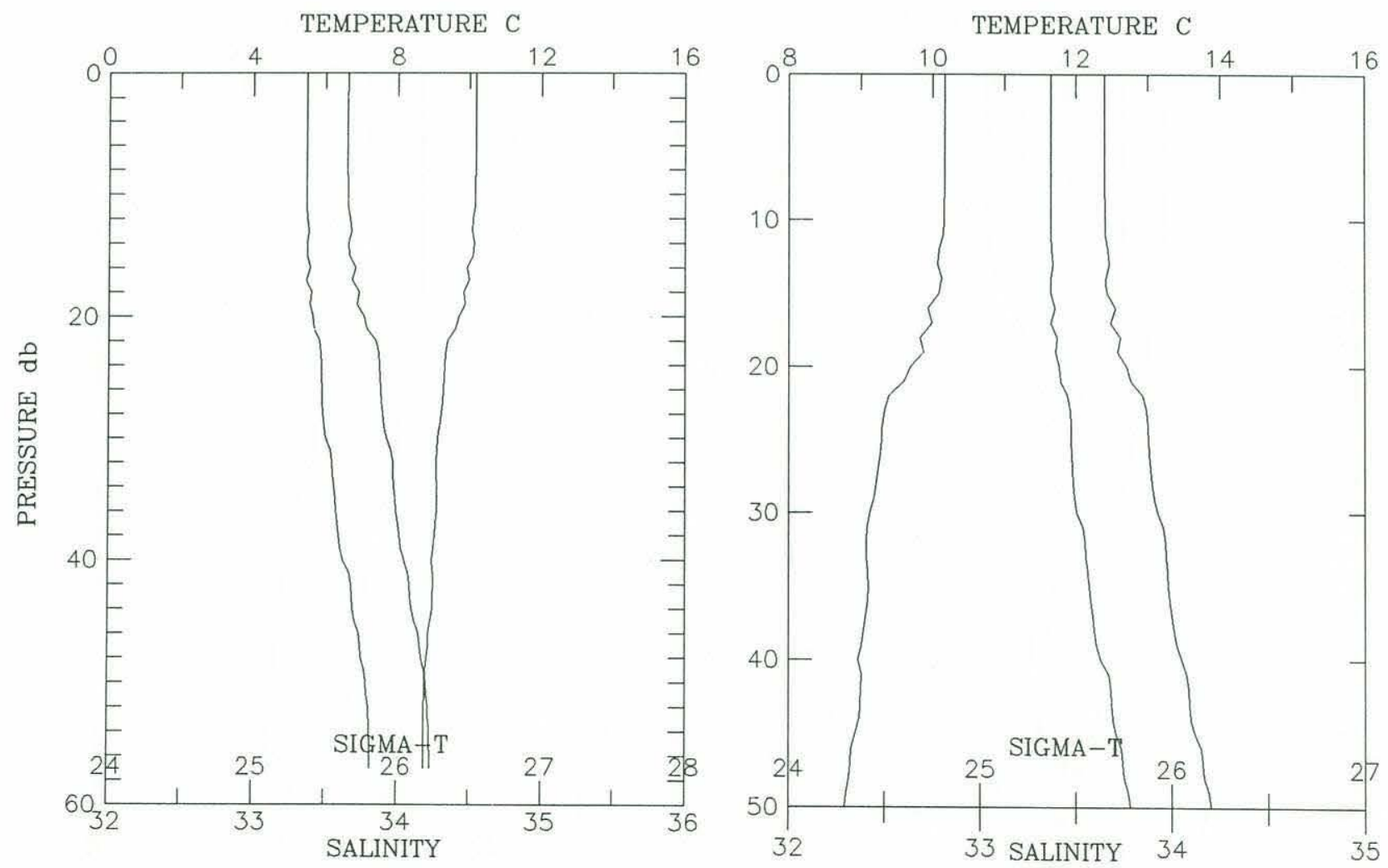
Cruise w8905 Station \# 81 Depth m: 94 Time: 1989-05-09 04:20 Position: $38 \quad 26.30^{\prime} \mathrm{N} 123 \quad 17.00$ ' W Depth Deck Offset: -0.30

\begin{tabular}{|c|c|c|c|c|c|c|}
\hline $\begin{array}{l}\text { PRESSURE } \\
\text { (d-bars) }\end{array}$ & Interp & $\begin{array}{l}\text { TEMP } \\
(\operatorname{deg} C)\end{array}$ & $\begin{array}{l}\text { SALINITY } \\
(0 / 00)\end{array}$ & SIGMA-T & DELTA-D & $\begin{array}{l}\text { CHANNELA } \\
\text { ( } \$ 1 \text { ight) }\end{array}$ \\
\hline 0.0 & E & 10.290 & 33.199 & 25.498 & 0.0000 & 0.0 \\
\hline 5.0 & & 10.276 & 33.198 & 25.499 & 0.0125 & 0.0 \\
\hline 10.0 & & 10.160 & 33.198 & 25.519 & 0.0251 & 0.0 \\
\hline 15.0 & & 9.890 & 33.237 & 25.595 & 0.0374 & 0.0 \\
\hline 20.0 & & 9.599 & 33.274 & 25.671 & 0.0494 & 0.0 \\
\hline 25.0 & & 9.347 & 33.329 & 25.755 & 0.0608 & 0.0 \\
\hline 30.0 & & 9.024 & 33.391 & 25.855 & 0.0719 & 0.0 \\
\hline 35.0 & & 8.817 & 33.409 & 25.901 & 0.0826 & 0.0 \\
\hline 40.0 & & 8.769 & 33.425 & 25.921 & 0.0933 & 0.0 \\
\hline 45.0 & & 8.734 & 33.451 & 25.947 & 0.1038 & 0.0 \\
\hline 50.0 & & 8.637 & 33.495 & 25.996 & 0.1141 & 0.0 \\
\hline 55.0 & & 8.611 & 33.507 & 26.010 & 0.1243 & 0.0 \\
\hline 60.0 & & 8.697 & 33.641 & 26.102 & 0.1342 & 0.0 \\
\hline 65.0 & & 8.641 & 33.666 & 26.130 & 0.1439 & 0.0 \\
\hline 70.0 & & 8.717 & 33.726 & 26.165 & 0.1534 & 0.0 \\
\hline 75.0 & & 8.815 & 33.794 & 26.203 & 0.1628 & 0.0 \\
\hline 80.0 & & 8.798 & 33.837 & 26.239 & 0.1720 & 0.0 \\
\hline 85.0 & & 8.797 & 33.837 & 26.240 & 0.1811 & 0.0 \\
\hline
\end{tabular}

TEMPERATURE C
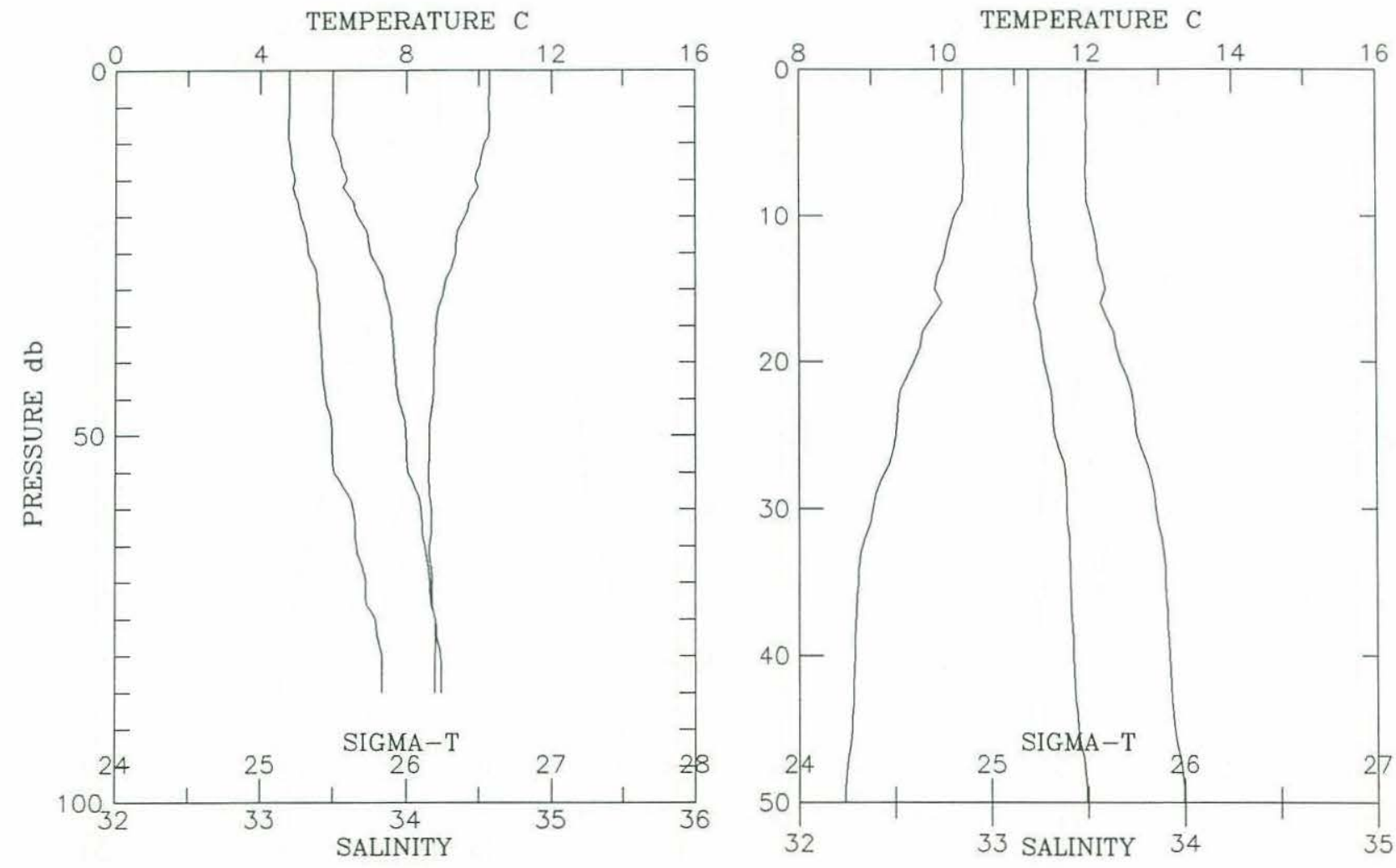


\begin{tabular}{|c|c|c|c|c|c|c|c|}
\hline Cruise W & $\begin{array}{r}8905 \\
\quad 38\end{array}$ & tation \# & 82 Dep & h m: 119 & Time: & 1989-05-09 & $05: 00$ \\
\hline $\begin{array}{l}\text { PRESSURE } \\
\text { (d-bars) }\end{array}$ & Interp & $\begin{array}{l}\text { TEMP } \\
(\operatorname{deg} C)\end{array}$ & $\begin{array}{c}\text { SALINITY } \\
(0 / 00)\end{array}$ & SIGMA-T & DELTA-D & $\begin{array}{l}\text { CHANNELA } \\
\text { ( } \delta 1 \text { ight) }\end{array}$ & \\
\hline 0.0 & E & 12.254 & 32.872 & 24.889 & 0.0000 & 0.0 & \\
\hline 10.0 & & 12.255 & 32.869 & 24.887 & 0.0309 & 0.0 & \\
\hline 20.0 & & 11.539 & 32.810 & 24.974 & 0.0615 & 0.0 & \\
\hline 30.0 & & 10.181 & 32.924 & 25.302 & 0.0892 & 0.0 & \\
\hline 40.0 & & 9.721 & 33.062 & 25.486 & 0.1153 & 0.0 & \\
\hline 50.0 & & 9.696 & 33.290 & 25.668 & 0.1397 & 0.0 & \\
\hline 60.0 & & 9.442 & 33.324 & 25.735 & 0.1630 & 0.0 & \\
\hline 70.0 & & 8.648 & 33.488 & 25.989 & 0.1844 & 0.0 & \\
\hline 80.0 & & 8.670 & 33.683 & 26.139 & 0.2042 & 0.0 & \\
\hline 90.0 & & 8.657 & 33.776 & 26.214 & 0.2229 & 0.0 & \\
\hline 100.0 & & 8.659 & 33.856 & 26.276 & 0.2411 & 0.0 & \\
\hline 110.0 & & 8.659 & 33.857 & 26.277 & 0.2590 & 0.0 & \\
\hline
\end{tabular}
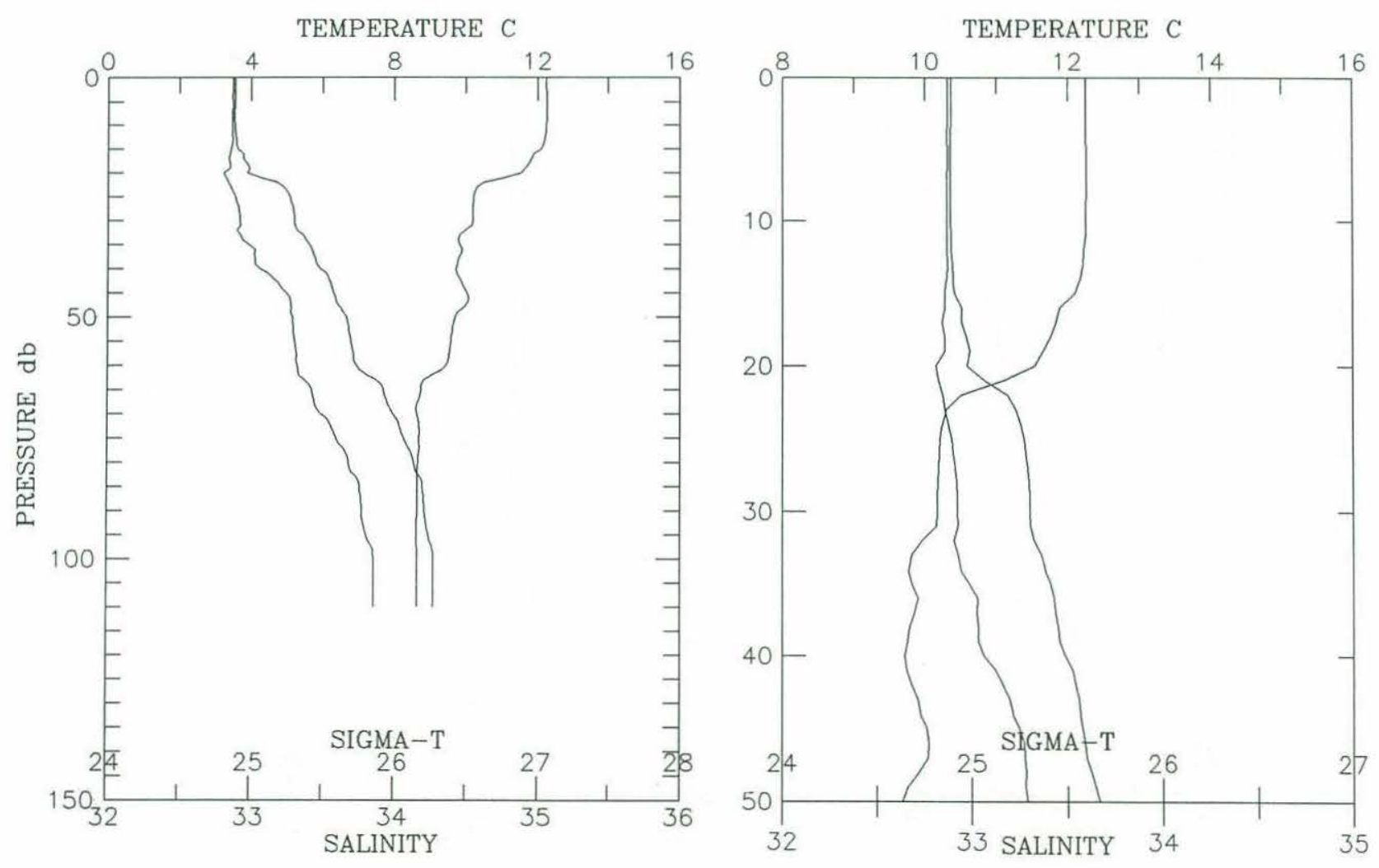
Cruise w8905 Station \# 83 Depth m: 138 Time: 1989-05-09 05:34 Position: $3821.20^{\prime} \mathrm{N} 12323.80^{\prime} \mathrm{W}$ Depth Deck Offset: -0.30

\begin{tabular}{|c|c|c|c|c|c|c|}
\hline $\begin{array}{l}\text { PRESSURE } \\
\text { (d-bars) }\end{array}$ & Interp & $\begin{array}{l}\text { TEMP } \\
(\operatorname{deg} C)\end{array}$ & $\begin{array}{l}\text { SALINITY } \\
(0 / 00)\end{array}$ & SIGMA-T & DELTA-D & $\begin{array}{l}\text { CHANNELA } \\
\text { ( }(\text { ight) }\end{array}$ \\
\hline 0.0 & E & 12.780 & 32.831 & 24.756 & 0.0000 & 0.0 \\
\hline 10.0 & & 12.761 & 32.829 & 24.759 & 0.0321 & 0.0 \\
\hline 20.0 & & 12.633 & 32.822 & 24.778 & 0.0642 & 0.0 \\
\hline 30.0 & & 10.319 & 32.874 & 25.239 & 0.0941 & 0.0 \\
\hline 40.0 & & 9.922 & 33.018 & 25.418 & 0.1207 & 0.0 \\
\hline 50.0 & & 9.999 & 33.277 & 25.608 & 0.1456 & 0.0 \\
\hline 60.0 & & 9.595 & 33.332 & 25.717 & 0.1692 & 0.0 \\
\hline 70.0 & & 8.712 & 33.429 & 25.933 & 0.1914 & 0.0 \\
\hline 80.0 & & 8.587 & 33.521 & 26.024 & 0.2122 & 0.0 \\
\hline 90.0 & & 8.766 & 33.670 & 26.114 & 0.2320 & 0.0 \\
\hline 100.0 & & 8.676 & 33.708 & 26.157 & 0.2512 & 0.0 \\
\hline 110.0 & & 8.429 & 33.846 & 26.303 & 0.2696 & 0.0 \\
\hline 120.0 & & 8.311 & 33.908 & 26.370 & 0.2870 & 0.0 \\
\hline 130.0 & & 8.047 & 33.970 & 26.458 & 0.3037 & 0.0 \\
\hline
\end{tabular}
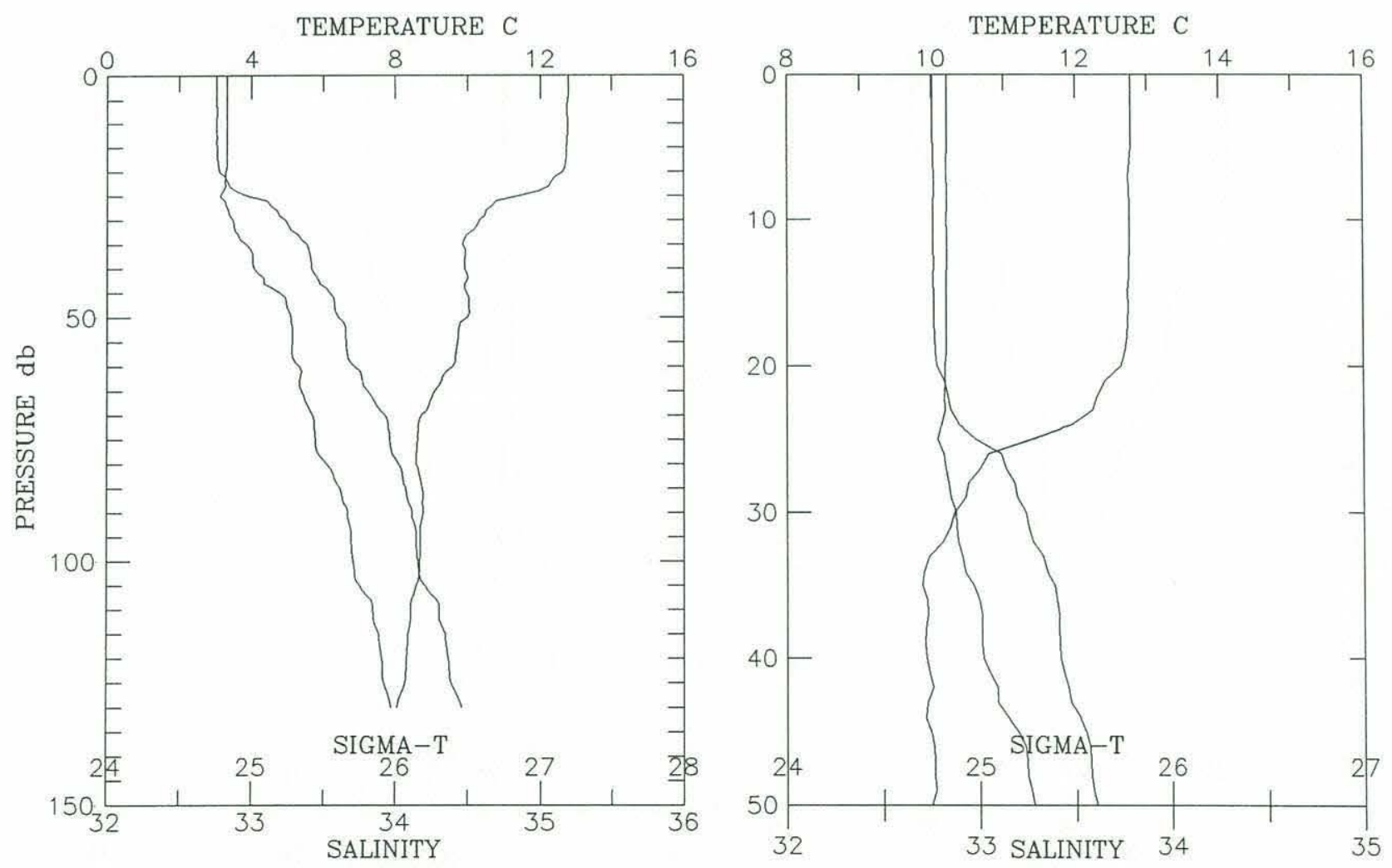
Cruise w8905 Station \# 84 Depth m: 163 Time: 1989-05-09 06:06

Position: $3819.80^{\prime} \mathrm{N} 12325.10^{\prime} \mathrm{W}$ Depth Deck Offset: -0.30

\begin{tabular}{|c|c|c|c|c|c|c|}
\hline $\begin{array}{l}\text { PRESSURE } \\
\text { (d-bars) }\end{array}$ & Interp & $\begin{array}{c}\text { TEMP } \\
(\operatorname{deg} C)\end{array}$ & $\begin{array}{c}\text { SALINITY } \\
(0 / 00)\end{array}$ & SIGMA-T & DELTA-D & $\begin{array}{l}\text { CHANNELA } \\
\text { ( }(\text { ilight) }\end{array}$ \\
\hline 0.0 & E & 13.002 & 32.876 & 24.748 & 0.0000 & 0.0 \\
\hline 10.0 & & 13.004 & 32.875 & 24.747 & 0.0322 & 0.0 \\
\hline 20.0 & & 13.003 & 32.875 & 24.747 & 0.0645 & 0.0 \\
\hline 30.0 & & 11.540 & 32.846 & 25.002 & 0.0963 & 0.0 \\
\hline 40.0 & & 9.916 & 32.858 & 25.294 & 0.1240 & 0.0 \\
\hline 50.0 & & 10.205 & 33.200 & 25.513 & 0.1504 & 0.0 \\
\hline 60.0 & & 9.731 & 33.315 & 25.681 & 0.1747 & 0.0 \\
\hline 70.0 & & 9.109 & 33.336 & 25.798 & 0.1977 & 0.0 \\
\hline 80.0 & & 8.766 & 33.430 & 25.926 & 0.2195 & 0.0 \\
\hline 90.0 & & 8.505 & 33.478 & 26.003 & 0.2404 & 0.0 \\
\hline 100.0 & & 8.475 & 33.614 & 26.115 & 0.2602 & 0.0 \\
\hline 110.0 & & 8.674 & 33.819 & 26.245 & 0.2791 & 0.0 \\
\hline 120.0 & & 8.563 & 33.856 & 26.291 & 0.2970 & 0.0 \\
\hline 130.0 & & 8.458 & 33.901 & 26.342 & 0.3146 & 0.0 \\
\hline 140.0 & & 8.281 & 33.975 & 26.427 & 0.3315 & 0.0 \\
\hline 150.0 & & 8.038 & 33.983 & 26.470 & 0.3479 & 0.0 \\
\hline 154.0 & & 7.884 & 33.992 & 26.500 & 0.3543 & 0.0 \\
\hline
\end{tabular}
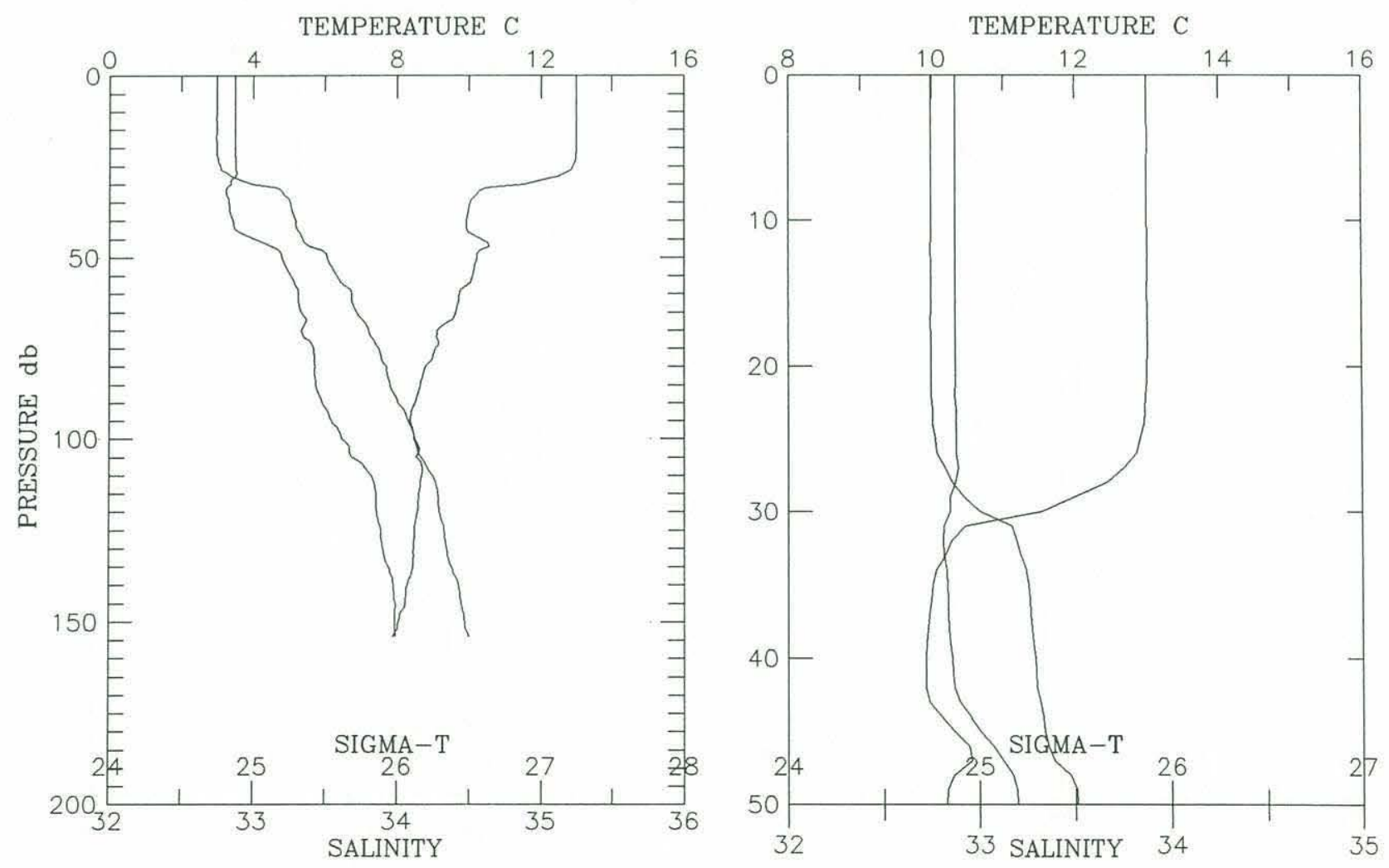


\begin{tabular}{|c|c|c|c|c|c|c|}
\hline $\begin{array}{l}\text { PRESSURE } \\
\text { (d-bars) }\end{array}$ & Interp & $\begin{array}{l}\text { TEMP } \\
(\operatorname{deg} C)\end{array}$ & $\begin{array}{c}\text { SALINITY } \\
(0 / 00)\end{array}$ & SIGMA-T & DELTA-D & $\begin{array}{l}\text { CHANNELA } \\
\text { (\&light) }\end{array}$ \\
\hline 0.0 & $\mathrm{E}$ & 13.225 & 32.853 & 24.686 & 0.0000 & 0.0 \\
\hline 10.0 & & 13.224 & 32.851 & 24.685 & 0.0328 & 0.0 \\
\hline 20.0 & & 13.224 & 32.852 & 24.685 & 0.0657 & 0.0 \\
\hline 30.0 & & 13.087 & 32.863 & 24.721 & 0.0985 & 0.0 \\
\hline 40.0 & & 11.221 & 32.981 & 25.165 & 0.1287 & 0.0 \\
\hline 50.0 & & 10.475 & 33.095 & 25.385 & 0.1557 & 0.0 \\
\hline 60.0 & & 9.576 & 33.129 & 25.561 & 0.1813 & 0.0 \\
\hline 80.0 & & 8.913 & 33.343 & 25.834 & 0.2283 & 0.0 \\
\hline 100.0 & & 9.092 & 33.720 & 26.101 & 0.2703 & 0.0 \\
\hline 150.0 & & 7.747 & 33.969 & 26.501 & 0.3581 & 0.0 \\
\hline 200.0 & & 7.381 & 34.032 & 26.604 & 0.4345 & 0.0 \\
\hline 250.0 & & 7.056 & 34.086 & 26.692 & 0.5075 & 0.0 \\
\hline 300.0 & & 6.773 & 34.121 & 26.758 & 0.5769 & 0.0 \\
\hline 371.0 & & 6.535 & 34.124 & 26.791 & 0.6730 & 0.0 \\
\hline
\end{tabular}
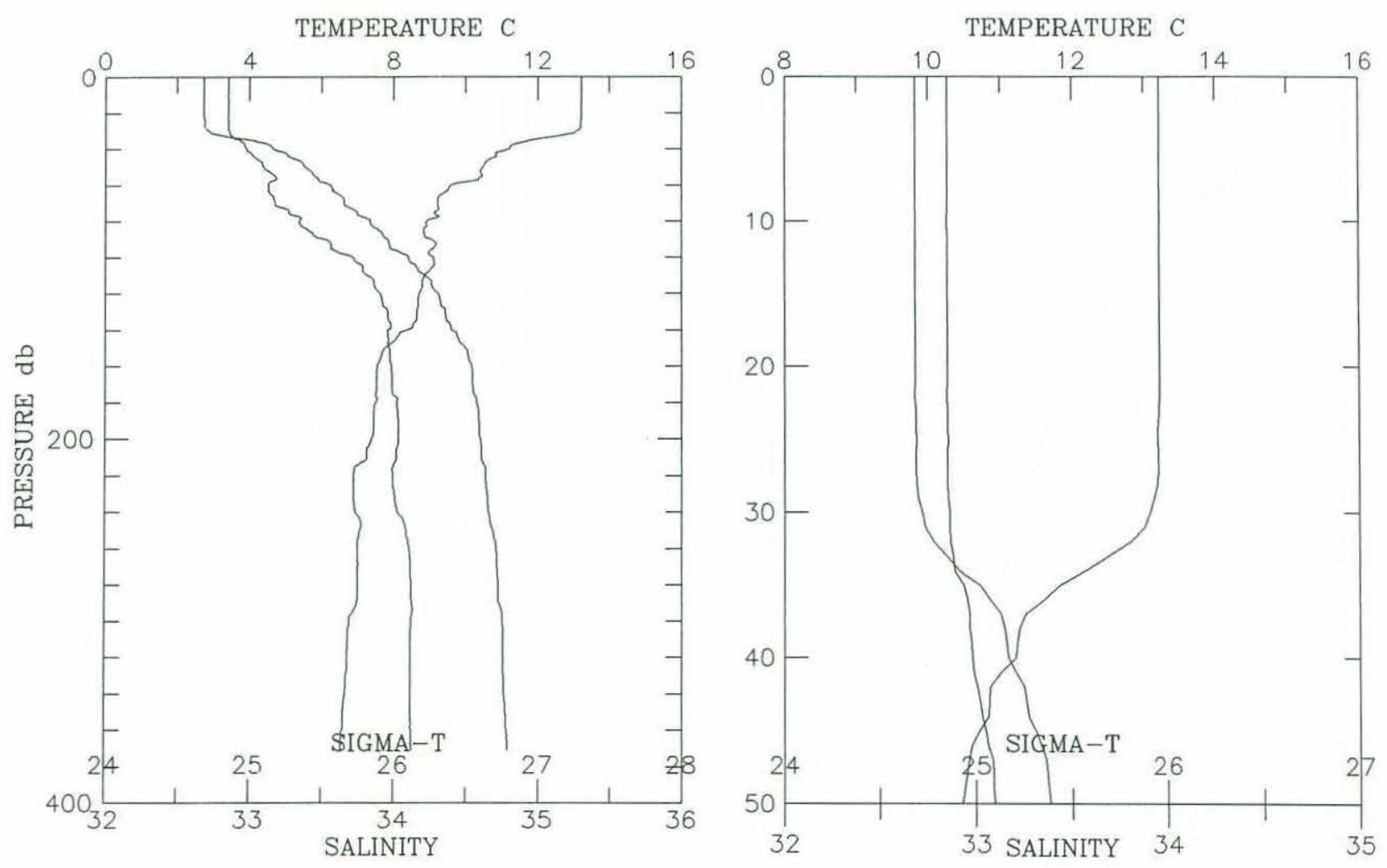
Cruise w8905 Station \# 86 Depth m: 745 Time: 1989-05-09 08:20 Position: $3813.70^{\prime} \mathrm{N} 123 \quad 33.80^{\prime} \mathrm{W}$ Depth Deck Offset: -0.20

\begin{tabular}{|c|c|c|c|c|c|c|}
\hline $\begin{array}{l}\text { PRESSURE } \\
\text { (d-bars) }\end{array}$ & Interp & $\begin{array}{l}\text { TEMP } \\
(\operatorname{deg} C)\end{array}$ & $\begin{array}{l}\text { SALINITY } \\
(0 / 00)\end{array}$ & SIGMA-T & DELTA-D & $\begin{array}{l}\text { CHANNELA } \\
\text { ( } \& 1 \text { ight) }\end{array}$ \\
\hline 0.0 & E & 13.332 & 32.833 & 24.649 & 0.0000 & 0.0 \\
\hline 10.0 & & 13.332 & 32.831 & 24.647 & 0.0332 & 0.0 \\
\hline 20.0 & & 13.227 & 32.837 & 24.673 & 0.0663 & 0.0 \\
\hline 30.0 & & 12.010 & 32.934 & 24.983 & 0.0986 & 0.0 \\
\hline 40.0 & & 11.178 & 33.015 & 25.199 & 0.1276 & 0.0 \\
\hline 50.0 & & 10.813 & 33.025 & 25.271 & 0.1552 & 0.0 \\
\hline 60.0 & & 10.374 & 33.160 & 25.453 & 0.1815 & 0.0 \\
\hline 80.0 & & 9.302 & 33.237 & 25.690 & 0.2306 & 0.0 \\
\hline 100.0 & & 9.023 & 33.554 & 25.982 & 0.2752 & 0.0 \\
\hline 150.0 & & 8.276 & 33.935 & 26.396 & 0.3673 & 0.0 \\
\hline 200.0 & & 7.487 & 34.007 & 26.569 & 0.4467 & 0.0 \\
\hline 250.0 & & 7.282 & 34.065 & 26.644 & 0.5212 & 0.0 \\
\hline 300.0 & & 7.011 & 34.120 & 26.724 & 0.5923 & 0.0 \\
\hline 400.0 & & 6.404 & 34.170 & 26.845 & 0.7262 & 0.0 \\
\hline 500.0 & & 5.977 & 34.207 & 26.929 & 0.8526 & 0.0 \\
\hline 600.0 & & 5.317 & 34.280 & 27.068 & 0.9676 & 0.0 \\
\hline 700.0 & & 4.998 & 34.320 & 27.136 & 1.0743 & 0.0 \\
\hline 737.0 & & 4.756 & 34.343 & 27.182 & 1.1123 & 0.0 \\
\hline
\end{tabular}
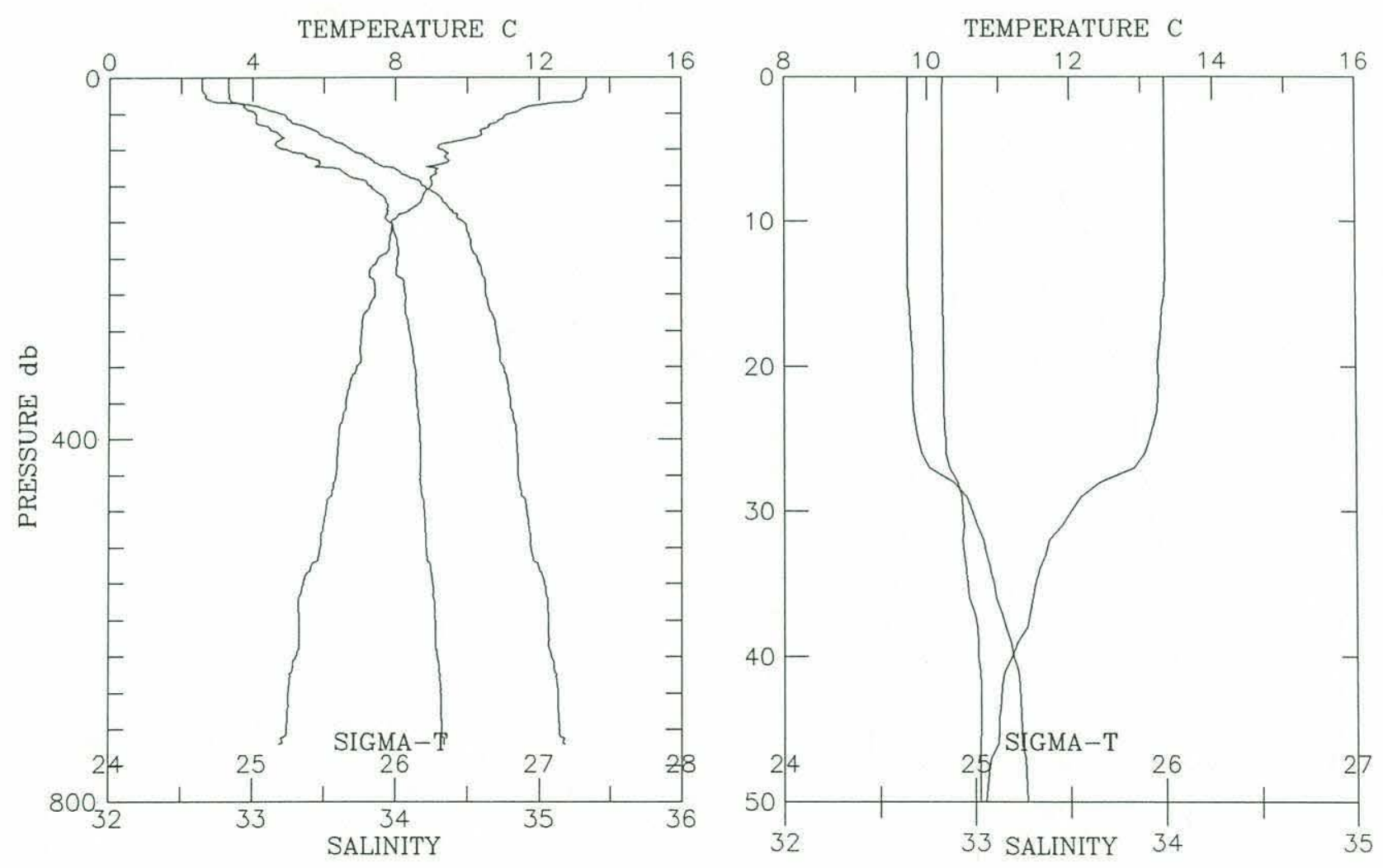
Cruise W8905

Position: 38
Station \# 87 Depth m: 965 $8.10^{\prime} \mathrm{N} 123 \quad 34.10^{\prime} \mathrm{W}$
Time: 1989-05-09 09:35

Depth Deck Offset: $\quad-0.20$

\begin{tabular}{|c|c|c|c|c|c|c|}
\hline $\begin{array}{l}\text { PRESSURE } \\
\text { (d-bars) }\end{array}$ & Interp & $\begin{array}{l}\text { TEMP } \\
(\operatorname{deg} C)\end{array}$ & $\begin{array}{c}\text { SALINITY } \\
(0 / 00)\end{array}$ & SIGMA-T & DELTA-D & $\begin{array}{l}\text { CHANNELA } \\
\text { ( } 81 \text { ight) }\end{array}$ \\
\hline 0.0 & E & 14.062 & 32.842 & 24.508 & 0.0000 & 0.0 \\
\hline 10.0 & & 14.062 & 32.841 & 24.507 & 0.0345 & 0.0 \\
\hline 20.0 & & 14.040 & 32.845 & 24.515 & 0.0691 & 0.0 \\
\hline 30.0 & & 12.206 & 32.991 & 24.991 & 0.1018 & 0.0 \\
\hline 40.0 & & 11.147 & 32.993 & 25.187 & 0.1307 & 0.0 \\
\hline 50.0 & & 10.724 & 33.069 & 25.321 & 0.1582 & 0.0 \\
\hline 60.0 & & 10.820 & 33.259 & 25.452 & 0.1844 & 0.0 \\
\hline 80.0 & & 10.076 & 33.372 & 25.669 & 0.2337 & 0.0 \\
\hline 100.0 & & 9.489 & 33.606 & 25.948 & 0.2786 & 0.0 \\
\hline 150.0 & & 8.266 & 33.923 & 26.389 & 0.3729 & 0.0 \\
\hline 200.0 & & 7.517 & 34.012 & 26.569 & 0.4525 & 0.0 \\
\hline 250.0 & & 7.362 & 34.089 & 26.651 & 0.5269 & 0.0 \\
\hline 300.0 & & 7.040 & 34.106 & 26.710 & 0.5986 & 0.0 \\
\hline 400.0 & & 6.417 & 34.163 & 26.838 & 0.7338 & 0.0 \\
\hline 500.0 & & 5.819 & 34.218 & 26.957 & 0.8573 & 0.0 \\
\hline 600.0 & & 5.432 & 34.267 & 27.043 & 0.9726 & 0.0 \\
\hline 700.0 & & 5.007 & 34.313 & 27.130 & 1.0804 & 0.0 \\
\hline 800.0 & & 4.645 & 34.362 & 27.210 & 1.1806 & 0.0 \\
\hline 900.0 & & 4.465 & 34.386 & 27.249 & 1.2767 & 0.0 \\
\hline 956.0 & & 4.118 & 34.426 & 27.318 & 1.3270 & 0.0 \\
\hline
\end{tabular}

TEMPERATURE C

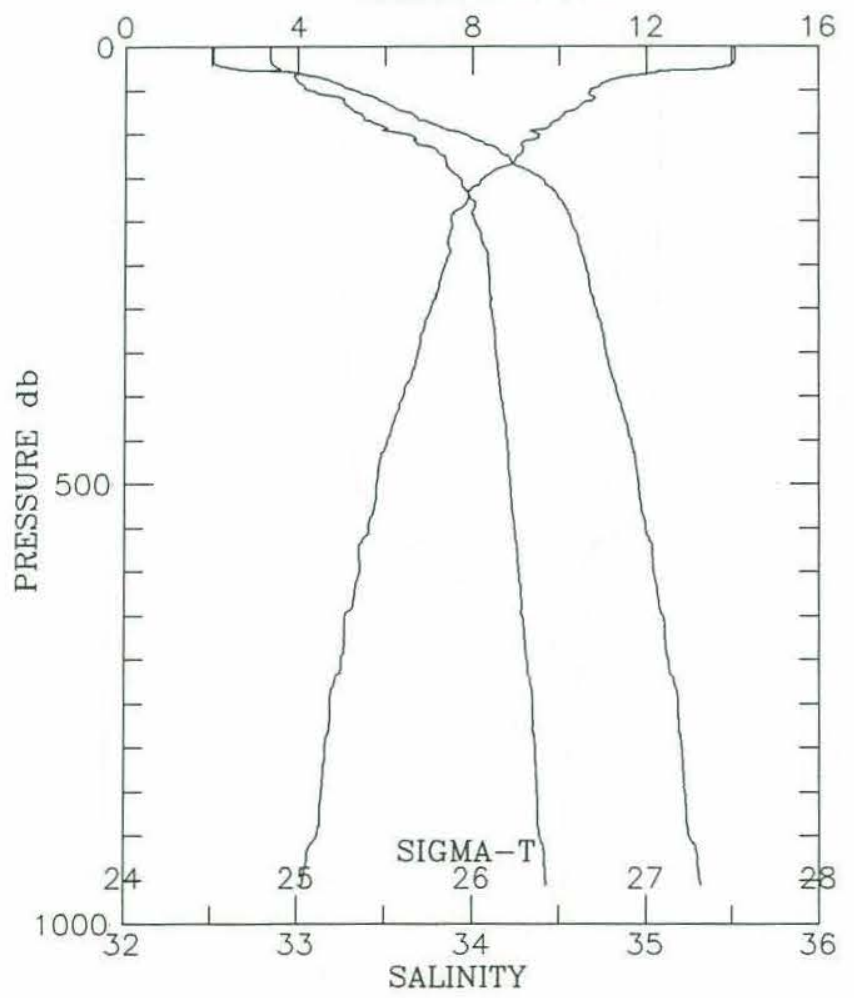

TEMPERATURE C

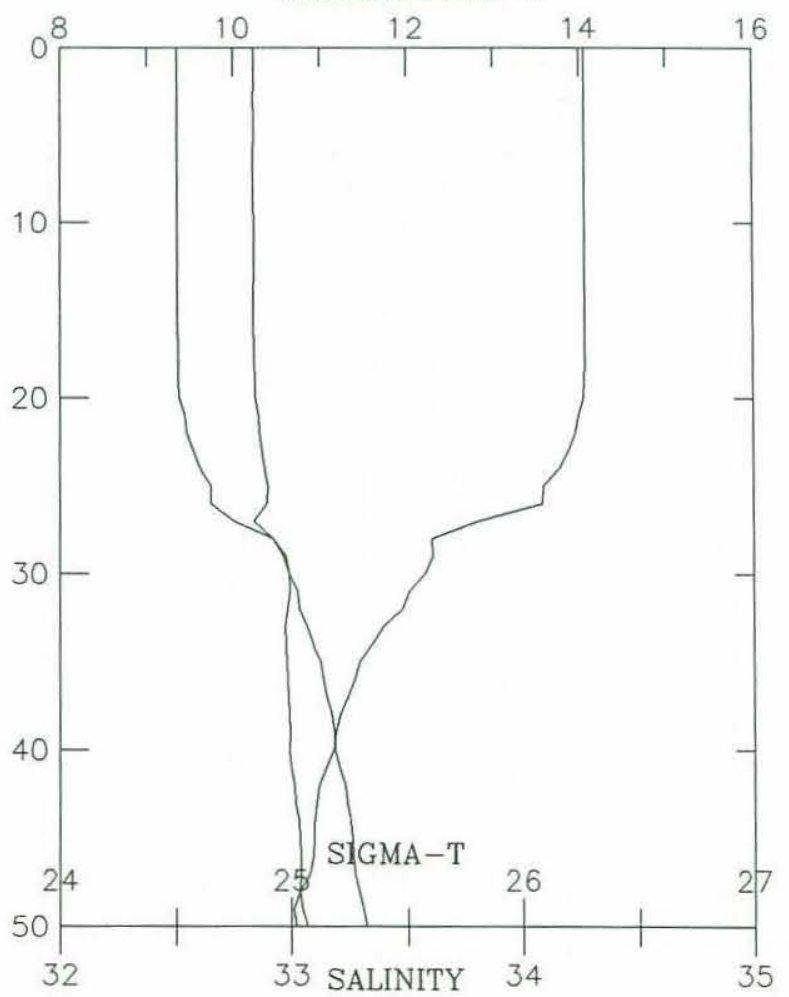


Cruise w8905 Station \# 88 Depth m: 379 Time: 1989-05-09 11:46 Position: $38 \quad 9.30^{\prime} \mathrm{N} 123 \quad 28.20^{\prime} \mathrm{W} \quad$ Depth Deck Offset: $\quad-0.20$

\begin{tabular}{|c|c|c|c|c|c|c|}
\hline $\begin{array}{l}\text { PRESSURE } \\
\text { (d-bars) }\end{array}$ & Interp & $\begin{array}{c}\text { TEMP } \\
(\operatorname{deg} C)\end{array}$ & $\begin{array}{c}\text { SALINITY } \\
(0 / 00)\end{array}$ & SIGMA-T & DELTA-D & $\begin{array}{l}\text { CHANNELA } \\
\text { ( } \delta 1 i g h t)\end{array}$ \\
\hline 0.0 & E & 13.845 & 32.843 & 24.553 & 0.0000 & 0.0 \\
\hline 10.0 & & 13.845 & 32.846 & 24.556 & 0.0341 & 0.0 \\
\hline 20.0 & & 13.847 & 32.851 & 24.559 & 0.0682 & 0.0 \\
\hline 30.0 & & 13.507 & 32.910 & 24.673 & 0.1020 & 0.0 \\
\hline 40.0 & & 11.410 & 32.974 & 25.125 & 0.1325 & 0.0 \\
\hline 50.0 & & 10.913 & 33.116 & 25.325 & 0.1601 & 0.0 \\
\hline 60.0 & & 10.719 & 33.240 & 25.455 & 0.1862 & 0.0 \\
\hline 80.0 & & 10.152 & 33.470 & 25.732 & 0.2352 & 0.0 \\
\hline 100.0 & & 8.905 & 33.494 & 25.954 & 0.2795 & 0.0 \\
\hline 150.0 & & 8.073 & 33.964 & 26.450 & 0.3693 & 0.0 \\
\hline 200.0 & & 7.419 & 34.024 & 26.592 & 0.4464 & 0.0 \\
\hline 250.0 & & 7.221 & 34.078 & 26.662 & 0.5204 & 0.0 \\
\hline 300.0 & & 6.848 & 34.101 & 26.732 & 0.5919 & 0.0 \\
\hline 361.0 & & 6.408 & 34.163 & 26.839 & 0.6715 & 0.0 \\
\hline
\end{tabular}
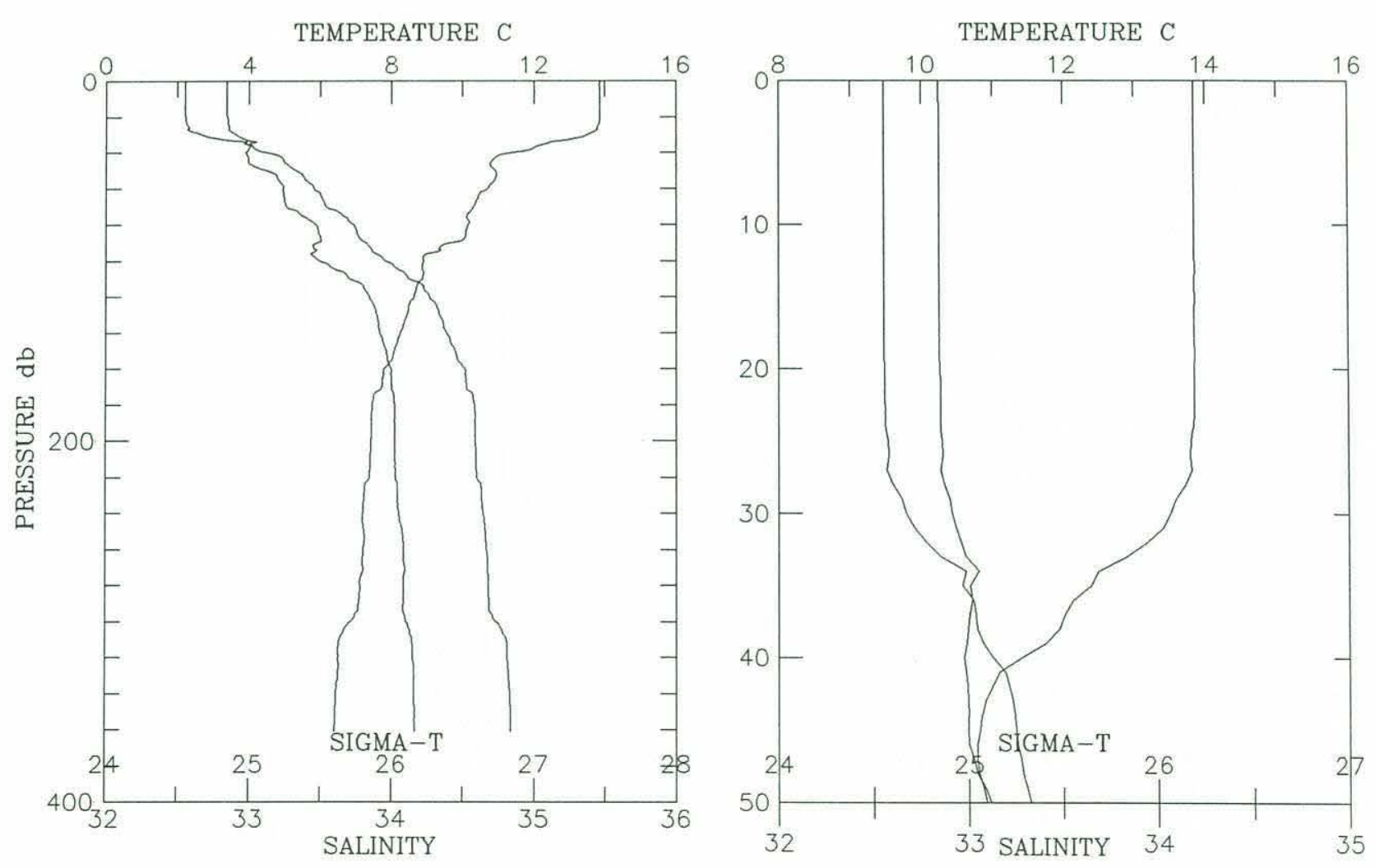
Cruise w8905 Station \# 89 Depth m: 201 Time: 1989-05-09 13:00 Position: 38 10.50'N $12322.10^{\prime} \mathrm{W}$ Depth Deck Offset: -0.20

\begin{tabular}{|c|c|c|c|c|c|c|}
\hline $\begin{array}{l}\text { PRESSURE } \\
\text { (d-bars) }\end{array}$ & Interp & $\begin{array}{c}\text { TEMP } \\
(\operatorname{deg} \quad \mathrm{C})\end{array}$ & $\begin{array}{c}\text { SALINITY } \\
(0 / 00)\end{array}$ & SIGMA-T & DELTA-D & $\begin{array}{l}\text { CHANNELA } \\
\text { ( } \& 1 \text { ight) }\end{array}$ \\
\hline 0.0 & E & 13.091 & 32.862 & 24.719 & 0.0000 & 0.0 \\
\hline 10.0 & & 13.087 & 32.861 & 24.719 & 0.0325 & 0.0 \\
\hline 20.0 & & 13.088 & 32.861 & 24.719 & 0.0651 & 0.0 \\
\hline 30.0 & & 13.068 & 32.865 & 24.726 & 0.0976 & 0.0 \\
\hline 40.0 & & 11.182 & 32.938 & 25.138 & 0.1285 & 0.0 \\
\hline 50.0 & & 10.656 & 33.062 & 25.328 & 0.1562 & 0.0 \\
\hline 60.0 & & 10.610 & 33.256 & 25.487 & 0.1824 & 0.0 \\
\hline 70.0 & & 10.152 & 33.363 & 25.649 & 0.2070 & 0.0 \\
\hline 80.0 & & 9.547 & 33.413 & 25.788 & 0.2302 & 0.0 \\
\hline 90.0 & & 9.439 & 33.447 & 25.832 & 0.2524 & 0.0 \\
\hline 100.0 & & 8.979 & 33.487 & 25.937 & 0.2740 & 0.0 \\
\hline 110.0 & & 8.834 & 33.647 & 26.085 & 0.2946 & 0.0 \\
\hline 120.0 & & 8.738 & 33.739 & 26.172 & 0.3138 & 0.0 \\
\hline 130.0 & & 8.589 & 33.860 & 26.290 & 0.3322 & 0.0 \\
\hline 140.0 & & 8.353 & 33.904 & 26.360 & 0.3497 & 0.0 \\
\hline 150.0 & & 8.066 & 33.931 & 26.425 & 0.3666 & 0.0 \\
\hline 160.0 & & 8.016 & 33.977 & 26.468 & 0.3830 & 0.0 \\
\hline 170.0 & & 7.895 & 33.998 & 26.503 & 0.3990 & 0.0 \\
\hline 180.0 & & 7.515 & 34.038 & 26.589 & 0.4143 & 0.0 \\
\hline 190.0 & & 7.461 & 34.035 & 26.595 & 0.4292 & 0.0 \\
\hline
\end{tabular}
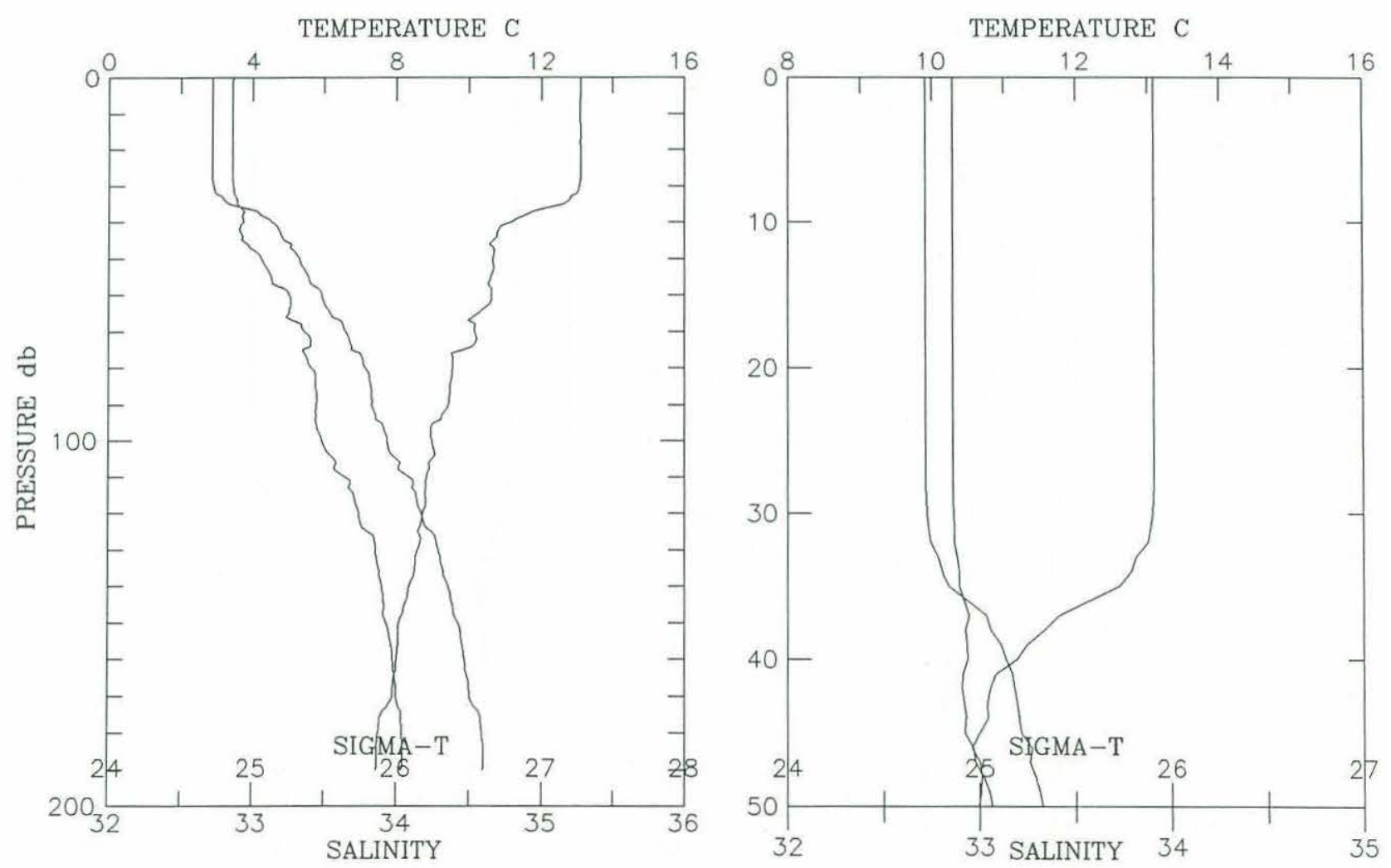
Cruise W8905 Station \# 90 Depth m: 116 Time: 1989-05-09 14:03 Position: $38 \quad 11.60^{\prime} \mathrm{N} 123 \quad 15.90^{\prime} \mathrm{W}$ Depth Deck Offset: -0.20

\begin{tabular}{|c|c|c|c|c|c|c|}
\hline $\begin{array}{l}\text { PRESSURE } \\
\text { (d-bars) }\end{array}$ & Interp & $\begin{array}{l}\text { TEMP } \\
(\operatorname{deg} C)\end{array}$ & $\begin{array}{c}\text { SALINITY } \\
(0 / 00)\end{array}$ & SIGMA-T & DELTA-D & $\begin{array}{l}\text { CHANNELA } \\
\text { ( } \% 1 \text { ight) }\end{array}$ \\
\hline 0.0 & $\mathrm{E}$ & 11.395 & 32.894 & 25.066 & 0.0000 & 0.0 \\
\hline 10.0 & & 11.398 & 32.896 & 25.067 & 0.0292 & 0.0 \\
\hline 20.0 & & 11.385 & 32.889 & 25.064 & 0.0584 & 0.0 \\
\hline 30.0 & & 11.388 & 32.890 & 25.064 & 0.0877 & 0.0 \\
\hline 40.0 & & 11.304 & 32.903 & 25.089 & 0.1170 & 0.0 \\
\hline 50.0 & & 10.014 & 33.046 & 25.425 & 0.1440 & 0.0 \\
\hline 60.0 & & 10.049 & 33.219 & 25.554 & 0.1692 & 0.0 \\
\hline 70.0 & & 9.490 & 33.251 & 25.671 & 0.1932 & 0.0 \\
\hline 80.0 & & 8.979 & 33.354 & 25.833 & 0.2159 & 0.0 \\
\hline 90.0 & & 9.119 & 33.666 & 26.055 & 0.2371 & 0.0 \\
\hline 100.0 & & 8.954 & 33.773 & 26.165 & 0.2566 & 0.0 \\
\hline 107.0 & & 8.941 & 33.778 & 26.171 & 0.2699 & 0.0 \\
\hline
\end{tabular}
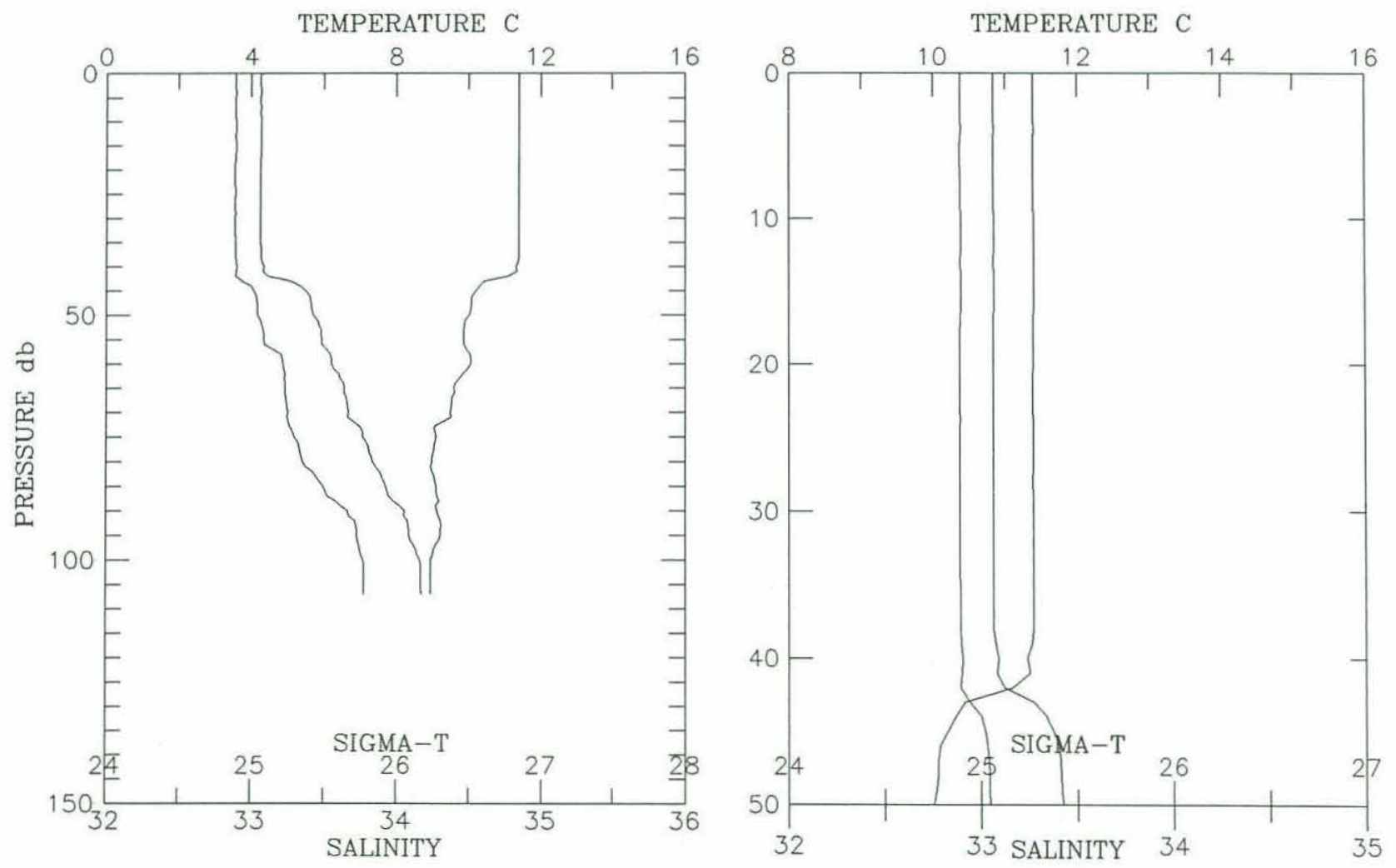


\begin{tabular}{|c|c|c|c|c|c|c|}
\hline $\begin{array}{l}\text { PRESSURE } \\
\text { (d-bars) }\end{array}$ & Interp & $\begin{array}{l}\text { TEMP } \\
(\operatorname{deg} C)\end{array}$ & $\begin{array}{l}\text { SALINITY } \\
(0 / 00)\end{array}$ & SIGMA-T & DELTA-D & $\begin{array}{l}\text { CHANNELA } \\
\text { ( }(1 \mathrm{ight})\end{array}$ \\
\hline 0.0 & $\mathrm{E}$ & 10.664 & 33.108 & 25.362 & 0.0000 & 0.0 \\
\hline 5.0 & & 10.659 & 33.107 & 25.362 & 0.0132 & 0.0 \\
\hline 10.0 & & 10.652 & 33.108 & 25.364 & 0.0264 & 0.0 \\
\hline 15.0 & & 10.644 & 33.109 & 25.367 & 0.0396 & 0.0 \\
\hline 20.0 & & 10.601 & 33.116 & 25.379 & 0.0528 & 0.0 \\
\hline 25.0 & & 10.081 & 33.141 & 25.488 & 0.0657 & 0.0 \\
\hline 30.0 & & 9.594 & 33.241 & 25.646 & 0.0779 & 0.0 \\
\hline 35.0 & & 9.435 & 33.280 & 25.702 & 0.0896 & 0.0 \\
\hline 40.0 & & 9.254 & 33.275 & 25.727 & 0.1012 & 0.0 \\
\hline 45.0 & & 8.804 & 33.303 & 25.820 & 0.1125 & 0.0 \\
\hline 50.0 & & 8.779 & 33.372 & 25.878 & 0.1234 & 0.0 \\
\hline 55.0 & & 8.779 & 33.431 & 25.924 & 0.1341 & 0.0 \\
\hline 60.0 & & 8.855 & 33.500 & 25.967 & 0.1446 & 0.0 \\
\hline 65.0 & & 9.027 & 33.610 & 26.026 & 0.1548 & 0.0 \\
\hline 70.0 & & 8.914 & 33.661 & 26.083 & 0.1648 & 0.0 \\
\hline 75.0 & & 8.847 & 33.759 & 26.171 & 0.1744 & 0.0 \\
\hline 80.0 & & 8.804 & 33.803 & 26.212 & 0.1837 & 0.0 \\
\hline 81.0 & & 8.798 & 33.808 & 26.217 & 0.1856 & 0.0 \\
\hline
\end{tabular}
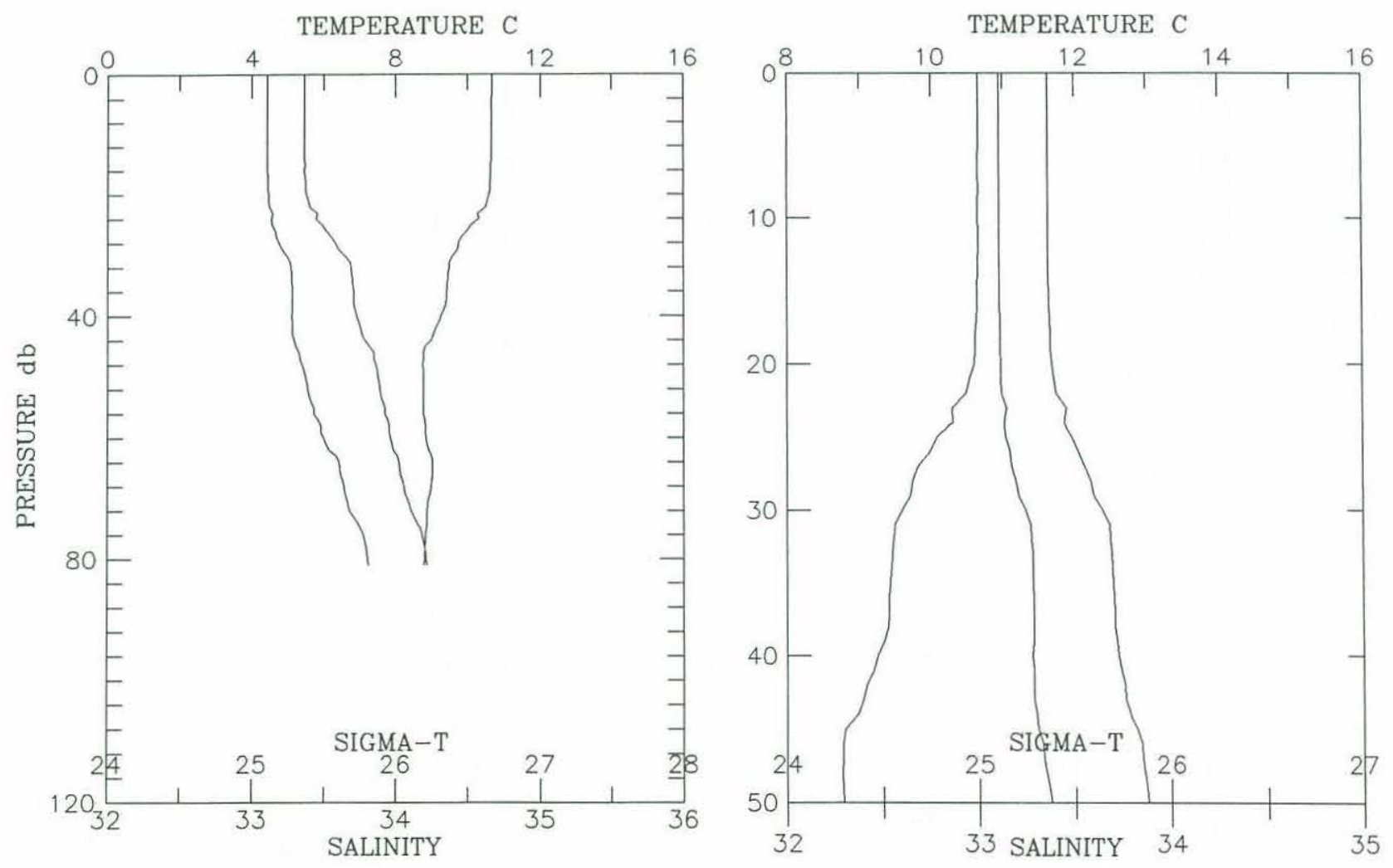
Cruise w8905 Station \# 92 Depth m: 75 Time: 1989-05-09 16:15 Position: $38 \quad 13.80^{\prime} \mathrm{N} 123 \quad 4.60$ ' W Depth Deck Offset: -0.20

\begin{tabular}{|c|c|c|c|c|c|c|}
\hline $\begin{array}{l}\text { PRESSURE } \\
\text { (d-bars) }\end{array}$ & Interp & $\begin{array}{l}\text { TEMP } \\
(\operatorname{deg} C)\end{array}$ & $\begin{array}{c}\text { SALINITY } \\
(0 / 00)\end{array}$ & SIGMA-T & DELTA-D & $\begin{array}{l}\text { CHANNELA } \\
\text { ( } \delta \text { light) }\end{array}$ \\
\hline 0.0 & E & 9.960 & 33.506 & 25.792 & 0.0000 & 0.0 \\
\hline 5.0 & & 9.958 & 33.504 & 25.791 & 0.0112 & 0.0 \\
\hline 10.0 & & 9.952 & 33.504 & 25.792 & 0.0223 & 0.0 \\
\hline 15.0 & & 9.882 & 33.515 & 25.812 & 0.0334 & 0.0 \\
\hline 20.0 & & 9.814 & 33.527 & 25.833 & 0.0445 & 0.0 \\
\hline 25.0 & & 9.803 & 33.529 & 25.836 & 0.0554 & 0.0 \\
\hline 30.0 & & 9.712 & 33.535 & 25.856 & 0.0664 & 0.0 \\
\hline 35.0 & & 9.213 & 33.547 & 25.947 & 0.0771 & 0.0 \\
\hline 40.0 & & 8.923 & 33.634 & 26.061 & 0.0873 & 0.0 \\
\hline 45.0 & & 8.988 & 33.705 & 26.106 & 0.0971 & 0.0 \\
\hline 50.0 & & 8.927 & 33.761 & 26.160 & 0.1066 & 0.0 \\
\hline 55.0 & & 8.891 & 33.775 & 26.176 & 0.1160 & 0.0 \\
\hline 60.0 & & 8.888 & 33.774 & 26.176 & 0.1254 & 0.0 \\
\hline 65.0 & & 8.887 & 33.775 & 26.177 & 0.1348 & 0.0 \\
\hline 66.0 & & 8.887 & 33.775 & 26.177 & 0.1366 & 0.0 \\
\hline
\end{tabular}
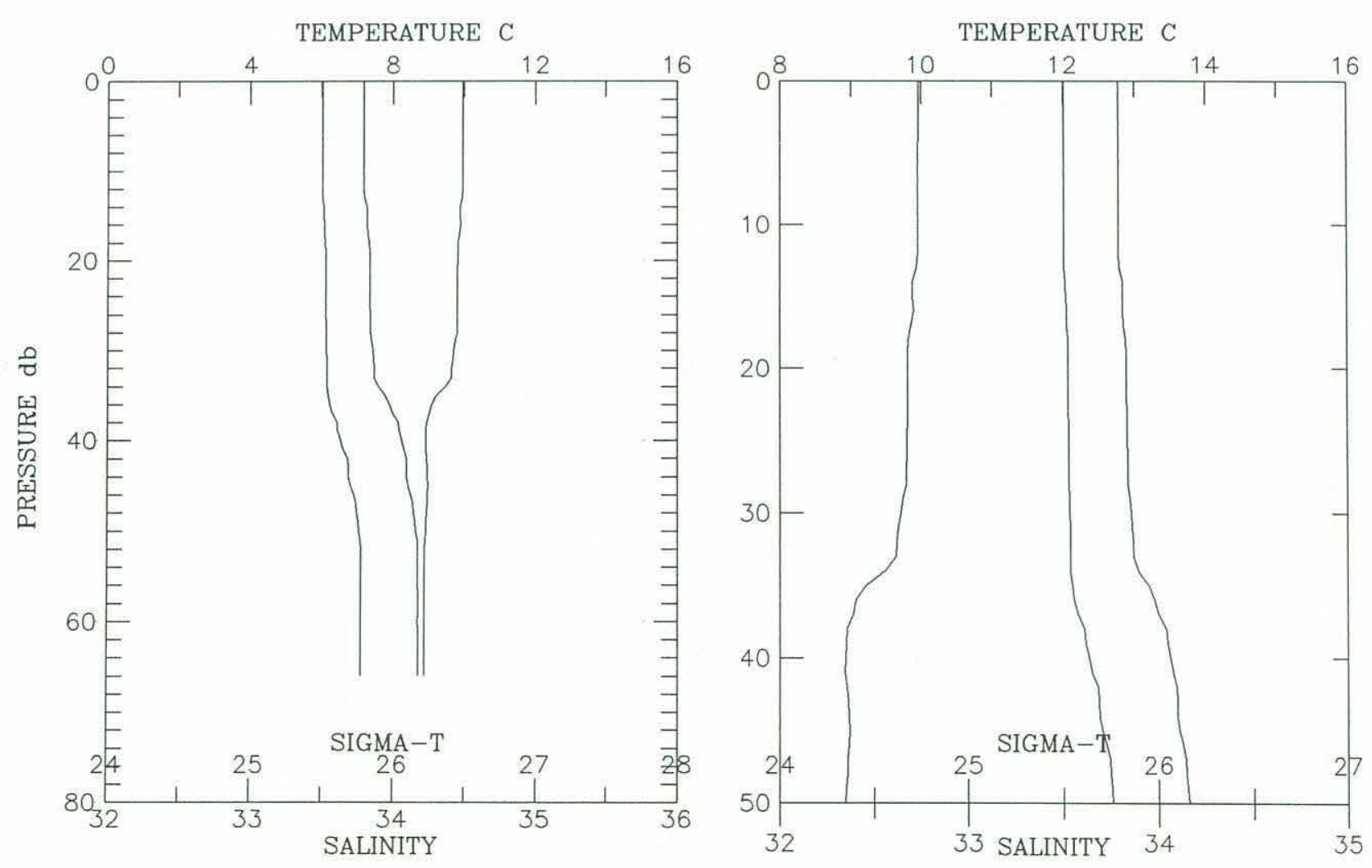
Cruise W8905 Station \# 93 Depth m: 61 Time: 1989-05-09 16:43 Position: $38 \quad 14.10^{\prime} \mathrm{N} 123 \quad 2.20^{\prime} \mathrm{W}$ Depth Deck Offset: -0.20

\begin{tabular}{|c|c|c|c|c|c|c|}
\hline $\begin{array}{l}\text { PRESSURE } \\
\text { (d-bars) }\end{array}$ & Interp & $\begin{array}{l}\text { TEMP } \\
(\operatorname{deg} C)\end{array}$ & $\begin{array}{c}\text { SALINITY } \\
(0 / 00)\end{array}$ & SIGMA-T & DELTA-D & $\begin{array}{l}\text { CHANNELA } \\
\text { (\&light) }\end{array}$ \\
\hline 0.0 & E & 10.000 & 33.489 & 25.772 & 0.0000 & 0.0 \\
\hline 5.0 & & 9.996 & 33.488 & 25.772 & 0.0112 & 0.0 \\
\hline 10.0 & & 9.992 & 33.490 & 25.774 & 0.0225 & 0.0 \\
\hline 15.0 & & 9.991 & 33.495 & 25.778 & 0.0337 & 0.0 \\
\hline 20.0 & & 9.974 & 33.501 & 25.786 & 0.0449 & 0.0 \\
\hline 25.0 & & 9.932 & 33.500 & 25.792 & 0.0561 & 0.0 \\
\hline 30.0 & & 9.031 & 33.607 & 26.023 & 0.0668 & 0.0 \\
\hline 35.0 & & 9.013 & 33.666 & 26.072 & 0.0768 & 0.0 \\
\hline 40.0 & & 8.983 & 33.688 & 26.094 & 0.0866 & 0.0 \\
\hline 45.0 & & 8.979 & 33.696 & 26.101 & 0.0963 & 0.0 \\
\hline 50.0 & & 8.977 & 33.709 & 26.111 & 0.1060 & 0.0 \\
\hline
\end{tabular}
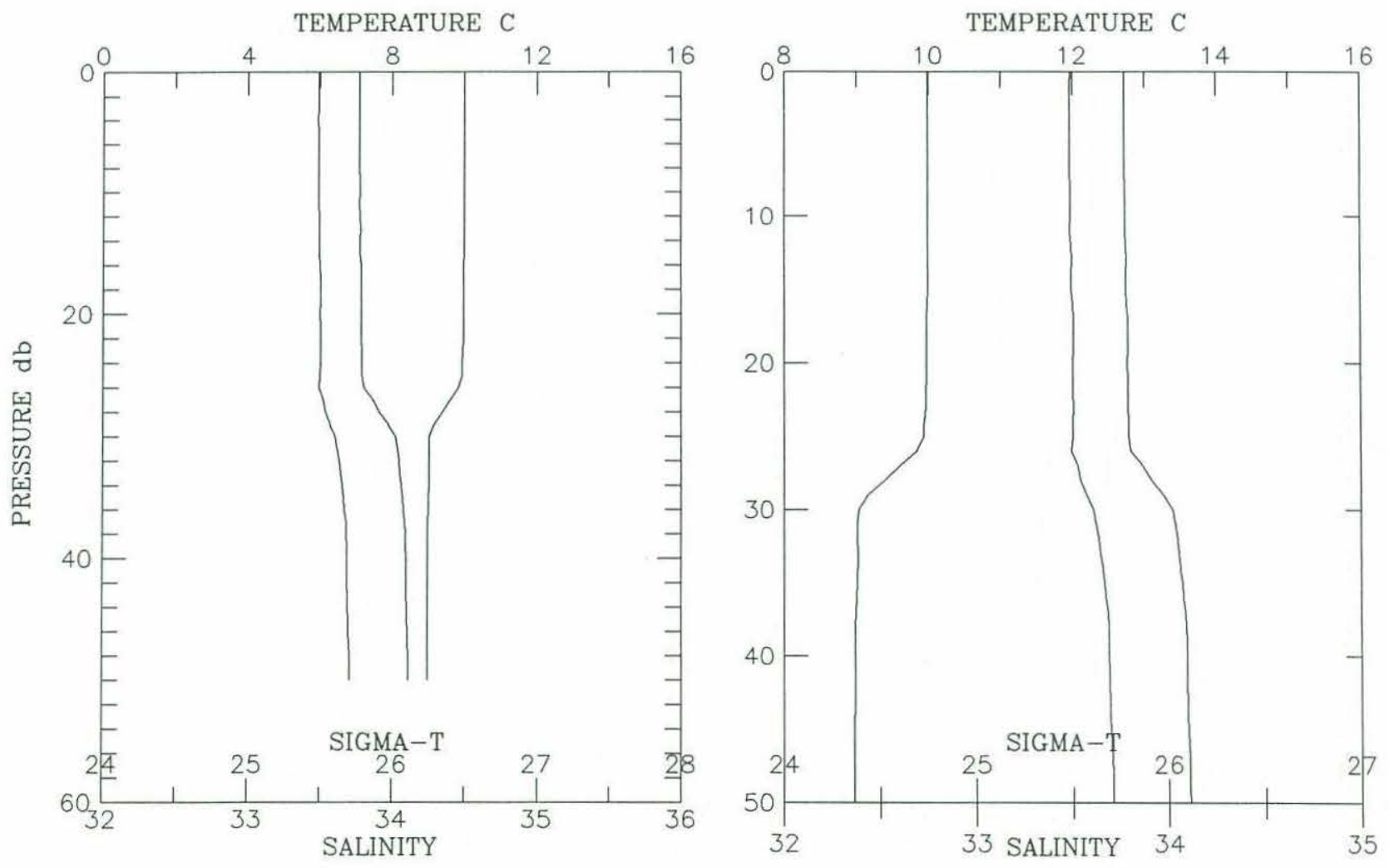


\begin{tabular}{|c|c|c|c|c|c|c|}
\hline \multicolumn{2}{|c|}{ Cruise W8905 } & tation \# & \multicolumn{2}{|r|}{$\mathrm{m}: 78$} & Time: & \multirow{2}{*}{$\begin{array}{l}1989-05-10 \\
\text { Offset: }\end{array}$} \\
\hline Position & 38 & $.90^{\prime} \mathrm{N}$ & 27.50 & & epth Decl & \\
\hline $\begin{array}{l}\text { PRESSURE } \\
\text { (d-bars) }\end{array}$ & Interp & $\begin{array}{l}\text { TEMP } \\
(\operatorname{deg} C)\end{array}$ & $\begin{array}{c}\text { SALINITY } \\
(0 / 00)\end{array}$ & SIGMA-T & DELTA-D & $\begin{array}{l}\text { CHANNELA } \\
\text { ( } \% 1 \text { ight) }\end{array}$ \\
\hline 0.0 & $\mathrm{E}$ & 9.009 & 33.697 & 26.097 & 0.0000 & 0.0 \\
\hline 5.0 & & 9.005 & 33.699 & 26.099 & 0.0097 & 0.0 \\
\hline 10.0 & & 9.006 & 33.700 & 26.099 & 0.0194 & 0.0 \\
\hline 15.0 & & 9.005 & 33.700 & 26.100 & 0.0291 & 0.0 \\
\hline 20.0 & & 9.006 & 33.700 & 26.099 & 0.0388 & 0.0 \\
\hline 25.0 & & 9.001 & 33.699 & 26.099 & 0.0485 & 0.0 \\
\hline 30.0 & & 8.982 & 33.701 & 26.104 & 0.0582 & 0.0 \\
\hline 35.0 & & 8.824 & 33.751 & 26.168 & 0.0678 & 0.0 \\
\hline 40.0 & & 8.626 & 33.831 & 26.261 & 0.0770 & 0.0 \\
\hline 45.0 & & 8.643 & 33.858 & 26.280 & 0.0859 & 0.0 \\
\hline 50.0 & & 8.653 & 33.866 & 26.285 & 0.0948 & 0.0 \\
\hline 55.0 & & 8.659 & 33.874 & 26.290 & 0.1036 & 0.0 \\
\hline 60.0 & & 8.650 & 33.881 & 26.297 & 0.1124 & 0.0 \\
\hline 65.0 & & 8.644 & 33.882 & 26.299 & 0.1213 & 0.0 \\
\hline 70.0 & & 8.639 & 33.882 & 26.299 & 0.1301 & 0.0 \\
\hline 73.0 & & 8.639 & 33.882 & 26.300 & 0.1353 & 0.0 \\
\hline
\end{tabular}
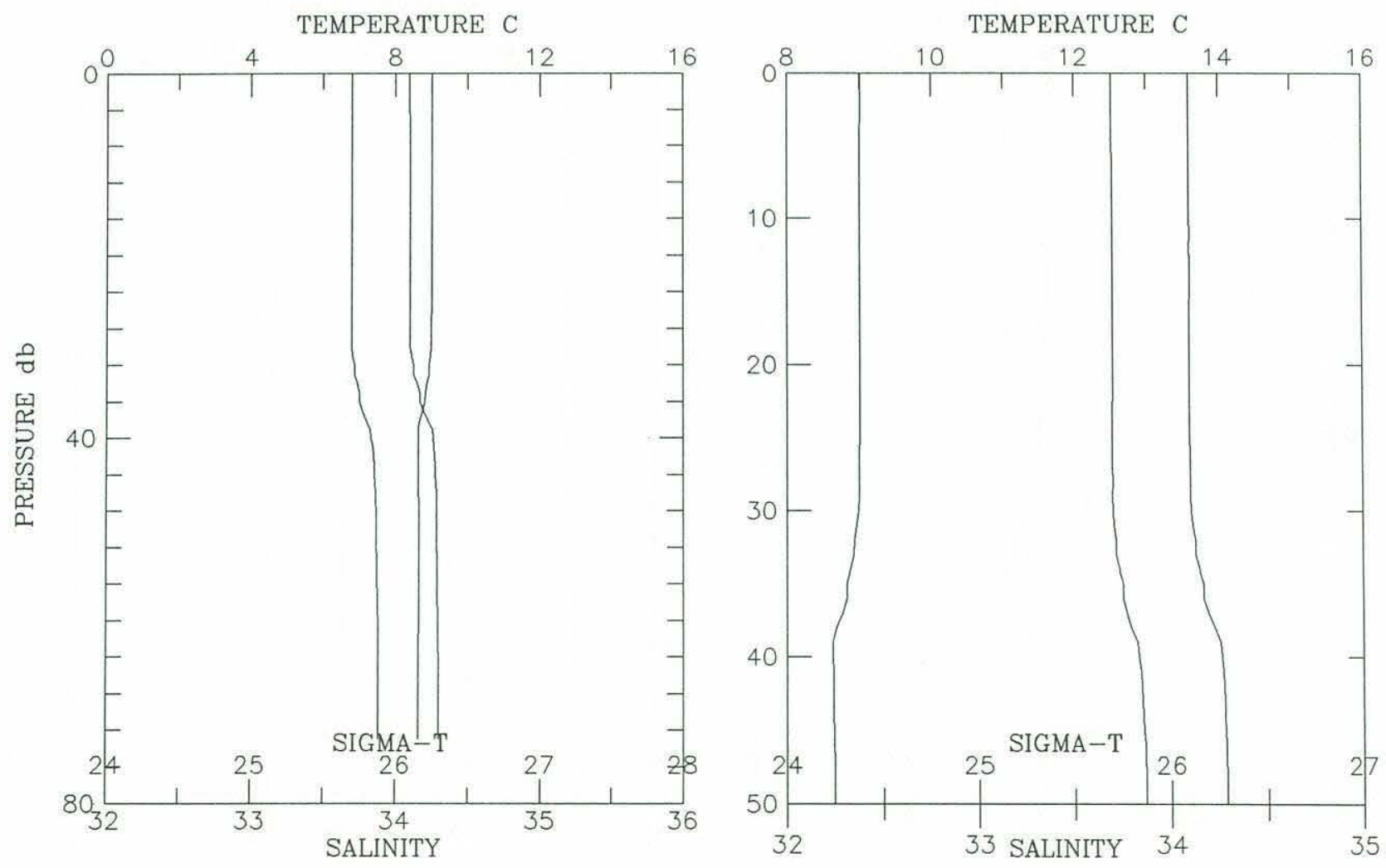
Cruise W8905 Station \# 95 Depth m: 93 Time: 1989-05-10 11:38

Position: $38 \quad 38.70^{\prime} \mathrm{N} 12329.10^{\prime} \mathrm{W}$ Depth Deck Offset: -0.38

\begin{tabular}{|c|c|c|c|c|c|c|}
\hline $\begin{array}{l}\text { PRESSURE } \\
\text { (d-bars) }\end{array}$ & Interp & $\begin{array}{c}\text { TEMP } \\
(\operatorname{deg} C)\end{array}$ & $\begin{array}{l}\text { SALINITY } \\
(0 / 00)\end{array}$ & SIGMA-T & DELTA-D & $\begin{array}{l}\text { CHANNELA } \\
\text { ( }(1 \text { ight) }\end{array}$ \\
\hline 0.0 & E & 9.264 & 33.590 & 25.972 & 0.0000 & 0.0 \\
\hline 5.0 & & 9.260 & 33.593 & 25.975 & 0.0103 & 0.0 \\
\hline 10.0 & & 9.268 & 33.589 & 25.971 & 0.0206 & 0.0 \\
\hline 15.0 & & 9.251 & 33.598 & 25.981 & 0.0309 & 0.0 \\
\hline 20.0 & & 9.234 & 33.606 & 25.990 & 0.0411 & 0.0 \\
\hline 25.0 & & 9.227 & 33.611 & 25.995 & 0.0514 & 0.0 \\
\hline 30.0 & & 9.225 & 33.615 & 25.998 & 0.0616 & 0.0 \\
\hline 35.0 & & 9.151 & 33.624 & 26.017 & 0.0718 & 0.0 \\
\hline 40.0 & & 9.076 & 33.637 & 26.039 & 0.0818 & 0.0 \\
\hline 45.0 & & 9.063 & 33.637 & 26.041 & 0.0919 & 0.0 \\
\hline 50.0 & & 9.042 & 33.641 & 26.048 & 0.1018 & 0.0 \\
\hline 55.0 & & 8.956 & 33.658 & 26.074 & 0.1118 & 0.0 \\
\hline 60.0 & & 8.781 & 33.716 & 26.147 & 0.1215 & 0.0 \\
\hline 65.0 & & 8.554 & 33.781 & 26.233 & 0.1308 & 0.0 \\
\hline 70.0 & & 8.473 & 33.835 & 26.288 & 0.1398 & 0.0 \\
\hline 75.0 & & 8.487 & 33.890 & 26.329 & 0.1485 & 0.0 \\
\hline 80.0 & & 8.388 & 33.907 & 26.358 & 0.1571 & 0.0 \\
\hline 85.0 & & 8.307 & 33.926 & 26.385 & 0.1656 & 0.0 \\
\hline 88.0 & & 8.286 & 33.931 & 26.392 & 0.1706 & 0.0 \\
\hline
\end{tabular}

TEMPERATURE C
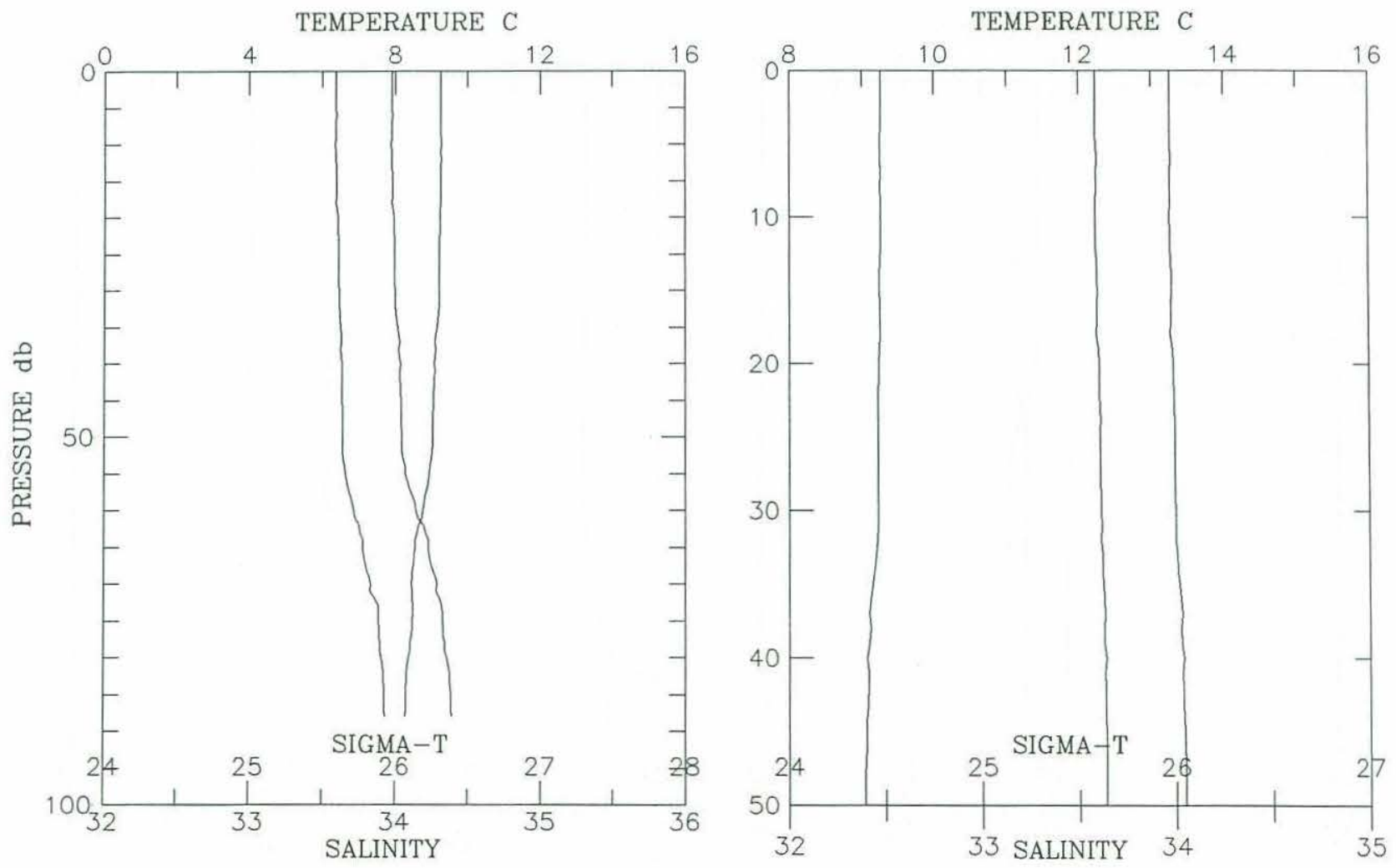
Cruise w8905 Station \# 96 Depth m: 118 Time: 1989-05-10 12:20

Position: $38 \quad 36.67^{\prime} \mathrm{N} 123 \quad 31.63^{\prime} \mathrm{W} \quad$ Depth Deck Offset: -0.38

PRESSURE Interp TEMP SALINITY SIGMA-T DELTA-D CHANNELA

(d-bars)

$(\operatorname{deg} C)(0 / 00) \quad$ (\&light)

\begin{tabular}{|c|c|c|c|c|c|c|}
\hline 0.0 & $\mathrm{E}$ & 9.750 & 33.401 & 25.745 & 0.0000 & 0.0 \\
\hline 10.0 & & 9.750 & 33.400 & 25.745 & 0.0228 & 0.0 \\
\hline 20.0 & & 9.597 & 33.429 & 25.792 & 0.0455 & 0.0 \\
\hline 30.0 & & 8.941 & 33.641 & 26.064 & 0.0659 & 0.0 \\
\hline 40.0 & & 8.828 & 33.696 & 26.124 & 0.0853 & 0.0 \\
\hline 50.0 & & 8.803 & 33.738 & 26.161 & 0.1044 & 0.0 \\
\hline 60.0 & & 8.491 & 33.800 & 26.258 & 0.1228 & 0.0 \\
\hline 70.0 & & 8.388 & 33.830 & 26.297 & 0.1407 & 0.0 \\
\hline 80.0 & & 8.227 & 33.886 & 26.365 & 0.1580 & 0.0 \\
\hline 90.0 & & 8.173 & 33.929 & 26.407 & 0.1748 & 0.0 \\
\hline 100.0 & & 8.055 & 33.971 & 26.458 & 0.1912 & 0.0 \\
\hline 110.0 & & 7.896 & 33.999 & 26.503 & 0.2072 & 0.0 \\
\hline 114.0 & & 7.841 & 34.006 & 26.517 & 0.2134 & 0.0 \\
\hline
\end{tabular}

TEMPERATURE C
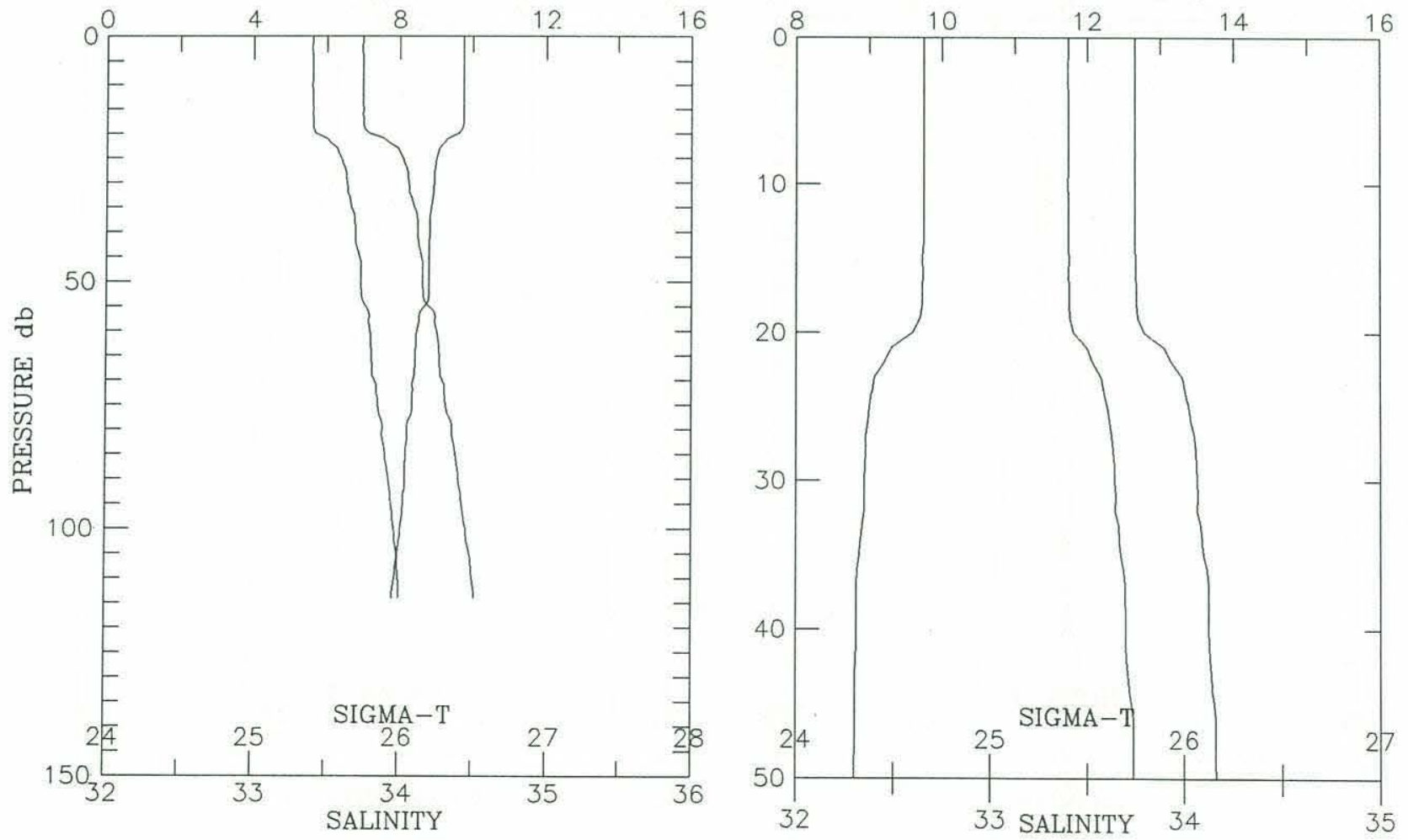
Cruise w8905 Station \# 97 Depth m: 141 Time: 1989-05-10 12:51 Position: 3835.32 'N $123 \quad 33.77^{\prime} \mathrm{W}$ Depth Deck Offset: -0.38

\begin{tabular}{|c|c|c|c|c|c|c|}
\hline $\begin{array}{l}\text { PRESSURE } \\
\text { (d-bars) }\end{array}$ & Interp & $\begin{array}{l}\text { TEMP } \\
(\operatorname{deg} C)\end{array}$ & $\begin{array}{c}\text { SALINITY } \\
(0 / 00)\end{array}$ & SIGMA-T & DELTA-D & $\begin{array}{l}\text { CHANNELA } \\
\text { (\&light) }\end{array}$ \\
\hline 0.0 & E & 9.913 & 33.382 & 25.703 & 0.0000 & 0.0 \\
\hline 10.0 & & 9.906 & 33.382 & 25.705 & 0.0231 & 0.0 \\
\hline 20.0 & & 8.979 & 33.589 & 26.017 & 0.0445 & 0.0 \\
\hline 30.0 & & 8.801 & 33.684 & 26.119 & 0.0642 & 0.0 \\
\hline 40.0 & & 8.788 & 33.688 & 26.124 & 0.0835 & 0.0 \\
\hline 50.0 & & 8.679 & 33.755 & 26.194 & 0.1024 & 0.0 \\
\hline 60.0 & & 8.420 & 33.841 & 26.301 & 0.1206 & 0.0 \\
\hline 70.0 & & 8.363 & 33.888 & 26.347 & 0.1379 & 0.0 \\
\hline 80.0 & & 8.345 & 33.926 & 26.379 & 0.1549 & 0.0 \\
\hline 90.0 & & 8.219 & 33.939 & 26.408 & 0.1717 & 0.0 \\
\hline 100.0 & & 8.101 & 33.957 & 26.440 & 0.1882 & 0.0 \\
\hline 110.0 & & 7.972 & 33.977 & 26.475 & 0.2044 & 0.0 \\
\hline 120.0 & & 7.898 & 33.988 & 26.494 & 0.2203 & 0.0 \\
\hline 130.0 & & 7.591 & 34.033 & 26.575 & 0.2357 & 0.0 \\
\hline 137.0 & & 7.585 & 34.034 & 26.576 & 0.2463 & 0.0 \\
\hline
\end{tabular}
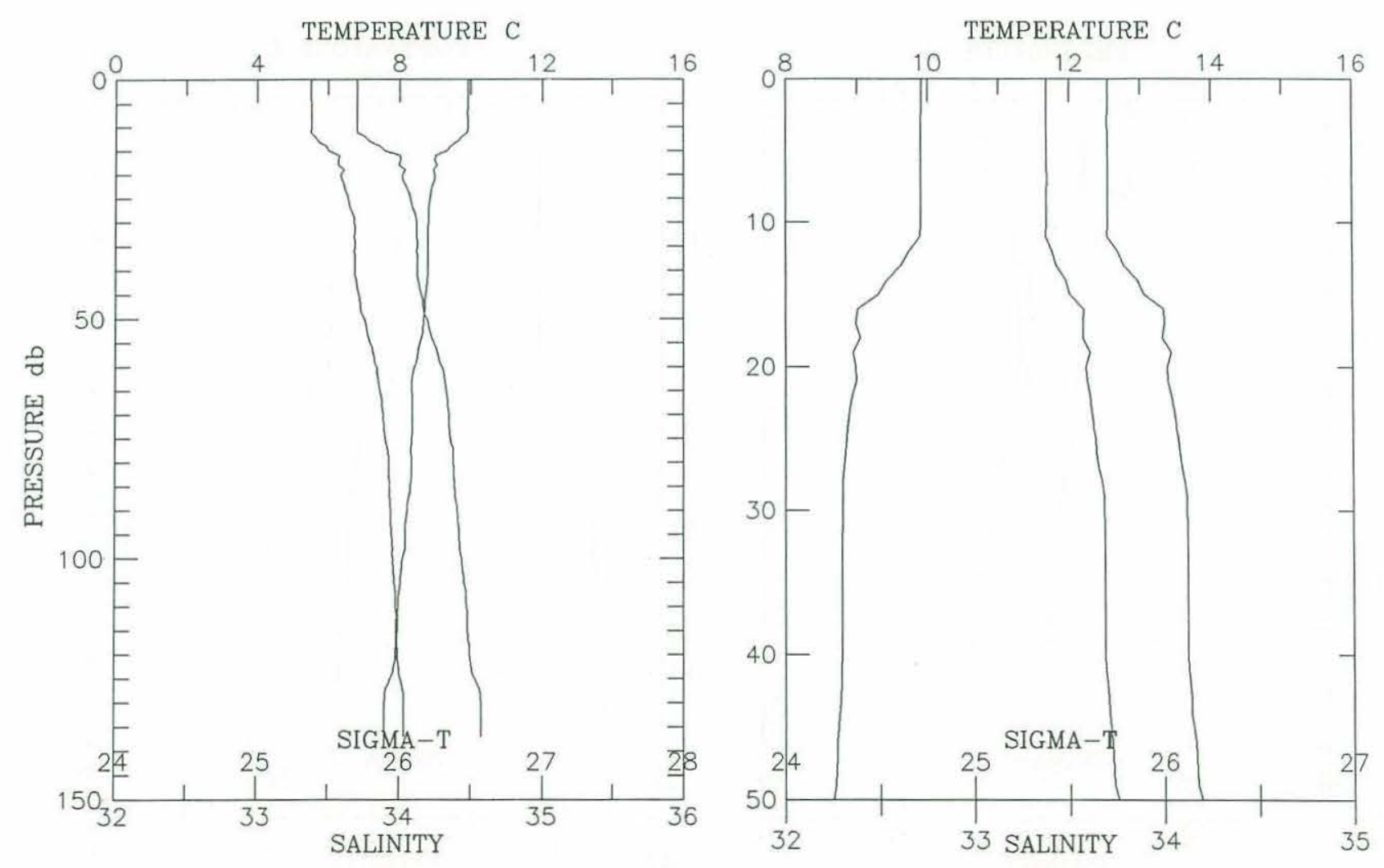
Cruise w8905 Station \# 98 Depth m: 162 Time: 1989-05-10 13:37

Position: $38 \quad 33.13^{\prime} \mathrm{N} 123 \quad 36.87^{\prime} \mathrm{W}$ Depth Deck Offset: -0.38

\begin{tabular}{|c|c|c|c|c|c|c|}
\hline $\begin{array}{l}\text { PRESSURE } \\
\text { (d-bars) }\end{array}$ & Interp & $\begin{array}{l}\text { TEMP } \\
(\operatorname{deg} C)\end{array}$ & $\begin{array}{c}\text { SALINITY } \\
(0 / 00)\end{array}$ & SIGMA-T & DELTA-D & $\begin{array}{l}\text { CHANNELA } \\
\text { ( }(z 1 \text { ight) }\end{array}$ \\
\hline 0.0 & E & 10.072 & 33.384 & 25.678 & 0.0000 & 0.0 \\
\hline 10.0 & & 10.078 & 33.383 & 25.676 & 0.0234 & 0.0 \\
\hline 20.0 & & 9.793 & 33.432 & 25.762 & 0.0464 & 0.0 \\
\hline 30.0 & & 9.255 & 33.520 & 25.919 & 0.0684 & 0.0 \\
\hline 40.0 & & 9.074 & 33.587 & 26.000 & 0.0893 & 0.0 \\
\hline 50.0 & & 8.918 & 33.646 & 26.071 & 0.1094 & 0.0 \\
\hline 60.0 & & 8.636 & 33.706 & 26.162 & 0.1287 & 0.0 \\
\hline 70.0 & & 8.416 & 33.788 & 26.260 & 0.1471 & 0.0 \\
\hline 80.0 & & 8.196 & 33.828 & 26.325 & 0.1648 & 0.0 \\
\hline 90.0 & & 8.039 & 33.914 & 26.415 & 0.1818 & 0.0 \\
\hline 100.0 & & 7.995 & 33.986 & 26.479 & 0.1980 & 0.0 \\
\hline 110.0 & & 7.906 & 34.017 & 26.516 & 0.2137 & 0.0 \\
\hline 120.0 & & 7.728 & 34.017 & 26.542 & 0.2293 & 0.0 \\
\hline 130.0 & & 7.513 & 34.012 & 26.569 & 0.2446 & 0.0 \\
\hline 140.0 & & 7.392 & 34.031 & 26.601 & 0.2596 & 0.0 \\
\hline 150.0 & & 7.349 & 34.045 & 26.619 & 0.2743 & 0.0 \\
\hline 157.0 & & 7.311 & 34.051 & 26.629 & 0.2846 & 0.0 \\
\hline
\end{tabular}

TEMPERATURE $\mathrm{C}$
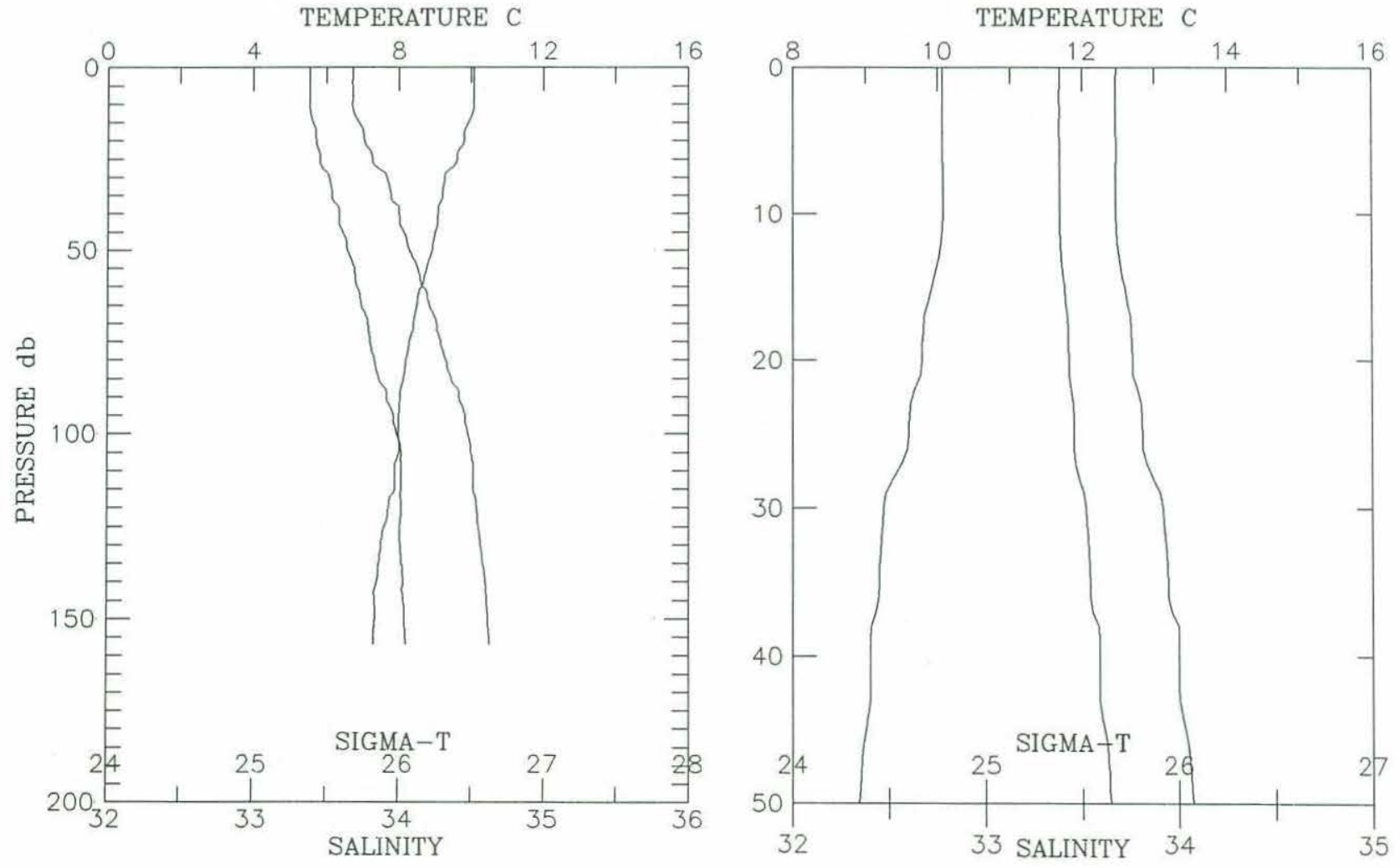


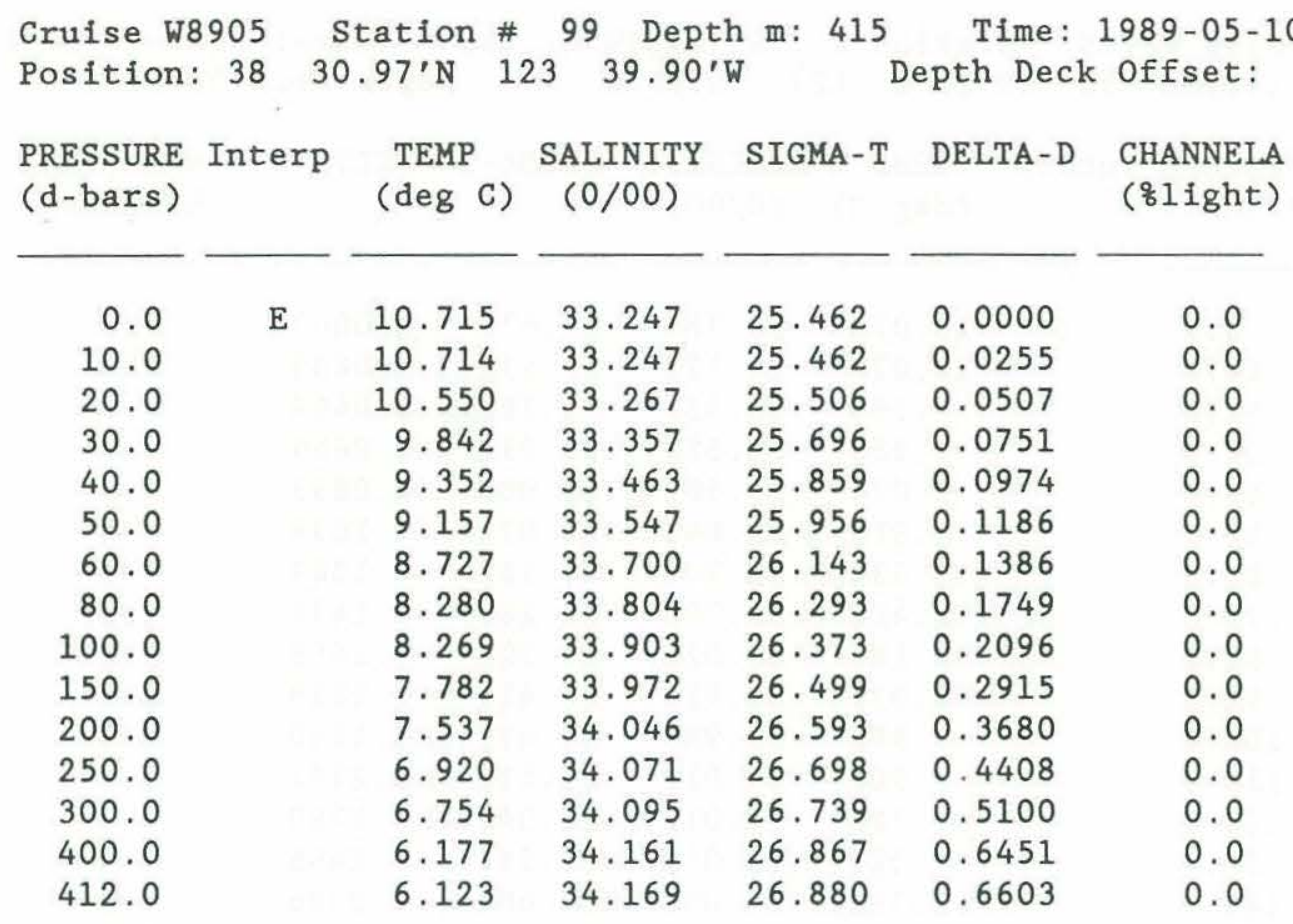
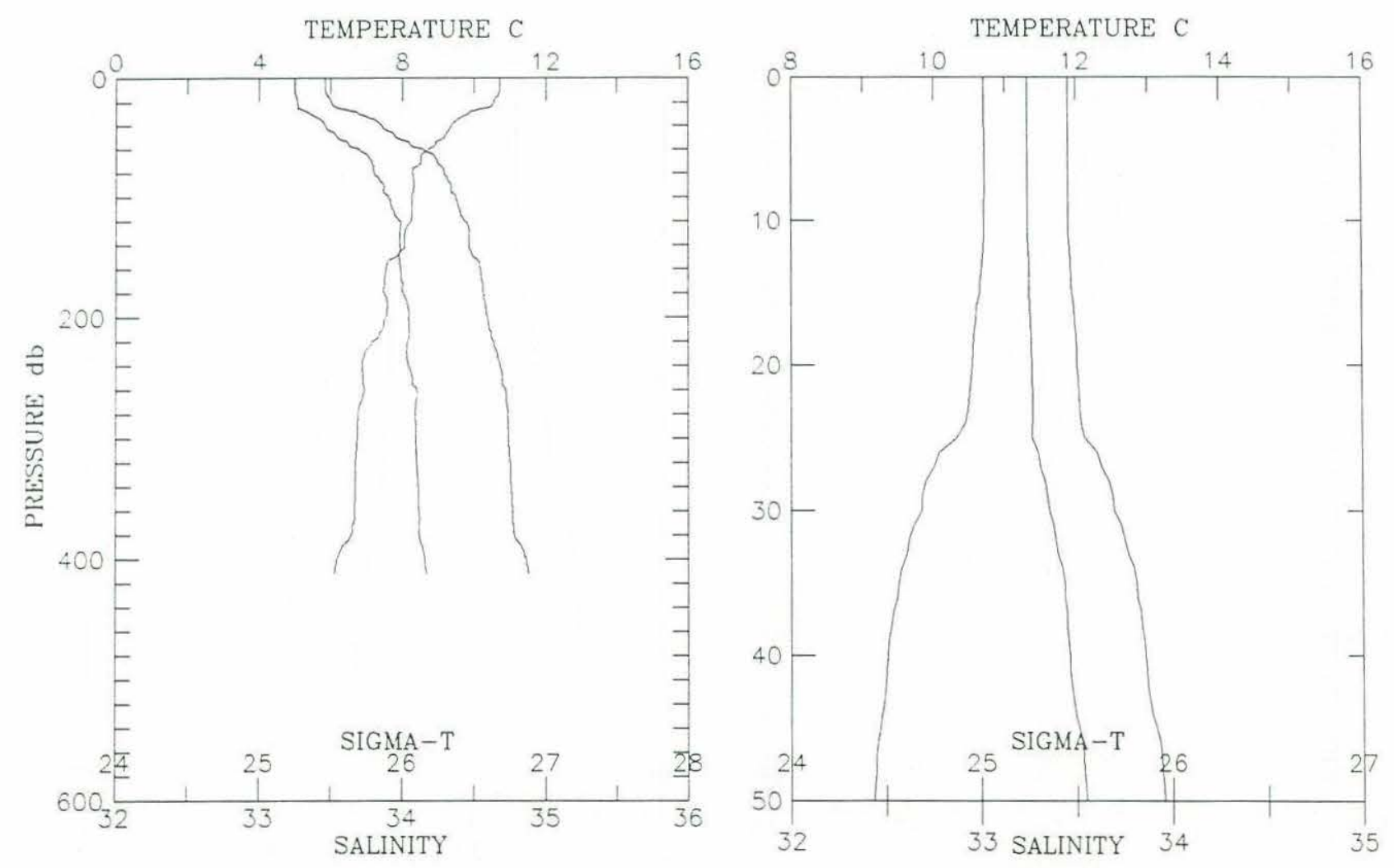


\begin{tabular}{|c|c|c|c|c|c|c|}
\hline $\begin{array}{l}\text { PRESSURE } \\
\text { (d-bars) }\end{array}$ & Interp & $\begin{array}{l}\text { TEMP } \\
(\operatorname{deg} C)\end{array}$ & $\begin{array}{c}\text { SALINITY } \\
(0 / 00)\end{array}$ & SIGMA-T & DELTA-D & $\begin{array}{l}\text { CHANNELA } \\
\text { (\&light) }\end{array}$ \\
\hline 0.0 & E & 11.880 & 33.020 & 25.075 & 0.0000 & 0.0 \\
\hline 10.0 & & 11.858 & 33.021 & 25.079 & 0.0291 & 0.0 \\
\hline 20.0 & & 11.819 & 33.027 & 25.091 & 0.0581 & 0.0 \\
\hline 30.0 & & 9.362 & 33.375 & 25.788 & 0.0823 & 0.0 \\
\hline 40.0 & & 9.130 & 33.519 & 25.938 & 0.1038 & 0.0 \\
\hline 50.0 & & 8.951 & 33.602 & 26.031 & 0.1244 & 0.0 \\
\hline 60.0 & & 8.863 & 33.641 & 26.076 & 0.1443 & 0.0 \\
\hline 80.0 & & 8.436 & 33.768 & 26.241 & 0.1823 & 0.0 \\
\hline 100.0 & & 8.146 & 33.846 & 26.346 & 0.2179 & 0.0 \\
\hline 150.0 & & 7.939 & 34.025 & 26.517 & 0.2991 & 0.0 \\
\hline 200.0 & & 7.311 & 34.041 & 26.620 & 0.3753 & 0.0 \\
\hline 250.0 & & 6.654 & 34.007 & 26.683 & 0.4478 & 0.0 \\
\hline 300.0 & & 6.962 & 34.126 & 26.735 & 0.5179 & 0.0 \\
\hline 400.0 & & 6.412 & 34.179 & 26.851 & 0.6512 & 0.0 \\
\hline 500.0 & & 6.035 & 34.204 & 26.919 & 0.7766 & 0.0 \\
\hline 600.0 & & 5.484 & 34.261 & 27.033 & 0.8964 & 0.0 \\
\hline 700.0 & & 5.131 & 34.313 & 27.116 & 1.0051 & 0.0 \\
\hline 800.0 & & 4.859 & 34.346 & 27.173 & 1.1080 & 0.0 \\
\hline 847.0 & & 4.662 & 34.369 & 27.214 & 1.1548 & 0.0 \\
\hline
\end{tabular}
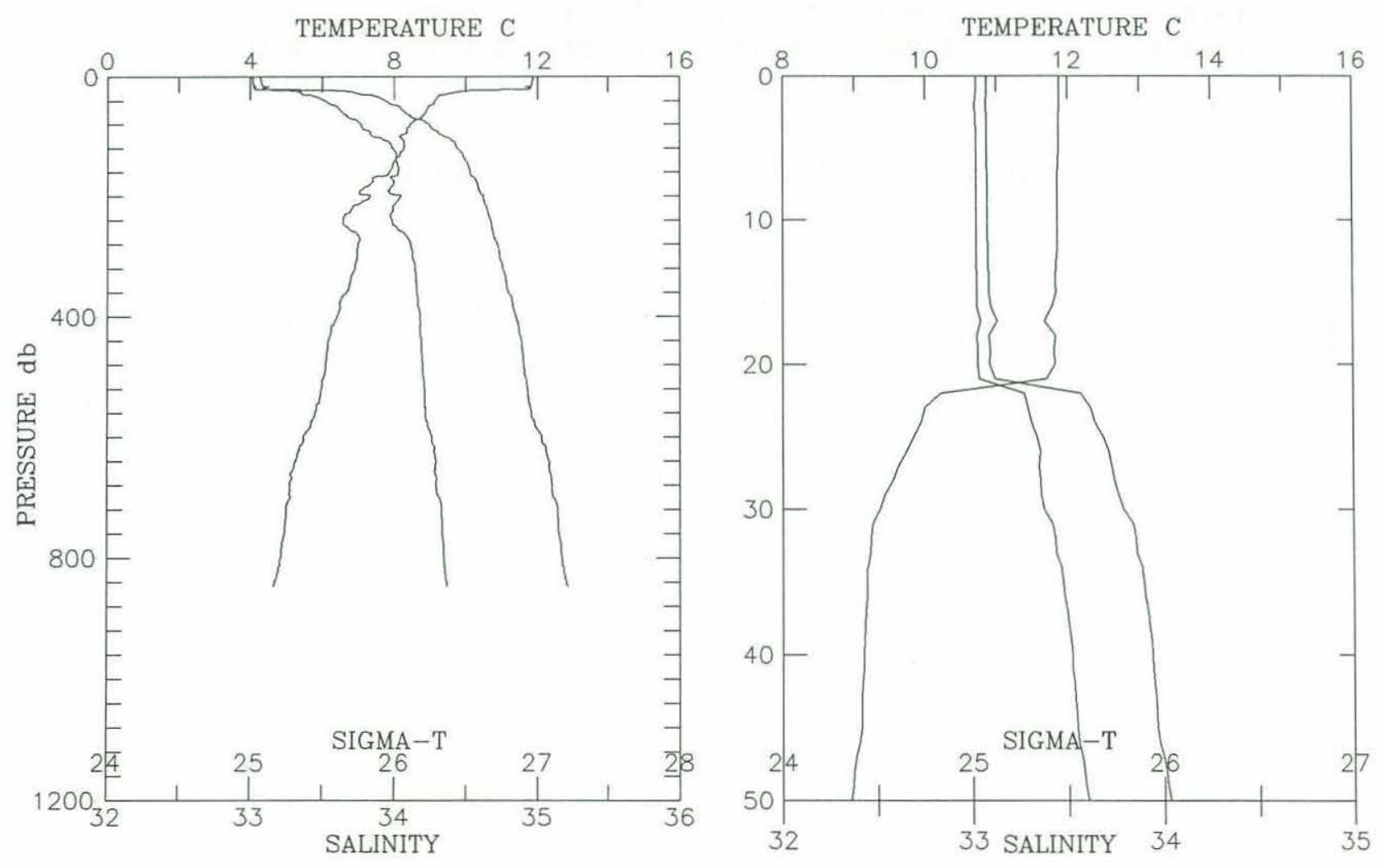
Cruise w8905 Station \# 123 Depth m: 1353 Time: 1989-05-11 05:15 Position: $3851.82^{\prime} \mathrm{N} 124 \quad 1.07^{\prime} \mathrm{W} \quad$ Depth Deck Offset: -0.15

\begin{tabular}{|c|c|c|c|c|c|c|}
\hline $\begin{array}{l}\text { PRESSURE } \\
\text { (d-bars) }\end{array}$ & Interp & $\begin{array}{c}\text { TEMP } \\
(\operatorname{deg} C)\end{array}$ & $\begin{array}{c}\text { SALINITY } \\
(0 / 00)\end{array}$ & SIGMA-T & DELTA-D & $\begin{array}{l}\text { CHANNELA } \\
\text { ( } \delta 1 \text { ight) }\end{array}$ \\
\hline 0.0 & $\mathrm{E}$ & 13.208 & 32.963 & 24.775 & 0.0000 & 0.0 \\
\hline 10.0 & & 13.211 & 32.963 & 24.774 & 0.0320 & 0.0 \\
\hline 20.0 & & 13.214 & 32.963 & 24.773 & 0.0640 & 0.0 \\
\hline 30.0 & & 13.145 & 32.960 & 24.785 & 0.0960 & 0.0 \\
\hline 40.0 & & 12.972 & 32.960 & 24.819 & 0.1278 & 0.0 \\
\hline 50.0 & & 11.941 & 32.973 & 25.027 & 0.1585 & 0.0 \\
\hline 60.0 & & 10.419 & 33.147 & 25.434 & 0.1859 & 0.0 \\
\hline 80.0 & & 9.843 & 33.489 & 25.799 & 0.2340 & 0.0 \\
\hline 100.0 & & 9.480 & 33.787 & 26.091 & 0.2757 & 0.0 \\
\hline 150.0 & & 8.495 & 33.953 & 26.378 & 0.3663 & 0.0 \\
\hline 200.0 & & 7.923 & 34.053 & 26.542 & 0.4469 & 0.0 \\
\hline 250.0 & & 7. 321 & 34.050 & 26.626 & 0.5225 & 0.0 \\
\hline 300.0 & & 6.320 & 34.012 & 26.731 & 0.5940 & 0.0 \\
\hline 400.0 & & 6.265 & 34.148 & 26.846 & 0.7267 & 0.0 \\
\hline 500.0 & & 5.843 & 34.214 & 26.952 & 0.8509 & 0.0 \\
\hline 600.0 & & 5.527 & 34.260 & 27.027 & 0.9675 & 0.0 \\
\hline 700.0 & & 4.953 & 34.333 & 27.152 & 1.0765 & 0.0 \\
\hline 800.0 & & 4.549 & 34.380 & 27.234 & 1.1750 & 0.0 \\
\hline 900.0 & & 4.292 & 34.409 & 27.285 & 1.2671 & 0.0 \\
\hline 1000.0 & & 3.952 & 34.430 & 27.338 & 1.3555 & 0.0 \\
\hline 1100.0 & & 3.685 & 34.464 & 27.392 & 1.4394 & 0.0 \\
\hline 1200.0 & & 3.381 & 34.492 & 27.444 & 1.5178 & 0.0 \\
\hline 1300.0 & & 3.096 & 34.515 & 27.489 & 1.5918 & 0.0 \\
\hline 1343.0 & & 2.982 & 34.525 & 27.508 & 1.6223 & 0.0 \\
\hline
\end{tabular}
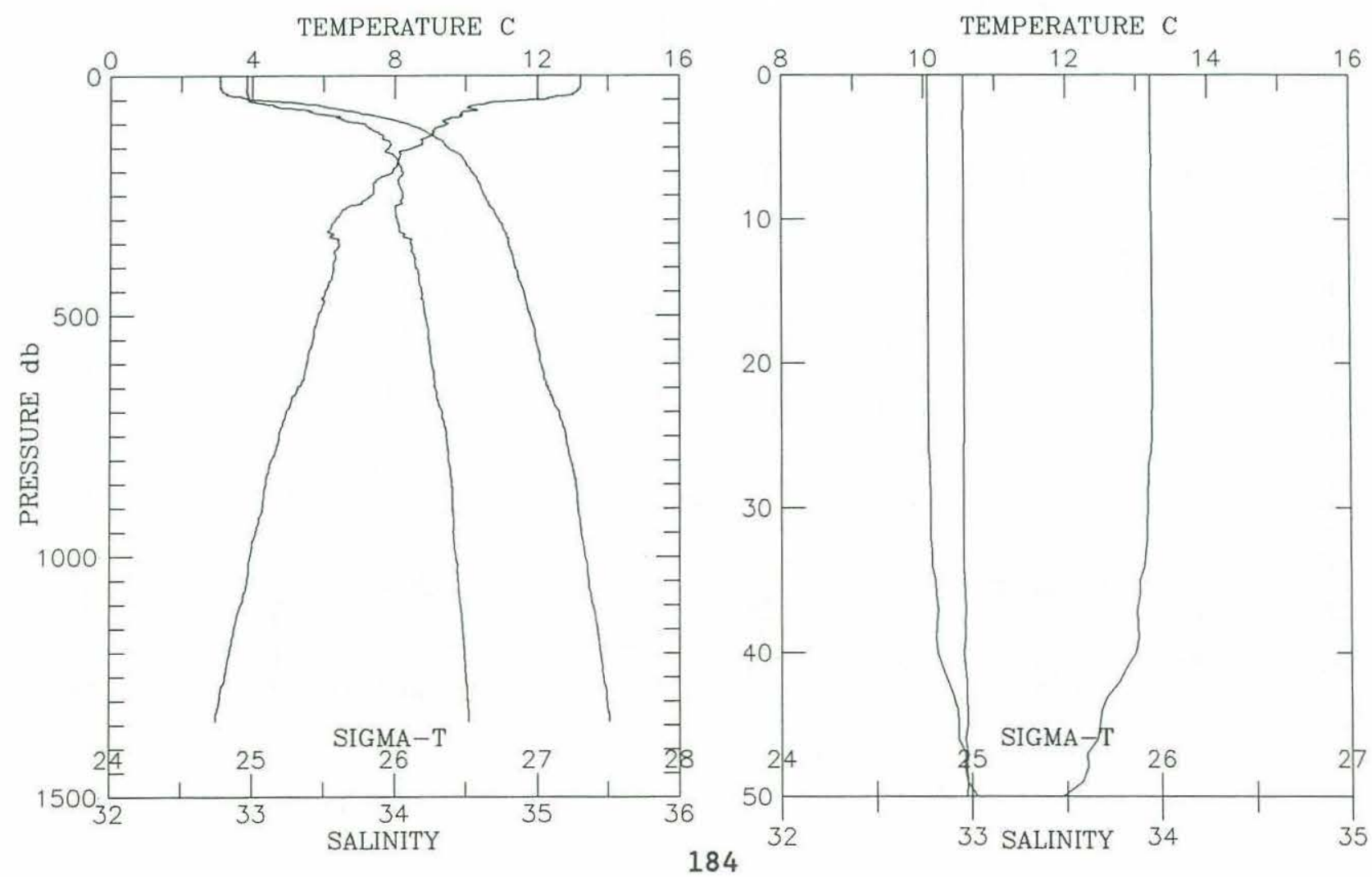
Cruise w8905 Station \# 124 Depth m: 519 Time: 1989-05-11 06:56

Position: $3852.80^{\prime} \mathrm{N} 12358.20^{\prime} \mathrm{W} \quad$ Depth Deck Offset: -0.15

\begin{tabular}{|c|c|c|c|c|c|c|}
\hline $\begin{array}{l}\text { PRESSURE } \\
\text { (d-bars) }\end{array}$ & Interp & $\begin{array}{l}\text { TEMP } \\
(\operatorname{deg} C)\end{array}$ & $\begin{array}{c}\text { SALINITY } \\
(0 / 00)\end{array}$ & SIGMA-T & DELTA-D & $\begin{array}{l}\text { CHANNELA } \\
\text { ( } \% 1 \text { ight) }\end{array}$ \\
\hline 0.0 & E & 11.963 & 33.044 & 25.078 & 0.0000 & 0.0 \\
\hline 10.0 & & 11.972 & 33.045 & 25.077 & 0.0291 & 0.0 \\
\hline 20.0 & & 11.967 & 33.045 & 25.078 & 0.0582 & 0.0 \\
\hline 30.0 & & 11.830 & 33.059 & 25.114 & 0.0873 & 0.0 \\
\hline 40.0 & & 10.574 & 33.110 & 25.379 & 0.1152 & 0.0 \\
\hline 50.0 & & 9.969 & 33.234 & 25.579 & 0.1405 & 0.0 \\
\hline 60.0 & & 9.691 & 33.300 & 25.676 & 0.1644 & 0.0 \\
\hline 80.0 & & 9.743 & 33.540 & 25.855 & 0.2100 & 0.0 \\
\hline 100.0 & & 9.247 & 33.703 & 26.064 & 0.2520 & 0.0 \\
\hline 150.0 & & 8.180 & 33.958 & 26.429 & 0.3425 & 0.0 \\
\hline 200.0 & & 7.497 & 34.027 & 26.583 & 0.4212 & 0.0 \\
\hline 250.0 & & 6.642 & 33.993 & 26.674 & 0.4943 & 0.0 \\
\hline 300.0 & & 6.737 & 34.140 & 26.777 & 0.5635 & 0.0 \\
\hline 400.0 & & 6.437 & 34.168 & 26.839 & 0.6950 & 0.0 \\
\hline 500.0 & & 5.997 & 34.189 & 26.912 & 0.8211 & 0.0 \\
\hline 511.0 & & 5.988 & 34.191 & 26.915 & 0.8347 & 0.0 \\
\hline
\end{tabular}

TEMPERATURE C

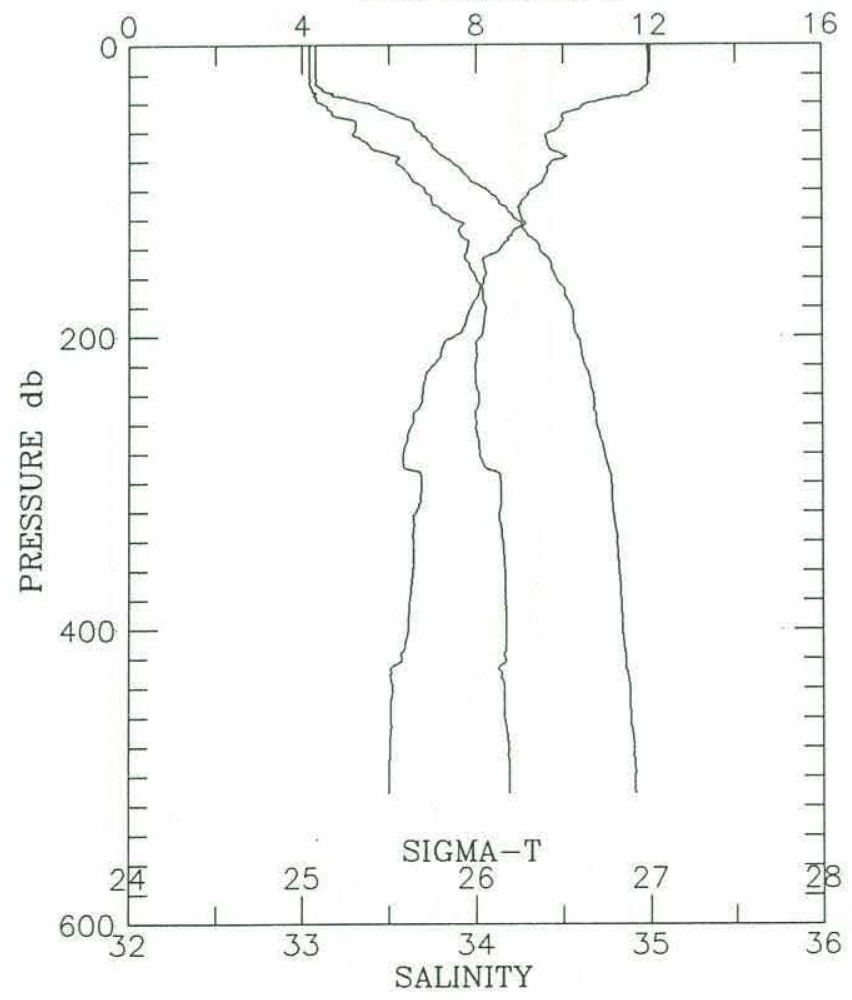

TEMPERATURE C

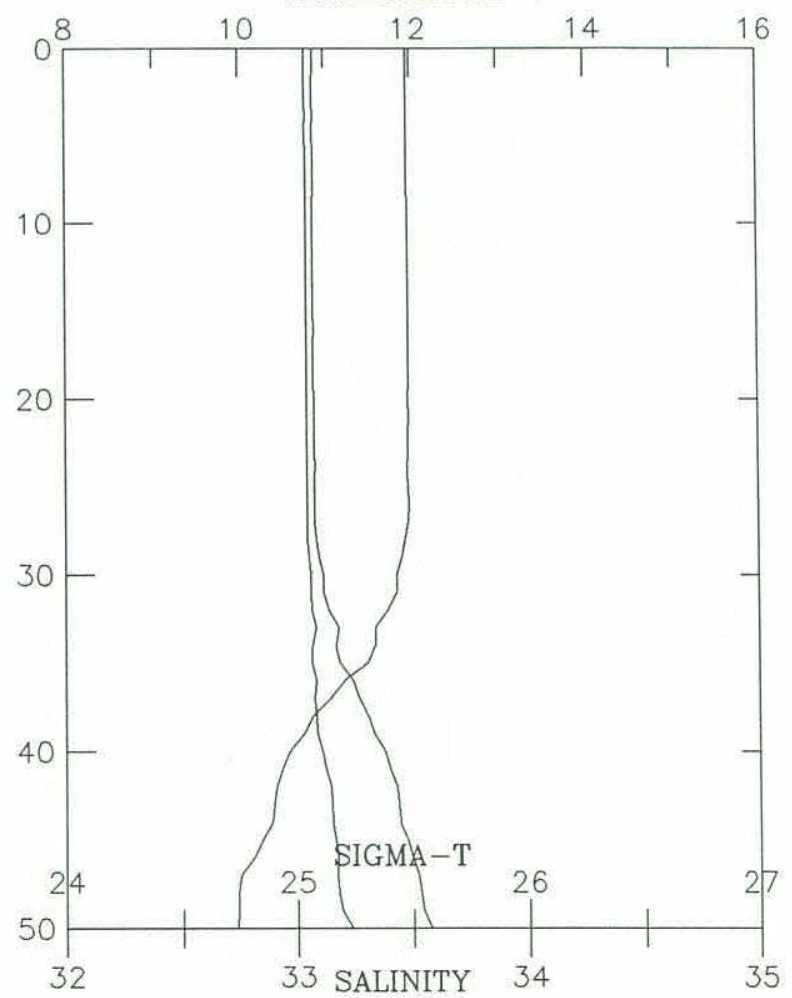


Cruise w8905 Station \# 125 Depth m: 257 Time: 1989-05-11 07:57

Position: $3853.40^{\prime} \mathrm{N} 12355.40^{\prime} \mathrm{W}$ Depth Deck Offset: -0.30

\begin{tabular}{|c|c|c|c|c|c|c|}
\hline $\begin{array}{l}\text { PRESSURE } \\
\text { (d-bars) }\end{array}$ & Interp & $\begin{array}{c}\text { TEMP } \\
(\operatorname{deg} C)\end{array}$ & $\begin{array}{c}\text { SALINITY } \\
(0 / 00)\end{array}$ & SIGMA-T & DELTA-D & $\begin{array}{l}\text { CHANNELA } \\
\text { ( } \delta 1 \text { ight) }\end{array}$ \\
\hline 0.0 & E & 11.376 & 33.117 & 25.243 & 0.0000 & 0.0 \\
\hline 10.0 & & 11.372 & 33.117 & 25.244 & 0.0275 & 0.0 \\
\hline 20.0 & & 11.362 & 33.119 & 25.247 & 0.0551 & 0.0 \\
\hline 30.0 & & 11.191 & 33.157 & 25.307 & 0.0823 & 0.0 \\
\hline 40.0 & & 10.408 & 33.200 & 25.478 & 0.1086 & 0.0 \\
\hline 50.0 & & 10.027 & 33.268 & 25.595 & 0.1333 & 0.0 \\
\hline 60.0 & & 9.689 & 33.380 & 25.739 & 0.1569 & 0.0 \\
\hline 80.0 & & 9.414 & 33.663 & 26.005 & 0.2005 & 0.0 \\
\hline 100.0 & & 9.075 & 33.823 & 26.185 & 0.2397 & 0.0 \\
\hline 150.0 & & 8.138 & 34.010 & 26.476 & 0.3266 & 0.0 \\
\hline 200.0 & & 6.811 & 34.034 & 26.684 & 0.4018 & 0.0 \\
\hline 250.0 & & 6.791 & 34.083 & 26.725 & 0.4716 & 0.0 \\
\hline 251.0 & & 6.788 & 34.082 & 26.725 & 0.4730 & 0.0 \\
\hline
\end{tabular}
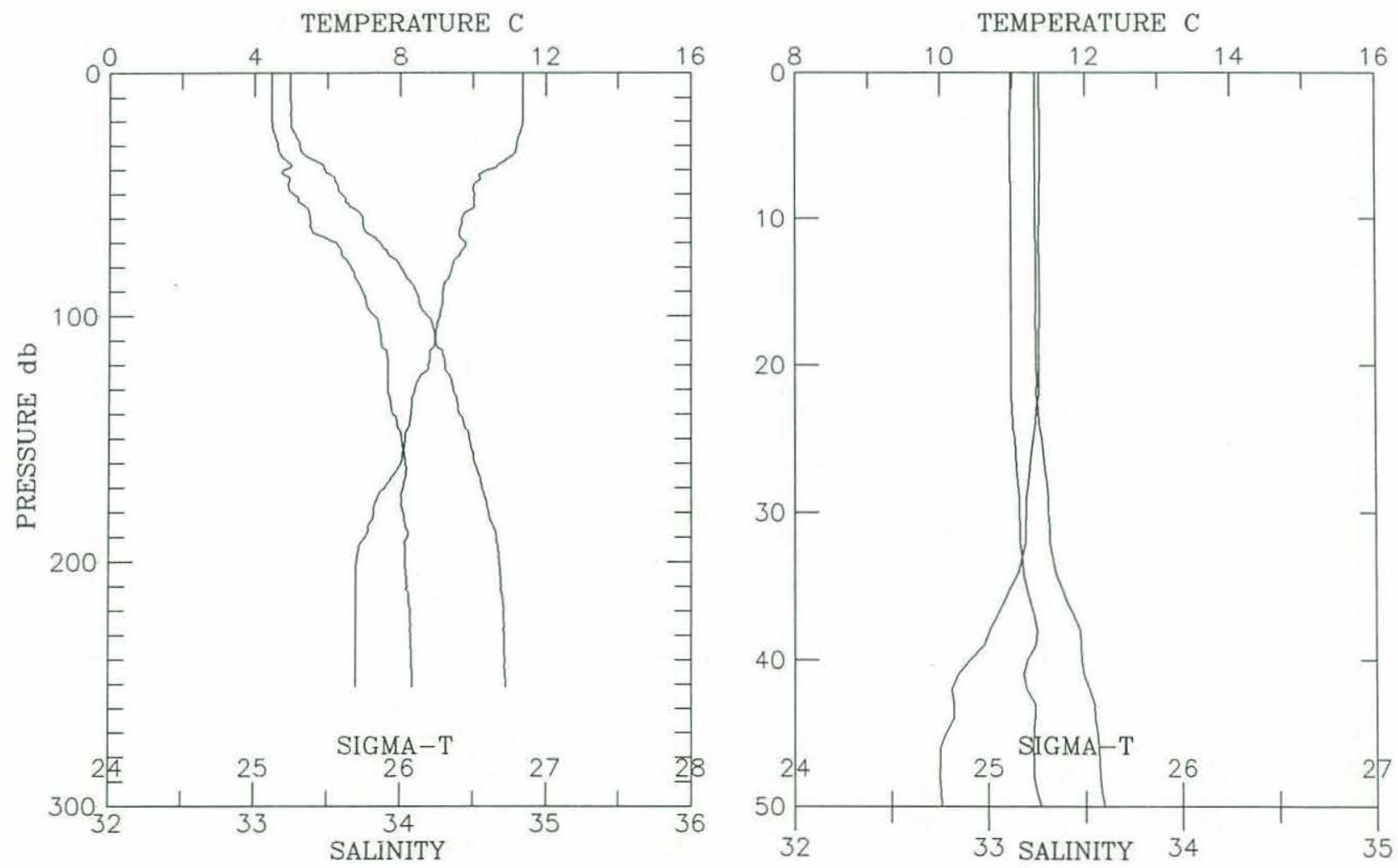
Cruise w8905 Station \# 126 Depth m: 141 Time: 1989-05-11 08:42

Position: $3854.10^{\prime} \mathrm{N} 12353.10^{\prime} \mathrm{W}$ Depth Deck Offset: -0.25

\begin{tabular}{|c|c|c|c|c|c|c|}
\hline $\begin{array}{l}\text { PRESSURE } \\
\text { (d-bars) }\end{array}$ & Interp & $\begin{array}{c}\text { TEMP } \\
(\operatorname{deg} C)\end{array}$ & $\begin{array}{c}\text { SALINITY } \\
(0 / 00)\end{array}$ & SIGMA-T & DELTA-D & $\begin{array}{l}\text { CHANNELA } \\
\text { ( }(\delta 1 i g h t)\end{array}$ \\
\hline 0.0 & E & 10.770 & 33.255 & 25.458 & 0.0000 & 0.0 \\
\hline 10.0 & & 10.768 & 33.255 & 25.458 & 0.0255 & 0.0 \\
\hline 20.0 & & 10.758 & 33.260 & 25.464 & 0.0509 & 0.0 \\
\hline 30.0 & & 10.701 & 33.279 & 25.488 & 0.0764 & 0.0 \\
\hline 40.0 & & 10.583 & 33.323 & 25.543 & 0.1013 & 0.0 \\
\hline 50.0 & & 10.510 & 33.348 & 25.575 & 0.1259 & 0.0 \\
\hline 60.0 & & 10.208 & 33.463 & 25.717 & 0.1499 & 0.0 \\
\hline 70.0 & & 9.819 & 33.553 & 25.853 & 0.1725 & 0.0 \\
\hline 80.0 & & 9.469 & 33.618 & 25.961 & 0.1938 & 0.0 \\
\hline 90.0 & & 9.206 & 33.778 & 26.129 & 0.2136 & 0.0 \\
\hline 100.0 & & 8.954 & 33.825 & 26.206 & 0.2325 & 0.0 \\
\hline 110.0 & & 8.571 & 33.904 & 26.328 & 0.2505 & 0.0 \\
\hline 120.0 & & 8.362 & 33.943 & 26.390 & 0.2676 & 0.0 \\
\hline 130.0 & & 8.005 & 33.986 & 26.478 & 0.2841 & 0.0 \\
\hline 136.0 & & 7.740 & 34.012 & 26.536 & 0.2935 & 0.0 \\
\hline
\end{tabular}

TEMPERATURE $\mathrm{C}$
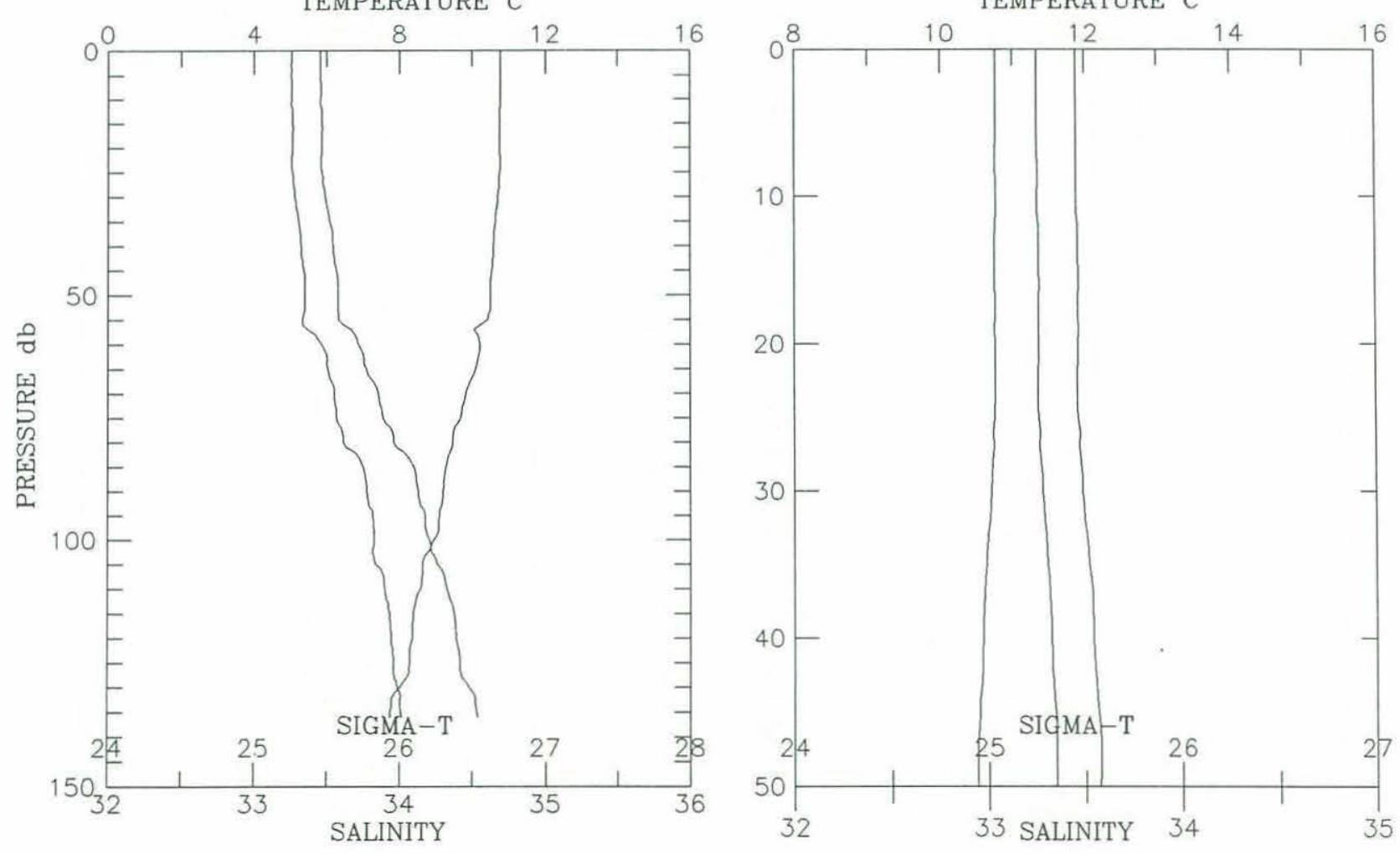


\begin{tabular}{|c|c|c|c|c|c|c|c|}
\hline Cruise W & $\begin{array}{r}3905 \\
38\end{array}$ & $\begin{array}{l}\text { ation \# } \\
90 \text { 'N } 1\end{array}$ & $\begin{array}{rr}127 & \text { Dep } \\
23 & 50.40\end{array}$ & $\mathrm{~h} \mathrm{~m}: 116$ & $\begin{array}{l}\text { Time: } \\
\text { epth Decl }\end{array}$ & $\begin{array}{l}1989-05-11 \\
\text { offset: }\end{array}$ & $\begin{array}{l}09: 22 \\
-0.35\end{array}$ \\
\hline $\begin{array}{l}\text { PRESSURE } \\
\text { (d-bars) }\end{array}$ & Interp & $\begin{array}{l}\text { TEMP } \\
(\operatorname{deg} C)\end{array}$ & $\begin{array}{c}\text { SALINITY } \\
(0 / 00)\end{array}$ & SIGMA-T & DELTA-D & $\begin{array}{l}\text { CHANNELA } \\
(\xi 1 i g h t)\end{array}$ & \\
\hline 0.0 & E & 9.867 & 33.467 & 25.778 & 0.0000 & 0.0 & \\
\hline 10.0 & & 9.884 & 33.463 & 25.772 & 0.0225 & 0.0 & \\
\hline 20.0 & & 9.834 & 33.470 & 25.786 & 0.0449 & 0.0 & \\
\hline 30.0 & & 9.784 & 33.481 & 25.802 & 0.0673 & 0.0 & \\
\hline 40.0 & & 9.759 & 33.495 & 25.818 & 0.0895 & 0.0 & \\
\hline 50.0 & & 9.778 & 33.569 & 25.872 & 0.1114 & 0.0 & \\
\hline 60.0 & & 9.278 & 33.709 & 26.063 & 0.1320 & 0.0 & \\
\hline 70.0 & & 8.967 & 33.754 & 26.148 & 0.1513 & 0.0 & \\
\hline 80.0 & & 8.874 & 33.774 & 26.179 & 0.1702 & 0.0 & \\
\hline 90.0 & & 8.789 & 33.808 & 26.218 & 0.1888 & 0.0 & \\
\hline 100.0 & & 8.363 & 33.922 & 26.373 & 0.2065 & 0.0 & \\
\hline 110.0 & & 8.170 & 33.946 & 26.421 & 0.2233 & 0.0 & \\
\hline
\end{tabular}
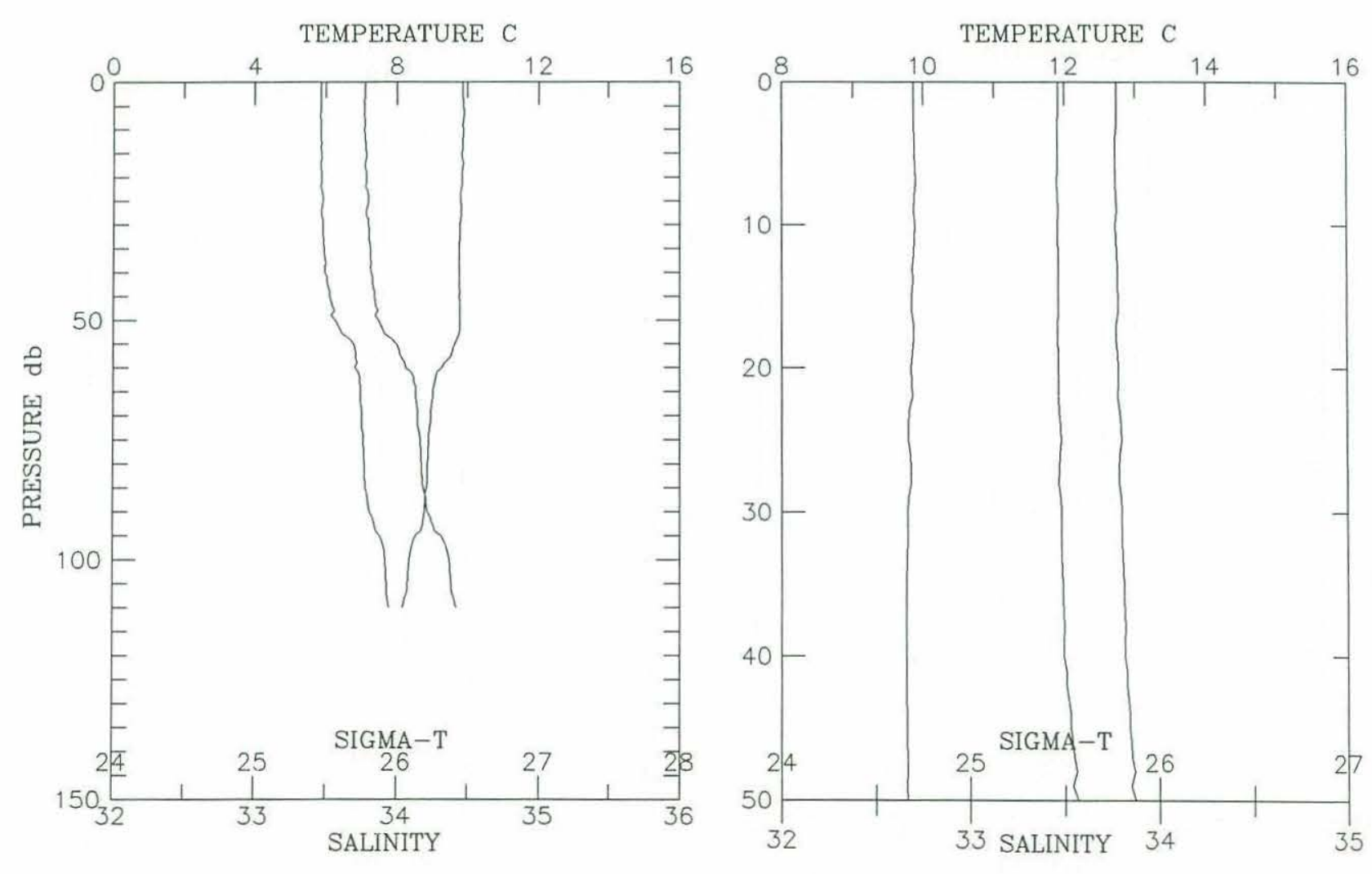
Cruise w8905 Station \# 128 Depth m: 100 Time: 1989-05-11 10:12 Position: $3855.90^{\prime} \mathrm{N} 123 \quad 47.70^{\prime} \mathrm{W} \quad$ Depth Deck Offset: -0.30

\begin{tabular}{|c|c|c|c|c|c|c|}
\hline $\begin{array}{l}\text { PRESSURE } \\
\text { (d-bars) }\end{array}$ & Interp & $\begin{array}{l}\text { TEMP } \\
(\operatorname{deg} C)\end{array}$ & $\begin{array}{c}\text { SALINITY } \\
(0 / 00)\end{array}$ & SIGMA-T & DELTA-D & $\begin{array}{l}\text { CHANNELA } \\
\text { ( } \% \text { ight) }\end{array}$ \\
\hline 0.0 & E & 9.131 & 33.654 & 26.044 & 0.0000 & 0.0 \\
\hline 5.0 & & 9.130 & 33.652 & 26.042 & 0.0100 & 0.0 \\
\hline 10.0 & & 9.134 & 33.652 & 26.042 & 0.0199 & 0.0 \\
\hline 15.0 & & 9.134 & 33.651 & 26.041 & 0.0299 & 0.0 \\
\hline 20.0 & & 9.133 & 33.654 & 26.043 & 0.0399 & 0.0 \\
\hline 25.0 & & 9.133 & 33.659 & 26.048 & 0.0499 & 0.0 \\
\hline 30.0 & & 9.129 & 33.657 & 26.046 & 0.0598 & 0.0 \\
\hline 35.0 & & 9.134 & 33.661 & 26.049 & 0.0698 & 0.0 \\
\hline 40.0 & & 9.132 & 33.665 & 26.052 & 0.0798 & 0.0 \\
\hline 45.0 & & 9.136 & 33.673 & 26.058 & 0.0897 & 0.0 \\
\hline 50.0 & & 9.085 & 33.703 & 26.090 & 0.0996 & 0.0 \\
\hline 55.0 & & 9.008 & 33.729 & 26.122 & 0.1093 & 0.0 \\
\hline 60.0 & & 8.931 & 33.748 & 26.149 & 0.1189 & 0.0 \\
\hline 65.0 & & 8.792 & 33.777 & 26.194 & 0.1283 & 0.0 \\
\hline 70.0 & & 8.721 & 33.794 & 26.218 & 0.1376 & 0.0 \\
\hline 75.0 & & 8.684 & 33.804 & 26.232 & 0.1467 & 0.0 \\
\hline 80.0 & & 8.565 & 33.827 & 26.268 & 0.1558 & 0.0 \\
\hline 85.0 & & 8.549 & 33.829 & 26.272 & 0.1647 & 0.0 \\
\hline 90.0 & & 8.402 & 33.871 & 26.328 & 0.1736 & 0.0 \\
\hline 95.0 & & 8.398 & 33.872 & 26.329 & 0.1823 & 0.0 \\
\hline 96.0 & & 8.397 & 33.872 & 26.329 & 0.1840 & 0.0 \\
\hline
\end{tabular}
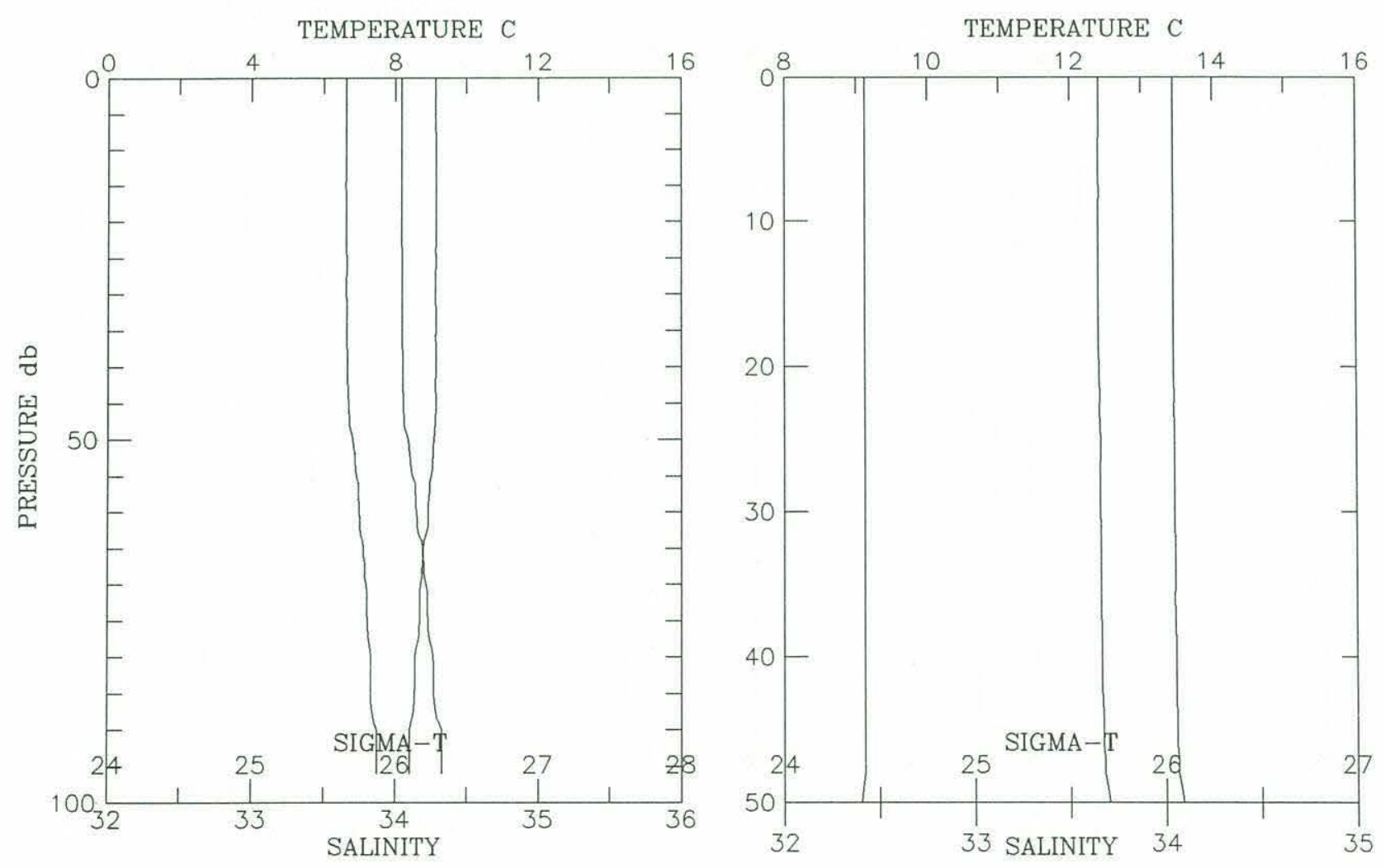
Cruise w8905 Station \# 129 Depth m: 78 Time: 1989-05-11 11:10 Position: $3851.06^{\prime} \mathrm{N} 123 \quad 42.52^{\prime} \mathrm{W}$ Depth Deck Offset: -0.30

\begin{tabular}{|c|c|c|c|c|c|c|}
\hline $\begin{array}{l}\text { PRESSURE } \\
\text { (d-bars) }\end{array}$ & Interp & $\begin{array}{l}\text { TEMP } \\
(\operatorname{deg} C)\end{array}$ & $\begin{array}{c}\text { SALINITY } \\
(0 / 00)\end{array}$ & SIGMA-T & DELTA-D & $\begin{array}{l}\text { CHANNELA } \\
\text { ( } 81 \text { ight) }\end{array}$ \\
\hline 0.0 & E & 8.933 & 33.738 & 26.141 & 0.0000 & 0.0 \\
\hline 5.0 & & 8.929 & 33.739 & 26.142 & 0.0095 & 0.0 \\
\hline 10.0 & & 8.934 & 33.736 & 26.139 & 0.0190 & 0.0 \\
\hline 15.0 & & 8.883 & 33.754 & 26.161 & 0.0284 & 0.0 \\
\hline 20.0 & & 8.750 & 33.763 & 26.189 & 0.0378 & 0.0 \\
\hline 25.0 & & 8.658 & 33.778 & 26.215 & 0.0470 & 0.0 \\
\hline 30.0 & & 8.605 & 33.798 & 26.239 & 0.0561 & 0.0 \\
\hline 35.0 & & 8.442 & 33.854 & 26.308 & 0.0650 & 0.0 \\
\hline 40.0 & & 8.397 & 33.869 & 26.327 & 0.0737 & 0.0 \\
\hline 45.0 & & 8.382 & 33.875 & 26.334 & 0.0823 & 0.0 \\
\hline 50.0 & & 8.338 & 33.884 & 26.347 & 0.0909 & 0.0 \\
\hline 55.0 & & 8.257 & 33.900 & 26.372 & 0.0994 & 0.0 \\
\hline 60.0 & & 8.220 & 33.911 & 26.386 & 0.1078 & 0.0 \\
\hline 65.0 & & 8.104 & 33.933 & 26.420 & 0.1161 & 0.0 \\
\hline 70.0 & & 8.048 & 33.944 & 26.437 & 0.1243 & 0.0 \\
\hline 74.0 & & 8.029 & 33.946 & 26.442 & 0.1308 & 0.0 \\
\hline
\end{tabular}
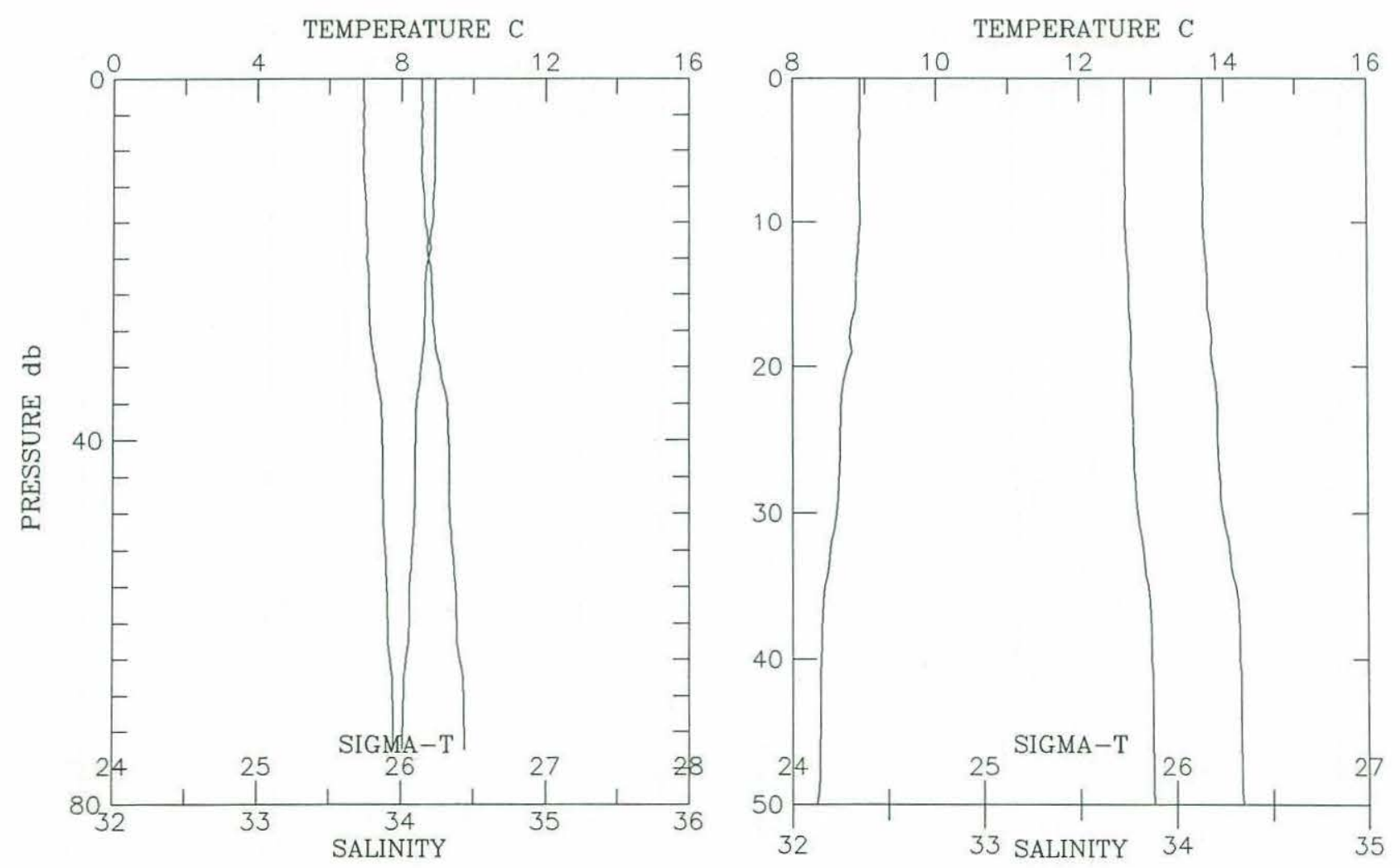


\begin{tabular}{|c|c|c|c|c|c|c|}
\hline $\begin{array}{l}\text { PRESSURE } \\
\text { (d-bars) }\end{array}$ & Interp & $\begin{array}{l}\text { TEMP } \\
(\operatorname{deg} C)\end{array}$ & $\begin{array}{l}\text { SALINITY } \\
(0 / 00)\end{array}$ & SIGMA-T & DELTA-D & $\begin{array}{l}\text { CHANNELA } \\
\text { ( } \delta 1 \text { ight) }\end{array}$ \\
\hline 0.0 & $\mathrm{E}$ & 9.019 & 33.648 & 26.057 & 0.0000 & 0.0 \\
\hline 5.0 & & 9.021 & 33.648 & 26.057 & 0.0099 & 0.0 \\
\hline 10.0 & & 9.019 & 33.647 & 26.056 & 0.0198 & 0.0 \\
\hline 15.0 & & 9.001 & 33.656 & 26.066 & 0.0297 & 0.0 \\
\hline 20.0 & & 8.938 & 33.684 & 26.098 & 0.0395 & 0.0 \\
\hline 25.0 & & 8.769 & 33.751 & 26.177 & 0.0490 & 0.0 \\
\hline 30.0 & & 8.709 & 33.766 & 26.198 & 0.0583 & 0.0 \\
\hline 35.0 & & 8.661 & 33.777 & 26.214 & 0.0676 & 0.0 \\
\hline 40.0 & & 8.630 & 33.789 & 26.228 & 0.0767 & 0.0 \\
\hline 45.0 & & 8.581 & 33.808 & 26.251 & 0.0858 & 0.0 \\
\hline 50.0 & & 8.507 & 33.835 & 26.283 & 0.0947 & 0.0 \\
\hline 55.0 & & 8.479 & 33.844 & 26.295 & 0.1035 & 0.0 \\
\hline 60.0 & & 8.440 & 33.860 & 26.313 & 0.1123 & 0.0 \\
\hline 65.0 & & 8.419 & 33.870 & 26.324 & 0.1210 & 0.0 \\
\hline 70.0 & & 8.410 & 33.872 & 26.327 & 0.1297 & 0.0 \\
\hline 75.0 & & 8.416 & 33.868 & 26.323 & 0.1384 & 0.0 \\
\hline 80.0 & & 8.293 & 33.899 & 26.366 & 0.1471 & 0.0 \\
\hline 85.0 & & 7.992 & 33.961 & 26.459 & 0.1553 & 0.0 \\
\hline 90.0 & & 7.788 & 33.984 & 26.507 & 0.1633 & 0.0 \\
\hline 93.0 & & 7.728 & 33.987 & 26.518 & 0.1680 & 0.0 \\
\hline
\end{tabular}

TEMPERATURE C
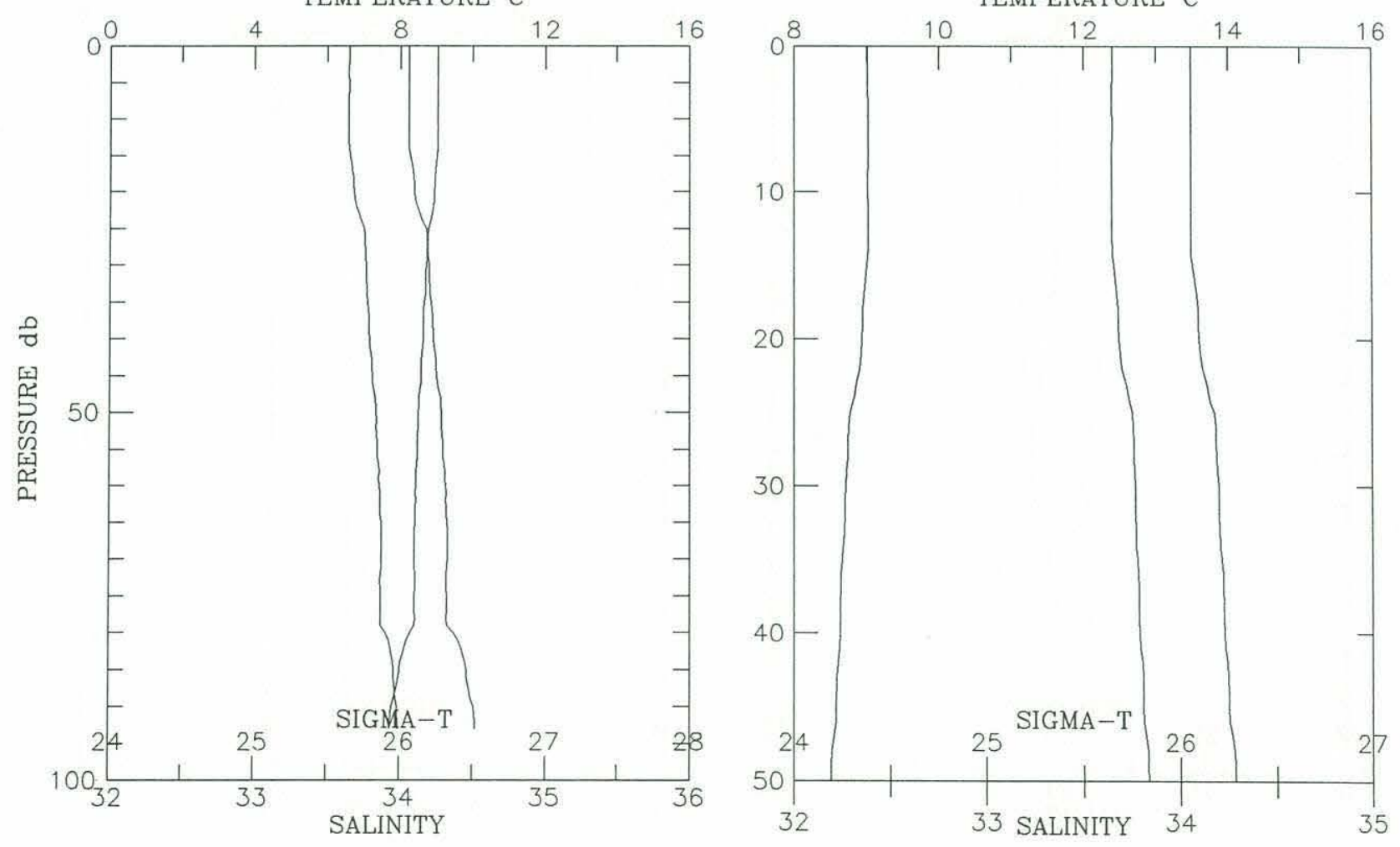
Cruise w8905 Station \# 131 Depth m: 127 Time: 1989-05-11 12:21

Position: $3848.38^{\prime} \mathrm{N} 123 \quad 45.59^{\prime} \mathrm{W}$ Depth Deck Offset: -0.30

\begin{tabular}{|c|c|c|c|c|c|c|}
\hline $\begin{array}{l}\text { PRESSURE } \\
\text { (d-bars) }\end{array}$ & Interp & $\begin{array}{c}\text { TEMP } \\
(\operatorname{deg} C)\end{array}$ & $\begin{array}{c}\text { SALINITY } \\
(0 / 00)\end{array}$ & SIGMA-T & DELTA-D & $\begin{array}{l}\text { CHANNEL } \\
\text { ( } \delta 1 \text { ight) }\end{array}$ \\
\hline 0.0 & E & 9.114 & 33.641 & 26.036 & 0.0000 & 0.0 \\
\hline 10.0 & & 9.117 & 33.640 & 26.035 & 0.0200 & 0.0 \\
\hline 20.0 & & 9.117 & 33.640 & 26.035 & 0.0400 & 0.0 \\
\hline 30.0 & & 9.110 & 33.642 & 26.038 & 0.0601 & 0.0 \\
\hline 40.0 & & 9.107 & 33.643 & 26.039 & 0.0801 & 0.0 \\
\hline 50.0 & & 9.096 & 33.644 & 26.042 & 0.1001 & 0.0 \\
\hline 60.0 & & 9.047 & 33.678 & 26.076 & 0.1201 & 0.0 \\
\hline 70.0 & & 8.963 & 33.748 & 26.144 & 0.1394 & 0.0 \\
\hline 80.0 & & 8.922 & 33.795 & 26.188 & 0.1584 & 0.0 \\
\hline 90.0 & & 8.786 & 33.832 & 26.238 & 0.1768 & 0.0 \\
\hline 100.0 & & 8.458 & 33.900 & 26.342 & 0.1946 & 0.0 \\
\hline 110.0 & & 8.104 & 33.951 & 26.434 & 0.2114 & 0.0 \\
\hline 120.0 & & 7.110 & 34.058 & 26.662 & 0.2266 & 0.0 \\
\hline 123.0 & & 7.108 & 34.060 & 26.664 & 0.2308 & 0.0 \\
\hline
\end{tabular}
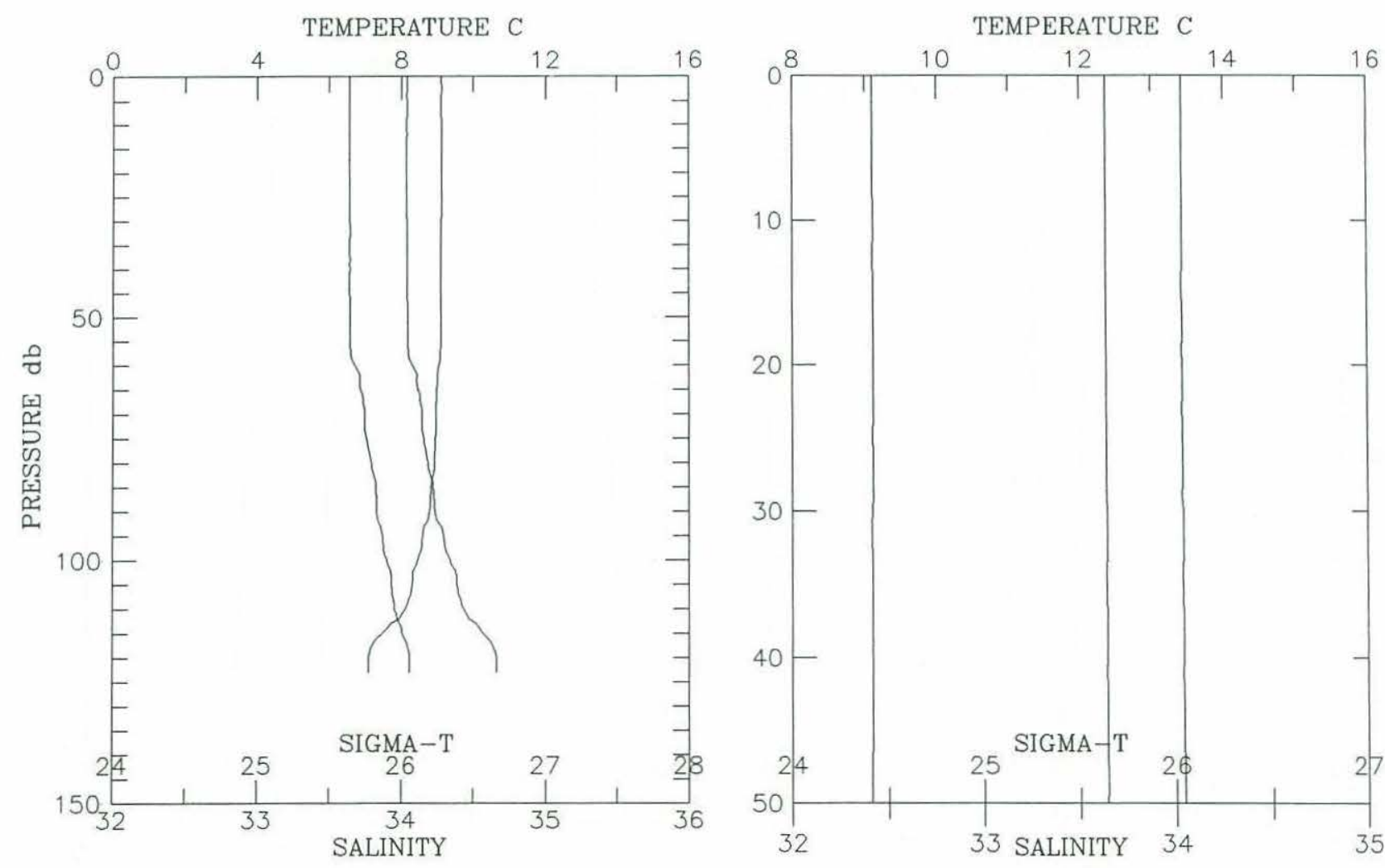
Cruise w8905 Station \# 132 Depth m: 135 Time: 1989-05-11 13:00 Position: $38 \quad 47.26^{\prime} \mathrm{N} 12347.12^{\prime} \mathrm{W}$ Depth Deck Offset: $\quad-0.30$

\begin{tabular}{|c|c|c|c|c|c|c|}
\hline $\begin{array}{l}\text { PRESSURE } \\
\text { (d-bars) }\end{array}$ & Interp & $\begin{array}{c}\text { TEMP } \\
(\operatorname{deg} C)\end{array}$ & $\begin{array}{c}\text { SALINITY } \\
(0 / 00)\end{array}$ & SIGMA-T & DELTA-D & $\begin{array}{l}\text { CHANNELA } \\
\text { ( } 81 \text { ight) }\end{array}$ \\
\hline 0.0 & $\mathrm{E}$ & 9.512 & 33.501 & 25.863 & 0.0000 & 0.0 \\
\hline 10.0 & & 9.504 & 33.502 & 25.865 & 0.0216 & 0.0 \\
\hline 20.0 & & 9.512 & 33.500 & 25.862 & 0.0433 & 0.0 \\
\hline 30.0 & & 9.492 & 33.506 & 25.870 & 0.0650 & 0.0 \\
\hline 40.0 & & 9.426 & 33.525 & 25.895 & 0.0865 & 0.0 \\
\hline 50.0 & & 9.404 & 33.541 & 25.912 & 0.1078 & 0.0 \\
\hline 60.0 & & 9.499 & 33.670 & 25.997 & 0.1289 & 0.0 \\
\hline 70.0 & & 8.986 & 33.764 & 26.153 & 0.1485 & 0.0 \\
\hline 80.0 & & 8.890 & 33.792 & 26.190 & 0.1673 & 0.0 \\
\hline 90.0 & & 8.693 & 33.857 & 26.272 & 0.1856 & 0.0 \\
\hline 100.0 & & 8.141 & 33.957 & 26.434 & 0.2025 & 0.0 \\
\hline 110.0 & & 7.518 & 34.017 & 26.572 & 0.2182 & 0.0 \\
\hline 120.0 & & 7.411 & 34.030 & 26.598 & 0.2331 & 0.0 \\
\hline 130.0 & & 7.271 & 34.047 & 26.631 & 0.2479 & 0.0 \\
\hline
\end{tabular}

TEMPERATURE C
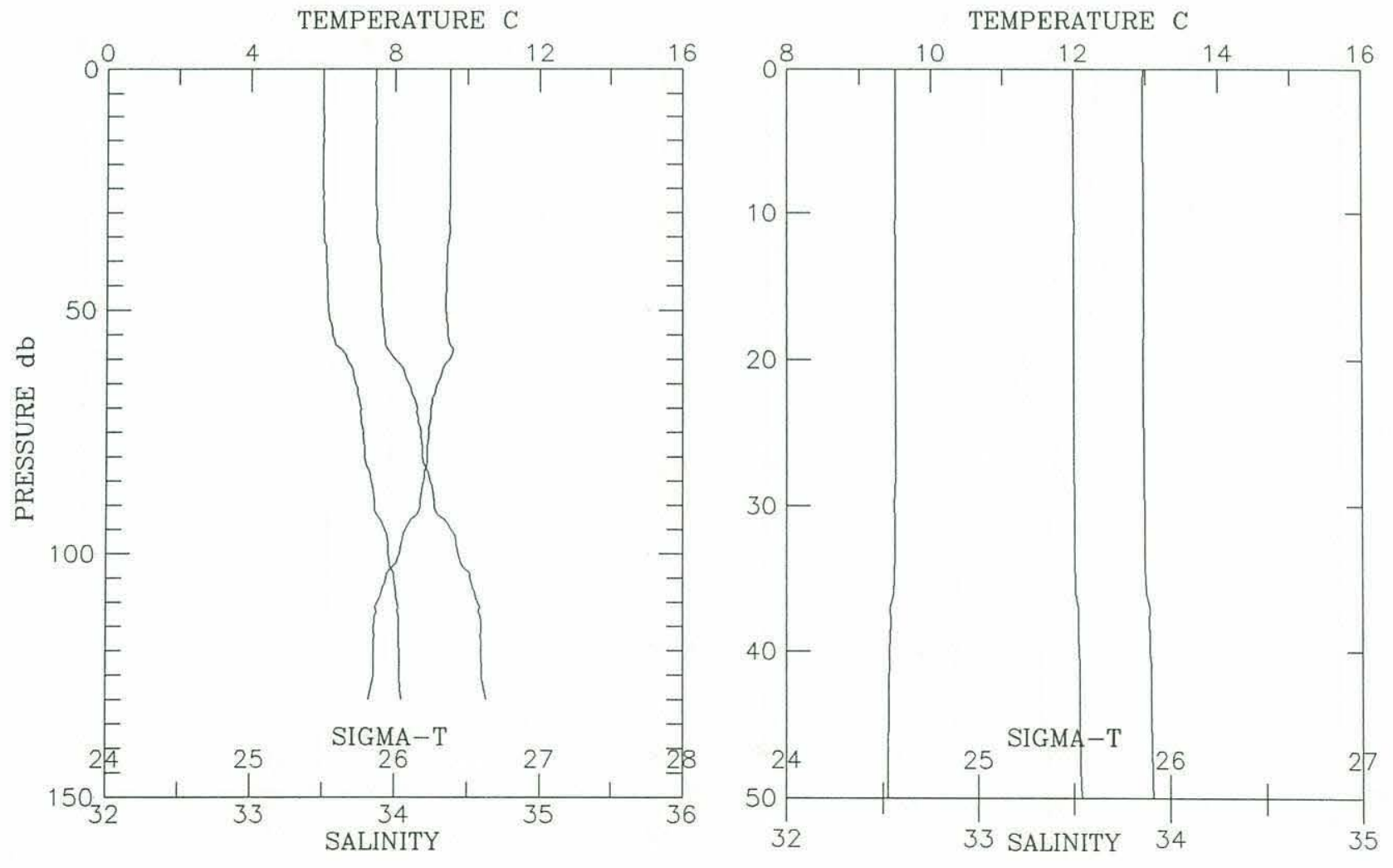
Cruise W8905 Station \# 133 Depth m: 189 Time: 1989-05-11 13:39

Position: $38 \quad 45.91^{\prime} \mathrm{N} 12348.82^{\prime} \mathrm{W}$ Depth Deck Offset: -0.30

\begin{tabular}{|c|c|c|c|c|c|c|}
\hline $\begin{array}{l}\text { PRESSURE } \\
\text { (d-bars) }\end{array}$ & Interp & $\begin{array}{l}\text { TEMP } \\
(\operatorname{deg} C)\end{array}$ & $\begin{array}{c}\text { SALINITY } \\
(0 / 00)\end{array}$ & SIGMA-T & DELTA-D & $\begin{array}{l}\text { CHANNELA } \\
\text { (\%1ight) }\end{array}$ \\
\hline 0.0 & $\mathrm{E}$ & 10.003 & 33.413 & 25.713 & 0.0000 & 0.0 \\
\hline 10.0 & & 9.997 & 33.414 & 25.714 & 0.0231 & 0.0 \\
\hline 20.0 & & 9.991 & 33.415 & 25.716 & 0.0461 & 0.0 \\
\hline 30.0 & & 9.948 & 33.423 & 25.730 & 0.0692 & 0.0 \\
\hline 40.0 & & 9.921 & 33.431 & 25.740 & 0.0920 & 0.0 \\
\hline 50.0 & & 9.805 & 33.492 & 25.807 & 0.1147 & 0.0 \\
\hline 60.0 & & 9.451 & 33.652 & 25.991 & 0.1356 & 0.0 \\
\hline 70.0 & & 9.244 & 33.770 & 26.117 & 0.1556 & 0.0 \\
\hline 80.0 & & 8.902 & 33.801 & 26.195 & 0.1747 & 0.0 \\
\hline 90.0 & & 8.685 & 33.843 & 26.262 & 0.1929 & 0.0 \\
\hline 100.0 & & 8.387 & 33.922 & 26.370 & 0.2106 & 0.0 \\
\hline 110.0 & & 7.878 & 33.994 & 26.502 & 0.2269 & 0.0 \\
\hline 120.0 & & 7.492 & 34.007 & 26.568 & 0.2423 & 0.0 \\
\hline 130.0 & & 7.220 & 34.044 & 26.635 & 0.2572 & 0.0 \\
\hline 140.0 & & 7.147 & 34.049 & 26.649 & 0.2716 & 0.0 \\
\hline 150.0 & & 7.098 & 34.060 & 26.665 & 0.2859 & 0.0 \\
\hline 160.0 & & 7.065 & 34.067 & 26.675 & 0.3001 & 0.0 \\
\hline 170.0 & & 7.084 & 34.079 & 26.682 & 0.3143 & 0.0 \\
\hline 180.0 & & 7.078 & 34.082 & 26.685 & 0.3284 & 0.0 \\
\hline 186.0 & & 7.075 & 34.082 & 26.685 & 0.3369 & 0.0 \\
\hline
\end{tabular}
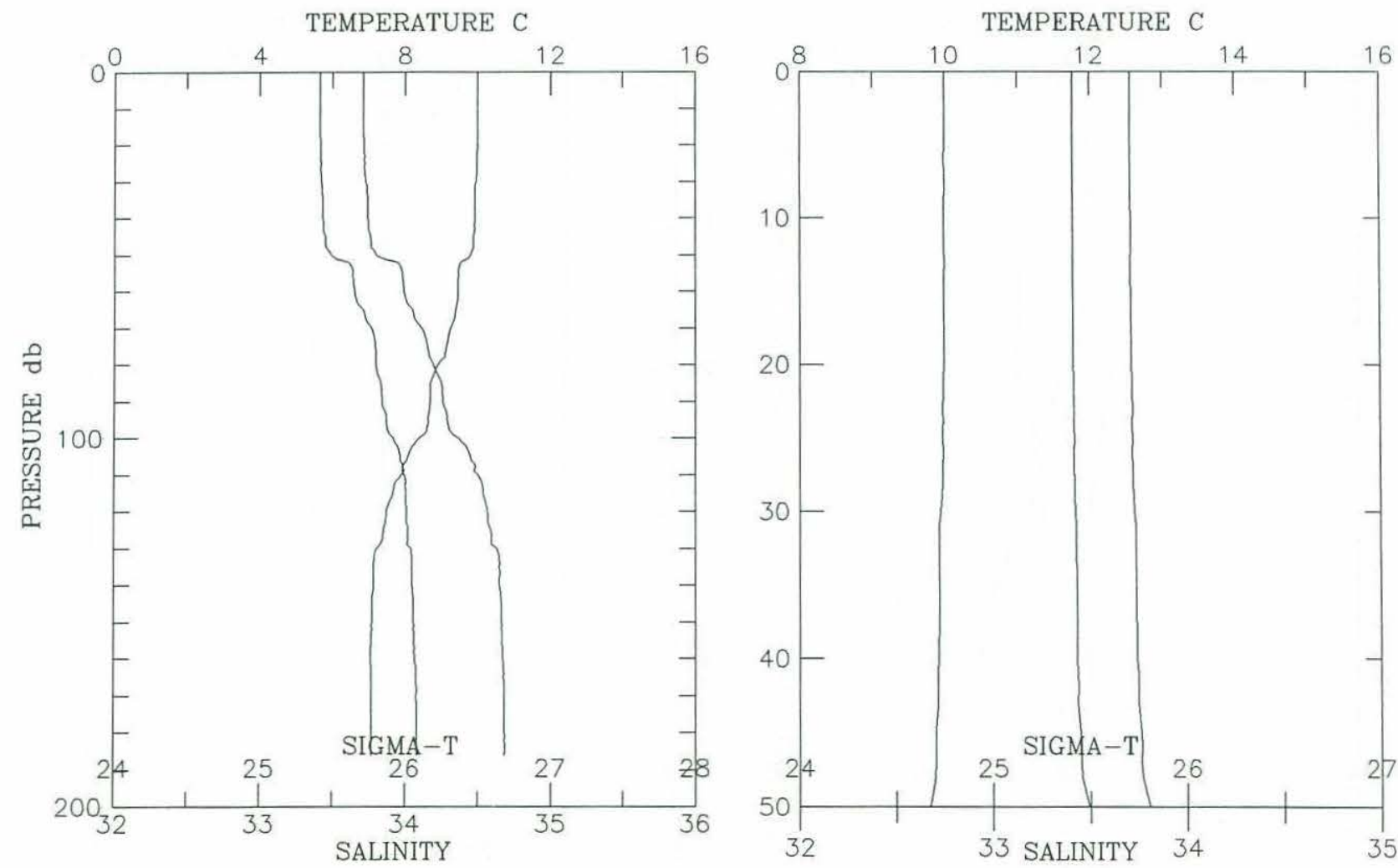
Cruise w8905 Station \# 134 Depth m: 316 Time: 1989-05-11 14:18

Position: $38 \quad 44.65^{\prime} \mathrm{N} 12350.63^{\prime} \mathrm{W}$ Depth Deck Offset: $\quad-0.30$

\begin{tabular}{|c|c|c|c|c|c|c|}
\hline $\begin{array}{l}\text { PRESSURE } \\
\text { (d-bars) }\end{array}$ & Interp & $\begin{array}{l}\text { TEMP } \\
(\operatorname{deg} C)\end{array}$ & $\begin{array}{l}\text { SALINITY } \\
(0 / 00)\end{array}$ & SIGMA-T & DELTA-D & $\begin{array}{l}\text { CHANNELA } \\
\text { ( } \delta 1 \text { ight) }\end{array}$ \\
\hline 0.0 & E & 10.359 & 33.349 & 25.602 & 0.0000 & 0.0 \\
\hline 10.0 & & 10.353 & 33.349 & 25.603 & 0.0241 & 0.0 \\
\hline 20.0 & & 10.357 & 33.349 & 25.603 & 0.0482 & 0.0 \\
\hline 30.0 & & 10.320 & 33.353 & 25.612 & 0.0724 & 0.0 \\
\hline 40.0 & & 10.170 & 33.348 & 25.634 & 0.0964 & 0.0 \\
\hline 50.0 & & 9.800 & 33.516 & 25.827 & 0.1194 & 0.0 \\
\hline 60.0 & & 9.389 & 33.664 & 26.010 & 0.1405 & 0.0 \\
\hline 80.0 & & 8.926 & 33.838 & 26.220 & 0.1790 & 0.0 \\
\hline 100.0 & & 8.299 & 33.941 & 26.398 & 0.2142 & 0.0 \\
\hline 150.0 & & 7.326 & 34.017 & 26.599 & 0.2915 & 0.0 \\
\hline 200.0 & & 7.060 & 34.077 & 26.684 & 0.3637 & 0.0 \\
\hline 250.0 & & 6.954 & 34.086 & 26.705 & 0.4343 & 0.0 \\
\hline 300.0 & & 6.648 & 34.096 & 26.755 & 0.5039 & 0.0 \\
\hline 312.0 & & 6.629 & 34.096 & 26.757 & 0.5203 & 0.0 \\
\hline
\end{tabular}
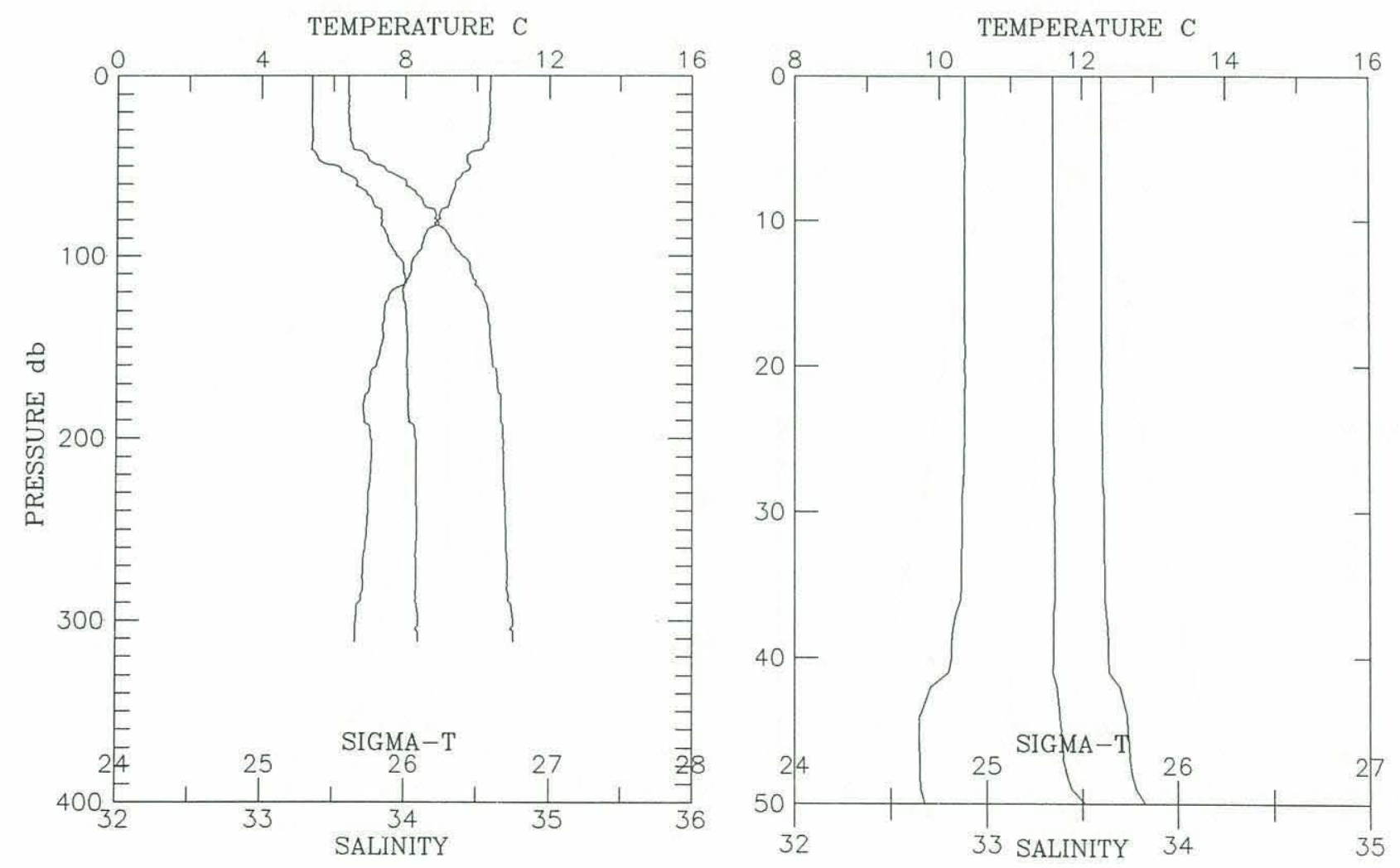
Cruise W8905 Station \# 135 Depth m: 965 Time: 1989-05-11 15:17

Position: $3843.07 ' \mathrm{~N} 12352.86^{\prime} \mathrm{W}$ Depth Deck Offset: -0.30

\begin{tabular}{|c|c|c|c|c|c|c|}
\hline $\begin{array}{l}\text { PRESSURE } \\
\text { (d-bars) }\end{array}$ & Interp & $\begin{array}{l}\text { TEMP } \\
(\operatorname{deg} G)\end{array}$ & $\begin{array}{c}\text { SALINITY } \\
(0 / 00)\end{array}$ & SIGMA-T & DELTA-D & $\begin{array}{l}\text { CHANNELA } \\
\text { ( } 81 \text { ight) }\end{array}$ \\
\hline 0.0 & $\mathrm{E}$ & 10.775 & 33.195 & 25.410 & 0.0000 & 0.0 \\
\hline 10.0 & & 10.785 & 33.194 & 25.408 & 0.0260 & 0.0 \\
\hline 20.0 & & 10.768 & 33.194 & 25.411 & 0.0519 & 0.0 \\
\hline 30.0 & & 10.726 & 33.193 & 25.417 & 0.0779 & 0.0 \\
\hline 40.0 & & 10.085 & 33.254 & 25.575 & 0.1033 & 0.0 \\
\hline 50.0 & & 9.793 & 33.308 & 25.666 & 0.1272 & 0.0 \\
\hline 60.0 & & 9.997 & 33.539 & 25.812 & 0.1501 & 0.0 \\
\hline 80.0 & & 9.239 & 33.725 & 26.082 & 0.1924 & 0.0 \\
\hline 100.0 & & 8.808 & 33.863 & 26.259 & 0.2302 & 0.0 \\
\hline 150.0 & & 7.611 & 33.978 & 26.528 & 0.3144 & 0.0 \\
\hline 200.0 & & 7.312 & 34.044 & 26.623 & 0.3899 & 0.0 \\
\hline 250.0 & & 6.905 & 34.062 & 26.693 & 0.4621 & 0.0 \\
\hline 300.0 & & 6.372 & 34.034 & 26.742 & 0.5319 & 0.0 \\
\hline 400.0 & & 6.284 & 34.159 & 26.852 & 0.6641 & 0.0 \\
\hline 500.0 & & 5.913 & 34.204 & 26.935 & 0.7892 & 0.0 \\
\hline 600.0 & & 5.603 & 34.252 & 27.011 & 0.9079 & 0.0 \\
\hline 700.0 & & 5.116 & 34.315 & 27.119 & 1.0183 & 0.0 \\
\hline 800.0 & & 4.654 & 34.374 & 27.218 & 1.1186 & 0.0 \\
\hline 900.0 & & 4.111 & 34.409 & 27.304 & 1.2116 & 0.0 \\
\hline 951.0 & & 4.037 & 34.433 & 27.331 & 1.2562 & 0.0 \\
\hline
\end{tabular}
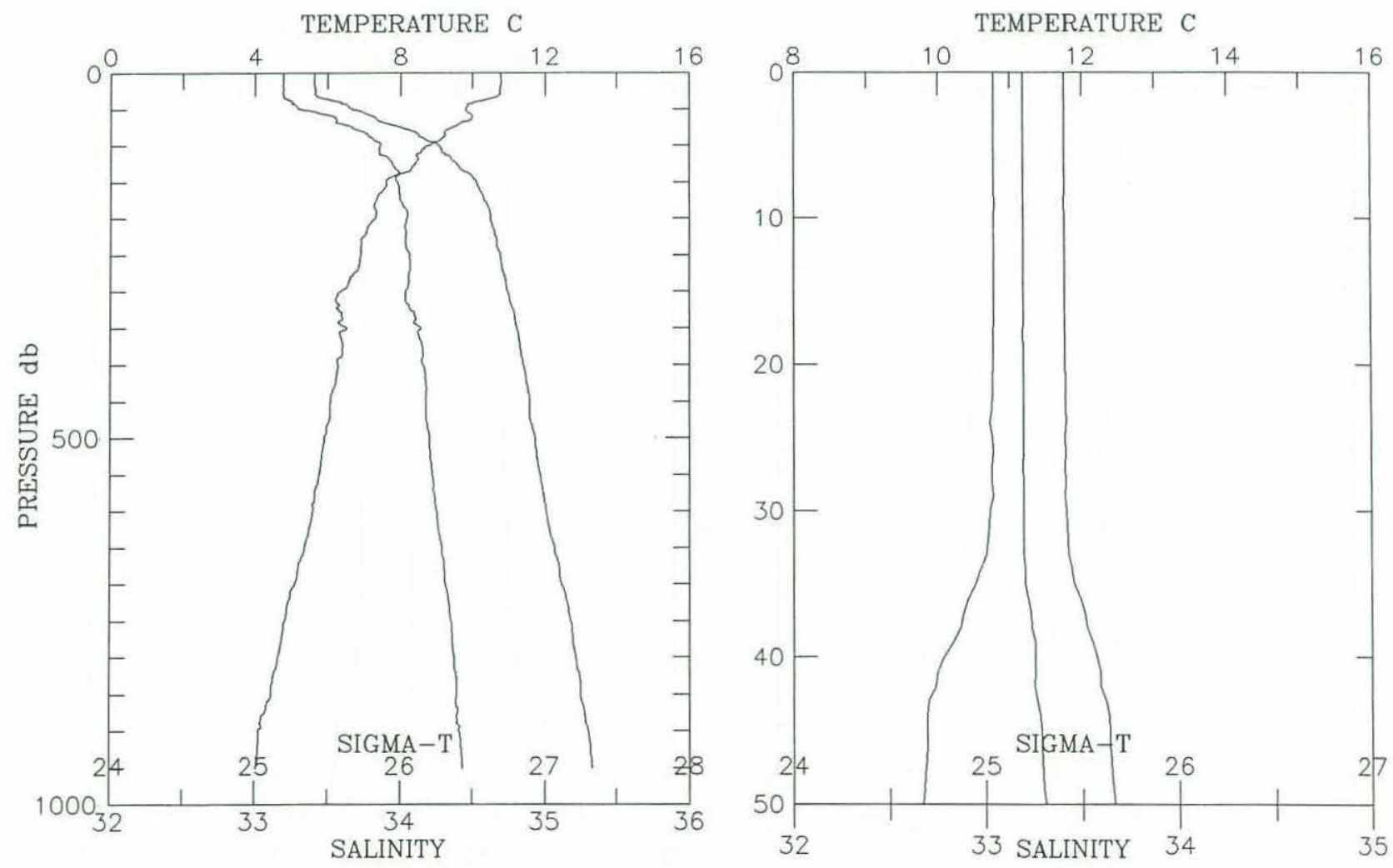


\begin{tabular}{|c|c|c|c|c|c|c|c|}
\hline $\begin{array}{l}\text { Cruise Wह } \\
\text { Position }\end{array}$ & $\begin{array}{l}8905 \\
: \quad 38\end{array}$ & $\begin{array}{l}\text { tation \# } \\
555^{\prime} \mathrm{N} 1\end{array}$ & $\begin{array}{r}136 \text { Dep } \\
23 \quad 47.22\end{array}$ & $\mathrm{~h} \mathrm{~m}: 885$ & $\begin{array}{l}\text { Time: } \\
\text { epth Decl }\end{array}$ & $\begin{array}{l}1989-05-11 \\
\text { Offset: }\end{array}$ & $\begin{array}{l}16: 50 \\
-0.30\end{array}$ \\
\hline $\begin{array}{l}\text { PRESSURE } \\
\text { (d-bars) }\end{array}$ & Interp & $\begin{array}{l}\text { TEMP } \\
(\operatorname{deg} C)\end{array}$ & $\begin{array}{l}\text { SALINITY } \\
(0 / 00)\end{array}$ & SIGMA-T & DELTA-D & $\begin{array}{l}\text { CHANNELA } \\
\text { ( } ₹ 1 \text { ight) }\end{array}$ & \\
\hline 0.0 & $\mathrm{E}$ & 9.541 & 33.506 & 25.862 & 0.0000 & 0.0 & \\
\hline 10.0 & & 9.569 & 33.503 & 25.855 & 0.0217 & 0.0 & \\
\hline 20.0 & & 9.548 & 33.504 & 25.859 & 0.0434 & 0.0 & \\
\hline 30.0 & & 9.425 & 33.520 & 25.892 & 0.0650 & 0.0 & \\
\hline 40.0 & & 9.356 & 33.537 & 25.916 & 0.0863 & 0.0 & \\
\hline 50.0 & & 9.252 & 33.568 & 25.957 & 0.1073 & 0.0 & \\
\hline 60.0 & & 8.947 & 33.646 & 26.067 & 0.1276 & 0.0 & \\
\hline 80.0 & & 8.482 & 33.846 & 26.296 & 0.1652 & 0.0 & \\
\hline 100.0 & & 8.064 & 33.900 & 26.400 & 0.1996 & 0.0 & \\
\hline 150.0 & & 7.742 & 33.998 & 26.525 & 0.2805 & 0.0 & \\
\hline 200.0 & & 7.276 & 34.035 & 26.620 & 0.3565 & 0.0 & \\
\hline 250.0 & & 7.045 & 34.107 & 26.709 & 0.4284 & 0.0 & \\
\hline 300.0 & & 6.689 & 34.144 & 26.787 & 0.4970 & 0.0 & \\
\hline 400.0 & & 6.362 & 34.152 & 26.837 & 0.6290 & 0.0 & \\
\hline 500.0 & & 5.967 & 34.194 & 26.920 & 0.7552 & 0.0 & \\
\hline 600.0 & & 5.495 & 34.266 & 27.036 & 0.8737 & 0.0 & \\
\hline 700.0 & & 5.039 & 34.326 & 27.136 & 0.9817 & 0.0 & \\
\hline 800.0 & & 4.709 & 34.368 & 27.207 & 1.0823 & 0.0 & \\
\hline 872.0 & & 4.568 & 34.384 & 27.235 & 1.1519 & 0.0 & \\
\hline
\end{tabular}
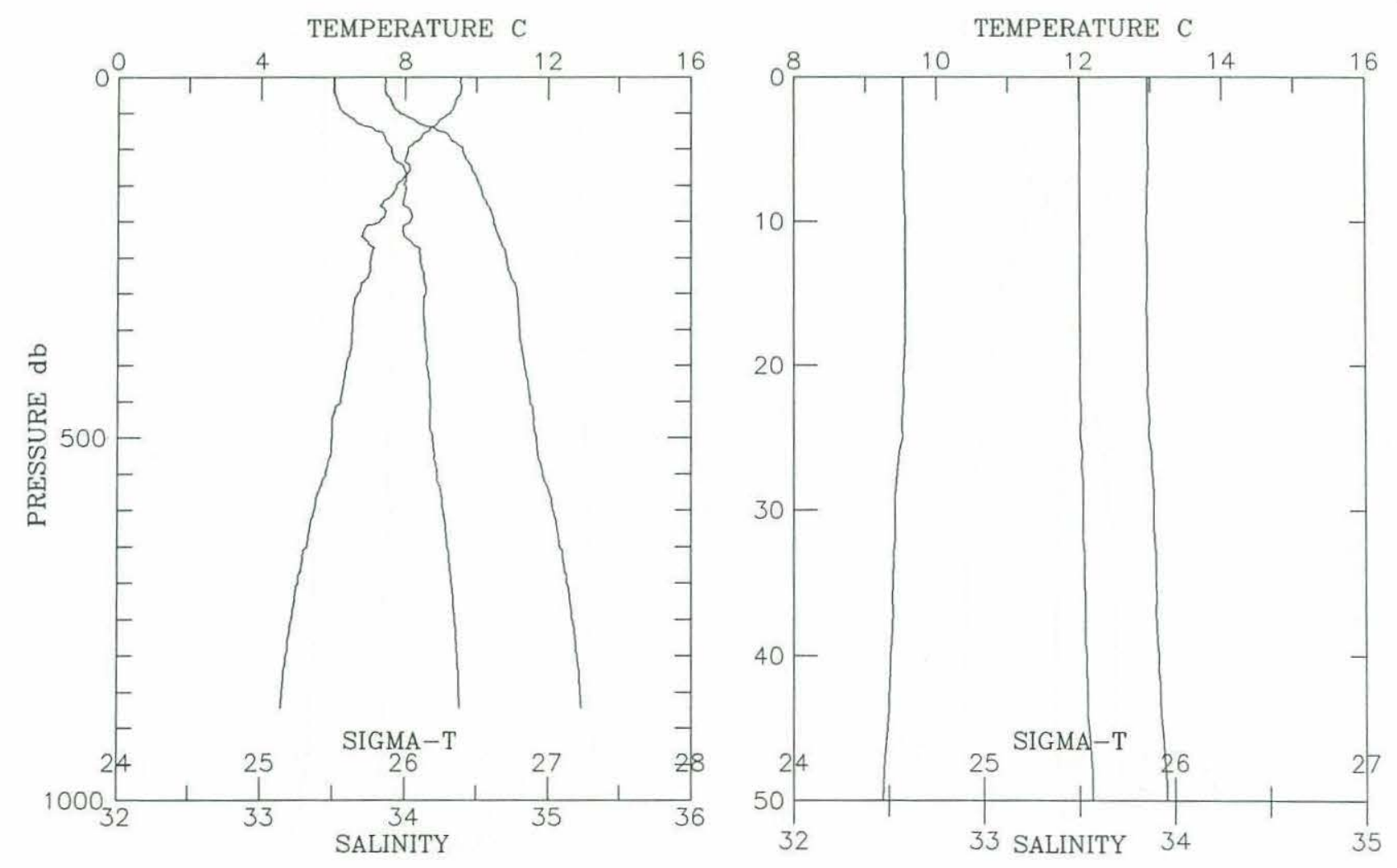
Cruise w8905 Station \# 137 Depth m: 400 Time: 1989-05-11 18:11

Position: $38 \quad 37.80^{\prime} \mathrm{N} 123 \quad 45.12^{\prime} \mathrm{W} \quad$ Depth Deck Offset: -0.30

\begin{tabular}{|c|c|c|c|c|c|c|}
\hline $\begin{array}{l}\text { PRESSURE } \\
\text { (d-bars) }\end{array}$ & Interp & $\begin{array}{c}\text { TEMP } \\
(\operatorname{deg} C)\end{array}$ & $\begin{array}{c}\text { SALINITY } \\
(0 / 00)\end{array}$ & SIGMA-T & DELTA-D & $\begin{array}{l}\text { CHANNELA } \\
\text { (§light) }\end{array}$ \\
\hline 0.0 & & 9.479 & 33.513 & 25.877 & 0.0000 & 0.0 \\
\hline 10.0 & & 9.469 & 33.530 & 25.893 & 0.0214 & 0.0 \\
\hline 20.0 & & 9.430 & 33.536 & 25.904 & 0.0427 & 0.0 \\
\hline 30.0 & & 9.347 & 33.552 & 25.929 & 0.0639 & 0.0 \\
\hline 40.0 & & 9.340 & 33.555 & 25.933 & 0.0849 & 0.0 \\
\hline 50.0 & & 9.225 & 33.588 & 25.977 & 0.1057 & 0.0 \\
\hline 60.0 & & 9.076 & 33.702 & 26.090 & 0.1261 & 0.0 \\
\hline 80.0 & & 8.476 & 33.857 & 26.305 & 0.1629 & 0.0 \\
\hline 100.0 & & 8.141 & 33.926 & 26.409 & 0.1972 & 0.0 \\
\hline 150.0 & & 7.509 & 33.992 & 26.553 & 0.2768 & 0.0 \\
\hline 200.0 & & 7.098 & 34.010 & 26.626 & 0.3519 & 0.0 \\
\hline 250.0 & & 6.881 & 34.081 & 26.711 & 0.4229 & 0.0 \\
\hline 300.0 & & 6.784 & 34.111 & 26.748 & 0.4918 & 0.0 \\
\hline 386.0 & & 6.676 & 34.112 & 26.763 & 0.6097 & 0.0 \\
\hline
\end{tabular}

TEMPERATURE C
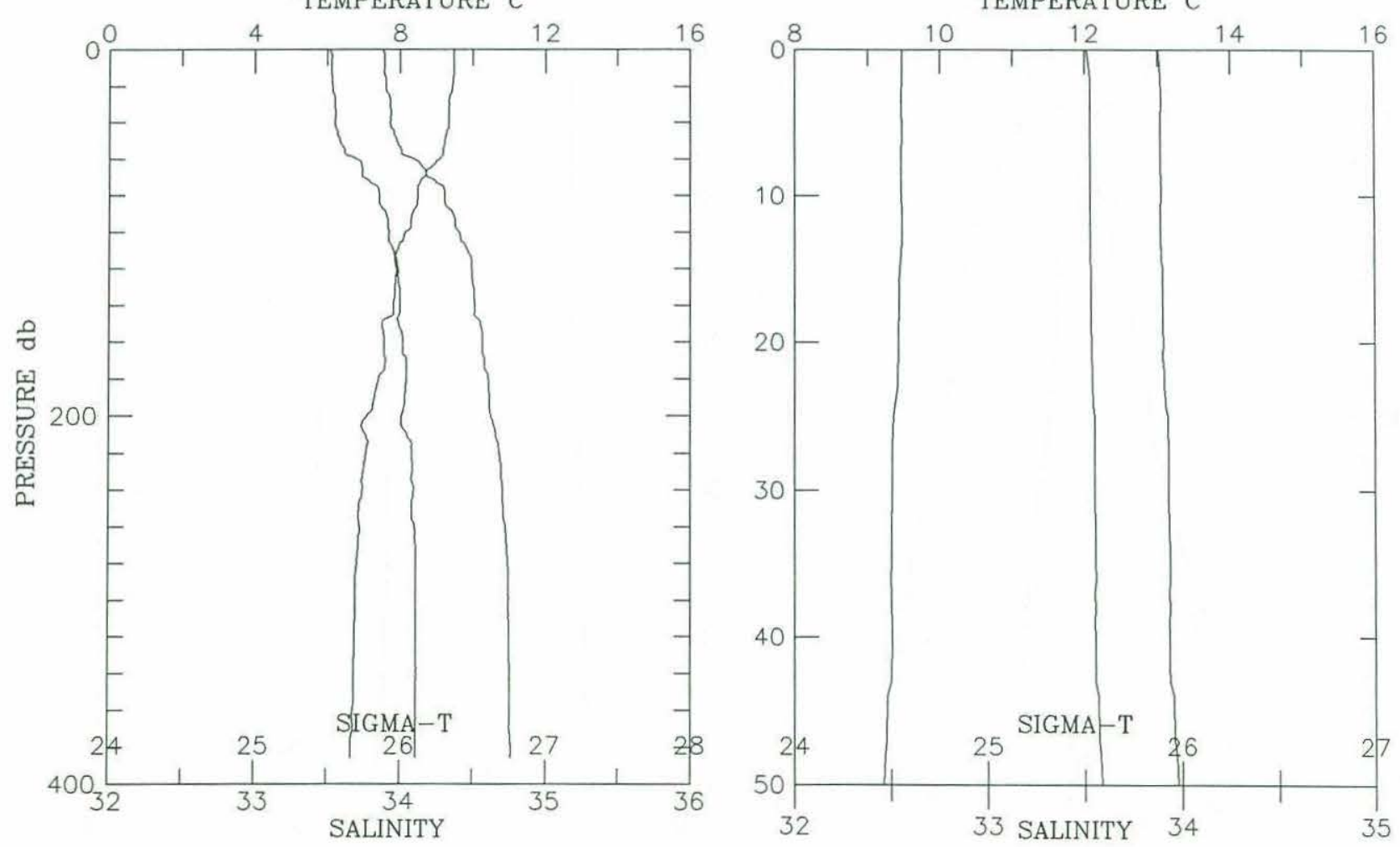
Cruise w8905 Station \# 138 Depth m: 180 Time: 1989-05-11 19:18 Position: $38 \quad 39.70^{\prime} \mathrm{N} 123 \quad 42.80^{\prime} \mathrm{W}$ Depth Deck Offset: -0.30

\begin{tabular}{|c|c|c|c|c|c|c|}
\hline $\begin{array}{l}\text { PRESSURE } \\
\text { (d-bars) }\end{array}$ & Interp & $\begin{array}{l}\text { TEMP } \\
(\operatorname{deg} C)\end{array}$ & $\begin{array}{c}\text { SALINITY } \\
(0 / 00)\end{array}$ & SIGMA-T & DELTA-D & $\begin{array}{l}\text { CHANNELA } \\
\text { ( } 81 \text { ight) }\end{array}$ \\
\hline 0.0 & E & 9.321 & 33.578 & 25.954 & 0.0000 & 0.0 \\
\hline 10.0 & & 9.312 & 33.579 & 25.956 & 0.0208 & 0.0 \\
\hline 20.0 & & 9.300 & 33.582 & 25.960 & 0.0415 & 0.0 \\
\hline 30.0 & & 9.241 & 33.598 & 25.983 & 0.0621 & 0.0 \\
\hline 40.0 & & 9.045 & 33.722 & 26.111 & 0.0823 & 0.0 \\
\hline 50.0 & & 8.514 & 33.837 & 26.284 & 0.1005 & 0.0 \\
\hline 60.0 & & 8.137 & 33.912 & 26.399 & 0.1177 & 0.0 \\
\hline 70.0 & & 8.082 & 33.925 & 26.417 & 0.1342 & 0.0 \\
\hline 80.0 & & 7.958 & 33.976 & 26.476 & 0.1504 & 0.0 \\
\hline 90.0 & & 7.828 & 34.003 & 26.516 & 0.1662 & 0.0 \\
\hline 100.0 & & 7.653 & 34.024 & 26.558 & 0.1816 & 0.0 \\
\hline 110.0 & & 7.564 & 34.028 & 26.574 & 0.1967 & 0.0 \\
\hline 120.0 & & 7.554 & 34.029 & 26.576 & 0.2118 & 0.0 \\
\hline 130.0 & & 7.490 & 34.029 & 26.585 & 0.2268 & 0.0 \\
\hline 140.0 & & 7.286 & 34.022 & 26.609 & 0.2417 & 0.0 \\
\hline 150.0 & & 7.277 & 34.026 & 26.613 & 0.2564 & 0.0 \\
\hline 160.0 & & 7.158 & 34.053 & 26.651 & 0.2709 & 0.0 \\
\hline 170.0 & & 7.157 & 34.054 & 26.652 & 0.2853 & 0.0 \\
\hline 171.0 & & 7.157 & 34.055 & 26.653 & 0.2867 & 0.0 \\
\hline
\end{tabular}
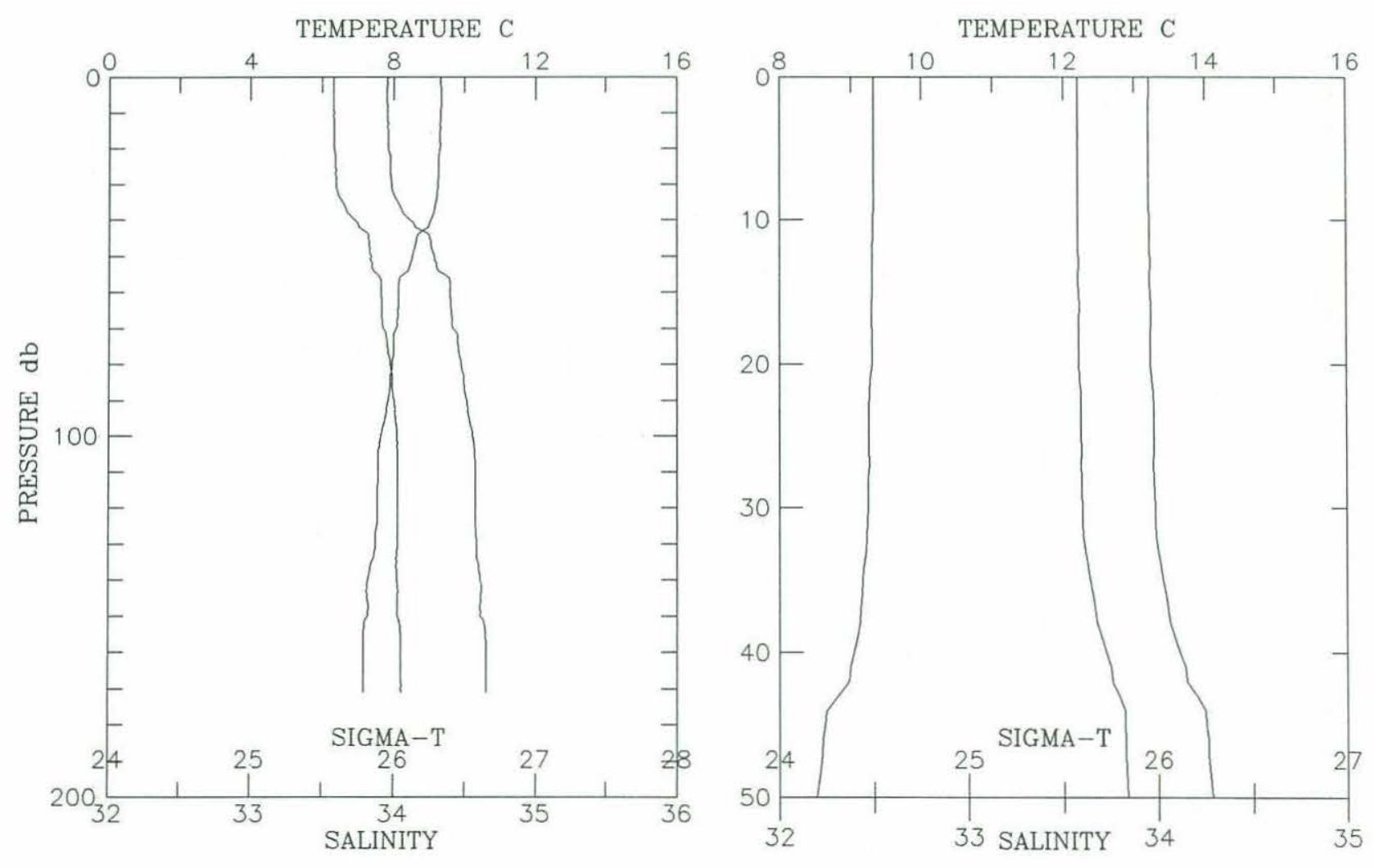
Cruise w8905 Station \# 139 Depth m: 135 Time: 1989-05-11 20:12 Position: $3841.50^{\prime} \mathrm{N} 12340.40^{\prime} \mathrm{W}$ Depth Deck Offset: -0.30

\begin{tabular}{|c|c|c|c|c|c|c|}
\hline $\begin{array}{l}\text { PRESSURE } \\
\text { (d-bars) }\end{array}$ & Interp & $\begin{array}{l}\text { TEMP } \\
(\operatorname{deg} C)\end{array}$ & $\begin{array}{l}\text { SALINITY } \\
(0 / 00)\end{array}$ & SIGMA-T & DELTA-D & $\begin{array}{l}\text { CHANNELA } \\
\text { ( } 81 \text { ight) }\end{array}$ \\
\hline 0.0 & E & 9.284 & 33.605 & 25.981 & 0.0000 & 0.0 \\
\hline 10.0 & & 9.291 & 33.601 & 25.977 & 0.0205 & 0.0 \\
\hline 20.0 & & 9.281 & 33.602 & 25.979 & 0.0411 & 0.0 \\
\hline 30.0 & & 8.987 & 33.743 & 26.136 & 0.0611 & 0.0 \\
\hline 40.0 & & 8.562 & 33.853 & 26.289 & 0.0794 & 0.0 \\
\hline 50.0 & & 8.384 & 33.887 & 26.342 & 0.0968 & 0.0 \\
\hline 60.0 & & 8.347 & 33.897 & 26.356 & 0.1139 & 0.0 \\
\hline 70.0 & & 8.172 & 33.920 & 26.400 & 0.1308 & 0.0 \\
\hline 80.0 & & 8.081 & 33.932 & 26.423 & 0.1474 & 0.0 \\
\hline 90.0 & & 8.002 & 33.946 & 26.446 & 0.1638 & 0.0 \\
\hline 100.0 & & 7.790 & 33.976 & 26.500 & 0.1799 & 0.0 \\
\hline 110.0 & & 7.603 & 34.018 & 26.561 & 0.1953 & 0.0 \\
\hline 120.0 & & 7.463 & 34.031 & 26.591 & 0.2105 & 0.0 \\
\hline 130.0 & & 7.317 & 34.059 & 26.634 & 0.2251 & 0.0 \\
\hline 131.0 & & 7.316 & 34.058 & 26.633 & 0.2265 & 0.0 \\
\hline
\end{tabular}
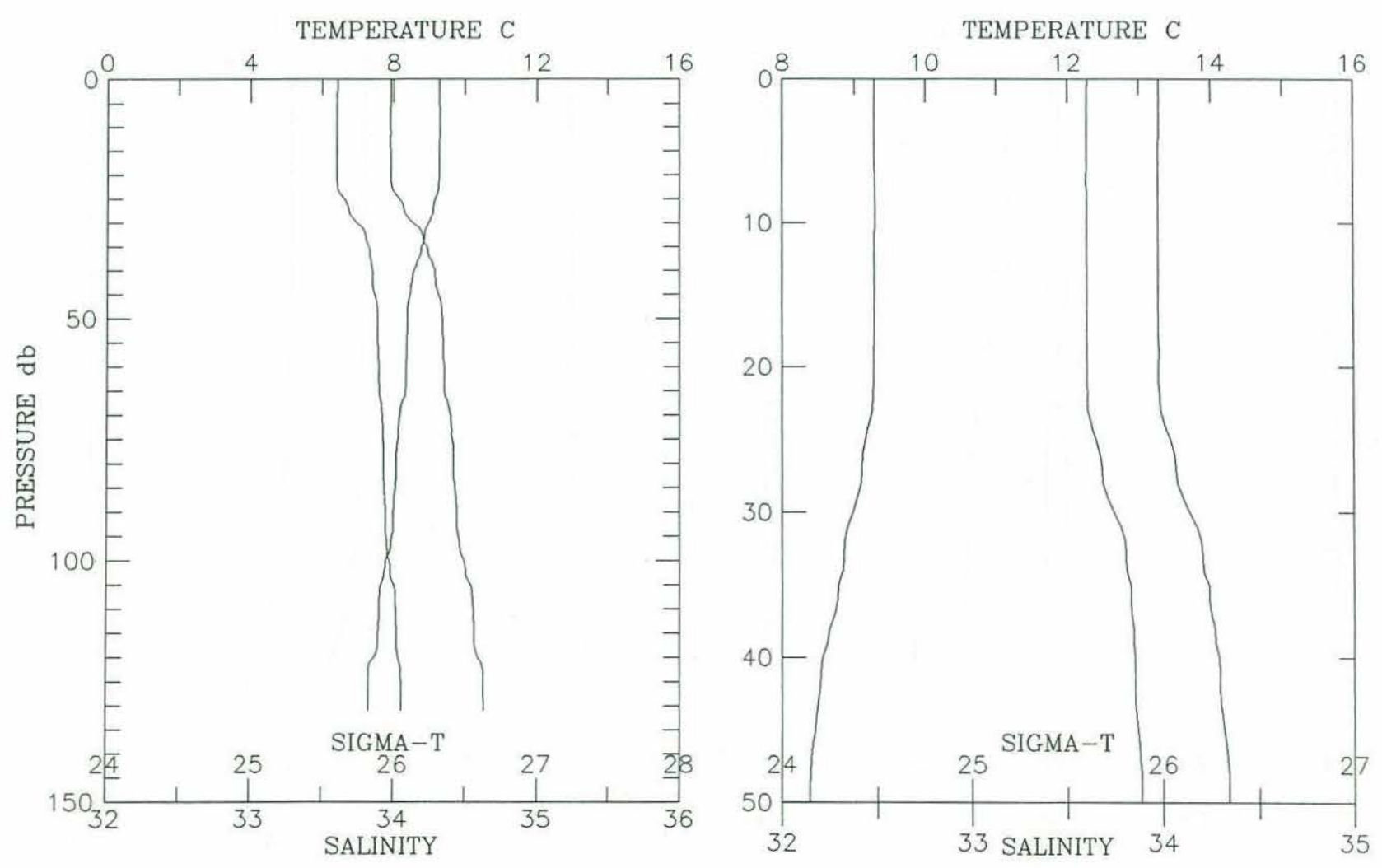
Cruise w8905 Station \# 140 Depth m: 118 Time: 1989-05-11 20:55

Position: $38 \quad 42.80^{\prime} \mathrm{N} 123 \quad 38.50^{\prime} \mathrm{W} \quad$ Depth Deck Offset: -0.30

\begin{tabular}{|c|c|c|c|c|c|c|}
\hline $\begin{array}{l}\text { PRESSURE } \\
\text { (d-bars) }\end{array}$ & Interp & $\begin{array}{l}\text { TEMP } \\
(\operatorname{deg} C)\end{array}$ & $\begin{array}{c}\text { SALINITY } \\
(0 / 00)\end{array}$ & SIGMA-T & DELTA-D & $\begin{array}{l}\text { CHANNELA } \\
\text { ( }(81 \text { ight) }\end{array}$ \\
\hline 0.0 & E & 9.140 & 33.646 & 26.036 & 0.0000 & 0.0 \\
\hline 10.0 & & 9.133 & 33.645 & 26.037 & 0.0200 & 0.0 \\
\hline 20.0 & & 9.113 & 33.651 & 26.045 & 0.0400 & 0.0 \\
\hline 30.0 & & 8.808 & 33.809 & 26.216 & 0.0594 & 0.0 \\
\hline 40.0 & & 8.514 & 33.849 & 26.292 & 0.0774 & 0.0 \\
\hline 50.0 & & 8.420 & 33.897 & 26.344 & 0.0947 & 0.0 \\
\hline 60.0 & & 8.412 & 33.898 & 26.347 & 0.1118 & 0.0 \\
\hline 70.0 & & 8.235 & 33.915 & 26.387 & 0.1289 & 0.0 \\
\hline 80.0 & & 8.050 & 33.932 & 26.428 & 0.1455 & 0.0 \\
\hline 90.0 & & 7.913 & 33.963 & 26.472 & 0.1617 & 0.0 \\
\hline 100.0 & & 7.691 & 33.996 & 26.531 & 0.1775 & 0.0 \\
\hline 110.0 & & 7.436 & 34.039 & 26.601 & 0.1926 & 0.0 \\
\hline 113.0 & & 7.375 & 34.049 & 26.618 & 0.1970 & 0.0 \\
\hline
\end{tabular}
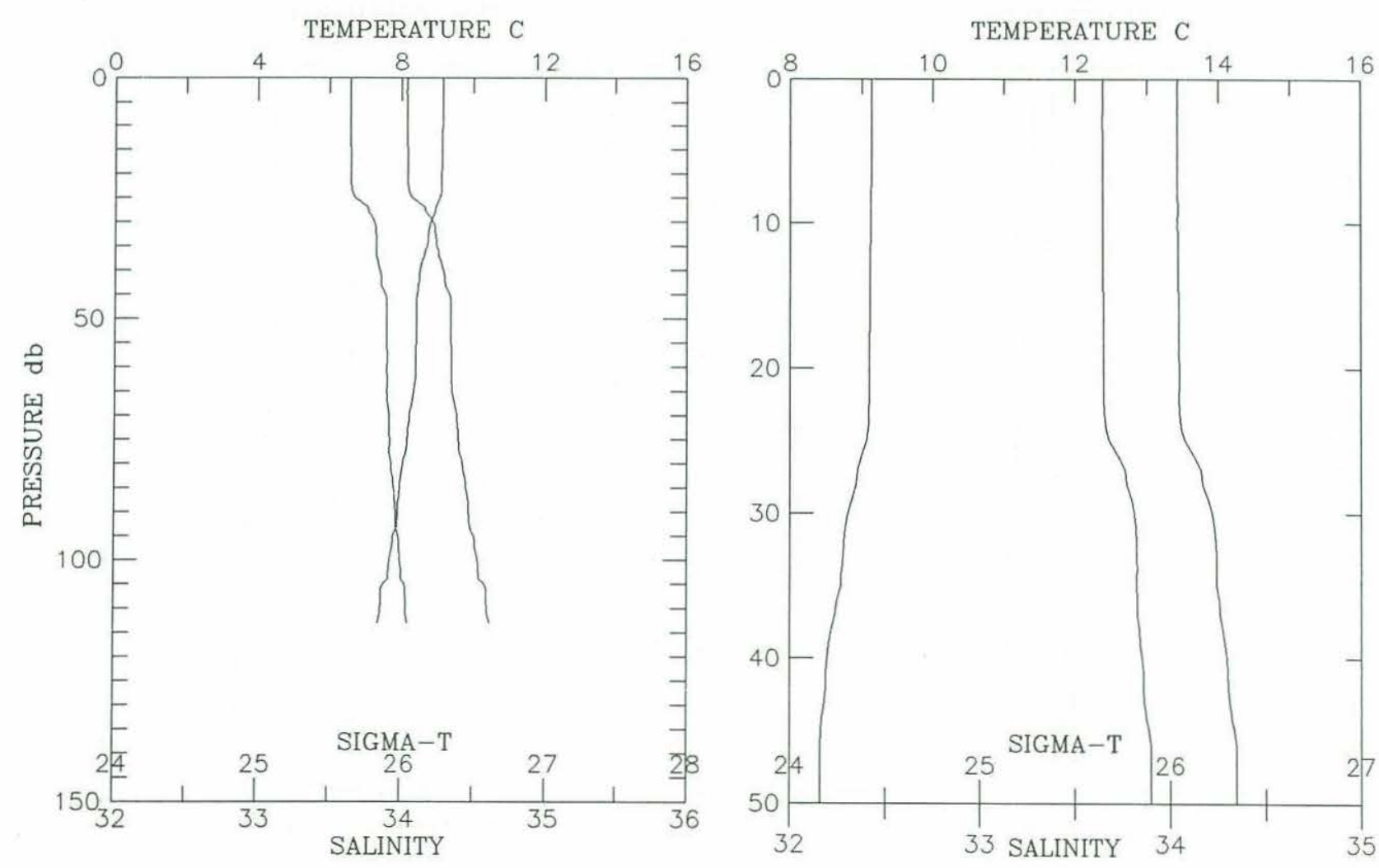
Cruise w8905 Station \# 141 Depth m: 95

Time: 1989-05-11 21:48

Position: $38 \quad 44.10^{\prime} \mathrm{N} \quad 123 \quad 36.10^{\prime} \mathrm{W}$

Depth Deck Offset: -0.30

\begin{tabular}{|c|c|c|c|c|c|c|}
\hline $\begin{array}{l}\text { PRESSURE } \\
\text { (d-bars) }\end{array}$ & Interp & $\begin{array}{l}\text { TEMP } \\
(\operatorname{deg} \quad C)\end{array}$ & $\begin{array}{c}\text { SALINITY } \\
(0 / 00)\end{array}$ & SIGMA-T & DELTA-D & $\begin{array}{l}\text { CHANNELA } \\
\text { ( }(1 \mathrm{light})\end{array}$ \\
\hline 0.0 & $\mathrm{E}$ & 8.997 & 33.708 & 26.108 & 0.0000 & 0.0 \\
\hline 5.0 & & 8.992 & 33.711 & 26.111 & 0.0097 & 0.0 \\
\hline 10.0 & & 9.009 & 33.708 & 26.106 & 0.0193 & 0.0 \\
\hline 15.0 & & 8.998 & 33.709 & 26.108 & 0.0290 & 0.0 \\
\hline 20.0 & & 8.953 & 33.714 & 26.119 & 0.0386 & 0.0 \\
\hline 25.0 & & 8.870 & 33.736 & 26.149 & 0.0482 & 0.0 \\
\hline 30.0 & & 8.695 & 33.792 & 26.221 & 0.0576 & 0.0 \\
\hline 35.0 & & 8.526 & 33.844 & 26.287 & 0.0665 & 0.0 \\
\hline 40.0 & & 8.497 & 33.858 & 26.302 & 0.0753 & 0.0 \\
\hline 45.0 & & 8.472 & 33.884 & 26.326 & 0.0840 & 0.0 \\
\hline 50.0 & & 8.417 & 33.902 & 26.349 & 0.0926 & 0.0 \\
\hline 55.0 & & 8.273 & 33.907 & 26.375 & 0.1011 & 0.0 \\
\hline 60.0 & & 8.221 & 33.918 & 26.391 & 0.1095 & 0.0 \\
\hline 65.0 & & 8.156 & 33.932 & 26.412 & 0.1178 & 0.0 \\
\hline 70.0 & & 8.134 & 33.935 & 26.417 & 0.1261 & 0.0 \\
\hline 75.0 & & 8.124 & 33.937 & 26.421 & 0.1343 & 0.0 \\
\hline 80.0 & & 8.101 & 33.939 & 26.426 & 0.1426 & 0.0 \\
\hline 85.0 & & 7.860 & 33.969 & 26.485 & 0.1506 & 0.0 \\
\hline 90.0 & & 7.584 & 34.009 & 26.556 & 0.1585 & 0.0 \\
\hline 92.0 & & 7.529 & 34.019 & 26.572 & 0.1615 & 0.0 \\
\hline
\end{tabular}

TEMPERATURE $\mathrm{C}$
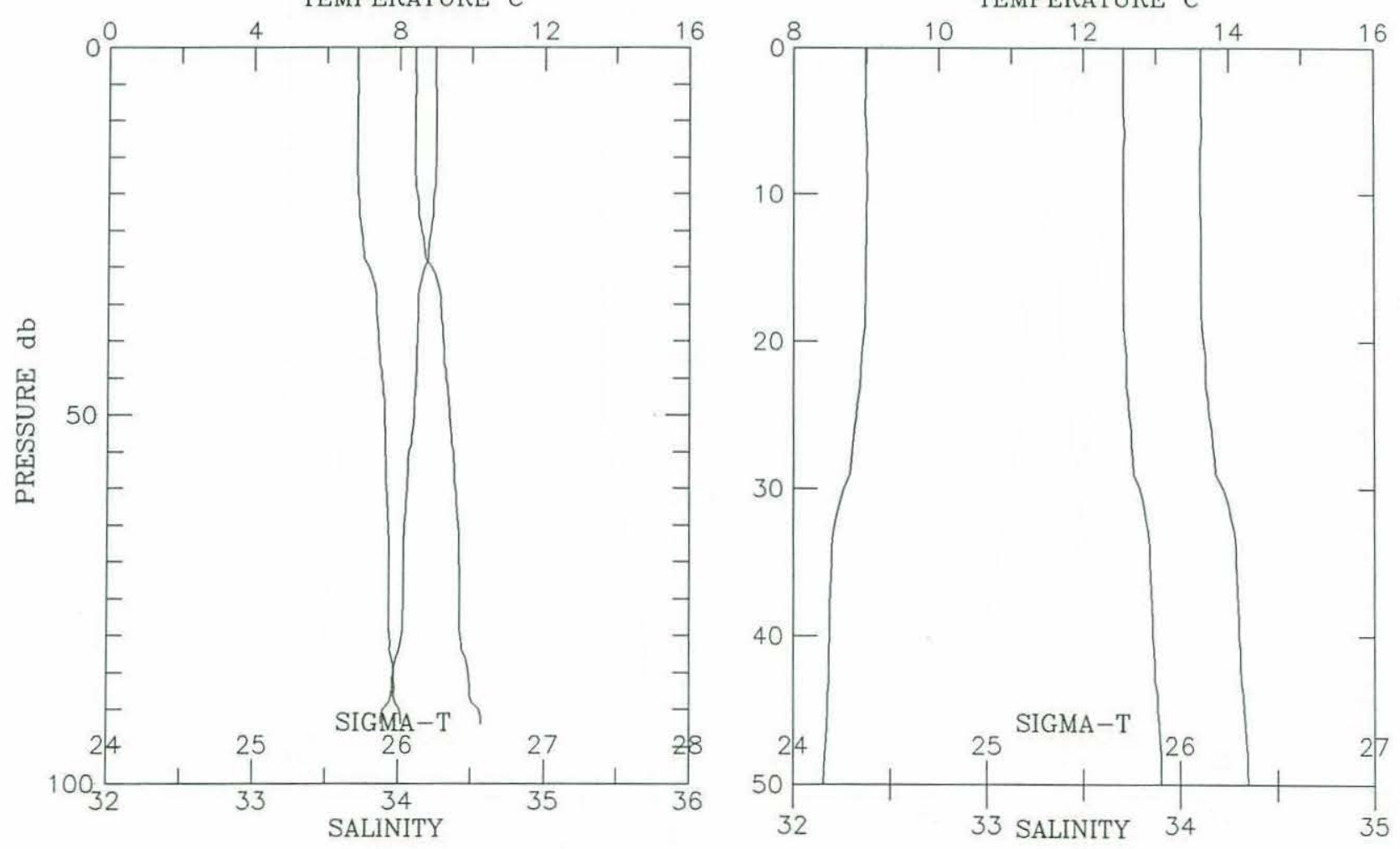


\begin{tabular}{|c|c|c|c|c|c|c|}
\hline $\begin{array}{l}\text { PRESSURE } \\
\text { (d-bars) }\end{array}$ & Interp & $\begin{array}{c}\text { TEMP } \\
(\operatorname{deg} C)\end{array}$ & $\begin{array}{c}\text { SALINITY } \\
(0 / 00)\end{array}$ & SIGMA-T & DELTA-D & $\begin{array}{l}\text { CHANNELA } \\
\text { ( }(\mathrm{i} 1 \mathrm{ght})\end{array}$ \\
\hline 0.0 & $\mathrm{E}$ & 8.997 & 33.708 & 26.108 & 0.0000 & 0.0 \\
\hline 5.0 & & 8.992 & 33.711 & 26.111 & 0.0097 & 0.0 \\
\hline 10.0 & & 9.009 & 33.708 & 26.106 & 0.0193 & 0.0 \\
\hline 15.0 & & 8.998 & 33.709 & 26.108 & 0.0290 & 0.0 \\
\hline 20.0 & & 8.953 & 33.714 & 26.119 & 0.0386 & 0.0 \\
\hline 25.0 & & 8.870 & 33.736 & 26.149 & 0.0482 & 0.0 \\
\hline 30.0 & & 8.695 & 33.792 & 26.221 & 0.0576 & 0.0 \\
\hline 35.0 & & 8.526 & 33.844 & 26.287 & 0.0665 & 0.0 \\
\hline 40.0 & & 8.497 & 33.858 & 26.302 & 0.0753 & 0.0 \\
\hline 45.0 & & 8.472 & 33.884 & 26.326 & 0.0840 & 0.0 \\
\hline 50.0 & & 8.417 & 33.902 & 26.349 & 0.0926 & 0.0 \\
\hline 55.0 & & 8.273 & 33.907 & 26.375 & 0.1011 & 0.0 \\
\hline 60.0 & & 8.221 & 33.918 & 26.391 & 0.1095 & 0.0 \\
\hline 65.0 & & 8.156 & 33.932 & 26.412 & 0.1178 & 0.0 \\
\hline 70.0 & & 8.134 & 33.935 & 26.417 & 0.1261 & 0.0 \\
\hline 75.0 & & 8.124 & 33.937 & 26.421 & 0.1343 & 0.0 \\
\hline 80.0 & & 8.101 & 33.939 & 26.426 & 0.1426 & 0.0 \\
\hline 85.0 & & 7.860 & 33.969 & 26.485 & 0.1506 & 0.0 \\
\hline 90.0 & & 7.584 & 34.009 & 26.556 & 0.1585 & 0.0 \\
\hline 92.0 & & 7.529 & 34.019 & 26.572 & 0.1615 & 0.0 \\
\hline
\end{tabular}
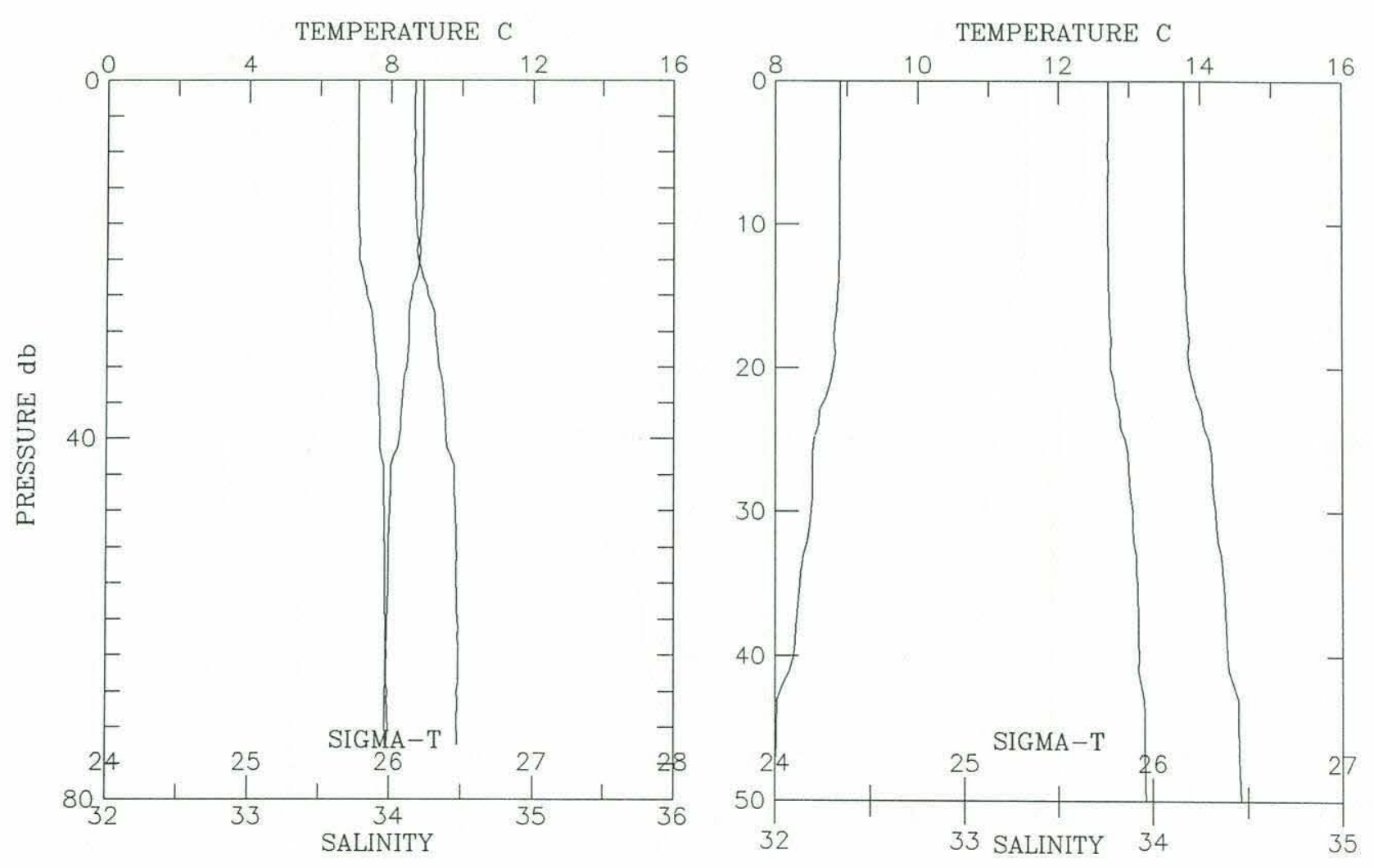
Cruise W8905 Station \# 142 Depth m: 80 Position: 38 $39.83^{\prime} \mathrm{N} \quad 123 \quad 27.67^{\prime} \mathrm{W}$
Time: 1989-05-11 23:49

Depth Deck Offset: -0.30

\begin{tabular}{|c|c|c|c|c|c|c|}
\hline $\begin{array}{l}\text { PRESSURE } \\
\text { (d-bars) }\end{array}$ & Interp & $\begin{array}{l}\text { TEMP } \\
(\operatorname{deg} C)\end{array}$ & $\begin{array}{c}\text { SALINITY } \\
(0 / 00)\end{array}$ & SIGMA-T & DELTA-D & $\begin{array}{l}\text { CHANNELA } \\
\text { ( } \% 1 \text { ight) }\end{array}$ \\
\hline 0.0 & $\mathrm{E}$ & 8.907 & 33.764 & 26.166 & 0.0000 & 0.0 \\
\hline 5.0 & & 8.905 & 33.762 & 26.164 & 0.0094 & 0.0 \\
\hline 10.0 & & 8.899 & 33.762 & 26.165 & 0.0188 & 0.0 \\
\hline 15.0 & & 8.866 & 33.767 & 26.174 & 0.0281 & 0.0 \\
\hline 20.0 & & 8.830 & 33.774 & 26.186 & 0.0375 & 0.0 \\
\hline 25.0 & & 8.552 & 33.846 & 26.284 & 0.0465 & 0.0 \\
\hline 30.0 & & 8.507 & 33.890 & 26.326 & 0.0553 & 0.0 \\
\hline 35.0 & & 8.344 & 33.916 & 26.371 & 0.0638 & 0.0 \\
\hline 40.0 & & 8.259 & 33.925 & 26.391 & 0.0722 & 0.0 \\
\hline 45.0 & & 8.018 & 33.954 & 26.450 & 0.0804 & 0.0 \\
\hline 50.0 & & 7.974 & 33.961 & 26.462 & 0.0884 & 0.0 \\
\hline 55.0 & & 7.960 & 33.962 & 26.465 & 0.0964 & 0.0 \\
\hline 60.0 & & 7.915 & 33.967 & 26.475 & 0.1044 & 0.0 \\
\hline 65.0 & & 7.908 & 33.968 & 26.477 & 0.1124 & 0.0 \\
\hline 70.0 & & 7.927 & 33.966 & 26.473 & 0.1204 & 0.0 \\
\hline 74.0 & & 7.929 & 33.967 & 26.473 & 0.1267 & 0.0 \\
\hline
\end{tabular}
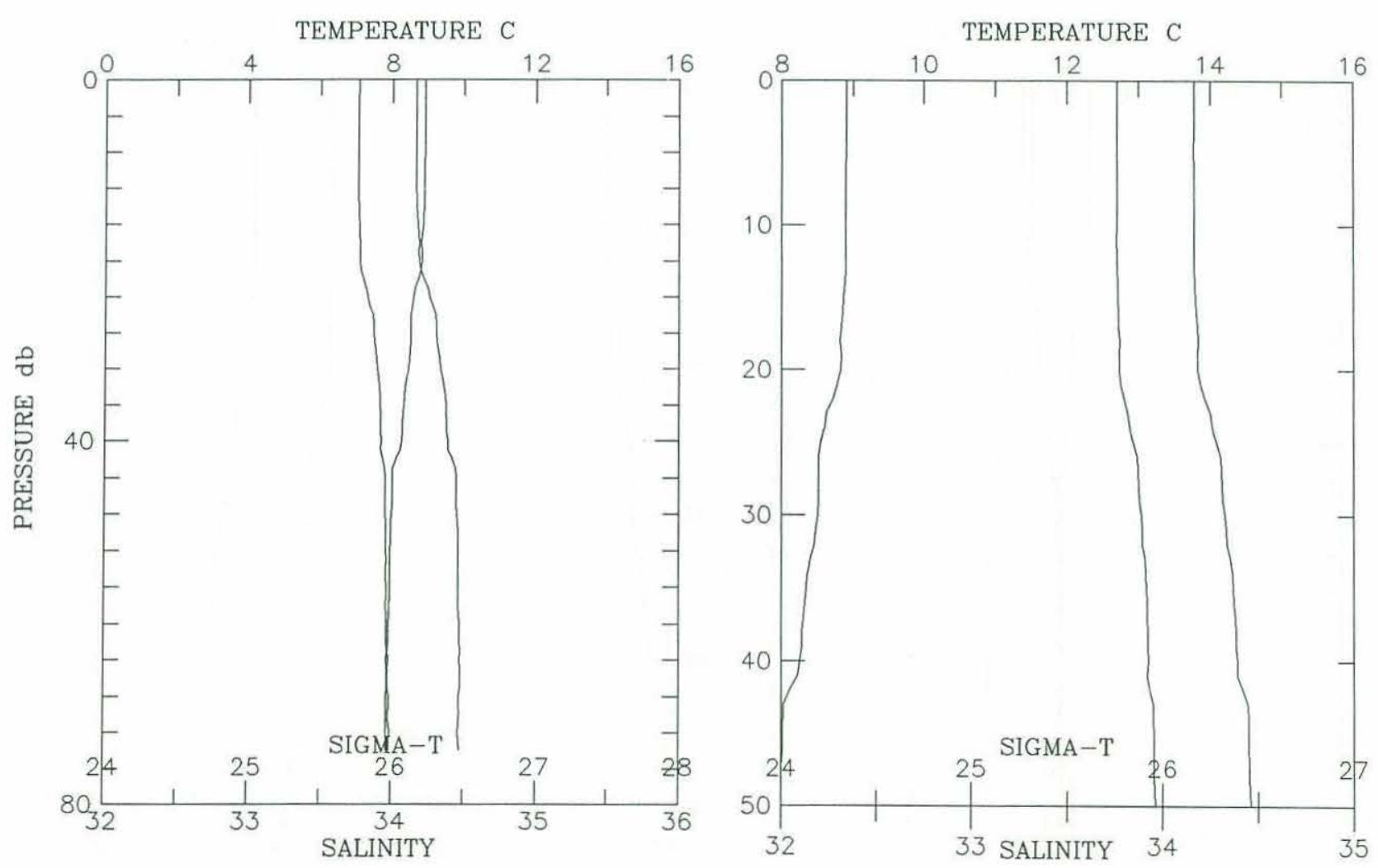
Cruise W8905 Station \# 143 Depth m: 95

Time: 1989-05-12 00:25

Position: $38 \quad 38.43^{\prime} \mathrm{N} 12329.24^{\prime} \mathrm{W}$ Depth Deck offset: -0.30

\begin{tabular}{|c|c|c|c|c|c|c|}
\hline $\begin{array}{l}\text { PRESSURE } \\
\text { (d-bars) }\end{array}$ & Interp & $\begin{array}{l}\text { TEMP } \\
(\operatorname{deg} C)\end{array}$ & $\begin{array}{c}\text { SALINITY } \\
(0 / 00)\end{array}$ & SIGMA-T & DELTA-D & $\begin{array}{l}\text { CHANNELA } \\
\text { ( } \$ 1 \text { ight) }\end{array}$ \\
\hline 0.0 & $\mathrm{E}$ & 9.016 & 33.775 & 26.157 & 0.0000 & 0.0 \\
\hline 5.0 & & 9.016 & 33.774 & 26.156 & 0.0094 & 0.0 \\
\hline 10.0 & & 9.011 & 33.775 & 26.158 & 0.0188 & 0.0 \\
\hline 15.0 & & 9.011 & 33.775 & 26.158 & 0.0283 & 0.0 \\
\hline 20.0 & & 8.996 & 33.778 & 26.163 & 0.0377 & 0.0 \\
\hline 25.0 & & 8.997 & 33.779 & 26.163 & 0.0471 & 0.0 \\
\hline 30.0 & & 8.996 & 33.779 & 26.163 & 0.0565 & 0.0 \\
\hline 35.0 & & 8.963 & 33.785 & 26.173 & 0.0659 & 0.0 \\
\hline 40.0 & & 8.914 & 33.797 & 26.190 & 0.0753 & 0.0 \\
\hline 45.0 & & 8.860 & 33.807 & 26.207 & 0.0845 & 0.0 \\
\hline 50.0 & & 8.747 & 33.825 & 26.238 & 0.0937 & 0.0 \\
\hline 55.0 & & 8.642 & 33.858 & 26.281 & 0.1027 & 0.0 \\
\hline 60.0 & & 8.559 & 33.893 & 26.320 & 0.1114 & 0.0 \\
\hline 65.0 & & 8.524 & 33.898 & 26.329 & 0.1201 & 0.0 \\
\hline 70.0 & & 8.469 & 33.907 & 26.345 & 0.1287 & 0.0 \\
\hline 75.0 & & 8.329 & 33.921 & 26.377 & 0.1373 & 0.0 \\
\hline 80.0 & & 8.111 & 33.956 & 26.437 & 0.1456 & 0.0 \\
\hline 85.0 & & 7.903 & 33.988 & 26.493 & 0.1536 & 0.0 \\
\hline 90.0 & & 7.814 & 34.006 & 26.521 & 0.1615 & 0.0 \\
\hline
\end{tabular}
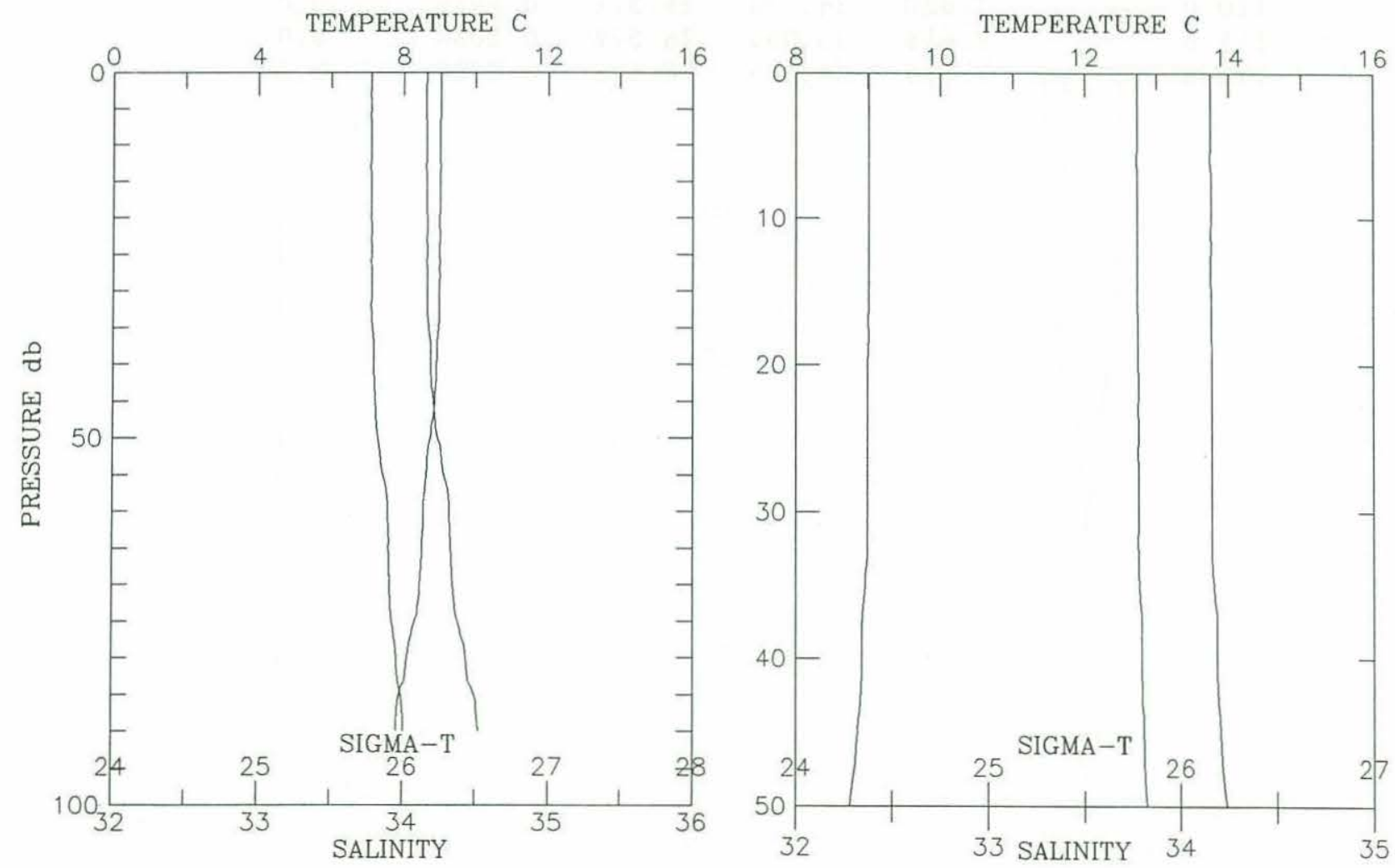


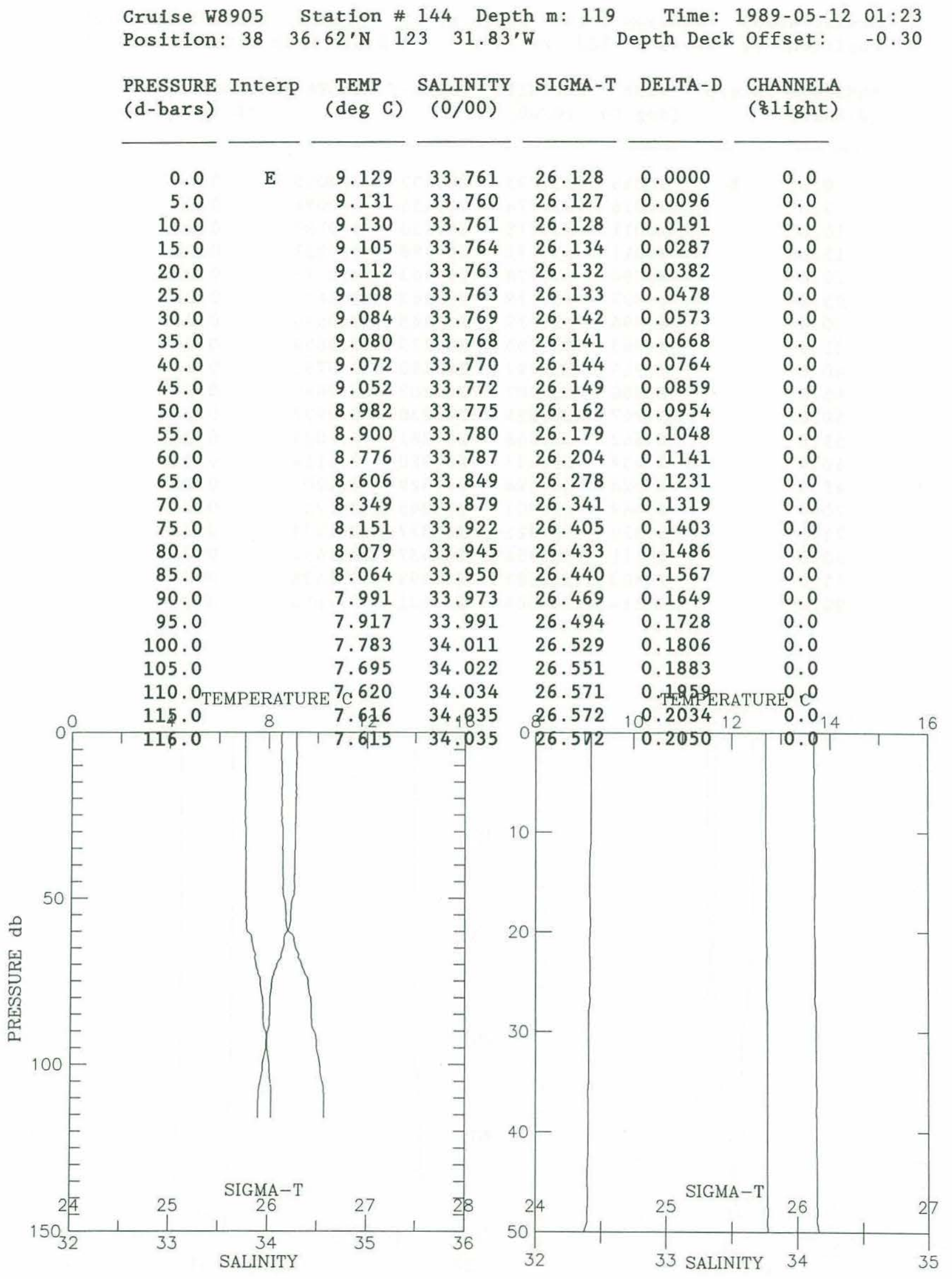




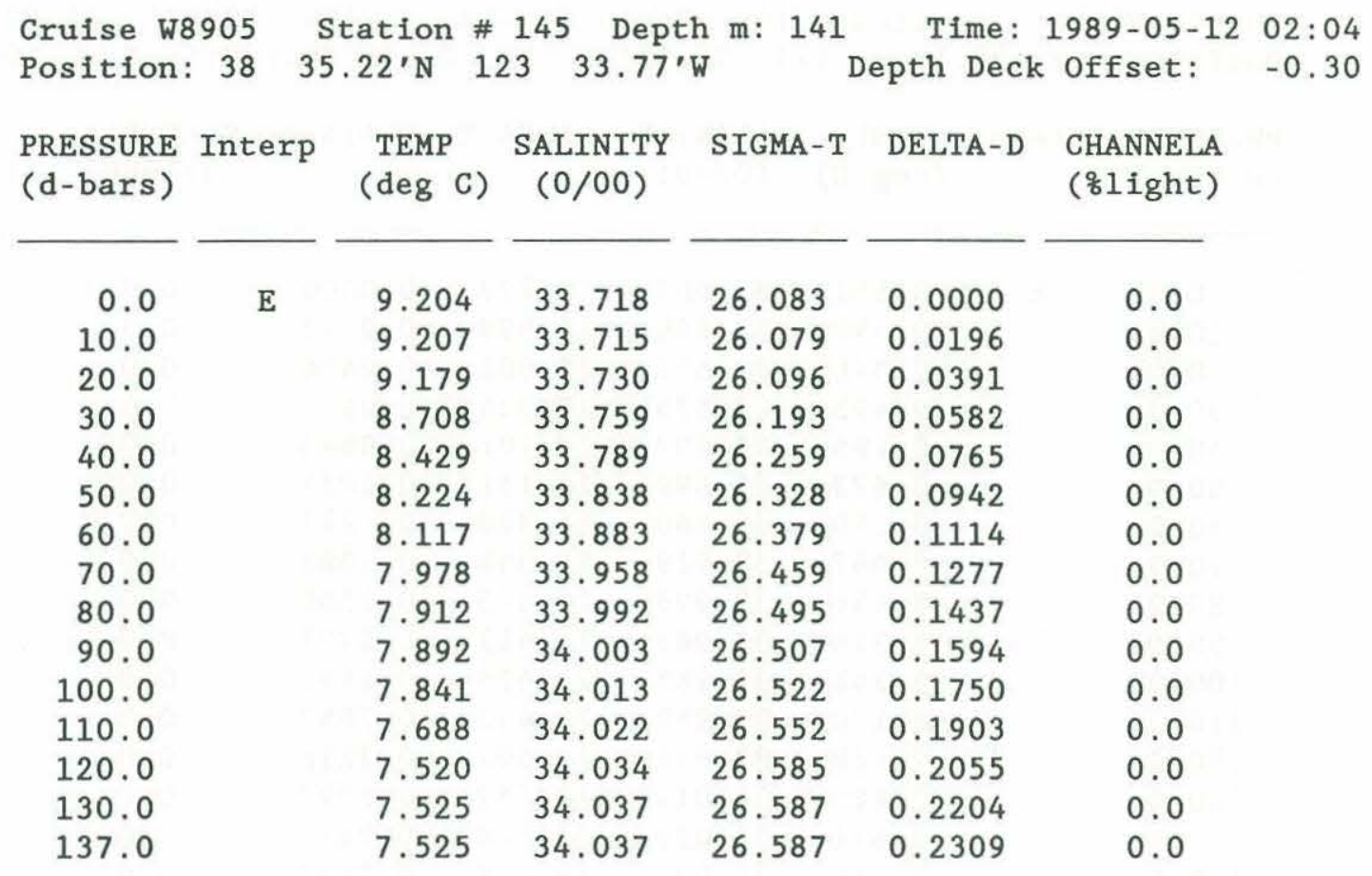
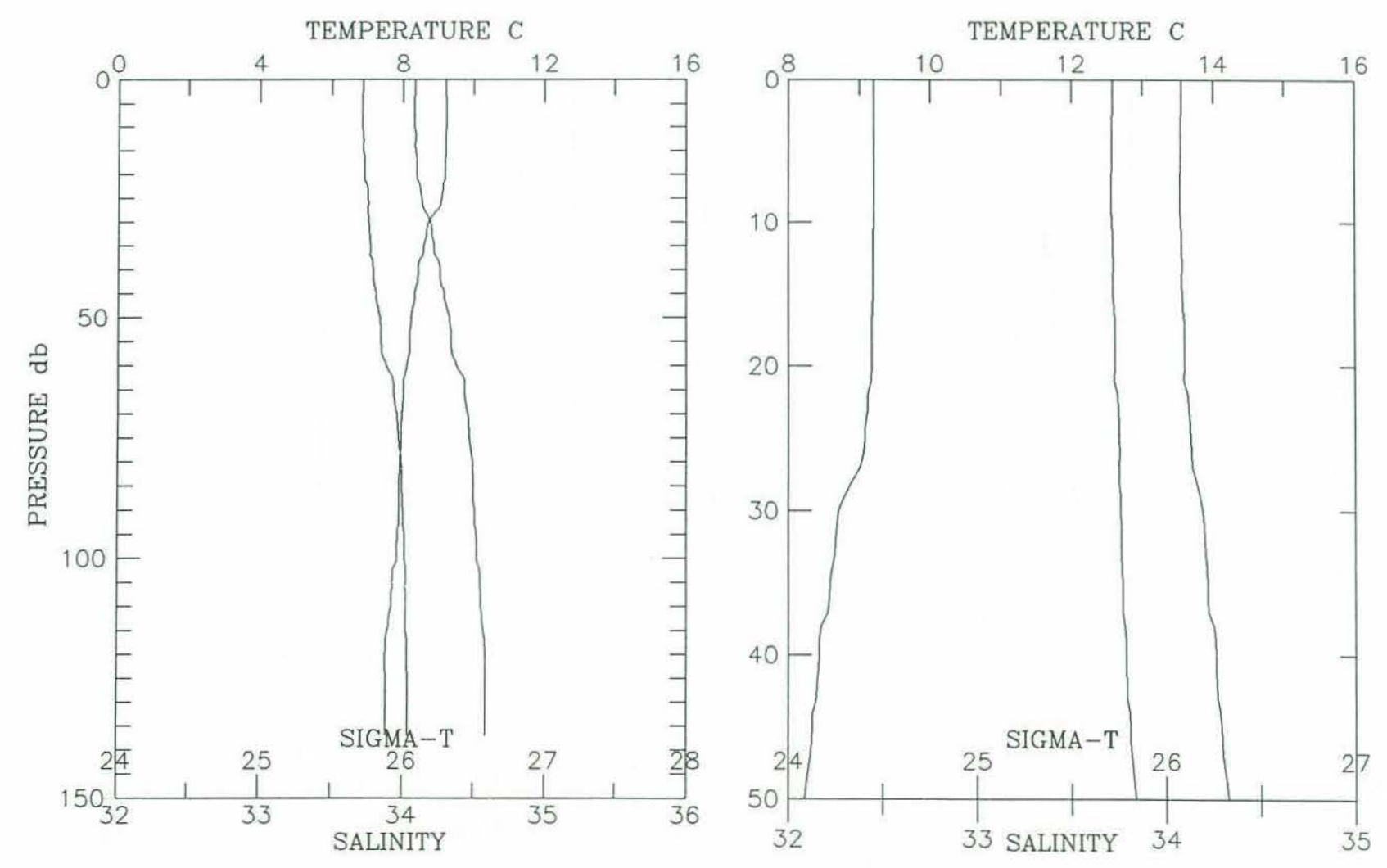


\begin{tabular}{|c|c|c|c|c|c|c|}
\hline $\begin{array}{l}\text { PRESSURE } \\
\text { (d-bars) }\end{array}$ & Interp & $\begin{array}{l}\text { TEMP } \\
(\operatorname{deg} C)\end{array}$ & $\begin{array}{l}\text { SALINITY } \\
(0 / 00)\end{array}$ & SIGMA-T & DELTA-D & $\begin{array}{l}\text { CHANNELA } \\
\text { ( }(81 \text { ight) }\end{array}$ \\
\hline 0.0 & $\mathrm{E}$ & 9.551 & 33.553 & 25.897 & 0.0000 & 0.0 \\
\hline 10.0 & & 9.552 & 33.554 & 25.898 & 0.0213 & 0.0 \\
\hline 20.0 & & 9.548 & 33.557 & 25.901 & 0.0426 & 0.0 \\
\hline 30.0 & & 9.493 & 33.575 & 25.924 & 0.0639 & 0.0 \\
\hline 40.0 & & 8.785 & 33.657 & 26.101 & 0.0843 & 0.0 \\
\hline 50.0 & & 8.673 & 33.699 & 26.151 & 0.1035 & 0.0 \\
\hline 60.0 & & 8.390 & 33.860 & 26.320 & 0.1217 & 0.0 \\
\hline 70.0 & & 8.447 & 33.919 & 26.358 & 0.1388 & 0.0 \\
\hline 80.0 & & 8.436 & 33.926 & 26.365 & 0.1559 & 0.0 \\
\hline 90.0 & & 8.314 & 33.963 & 26.412 & 0.1727 & 0.0 \\
\hline 100.0 & & 8.248 & 33.965 & 26.424 & 0.1892 & 0.0 \\
\hline 110.0 & & 8.178 & 33.959 & 26.430 & 0.2057 & 0.0 \\
\hline 120.0 & & 7.768 & 33.974 & 26.502 & 0.2218 & 0.0 \\
\hline 130.0 & & 7.665 & 34.019 & 26.552 & 0.2373 & 0.0 \\
\hline 140.0 & & 7.471 & 34.029 & 26.588 & 0.2524 & 0.0 \\
\hline 150.0 & & 7.319 & 34.035 & 26.615 & 0.2673 & 0.0 \\
\hline 156.0 & & 7.331 & 34.040 & 26.617 & 0.2761 & 0.0 \\
\hline
\end{tabular}
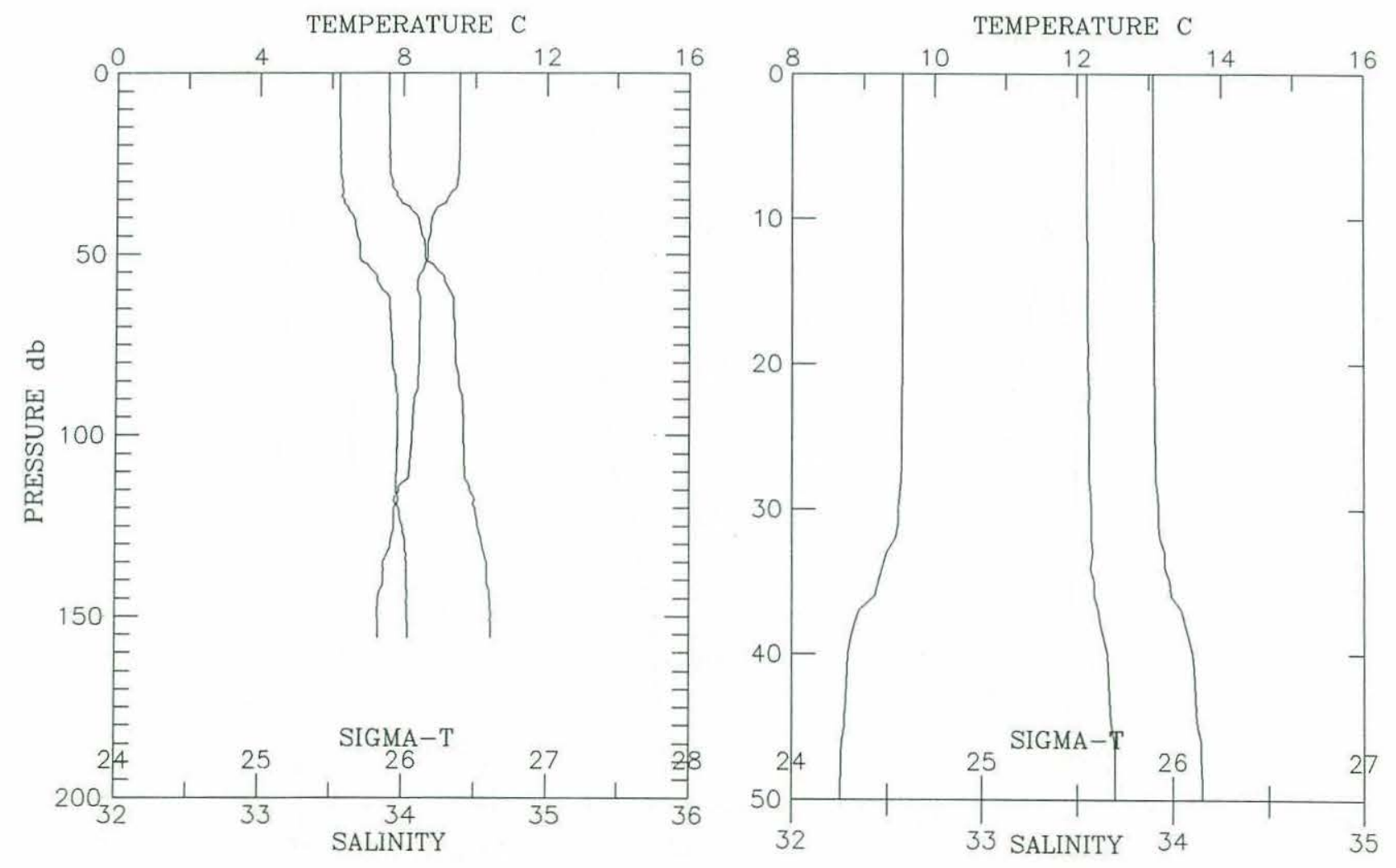


\begin{tabular}{|c|c|c|c|c|c|c|}
\hline $\begin{array}{l}\text { PRESSURE } \\
\text { (d-bars) }\end{array}$ & Interp & $\begin{array}{c}\text { TEMP } \\
(\operatorname{deg} C)\end{array}$ & $\begin{array}{c}\text { SALINITY } \\
(0 / 00)\end{array}$ & SIGMA-T & DELTA-D & $\begin{array}{l}\text { CHANNELA } \\
\text { ( } \% \text { ight) }\end{array}$ \\
\hline 0.0 & $\mathrm{E}$ & 9.632 & 33.434 & 25.791 & 0.0000 & 0.0 \\
\hline 10.0 & & 9.631 & 33.434 & 25.791 & 0.0223 & 0.0 \\
\hline 20.0 & & 9.632 & 33.433 & 25.790 & 0.0447 & 0.0 \\
\hline 30.0 & & 9.601 & 33.436 & 25.797 & 0.0670 & 0.0 \\
\hline 40.0 & & 9.237 & 33.532 & 25.932 & 0.0888 & 0.0 \\
\hline 50.0 & & 8.911 & 33.618 & 26.051 & 0.1093 & 0.0 \\
\hline 60.0 & & 8.604 & 33.726 & 26.182 & 0.1288 & 0.0 \\
\hline 80.0 & & 8.540 & 33.804 & 26.253 & 0.1653 & 0.0 \\
\hline 100.0 & & 8.478 & 33.950 & 26.377 & 0.2003 & 0.0 \\
\hline 150.0 & & 7.497 & 34.026 & 26.582 & 0.2802 & 0.0 \\
\hline 200.0 & & 7.220 & 34.080 & 26.664 & 0.3536 & 0.0 \\
\hline 250.0 & & 6.949 & 34.109 & 26.724 & 0.4242 & 0.0 \\
\hline 300.0 & & 6.848 & 34.124 & 26.750 & 0.4931 & 0.0 \\
\hline 400.0 & & 6.360 & 34.167 & 26.849 & 0.6256 & 0.0 \\
\hline 472.0 & & 6.176 & 34.188 & 26.889 & 0.7170 & 0.0 \\
\hline
\end{tabular}

TEMPERATURE C
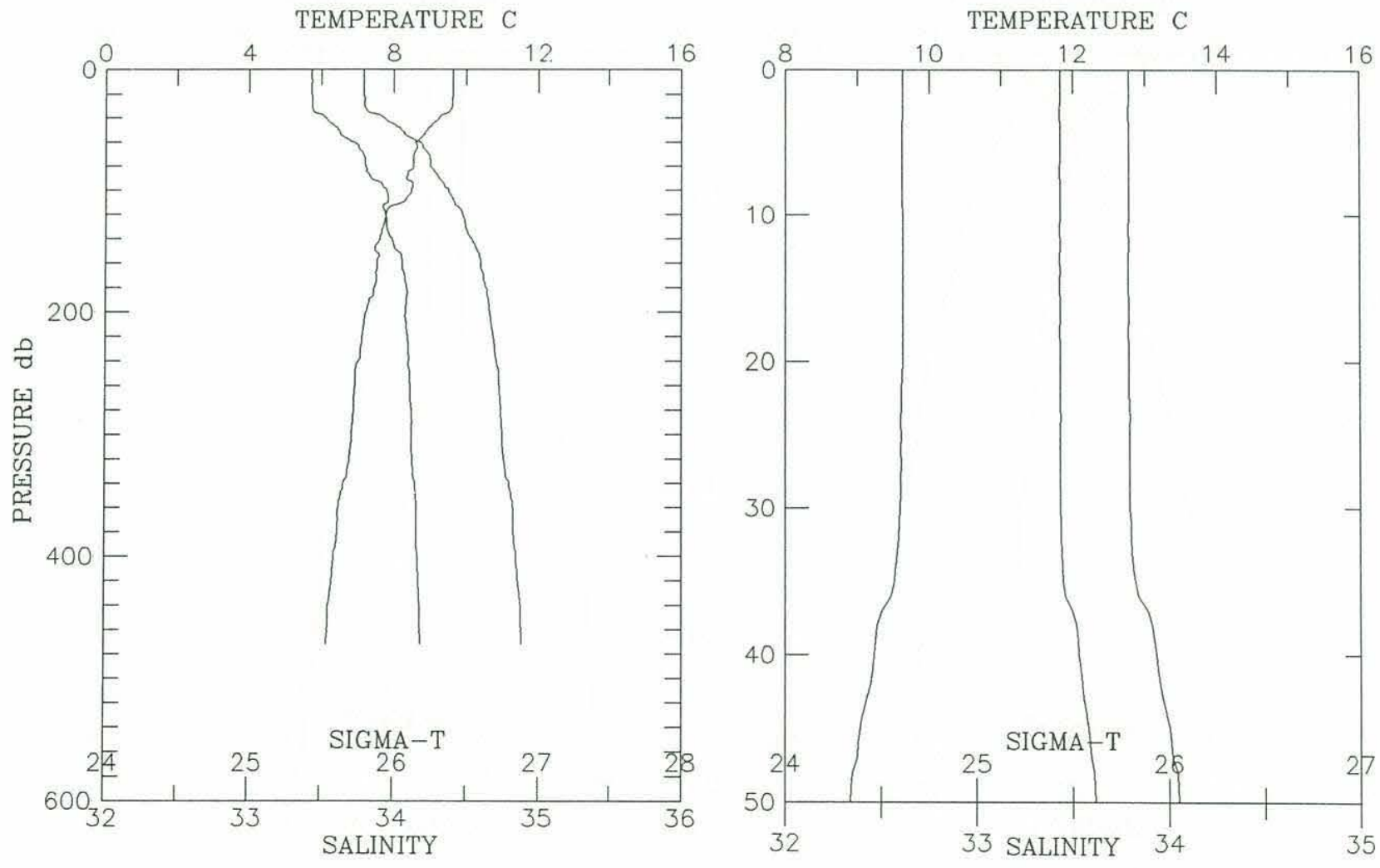


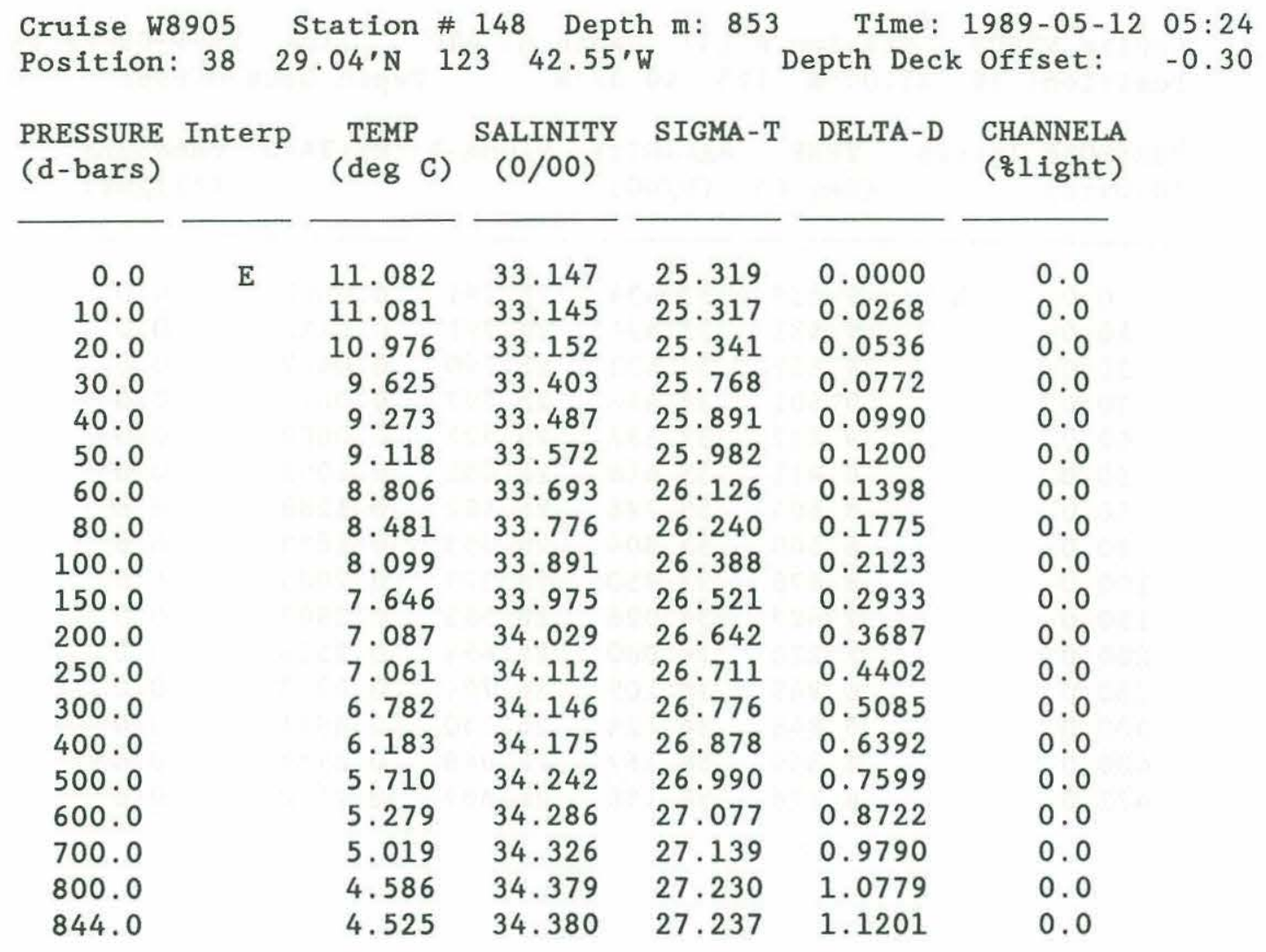
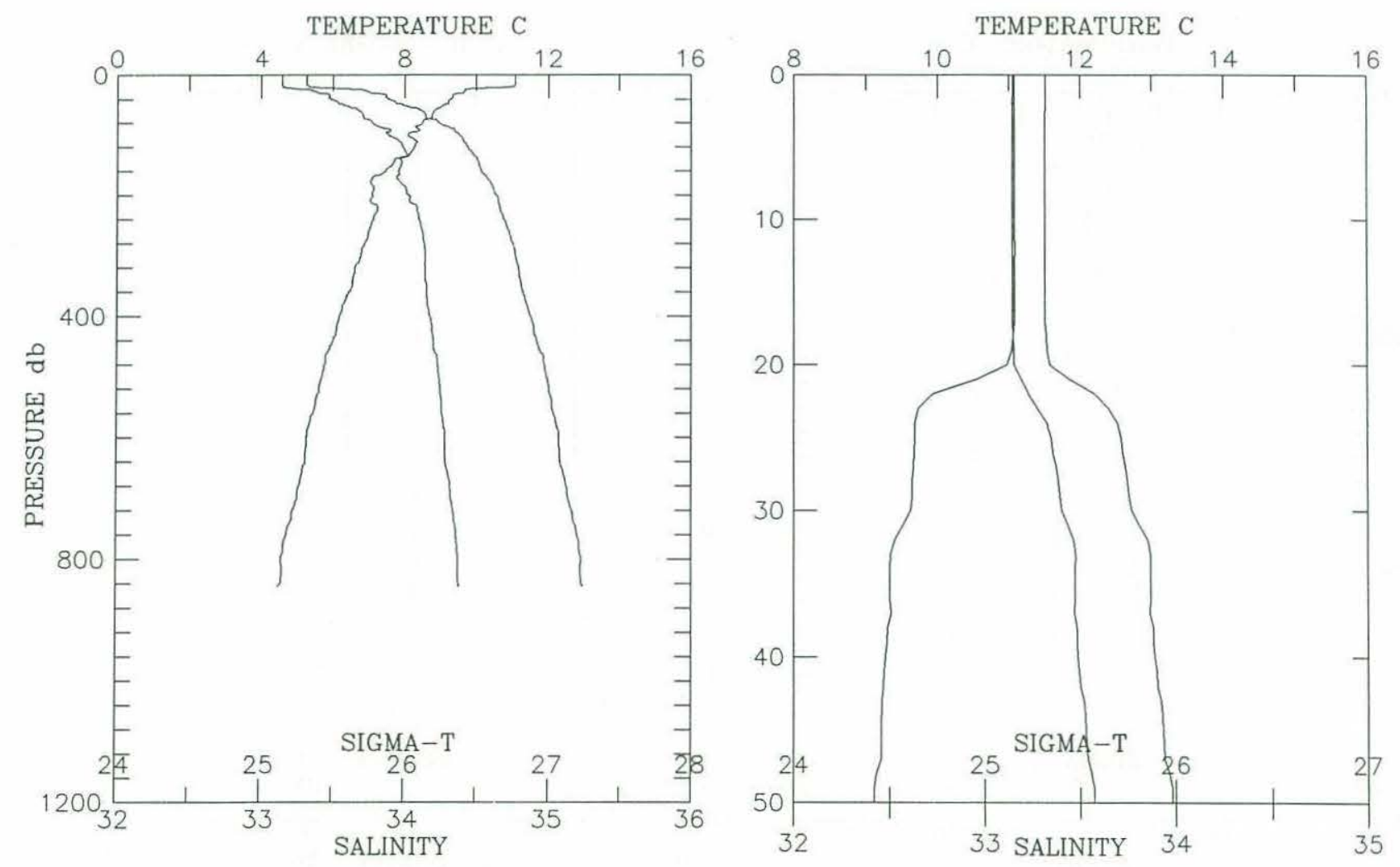
Cruise w8905 Station \# 149 Depth m: 1013 Time: 1989-05-12 07:13 Position: 38 20.00'N 12340.00 'W Depth Deck Offset: -0.30

\begin{tabular}{|c|c|c|c|c|c|c|}
\hline $\begin{array}{l}\text { PRESSURE } \\
\text { (d-bars) }\end{array}$ & Interp & $\begin{array}{l}\text { TEMP } \\
(\operatorname{deg} C)\end{array}$ & $\begin{array}{l}\text { SALINITY } \\
(0 / 00)\end{array}$ & SIGMA-T & DELTA-D & $\begin{array}{l}\text { CHANNELA } \\
\text { ( } \delta 1 \text { ight) }\end{array}$ \\
\hline 0.0 & E & 12.039 & 32.985 & 25.017 & 0.0000 & 0.0 \\
\hline 10.0 & & 12.040 & 32.984 & 25.016 & 0.0297 & 0.0 \\
\hline 20.0 & & 12.023 & 32.986 & 25.021 & 0.0594 & 0.0 \\
\hline 30.0 & & 11.969 & 32.993 & 25.036 & 0.0890 & 0.0 \\
\hline 40.0 & & 10.064 & 33.286 & 25.604 & 0.1152 & 0.0 \\
\hline 50.0 & & 9.652 & 33.292 & 25.677 & 0.1392 & 0.0 \\
\hline 60.0 & & 9.424 & 33.461 & 25.846 & 0.1620 & 0.0 \\
\hline 80.0 & & 8.706 & 33.688 & 26.137 & 0.2034 & 0.0 \\
\hline 100.0 & & 8.431 & 33.816 & 26.279 & 0.2405 & 0.0 \\
\hline 150.0 & & 8.148 & 34.023 & 26.485 & 0.3238 & 0.0 \\
\hline 200.0 & & 7.109 & 33.962 & 26.586 & 0.4017 & 0.0 \\
\hline 250.0 & & 6.595 & 34.002 & 26.688 & 0.4746 & 0.0 \\
\hline 300.0 & & 6.760 & 34.131 & 26.767 & 0.5439 & 0.0 \\
\hline 400.0 & & 6.161 & 34.194 & 26.896 & 0.6734 & 0.0 \\
\hline 500.0 & & 5.643 & 34.239 & 26.995 & 0.7933 & 0.0 \\
\hline 600.0 & & 5.192 & 34.294 & 27.093 & 0.9047 & 0.0 \\
\hline 700.0 & & 4.866 & 34.338 & 27.166 & 1.0087 & 0.0 \\
\hline 800.0 & & 4.492 & 34.383 & 27.243 & 1.1055 & 0.0 \\
\hline 900.0 & & 4.274 & 34.411 & 27.289 & 1.1973 & 0.0 \\
\hline 1000.0 & & 3.957 & 34.442 & 27.347 & 1.2846 & 0.0 \\
\hline 1003.0 & & 3.958 & 34.442 & 27.347 & 1.2872 & 0.0 \\
\hline
\end{tabular}
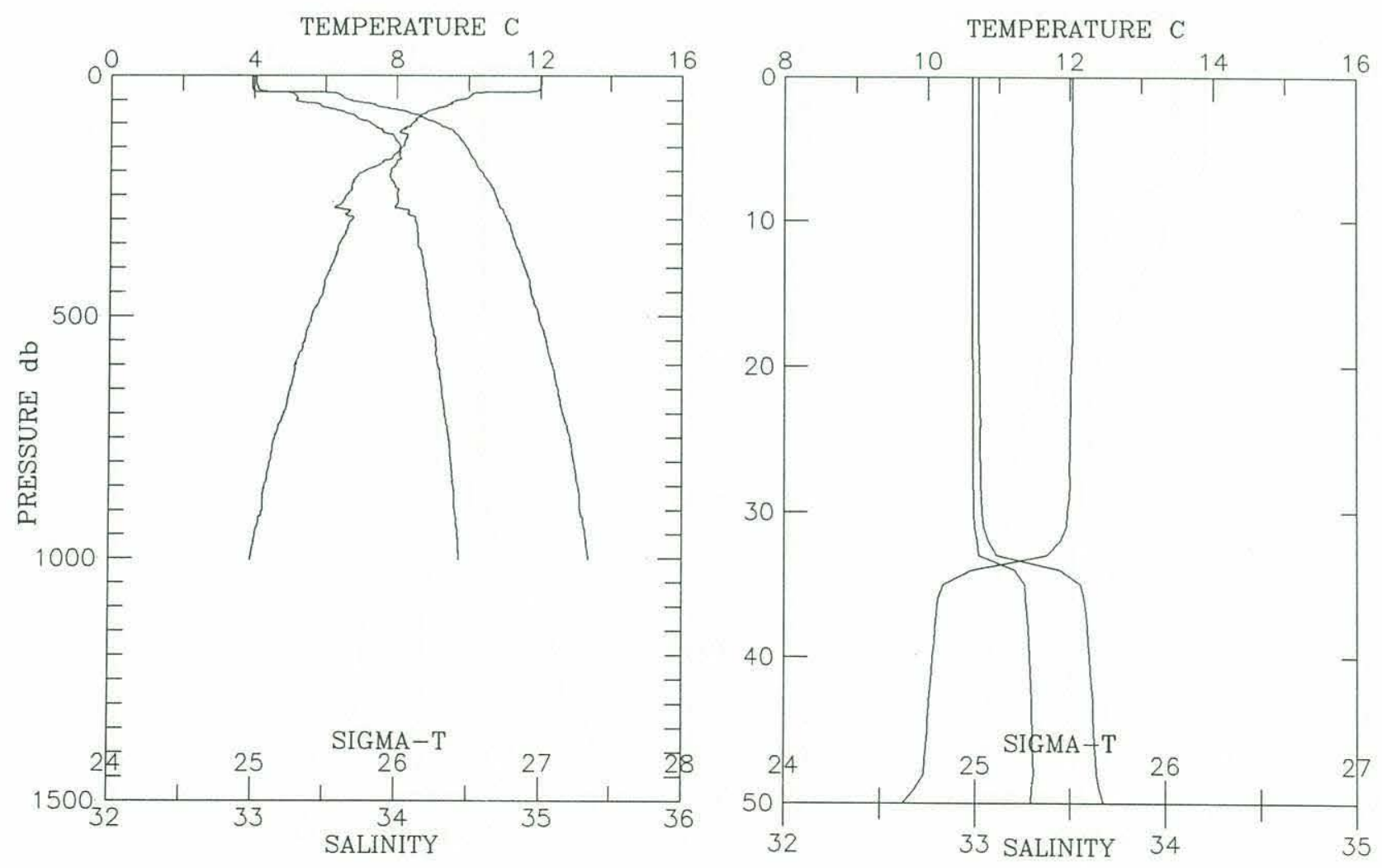


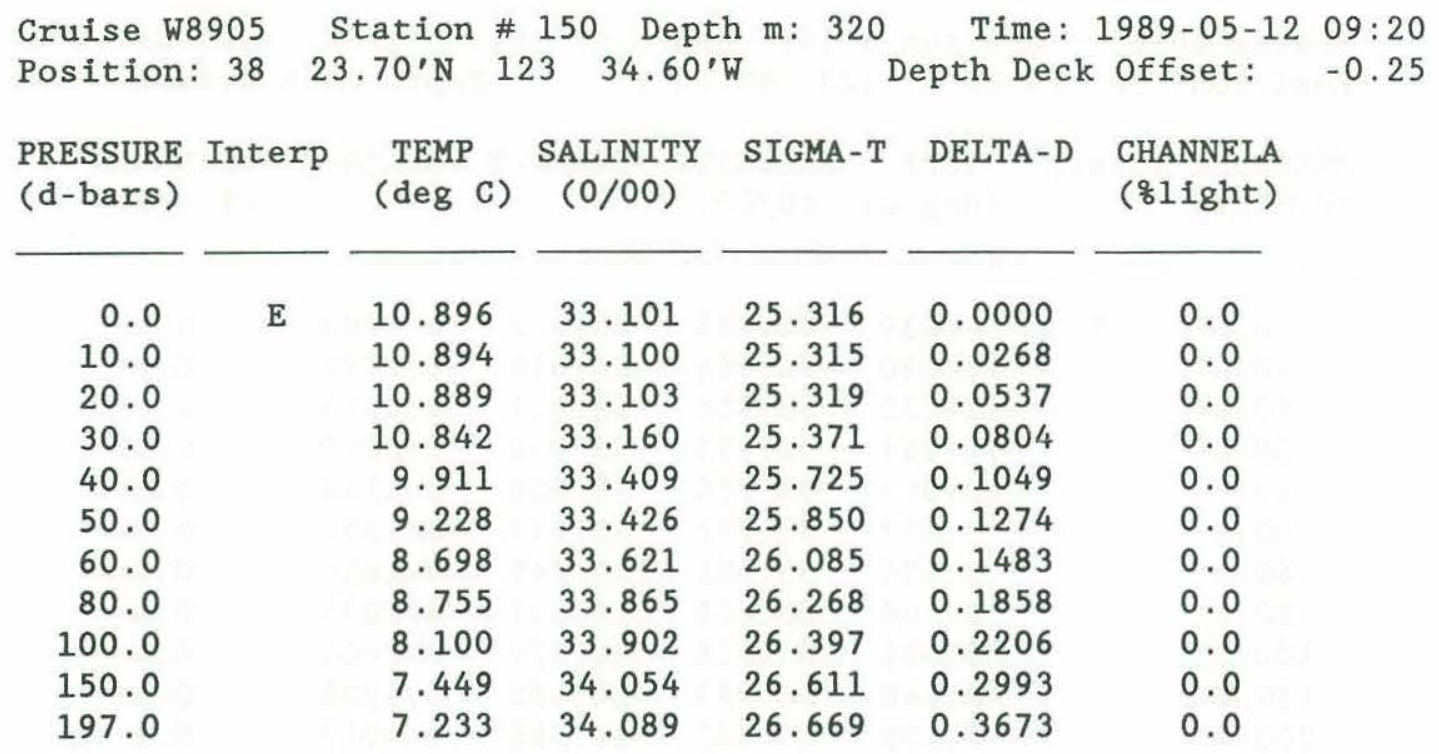
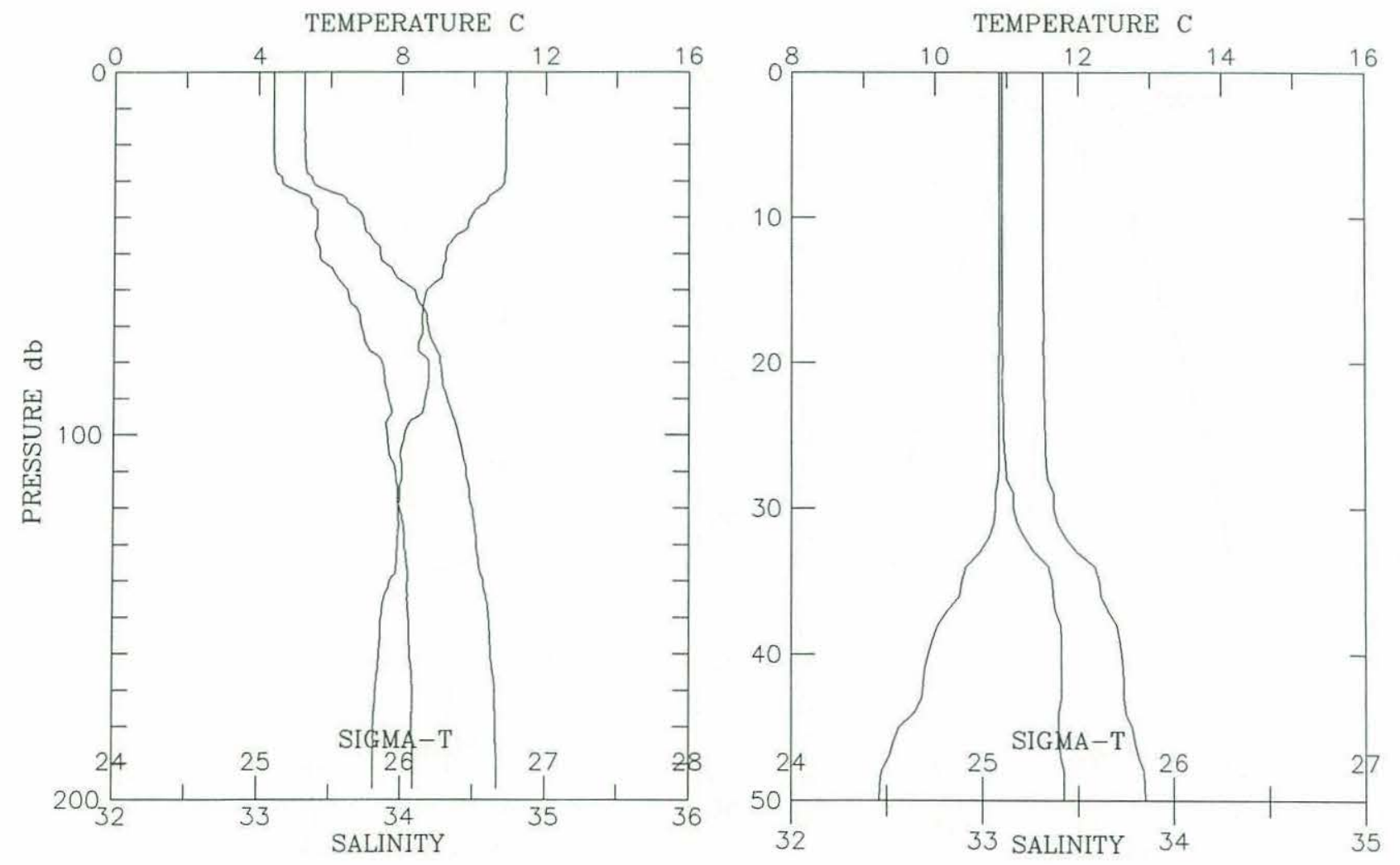
Cruise w8905 Station \# 151 Depth m: 155 Time: 1989-05-12 11:04

Position: 38 27.30'N $123 \quad 30.20^{\prime} \mathrm{W}$ Depth Deck Offset: -0.25

\begin{tabular}{|c|c|c|c|c|c|c|}
\hline $\begin{array}{l}\text { PRESSURE } \\
\text { (d-bars) }\end{array}$ & Interp & $\begin{array}{l}\text { TEMP } \\
(\operatorname{deg} G)\end{array}$ & $\begin{array}{c}\text { SALINITY } \\
(0 / 00)\end{array}$ & SIGMA-T & DELTA-D & $\begin{array}{l}\text { CHANNELA } \\
\text { ( }(z 1 \text { light) }\end{array}$ \\
\hline 0.0 & E & 9.394 & 33.617 & 25.973 & 0.0000 & 0.0 \\
\hline 10.0 & & 9.397 & 33.617 & 25.972 & 0.0206 & 0.0 \\
\hline 20.0 & & 9.397 & 33.618 & 25.973 & 0.0412 & 0.0 \\
\hline 30.0 & & 9.354 & 33.628 & 25.988 & 0.0618 & 0.0 \\
\hline 40.0 & & 9.245 & 33.637 & 26.013 & 0.0822 & 0.0 \\
\hline 50.0 & & 8.657 & 33.662 & 26.124 & 0.1020 & 0.0 \\
\hline 60.0 & & 8.724 & 33.876 & 26.281 & 0.1204 & 0.0 \\
\hline 70.0 & & 8.574 & 33.935 & 26.351 & 0.1378 & 0.0 \\
\hline 80.0 & & 8.084 & 33.928 & 26.420 & 0.1548 & 0.0 \\
\hline 90.0 & & 7.939 & 33.987 & 26.487 & 0.1709 & 0.0 \\
\hline 100.0 & & 7.920 & 33.995 & 26.496 & 0.1867 & 0.0 \\
\hline 110.0 & & 7.831 & 34.009 & 26.521 & 0.2025 & 0.0 \\
\hline 120.0 & & 7.772 & 34.021 & 26.539 & 0.2180 & 0.0 \\
\hline 130.0 & & 7.533 & 34.019 & 26.572 & 0.2333 & 0.0 \\
\hline 140.0 & & 7.501 & 34.041 & 26.593 & 0.2483 & 0.0 \\
\hline 150.0 & & 7.505 & 34.048 & 26.598 & 0.2632 & 0.0 \\
\hline 151.0 & & 7.505 & 34.048 & 26.598 & 0.2647 & 0.0 \\
\hline
\end{tabular}

TEMPERATURE $\mathrm{C}$
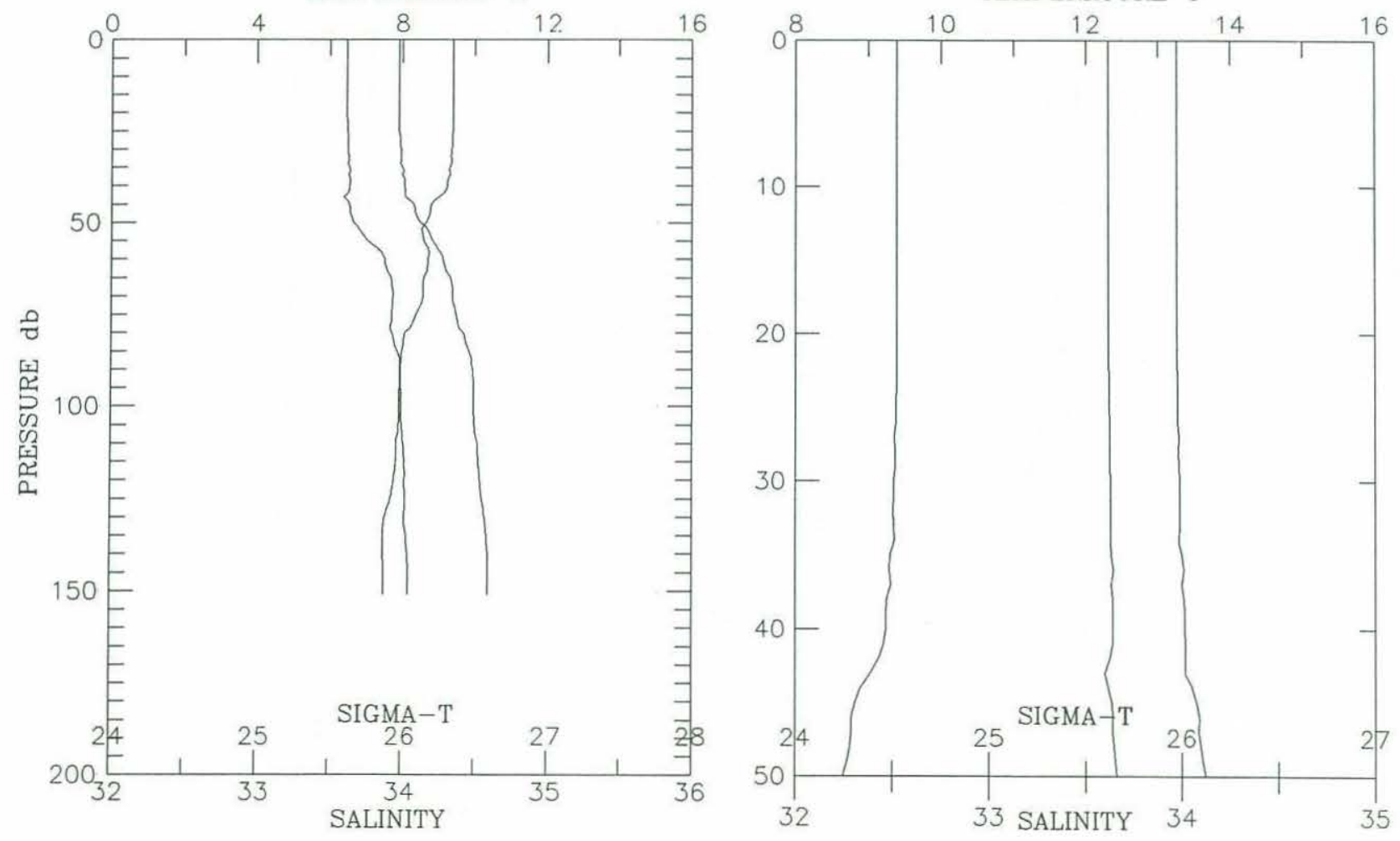
Cruise w8905 Station \# 152 Depth m: 136 Time: 1989-05-12 11:55 Position: $38 \quad 29.28^{\prime} \mathrm{N} 123 \quad 27.42^{\prime} \mathrm{W} \quad$ Depth Deck Offset: -0.25

\begin{tabular}{|c|c|c|c|c|c|c|}
\hline $\begin{array}{l}\text { PRESSURE } \\
\text { (d-bars) }\end{array}$ & Interp & $\begin{array}{l}\text { TEMP } \\
(\operatorname{deg} \mathrm{C})\end{array}$ & $\begin{array}{l}\text { SALINITY } \\
(0 / 00)\end{array}$ & SIGMA-T & DELTA-D & $\begin{array}{l}\text { CHANNELA } \\
\text { ( }(1 \text { ight) }\end{array}$ \\
\hline 0.0 & E & 9.087 & 33.675 & 26.067 & 0.0000 & 0.0 \\
\hline 10.0 & & 9.078 & 33.679 & 26.072 & 0.0197 & 0.0 \\
\hline 20.0 & & 9.012 & 33.695 & 26.095 & 0.0393 & 0.0 \\
\hline 30.0 & & 8.910 & 33.752 & 26.155 & 0.0587 & 0.0 \\
\hline 40.0 & & 8.903 & 33.769 & 26.170 & 0.0776 & 0.0 \\
\hline 50.0 & & 8.931 & 33.783 & 26.176 & 0.0964 & 0.0 \\
\hline 60.0 & & 8.793 & 33.779 & 26.194 & 0.1150 & 0.0 \\
\hline 70.0 & & 8.501 & 33.841 & 26.288 & 0.1334 & 0.0 \\
\hline 80.0 & & 8.550 & 33.893 & 26.322 & 0.1509 & 0.0 \\
\hline 90.0 & & 8.384 & 33.969 & 26.406 & 0.1679 & 0.0 \\
\hline 100.0 & & 8.042 & 33.988 & 26.473 & 0.1843 & 0.0 \\
\hline 110.0 & & 7.796 & 34.011 & 26.527 & 0.2001 & 0.0 \\
\hline 120.0 & & 7.579 & 34.017 & 26.563 & 0.2154 & 0.0 \\
\hline 130.0 & & 7.576 & 34.017 & 26.564 & 0.2306 & 0.0 \\
\hline 131.0 & & 7.577 & 34.018 & 26.565 & 0.2321 & 0.0 \\
\hline
\end{tabular}
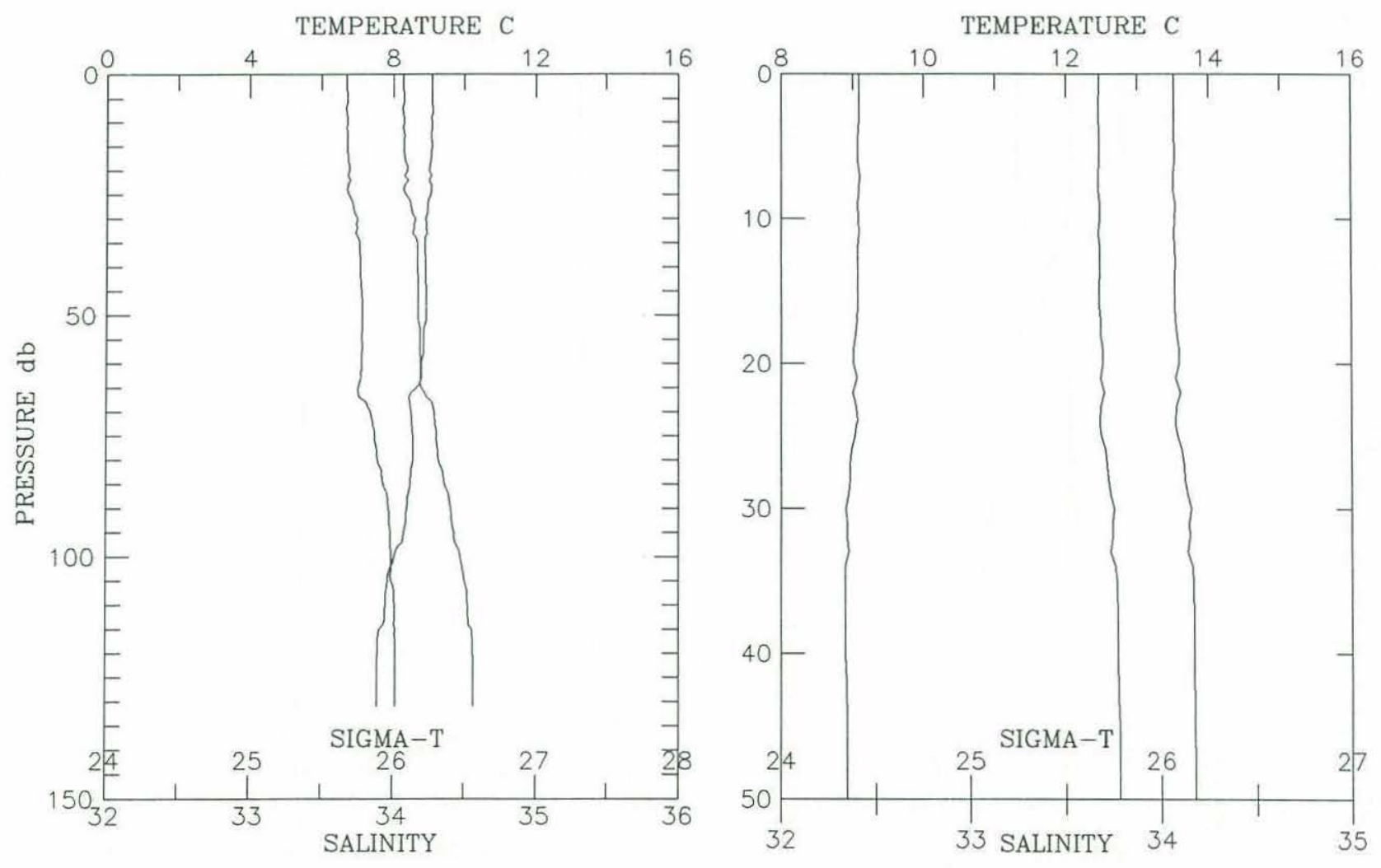


\begin{tabular}{|c|c|c|c|c|c|c|c|}
\hline Cruise wE & $\begin{array}{r}3905 \\
\quad 38\end{array}$ & cation \# & 153 Dept & $\mathrm{h}: 113$ & Time: & 1989-05-12 & $\begin{array}{l}12: 37 \\
-0.25\end{array}$ \\
\hline $\begin{array}{l}\text { PRESSURE } \\
\text { (d-bars) }\end{array}$ & Interp & $\begin{array}{l}\text { TEMP } \\
(\operatorname{deg} C)\end{array}$ & $\begin{array}{c}\text { SALINITY } \\
(0 / 00)\end{array}$ & SIGMA-T & DELTA-D & $\begin{array}{l}\text { CHANNELA } \\
\text { ( } 81 \text { ight) }\end{array}$ & \\
\hline 0.0 & E & 8.951 & 33.766 & 26.160 & 0.0000 & 0.0 & \\
\hline 10.0 & & 8.955 & 33.764 & 26.157 & 0.0188 & 0.0 & \\
\hline 20.0 & & 8.957 & 33.764 & 26.157 & 0.0377 & 0.0 & \\
\hline 30.0 & & 8.926 & 33.766 & 26.163 & 0.0566 & 0.0 & \\
\hline 40.0 & & 8.873 & 33.769 & 26.174 & 0.0753 & 0.0 & \\
\hline 50.0 & & 8.568 & 33.780 & 26.230 & 0.0939 & 0.0 & \\
\hline 60.0 & & 8.120 & 33.888 & 26.383 & 0.1112 & 0.0 & \\
\hline 70.0 & & 8.063 & 33.917 & 26.414 & 0.1278 & 0.0 & \\
\hline 80.0 & & 8.024 & 33.969 & 26.461 & 0.1441 & 0.0 & \\
\hline 90.0 & & 8.032 & 33.989 & 26.475 & 0.1602 & 0.0 & \\
\hline 100.0 & & 7.703 & 34.027 & 26.553 & 0.1759 & 0.0 & \\
\hline 109.0 & & 7.683 & 34.030 & 26.559 & 0.1896 & 0.0 & \\
\hline
\end{tabular}
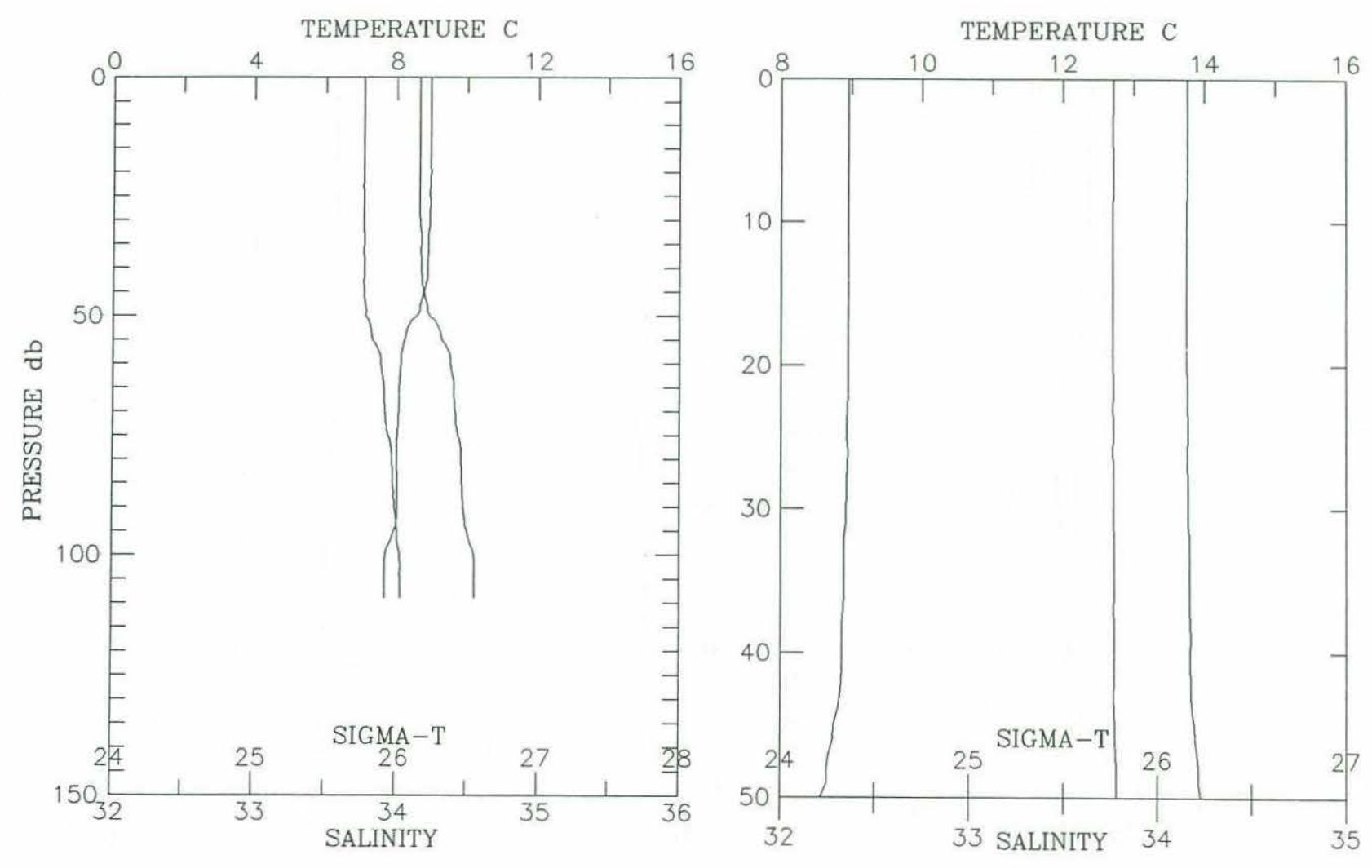


\begin{tabular}{|c|c|c|c|c|c|c|}
\hline $\begin{array}{l}\text { PRESSURE } \\
\text { (d-bars) }\end{array}$ & Interp & $\begin{array}{l}\text { TEMP } \\
(\operatorname{deg} C)\end{array}$ & $\begin{array}{c}\text { SALINITY } \\
(0 / 00)\end{array}$ & SIGMA-T & DELTA-D & $\begin{array}{l}\text { CHANNELA } \\
\text { ( } \delta 1 \text { ight) }\end{array}$ \\
\hline 0.0 & E & 8.695 & 33.833 & 26.252 & 0.0000 & 0.0 \\
\hline 5.0 & & 8.697 & 33.831 & 26.250 & 0.0090 & 0.0 \\
\hline 10.0 & & 8.695 & 33.835 & 26.254 & 0.0179 & 0.0 \\
\hline 15.0 & & 8.697 & 33.834 & 26.253 & 0.0269 & 0.0 \\
\hline 20.0 & & 8.693 & 33.837 & 26.256 & 0.0359 & 0.0 \\
\hline 25.0 & & 8.656 & 33.850 & 26.271 & 0.0448 & 0.0 \\
\hline 30.0 & & 8.547 & 33.888 & 26.318 & 0.0536 & 0.0 \\
\hline 35.0 & & 8.538 & 33.895 & 26.325 & 0.0623 & 0.0 \\
\hline 40.0 & & 8.450 & 33.910 & 26.350 & 0.0709 & 0.0 \\
\hline 45.0 & & 8.397 & 33.918 & 26.365 & 0.0794 & 0.0 \\
\hline 50.0 & & 8.365 & 33.918 & 26.370 & 0.0879 & 0.0 \\
\hline 55.0 & & 8.312 & 33.922 & 26.381 & 0.0963 & 0.0 \\
\hline 60.0 & & 8.217 & 33.933 & 26.404 & 0.1047 & 0.0 \\
\hline 65.0 & & 8.192 & 33.940 & 26.413 & 0.1130 & 0.0 \\
\hline 70.0 & & 8.112 & 33.958 & 26.439 & 0.1212 & 0.0 \\
\hline 75.0 & & 8.047 & 33.968 & 26.456 & 0.1293 & 0.0 \\
\hline 80.0 & & 7.936 & 33.983 & 26.485 & 0.1373 & 0.0 \\
\hline 85.0 & & 7.658 & 34.026 & 26.559 & 0.1450 & 0.0 \\
\hline 90.0 & & 7.642 & 34.029 & 26.564 & 0.1526 & 0.0 \\
\hline 92.0 & & 7.641 & 34.029 & 26.564 & 0.1556 & 0.0 \\
\hline
\end{tabular}

TEMPERATURE C

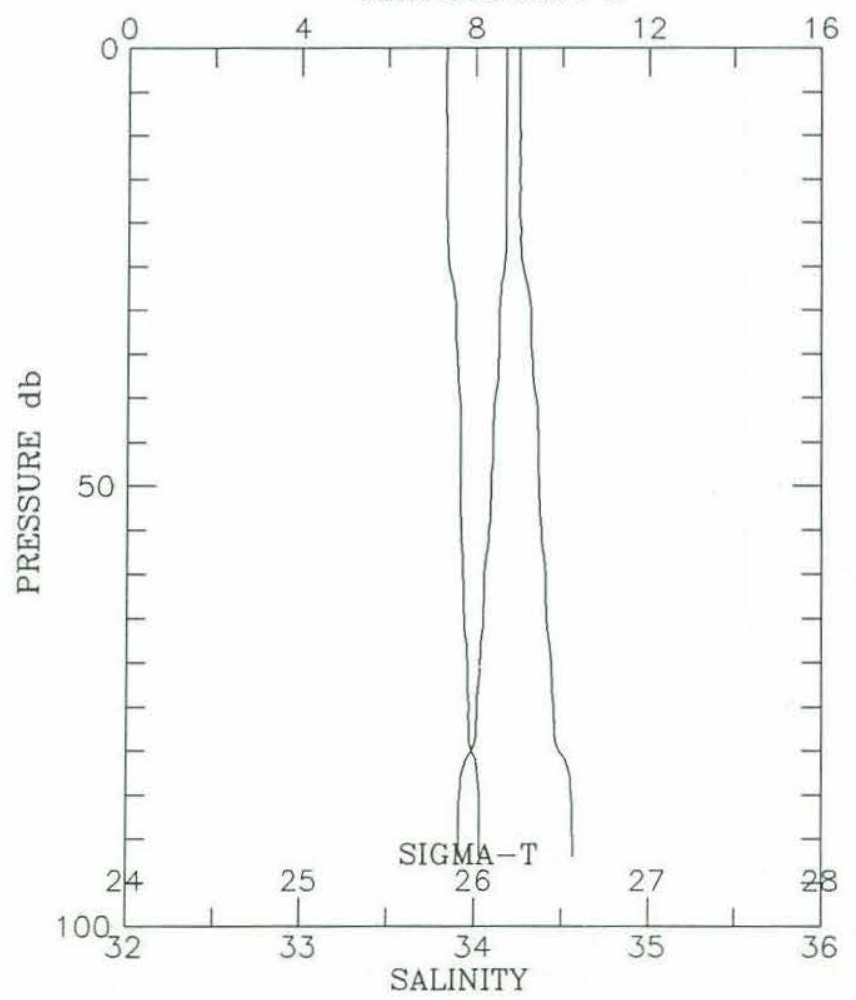

TEMPERATURE C

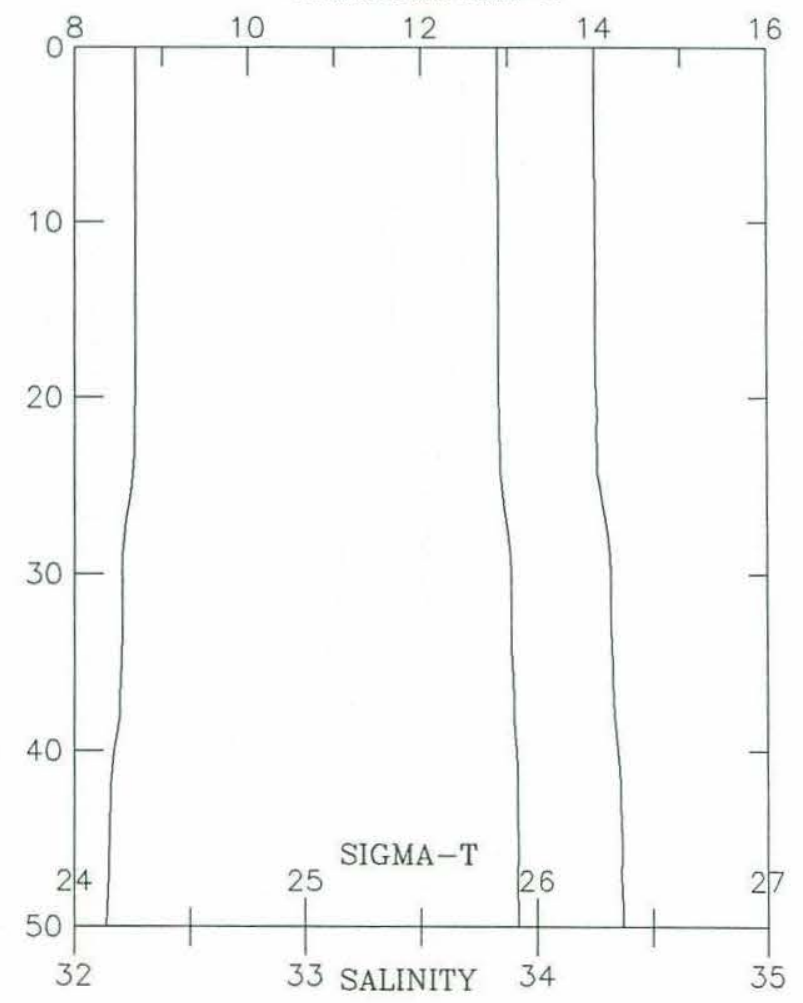


Cruise W8905 Station \# 155 Depth m: 78 Time: 1989-05-12 13:32 Position: 38 33.56'N $12321.55^{\prime} \mathrm{W}$ Depth Deck Offset: -0.25

\begin{tabular}{|c|c|c|c|c|c|c|}
\hline $\begin{array}{l}\text { PRESSURE } \\
\text { (d-bars) }\end{array}$ & Interp & $\begin{array}{l}\text { TEMP } \\
(\operatorname{deg} C)\end{array}$ & $\begin{array}{l}\text { SALINITY } \\
(0 / 00)\end{array}$ & SIGMA-T & DELTA-D & $\begin{array}{l}\text { CHANNELA } \\
\text { ( }(\delta 1 \text { ight) }\end{array}$ \\
\hline 0.0 & E & 8.695 & 33.864 & 26.276 & 0.0000 & 0.0 \\
\hline 5.0 & & 8.697 & 33.864 & 26.276 & 0.0089 & 0.0 \\
\hline 10.0 & & 8.699 & 33.864 & 26.276 & 0.0177 & 0.0 \\
\hline 15.0 & & 8.693 & 33.863 & 26.276 & 0.0266 & 0.0 \\
\hline 20.0 & & 8.467 & 33.870 & 26.316 & 0.0354 & 0.0 \\
\hline 25.0 & & 8.274 & 33.894 & 26.365 & 0.0439 & 0.0 \\
\hline 30.0 & & 8.208 & 33.929 & 26.402 & 0.0523 & 0.0 \\
\hline 35.0 & & 8.106 & 33.948 & 26.432 & 0.0605 & 0.0 \\
\hline 40.0 & & 8.068 & 33.958 & 26.446 & 0.0686 & 0.0 \\
\hline 45.0 & & 8.024 & 33.968 & 26.460 & 0.0767 & 0.0 \\
\hline 50.0 & & 7.995 & 33.973 & 26.468 & 0.0847 & 0.0 \\
\hline 55.0 & & 7.975 & 33.978 & 26.475 & 0.0927 & 0.0 \\
\hline 60.0 & & 7.935 & 33.985 & 26.487 & 0.1006 & 0.0 \\
\hline 65.0 & & 7.925 & 33.988 & 26.490 & 0.1085 & 0.0 \\
\hline 70.0 & & 7.926 & 33.987 & 26.489 & 0.1164 & 0.0 \\
\hline 74.0 & & 7.932 & 33.986 & 26.488 & 0.1227 & 0.0 \\
\hline
\end{tabular}

TEMPERATURE C
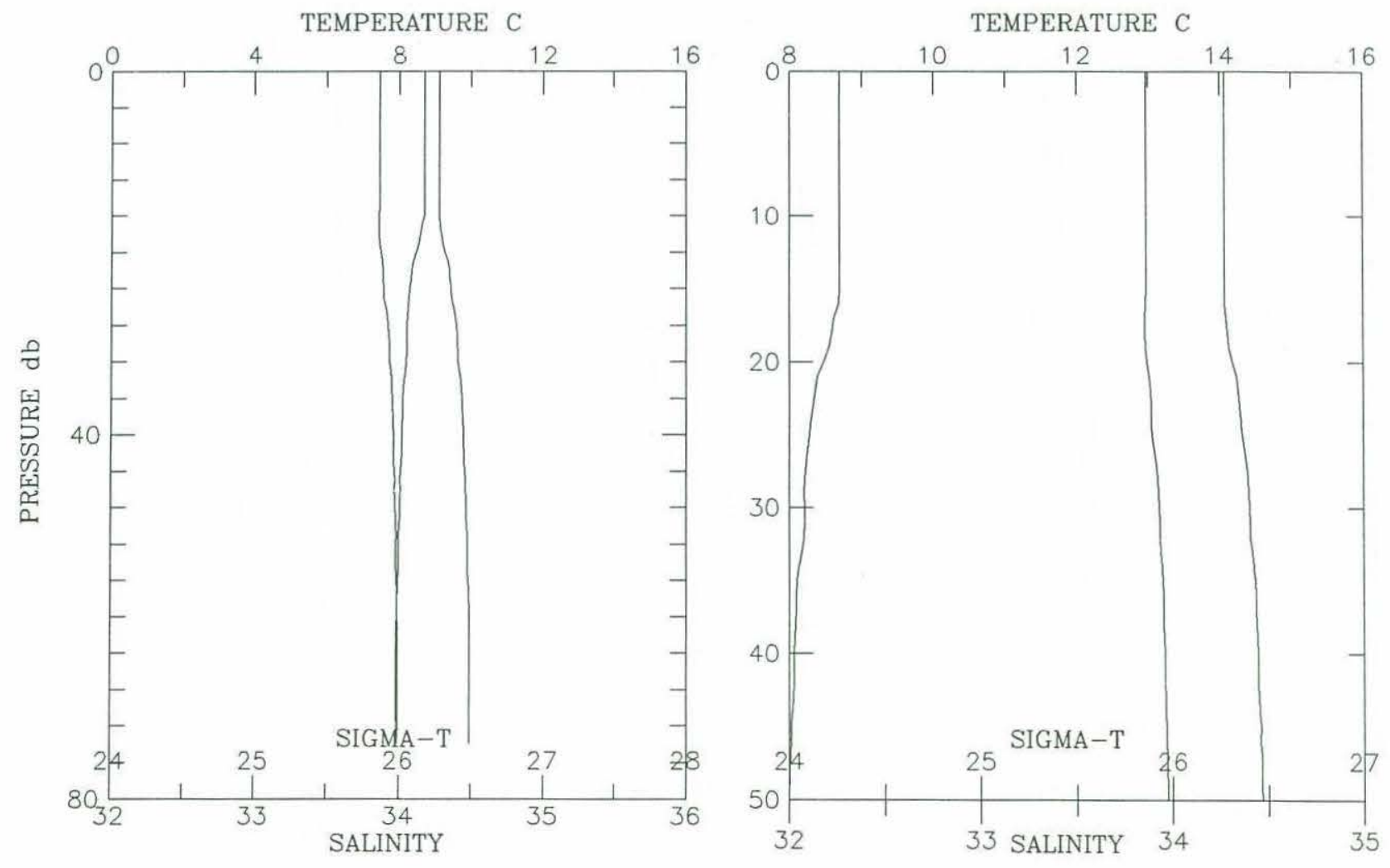


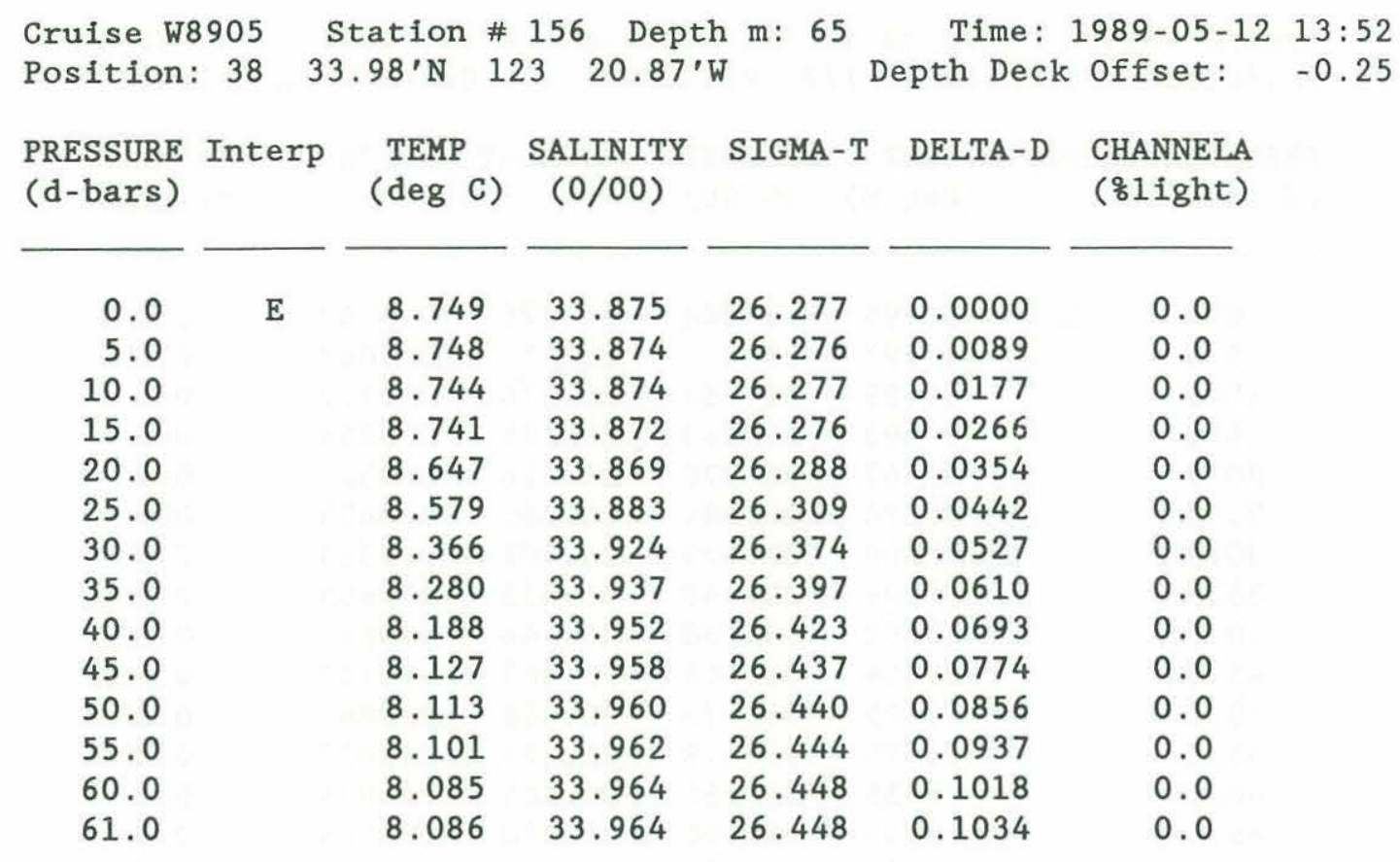
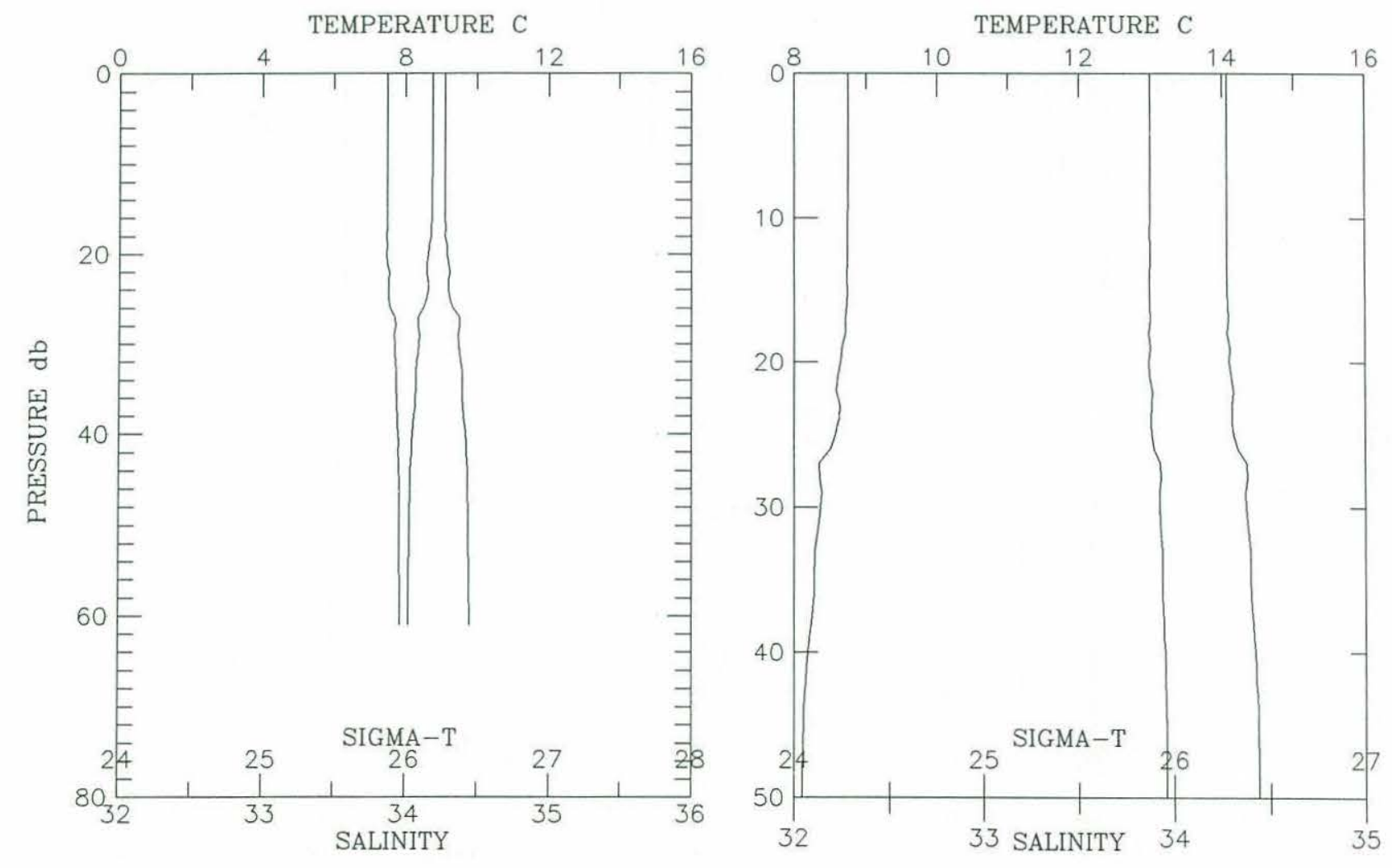


\begin{tabular}{|c|c|c|c|c|c|c|}
\hline $\begin{array}{l}\text { PRESSURE } \\
\text { (d-bars) }\end{array}$ & Interp & $\begin{array}{l}\text { TEMP } \\
(\operatorname{deg} C)\end{array}$ & $\begin{array}{l}\text { SALINITY } \\
(0 / 00)\end{array}$ & SIGMA-T & DELTA-D & $\begin{array}{l}\text { CHANNELA } \\
\text { ( } \& 1 \text { ight) }\end{array}$ \\
\hline 0.0 & E & 8.603 & 33.892 & 26.313 & 0.0000 & 0.0 \\
\hline 5.0 & & 8.598 & 33.891 & 26.313 & 0.0087 & 0.0 \\
\hline 10.0 & & 8.600 & 33.891 & 26.312 & 0.0174 & 0.0 \\
\hline 15.0 & & 8.593 & 33.892 & 26.314 & 0.0261 & 0.0 \\
\hline 20.0 & & 8.321 & 33.933 & 26.388 & 0.0346 & 0.0 \\
\hline 25.0 & & 8.052 & 33.976 & 26.462 & 0.0427 & 0.0 \\
\hline 30.0 & & 8.039 & 33.978 & 26.466 & 0.0507 & 0.0 \\
\hline 35.0 & & 8.019 & 33.981 & 26.471 & 0.0587 & 0.0 \\
\hline 40.0 & & 7.993 & 33.985 & 26.478 & 0.0666 & 0.0 \\
\hline 45.0 & & 7.972 & 33.988 & 26.483 & 0.0746 & 0.0 \\
\hline 50.0 & & 7.956 & 33.990 & 26.487 & 0.0825 & 0.0 \\
\hline 55.0 & & 7.952 & 33.991 & 26.489 & 0.0903 & 0.0 \\
\hline 58.0 & & 7.953 & 33.991 & 26.489 & 0.0951 & 0.0 \\
\hline
\end{tabular}

TEMPERATURE C
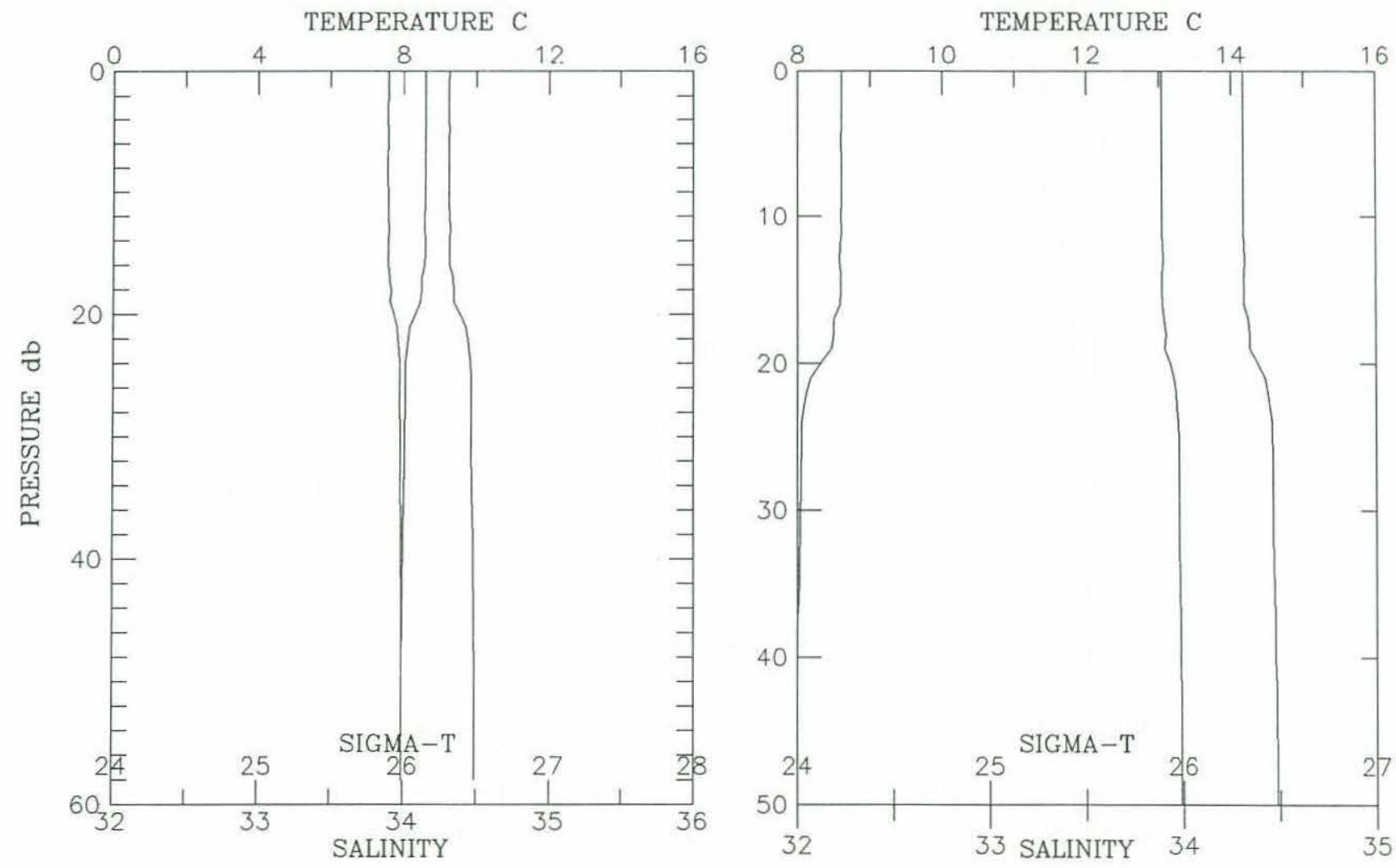
Cruise w8905 Station \# 158 Depth m: 77 Time: 1989-05-12 15:22

Position: $38 \quad 27.53^{\prime} \mathrm{N} 123 \quad 15.20^{\prime} \mathrm{W} \quad$ Depth Deck Offset: -0.25

PRESSURE Interp TEMP SALINITY SIGMA-T DELTA-D CHANNELA

(d-bars) ( $\quad(\operatorname{deg} G) \quad(0 / 00) \quad$ (olight)

\begin{tabular}{|c|c|c|c|c|c|c|}
\hline 0.0 & $\mathrm{E}$ & 8.723 & 33.883 & 26.287 & 0.0000 & 0.0 \\
\hline 5.0 & & 8.724 & 33.883 & 26.287 & 0.0088 & 0.0 \\
\hline 10.0 & & 8.723 & 33.883 & 26.287 & 0.0176 & 0.0 \\
\hline 15.0 & & 8.701 & 33.884 & 26.291 & 0.0264 & 0.0 \\
\hline 20.0 & & 8.640 & 33.890 & 26.305 & 0.0352 & 0.0 \\
\hline 25.0 & & 8.522 & 33.910 & 26.339 & 0.0439 & 0.0 \\
\hline 30.0 & & 8.347 & 33.934 & 26.385 & 0.0523 & 0.0 \\
\hline 35.0 & & 8.281 & 33.944 & 26.403 & 0.0607 & 0.0 \\
\hline 40.0 & & 8.264 & 33.946 & 26.407 & 0.0689 & 0.0 \\
\hline 45.0 & & 8.237 & 33.950 & 26.414 & 0.0772 & 0.0 \\
\hline 50.0 & & 8.203 & 33.955 & 26.423 & 0.0854 & 0.0 \\
\hline 55.0 & & 8.064 & 33.970 & 26.456 & 0.0936 & 0.0 \\
\hline 60.0 & & 7.903 & 33.998 & 26.501 & 0.1014 & 0.0 \\
\hline 65.0 & & 7.892 & 33.999 & 26.504 & 0.1093 & 0.0 \\
\hline 70.0 & & 7.888 & 34.000 & 26.505 & 0.1171 & 0.0 \\
\hline 71.0 & & 7.887 & 34.000 & 26.505 & 0.1187 & 0.0 \\
\hline
\end{tabular}
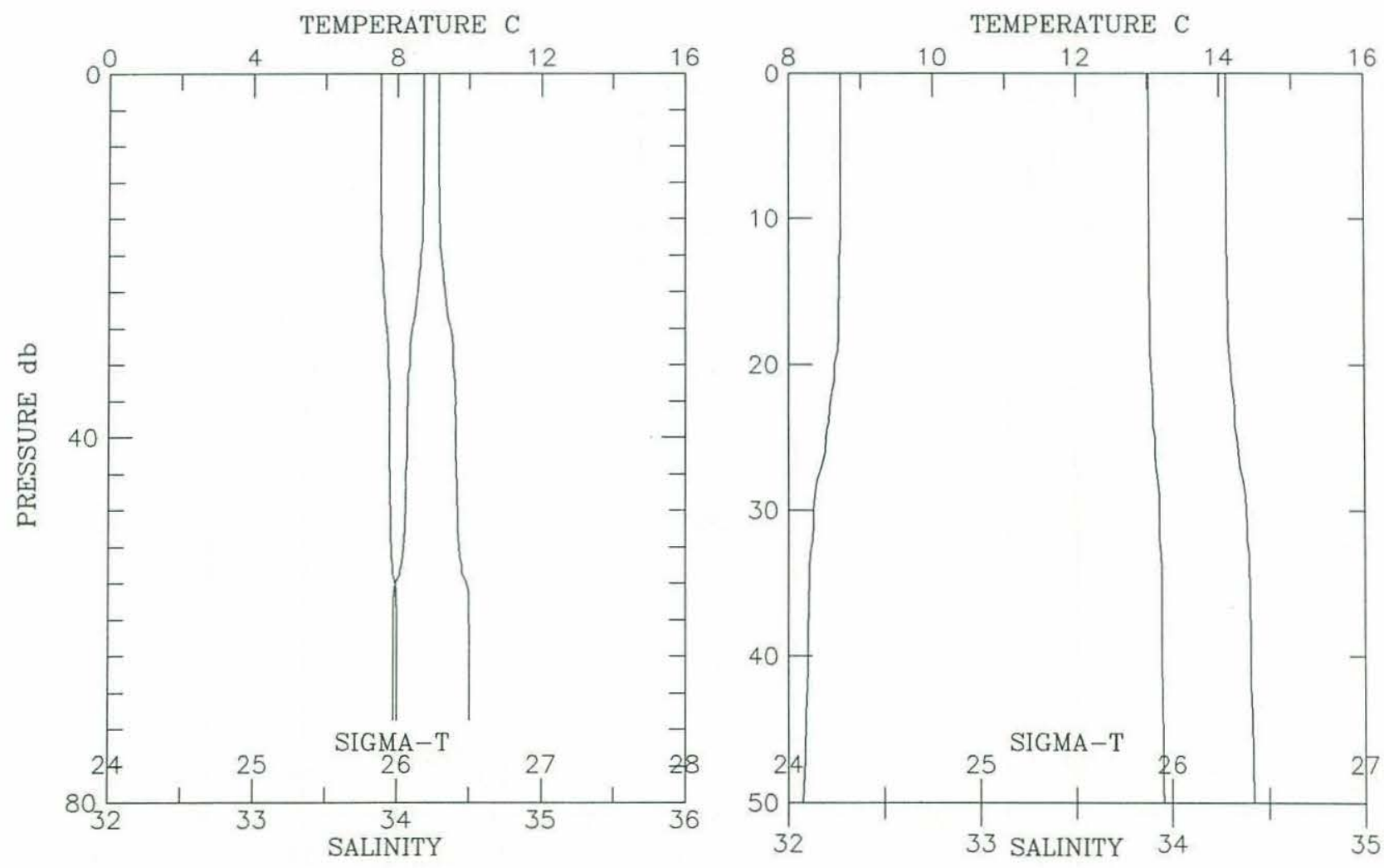
Cruise w8905 Station \# 159 Depth m: 95 Time: 1989-05-12 16:05

Position: $3826.21^{\prime} \mathrm{N} 12316.76^{\prime} \mathrm{W}$ Depth Deck Offset: -0.25

\begin{tabular}{|c|c|c|c|c|c|c|}
\hline $\begin{array}{l}\text { PRESSURE } \\
\text { (d-bars) }\end{array}$ & Interp & $\begin{array}{c}\text { TEMP } \\
(\operatorname{deg} C)\end{array}$ & $\begin{array}{c}\text { SALINITY } \\
(0 / 00)\end{array}$ & SIGMA-T & DELTA-D & $\begin{array}{l}\text { CHANNELA } \\
\text { ( } 81 \text { ight) }\end{array}$ \\
\hline 0.0 & E & 8.768 & 33.872 & 26.271 & 0.0000 & 0.0 \\
\hline 5.0 & & 8.769 & 33.871 & 26.270 & 0.0089 & 0.0 \\
\hline 10.0 & & 8.755 & 33.871 & 26.273 & 0.0178 & 0.0 \\
\hline 15.0 & & 8.755 & 33.872 & 26.273 & 0.0266 & 0.0 \\
\hline 20.0 & & 8.751 & 33.871 & 26.273 & 0.0355 & 0.0 \\
\hline 25.0 & & 8.746 & 33.871 & 26.274 & 0.0444 & 0.0 \\
\hline 30.0 & & 8.747 & 33.871 & 26.274 & 0.0533 & 0.0 \\
\hline 35.0 & & 8.743 & 33.871 & 26.274 & 0.0622 & 0.0 \\
\hline 40.0 & & 8.738 & 33.871 & 26.275 & 0.0711 & 0.0 \\
\hline 45.0 & & 8.424 & 33.906 & 26.351 & 0.0798 & 0.0 \\
\hline 50.0 & & 8.337 & 33.924 & 26.378 & 0.0883 & 0.0 \\
\hline 55.0 & & 8.289 & 33.934 & 26.394 & 0.0967 & 0.0 \\
\hline 60.0 & & 8.172 & 33.950 & 26.424 & 0.1050 & 0.0 \\
\hline 65.0 & & 8.110 & 33.960 & 26.441 & 0.1132 & 0.0 \\
\hline 70.0 & & 7.961 & 33.986 & 26.483 & 0.1212 & 0.0 \\
\hline 75.0 & & 7.874 & 34.001 & 26.508 & 0.1290 & 0.0 \\
\hline 80.0 & & 7.840 & 34.007 & 26.518 & 0.1368 & 0.0 \\
\hline 85.0 & & 7.837 & 34.008 & 26.519 & 0.1446 & 0.0 \\
\hline 90.0 & & 7.838 & 34.008 & 26.519 & 0.1524 & 0.0 \\
\hline 91.0 & & 7.837 & 34.008 & 26.519 & 0.1539 & 0.0 \\
\hline
\end{tabular}

TEMPERATURE C
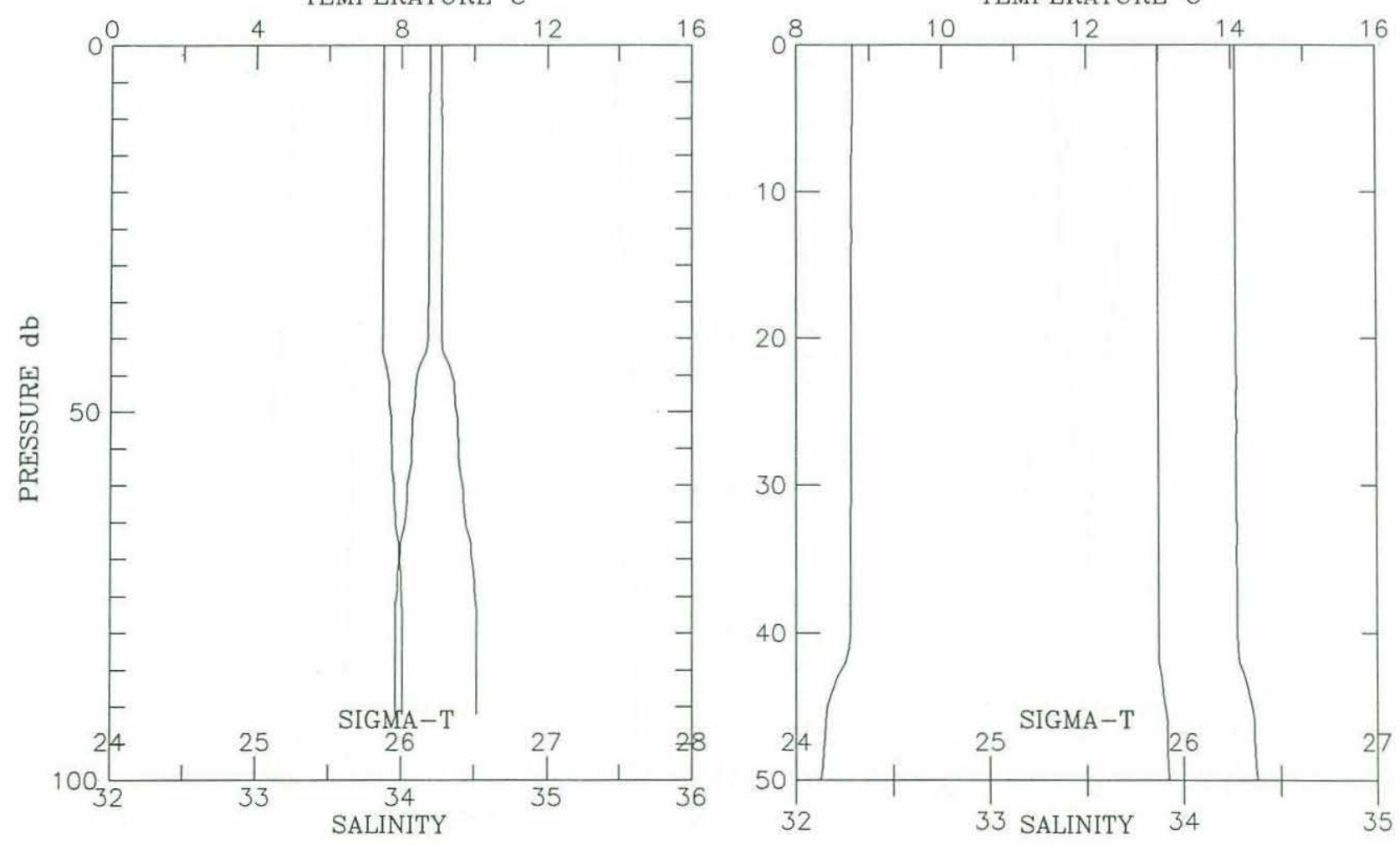
Cruise w8905 Station \# 160 Depth m: 116 Time: 1989-05-12 16:44 Position: 38 23.29'N $12320.68^{\prime} \mathrm{W}$ Depth Deck Offset: -0.25

\begin{tabular}{|c|c|c|c|c|c|c|}
\hline $\begin{array}{l}\text { PRESSURE } \\
\text { (d-bars) }\end{array}$ & Interp & $\begin{array}{l}\text { TEMP } \\
(\operatorname{deg} C)\end{array}$ & $\begin{array}{c}\text { SALINITY } \\
(0 / 00)\end{array}$ & SIGMA-T & DELTA-D & $\begin{array}{l}\text { CHANNELA } \\
\text { ( } \% \text { ight) }\end{array}$ \\
\hline 0.0 & E & 9.112 & 33.691 & 26.075 & 0.0000 & 0.0 \\
\hline 10.0 & & 9.054 & 33.698 & 26.090 & 0.0196 & 0.0 \\
\hline 20.0 & & 8.927 & 33.737 & 26.141 & 0.0389 & 0.0 \\
\hline 30.0 & & 8.832 & 33.789 & 26.196 & 0.0576 & 0.0 \\
\hline 40.0 & & 8.842 & 33.801 & 26.204 & 0.0761 & 0.0 \\
\hline 50.0 & & 8.829 & 33.808 & 26.212 & 0.0945 & 0.0 \\
\hline 60.0 & & 8.587 & 33.871 & 26.299 & 0.1126 & 0.0 \\
\hline 70.0 & & 8.564 & 33.923 & 26.343 & 0.1300 & 0.0 \\
\hline 80.0 & & 8.484 & 33.974 & 26.395 & 0.1470 & 0.0 \\
\hline 90.0 & & 8.253 & 33.991 & 26.444 & 0.1635 & 0.0 \\
\hline 100.0 & & 8.126 & 34.020 & 26.486 & 0.1796 & 0.0 \\
\hline 110.0 & & 8.128 & 34.020 & 26.485 & 0.1955 & 0.0 \\
\hline
\end{tabular}
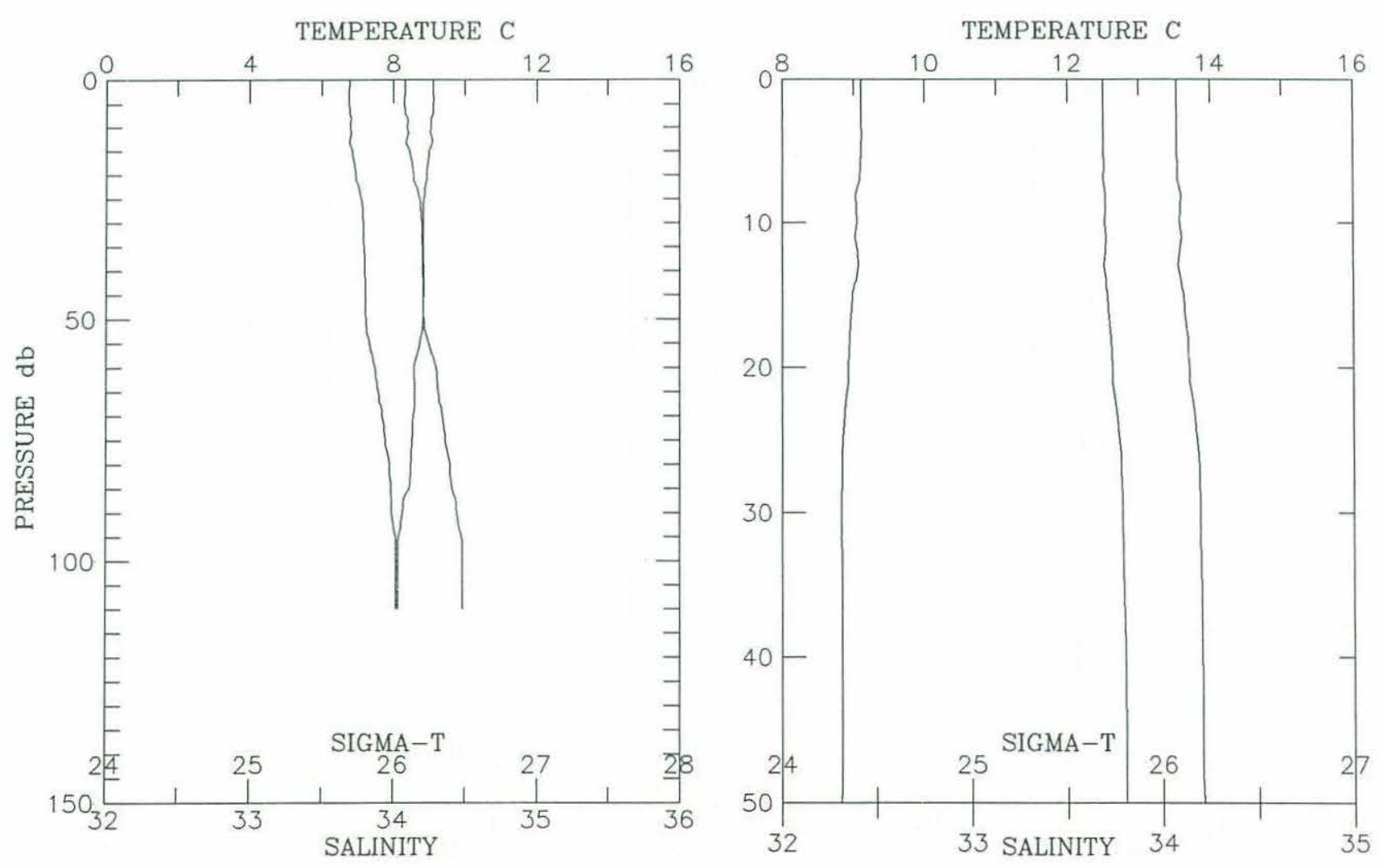


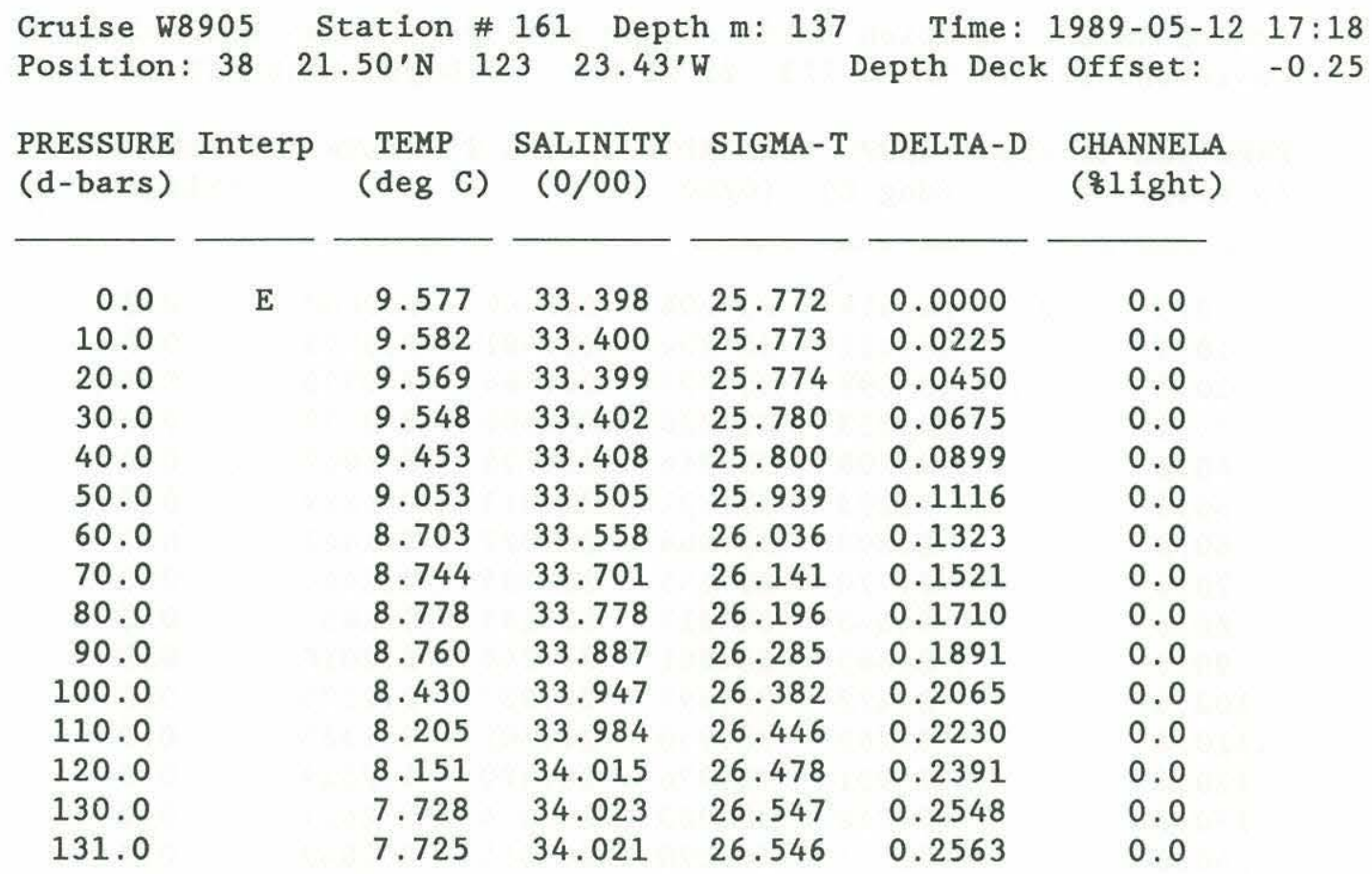
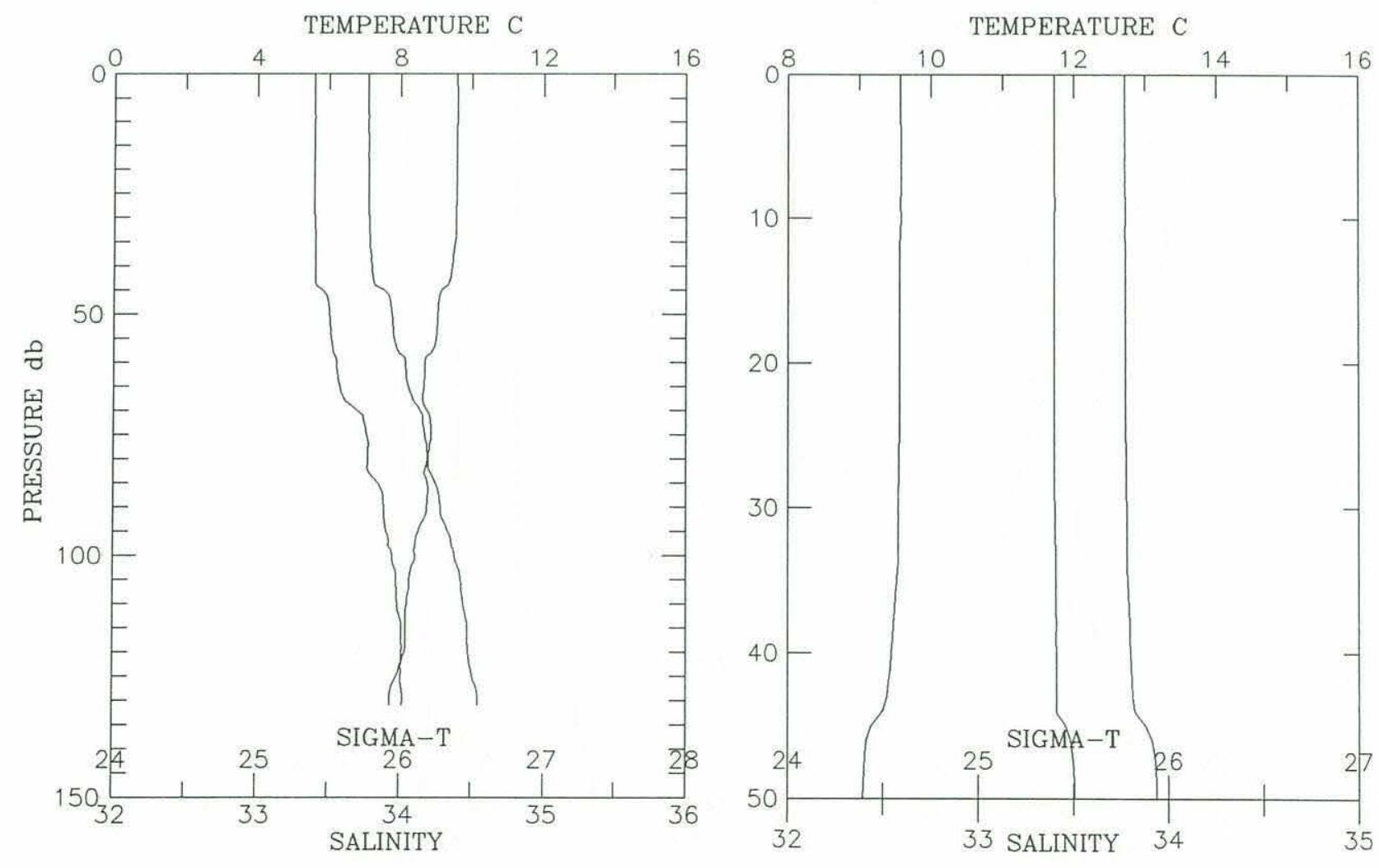


\begin{tabular}{|c|c|c|c|c|c|c|}
\hline $\begin{array}{l}\text { PRESSURE } \\
\text { (d-bars) }\end{array}$ & Interp & $\begin{array}{l}\text { TEMP } \\
(\operatorname{deg} C)\end{array}$ & $\begin{array}{c}\text { SALINITY } \\
(0 / 00)\end{array}$ & SIGMA-T & DELTA-D & $\begin{array}{l}\text { CHANNELA } \\
\text { ( } \delta 1 \text { ight) }\end{array}$ \\
\hline 0.0 & $\mathrm{E}$ & 10.414 & 33.204 & 25.480 & 0.0000 & 0.0 \\
\hline 10.0 & & 10.411 & 33.204 & 25.481 & 0.0253 & 0.0 \\
\hline 20.0 & & 10.398 & 33.206 & 25.484 & 0.0506 & 0.0 \\
\hline 30.0 & & 10.353 & 33.226 & 25.508 & 0.0758 & 0.0 \\
\hline 40.0 & & 9.708 & 33.366 & 25.725 & 0.1002 & 0.0 \\
\hline 50.0 & & 9.293 & 33.391 & 25.813 & 0.1229 & 0.0 \\
\hline 60.0 & & 8.800 & 33.566 & 26.027 & 0.1442 & 0.0 \\
\hline 70.0 & & 8.770 & 33.635 & 26.085 & 0.1641 & 0.0 \\
\hline 80.0 & & 9.043 & 33.817 & 26.185 & 0.1834 & 0.0 \\
\hline 90.0 & & 8.863 & 33.861 & 26.248 & 0.2018 & 0.0 \\
\hline 100.0 & & 8.472 & 33.897 & 26.337 & 0.2195 & 0.0 \\
\hline 110.0 & & 8.266 & 33.940 & 26.402 & 0.2365 & 0.0 \\
\hline 120.0 & & 7.931 & 33.976 & 26.480 & 0.2529 & 0.0 \\
\hline 130.0 & & 7.718 & 33.980 & 26.514 & 0.2687 & 0.0 \\
\hline 140.0 & & 7.655 & 34.020 & 26.555 & 0.2842 & 0.0 \\
\hline 150.0 & & 7.460 & 34.057 & 26.612 & 0.2994 & 0.0 \\
\hline 156.0 & & 7.389 & 34.060 & 26.625 & 0.3082 & 0.0 \\
\hline
\end{tabular}
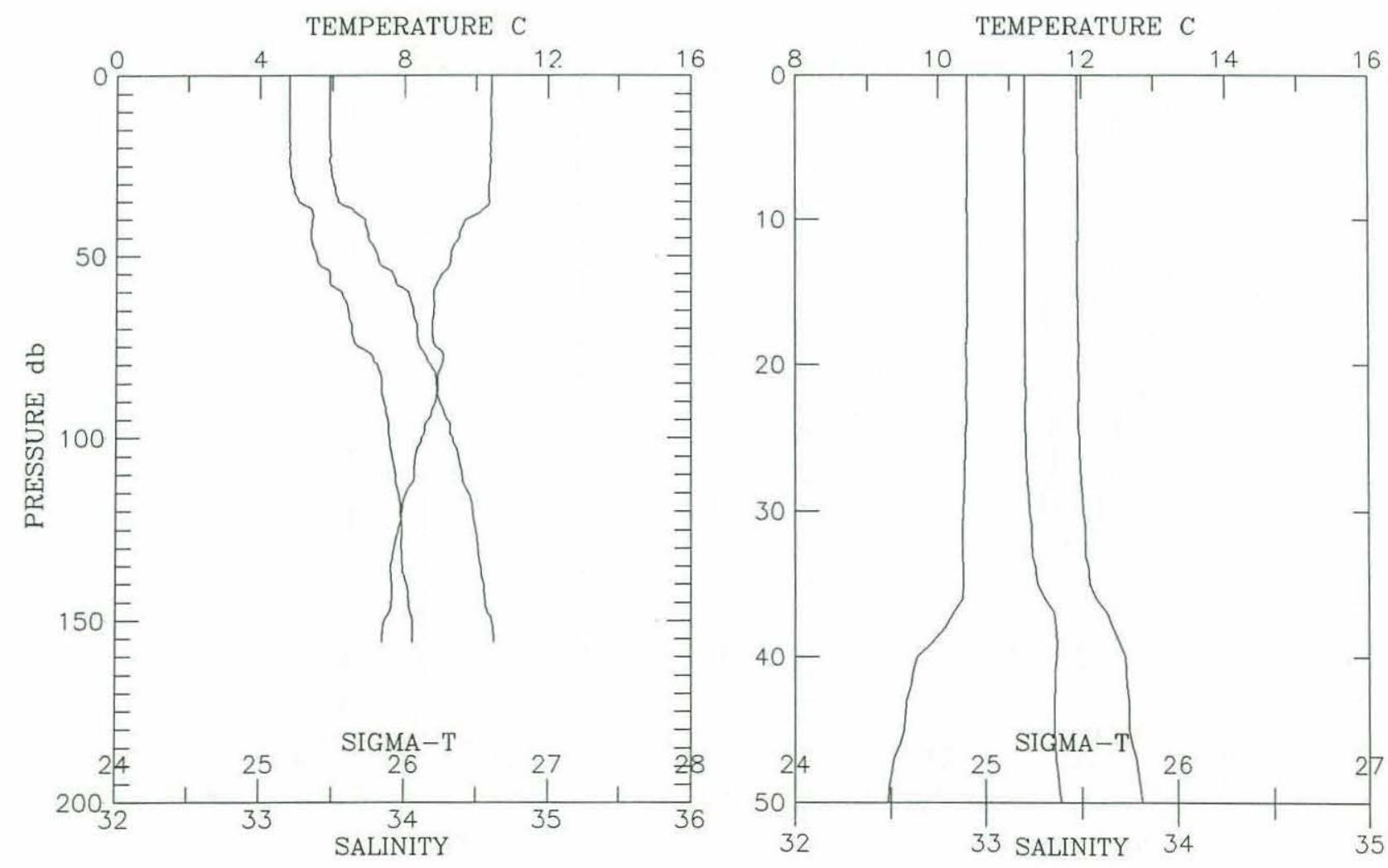
Cruise W8905 Station \# 163 Depth m: 380 Time: 1989-05-12 18:47 Position: $38 \quad 15.75^{\prime} \mathrm{N} 123 \quad 30.85^{\prime} \mathrm{W} \quad$ Depth Deck Offset: -0.25

\begin{tabular}{|c|c|c|c|c|c|c|}
\hline $\begin{array}{l}\text { PRESSURE } \\
\text { (d-bars) }\end{array}$ & Interp & $\begin{array}{c}\text { TEMP } \\
(\operatorname{deg} C)\end{array}$ & $\begin{array}{c}\text { SALINITY } \\
(0 / 00)\end{array}$ & SIGMA-T & DELTA-D & $\begin{array}{l}\text { CHANNELA } \\
\text { ( } \delta 1 i g h t)\end{array}$ \\
\hline 0.0 & E & 11.571 & 33.018 & 25.130 & 0.0000 & 0.0 \\
\hline 10.0 & & 11.566 & 33.018 & 25.131 & 0.0286 & 0.0 \\
\hline 20.0 & & 11.558 & 33.019 & 25.133 & 0.0572 & 0.0 \\
\hline 30.0 & & 11.435 & 33.031 & 25.165 & 0.0858 & 0.0 \\
\hline 40.0 & & 10.510 & 33.176 & 25.442 & 0.1128 & 0.0 \\
\hline 50.0 & & 10.389 & 33.332 & 25.584 & 0.1379 & 0.0 \\
\hline 60.0 & & 9.656 & 33.305 & 25.686 & 0.1620 & 0.0 \\
\hline 80.0 & & 9.125 & 33.512 & 25.933 & 0.2067 & 0.0 \\
\hline 100.0 & & 8.920 & 33.830 & 26.215 & 0.2461 & 0.0 \\
\hline 150.0 & & 7.964 & 34.013 & 26.504 & 0.3297 & 0.0 \\
\hline 200.0 & & 7.616 & 34.061 & 26.593 & 0.4063 & 0.0 \\
\hline 250.0 & & 7.324 & 34.082 & 26.651 & 0.4800 & 0.0 \\
\hline 300.0 & & 6.924 & 34.095 & 26.717 & 0.5517 & 0.0 \\
\hline 373.0 & & 6.817 & 34.105 & 26.739 & 0.6532 & 0.0 \\
\hline
\end{tabular}
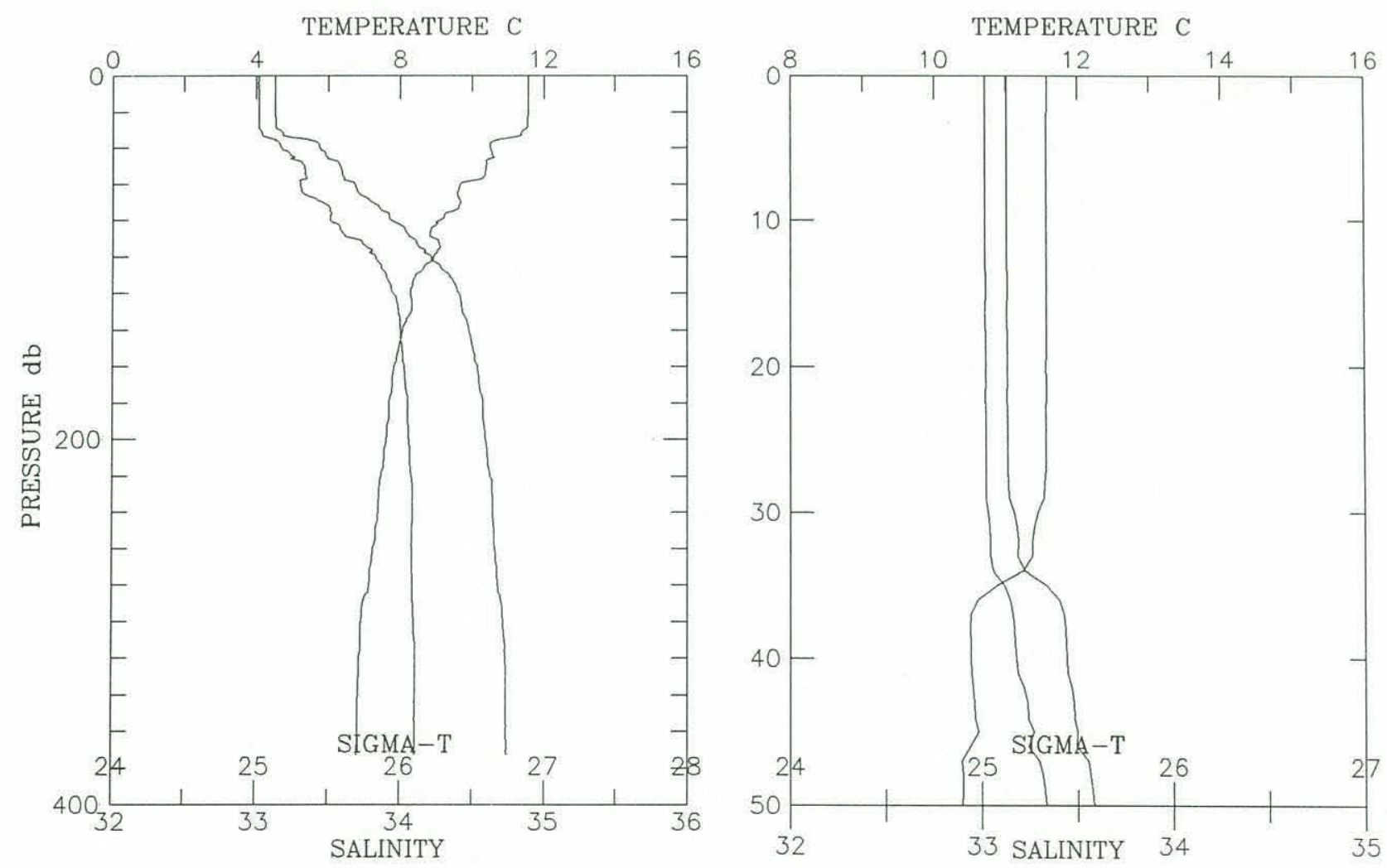


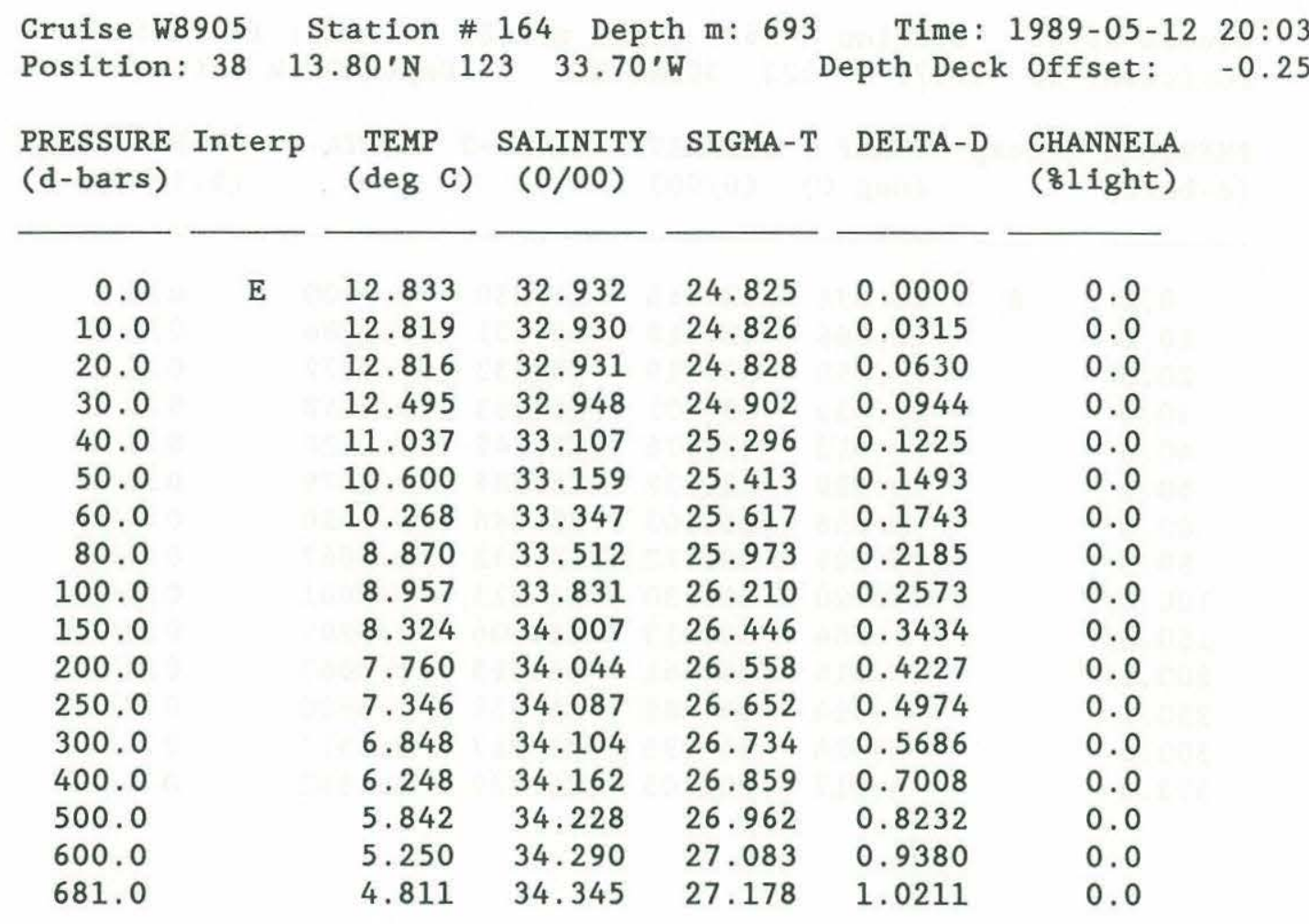
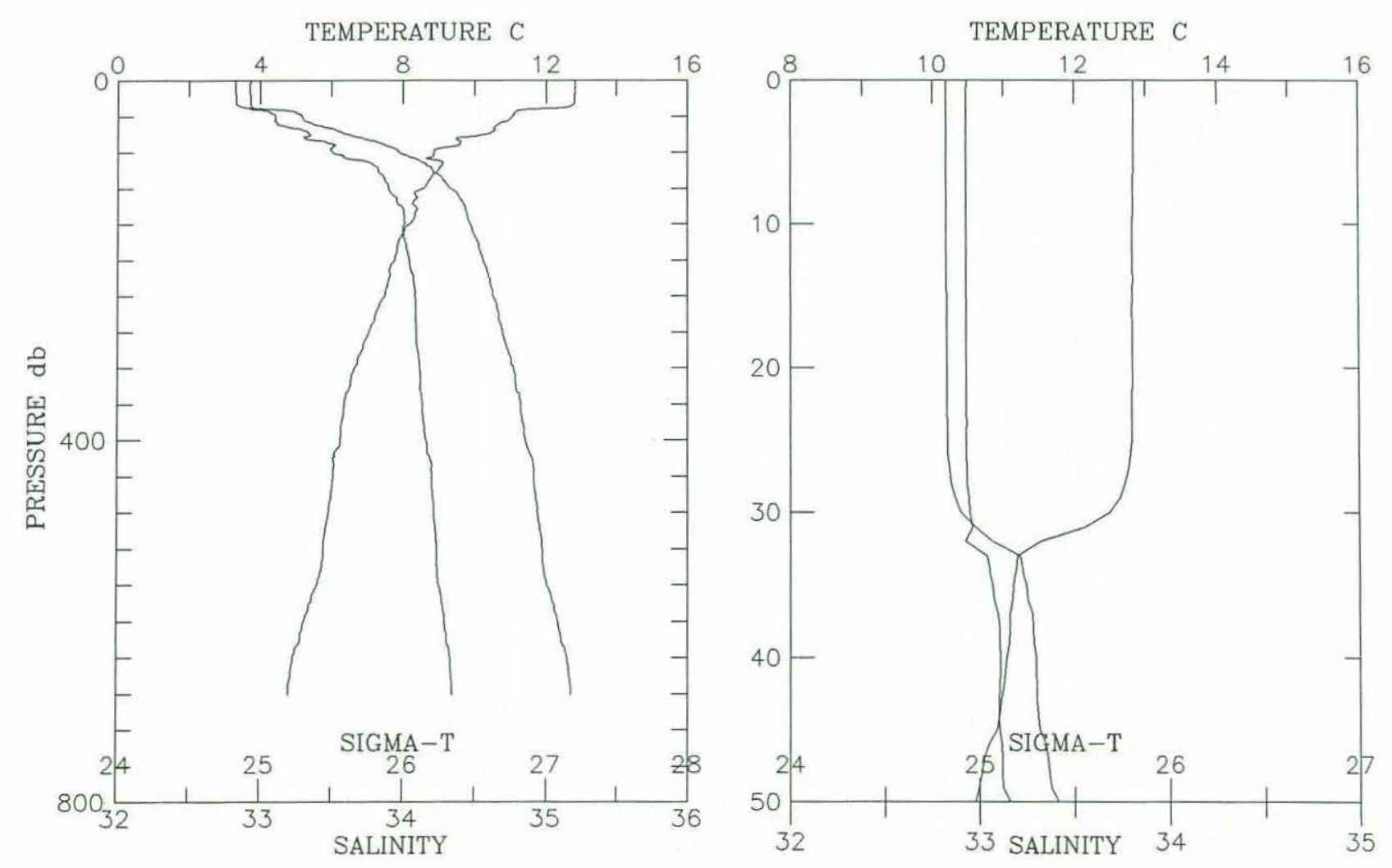
Cruise W8905 Station \# 165 Depth m: 973 Time: 1989-05-12 21:23

Position: $38 \quad 8.20^{\prime} \mathrm{N} 123 \quad 34.10^{\prime} \mathrm{W} \quad$ Depth Deck Offset: -0.20

\begin{tabular}{|c|c|c|c|c|c|c|}
\hline $\begin{array}{l}\text { PRESSURE } \\
\text { (d-bars) }\end{array}$ & Interp & $\begin{array}{l}\text { TEMP } \\
(\operatorname{deg} \quad C)\end{array}$ & $\begin{array}{l}\text { SALINITY } \\
(0 / 00)\end{array}$ & SIGMA-T & DELTA-D & $\begin{array}{l}\text { CHANNELA } \\
\text { ( }(z 1 \text { ight) }\end{array}$ \\
\hline 0.0 & E & 13.060 & 32.896 & 24.752 & 0.0000 & 0.0 \\
\hline 10.0 & & 13.061 & 32.895 & 24.751 & 0.0322 & 0.0 \\
\hline 20.0 & & 13.061 & 32.895 & 24.751 & 0.0644 & 0.0 \\
\hline 30.0 & & 13.041 & 32.896 & 24.756 & 0.0967 & 0.0 \\
\hline 40.0 & & 12.665 & 32.909 & 24.839 & 0.1287 & 0.0 \\
\hline 50.0 & & 10.604 & 32.988 & 25.279 & 0.1570 & 0.0 \\
\hline 60.0 & & 10.447 & 33.171 & 25.449 & 0.1836 & 0.0 \\
\hline 80.0 & & 9.656 & 33.400 & 25.760 & 0.2318 & 0.0 \\
\hline 100.0 & & 9.371 & 33.693 & 26.035 & 0.2754 & 0.0 \\
\hline 150.0 & & 8.318 & 33.972 & 26.419 & 0.3656 & 0.0 \\
\hline 200.0 & & 7.847 & 34.052 & 26.552 & 0.4456 & 0.0 \\
\hline 250.0 & & 7.368 & 34.071 & 26.636 & 0.5212 & 0.0 \\
\hline 300.0 & & 6.847 & 34.093 & 26.726 & 0.5931 & 0.0 \\
\hline 400.0 & & 6.408 & 34.175 & 26.848 & 0.7259 & 0.0 \\
\hline 500.0 & & 5.729 & 34.236 & 26.983 & 0.8493 & 0.0 \\
\hline 600.0 & & 5.314 & 34.294 & 27.079 & 0.9622 & 0.0 \\
\hline 700.0 & & 4.830 & 34.342 & 27.173 & 1.0661 & 0.0 \\
\hline 800.0 & & 4.392 & 34.388 & 27.258 & 1.1618 & 0.0 \\
\hline 900.0 & & 4.198 & 34.414 & 27.300 & 1.2525 & 0.0 \\
\hline 967.0 & & 4.097 & 34.426 & 27.320 & 1.3118 & 0.0 \\
\hline
\end{tabular}
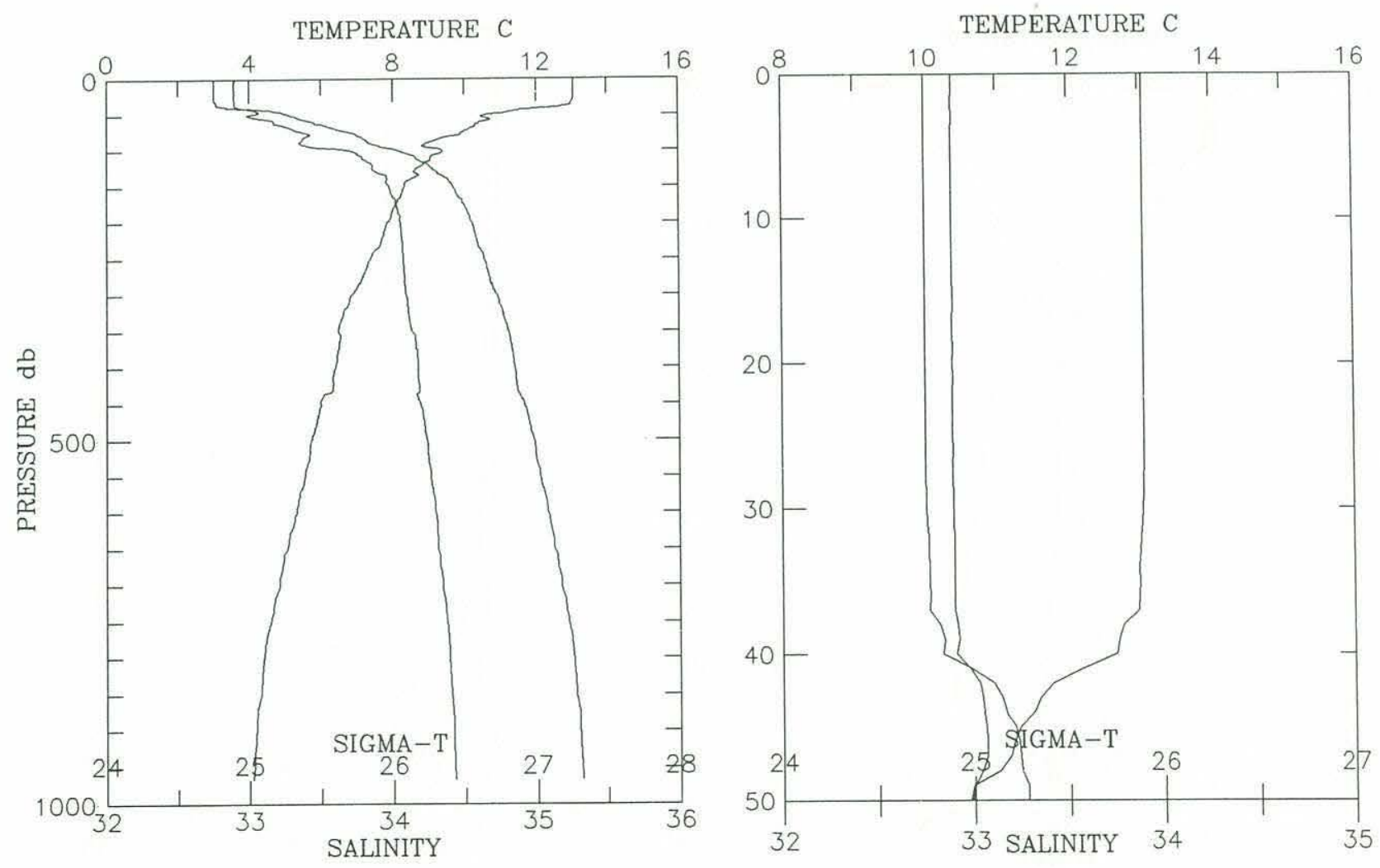


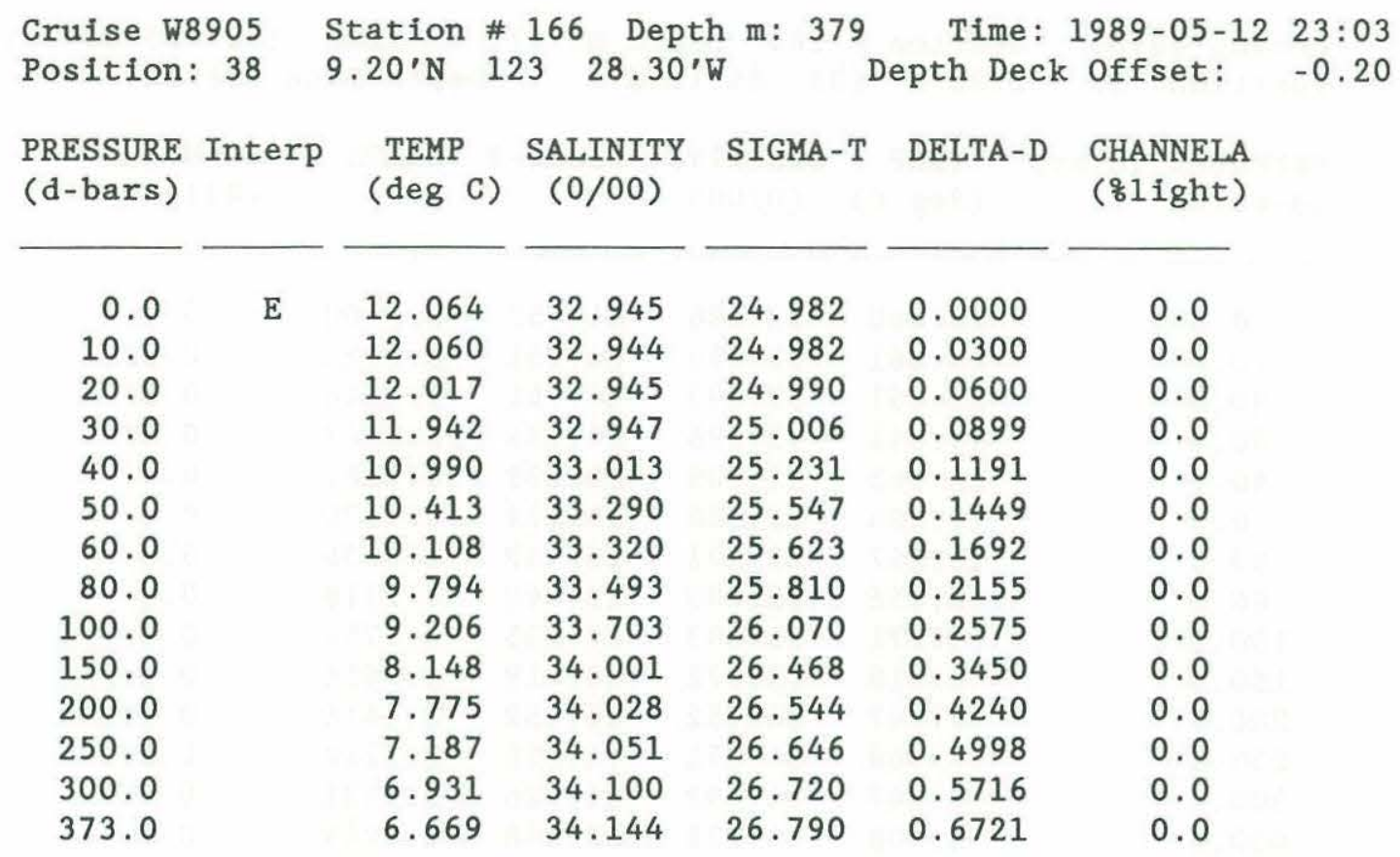

TEMPERATURE C

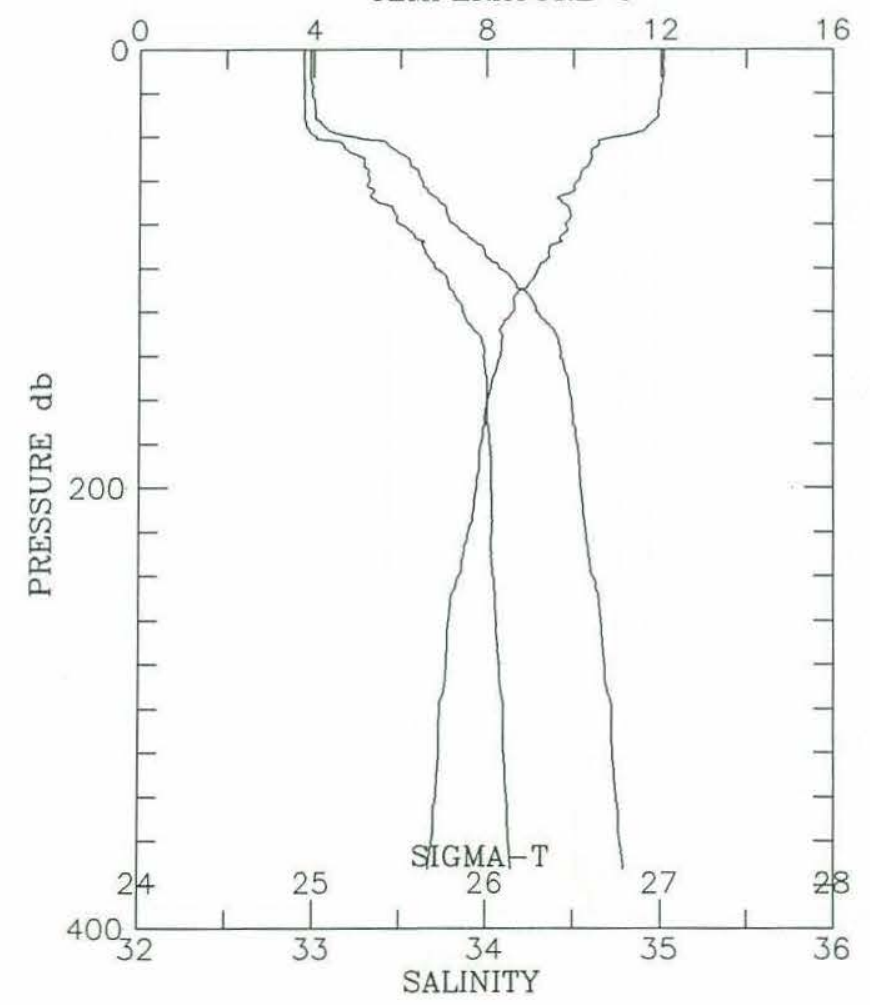

TEMPERATURE C

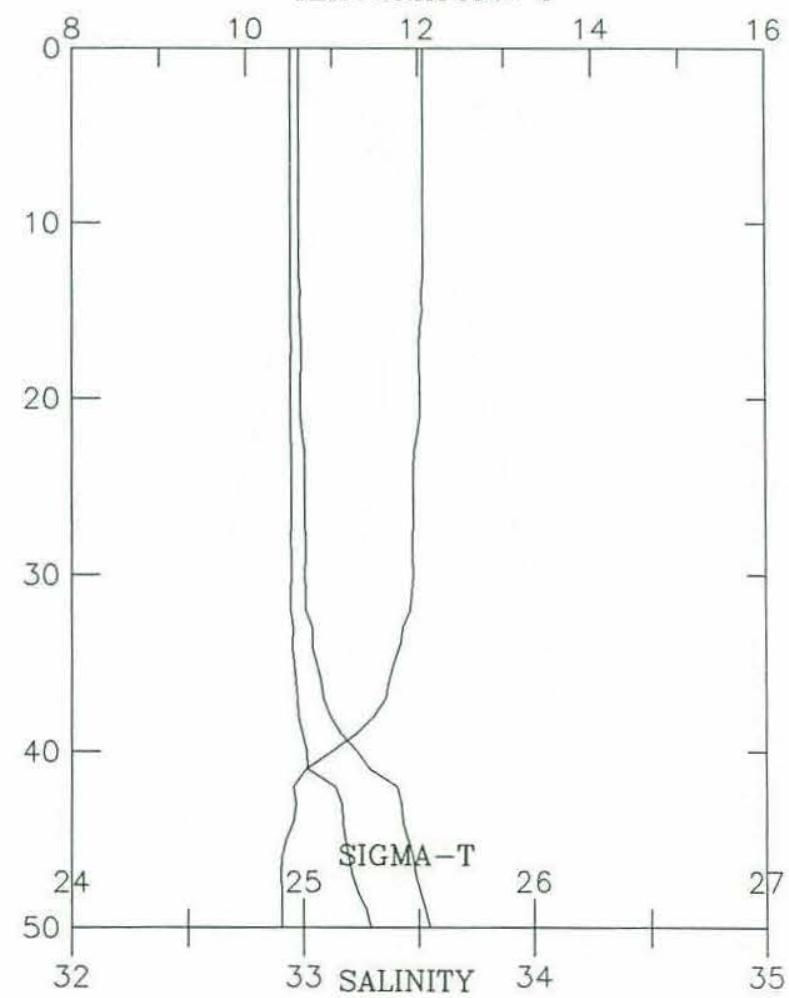


Cruise W8905 Station \# 167 Depth m: 192 Time: 1989-05-13 00:28 Position: $3810.39^{\prime} \mathrm{N} 12322.02^{\prime} \mathrm{W}$ Depth Deck Offset: -0.20

\begin{tabular}{|c|c|c|c|c|c|c|}
\hline $\begin{array}{l}\text { PRESSURE } \\
\text { (d-bars) }\end{array}$ & Interp & $\begin{array}{l}\text { TEMP } \\
(\operatorname{deg} C)\end{array}$ & $\begin{array}{c}\text { SALINITY } \\
(0 / 00)\end{array}$ & SIGMA-T & DELTA-D & $\begin{array}{l}\text { CHANNELA } \\
\text { ( }(1 \text { light) }\end{array}$ \\
\hline 0.0 & E & 10.827 & 33.139 & 25.358 & 0.0000 & 0.0 \\
\hline 10.0 & & 10.824 & 33.139 & 25.358 & 0.0264 & 0.0 \\
\hline 20.0 & & 10.821 & 33.139 & 25.359 & 0.0529 & 0.0 \\
\hline 30.0 & & 10.815 & 33.140 & 25.360 & 0.0794 & 0.0 \\
\hline 40.0 & & 10.646 & 33.165 & 25.410 & 0.1057 & 0.0 \\
\hline 50.0 & & 9.576 & 33.342 & 25.728 & 0.1302 & 0.0 \\
\hline 60.0 & & 9.254 & 33.350 & 25.786 & 0.1529 & 0.0 \\
\hline 70.0 & & 9.667 & 33.637 & 25.944 & 0.1748 & 0.0 \\
\hline 80.0 & & 9.247 & 33.631 & 26.007 & 0.1955 & 0.0 \\
\hline 90.0 & & 8.932 & 33.813 & 26.199 & 0.2148 & 0.0 \\
\hline 100.0 & & 8.718 & 33.917 & 26.314 & 0.2330 & 0.0 \\
\hline 110.0 & & 8.540 & 33.929 & 26.351 & 0.2503 & 0.0 \\
\hline 120.0 & & 8.423 & 33.961 & 26.394 & 0.2673 & 0.0 \\
\hline 130.0 & & 8.157 & 33.961 & 26.435 & 0.2840 & 0.0 \\
\hline 140.0 & & 8.001 & 33.969 & 26.464 & 0.3003 & 0.0 \\
\hline 150.0 & & 7.827 & 33.995 & 26.510 & 0.3162 & 0.0 \\
\hline 160.0 & & 7.750 & 34.011 & 26.534 & 0.3319 & 0.0 \\
\hline 170.0 & & 7.545 & 34.020 & 26.571 & 0.3473 & 0.0 \\
\hline 180.0 & & 7.390 & 34.029 & 26.600 & 0.3623 & 0.0 \\
\hline 188.0 & & 7.382 & 34.031 & 26.603 & 0.3743 & 0.0 \\
\hline
\end{tabular}
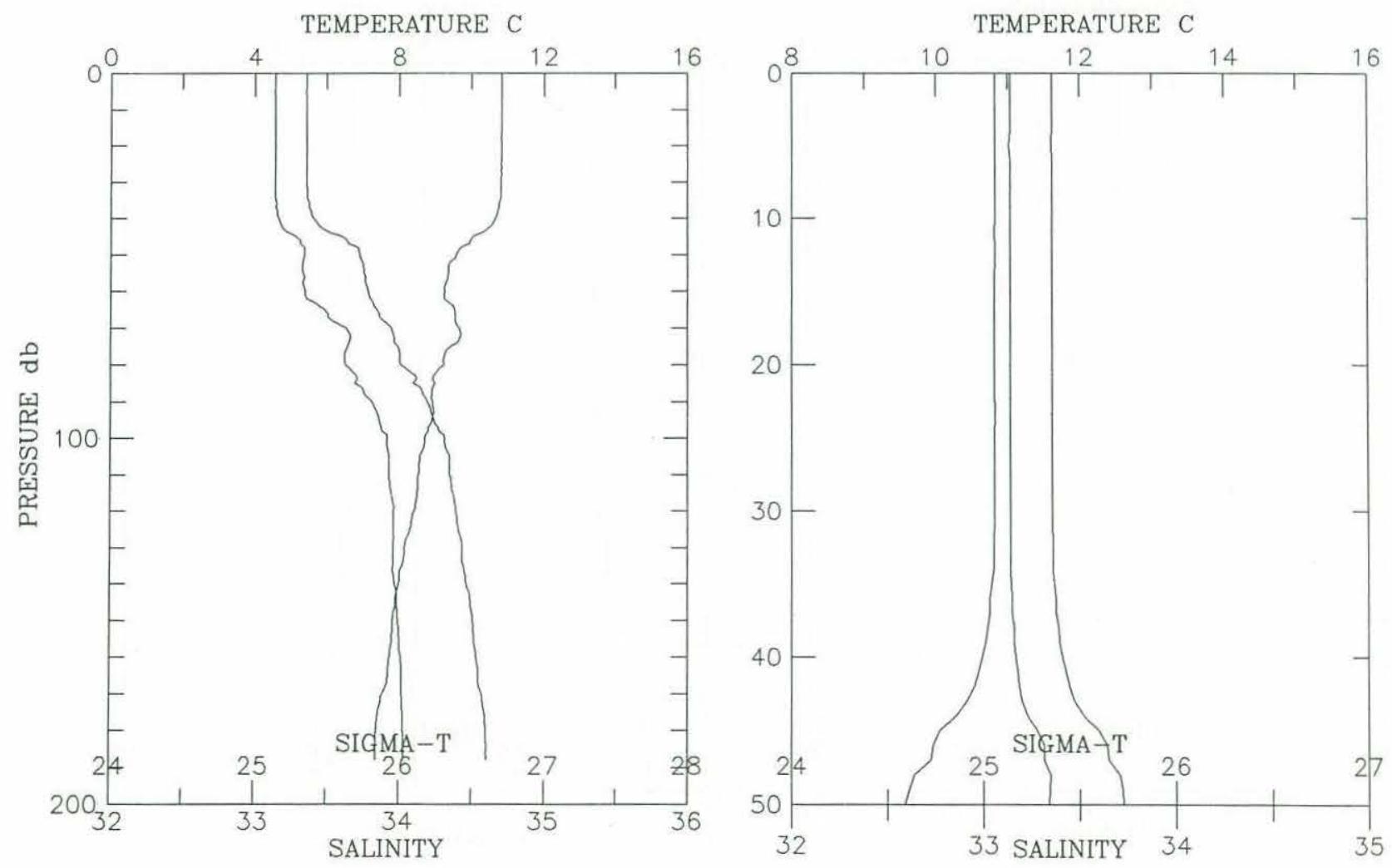


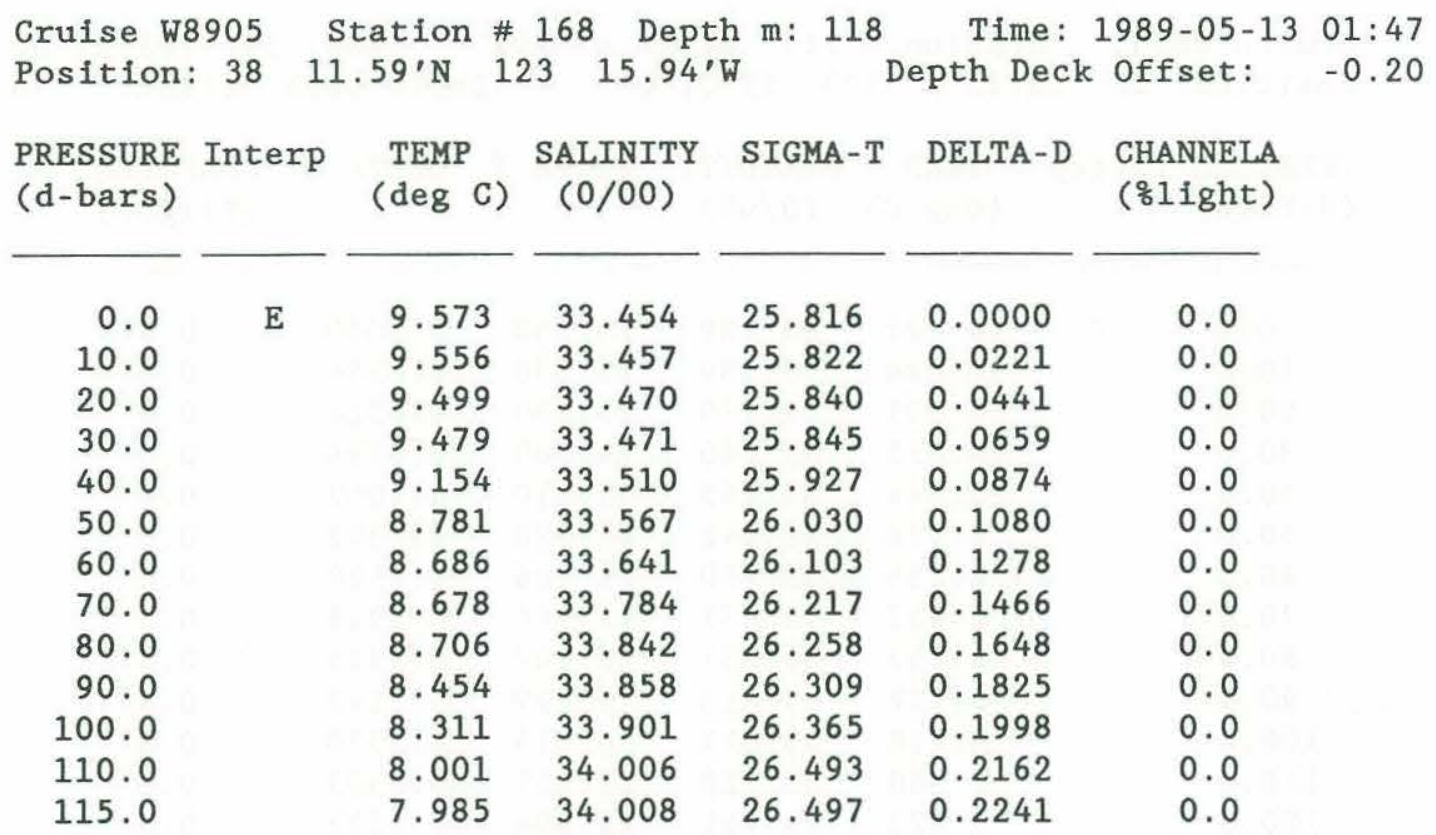
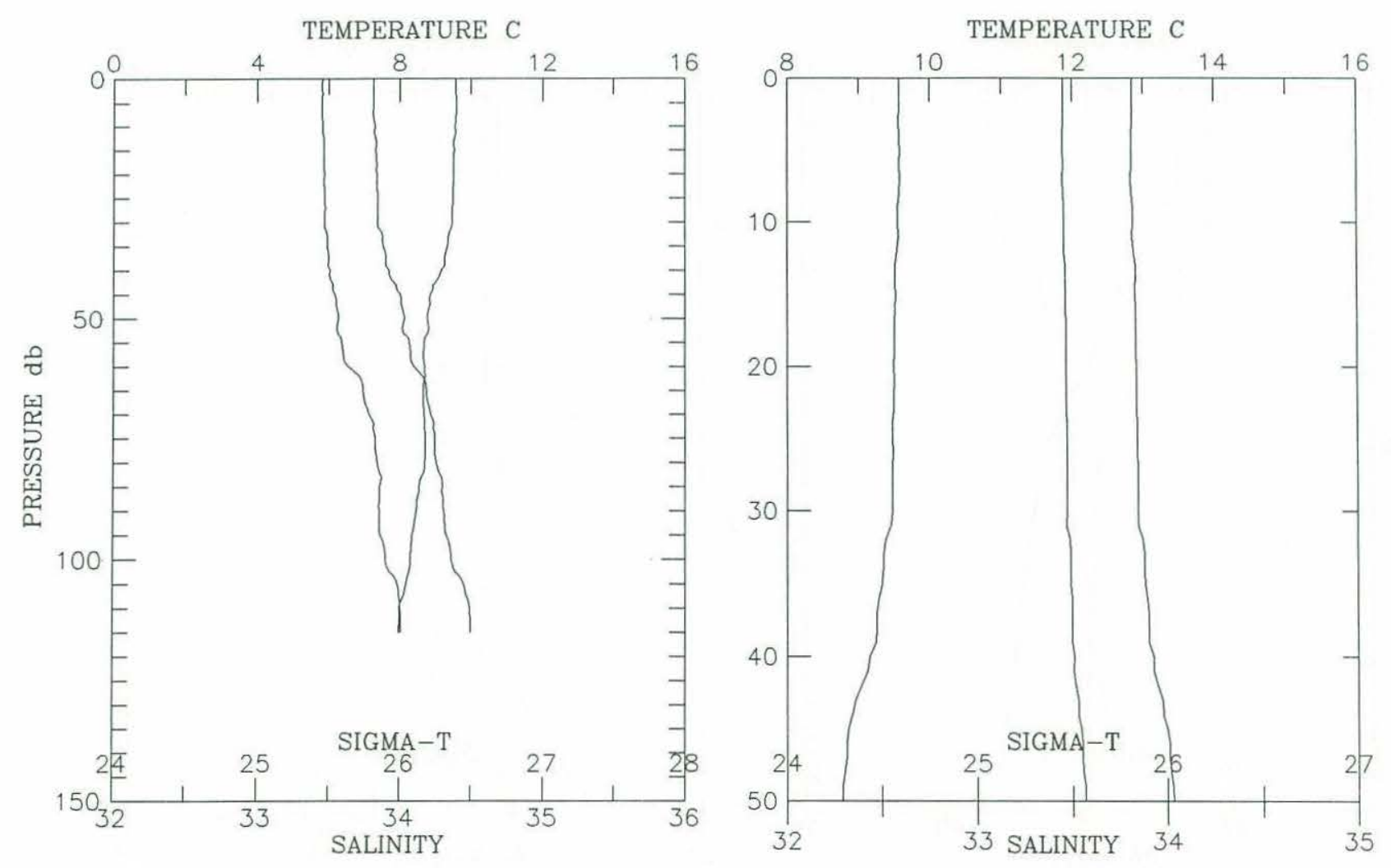
Cruise W8905 Station \# 169 Depth m: 95 Time: 1989-05-13 02:58

Position: $38 \quad 12.75^{\prime} \mathrm{N} 123 \quad 9.70^{\prime} \mathrm{W}$ Depth Deck Offset: $\quad-0.20$

\begin{tabular}{|c|c|c|c|c|c|c|}
\hline $\begin{array}{l}\text { PRESSURE } \\
\text { (d-bars) }\end{array}$ & Interp & $\begin{array}{l}\text { TEMP } \\
(\operatorname{deg} C)\end{array}$ & $\begin{array}{c}\text { SALINITY } \\
(0 / 00)\end{array}$ & SIGMA-T & DELTA-D & $\begin{array}{l}\text { CHANNELA } \\
\text { (\&light) }\end{array}$ \\
\hline 0.0 & E & 8.878 & 33.819 & 26.213 & 0.0000 & 0.0 \\
\hline 5.0 & & 8.878 & 33.817 & 26.211 & 0.0092 & 0.0 \\
\hline 10.0 & & 8.873 & 33.816 & 26.211 & 0.0183 & 0.0 \\
\hline 15.0 & & 8.870 & 33.815 & 26.211 & 0.0275 & 0.0 \\
\hline 20.0 & & 8.877 & 33.818 & 26.212 & 0.0367 & 0.0 \\
\hline 25.0 & & 8.874 & 33.818 & 26.213 & 0.0459 & 0.0 \\
\hline 30.0 & & 8.881 & 33.820 & 26.213 & 0.0551 & 0.0 \\
\hline 35.0 & & 8.878 & 33.825 & 26.218 & 0.0642 & 0.0 \\
\hline 40.0 & & 8.835 & 33.826 & 26.225 & 0.0734 & 0.0 \\
\hline 45.0 & & 8.780 & 33.828 & 26.235 & 0.0825 & 0.0 \\
\hline 50.0 & & 8.727 & 33.827 & 26.243 & 0.0916 & 0.0 \\
\hline 55.0 & & 8.605 & 33.832 & 26.265 & 0.1006 & 0.0 \\
\hline 60.0 & & 8.253 & 33.887 & 26.362 & 0.1094 & 0.0 \\
\hline 65.0 & & 8.210 & 33.916 & 26.391 & 0.1178 & 0.0 \\
\hline 70.0 & & 8.207 & 33.918 & 26.393 & 0.1262 & 0.0 \\
\hline 75.0 & & 8.206 & 33.920 & 26.395 & 0.1345 & 0.0 \\
\hline 80.0 & & 8.210 & 33.918 & 26.393 & 0.1429 & 0.0 \\
\hline 85.0 & & 8.209 & 33.918 & 26.393 & 0.1513 & 0.0 \\
\hline 90.0 & & 8.212 & 33.918 & 26.393 & 0.1596 & 0.0 \\
\hline 91.0 & & 8.212 & 33.918 & 26.393 & 0.1613 & 0.0 \\
\hline
\end{tabular}

TEMPERATURE C
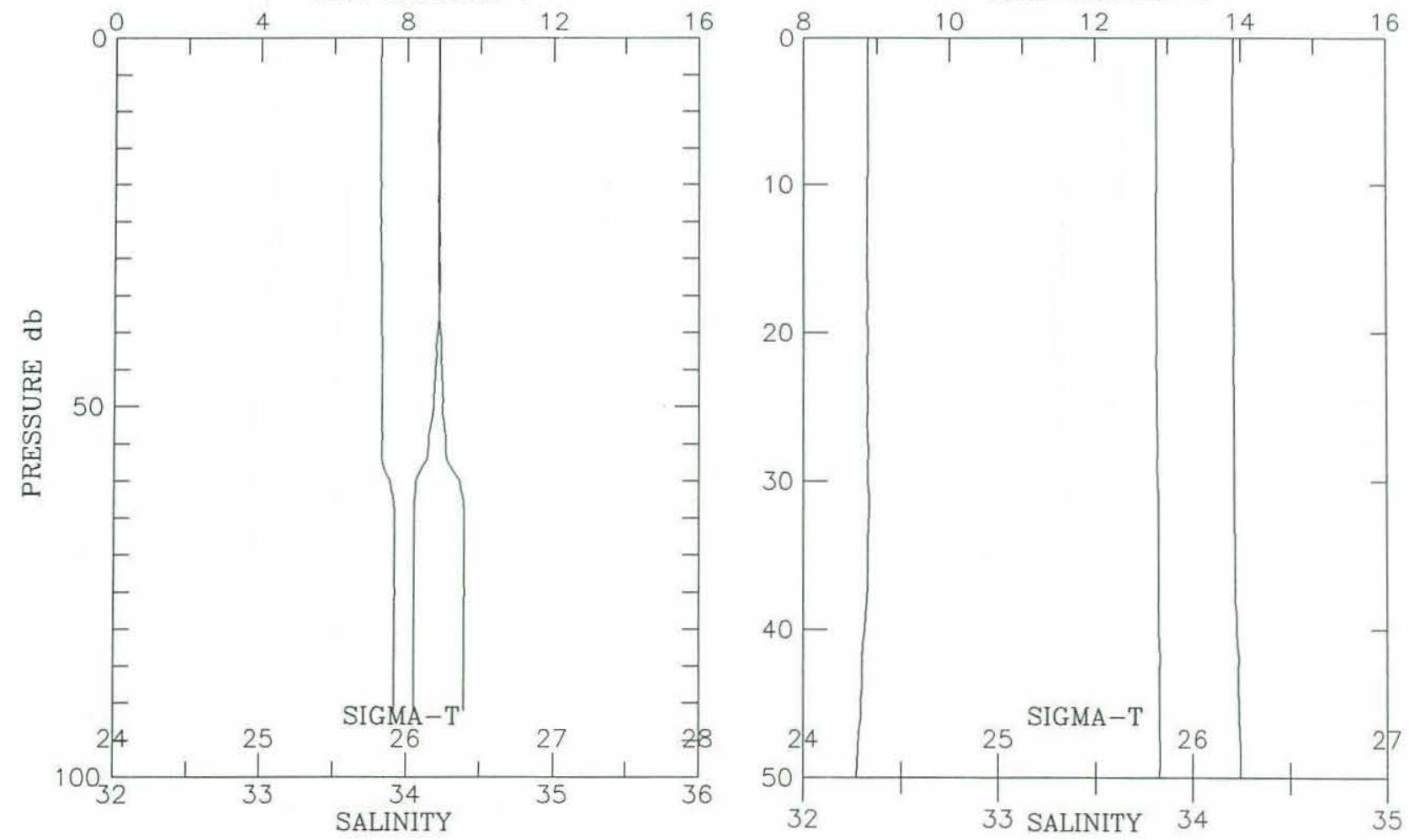


\begin{tabular}{|c|c|c|c|c|c|c|}
\hline $\begin{array}{l}\text { PRESSURE } \\
\text { (d-bars) }\end{array}$ & Interp & $\begin{array}{l}\text { TEMP } \\
(\operatorname{deg} C)\end{array}$ & $\begin{array}{c}\text { SALINITY } \\
(0 / 00)\end{array}$ & SIGMA-T & DELTA-D & $\begin{array}{l}\text { CHANNELA } \\
\text { (\&light) }\end{array}$ \\
\hline 0.0 & $\mathrm{E}$ & 9.214 & 33.792 & 26.138 & 0.0000 & 0.0 \\
\hline 5.0 & & 9.214 & 33.790 & 26.137 & 0.0095 & 0.0 \\
\hline 10.0 & & 9.218 & 33.790 & 26.136 & 0.0190 & 0.0 \\
\hline 15.0 & & 9.219 & 33.790 & 26.136 & 0.0286 & 0.0 \\
\hline 20.0 & & 8.784 & 33.825 & 26.232 & 0.0379 & 0.0 \\
\hline 25.0 & & 8.752 & 33.832 & 26.243 & 0.0470 & 0.0 \\
\hline 30.0 & & 8.733 & 33.832 & 26.246 & 0.0560 & 0.0 \\
\hline 35.0 & & 8.721 & 33.832 & 26.248 & 0.0650 & 0.0 \\
\hline 40.0 & & 8.711 & 33.832 & 26.249 & 0.0741 & 0.0 \\
\hline 45.0 & & 8.688 & 33.835 & 26.255 & 0.0831 & 0.0 \\
\hline 50.0 & & 8.522 & 33.866 & 26.305 & 0.0920 & 0.0 \\
\hline 55.0 & & 8.247 & 33.915 & 26.385 & 0.1006 & 0.0 \\
\hline 60.0 & & 8.150 & 33.936 & 26.416 & 0.1089 & 0.0 \\
\hline 65.0 & & 8.144 & 33.938 & 26.419 & 0.1171 & 0.0 \\
\hline 70.0 & & 8.143 & 33.938 & 26.419 & 0.1253 & 0.0 \\
\hline 71.0 & & 8.143 & 33.938 & 26.419 & 0.1270 & 0.0 \\
\hline
\end{tabular}

TEMPERATURE C
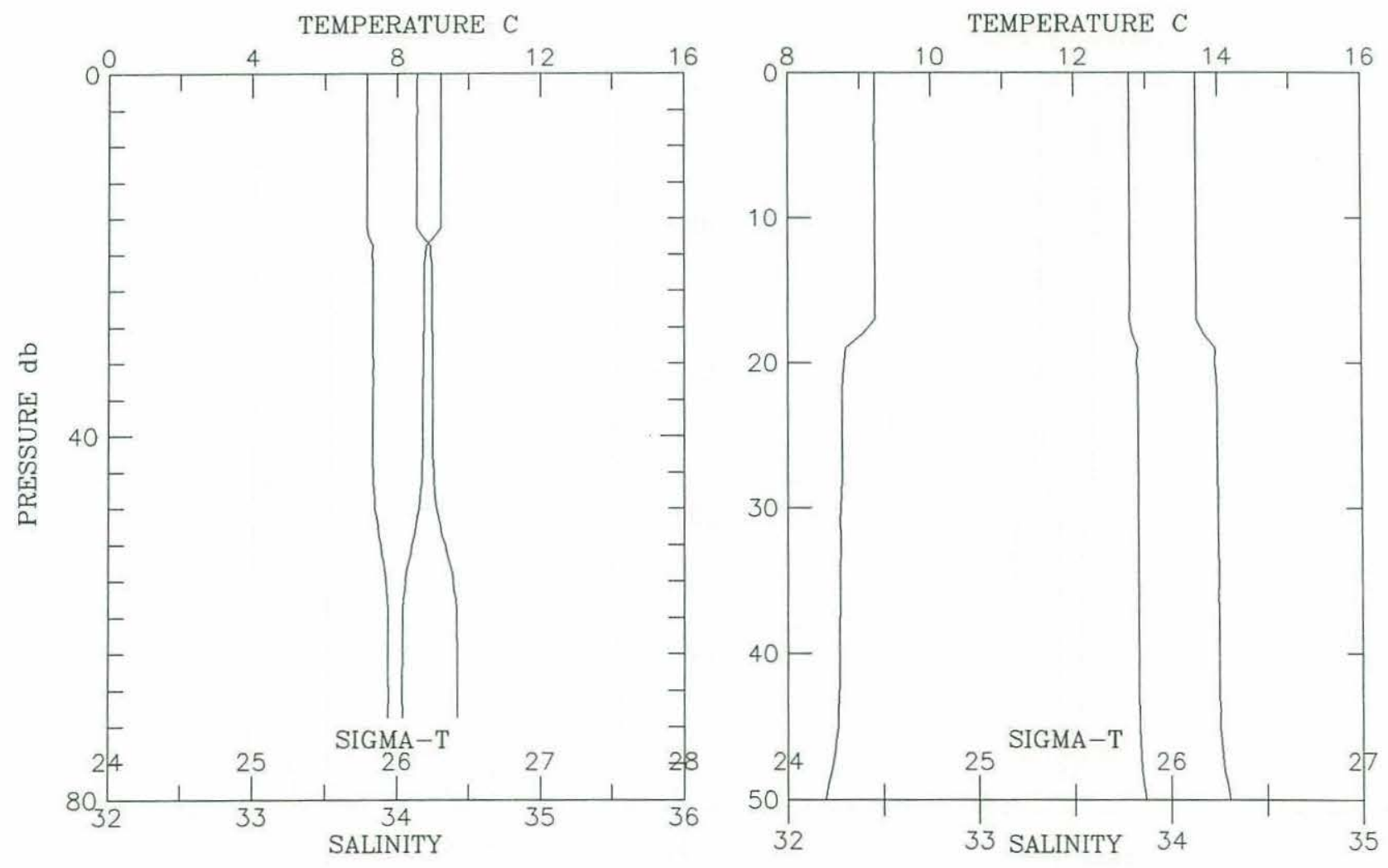
Cruise w8905 Station \# 256 Depth m: 78 Time: 1989-05-14 12:05

Position: $3839.99^{\prime} \mathrm{N} 12327.55^{\prime} \mathrm{W}$ Depth Deck Offset: -0.20

\begin{tabular}{|c|c|c|c|c|c|c|}
\hline $\begin{array}{l}\text { PRESSURE } \\
\text { (d-bars) }\end{array}$ & Interp & $\begin{array}{l}\text { TEMP } \\
(\operatorname{deg} C)\end{array}$ & $\begin{array}{c}\text { SALINITY } \\
(0 / 00)\end{array}$ & SIGMA-T & DELTA-D & $\begin{array}{l}\text { CHANNEL } \\
\text { ( } \delta 1 \text { ight) }\end{array}$ \\
\hline 0.0 & E & 8.123 & 33.967 & 26.445 & 0.0000 & 0.0 \\
\hline 5.0 & & 8.117 & 33.966 & 26.445 & 0.0081 & 0.0 \\
\hline 10.0 & & 8.080 & 33.967 & 26.451 & 0.0161 & 0.0 \\
\hline 15.0 & & 7.907 & 33.985 & 26.491 & 0.0240 & 0.0 \\
\hline 20.0 & & 7.824 & 33.992 & 26.508 & 0.0319 & 0.0 \\
\hline 25.0 & & 7.759 & 33.996 & 26.521 & 0.0396 & 0.0 \\
\hline 30.0 & & 7.548 & 34.010 & 26.563 & 0.0472 & 0.0 \\
\hline 35.0 & & 7.391 & 34.024 & 26.596 & 0.0546 & 0.0 \\
\hline 40.0 & & 7.298 & 34.035 & 26.618 & 0.0619 & 0.0 \\
\hline 45.0 & & 7.201 & 34.046 & 26.640 & 0.0692 & 0.0 \\
\hline 50.0 & & 7.138 & 34.052 & 26.653 & 0.0763 & 0.0 \\
\hline 55.0 & & 7.079 & 34.061 & 26.669 & 0.0834 & 0.0 \\
\hline 60.0 & & 7.064 & 34.064 & 26.673 & 0.0904 & 0.0 \\
\hline 65.0 & & 7.061 & 34.064 & 26.674 & 0.0974 & 0.0 \\
\hline 70.0 & & 7.060 & 34.065 & 26.675 & 0.1045 & 0.0 \\
\hline 75.0 & & 7.060 & 34.065 & 26.674 & 0.1115 & 0.0 \\
\hline
\end{tabular}

TEMPERATURE C
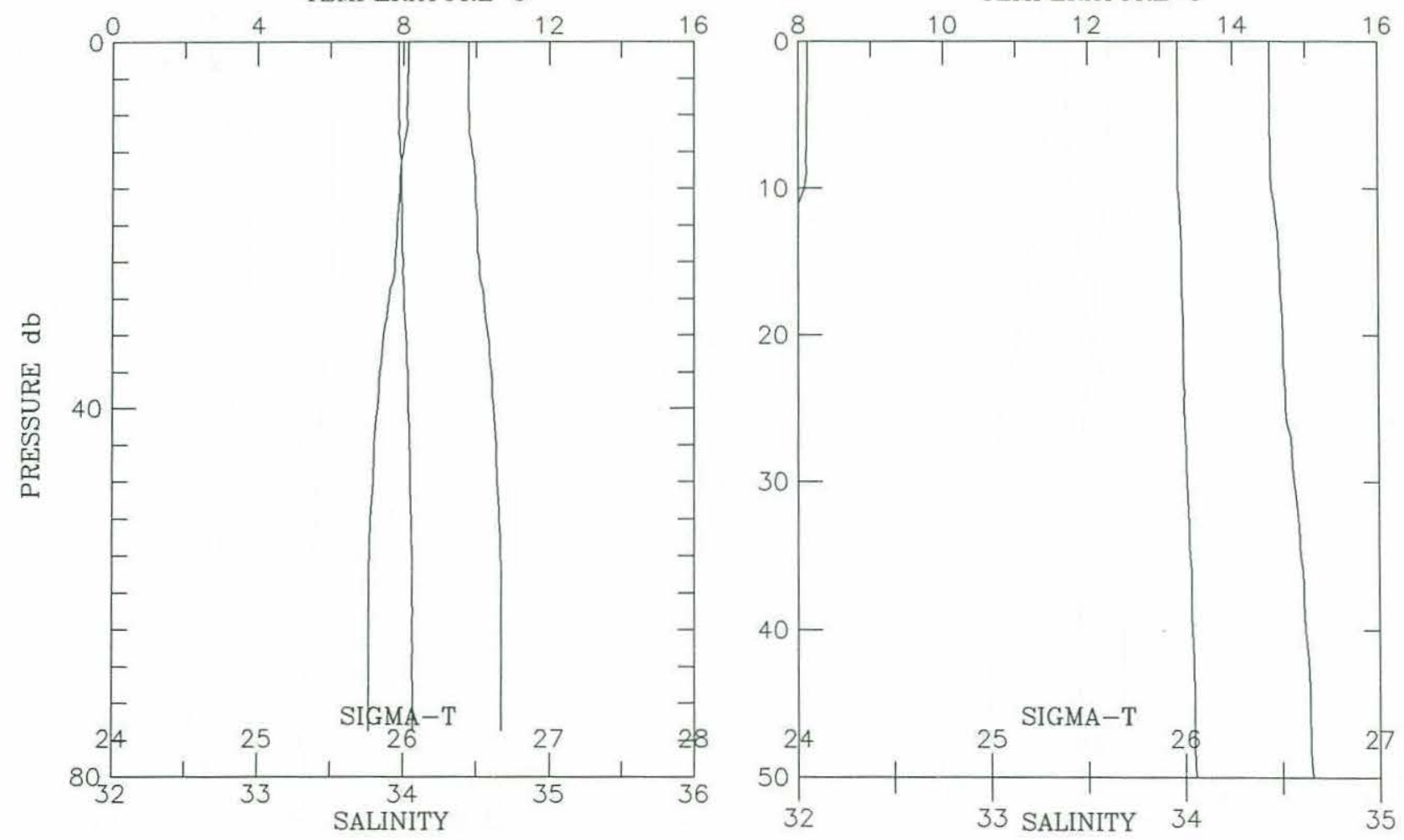
Cruise W8905 Station \# 257 Depth m: 95 Time: 1989-05-14 12:34

Position: $38 \quad 38.55^{\prime} \mathrm{N} 123 \quad 29.38^{\prime} \mathrm{W}$ Depth Deck Offset: $\quad-0.20$

PRESSURE Interp TEMP SALINITY SIGMA-T DELTA-D CHANNELA

(d-bars) ( $\quad(\operatorname{deg} C) \quad(0 / 00) \quad$ (olight)

\begin{tabular}{|c|c|c|c|c|c|c|}
\hline 0.0 & E & 8.640 & 33.880 & 26.298 & 0.0000 & 0.0 \\
\hline 5.0 & & 8.637 & 33.878 & 26.297 & 0.0088 & 0.0 \\
\hline 10.0 & & 8.629 & 33.880 & 26.299 & 0.0175 & 0.0 \\
\hline 15.0 & & 8.630 & 33.880 & 26.299 & 0.0263 & 0.0 \\
\hline 20.0 & & 8.546 & 33.893 & 26.322 & 0.0350 & 0.0 \\
\hline 25.0 & & 8.349 & 33.926 & 26.378 & 0.0435 & 0.0 \\
\hline 30.0 & & 8.210 & 33.946 & 26.415 & 0.0518 & 0.0 \\
\hline 35.0 & & 8.137 & 33.955 & 26.433 & 0.0600 & 0.0 \\
\hline 40.0 & & 7.882 & 33.984 & 26.494 & 0.0680 & 0.0 \\
\hline 45.0 & & 7.589 & 34.014 & 26.560 & 0.0757 & 0.0 \\
\hline 50.0 & & 7.355 & 34.039 & 26.613 & 0.0831 & 0.0 \\
\hline 55.0 & & 7.270 & 34.047 & 26.631 & 0.0904 & 0.0 \\
\hline 60.0 & & 7.099 & 34.058 & 26.664 & 0.0976 & 0.0 \\
\hline 65.0 & & 7.018 & 34.059 & 26.676 & 0.1046 & 0.0 \\
\hline 70.0 & & 6.997 & 34.059 & 26.678 & 0.1116 & 0.0 \\
\hline 75.0 & & 6.993 & 34.059 & 26.679 & 0.1186 & 0.0 \\
\hline 80.0 & & 6.988 & 34.059 & 26.680 & 0.1256 & 0.0 \\
\hline 85.0 & & 6.985 & 34.059 & 26.680 & 0.1326 & 0.0 \\
\hline 90.0 & & 6.986 & 34.060 & 26.681 & 0.1396 & 0.0 \\
\hline 92.0 & & 6.987 & 34.059 & 26.680 & 0.1424 & 0.0 \\
\hline
\end{tabular}

TEMPERATURE C
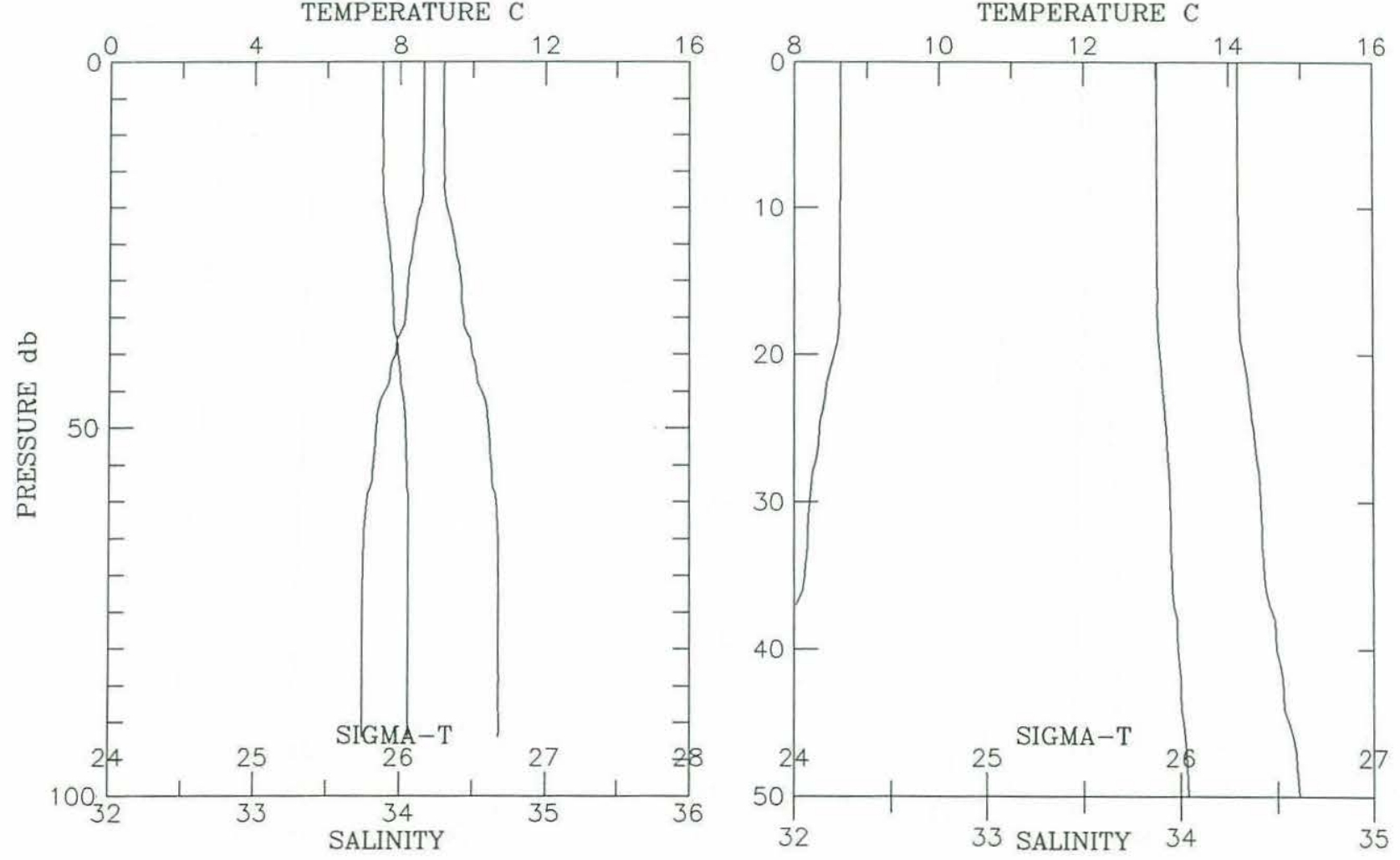
Cruise w8905 Station \# 258 Depth m: 119 Time: 1989-05-14 13:08

Position: $38 \quad 36.64^{\prime} \mathrm{N} 123 \quad 31.80^{\prime} \mathrm{W}$ Depth Deck Offset: $\quad-0.20$

\begin{tabular}{|c|c|c|c|c|c|c|}
\hline $\begin{array}{l}\text { PRESSURE } \\
\text { (d-bars) }\end{array}$ & Interp & $\begin{array}{l}\text { TEMP } \\
(\operatorname{deg} C)\end{array}$ & $\begin{array}{c}\text { SALINITY } \\
(0 / 00)\end{array}$ & SIGMA-T & DELTA-D & $\begin{array}{l}\text { CHANNELA } \\
(\& 1 i g h t)\end{array}$ \\
\hline 0.0 & E & 8.706 & 33.872 & 26.281 & 0.0000 & 0.0 \\
\hline 10.0 & & 8.707 & 33.870 & 26.279 & 0.0177 & 0.0 \\
\hline 20.0 & & 8.704 & 33.870 & 26.280 & 0.0354 & 0.0 \\
\hline 30.0 & & 8.700 & 33.871 & 26.281 & 0.0531 & 0.0 \\
\hline 40.0 & & 7.938 & 33.977 & 26.480 & 0.0700 & 0.0 \\
\hline 50.0 & & 7.755 & 34.012 & 26.534 & 0.0855 & 0.0 \\
\hline 60.0 & & 7.425 & 34.019 & 26.587 & 0.1006 & 0.0 \\
\hline 70.0 & & 7.333 & 34.026 & 26.606 & 0.1154 & 0.0 \\
\hline 80.0 & & 7.205 & 34.034 & 26.630 & 0.1300 & 0.0 \\
\hline 90.0 & & 7.121 & 34.055 & 26.658 & 0.1443 & 0.0 \\
\hline 100.0 & & 7.093 & 34.063 & 26.668 & 0.1585 & 0.0 \\
\hline 110.0 & & 7.096 & 34.069 & 26.673 & 0.1726 & 0.0 \\
\hline 115.0 & & 7.099 & 34.069 & 26.672 & 0.1797 & 0.0 \\
\hline
\end{tabular}
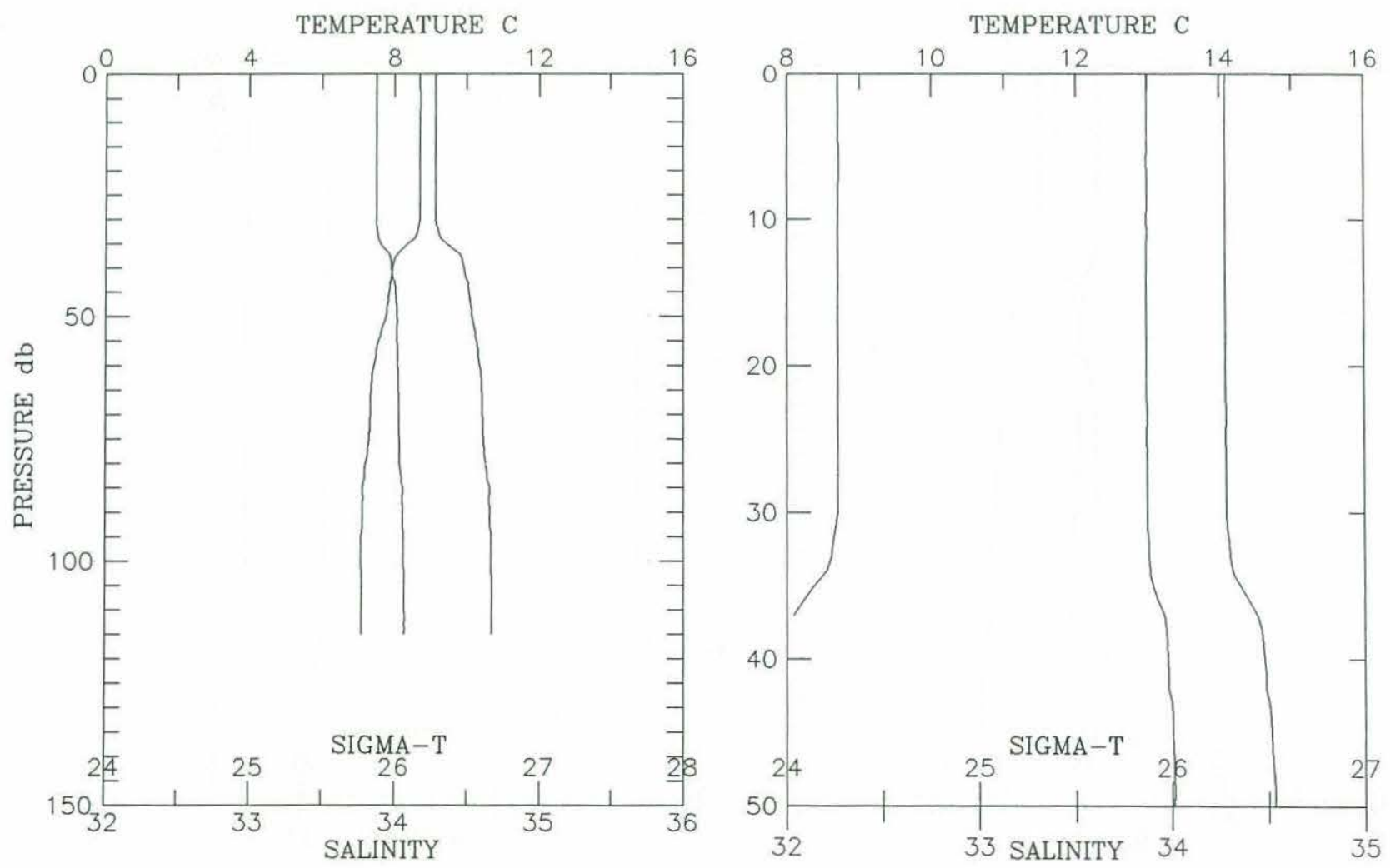


\begin{tabular}{|c|c|c|c|c|c|c|c|}
\hline Cruise W & $\begin{array}{r}3905 \\
38\end{array}$ & Eation \# & 259 Dep & $\mathrm{m}: 140$ & Time: & 1989-05-14 & $\begin{array}{l}13: 50 \\
-0.20\end{array}$ \\
\hline $\begin{array}{l}\text { PRESSURE } \\
\text { (d-bars) }\end{array}$ & Interp & $\begin{array}{c}\text { TEMP } \\
(\operatorname{deg} C)\end{array}$ & $\begin{array}{c}\text { SALINITY } \\
(0 / 00)\end{array}$ & SIGMA-T & DELTA-D & $\begin{array}{l}\text { CHANNELA } \\
\text { ( } \delta 1 \text { ight) }\end{array}$ & \\
\hline 0.0 & $\mathrm{E}$ & 8.704 & 33.877 & 26.285 & 0.0000 & 0.0 & \\
\hline 10.0 & & 8.703 & 33.876 & 26.285 & 0.0176 & 0.0 & \\
\hline 20.0 & & 8.701 & 33.876 & 26.285 & 0.0353 & 0.0 & \\
\hline 30.0 & & 8.694 & 33.877 & 26.287 & 0.0530 & 0.0 & \\
\hline 40.0 & & 8.516 & 33.903 & 26.335 & 0.0705 & 0.0 & \\
\hline 50.0 & & 8.084 & 33.990 & 26.469 & 0.0869 & 0.0 & \\
\hline 60.0 & & 7.812 & 33.991 & 26.509 & 0.1027 & 0.0 & \\
\hline 70.0 & & 7.587 & 34.008 & 26.555 & 0.1181 & 0.0 & \\
\hline 80.0 & & 7.520 & 34.025 & 26.578 & 0.1332 & 0.0 & \\
\hline 90.0 & & 7.537 & 34.034 & 26.583 & 0.1482 & 0.0 & \\
\hline 100.0 & & 7.482 & 34.066 & 26.616 & 0.1630 & 0.0 & \\
\hline 110.0 & & 7.402 & 34.073 & 26.633 & 0.1776 & 0.0 & \\
\hline 120.0 & & 7.306 & 34.086 & 26.657 & 0.1920 & 0.0 & \\
\hline 130.0 & & 7.150 & 34.088 & 26.680 & 0.2062 & 0.0 & \\
\hline 136.0 & & 7.149 & 34.087 & 26.679 & 0.2147 & 0.0 & \\
\hline
\end{tabular}
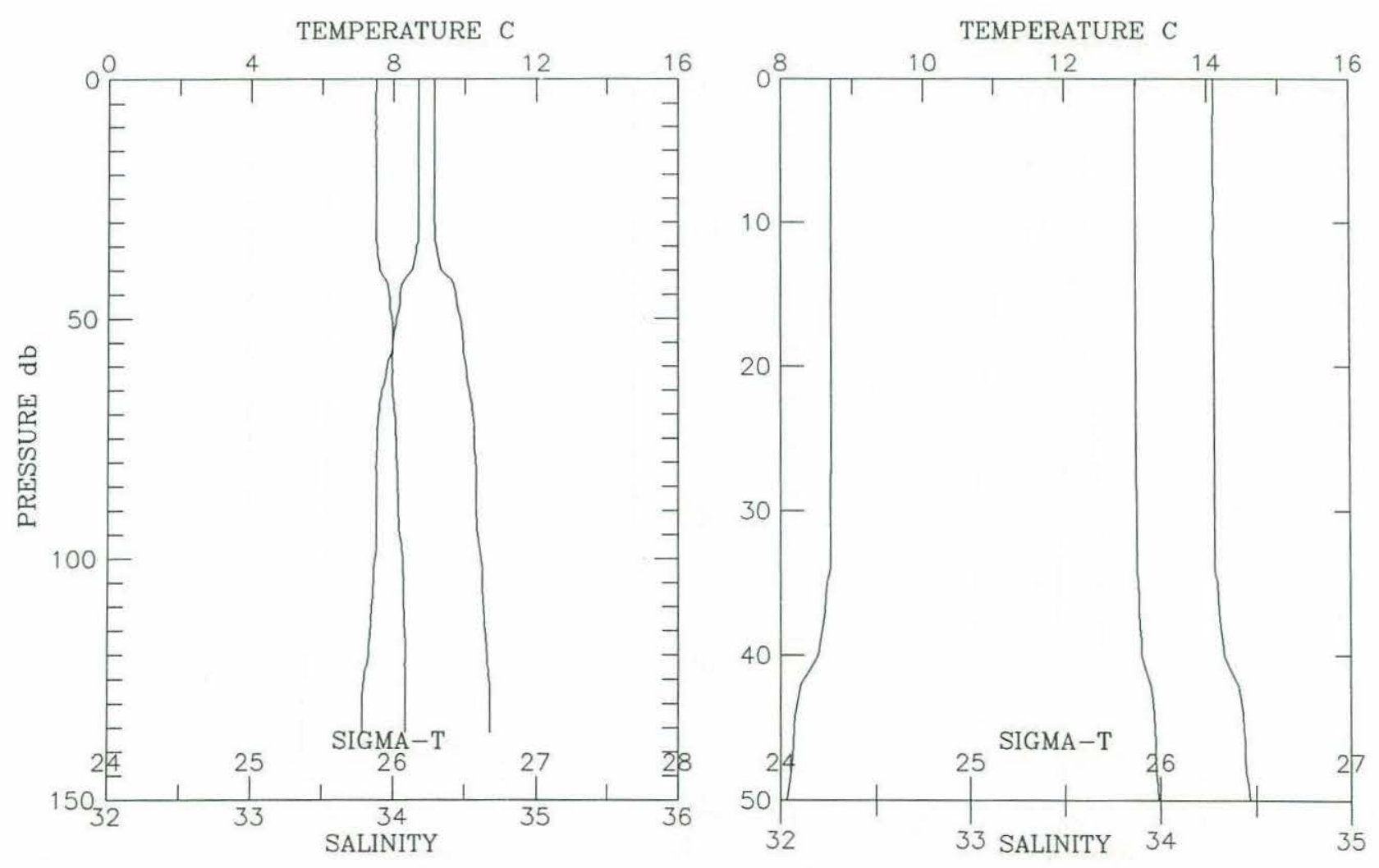


\begin{tabular}{|c|c|c|c|c|c|c|c|}
\hline \multicolumn{2}{|c|}{ Cruise W8905 } & Station \# 26 & $\begin{array}{r}260 \text { Dep } \\
23 \quad 36.72\end{array}$ & $\mathrm{~m}: 162$ & Time: & $\begin{array}{l}1989-05-14 \\
\text { Offset: }\end{array}$ & $\begin{array}{l}14: 34 \\
-0.20\end{array}$ \\
\hline $\begin{array}{l}\text { PRESSURE } \\
\text { (d-bars) }\end{array}$ & Interp & $\begin{array}{c}\text { TEMP } \\
(\operatorname{deg} C)\end{array}$ & $\begin{array}{c}\text { SALINITY } \\
(0 / 00)\end{array}$ & SIGMA-T & DELTA-D & $\begin{array}{l}\text { CHANNELA } \\
\text { ( } \delta 1 \text { ight) }\end{array}$ & \\
\hline 0.0 & $\mathrm{E}$ & 9.048 & 33.805 & 26.175 & 0.0000 & 0.0 & \\
\hline 10.0 & & 9.043 & 33.802 & 26.173 & 0.0187 & 0.0 & \\
\hline 20.0 & & 9.047 & 33.799 & 26.170 & 0.0374 & 0.0 & \\
\hline 30.0 & & 9.045 & 33.800 & 26.171 & 0.0562 & 0.0 & \\
\hline 40.0 & & 9.035 & 33.804 & 26.176 & 0.0750 & 0.0 & \\
\hline 50.0 & & 9.020 & 33.810 & 26.183 & 0.0937 & 0.0 & \\
\hline 60.0 & & 8.798 & 33.830 & 26.234 & 0.1121 & 0.0 & \\
\hline 70.0 & & 8.639 & 33.858 & 26.281 & 0.1302 & 0.0 & \\
\hline 80.0 & & 8.410 & 33.904 & 26.352 & 0.1477 & 0.0 & \\
\hline 90.0 & & 7.971 & 33.955 & 26.458 & 0.1644 & 0.0 & \\
\hline 100.0 & & 7.796 & 34.019 & 26.534 & 0.1801 & 0.0 & \\
\hline 110.0 & & 7.657 & 34.056 & 26.583 & 0.1953 & 0.0 & \\
\hline 120.0 & & 7.526 & 34.055 & 26.601 & 0.2102 & 0.0 & \\
\hline 130.0 & & 7.532 & 34.057 & 26.602 & 0.2251 & 0.0 & \\
\hline 140.0 & & 7.386 & 34.068 & 26.631 & 0.2398 & 0.0 & \\
\hline 150.0 & & 7.296 & 34.071 & 26.646 & 0.2543 & 0.0 & \\
\hline 158.0 & & 7.285 & 34.072 & 26.649 & 0.2659 & 0.0 & \\
\hline
\end{tabular}

TEMPERATURE C
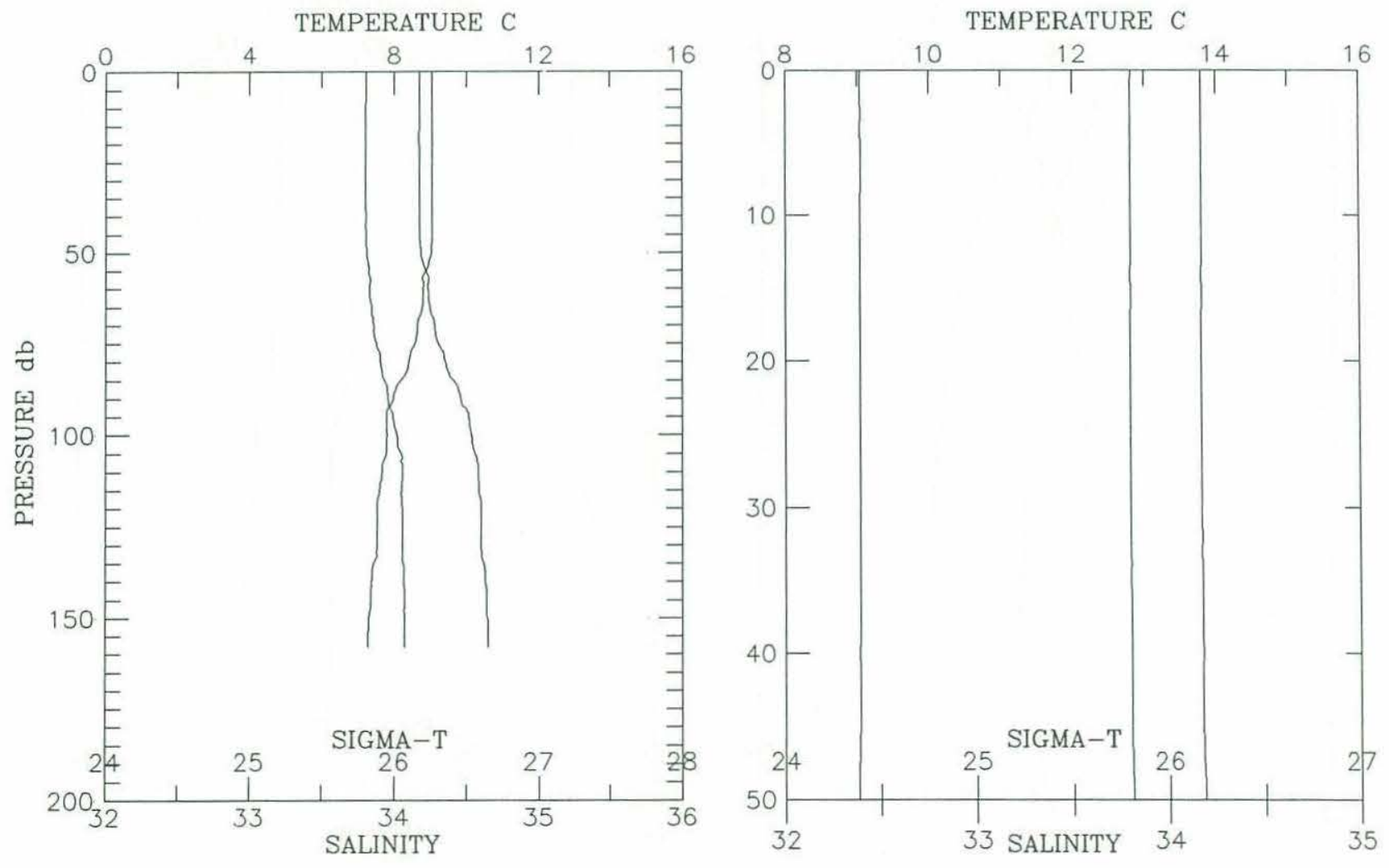


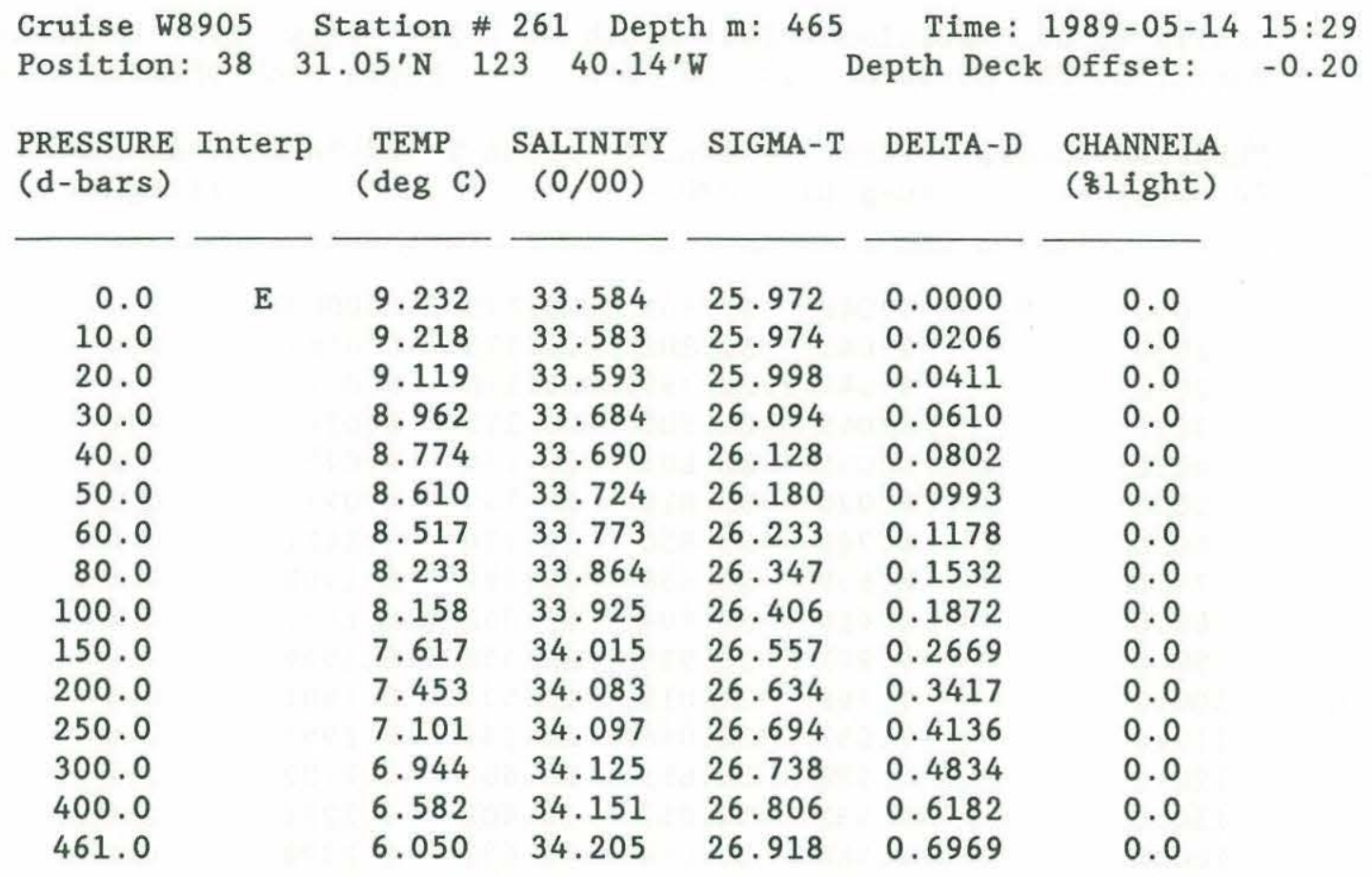
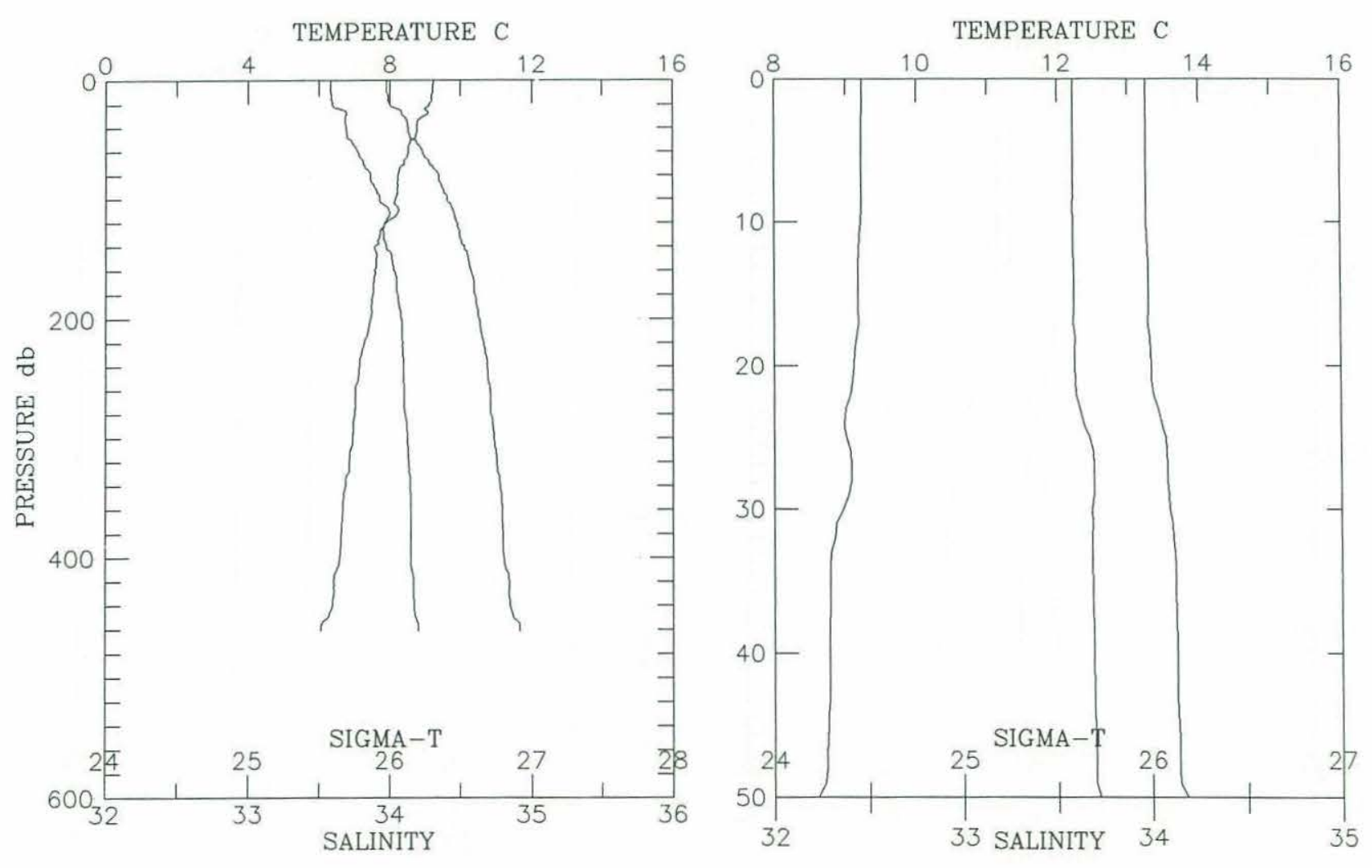


\begin{tabular}{|c|c|c|c|c|c|c|}
\hline $\begin{array}{l}\text { PRESSURE } \\
\text { (d-bars) }\end{array}$ & Interp & $\begin{array}{l}\text { TEMP } \\
(\operatorname{deg} C)\end{array}$ & $\begin{array}{c}\text { SALINITY } \\
(0 / 00)\end{array}$ & SIGMA-T & DELTA-D & $\begin{array}{l}\text { CHANNELA } \\
\text { ( }(\text { light) }\end{array}$ \\
\hline 0.0 & E & 11.570 & 33.103 & 25.196 & 0.0000 & 0.0 \\
\hline 10.0 & & 11.202 & 33.161 & 25.308 & 0.0279 & 0.0 \\
\hline 20.0 & & 9.840 & 33.387 & 25.720 & 0.0524 & 0.0 \\
\hline 30.0 & & 9.162 & 33.557 & 25.963 & 0.0742 & 0.0 \\
\hline 40.0 & & 9.143 & 33.650 & 26.038 & 0.0945 & 0.0 \\
\hline 50.0 & & 9.229 & 33.679 & 26.047 & 0.1145 & 0.0 \\
\hline 60.0 & & 9.273 & 33.711 & 26.065 & 0.1344 & 0.0 \\
\hline 80.0 & & 8.651 & 33.768 & 26.208 & 0.1730 & 0.0 \\
\hline 100.0 & & 8.272 & 33.834 & 26.318 & 0.2091 & 0.0 \\
\hline 150.0 & & 8.142 & 34.015 & 26.479 & 0.2925 & 0.0 \\
\hline 200.0 & & 7.428 & 34.001 & 26.573 & 0.3703 & 0.0 \\
\hline 250.0 & & 7.221 & 34.098 & 26.678 & 0.4444 & 0.0 \\
\hline 300.0 & & 6.894 & 34.114 & 26.735 & 0.5147 & 0.0 \\
\hline 400.0 & & 6.207 & 34.177 & 26.876 & 0.6466 & 0.0 \\
\hline 500.0 & & 5.828 & 34.228 & 26.964 & 0.7689 & 0.0 \\
\hline 600.0 & & 5.378 & 34.274 & 27.056 & 0.8840 & 0.0 \\
\hline 700.0 & & 4.956 & 34.331 & 27.150 & 0.9906 & 0.0 \\
\hline 800.0 & & 4.566 & 34.378 & 27.231 & 1.0900 & 0.0 \\
\hline 826.0 & & 4.497 & 34.388 & 27.247 & 1.1146 & 0.0 \\
\hline
\end{tabular}
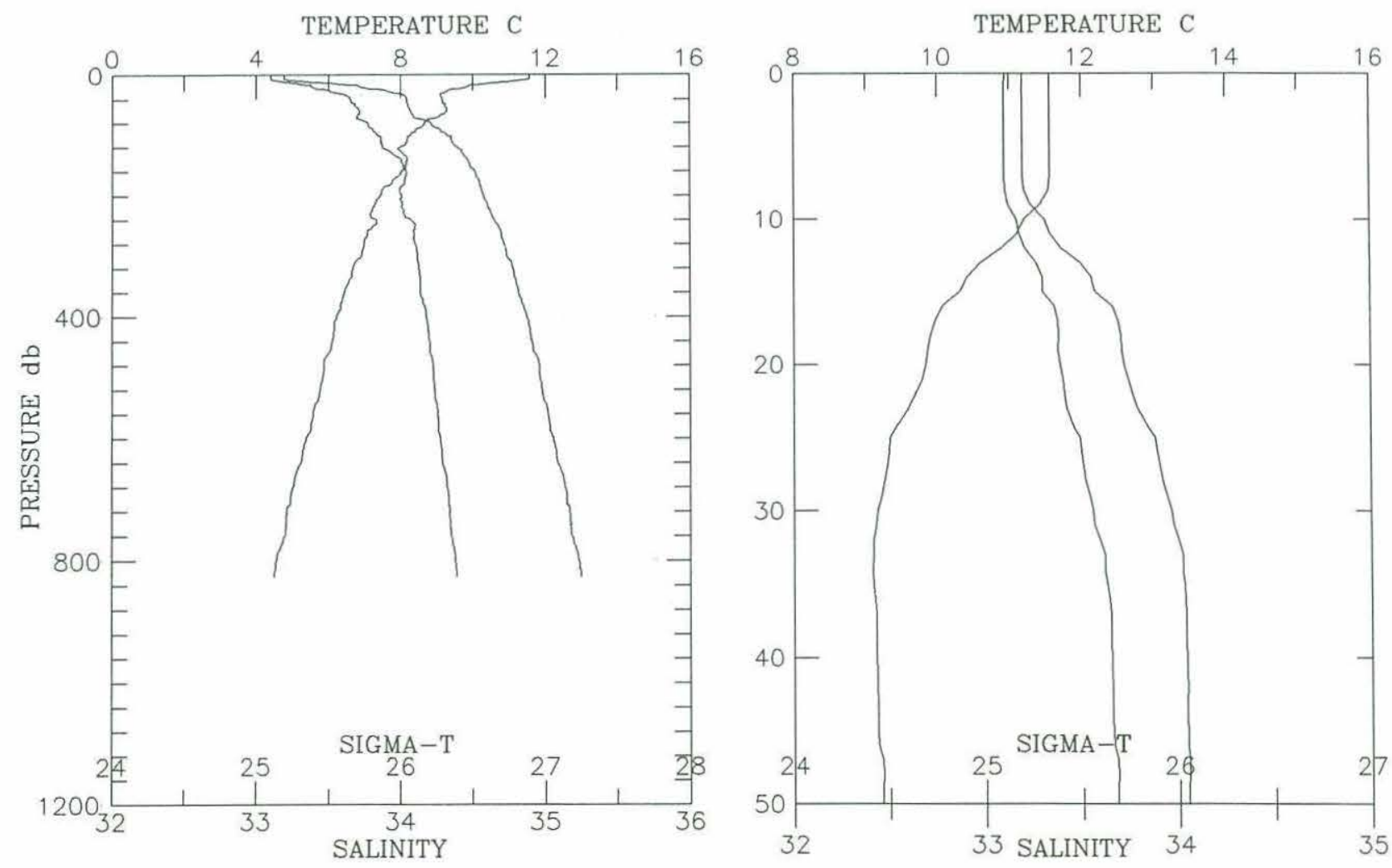


\section{DOCUMENT LIBRARY}

July 5,1989

Distribution List for Technical Report Exchange

Attn: Stella Sanchez-Wade

Documents Section

Scripps Institution of Oceanography

Library, Mail Code C-075C

La Jolla, CA 92093

Hancock Library of Biology \& Oceanography

Alan Hancock Laboratory

University of Southern California

University Park

Los Angeles, CA 90089-0371

Gifts \& Exchanges

Library

Bedford Institute of Oceanography

P.O. Box 1006

Dartmouth, NS, B2Y 4A2, CANADA

Office of the International Ice Patrol

c/o Coast Guard R \& D Center

Avery Point

Groton, CT 06340

Library

Physical Oceanographic Laboratory

Nova University

8000 N. Ocean Drive

Dania, FL 33304

NOAA/EDIS Miami Library Center

4301 Rickenbacker Causeway

Miami, FL 33149

Library

Skidaway Institute of Oceanography

P.O. Box 13687

Savannah, GA 31416

Institute of Geophysics

University of Hawaii

Library Room 252

2525 Correa Road

Honolulu, HI 96822

Library

Chesapeake Bay Institute

4800 Atwell Road

Shady Side, MD 20876

MIT Libraries

Serial Journal Room 14E-210

Cambridge, MA 02139
Director, Ralph M. Parsons Laboratory

Room 48-311

MIT

Cambridge, MA 02139

Marine Resources Information Center

Building E38-320

MIT

Cambridge, MA 02139

Library

Lamont-Doherty Geological Observatory

Colombia University

Palisades, NY 10964

Library

Serials Department

Oregon State University

Corvallis, OR 97331

Pell Marine Science Library

University of Rhode Island

Narragansett Bay Campus

Narragansett, RI 02882

Working Collection

Texas A\&M University

Dept. of Oceanography

College Station, TX 77843

Library

Virginia Institute of Marine Science

Gloucester Point, VA 23062

Fisheries-Oceanography Library

151 Oceanography Teaching Bldg.

University of Washington

Seattle, WA 98195

Library

R.S.M.A.S.

University of Miami

4600 Rickenbacker Causeway

Miami, FL 33149

Maury Oceanographic Library

Naval Oceanographic Office

Bay St. Louis

NSTL, MS 39522-5001

Marine Sciences Collection

Mayaguez Campus Library

University of Puerto Rico

Mayagues, Puerto Rico 00708 


\begin{tabular}{|c|c|c|c|}
\hline $\begin{array}{l}\text { REPORT DOCUMENTATION } \\
\text { PAGE }\end{array}$ & $\begin{array}{l}\text { 1. REPORT NO. } \\
\text { WHOI-89-42 }\end{array}$ & 2. & 3. Recipient's Accession No. \\
\hline \multirow{2}{*}{\multicolumn{3}{|c|}{$\begin{array}{l}\text { 4. Title and Subtitle } \\
\text { CTD Observations Off Northern California During the Shelf Mixed Layer Experiment, } \\
\text { SMILE, May } 1989\end{array}$}} & $\begin{array}{l}\text { 5. Report Date } \\
\text { September, } 1989\end{array}$ \\
\hline & & & 6. \\
\hline \multicolumn{3}{|c|}{$\begin{array}{l}\text { 7. Author(s) } \\
\text { Richard Limeburner and Robert C. Beardsley }\end{array}$} & $\begin{array}{l}\text { 8. Performing Organization Rept. No. } \\
\text { WHOI-89-42 }\end{array}$ \\
\hline \multirow{2}{*}{\multicolumn{3}{|c|}{$\begin{array}{l}\text { 9. Performing Organization Name and Address } \\
\text { The Woods Hole Oceanographic Institution } \\
\text { Woods Hole, Massachusetts } 02543\end{array}$}} & 10. Project/Task/Work Unit No. \\
\hline & & & $\begin{array}{l}\text { 11. Contract(C) or Grant(G) No. } \\
\text { (C) } \\
\text { (G) OCE } 87-16937\end{array}$ \\
\hline \multicolumn{3}{|c|}{ 12. Sponsoring Organization Name and Address } & $\begin{array}{l}\text { 13. Type of Report \& Period Covered } \\
\text { Technical Report }\end{array}$ \\
\hline \multicolumn{3}{|c|}{ The National Science Foundation } & 14. \\
\hline
\end{tabular}

15. Supplementary Notes

This report should be cited as: Woods Hole Oceanog. Inst. Tech. Rept., WHOI-89-42,

16. Abstract (Limit: 200 words)

CTD observations were made off the northern California coast during R/V Wecoma cruise W8905 May 5-14, 1989 as part of the Shelf MIxed Layer Experiment (SMILE). The surveys consisted of two sampling plans - a large-scale grid of four cross-shelf transects extending to both sides of Point Arena and Point Reyes, and a small scale grid of six cross-shelf transects located near the central SMILE mooring site. All of the cross-shelf transects extended beyond the shelf break and the maximum sampling depth at each station was near-bottom or $1500 \mathrm{~m}$. The average along-shelf separation between cross-shelf transects was about $15 \mathrm{~km}$ for the small-scale surveys and $50 \mathrm{~km}$ for the large-scale grid.

The primary objectives of the hydrographic measurement program were to observe and characterize the temperature, salinity, density, and light transmission fields and their temporal and spatial variability in the surface boundary layer along the continental shelf and slope near the SMILE moored array, and to acquire estimates of the cross- and along-shelf scales over which the mixed-layer depth varies. This report presents a summary in graphic and tabular form of the hydrographic observations made during cruise W8905 on the R/V Wecoma.

\section{Document Analysis a. Descriptors}

1. hydrography/CTD

2. N. California coastal region

3. SMILE (Shelf Mixed Layer Experiment)

b. Identifiers/Open-Ended Terms

c. COSATI Field/Group

18. Availability Statement

Approved for publication; distribution unlimited.

\begin{tabular}{|c|} 
19. Security Class (This Report) \\
UNCLASSIFIED
\end{tabular}

